\title{
Measurement of the Total Hadronic Cross-Section Below the Upsilon(4S) Resonance at BaBar Using Initial-State Radiation
}

\author{
Nocolas J. P. Berger
}

SLAC-R-874

Prepared for the Department of Energy

under contract number DE-AC02-76SF00515

Printed in the United States of America. Available from the National Technical Information Service, U.S. Department of Commerce, 5285 Port Royal Road, Springfield, VA 22161. 
This document, and the material and data contained therein, was developed under sponsorship of the United States Government. Neither the United States nor the Department of Energy, nor the Leland Stanford Junior University, nor their employees, nor their respective contractors, subcontractors, or their employees, makes an warranty, express or implied, or assumes any liability of responsibility for accuracy, completeness or usefulness of any information, apparatus, product or process disclosed, or represents that its use will not infringe privately owned rights. Mention of any product, its manufacturer, or suppliers shall not, nor is it intended to, imply approval, disapproval, or fitness of any particular use. A royalty-free, nonexclusive right to use and disseminate same of whatsoever, is expressly reserved to the United States and the University. 
MEASUREMENT OF THE TOTAL HADRONIC CROSS-SECTION BELOW THE $\Upsilon(4 S)$ RESONANCE AT BABAR USING INITIAL-STATE RADIATION

\author{
A DISSERTATION \\ SUBMITTED TO THE DEPARTMENT OF PHYSICS \\ AND THE COMMITTEE ON GRADUATE STUDIES \\ OF STANFORD UNIVERSITY \\ IN PARTIAL FULFILLMENT OF THE REQUIREMENTS \\ FOR THE DEGREE OF \\ DOCTOR OF PHILOSOPHY
}

Nicolas J. P. Berger

June 2006 
(C) Copyright by Nicolas J. P. Berger 2006

All Rights Reserved 


\section{Preface}

We present an inclusive measurement of $\Delta \alpha_{\text {had }}^{(5)}\left(m_{Z}^{2}\right)$ at BABAR using the Initial State Radiation (ISR) technique in $e^{+} e^{-}$interactions to simultaneously explore the whole low energy range at reduced center-of-mass energies below $7 \mathrm{GeV}$, where the current knowledge of $e^{+} e^{-} \rightarrow$ hadrons production limits the precision of the prediction of the running of $\alpha$. The BABAR ISR data sample is considerably larger than existing $e^{+} e^{-} \mathrm{R}$ scan measurement data over most of the low energy range, and there are also many systematic advantages with the ISR technique to allow significantly improved precision on the integral for $\Delta \alpha_{h a d}^{(5)}\left(m_{Z}^{2}\right)$. This thesis reports on a measurement of $\Delta \alpha_{h a d}^{(5)}\left(m_{Z}^{2}\right)$ at the $3 \%$ precision level, improving on the current knowledge of this quantity. 


\section{Acknowledgments}

First and foremost, my thanks go to my adviser, Su Dong, for giving me the wonderful opportunity to work in this project; for providing the original impetus for this work as well as many of the insights that led to its realization; for his constant availability in providing assistance at all hours of day and night; and most importantly for his unwavering and communicative enthusiasm for all aspects of experimental physics. If after these few years at SLAC I know what it means to be an experimental physicist, it is to him that I owe it.

I extend my sincere appreciation to the members of the review committee, Patricia Burchat, David Leith and Michael Peskin, for their careful reading of this far-fromconcise document and their many constructive comments, as well as for the help they have given me throughout my graduate education. Many thanks to Trevor Hastie for agreeing to chair this committee.

I am also indebted to the many professors that have contributed to my education. I would particularly like to thank Bill Dunwoodie for sharing his neverending knowledge of physics and its lore, as well as Henri Videau and Gérard Bonneaud of Ecole Polytechnique who provided the opportunity to discover SLAC and BABAR. I am also grateful to the Fondation de l'Ecole Polytechnique for its help in financing my graduate education.

I would also like to thank the members of Group $\mathrm{E}$ for providing a wonderful working environment: Martin Perl and Rafe Schindler, for their guidance and support during these years, Bill Wisniewski, Martin Kocian, Valerie Halyo and Eric Lee for their expertise and their friendship, and particularly Melodi Masaiani and Judy Meo, who made Group E a home away from home for the expatriates among us. 
Many thanks to Rainer Bartoldus, Anders Borgland, Jim Panetta, Xuedong Chai and other members of the trigger group for their patient teaching of the working of trigger software and hardware, and in the latter case for their understanding when it literally all went up in smoke.

Thanks to Hojeong Kim, Erhan Yenilmez, Anatoly Svidzinski, Alexandre Ivrii, Josh and Evonne Thomson, Mark Allen, Boaz Nash and other graduate students and young doctors for their companionship during these years.

Special thanks also go to Peter Kim, for his kindess, his support, his deep knowledge of physics and also for never forgetting what it is like to be a graduate student. It is impossible to express the debt I owe to Joerg Stelzer and Erkcan Özcan. If nothing else, meeting them made those years at Stanford worthwhile. But most of all I would like to thank my family, and especially my dear wife Tanya for her patience and support during all these years. 


\section{Contents}

Preface $\quad$ v

Acknowledgments $\quad$ vi

1 Introduction 1

2 Theory 5

2.1 The Standard Model . . . . . . . . . . . . . . . . . . 5 5

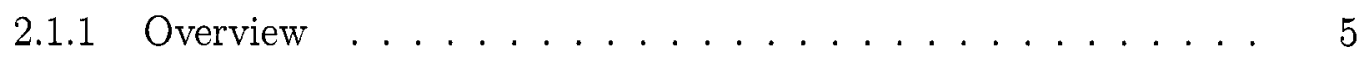

2.1.2 Electroweak symmetry breaking, Global fits . . . . . . . 8

2.2 Determining $\Delta \alpha_{\text {had }}^{(5)}\left(m_{Z}^{2}\right) \ldots \ldots \ldots \ldots$

2.2.1 The Running of $\alpha_{Q E D} \ldots \ldots \ldots 13$

2.2.2 Hadronic production in $e^{+} e^{-}$annihilation . . . . . . . 16

2.2.3 Calculation of $\Pi_{\text {Had }}(s) \ldots \ldots \ldots 18$

2.2.4 ITEP Sum Rules and Global Duality . . . . . . . . . . . 23

2.2.5 Correction to $R_{\text {had }}(s)$ due to the running of $\alpha_{s} \ldots \ldots 24$

2.3 Measuring $\Delta \alpha_{\text {had }}^{(5)}\left(m_{Z}^{2}\right)$ using the Radiative Return . . . . . . . . . . . 29

2.3.1 Initial-State Radiation . . . . . . . . . . . . . . . . . 29

2.3.2 $\Delta \alpha_{\text {had }}^{(5)}\left(m_{Z}^{2}\right)$ from ISR Processes . . . . . . . . . . . . . 32

2.3.3 Radiative Corrections . . . . . . . . . . . . . . 33

2.3.4 Forward-Backward Asymmetry in Two-prong Events . . . . . 36

2.3.5 Experimental Status of $\Delta \alpha_{h a d}^{(5)}\left(m_{Z}^{2}\right) \ldots \ldots \ldots$ 
3 The BABAR Detector $\quad 39$

3.1 Overview . . . . . . . . . . . . . . . . . . . . . . . 39

3.2 PEP-II and the Interaction Region $\ldots \ldots \ldots \ldots$

3.2.1 Choice of Beam Energies . . . . . . . . . . . . . . . 40

3.2 .2 Luminosity Considerations . . . . . . . . . . . . . . . 44

3.2.3 BABAR Coordinate System . . . . . . . . . . . . . . . 48

3.3 Vertex Detector and Interaction Region . . . . . . . . . . . . 49

3.3 .1 Interaction region . . . . . . . . . . . . . . . . 49

3.3.2 SVT Layout and Electronics . . . . . . . . . . . . . . 50

3.3.3 Performance . . . . . . . . . . . . . . . . . . . . 51

3.4 Drift Chamber $(\mathrm{DCH}) \ldots \ldots \ldots \ldots \ldots \ldots \ldots$

3.4.1 Design and Geometry . . . . . . . . . . . . . . . . 53

3.4 .2 Single-Cell Performance . . . . . . . . . . . . . . 55

3.4.3 Tracking Performance . . . . . . . . . . . . . . 56

3.5 Electromagnetic Calorimeter (EMC) $\ldots \ldots \ldots \ldots \ldots$

3.5 .1 Design . . . . . . . . . . . . . . . . 58

3.5 .2 Readout . . . . . . . . . . . . . . . . . . . . 59

3.5.3 Reconstruction and Performance . . . . . . . . . . 60

3.6 Muon Chambers (IFR) . . . . . . . . . . . . . . . . 61

3.6.1 The Resistive Plate Chambers . . . . . . . . . . . . . . . 62

3.6.2 Operational Experience . . . . . . . . . . . . . . . 63

3.7 Particle Identification System (DIRC) . . . . . . . . . . . . . 63

3.7.1 Design and Geometry . . . . . . . . . . . . . . . . . . . 64

3.7.2 Electronics and Reconstruction . . . . . . . . . . . 66

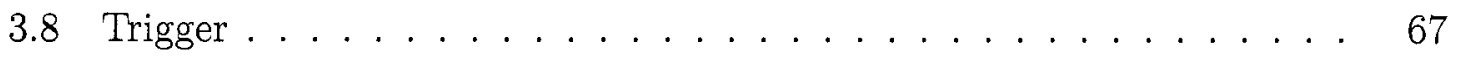

3.8.1 Data Acquisition and Trigger requirements . . . . . . . . 67

3.8 .2 Trigger Design . . . . . . . . . . . . . . . 68

3.8.3 Drift Chamber Trigger . . . . . . . . . . . . . 71

3.8 .4 Software Trigger . . . . . . . . . . . . . . . . 83

3.8 .5 Trigger Performance . . . . . . . . . . . . . . 88

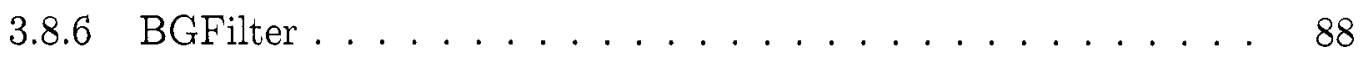


4 Analysis Overview $\quad 94$

4.1 ISR process $\ldots \ldots \ldots \ldots \ldots \ldots \ldots \ldots \ldots$

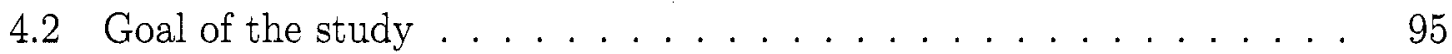

4.3 Photon Energy Resolution . . . . . . . . . . . . . . . . . 96

4.4 Backgrounds . . . . . . . . . . . . . . . . . . . . . . . . . 99

4.5 Analysis Strategy . . . . . . . . . . . . . . . . . . . . . . 101

4.6 Event Generators . . . . . . . . . . . . . . . . . . . . . . . . . 102

4.6 .1 Signal . . . . . . . . . . . . . . . . . . . . . . 102

4.6 .2 QED modes . . . . . . . . . . . . . . . . 102

4.6.3 $B \bar{B}$ and continuum production . . . . . . . . . 103

4.6.4 Detector Simulation . . . . . . . . . . . . . . . 103

4.7 Data and Simulation Samples . . . . . . . . . . . . . . . 103

4.8 ISR Pre-selection . . . . . . . . . . . . . . . . . . . . . . . . 104

4.9 Data Analysis . . . . . . . . . . . . . . . . . . . . . . . 105

4.10 Trigger/Filter Issues . . . . . . . . . . . . . . . 106

4.11 Single and Multiple Radiation . . . . . . . . . . . . . . . . 109

5 Hadronic Event Selection 111

5.1 Preselection cuts . . . . . . . . . . . . . . . 111

5.2 Photon Selection . . . . . . . . . . . . . . . . 113

5.3 Track requirements . . . . . . . . . . . . . . . . 116

5.4 Selection of Electron and Non-Electron tracks . . . . . . . . . 120

5.5 Virtual Compton Veto . . . . . . . . . . . . . . . . . . . 121

$5.6 \gamma \gamma$ Veto . . . . . . . . . . . . . . . . . . . . . . 121

$5.7 \gamma \gamma$ Conversion Veto . . . . . . . . . . . . . . . . . . . . 121

5.8 Radiative Bhabha Veto . . . . . . . . . . . . . . . . . . 125

5.9 All-Neutral QED Veto . . . . . . . . . . . . . . . . . . 125

5.101 -Track QED Veto . . . . . . . . . . . . . . . 126

5.11 Summary of QED background rejection . . . . . . . . . . 128

6 Rejection of Hadronic Backgrounds 137

6.1 Nature of backgrounds . . . . . . . . . . . . . . . . . 137 


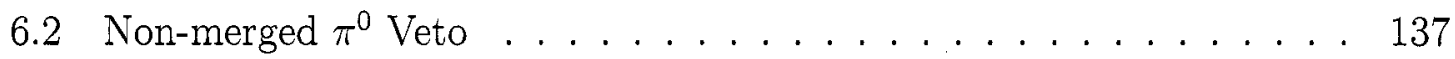

6.3 Other resonances . . . . . . . . . . . . . . . . . . 140

6.4 Merged $\pi^{0}$ and Neutral Hadron Rejection . . . . . . . . . . 140

6.5 Event-Shape Selection . . . . . . . . . . . . . . . . . . . 142

6.5.1 Thrust and Moments . . . . . . . . . . . . . . . . . 142

6.5 .2 Event-Shape Variables . . . . . . . . . . . . . . . . 144

6.5.3 Fisher Discriminant . . . . . . . . . . . . . . . . . . 145

6.5 .4 Variable Selection . . . . . . . . . . . . . . . . . 146

6.5.5 Fisher coefficients and cut values . . . . . . . . . . 151

6.6 Rejection Summary . . . . . . . . . . . . . . . . . . . . . 158

7 Photon Calibration $\quad 164$

7.1 Event Selection . . . . . . . . . . . . . . . . . . 165

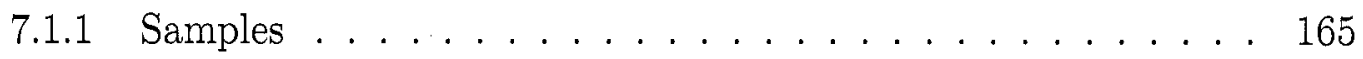

7.1 .2 General Selection . . . . . . . . . . . . . . 166

7.1.3 Two-prong cuts . . . . . . . . . . . . . . . . 168

7.1.4 Photon cuts . . . . . . . . . . . . . . . . . . 168

7.1 .5 Backgrounds . . . . . . . . . . . . . . . . . 170

7.1.6 Data-MC Comparison . . . . . . . . . . . . . . . 172

7.2 Correction Procedure . . . . . . . . . . . . . . . . 175

7.2.1 Fitted Missing Momentum . . . . . . . . . . . . . . . 175

7.2 .2 "Sculpting" distributions . . . . . . . . . . . . . . . 176

7.2 .3 Implementation . . . . . . . . . . . . . . . 177

7.2 .4 Smoothing . . . . . . . . . . . . . . . . . . . . . 179

7.3 Results . . . . . . . . . . . . . . . . . . . . 179

7.3.1 Energy Correction . . . . . . . . . . . . . . . . 179

$7.3 .2 \quad \theta$ Correction . . . . . . . . . . . . . . . . 182

8 Photon Efficiency 185

8.1 Determination of the Efficiency in MC Truth . . . . . . . . . 186

8.1 .1 Naive method . . . . . . . . . . . . . . . 186

8.1 .2 Improved method . . . . . . . . . . . . . . . . 187 
8.1.3 Truth Resolution Functions . . . . . . . . . . . . . . . . . 189

8.1 .4 True Photon Efficiency . . . . . . . . . . . . . . . . . . . 192

8.2 Correction of $\epsilon_{0}$ using an Unbiased Photon Sample . . . . . . . . 195

8.2 .1 Preselection . . . . . . . . . . . . . . . 195

8.2 .2 Event Selection . . . . . . . . . . . . . . . . . . . . . 198

$8.2 .3 \mathrm{MC} /$ Data Comparisons . . . . . . . . . . . . . . 200

8.2 .4 Correction to $\epsilon_{0} \ldots \ldots \ldots \ldots \ldots \ldots$

8.3 Correction of $\epsilon_{2}$ using Conversion Events . . . . . . . . . . . . 204

8.3.1 Reconstruction of Conversions . . . . . . . . . . . . 204

8.3 .2 Event Selection . . . . . . . . . . . . . . . . 205

8.3.3 Data-MC comparison . . . . . . . . . . . . . . 206

8.3 .4 Correction . . . . . . . . . . . . . . . . . . 210

8.4 Corrected photon efficiency . . . . . . . . . . . . . 210

9 Cross-Checks of Background Levels $\quad 215$

9.1 Calibration of Resonance Production Rates . . . . . . . . . . . . . . . 215

9.1 .1 Technique . . . . . . . . . . . . . . . . 215

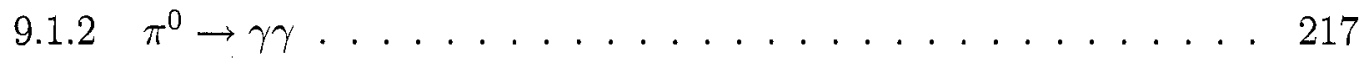

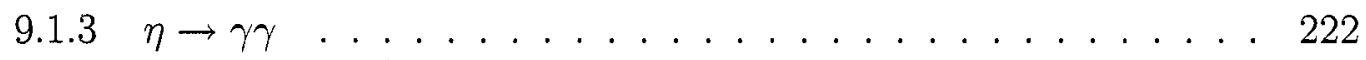

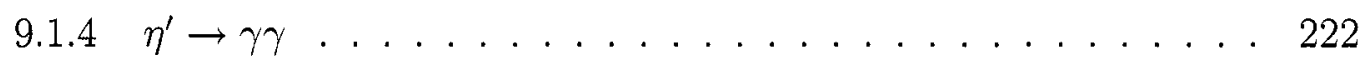

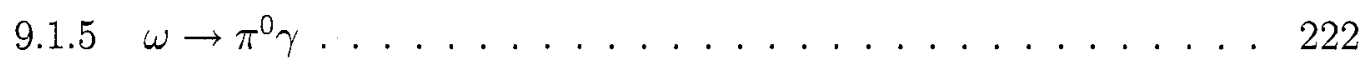

9.2 Cross-check of the $\pi^{0} \rightarrow \gamma \gamma$ yield in $\tau^{+} \tau^{-}$events . . . . . . . . 229

9.2.1 ISR Backgrounds from $\tau^{+} \tau^{-} \ldots \ldots \ldots \ldots \ldots . \ldots . \ldots 229$

9.2.2 Selection of $\tau \rightarrow \rho \nu$ events . . . . . . . . . . . . . 229

9.2 .3 Production rate . . . . . . . . . . . . . . . . . 232

9.2.4 Cross-check of Helicity distributions . . . . . . . . . . . . . 237

9.2.5 Validation of the $\pi^{0}$ Lateral Shape . . . . . . . . . . . 238

9.3 Cross-check of ISR cross-feed from antineutrons . . . . . . . . . . 246

9.3 .1 Strategy . . . . . . . . . . . . . . . . . . . 246

9.3.2 Selection of $\Lambda \rightarrow p^{+} \pi^{-}$events . . . . . . . . . . 247

9.3.3 Comparison of Antiprotons and Antineutrons in MC . . . . 249 
9.3.4 Validation of Antiproton Shower Parameters . . . . . . . . . 249

9.4 Study of $K_{L}^{0}$ background using $\phi \rightarrow K_{S}^{0} K_{L}^{0}$ events . . . . . . . . 253

9.4.1 Event Selection . . . . . . . . . . . . . . . . 253

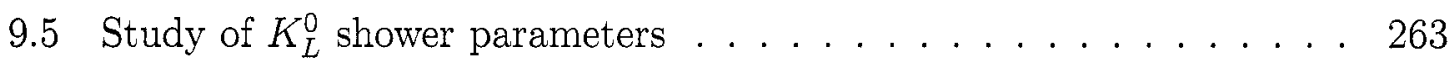

9.6 Validation of Continuum Fisher Distributions . . . . . . . . . . . 264

9.7 EMC Hot Towers . . . . . . . . . . . . . . . . . . . . . 268

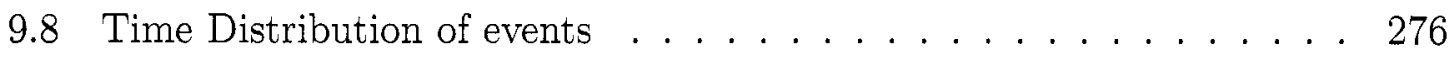

9.9 Overlapping Bhabha Events . . . . . . . . . . . . . . . . 277

9.10 Residual $e^{+} e^{-} \gamma$ cross-feed . . . . . . . . . . . . . . . 283

9.11 Residual $\gamma \gamma$ cross-feed . . . . . . . . . . . . . . . . . . . . 289

$9.12 \Upsilon$ resonance decays . . . . . . . . . . . . . . . . . . . . 291

9.12 .1 Order of magnitude estimate . . . . . . . . . . . . . . 291

9.12 .2 Measurement . . . . . . . . . . . . . . . . . . . . . 292

9.13 Two-photon collisions . . . . . . . . . . . . . . . . . . . . . 294

9.13.1 Events with less than two detected electrons . . . . . . . . . . 294

9.13.2 Events with two detected electrons . . . . . . . . . . . . 297

$9.14 e^{+} e^{-}$Annihilation to Two Virtual photons . . . . . . . . . . . 298

10 Signal Efficiency Determination $\quad 299$

10.1 Lateral Moment . . . . . . . . . . . . . . . . . . . . . . . 299

10.2 Fisher Selection . . . . . . . . . . . . . . . . . 300

10.3 Calibration of Particle Identification (PID) rates . . . . . . . . . 304

10.4 Tracking Efficiency Correction . . . . . . . . . . . . . 305

10.5 Efficiency Spectrum . . . . . . . . . . . . . . . . . . . 308

11 Results $\quad 313$

11.1 Data-MC comparisons in the final sample . . . . . . . . . . . 313

11.2 Systematic errors on Signal Efficiency . . . . . . . . . . . . . . . 319

11.3 Systematic Errors on Background Levels . . . . . . . . . . . . 327

11.4 Final Spectrum . . . . . . . . . . . . . . . . . . . . . 330

12 Summary and Outlook 336 
A The KKMC event Generator $\quad 338$

A.1 ISR emission model . . . . . . . . . . . . . . . . . . . . . 338

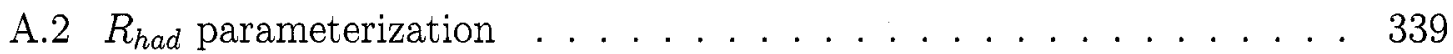

A.3 Hadronization . . . . . . . . . . . . . . . . . . . . . . 342

A.4 Comparison with PHOKHARA $\ldots \ldots \ldots \ldots \ldots \ldots$. . . . . 344

A.5 Comparison of the pion form factor . . . . . . . . . . 348

B Calculation of the Radiator Function $\xi(x) \quad 349$

C Hadronic Production from two Virtual Photons 354

C.1 Preliminaries: one virtual photon . . . . . . . . . . . . . 354

C.1.1 Cross-section calculation . . . . . . . . . . . . . . 354

C.1.2 Applications . . . . . . . . . . . . . . . . . 357

C.2 Two virtual photons . . . . . . . . . . . . . . . 358

C.2.1 "Parallel" Diagram . . . . . . . . . . . . . . . . . 359

C.2.2 Both diagrams together . . . . . . . . . . . . . . 362

C.2.3 Calculation of $\tilde{\mathcal{L}}_{2} \ldots \ldots \ldots \ldots 63$

C.2.4 Cross-Check . . . . . . . . . . . . . . . . . . . 366

C.2.5 Fiducial Cross-section . . . . . . . . . . . . . . 367

C.2.6 Applications . . . . . . . . . . . . . . . . 368

D Factorization of the Radiative Factor $\quad 371$

E Tracking and PID Requirements $\quad 373$

E.1 Track requirements . . . . . . . . . . . . . . . . . . . 373

E.2 Particle Identification (PID) $\ldots \ldots \ldots \ldots \ldots \ldots$

E.2.1 Hadron Identification . . . . . . . . . . . . . . . . . 374

E.2.2 Electron Identification $\ldots \ldots \ldots \ldots$

E.2.3 Muon Identification $\ldots \ldots \ldots 776$

$\begin{array}{ll}\text { Bibliography } & 378\end{array}$ 


\section{List of Tables}

2.1 Properties of Particles in the Standard Model . . . . . . . . . . 6

2.2 Results of the Global EW fit . . . . . . . . . . . . . . . . 11

2.3 Contributions to $\Delta \alpha_{h a d}^{(5)}\left(m_{Z}^{2}\right)$ per energy range $\ldots \ldots \ldots \ldots . \ldots 37$

3.1 Cross-sections on the $\Upsilon(4 S)$ resonance $\ldots \ldots \ldots \ldots \ldots$

3.2 Operating parameters for the PEP-II accelerator . . . . . . . . . 49

3.3 Properties of $\mathrm{CsI}(\mathrm{Tl}) \ldots \ldots \ldots \ldots \ldots \ldots$

3.4 DAQ parameters for the subdetectors . . . . . . . . . . . . 68

3.5 IFT trigger objects $\ldots \ldots \ldots \ldots \ldots \ldots \ldots \ldots \ldots \ldots$

3.6 GLT trigger objects and counting rules. . . . . . . . . . . . . 79

$3.7 \mathrm{~L} 1$ trigger configuration $\ldots \ldots \ldots \ldots \ldots$

3.8 Level 1 latency summary . . . . . . . . . . . . . . . . . . . . . 82

3.9 Level 1 Trigger efficiencies for various physics processes. . . . . . . . 82

$3.10 \mathrm{~L} 3$ trigger configuration . . . . . . . . . . . . . . . 86

3.11 Level 3 Trigger efficiencies for various physics processes. . . . . . . . 87

3.12 BGFilter efficiencies on ISR events . . . . . . . . . . . . . . 91

4.1 Datasets used for the analysis . . . . . . . . . . . . . . . . . 104

4.2 Summary of the data samples using in the ISR analysis. . . . . . . 105

4.3 Trigger and Filter efficiencies for signal samples . . . . . . . . . 108

5.1 Efficiencies for the main ISR event selection . . . . . . . . . . 128

5.2 Efficiencies for the main ISR event selection . . . . . . . . . . . 130

5.3 Efficiencies for the main ISR event selection . . . . . . . . . . . 130 
5.4 Efficiencies for the main ISR event selection . . . . . . . . . . . . 131

5.5 Efficiencies for the main ISR event selection . . . . . . . . . . . 131

5.6 Efficiencies for the main ISR event selection broken down by number

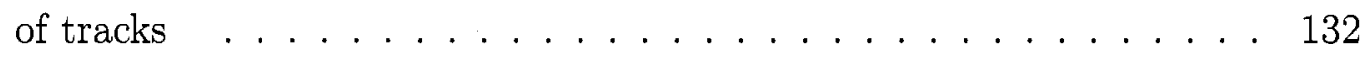

5.7 Summary of passing cross-sections after preselection cuts and after $e^{+} e^{-} \gamma, \gamma \gamma$, 1-track, neutral and virtual Compton vetos. . . . . . 133

5.8 Efficiencies for the main ISR event selection . . . . . . . . . . 136

6.1 Processes in $u d s, c \bar{c}$ and $\tau^{+} \tau^{-} \mathrm{MC}$ samples contributing to background. The samples correspond to events passing the QED rejection cuts. . 138

6.2 Minimum photon energy and $h$ mass interval used for the veto of $h \rightarrow$

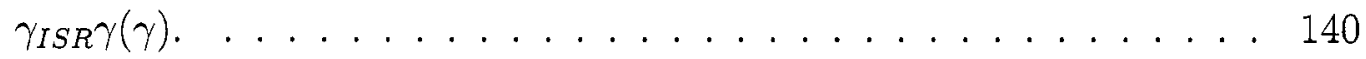

6.3 Boundaries of the $s^{\prime}$ bins used for Fisher discriminants . . . . . . 147

6.4 Value of the Fisher cut position for each bin, and corresponding values of the signal and background efficiencies and associated error as described in the text . . . . . . . . . . . . . 158

6.5 Summary of selection efficiencies broken down by signal MC mode. All ratios are given in percent. . . . . . . . . . . . . . . 159

6.6 Summary of selection efficiencies broken down by signal MC mode. All ratios are given in percent. . . . . . . . . . . 160

6.7 Summary of passing cross-sections after QED vetos, after $m_{\gamma \gamma}$ veto, lateral moment cut and Fisher cut. . . . . . . . . . . . . . 161

6.8 Effects of the rejection cuts on selected background modes, combined across $u d s, c \bar{c}$ and $\tau^{+} \tau^{-}$samples $\ldots \ldots \ldots \ldots 16 \ldots$

7.1 Efficiency of the selection of Section 7.1 on $\mu^{+} \mu^{-} \gamma$ MC . . . . . . 170

7.2 Efficiency for the selection of Section 7.1 on background MC samples. 171

7.3 Efficiency for the selection of Section 7.1 on background MC samples. 171

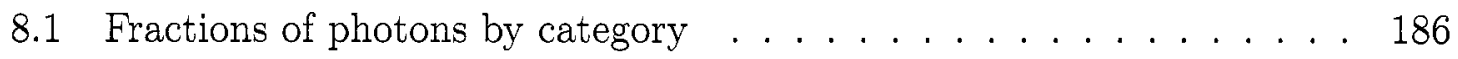

8.2 Efficiency and photon-finding bias in $\mu^{+} \mu^{-} \gamma \mathrm{MC}$ for the selection of Section $8.2 .2 \ldots \ldots \ldots \ldots$. . . . . . . . . . . 199 
8.3 Efficiency for the selection of Section 8.2.2 on background MC samples. 199

8.4 Efficiency for the selection of Section 8.2.2 on background MC samples. 200

8.5 Efficiency for the selection of Section 8.3.2 . . . . . . . . . . 207

8.6 Efficiency for the event selection of Section 8.3.2. . . . . . . . . . . 207

8.7 Efficiency for the event selection of Section 8.3.2. . . . . . . . . 207

9.1 Peak and combinatorics corrections for higher resonances . . . . . . 224

9.2 Breakdown of of the generic $\tau^{+} \tau^{-}$sample according to $\tau$ decay modes 229

9.3 Efficiencies for the $\tau \rightarrow \pi^{0} \pi \nu$ event selection . . . . . . . . . . . 231

9.4 Efficiencies for the $\tau \rightarrow \pi^{0} \pi \nu$ event selection . . . . . . . . . . . 231

9.5 Efficiencies for the $\tau \rightarrow \pi^{0} \pi \nu$ event selection . . . . . . . . . . 231

9.6 Efficiencies for the $\Lambda \rightarrow p^{ \pm} \pi^{\mp}$ event selection . . . . . . . . . . 248

9.7 Efficiencies for the $\phi \rightarrow K_{S}^{0} K_{L}^{0}$ event selection . . . . . . . . 261

9.8 Efficiencies for the $\phi \rightarrow K_{S}^{0} K_{L}^{0}$ event selection . . . . . . . . . 262

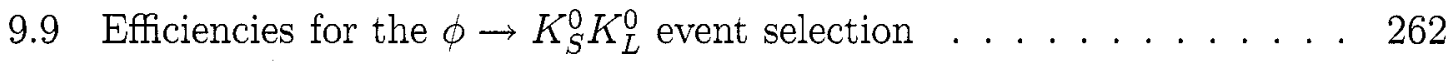

9.10 Efficiencies for the $\phi \rightarrow K_{S}^{0} K_{L}^{0}$ event selection . . . . . . . . 263

9.11 List of EMC $\phi$ and $\theta$ bin indices for crystals flagged as "hot". . . . . 272

9.12 Scale factors applied to $e^{+} e^{-} \gamma \mathrm{MC}$ as described in the text $\ldots . .287$

9.13 Cross-section for the ISR production of $\Upsilon$ resonances . . . . . . . . 291

9.14 Cross-section for the ISR production of $\Upsilon$ resonances . . . . . . . 293

9.15 Cross-feed from two-photon collision events . . . . . . . . . . 298

10.1 Average efficiencies for signal modes. . . . . . . . . . . . . . . . 309

11.1 Systematic errors on the signal efficiency. . . . . . . . . . 325

11.2 Values of fixed systematics applied to all $s^{\prime}$ bins. . . . . . . . . 328

11.3 Systematic errors on background. . . . . . . . . . . . . . 328

11.4 Breakdown of total systematics by source (\%). . . . . . . . . 333

A.1 Resonance parameters used in KKMC. . . . . . . . . . . . . . . . 339 


\section{List of Figures}

2.1 Higher-order corrections to the gauge boson propagators $\ldots \ldots .9$

2.2 Pull distribution for th Global SM fit . . . . . . . . . . . . . . 12

$2.3 \chi^{2}$ distribution for the Higgs mass . . . . . . . . . . . . . . . 14

2.4 One-loop diagrams for QED . . . . . . . . . . . . . . 15

2.5 A term in the photon propagator perturbative series. . . . . . . . 15

2.6 Hadronic cross-section as a function of collision CM energy at lowest

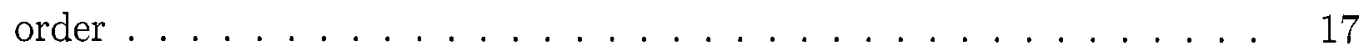

2.7 Parameterization of $R_{\text {had }}$ used by the KKMC event generator. . . . . 18

2.8 Measurements of the total hadronic cross-section and $R_{\text {had }}$ as a function of energy . . . . . . . . . . . . . . . . . . . . . . . . 19

2.9 QCD corrections to the photon propagator. . . . . . . . . 20

2.10 Diagrammatic relation for the photon vacuum polarization. . . . . . 20

2.11 Integration contour for $\Pi(s)$ in the complex $s$ plane . . . . . . . 22

2.12 The Optical Theorem in diagrammatic form . . . . . . . . . . 23

2.13 Perturbative QCD corrections to $R_{\text {had }} \ldots \ldots \ldots \ldots \ldots \ldots$

2.14 Variation of $\alpha_{s}$ as a function of $s=q^{2} \ldots \ldots \ldots \ldots \ldots 28$

$2.15 R_{\text {had }}(\sqrt{s})$ shape before the $\alpha_{s}$ correction (dashed line) and after (solid line) . . . . . . . . . . . . . . . . . . . 28

2.16 Leading-order diagram for ISR emission . . . . . . . . . . . . . . 29

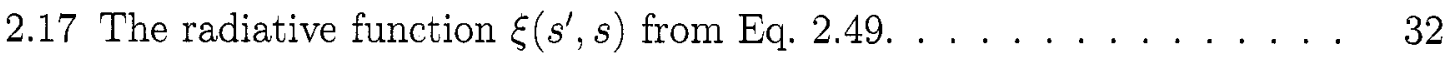

2.18 Weight function $w\left(s^{\prime}, s\right)$ for the $\Delta \alpha_{\text {had }}^{(5)}\left(m_{Z}^{2}\right)$ integral, for $\cos \theta_{\text {min }}=0.8034$

2.19 The Optical Theorem for hadronic events with final-state photons . . 35

3.1 Longitudinal cross-section view of the BABAR detector . . . . . . . 41 
3.2 $e^{+} e^{-} \rightarrow$ hadrons cross-section in the vicinity of the $\Upsilon(1 S), \Upsilon(2 S), \Upsilon(3 S)$ and $\Upsilon(4 S)$ resonances, as measured by the CUSB and CLEO collabo-

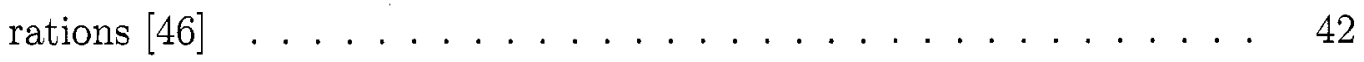

3.3 Correspondance between the polar angle $\theta_{L a b}$ in the laboratory frame and the cosine of the polar angle $\cos \theta_{c m}$ in the CM frame. . . . . 44

3.4 Schematic view of the PEP-II interaction region . . . . . . . . 46

3.5 Longitudinal cross-section of the SVT. . . . . . . . . . . . 51

3.6 Longitudinal cross-section of the DCH. . . . . . . . . . . 52

3.7 Schematic layout of the drift cells in the four inner DCH superlayers. 54

3.8 DCH single cell resolution. . . . . . . . . . . . . . . . 55

3.9 (a) Longitudinal section of EMC. (b) Crystal housing with front-end

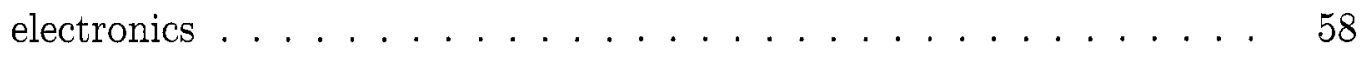

3.10 Geometry of the IFRbarrel and endcaps $\ldots \ldots \ldots \ldots$. . . . . 62

3.11 (a) Elevated view of overall DIRC geometry. (b) Bar/SOB transition

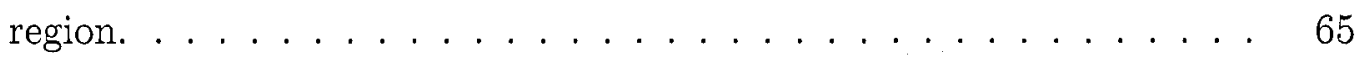

3.12 Representation of a TSF pivot group. Wire \#4 is the pivot wire. . . . 72

3.13 Envelopes for high- $p_{t}$ tracks with hits in SL 7 and SL 10 . . . . . 74

3.14 Detection efficiencies for the BLT A and B tracks and PTD A' track as a function of track $p_{t} \ldots \ldots \ldots \ldots \ldots \ldots$

3.15 Detection efficiency for the EMT M object as a function of cluster energy 77

3.16 BGFilter configuration for the data used in this document . . . . . 93

4.1 Beam (left) and top (right) view of a typical ISR event $\ldots \ldots . .95$

4.2 Integrand and Cumulative integral of Eq. 2.53 in signal MC using the

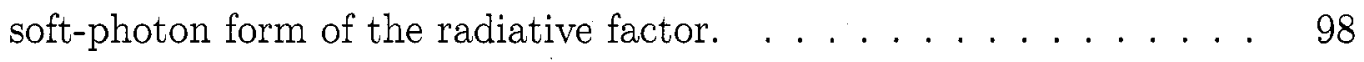

4.3 Triggering efficiencies versus $s^{\prime}$ for $q \bar{q} \gamma, \mu^{+} \mu^{-} \gamma$ and $\tau^{+} \tau^{-} \gamma \ldots \ldots . . .107$

5.1 Example of a background event resulting from a beam "accident". . . 114

$5.2 E^{*}, \theta, N_{C r y}$ and Lat distributions for the highest-energy cluster in the event in signal MC . . . . . . . . . . . . . . . 115

$5.3 s^{\prime}$ distribution for data and stacked MC samples after preselection cuts

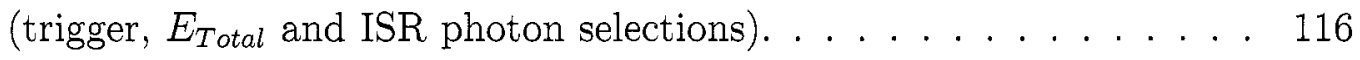


$5.4 \theta_{\gamma}$ distribution for data and stacked $\mathrm{MC}$ samples after preselection cuts

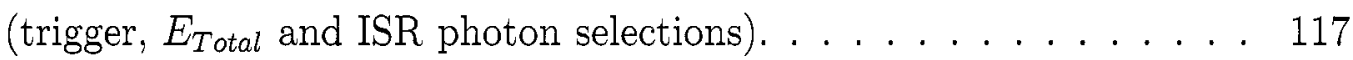

5.5 Number of tracks in event for simulated samples of signal, $\mu^{+} \mu^{-} \gamma, \gamma \gamma$ and radiative Bhabha. . . . . . . . . . . . . . . . . . . 118

5.6 Center-of-mass energy of the second-highest energy cluster in the event and $\Delta \theta, \Delta \phi$ with respect to the ISR photon back-to-back direction. in non-converted $\gamma \gamma \mathrm{MC}$, and KKMC. . . . . . . . . . . .

5.7 Conversion invariant mass (left), $d_{x y}$ (center) and $d_{z}$ (right) distributions. Top row is converted $\gamma \gamma \mathrm{MC}$, bottom is KKMC. . . . . . . . 124

5.8 Conversion vertex position in $x-y$ (left) and $r-z$ (right) coordinates for converted $\gamma \gamma$ MC . . . . . . . . . . . . . . . . . . . . 124

5.9 Distributions of $\pi^{0} \rightarrow \gamma \gamma$ candidate parameters in non-converted $\gamma \gamma$

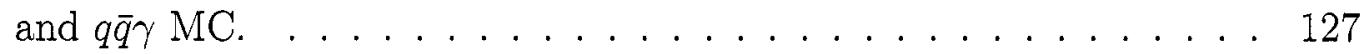

5.10 Variables used in the one-track veto algorithm, in $e^{+} e^{-} \gamma$ (left column) and $q \bar{q} \gamma \mathrm{MC}$ (right column). From top to bottom, the variables are the track $E_{C a l}, \Delta \phi, \Delta \theta$ and front-fact position. . . . . . . . .

$5.11 s^{\prime}$ distribution for data (round dots) and stacked MC samples after hadronic selection cuts. . . . . . . . . . . . . . . . . . . . 134

$5.12 \theta_{\gamma}$ distribution for data (round dots) and stacked $\mathrm{MC}$ samples after hadronic selection cuts. . . . . . . . . . . . . . . .

6.1 Distribution for the invariant mass, CM energy and lab polar angle for the $\gamma_{I S R} \gamma$ combination with mass closest to the nominal $\pi^{0}$ mass .

6.2 Lateral moment distribution for the ISR photon candidate in $u d s$ (left) and signal (right) MC . . . . . . . . . . . . . . . . . 141

6.3 Pruning sequence for Fisher discriminants in the regions $s^{\prime}=15-$ $25 \mathrm{GeV}^{2}$ and $s^{\prime}=35-40 \mathrm{GeV}^{2}$. . . . . . . . . . . . . 149

6.4 Background efficiency versus signal efficiency for pruned Fishers with given numbers of variables, for $s^{\prime}=15-25 \mathrm{GeV}^{2}$ (top) and $s^{\prime}=$ $35-40 \mathrm{GeV}^{2}$ (bottom). . . . . . . . . . . . . 150

6.5 Correlation matrices for the selected Fisher variables and $s^{\prime} \ldots \ldots .150$ 
6.6 Distributions for the variables used in the Fisher discriminant for signal

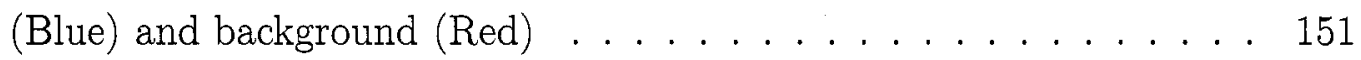

6.7 Values of the Fisher coefficients for versus $s^{\prime} \ldots \ldots \ldots \ldots \ldots$

6.8 Distributions of the Fisher discriminant for signal (Blue) and background (Red) in bins 1 (to left) to 8 (bottom right) . . . . . . . 154

6.9 Signal (Blue) and background (Red) efficiencies versus Fisher cut position 155

6.10 Background versus signal efficiency . . . . . . . . . . 156

6.11 Total Error $\delta N_{S} / N_{S}$ versus Fisher cut position for $\eta_{S}=\eta_{B}=25 \%$. . 157

$6.12 s^{\prime}$ distribution for data (round dots) and stacked MC samples after the

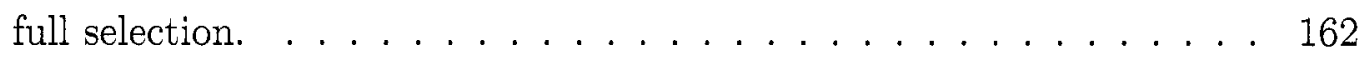

$6.13 \theta_{\gamma}$ distribution for data (round dots) and stacked MC samples after the

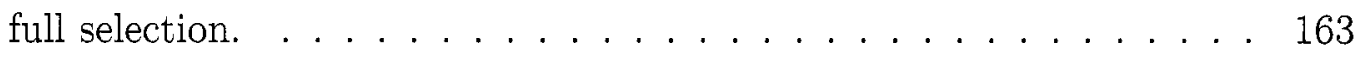

7.1 s' distribution with no energy correction, for data (points) and MC

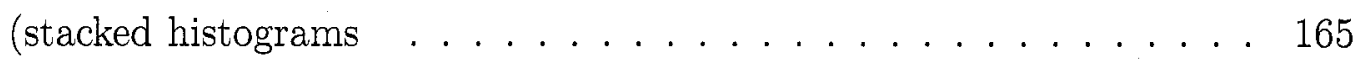

7.2 Number of good muon candidate (left) and missing mass (right) in

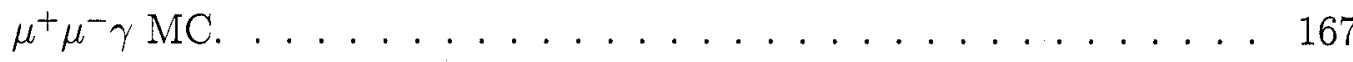

7.3 Angular separation between the missing momentum and the closest muon track (left) and energy outside a cone of $250 \mathrm{mrad}$ opening angle around the missing momentum (right). . . . . . . . . . . . 169

$7.4 E_{\gamma}^{*}-E_{M i s}^{*}$ (left), $\theta_{\gamma}-\theta_{M i s}$ (center) and $\phi_{\gamma}-\phi_{M i s}$ (right) for Raw (top row) and Fitted (bottom row) missing momenta. . . . . . . . . . . 169

7.5 Distribution of $E_{F i t}^{*}$ in data, showing from right to left the $\psi, \psi$ in off-resonance data and $\psi^{\prime}$ resonances. . . . . . . . . . . . . 172

7.6 Comparison between data and $\mu^{+} \mu^{-} \gamma \mathrm{MC}$ for missing momentum quantities. . . . . . . . . . . . . . . . . . 173

7.7 Comparison between data and $\mu^{+} \mu^{-} \gamma \mathrm{MC}$ for muon quantities. . . . 174

7.8 Energy correction distributions for the bin $0.386<\theta_{\gamma}<0.468$, in bins

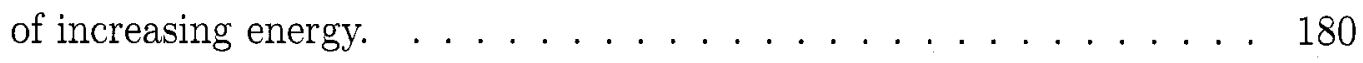

7.9 Energy resolution parameters for the bin $1.40<\theta_{\gamma}<1.90$, in bins of increasing energy. . . . . . . . . . . . . . . 181 
$7.10 \theta$ correction distributions for the bin $0.386<\theta_{\gamma}<0.468$, in bins of

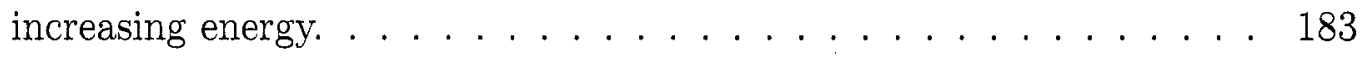

$7.11 \theta$ resolution parameters for the bin $1.40<\theta_{\gamma}<1.90$, in bins of increasing energy. . . . . . . . . . . . . . . . . . . . . 184

8.1 E* distributions for the numerator sample (left), denominator sample (center) and their ratio (right), for the range $0.35<\theta<2.2 \ldots \ldots 187$

8.2 $E^{*}$ distributions for the numerator sample (left), denominator sample (center) and their ratio (right), for the range $0.35<\theta<2.2 \ldots \ldots 189$

8.3 $E_{\text {reco }}^{*} E_{\text {true }}^{*}$ distributions without (left) and with (right) a photon separation cut (left) for the range $0.35<\theta<2.2 \ldots \ldots \ldots \ldots$

8.4 Ratio between the fraction of the $E_{\text {reco }}^{*} / E_{\text {true }}^{*}$ curve in the high-side tail to the fraction in the peak region, as a function of $E_{\text {true }}^{*} \ldots \ldots 191$

8.5 Resolution functions for $E^{*}$ in MC Truth, in bins of energy (increasing from left to right) and $\theta$ (increasing from top to bottom). . . . . . 192

8.6 Resolution functions for $\theta$ in MC Truth, in bins of energy (increasing from left to right) and $\theta$ (increasing from top to bottom). . . . . . 193

8.7 Photon Efficiency (left) and acceptance fraction (right) in MC Truth, in bins of $E^{*}$ and $\theta \ldots \ldots \ldots \ldots \ldots \ldots$

8.8 Efficiency in MC Truth in bins of $E^{*}$ and $\theta$ for different photon outcomes. 196

8.9 Comparison between data and $\mu^{+} \mu^{-} \gamma \mathrm{MC}$ missing momentum quantities. 201

8.10 Comparison between data and $\mu^{+} \mu^{-} \gamma \mathrm{MC}$ muon quantities. . . . . . 202

8.11 Values of $\epsilon^{\prime}$ in MC (left) and data (right), and their ratio $c_{0} \ldots 203$

8.12 Value $\epsilon_{0}$ extracted from $\epsilon^{\prime}$. Good agreement is seen with the values from the bottom left plot of Fig. 8.8. . . . . . . . . . . . . 204

8.13 Diagram illustrating the method used to reconstruct conversions. . . 205

8.14 Comparison between data and $\mu^{+} \mu^{-} \gamma \mathrm{MC}$ for converted photon quantities. . . . . . . . . . . . . . . . . . . . . . 208

8.15 Comparison between data and $\mu^{+} \mu^{-} \gamma \mathrm{MC}$ for converted photon quantities. . . . . . . . . . . . . . . . . . . . . . 208 
8.16 Comparison of conversion positions in the $x-y$ and $r-\phi$ planes in $\mu^{+} \mu^{-} \gamma \mathrm{MC}$ and data. . . . . . . . . . . . . . . . 209

8.17 Histograms for $N_{2}^{S e l} / N_{\gamma}^{\text {Sel }}$ in MC (left) and data (right) and the ratio $c_{12}$ of data to MC.

8.18 Histograms for the reconstruction efficiencies of events with a photon (top) and a conversion (middle), and the extracted value $\epsilon_{2}$ (bottom).

8.19 Histograms for the correction factor $c_{\gamma}$ applied on the photon efficiency (left) and the corrected efficiency (right). . . . . . . . . . . 214

9.1 Distributions of $m_{\pi^{0}}$ in data and MC . . . . . . . . . . 218

9.2 Fits of the $m_{\pi^{\circ}}$ distribution for the sum of all MC samples. . . . . . . 219

9.3 Fits of the $m_{\pi^{0}}$ distribution in data. . . . . . . . . . . . 220

9.4 Peak (top) and combinatorics (bottom) corrections for the $\pi^{0}$ region 221

9.5 Distributions of $m_{\eta}$ in data and MC . . . . . . . . . . 223

9.6 Fits of the $m_{\eta}$ distribution for the sum of all $\mathrm{MC}$ samples, in bins of $E_{\eta}^{*}$ (increasing from left to right) and $\theta_{\eta}$ (increasing from top to bottom). Histograms are scaled to $250 \mathrm{fb}^{-1} \ldots \ldots \ldots \ldots$. . . . . . . 224

9.7 Fits of the $m_{\eta}$ distribution in data, in bins of $E_{\eta}^{*}$ (increasing from left to right) and $\theta_{\eta}$ (increasing from top to bottom). Histograms are scaled

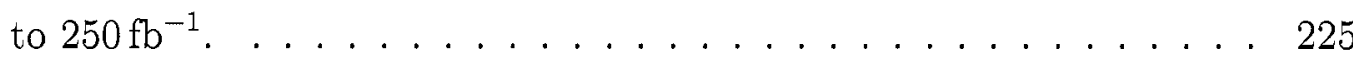

9.8 Peak (top) and combinatorics (bottom) corrections for the $\eta$ region . 226

9.9 Fit of $m_{\eta^{\prime}}$ from $u d s \mathrm{MC}$ events with a true $\eta^{\prime} \rightarrow \gamma \gamma$ decay. . . . . . 227

9.10 Distribution of $m_{\eta^{\prime}}$ in data and MC. . . . . . . . . . . 227

9.11 Fit of $m_{\omega}$ from $u d s \mathrm{MC}$ events with a true $\omega \rightarrow \pi^{0} \gamma$ decay. . . . . 228

9.12 Distribution of $m_{\omega}$ in data and MC. . . . . . . . . . . . . 228

9.13 Distributions of number of clusters on tag side (top left), tag $m_{\gamma \gamma}$ (top right), tag $m_{\gamma \gamma \pi}$ (bottom left) and signal $m_{\gamma \gamma \pi}$ (bottom right). . . . 230

9.14 Fits of the $m_{\pi^{0}}$ distribution with double $\tau \rightarrow \pi \pi^{0} \nu$ selection for $\tau^{+} \tau^{-}$ $\mathrm{MC}$, in bins of $E_{\pi^{0}}^{*}$ (increasing from left to right) and $\theta_{\pi^{0}}$ (increasing from top to bottom). Histograms are scaled to $250 \mathrm{fb}^{-1} \ldots \ldots \ldots . .232$ 
9.15 Fits of the $m_{\pi^{0}}$ distribution with double $\tau \rightarrow \pi \pi^{0} \nu$ selection in MC (shaded histogram) and data, in bins of $E_{\pi^{0}}^{*}$ (increasing from left to right) and $\theta_{\pi^{0}}$ (increasing from top to bottom). Histograms are scaled to $250 \mathrm{fb}^{-1}$. . . . . . . . . . . . . . . . . .

9.16 Fits of the $m_{\pi^{0}}$ distribution with double $\tau \rightarrow \pi \pi^{0} \nu$ selection in data, in bins of $E_{\pi^{0}}^{*}$ (increasing from left to right) and $\theta_{\pi^{0}}$ (increasing from top to bottom). Histograms are scaled to $250 \mathrm{fb}^{-1} \ldots \ldots$. . . . . . 234

9.17 Peak correction for the $\pi^{0}$ region in double $\tau \rightarrow \pi \pi^{0} \nu$ events . . . . 235

9.18 Left: Fraction of $\tau^{+} \tau^{-}$events in the $\pi^{0}$ mass peak of the inclusive $\pi^{0}$ sample. Right: Correction for the non- $\tau^{+} \tau^{-}$events in the inclusive $\pi^{0}$ sample. . . . . . . . . . . . . . . . .

$9.19 E_{\gamma}^{*}$ (left) and $\theta$ (right) distributions for the highest-energy cluster in the event, in data (blue), MC (red) and reweighed MC (black), normalized by luminosity . . . . . . . . . . . . . . . . . . 2 238

$9.20 \cos \theta_{h e l}$ distributions in data and reweighted $\mathrm{MC}$ in bins of $E_{\pi^{\circ}}^{*} . \ldots 239$

9.21 Left: ratio of merged $\pi^{0}$ yields in $\mathrm{MC}$ and data in bins of $E_{\pi^{0}}^{*}$, with the rightmost bins representing all events. Right: ratio of merged $\pi^{0} \mathrm{~s}$ to total $\pi^{0} \mathrm{~s}$ in $\mathrm{MC}$ (red) and data (blue). . . . . . . . . . 240

9.22 Left: difference between true and reco $\pi^{0}$ helicities in $\mathrm{MC}$ versus the lab energy of the softer photon. Right: helicity distributions in MC truth (blue) and $\mathrm{MC}$ reco (red). . . . . . . . . . . . . . . 240

9.23 Cluster lateral shape distributions (left column) and number of crystals (right column) for the hard photons in unmerged $\pi^{0}$ decays and merged $\pi^{0} \mathrm{~s}$, for data (blue) and reweighted MC (red). The top row corresponds to all events, while subsequent rows correspond to the usual $E_{\pi^{0}}^{*}$ bins with boundaries at $3.3,3.8,4.3,4.8$ and $5.3 \mathrm{GeV} \ldots \ldots \ldots \ldots 242$

9.24 Cluster lateral shape distributions for the hard photons in unmerged $\pi^{0}$ decays (left) and merged $\pi^{0} \mathrm{~s}$ (right). The histograms are data (blue) and reweighted $\mathrm{MC}$ (red). The top row corresponds to all events, while subsequent rows correspond to the usual $E_{\pi^{0}}^{*}$ bins with boundaries at $3.3,3.8,4.3,4.8$ and $5.3 \mathrm{GeV} \ldots \ldots \ldots \ldots \ldots \ldots$ 
9.25 Left: lateral moment of the hard photon versus energy of the soft photon for reconstructed $\pi^{0} \mathrm{~S}$ in data Right: lateral moment of the cluster versus true $\cos \theta_{\text {hel }}$ for merged $\pi^{0} \mathrm{~s}$ in data. . . . . . . . . 244

9.26 Cluster lateral shape distributions for the hard photons in merged $\pi^{0} \mathrm{~s}$ and unmerged with an invariant mass outside the interval $100<m_{\gamma \gamma}<$ $170 \mathrm{MeV}$. The left column shows the data and raw MC distribution, the middle column shows the same with the MC shifted up and the right column the same with the MC scaled up The histograms are data (blue) and reweighted MC (red). The top row corresponds to all events, while subsequent rows correspond to the usual $E_{\pi^{0}}^{*}$ bins with boundaries at $3.3,3.8,4.3,4.8$ and $5.3 \mathrm{GeV} \ldots \ldots \ldots \ldots$

9.27 Left: magnitude of the shift for the MC lateral moment distribution. The last bin corresponds to all events. Right:Efficiency correction for the lateral moment cut on $\pi^{0}$ s passing the mass veto. . . . . . . 246

9.28 $\Lambda$ mass distribution before (left) and after (right) extra selection cuts 248

9.29 Distributions for the lab energy deposition (left), CM energy deposition (center) and cluster lateral moment (right) for antiprotons (red) and antineutrons (blue) produced in simulated $\Lambda$ decays. The antiproton curves are shown rescaled by a factor $35.8 / 63.9$ to account for the difference in $\Lambda$ branching fractions. . . . . . . . . . . . . 249

9.30 Fits of the $m_{\Lambda}$ distributions in MC. The left column in for antiprotons, right for protons, and rows show bins of $p_{\text {proton }}$, increasing downward.

9.31 Fits of the $m_{\Lambda}$ distributions in data. The left column in for antiprotons, right for protons, and rows show bins of the proton momentum $p_{p}$, increasing downward. . . . . . . . . . . . . . . . 252

9.32 Distribution of the proton momentum $p_{p}$, signed with the charge of the particle. . . . . . . . . . . . . . . . . 253 
9.33 Distributions of the energy deposited by protons and antiprotons in the calorimeter, for MC (red) and data (blue). The left column in for antiprotons, right for protons. The first row shows all momenta, and rows below show bins of the proton momentum $p_{p}$, increasing

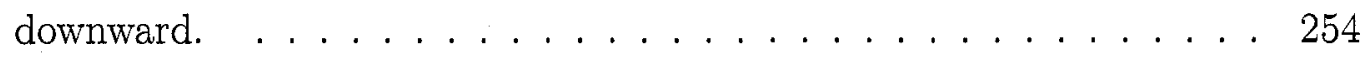

9.34 Distributions of the ratio of the energy deposited by protons and antiprotons in the calorimeter to the maximum value $m_{p}+\sqrt{p_{p}^{2}+m_{p}^{2}}$ for $\mathrm{MC}$ (red) and data (blue). The left column in for antiprotons, right for protons. The first row shows all momenta, and rows below show bins of the proton momentum $p_{p}$, increasing downward. . . . . . .

9.35 Top Left: Distributions of the CM energy deposited by antiprotons of all momenta in the calorimeter, for $\mathrm{MC}$ (red) and data (blue). Top Right: closeup of the region above $3.3 \mathrm{GeV}$ which is relevant for the ISR analysis. Bottom: ratio of data to MC distribution. . . . . . . 256

9.36 Distributions of the cluster lateral moment for MC (red) and data (blue). The left column in for antiprotons, right for protons. The first row shows all momenta, and rows below show bins of the proton momentum $p_{p}$, increasing downward. . . . . . . . . 257

9.37 Distribution of cluster lateral moment for cluster CM energies $E_{D e p}^{*}$ in the range $2<E_{\text {Dep }}^{*}<3 \mathrm{GeV}$ (black), $3<E_{\text {Dep }}^{*}<4 \mathrm{GeV}$ (blue) and $E_{\text {Dep }}^{*}>4 \mathrm{GeV}(\mathrm{red}) \ldots \ldots \ldots \ldots \ldots \ldots \ldots$

9.38 Left: distributions of the cluster CM energy $E_{D e p}^{*}$ for data (blue) and reweighted MC (red). Right: close-up of the region $E_{D e p}^{*}>3.3 \mathrm{GeV}$. 258

9.39 Left: distributions of cluster lateral moment for antiprotons with $E_{D e p}^{*}>$ $3.3 \mathrm{GeV}$ in data (blue) and reweighted $\mathrm{MC}$ (red). Left: Same plot with a shifted MC distribution. . . . . . . . . . . . . . . . . . 259

9.40 Distributions of the cut variables for signal signal (top row) and $u d s$ background (bottom row). From left to right the variables are ISR photon $E^{*}, K_{S}^{0}$ mass and angle between the $K_{S}^{0}$ and the ISR photon recoil direction in the $\mathrm{CM}$ frame. . . . . . . . . . . . 260 
9.41 Distributions of the cut variables for signal signal (top row) and background (bottom row). From left to right the variables are $\left|E_{\gamma}^{*}-2 E_{K}^{*}\right|$; the energy of the highest-energy cluster outside a $150 \mathrm{mrad}$ cone around the $K_{S}^{0}$ direction and the $K_{S}^{0}$ flight distance in units of $c \tau$. The background sample is $u d s \mathrm{MC}$ for the first two variables and non- $\gamma K_{S}^{0} K_{L}^{0}$ ISR events for the last one. . . . . . . . . . . . . . . . . . . 261

9.42 Expected lab energy spectrum of $K_{L}^{0}$ from $e^{+} e^{-} \rightarrow \gamma \phi\left(K_{S}^{0} K_{L}^{0}\right)$ based on the reconstructed $\gamma$ and $K_{S}^{0}$ momenta. . . . . . . . . 264

9.43 Left: $K_{L}^{0}$ center-of-mass deposited energy $E_{K L}^{* E M C}$. Center: ratio $E_{K L}^{E M C} / E_{K L}^{E x p}$ of deposited to expected energy in the lab frame. Right: lateral moment of the $K_{L}^{0}$ cluster for $E_{K L}^{* E M C}>1 \mathrm{GeV}$. The top row includes all events, and the next rows corresponds from top to bottom to the $E_{K L}^{E x p}$ intervals $1-2,2-3,3-4$ and $4-6 \mathrm{GeV} \ldots \ldots \ldots 265$

9.44 Left: Weight function applied to the $\mathrm{MC}$ so that the $E_{K L}^{E M C}$ distribution matched that of data. Right: $E_{K L}^{E M C}$ distributions in data (blue) and reweighted MC (red). . . . . . . . . . . . . . . . 266

9.45 Lateral moment distribution in data (blue) and reweighted MC (red) without (left) and with (right) a shift in the MC corresponding to the difference in the histogram means. . . . . . . . . . . . . 266

9.46 Fisher cut correction for continuum background . . . . . . . . 267

9.47 Left, Center: Fits of the $m_{\pi^{0}}$ distribution in MC (left) and data (right). Right: background-subtracted Fisher distributions in MC (shaded histograms) and data (points with errors). Rows correspond to $s^{\prime}$ bins, increasing from top to bottom . . . . . . . . . . . . . . . 269

9.48 Left, Center: Fits of the $m_{\pi^{\circ}}$ distribution in MC (left) and data (right). Right: background-subtracted Fisher distributions in MC (shaded histograms) and data (points with errors). Rows correspond to $s^{\prime}$ bins, increasing from top to bottom. All plots have the selection $N_{G T} \leq 2$ applied. . . . . . . . . . . . . . . . . . . 270 
9.49 Left, Center: Fits of the $m_{\pi^{0}}$ distribution in MC (left) and data (right). Right: background-subtracted Fisher distributions in MC (shaded histograms) and data (points with errors). Rows correspond to $s^{\prime}$ bins, increasing from top to bottom. All plots have the selection $N_{G T} \geq 3$ applied. . . . . . . . . . . . . . . . . . 271

9.50 Typical hitmaps for blocks with one or more hot crystals . . . . . 273

9.51 Distribution of hot crystals in the EMC . . . . . . . . . 274

$9.52 \theta_{\gamma}$ distribution for the ISR photon in data (points with error bars) and MC (filled histograms) after applying the weighting procedure of

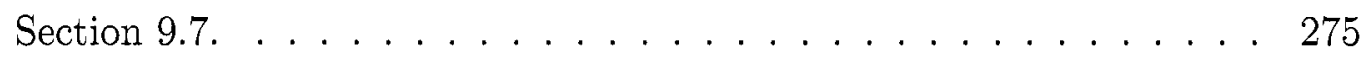

9.53 Fraction of signal event in each data block after hot tower removal. . 276

9.54 Beam (left) and top (right) view of a probable stale Bhabha event . . 277

9.55 $E^{*}$ versus time for the ISR photon in data (top) and signal MC (bottom). 279

$9.56 E^{*}$ versus time for the second-highest- $E^{*}$ cluster in the event in data (top) and signal MC (bottom). . . . . . . . . . . . 280

9.57 $E^{*}$ of the second-highest $\mathrm{CM}$ energy cluster in the event (top), same versus the ISR photon $E^{*}$ (top center), polar angle distribution for the ISR photon (bottom center) and $s^{\prime}$ (bottom). The left and right columns correspond to the signal and sideband regions respectively. .

9.58 Fit of the time distribution to an exponential distribution plus a constant outside the signal region. The fit favors a linear shape . . . . 282

$9.59 p^{*}$ of the highest- $p^{*}$ track in the event. . . . . . . . . . . 283

9.60 Number of IsrGoodTracks (top left), $E / p$ (top right) and crystal front-face position (bottom left) for the highest- $p^{*}$ track, and $p^{*}$ of the second-highest- $p^{*}$ track, in events where the highest- $p^{*}$ track has $p^{*}>5.1 \mathrm{GeV} \ldots \ldots \ldots \ldots \ldots \ldots \ldots$

$9.61 p^{*}$ of the highest- $p^{*}$ track in the event for events with one track, not identified as an electron (top left), with multiple tracks, and the highest- $p^{*}$ track not identified as an electron (top right) and for events with one track, not identified as an electron and passing the single-track veto of Section 5.10 (bottom) $\ldots \ldots \ldots \ldots$ 
9.62 Number of IsrGoodTracks (top left), $E / p$ (top right) and crystal front-face position (bottom left) for the highest- $p^{*}$ track, and $p^{*}$ of the second-highest- $p^{*}$ track, in events where the highest- $p^{*}$ track has $p^{*}>5.1 \mathrm{GeV} \ldots \ldots \ldots \ldots \ldots \ldots \ldots \ldots \ldots \ldots$

$9.63 s^{\prime}$ distribution for $N_{G T}=0 \ldots \ldots \ldots \ldots \ldots \ldots \ldots$

9.64 Distribution of $F_{2}$ for $N_{G T}=0 \ldots \ldots \ldots \ldots \ldots \ldots$

9.65 Distribution of $s^{\prime}$ (left) and $F_{2}$ (right) for $N_{G T}=0$ after rescaling the $q \bar{q} \gamma$ component as described in the text. . . . . . . . . . . . 290

9.66 Left: $s^{\prime}$ distribution for on-peak (blue curve) and rescaled off-peak (red curve) data. Right: Difference in production cross-section between the two, with a linear fit superimposed. . . . . . . . . . . . . . . . 293

9.67 Diagram for the radiative two-photon collision process described in Section $9.13 \ldots \ldots \ldots \ldots \ldots \ldots$

$9.68 x_{t}$ distribution for data (points) and MC (histograms). . . . . . . 296

$9.69 x_{l}^{\text {Mis }}$ distributions for data (points) and MC (histograms) for no-electron sample (left) and the one-electron sample (right). . . . . . . . . . 296

9.70 Back-to-back $\Delta \theta$ (left) and $\Delta \phi$ (right) distributions for electrons in data and MC for events with exactly two electrons where the electrons are the highest-momentum particles in the recoil frame. . . . . . . . . 297

$10.1 E_{\gamma}^{*}$ distribution for the $\mu \mu \gamma$ sample used in this study . . . . . . 300

10.2 Left: shift applied to the MC lateral moment distribution. Right: weighting to be applied to $\mathrm{MC}$ to correct for the difference in the efficiency of the lateral moment shape. . . . . . . . . . . . . . 300

10.3 Left: photon resolution function in MC (red) and data (blue). Center: lateral moment distributions for $E_{\gamma}^{*}>3.3 \mathrm{GeV}$. Right: same with shifted $\mathrm{MC} \ldots \ldots \ldots \ldots 1 . \ldots \ldots 1 . \ldots \ldots$

10.4 Fisher cut correction for signal . . . . . . . . . . . . . . . 302 
10.5 Fisher distributions after continuum-background rejection cuts in MC (stacked shaded histograms) and data (points with errors), for all events (left column), events with $N_{G T} \leq 2$ (middle column) and events with $N_{G T} \geq 3$ (right column). Rows correspond to $s^{\prime}$ bins, increasing from top to bottom . . . . . . . . . . . . . . . 303

10.6 $K_{S}^{0}$ mass distributions for $K_{S}^{0} \rightarrow \pi^{+} \pi^{-}$decays in $e^{+} e^{-} \rightarrow \phi\left(K_{S}^{0} K_{L}^{0}\right) \gamma$ events, in MC (left column) and data (center column), and PID category of the highest- $p^{*} K_{S}^{0}$ daughter as described in the text (right column). The vertical bins corresponds to $0<p^{*}<1 \mathrm{GeV}, 1<p^{*}<$ $2 \mathrm{GeV}, 2<p^{*}<2.5 \mathrm{GeV}$, and $2.5<p^{*}<3 \mathrm{GeV}$ from top to bottom, where $p^{*}$ id the $\mathrm{CM}$ momentum of the highest- $p^{*} K_{S}^{0}$ daughter. . . . . 306

10.7 Difference between the $q \bar{q} \gamma$ efficiency with the PID correction not applied and PID correction applied . . . . . . . . . . . . . . 307

10.8 Tracking efficiency correction vs. $s^{\prime}$ for $q \bar{q} \gamma$ MC . . . . . . . . 308

10.9 Efficiency in $q \bar{q} \gamma$ signal $\mathrm{MC}$ after all corrections. From top to bottom the cuts are photon-finding (closed circles) $h \rightarrow n \gamma$ Veto (closed squares), Lat Veto (upward-pointing closed triangles) Event shape (downpointing closed triangles), Rad. Bhabha veto (open circles), Other QED vetos (open squares), $p_{1}^{*}$ Cut (open triangles) and trigger and precuts (open lozenges). . . . . . . . . . . . . . . . . . 310

10.10Efficiency in $\mu^{+} \mu^{-} \gamma$ signal MC after all corrections.From top to bottom the cuts are photon-finding (closed circles) $h \rightarrow n \gamma$ Veto (closed squares), Lat Veto (upward-pointing closed triangles) Event shape (downpointing closed triangles), Rad. Bhabha veto (open circles), Other QED vetos (open squares), $p_{1}^{*}$ Cut (open triangles) and trigger and precuts (open lozenges). . . . . . . . . . . . . . . . 311 
10.11Efficiency in $\tau^{+} \tau^{-} \gamma$ signal MC after all corrections.From top to bottom the cuts are photon-finding (closed circles) $h \rightarrow n \gamma$ Veto (closed squares), Lat Veto (upward-pointing closed triangles) Event shape (downpointing closed triangles), Rad. Bhabha veto (open circles), Other QED vetos (open squares), $p_{1}^{*}$ Cut (open triangles) and trigger and precuts (open lozenges). . . . . . . . . . . . . . . . . . . . . 312

$11.1 s^{\prime}$ (top) and $\theta_{\gamma}$ (bottom) distributions in the final sample. Both $\mathrm{MC}$ and data samples are absolutely normalized and all the corrections are applied. . . . . . . . . . . . . . . . . . . . . . . . . . 314

11.2 $N_{G T}$ (top) and number of unmatched clusters with $E>100 \mathrm{MeV}$ (bottom) in the final sample, with all corrections applied and all samples absolutely normalized. . . . . . . . . . . . . . . . . . . 315

11.3 Fisher (top left) and $F_{2}$ (top right), angle between the ISR photon and the nearest track (bottom left) and the total neutral energy in the event (bottom right) in the final sample, with all corrections applied and all samples absolutely normalized. The Fisher distribution is represented

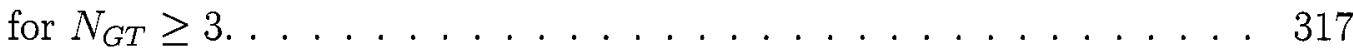

11.4 Distributions of $s^{\prime}$ (top left) and the invariant mass of the hadronic system (top right) in the $\psi$ region, and hadronic invariant mass for 2 (bottom left) and 4-track (bottom right) events in the $c \bar{c}$ threshold region. . . . . . . . . . . . . . . . . . . . . . 318

11.5 Hadronic system invariant mass (top) and two-track invariant mass with a pion mass hypothesis in the $\rho-\phi$ region with the requirement $N_{G T}=2$ (bottom).

11.6 Systematic uncertainties versus $s^{\prime}$ for $q \bar{q} \gamma$ signal. From top to bottom, the quantities are: Left column: $h \rightarrow n \gamma$ Veto, Event Shape, Tracking and QED systematics Right Column: Lateral Moment, Photon, PID and $p_{1}^{*}$ cut systematics $\ldots \ldots \ldots \ldots \ldots \ldots \ldots$ 
11.7 Systematic uncertainties versus $s^{\prime}$ for $\mu^{+} \mu^{-} \gamma$ signal. From top to bottom, the quantities are: Left column: $h \rightarrow n \gamma$ Veto, Event Shape, Tracking and QED systematics Right Column: Lateral Moment, Photon, PID and $p_{1}^{*}$ cut systematics . . . . . . . . . . . . 323

11.8 Systematic uncertainties versus $s^{\prime}$ for $\tau^{+} \tau^{-} \gamma$ signal. From top to bottom, the quantities are: Left column: $h \rightarrow n \gamma$ Veto, Event Shape, Tracking and QED systematics Right Column: Lateral Moment, Photon, PID and $p_{1}^{*}$ cut systematics . . . . . . . . . . . . . . 324

11.9 Total systematic uncertainties versus $s^{\prime}$ for $q \bar{q} \gamma$ signal. . . . . . . . 325

11.10Total systematic uncertainties versus $s^{\prime}$ for $\mu^{+} \mu^{-} \gamma$ signal, as fractions

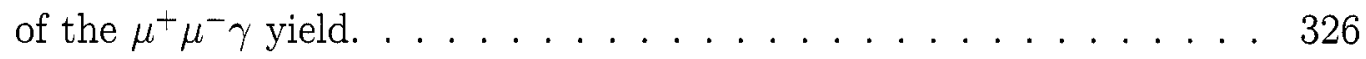

11.11Total systematic uncertainties versus $s^{\prime}$ for $\tau^{+} \tau^{-} \gamma$ signal, as fractions of the $\tau^{+} \tau^{-} \gamma$ yield. . . . . . . . . . . . . . .

11.12Systematic uncertainties versus $s^{\prime}$ for $u d s \mathrm{MC}$, as fractions of the $u d s$ yield. From left to right, the production, lateral moment and event shape systematics are shown $\ldots \ldots \ldots \ldots \ldots \ldots$

11.13Systematic uncertainties versus $s^{\prime}$ for $c \bar{c} \mathrm{MC}$, as fractions of the $c \bar{c}$ yield. From left to right, the production, lateral moment and event shape systematics are shown. . . . . . . . . . . .

11.14Systematic uncertainties versus $s^{\prime}$ for $\tau^{+} \tau^{-} \mathrm{MC}$, as fractions of the $\tau^{+} \tau^{-}$yield. From left to right, the production, lateral moment and event shape systematics are shown. . . . . . . . . . . . . . 329

11.15Production systematic versus $s^{\prime}$ for radiative Bhabha MC, as fractions of the $e^{+} e^{-} \gamma$ yield. . . . . . . . . . . . . . . . . . 330

11.16MC spectra subtracted from the data, with both systematic and statistical errors, for $u d s$ (top left), $c \bar{c}$ (top right), $\tau^{+} \tau^{-}$(bottom left) and $B \bar{B}$ (bottom right) samples. . . . . . . . . . . . . . 331

11.17MC spectra subtracted from the data, with both systematic and statistical errors, for $\gamma \gamma$ (left) and $e^{+} e^{-} \gamma$ (right) samples. . . . . . . 331

11.18MC spectra subtracted from the data, with both systematic and statistical errors, for $\mu^{+} \mu^{-} \gamma$ (top) and $\tau^{+} \tau^{-} \gamma$ (bottom) samples. . . . 332 
11.19Raw data spectrum before background subtraction. . . . . . . . . 333

11.20Final spectrum after background subtraction with no efficiency correction. . . . . . . . . . . . . . . . . . . 334

11.21Final spectrum after background subtraction and efficiency correction. 335

A.1 R function at low $s^{\prime}$ (left) and near the $c \bar{c}$ threshold (right). . . . . 340

A.2 Pion form-factor parametrization used in KKMC . . . . . . . . 341

A.3 R parametrization used in KKMC . . . . . . . . . . . . . . 341

A.4 Cross-section for $e^{+} e^{-} \rightarrow \pi^{+} \pi^{-} \pi^{+} \pi^{-}$used in KKMC. . . . . . . . . 343

A.5 Generated $s^{\prime}$ spectrum for KKMC (solid line) and the naive expectation of Eq. A.4. The solid triangles show the Phokhara spectrum in the mode with two hard photons enabled, while the empty triangles show the case of only one photon enabled. The plots show the full spectrum (top), the $\rho-\phi$ region (bottom left) and the $c \bar{c}$ threshold region (bottom

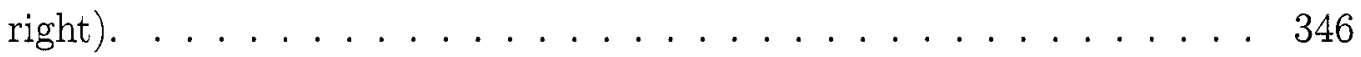

A.6 CM polar angle distribution for the hadronic system in KKMC . . . . 347

A.7 $s^{\prime}$ distribution for $\pi^{+} \pi^{-}$events as generated by KKMC (solid histogram) and PHOKHARA (triangles). . . . . . . . . . . . . 348

B.1 Diagram for the radiative factor $\xi \ldots \ldots \ldots \ldots \ldots$

C.1 One-photon exchange diagram for $e^{+} e^{-} \rightarrow F \ldots \ldots \ldots \ldots$. . . 354

C.2 Two-photon exchange diagrams for $e^{+} e^{-} \rightarrow F F^{\prime} \ldots \ldots \ldots \ldots . \ldots 358$ 
xxxiv 


\section{Chapter 1}

\section{Introduction}

The Standard Model (SM) of particle physics has been an extraordinarily successful theory, both in the field of flavor physics which constitutes the usual domain of BABAR experiment and at the electroweak scale, where LEP and SLD have confirmed Standard Model predictions to exceptional accuracy.

Outside the neutrino sector, the main remaining open question in the Standard Model is the status of the Higgs boson. This particle, which plays a crucial role in the theory, has not been observed so far, although direct observations may be just around the corner, perhaps at Fermilab and almost certainly (if it exists!) at LHC. However it can also be searched for indirectly, by measuring the radiative effects occurring from virtual Higgs bosons. Such measurements of radiative corrections are challenging but feasible thanks to the unprecedented precision of the $Z$-pole data and the fact that a single SM parameter, the mass of the Higgs boson, remains unknown.

In practice this is done by performing global fits of the electroweak (EW) sector of the SM [1], which use a number of electroweak observables to fit for 6 parameters: the top quark mass $m_{t}$, the $Z$ boson mass $m_{Z}$, the mass $m_{H}$ of the SM Higgs boson, the Fermi constant $G_{F}$, the value $\alpha_{s}\left(m_{Z}^{2}\right)$ of the strong coupling constant at $m_{Z}$ and the value $\alpha\left(m_{Z}^{2}\right)$ of the QED coupling constant at $m_{Z}$.

The error on the obtained Higgs mass value has two sources: uncertainties on the electroweak inputs to the fit on the one hand, and uncertainties on the remaining fit parameters on the other hand. The latter category is dominated by two quantities: the 
top quark mass and $\Delta \alpha_{h a d}^{(5)}\left(m_{Z}^{2}\right)$, which expresses the evolution of the QED coupling constant $\alpha$ from $Q^{2}=0$ to $Q^{2}=m_{Z}^{2}$ due to radiative corrections involving the five lightest quark flavors. The value of $\Delta \alpha_{\text {had }}^{(5)}\left(m_{Z}^{2}\right)$ depends on low-energy $Q C D$ processes that are not calculable from first principles. Fortunately, this quantity can be measured experimentally as an integral of the Drell ratio $R_{\text {had }}$, defined as the ratio of cross-sections

$$
R_{h a d}(s)=\frac{\sigma\left(e^{+} e^{-} \rightarrow \text { hadrons }\right)}{\sigma_{0}\left(e^{+} e^{-} \rightarrow \mu^{+} \mu^{-}\right)},
$$

as described in more detail in section 2.2.1.

The most recent determination of $\Delta \alpha_{h a d}^{(5)}\left(m_{Z}^{2}\right)$ is [2]

$$
\Delta \alpha_{h a d}^{(5)}\left(m_{Z}^{2}\right)=(2758 \pm 35) \times 10^{-5}
$$

which corresponds to a precision of about $1.3 \%$. Above $\sqrt{s} \approx 12 \mathrm{GeV}, R_{\text {had }}(s)$ can be reliably calculated using perturbative QCD with negligible errors of $0.2 \%$. This is also true to a lesser extent for the region $7<\sqrt{s^{\prime}}<12 \mathrm{GeV}$, which is known to a precision of about $1.4 \%$. The $\rho$ peak region is well measured, with a relative error of $0.9 \%$. The issue is therefore mainly with the $\sqrt{s}<7 \mathrm{GeV}$ region, excluding the $\rho$, which represents about $33 \%$ of the total, and has a relative error of about $4 \%$. The goal of this analysis is to bring this uncertainty down to $3 \%$ or less.

$R$ has traditionally been measured at low-energy colliders using energy scan techniques where data is gathered at various values of the beam energy. Recent examples include the measurements of $R$ near the $\rho$ peak by the CMD-2 collaboration [3] and the measurement by BES over the range $\sqrt{s}=2-5 \mathrm{GeV}[4]$.

However the scan technique has several limitations:

- Colliders perform optimally only in a limited range of beam energies. It is therefore necessary to combine data from several experiments operating at different machines in order to measure $R_{\text {had }}$ over a large energy range. This involves the combination of a variety of systematic uncertainties associated with different experiments. 
- Even within a single experiment, changes in the beam energy can alter the machine environment, leading to "point-to-point systematics" between data taken at different energy values. This is particularly true at low energies, where detector performance is strongly dependent on the beam energy.

- Beam-wall and beam-gas events are a background that is difficult to separate from hadronic events at low energies.

- The measurement is dependent on details of the hadronic model, such as the soft particle spectrum, used to model the detector acceptance.

- Accumulating large datasets at many energy points, as in the BES measurement, is impractical since it requires dedicated data-taking over long periods of time, producing data that is of limited use for other fields of study.

There are therefore significant limitation to measuring $\Delta \alpha_{\text {had }}^{(5)}\left(m_{Z}^{2}\right)$ using energy scan techniques.

Another technique, proposed a decade ago $[5,6,7]$ for $e^{+} e^{-}$machines, is to use initial-state radiation (ISR) to reduce the center-of-mass energy of the collision without modifying the beam energy. In this process, an energetic ISR photon is emitted from one of the incoming leptons before collision. In the case where the collision produces a hadronic event, the hadronic system is produced at a center-of-mass energy $\sqrt{s^{\prime}}$ below the nominal collision energy $\sqrt{s}$, recoiling against the ISR photon.

The method, detailed in Chapter 4, has already been successfully used by the KLOE collaboration to measure the pion form factor by radiating down from $\phi$ energies [11]. However it is particularly well-suited to the $B$-factory environment, where the entire interesting energy range $\sqrt{s^{\prime}}<7 \mathrm{GeV}$ is accessible by radiating down from $\sqrt{s}=10.58 \mathrm{GeV}$. A program to measure cross-sections of exclusive modes has been undertaken at $B A B A R$, with results for final states with up to six pions and kaons $[8,9,10]$. By analyzing final states of increasing multiplicity one can build up the full $R_{\text {had }}$, but this path becomes increasingly difficult for higher $s^{\prime}$.

This analysis instead relies on an inclusive approach, relying mainly on the ISR photon. It is required to be detected in the calorimeter, and is used to both reduce 
backgrounds and determine the value of $\sqrt{s^{\prime}}$ for the event. In order to improve the precision of the measurement, this analysis is made as inclusive as possible, with minimal dependence on details of the recoiling hadronic system. 


\section{Chapter 2}

\section{Theory}

\subsection{The Standard Model}

\subsubsection{Overview}

Our current best knowledge of the fundamental constituents of matter and their interactions is a theory commonly referred to as the Standard Model of particle physics. Its matter content is formed by three generations of particles, each composed of two flavors of left-handed and right-handed quarks (referred to as u-type and d-type after the members of the first generation), a charged lepton also coming in both left-handed and right-handed varieties, and a left-handed neutrino.

The properties of these particles are summarized in Table 2.1. Each particle carries an integer quantum number called the hypercharge and denoted as Y. Within each generation the left-handed u-type and d-type quarks form a doublet of the isospin group $S U(2)_{L}$, as do the left handed leptons. The right-handed particles form isospin singlets. The quarks also carry an additional quantum number, color, associated with the group $S U(3)_{C}$, while leptons are color singlets.

Interactions are obtained by building a non-Abelian gauge theory from the group $S U(3)_{C} \times S U(2)_{L} \times U(1)_{Y}$. Electroweak interactions are associated with the $S U(2)_{L} \times$ $U(1)_{Y}$ subgroup. An additional particle, the Higgs boson, is introduced to spontaneously break the $S U(2)_{L} \times U(1)_{Y}$ invariance down to a $U(1)$ subgroup representing 
Table 2.1: Properties of Particles in the Standard Model

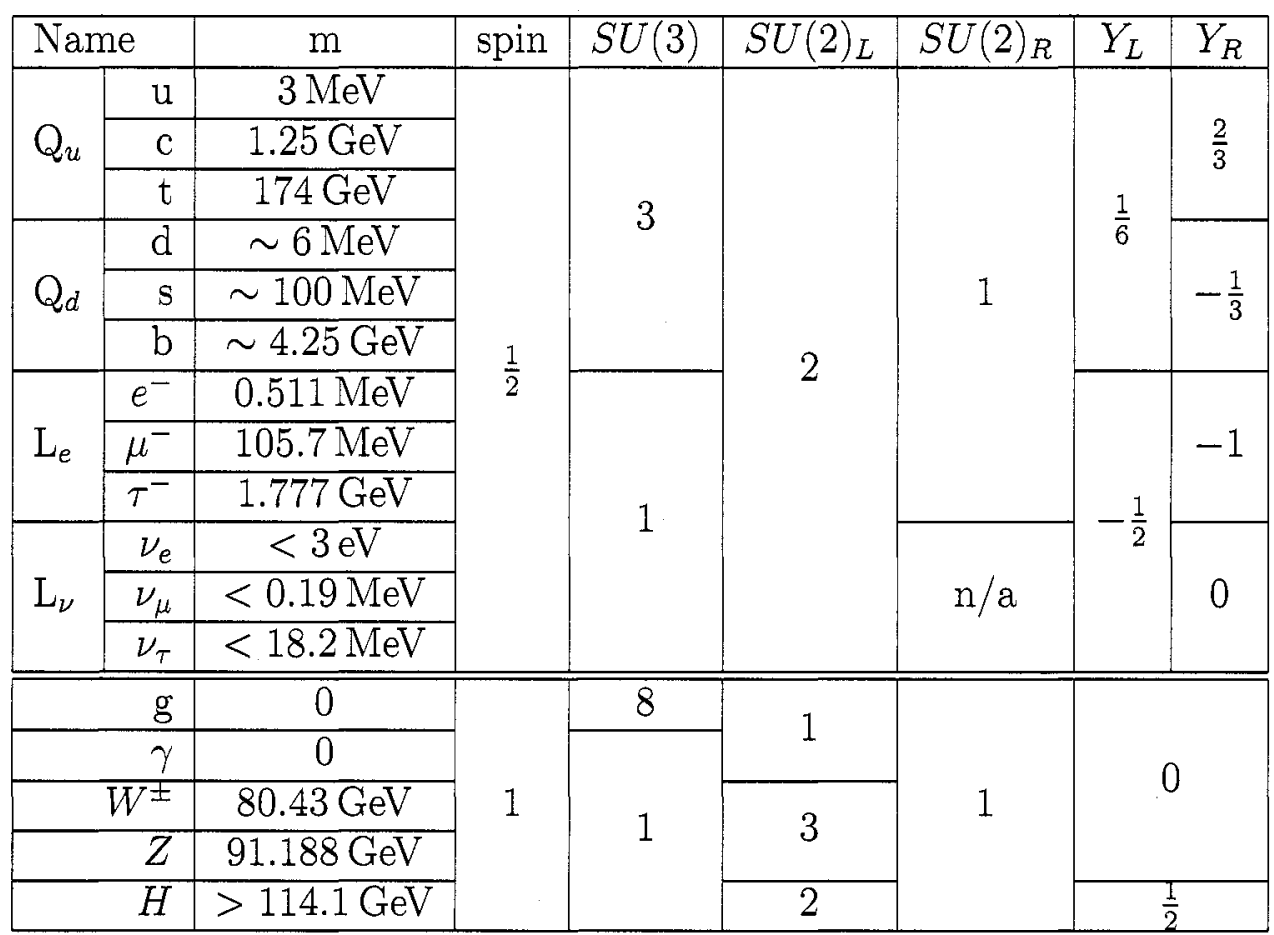


QED gauge invariance. This mechanism gives mass to the mediators of the weak interactions while keeping the photon massless, which accounts for the short range (and therefore weakness) of the weak interactions.

In this form, the Standard Model consists of 18 parameters: three coupling constants, nine particle masses, four parameters describing the mass mixing matrix between $u$ and d-type quarks of different generations (three mixing angles and a phase), and two parameters describing the Higgs sector, which can be taken to be the vacuum expectation value of the original Higgs field and the mass of the Higgs boson after symmetry breaking. Of these parameters, the first 17 have been measured to varying levels of precision, while there are only loose bounds on the Higgs mass, as described in Section 2.1.2.

In spite of this wealth of parameters, this minimal version of the Standard Model needs several extensions: first, the recent measurement of flavor oscillations between different neutrino flavors $[12,13]$ opens the possibility that right-handed neutrinos could also exist, which would require an additional set of 3 masses and possibly a mass mixing matrix. However due to the smallness of the neutrino masses, these right-handed neutrinos would couple extremely weakly to the other SM fields; their presence thus would not change the Standard Model in a fundamental way and they are ignored in the rest of this document. Secondly, to account for the absence of a $\mathrm{CP}$-breaking term in the strong interactions, an additional particle, the axion needs to be introduced, along with a parameter describing its potential. Again, no measurable effects are expected in the processes described in this work. Finally, the SM is divergent at high energy, and therefore must cease to be valid above a certain mass scale $M$. In the past it was hypothesized that this would be associated with the onset of gravitational effects, with $M$ of the order of the Planck mass, and that the physics at energies above $M$ would be described by a "Grand Unified Theory" that described the strong, weak and electromagnetic forces together with gravity in a consistent manner. However this met with two problems:

- If a "Grand Unified Theory" is to be found, the strength of the three usual interactions must match at some mass scale $M_{G U T}$. For the Standard Model, such a mass does not seem to exist. 
- If the mass scale for the GUT is of the order of the Planck mass, there is no reason for the Higgs boson mass to be at the $100 \mathrm{GeV}$ scale that is necessary for electroweak symmetry breaking. In a renormalizable quantum field theory the

physical mass is the sum of the original mass appearing in the Lagrangian and radiative corrections, which should be of the order of the Planck scale. For the physical mass to be small, a cancellation of 34 orders of magnitudes is required; while this is not impossible per se, it is "unnatural". The same problem does not occur for fermion masses since the radiative corrections would only scale as the logarithm of the Planck mass.

Several models have been put forward to remedy these problems, such as supersymmetry, large extra dimensions, etc. Most predict that New Physics beyond the SM should occur at a mass scale of the order of $1 \mathrm{TeV}$.

\subsubsection{Electroweak symmetry breaking, Global fits}

As described in the previous section, the current consensus is that beyond-the-SM physics should occur around the $\mathrm{TeV}$ scale. While these energies will soon be accessible directly at LHC, the effects of this new physics may already be observable indirectly through the radiative corrections that they induce in lower-energy processes. This is illustrated in Fig. 2.1, which shows the leading contributions to the radiative corrections on the $W$ boson mass, due to effects of the Higgs boson and top quark.

The precision electroweak measurements done at LEP, SLD [14] and Fermilab are particularly well-suited for this task, since they are both very precise and at high enough energy to be potentially sensitive to physics at $\mathrm{TeV}$ mass scales. This has led to the effort to perform "Global Fits" to the electroweak sector of the Standard Model, where a number of electroweak observables are fitted to theory predictions based on the SM [1].

Not all the 18 SM parameters listed above need to be included in such a treatment: the masses of all the fermions except the top quark are sufficiently small and well-known that they can be treated as constants, and the mass mixing matrix (or 

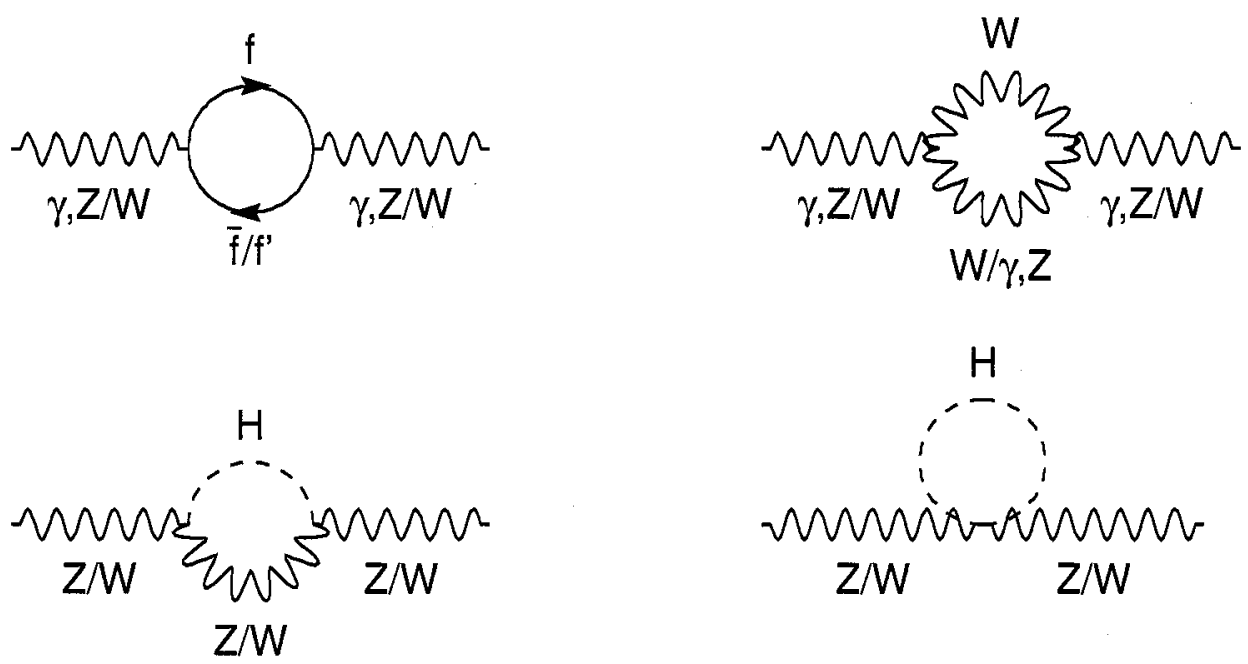

Figure 2.1: Radiative corrections to the gauge boson propagators due to boson and fermion loops.

matrices) similarly decouples. The Fermi constant $G_{F}$, which gives the strength of the weak interaction, is known to $9 \mathrm{ppm}$ [15] through measurements of the muon lifetime and can also be treated as a constant. This leaves 5 parameters: the top quark mass $m_{t}$, the $Z$ boson mass $m_{Z}$, the mass of the SM Higgs boson $m_{H}$, and the values $\alpha_{S}\left(m_{Z}^{2}\right)$ and $\alpha\left(m_{Z}^{2}\right)$ of the QCD and QED coupling constants at $q^{2}=m_{Z}^{2}$. It may seem surprising at first that $\alpha\left(m_{Z}^{2}\right)$ should not be categorized among the better known quantities: $\alpha\left(q^{2}=0\right)$ is know to about 3 ppb [16], mainly from measurements of the anomalous magnetic moment of the electron [17] and the study of radiative transitions of ${ }^{133} \mathrm{Cs}$ and ${ }^{87} \mathrm{Rb}$ atoms $[18,19]$. However as described in section 2.2.1, the value of $\alpha$ increases slightly with increasing energy, primarily due to the effect of virtual fermions. These effects can be calculated from first principles in the case of leptons and for the top quark (which decays before having time to hadronize), but in the case of the five lightest quark flavors the effects must be determined from data. For this reason it is not $\alpha\left(m_{Z}^{2}\right)$ itself that enters the fit, but rather the quantity $\Delta \alpha_{\text {had }}^{(5)}\left(m_{Z}^{2}\right)$ expressing the variation in $\alpha$ between $q^{2}=0$ and $q^{2}=m_{Z}^{2}$ that is 
attributable to these five flavors.

These parameters are fitted from a total of 18 observables, which can be categorized as follows:

- $Z$ lineshape and decay parameters: the $Z$ lineshape and partial widths are parameterized by the mass $m_{Z}$, full width $\Gamma_{Z}$, the total hadronic cross-section $\sigma_{\text {had }}^{0}$ at the peak and the ratios $R_{l}^{0}=\Gamma_{\text {had }} / \Gamma_{\ell \ell}[20]$ of hadronic to leptonic decay widths for $\ell=e, \mu, \tau$. This particular set of variables is used due to the fact that the correlations between the variables are small.

- Leptonic forward-backward asymmetries: the forward-backward asymmetry $A_{F B}^{0, l}$ in $Z \rightarrow \ell^{+} \ell^{-}$decays are defined, for each charged lepton, as the ratio $A_{F B}=$ $\left(\sigma_{F}-\sigma_{B}\right) /\left(\sigma_{F}+\sigma_{B}\right)$ where $\sigma_{F}$ is the "forward" cross-section corresponding to the case where the outgoing $\ell^{-}$direction forms an acute angle with the incoming $e^{-}$direction and vice versa for the backward cross-section [20].

- Lepton polarization asymmetries: the measured asymmetries consist of the $\tau$ polarization asymmetry $A_{\ell}\left(P_{\tau}\right)$, measured at LEP [21] and the left-right asymmetry $A_{\ell}(S L D)$ measured at SLD [22] with polarized electron beams. $A_{\ell}\left(P_{\tau}\right)$ is defined as the ratio $\left(\sigma_{-}-\sigma_{+}\right) /\left(\sigma_{-}+\sigma_{+}\right)$of $Z \rightarrow \tau^{+} \tau^{-}$events with $\tau$ s of negative and positive helicity. The helicity can be inferred from the spectrum of the $\tau$ decay products (for instance in $\tau^{+} \rightarrow \pi^{+} \nu$ the pion is emitted preferentially in the $\tau$ spin direction). $A_{\ell}(S L D)$ is defined (in the case of $100 \%$ beam polarization) as $\left(\sigma_{L}-\sigma_{R}\right) /\left(\sigma_{L}+\sigma_{R}\right)$, where $\sigma_{L}$ is the production cross-section for $Z$ 's from a left-polarized electron bunch and vice versa. It provides the most sensitive single measurement available for the effective weak mixing angle $\sin ^{2} \theta_{e f f}^{l e p t}$.

- Heavy Flavors: for the processes $Z \rightarrow c \bar{c}$ and $Z \rightarrow b \bar{b}$, the quantities used are the ratios $R_{c}^{0}$ and $R_{b}^{0}$ of the partial widths to the total hadronic width [23], and the forward-backward asymmetries $A_{F B}^{c \bar{c}}$ and $A_{F B}^{b \bar{b}}$ [24], in both cases defined similarly to the case of leptons above. At SLD the asymmetry $A_{F B}^{c \bar{c}}$ and $A_{F B}^{b \bar{b}}$ are calculated with respect to the left- or right-handed polarization of the colliding 
Table 2.2: Results of the global EW fit from Ref. [30]

\begin{tabular}{lccccc}
\hline \hline \multirow{2}{*}{ Parameter } & \multirow{2}{*}{ Value } & \multicolumn{5}{c}{ Correlations } \\
& & $\Delta \alpha_{\text {had }}^{(5)}\left(m_{Z}^{2}\right)$ & $\alpha_{s}\left(m_{Z}^{2}\right)$ & $m_{Z}$ & $m_{t}$ \\
\hline$\Delta \alpha_{\text {had }}^{(5)}\left(m_{Z}^{2}\right)$ & $0.02767 \pm 0.00034$ & & & & \\
$\alpha_{s}\left(m_{Z}^{2}\right)$ & $0.1186 \pm 0.0027$ & +0.01 & & & \\
$m_{Z}$ & $91.1874 \pm 0.0021 \mathrm{GeV}$ & -0.01 & -0.02 & & \\
$m_{t}$ & $173.3 \pm 2.7 \mathrm{GeV}$ & -0.02 & +0.05 & -0.03 & \\
$\log m_{H}$ & $1.96 \pm 0.18$ & -0.51 & +0.11 & +0.07 & +0.52 \\
\hline \hline
\end{tabular}

bunch [25]. These left-right-forward-backward asymmetries, denoted by $A_{L R F B}^{c \bar{c}}$ and $A_{L R F B}^{b \bar{b}}$, have the advantage of probing the couplings of the $Z$ to quarks without involving its coupling to electrons.

- $W$ lineshape parameters: the mass $m_{W}$ and full width $\Gamma_{W}$ of the $W$, measured at Fermilab and LEP-II [26].

- top quark mass $m_{t}$, measured at Fermilab [27, 28].

- Hadronic forward-backward asymmetry: the quantity $Q_{F B}$ measured at LEP [29] is a forward-backward asymmetry measured in hadronic decays of the $\mathrm{Z}$ using jet charge techniques.

The fit results from Ref. [30] are shown in Table 2.2.

The object of these fits is twofold: mainly, it may indicate that the precision EW data is incompatible with the SM as described currently, which would constitute evidence for new physics. However, as shown in Fig. 2.2 there are no strong disagreements between the fitted and measured values for any of the observables. The pulls are dominated by the tension between $A_{f b}^{0, b}$ and $A_{\ell}(S L D)$, but although $A_{f b}^{0, b}$ is somewhat marginalized it is less than $3 \sigma$ from its central value. This particular discrepancy is also difficult to explain in terms of new physics.

More modestly, the fit provides an indirect measurement of $m_{H}$. The sensitivity of this determination is unfortunately decreased due to the fact that the radiative 


\begin{tabular}{|c|c|c|c|}
\hline & Measurement & Fit & $\left|O^{\text {meas }}-O^{\text {fit }}\right| / \sigma^{\text {meas }}$ \\
\hline$\Delta \alpha_{\text {had }}^{(5)}\left(m_{z}\right)$ & $0.02758 \pm 0.00035$ & 0.02767 & 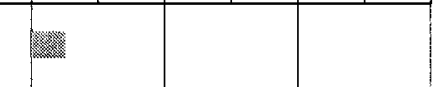 \\
\hline$m_{z}[\mathrm{GeV}]$ & $91.1875 \pm 0.0021$ & 91.1874 & 1 \\
\hline$\Gamma_{Z}[\mathrm{GeV}]$ & $2.4952 \pm 0.0023$ & 2.4959 & 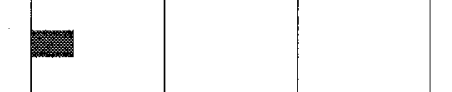 \\
\hline$\sigma_{\text {had }}^{0}[n b]$ & $41.540 \pm 0.037$ & 41.478 & $=$ \\
\hline $\mathrm{R}_{1}$ & $20.767 \pm 0.025$ & 20.742 & 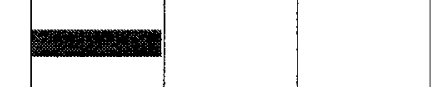 \\
\hline$A_{\mathrm{fb}}^{0,1}$ & $0.01714 \pm 0.00095$ & 0.01643 & m \\
\hline$A_{1}\left(P_{\tau}\right)$ & $0.1465 \pm 0.0032$ & 0.1480 & and \\
\hline $\mathrm{R}_{\mathrm{b}}$ & $0.21629 \pm 0.00066$ & 0.21579 & \\
\hline$R_{c}$ & $0.1721 \pm 0.0030$ & 0.1723 & I \\
\hline$A_{f b}^{0, b}$ & $0.0992 \pm 0.0016$ & 0.1038 & \\
\hline$A_{f b}^{0, c}$ & $0.0707 \pm 0.0035$ & 0.0742 & \\
\hline$A_{b}$ & $0.923 \pm 0.020$ & 0.935 & \\
\hline$A_{c}$ & $0.670 \pm 0.027$ & 0.668 & 1 \\
\hline$A_{1}(S L D)$ & $0.1513 \pm 0.0021$ & 0.1480 & \\
\hline $\sin ^{2} \theta_{\text {eff }}^{\text {lept }}\left(Q_{\mathrm{fb}}\right)$ & $0.2324 \pm 0.0012$ & 0.2314 & 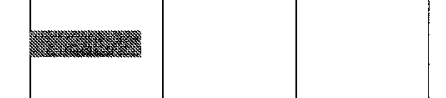 \\
\hline$m_{W}[\mathrm{GeV}]$ & $80.410 \pm 0.032$ & 80.377 & \\
\hline$\Gamma_{W}[\mathrm{GeV}]$ & $2.123 \pm 0.067$ & 2.092 & Ded \\
\hline \multirow[t]{2}{*}{$m_{t}[\mathrm{GeV}]$} & $172.7 \pm 2.9$ & 173.3 & a \\
\hline & & & 2 \\
\hline
\end{tabular}

Figure 2.2: Pull distribution for the global SM fit taken from Ref. [30]. 
corrections only depend on $\log m_{H}$, whereas their dependence on the top quark mass is quadratic. The result is illustrated in Fig. 2.3, taken from Ref [30].

The fitted Higgs mass is $m_{H}=91_{-32}^{+45} \mathrm{GeV}$, compared to the $114.1 \mathrm{GeV}$ lower bound set in direct searches. A light Higgs boson is therefore favored. As shown in Table 2.2, this value is mostly correlated with $m_{t}$ and $\Delta \alpha_{h a d}^{(5)}\left(m_{Z}^{2}\right)$, both of which have about a $50 \%$ correlation with $m_{H}$. If the measured value of $m_{t}$ were to decrease by $3 \mathrm{GeV}, m_{H}$ would shift downward by about $20 \mathrm{GeV}$, more than $20 \%$. The same effect would also be caused by a decrease of 0.00038 in $\Delta \alpha_{\text {had }}^{(5)}\left(m_{Z}^{2}\right)$, corresponding to a $1.4 \%$ variation. Since the precision in the top quark mass value is expected to improve as more data is collected by CDF and D0 [28], improving the determination of $\Delta \alpha_{\text {had }}^{(5)}\left(m_{Z}^{2}\right)$ is crucial in narrowing the allowed $m_{H}$ range.

\subsection{Determining $\Delta \alpha_{\text {had }}^{(5)}\left(m_{Z}^{2}\right)$}

\subsubsection{The Running of $\alpha_{Q E D}$}

As in any field theory, the effect of quantum corrections must be taken into account. We shall be mostly concerned with the QED sector of the SM. The one-loop-order corrections to QED are shown in Fig. 2.4.

These corrections affect the form of the fermion and photon propagators and of the vertex term. However the fermion current $J^{\mu}=\bar{\psi} \gamma^{\mu} \psi$ has the special property that its components are unambiguously normalized (for instance $J^{0}$ is the charge density associated with $\psi$ ) and it must therefore be unaffected by these corrections. If we write $\tilde{A}^{\mu}$ for the modified photon field, the effects of quantum corrections can be expressed as

$$
e J_{\mu} A^{\mu}(q) \rightarrow e J_{\mu} \tilde{A}^{\mu}(q)=e(q) J_{\mu} A^{\mu}(q),
$$

where the last relation defines an effective coupling constant $e\left(q^{2}\right)$. This "running" coupling constant is a function of energy, and arises only from corrections to the photon fields.

Let $\Pi^{\mu \nu}(q)$ be the most general insertion into the photon propagator that is "oneparticle irreducible" - that is, cannot be split into two insertions linked by a photon 


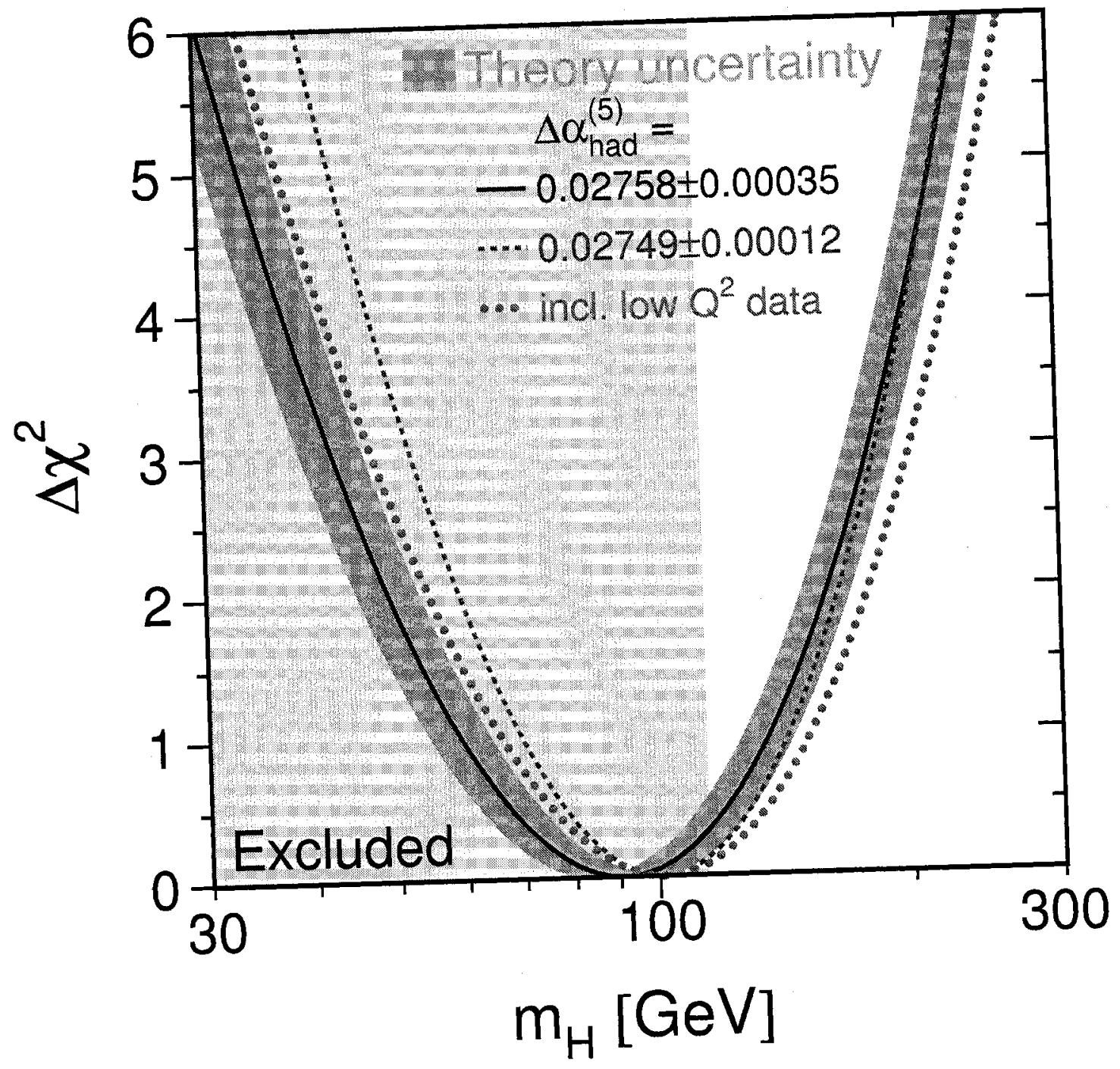

Figure 2.3: $\chi^{2}$ distribution for the Higgs mass, taken from Ref [30]. 

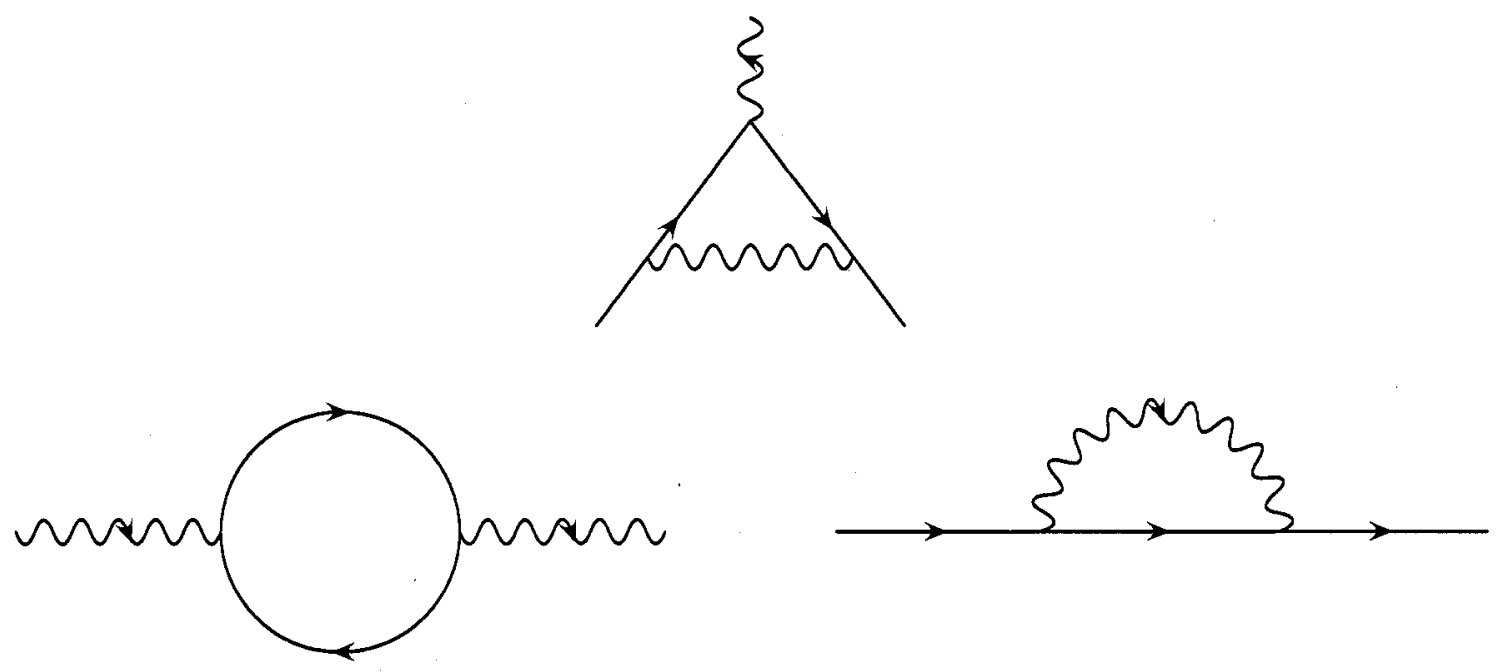

Figure 2.4: One-loop diagrams for QED

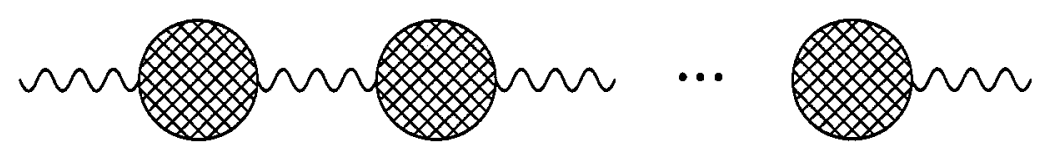

Figure 2.5: A term in the photon propagator perturbative series.

propagator. The most general photon propagator therefore corresponds to the sum of terms such as the ones shown in Fig. 2.5, with an arbitrary number of insertions. Using the expression

$$
D_{F}^{\mu \nu}(q)=\frac{-i g^{\mu \nu}}{q^{2}}
$$

for the tree-level photon propagator, the corrected propagator can be expressed as

$$
\tilde{D}_{F}^{\mu \nu}(q)=\frac{-i g^{\mu \nu}}{q^{2}}+\frac{-i g^{\mu \rho}}{q^{2}} \Pi^{\rho \sigma}(q) \frac{-i g^{\sigma \nu}}{q^{2}}+\frac{-i g^{\mu \rho}}{q^{2}} \Pi^{\rho \sigma}(q) \frac{-i g^{\sigma \lambda}}{q^{2}} \Pi^{\lambda \xi}(q) \frac{-i g^{\xi \nu}}{q^{2}}+\ldots
$$

By current conservation we must have $q^{\mu} \Pi^{\mu \nu}(q)=0$, which implies

$$
\Pi^{\mu \nu}(q)=\left(q^{2} g^{\mu \nu}-q^{\mu} q^{\nu}\right) \Pi\left(q^{2}\right)
$$

where $\Pi\left(q^{2}\right)$ is a scalar function of the momentum squared only. Then Eq. 2.3 can 
be summed to obtain

$$
\tilde{D}_{F}^{\mu \nu}(q)=\frac{-i g^{\mu \nu}}{q^{2}\left(1-\Pi\left(q^{2}\right)\right)} .
$$

We can therefore write

$$
\begin{aligned}
D_{F}^{\mu \nu}(q) \rightarrow \tilde{D}_{F}^{\mu \nu}(q) & =\frac{1}{1-\Pi\left(q^{2}\right)} D_{F}^{\mu \nu}(q) \\
A^{\mu}(q) \rightarrow \tilde{A}^{\mu}(q) & =A^{\mu}(q) \frac{1}{\sqrt{1-\Pi\left(q^{2}\right)}} \\
e_{0} \rightarrow e\left(q^{2}\right) & =\frac{e_{0}}{\sqrt{1-\Pi\left(q^{2}\right)}} \\
\alpha_{0} \rightarrow \alpha\left(q^{2}\right) & =\frac{\alpha_{0}}{1-\Pi\left(q^{2}\right)}=\frac{\alpha}{1-\left[\Pi\left(q^{2}\right)-\Pi(0)\right]},
\end{aligned}
$$

where $e_{0}$ is the bare electromagnetic coupling, $\alpha_{0}=e_{0}^{2} / 4 \pi$, and $\alpha$ the corresponding renormalized quantity. We therefore obtain

$$
\alpha\left(q^{2}\right)=\frac{\alpha}{1-\left[\Pi\left(q^{2}\right)-\Pi(0)\right]}=\frac{\alpha}{1-\Delta \alpha\left(q^{2}\right)} .
$$

\subsubsection{Hadronic production in $e^{+} e^{-}$annihilation}

The annihilation process $e^{+} e^{-} \rightarrow f \bar{f}$ is calculable from first principles if the outgoing fermions are leptons, with the well-known formula

$$
\sigma_{e^{+} e^{-\rightarrow \ell^{+} \ell^{-}}}^{0}(s)=\frac{4 \pi \alpha^{2}}{3 s}
$$

for the massless case and in the Born approximation. For quarks however, hadronization effects occur simultaneously with the scattering, and the annihilation crosssection is shaped by strong resonances. This is illustrated in Fig. 2.6 : near the production thresholds, the cross-section is shaped by numerous sharp resonances. However the resonant effects are damped as the center-of-mass energy $\sqrt{s}$ of the collision rises. This is a consequence of the fact that QCD is an asymptotically free theory: as the energy rises the coupling constant $\alpha_{s}$ decreases, and strong interactions become increasingly small corrections to the hard scattering process. Hadronization 


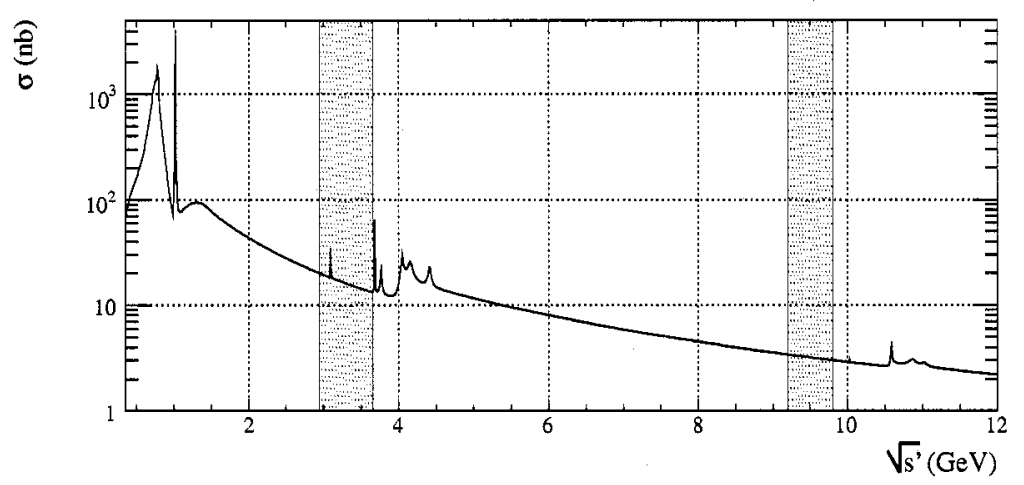

Figure 2.6: Hadronic cross-section as a function of collision CM energy at lowest order. The shaded regions show the $c \bar{c}$ and $b \bar{b}$ mass thresholds, with the lower and upper bounds of the shadings given by the $\overline{\mathrm{MS}}$ and pole masses respectively.

still occurs, but on a much longer time scale than the scattering and does not affect the total cross-section.

In the high-s limit ("2-jet" events) the quarks can be treated as free particles and the cross-section calculated in the same way as for leptons. One must include a factor $q_{f}^{2}$ for the square of the quark charge and an additional factor 3 for color, so that

$$
\sigma_{e^{+} e^{-} \rightarrow \text { hadrons }}^{0, \text { Asmp. }}(s)=\left(3 \sum_{f} q_{f}^{2}\right) \sigma_{e^{+} e^{-} \rightarrow \ell^{+} \ell^{-}}^{0}(s)
$$

with the sum over the number of accessible quark flavors. The exponent " 0 " is used to indicate to indicate the Born-level (first order) expression only. For quarks the Bornlevel expression corresponds to the parton model [31], where quarks are assumed to interact only electromagnetically. Additional corrections must also be applied, as described in Section 2.2.5

For arbitrary $s$, it is customary to introduce the Drell Ratio $R_{\text {had }}(s)$ by

$$
R_{\text {had }}(s)=\frac{\sigma_{e^{+} e^{-} \rightarrow \text { hadrons }}(s)}{\sigma_{e^{+} e^{-} \rightarrow \ell^{+} \ell^{-}}^{0}(s)}
$$

The variations of $R_{\text {had }}$, as modeled by the KKMC generator (see Appendix A) are 
shown in Fig. 2.7. Measured values of $R_{\text {had }}$ and the total hadronic cross-sections, as compiled by the Particle Data Group [15], are shown in Fig. 2.8. As expected, the resonances cluster around the production thresholds, separated by plateaus at $R_{\text {had }}=2,10 / 3$ and $11 / 3$ corresponding to the asymptotic values

$$
R_{0}=3 \sum_{f} q_{f}^{2}
$$

for 3,4 and 5 quark flavors.

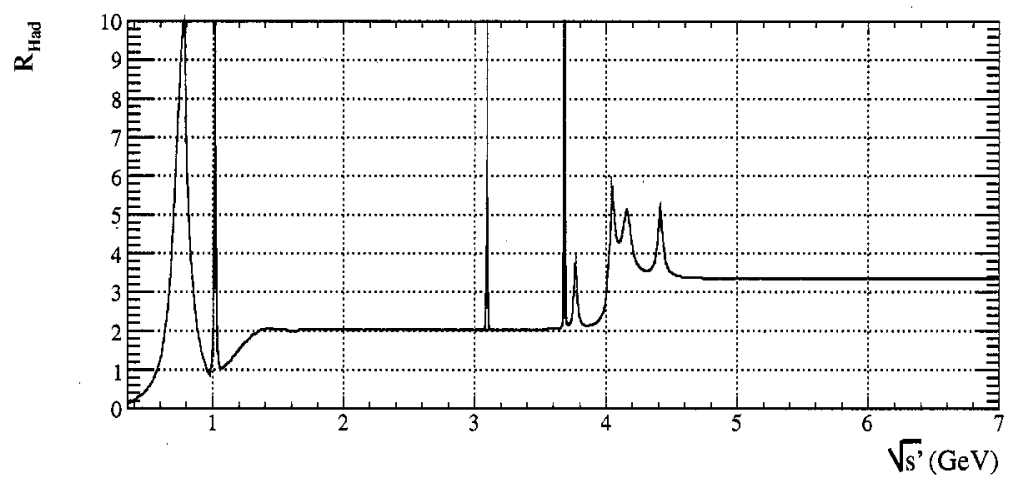

Figure 2.7: Parameterization of $R_{\text {had }}$ used by the KKMC event generator.

\subsubsection{Calculation of $\Pi_{H a d}(s)$}

In the Standard Model the first-order contributions are fermion loop corrections to the photon propagator, such as the one shown in the bottom left of Fig. 2.4, where the fermion in the loop is either a charged lepton or a quark. One can therefore decompose $\Delta \alpha\left(q^{2}\right)$ as

$$
\Delta \alpha\left(q^{2}\right)=\Delta \alpha_{L e p}\left(q^{2}\right)+\Delta \alpha_{T o p}\left(q^{2}\right)+\Delta \alpha_{H a d}^{(5)}\left(q^{2}\right) .
$$

This is motivated by the fact that, as for $e^{+} e^{-}$annihilation, the calculation of such diagrams is straightforward for leptons and for the top quark, which is too short-lived to be affected by hadronization. The leptonic contribution has been calculated to 

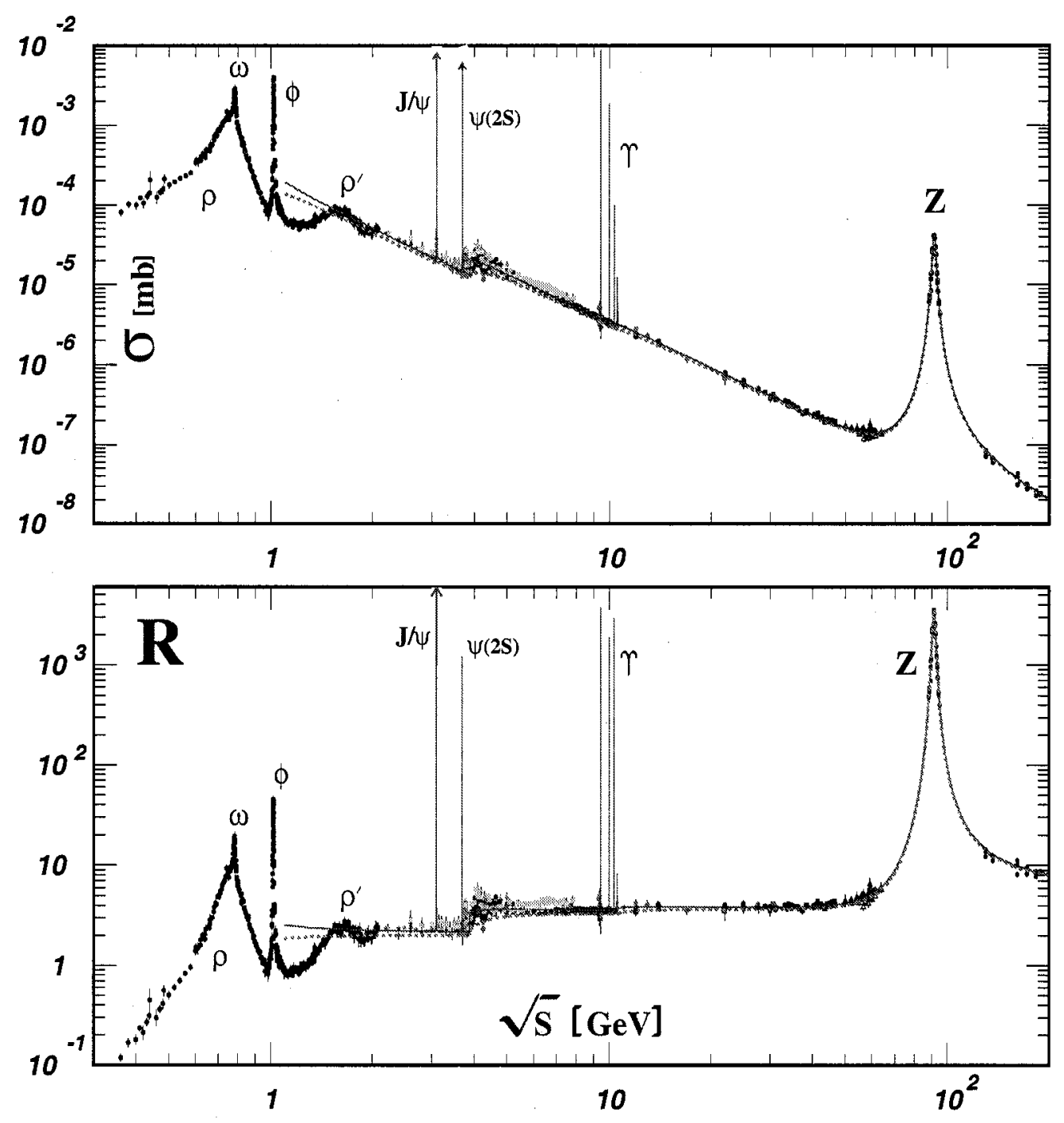

Figure 2.8: Measurements of the total hadronic cross-section and $R_{\text {had }}$ as a function of collision center-of-mass energy, taken from Ref. [15]. 
three loops [32], as has the top contribution [33], with the values

$$
\begin{aligned}
\Delta \alpha_{\text {Lep }}\left(m_{Z}^{2}\right) & =3149.7686 \times 10^{-5} \\
\Delta \alpha_{\text {Top }}\left(m_{Z}^{2}\right) & =(-7.0 \pm 0.5) \times 10^{-5}
\end{aligned}
$$

However the $\Delta \alpha_{\text {Had }}^{(5)}\left(q^{2}\right)$ term is problematic since it is affected by non-perturbative QCD, as illustrated in Fig. 2.9, which prevents a calculation from first principles.
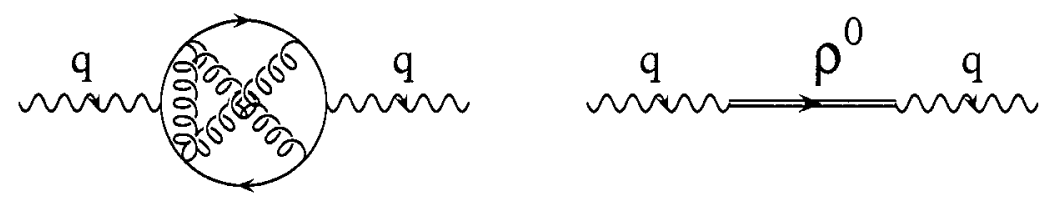

Figure 2.9: QCD corrections to the photon propagator.

However one can go further by relying on the general properties of $\Pi(s)$. These fall into two categories, analyticity and unitarity.

\section{Analyticity}

Since in the Standard Model photons only couple to fermions, the diagrammatic relation shown in Fig. 2.10 holds. Defining

$$
i \Pi^{\mu \nu}(q)=-e^{2} \int d^{4} x e^{-i q x}\left\langle 0\left|T J^{\mu}(x) J^{\nu}(0)\right| 0\right\rangle,
$$

it can be shown that

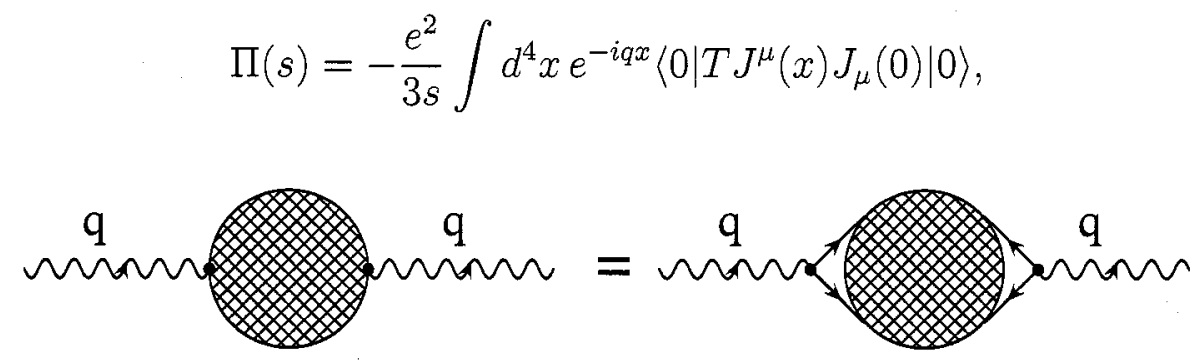

Figure 2.10: Diagrammatic relation for the photon vacuum polarization. 
where $T$ expresses time-ordering and $s=q^{2}$. Since the vacuum is translationinvariant,

$$
\left\langle 0\left|T J^{\mu}(x) J_{\mu}(0)\right| 0\right\rangle=\left\langle 0\left|T J^{\mu}(0) J_{\mu}(-x)\right| 0\right\rangle=\left\langle 0\left|T J_{\mu}(-x) J^{\mu}(0)\right| 0\right\rangle
$$

and $\Pi(s)$ is a real function, as it should be. It also has the analytic properties of a two-point function [34].

However for $s>0$, real particles such as leptons and hadrons may be created in the loop. If we limit ourselves to hadrons, this first occurs for $q^{2}=4 m_{\pi}^{2}$, when a real two-pion state becomes allowed. This introduces a branch cut singularity in $\Pi(s)$ for $s>4 m_{\pi}^{2}$. In principle pole singularities could also be created for bound states below threshold, but this doesn't happen for hadrons (although it would if charm was the lightest quark).

The region where we need to evaluate $\Pi(s)$ is precisely in the physical region where the branch cut occurs. The way around this difficulty is to analytically continue $\Pi(s)$ to a complex function of a complex variable. Since the original $\Pi(s)$ was analytic and real the continuation is also real, in the sense that $\Pi\left(s^{*}\right)=\Pi^{*}(s)$, and analytic everywhere except on the branch cut.

Using the analyticity, $\Pi\left(s_{0}<4 m^{2}\right)$ can be written as a contour integral

$$
\Pi\left(s_{0}\right)=\frac{1}{2 i \pi} \int_{\mathcal{C}} \frac{\Pi(s)}{s-s_{0}} d s .
$$

We use the contour $\mathcal{C}$ shown in Fig. 2.11, with the straight section at an arbitrarily small distance $\epsilon$ to the branch cut. The integral over the arc that closes the contour can be ignored since it vanishes once the subtraction $\Pi\left(s_{0}\right)-\Pi(0)$ is performed. We have:

$$
\begin{aligned}
\Pi\left(s_{0}\right) & =\frac{1}{2 i \pi} \int_{4 m_{\pi}^{2}}^{\infty} \frac{\Pi(s+i \epsilon)}{s+i \epsilon-s_{0}}-\frac{\Pi(s-i \epsilon)}{s-i \epsilon-s_{0}} d s \\
& \stackrel{\epsilon \rightarrow 0}{=} \frac{1}{2 i \pi} \int_{4 m_{\pi}^{2}}^{\infty} \frac{\left(s-s_{0}\right)(\Pi(s+i \epsilon)-\Pi(s-i \epsilon))}{\left(s-s_{0}\right)^{2}+\epsilon^{2}} d s \\
& =\frac{1}{2 i \pi} P \int_{4 m_{\pi}^{2}}^{\infty} \frac{\operatorname{Disc} \Pi(s)}{s-s_{0}} d s,
\end{aligned}
$$




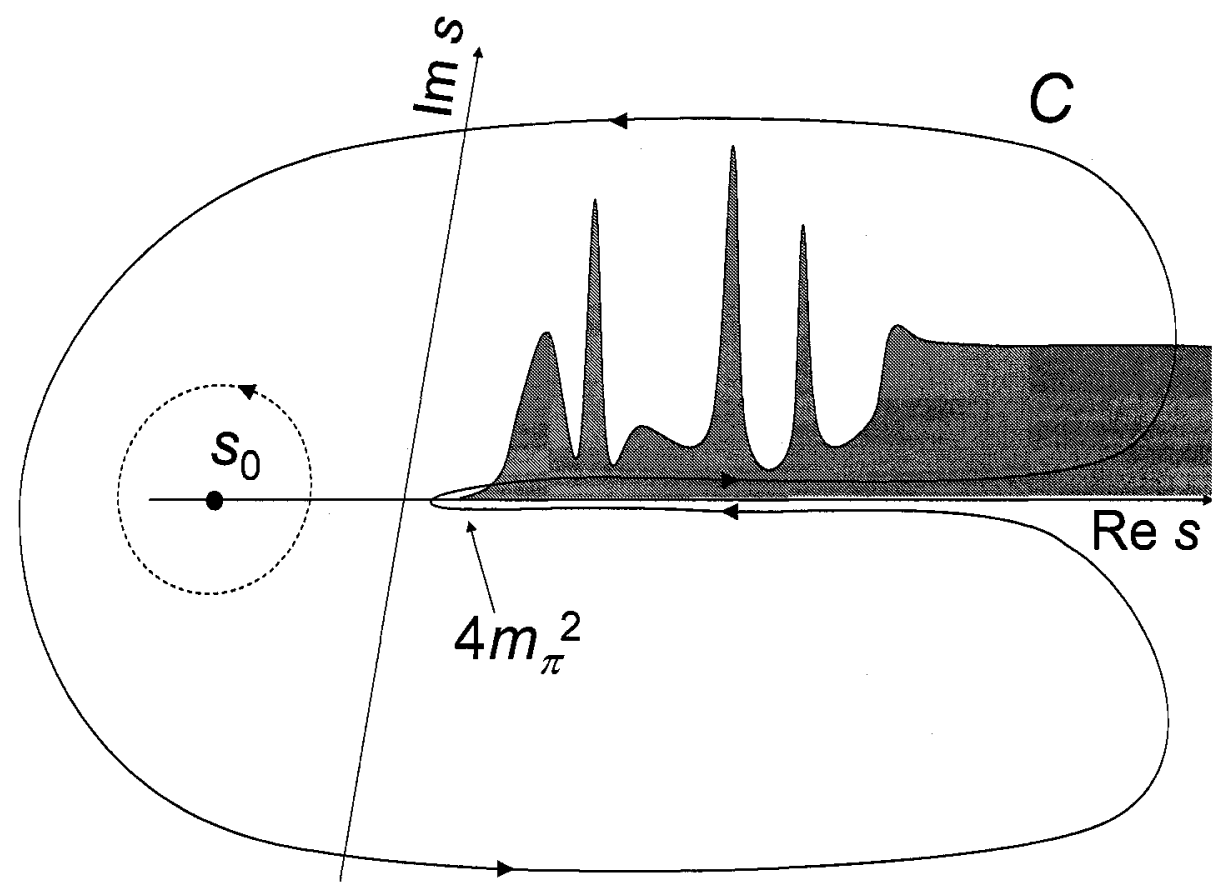

Figure 2.11: Integration contour for $\Pi(s)$ in the complex $s$ plane

where $P$ denotes the principal value of the integral and Disc $\Pi(s)$ is the discontinuity of $\Pi$ across the branch cut, which is $\operatorname{Disc} \Pi(s)=2 i \operatorname{Im} \Pi(s)$ since $\Pi$ is real.

\section{Unitarity}

The other constraint is unitarity, which as expressed by the Optical Theorem relates the imaginary part of amplitudes for physical process to the forward scattering crosssection of split diagrams, as illustrated in Fig. 2.12. This translates to

$$
\operatorname{Im} \mathcal{M}_{H a d}=2 s \sigma_{e^{+} e^{-} \rightarrow \operatorname{hadrons}}(s),
$$

where $\mathcal{M}_{\text {Had }}$ is the amplitude for the diagram of Fig. 2.12 in the forward scattering limit. After a straightforward calculation, we get

$$
\operatorname{Im} \mathcal{M}_{H a d}=-8 \pi \alpha \operatorname{Im} \Pi_{H a d}(s)
$$




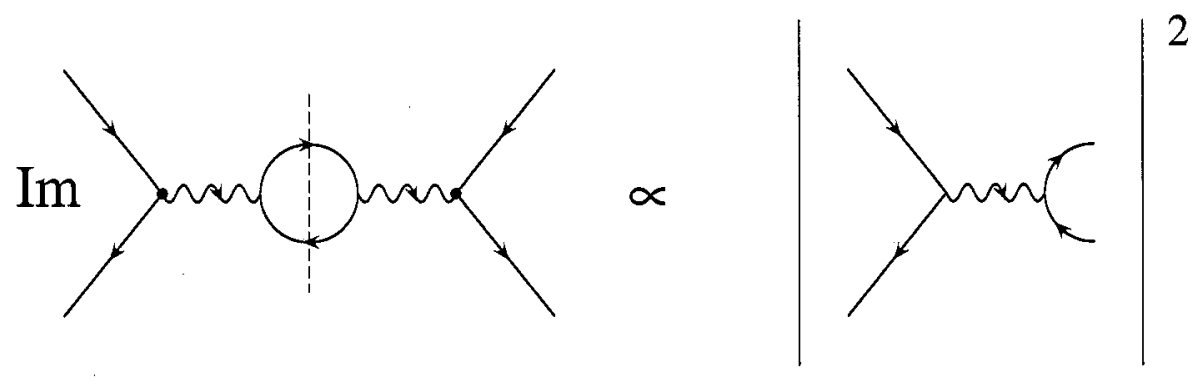

Figure 2.12: The Optical Theorem in diagrammatic form

and so

$$
\operatorname{Im} \Pi_{H a d}(s)=-\frac{s}{4 \pi \alpha} \sigma_{e^{+} e^{-} \rightarrow \text { hadrons }}(s) .
$$

We can rewrite the integral for the hadronic contribution as

$$
\begin{aligned}
\Pi_{H a d}\left(s_{0}\right) & =\frac{1}{\pi} P \int_{4 m_{\pi}^{2}}^{\infty} \frac{\operatorname{Im} \Pi_{H a d}(s)}{s-s_{0}} d s \\
& =-\frac{1}{4 \pi^{2} \alpha} P \int_{4 m_{\pi}^{2}}^{\infty} \frac{s d s}{s-s_{0}} \sigma_{e^{+} e^{-} \rightarrow \text { hadrons }}(s)
\end{aligned}
$$

and finally

$$
\Delta \alpha_{H a d}\left(s_{0}\right)=-\frac{s_{0}}{4 \pi^{2} \alpha} P \int_{4 m_{\pi}^{2}}^{\infty} \frac{\sigma_{e^{+} e^{-} \rightarrow \text { hadrons }}(s)}{s-s_{0}} d s=-\frac{\alpha s_{0}}{3 \pi} P \int_{4 m_{\pi}^{2}}^{\infty} \frac{R_{h a d}(s)}{s\left(s-s_{0}\right)} d s
$$

We have therefore obtained a formula for $\Delta \alpha_{\text {Had }}\left(s_{0}<4 m_{\pi}^{2}\right)$ in terms of $\sigma_{e^{+} e^{-} \rightarrow \text { hadrons }}(s)$, which can be obtained from experiment - the next best thing after a calculation from first principles. Furthermore, the expression is regular even for $s_{0}>4 m_{\pi}^{2}$ since the simple pole at $s_{0}$ is removed by the principal value. In the rest of this document we will use $s_{0}=m_{Z}^{2}$, which is the quantity relevant for standard model fits.

\subsubsection{ITEP Sum Rules and Global Duality}

An interesting property of $R_{\text {had }}(s)$ can be obtained in similar fashion. If we take $s_{0}=-Q_{0}^{2}$ with $Q_{0}$ large enough that $\mathrm{pQCD}$ is applicable, then $\Pi_{H a d}\left(s_{0}\right)$ can be calculated from first principles. It is equal to the QED value $\Pi_{Q E D}\left(s_{0}\right)=-\frac{\alpha}{3 \pi} \log \left(s_{0}\right)$ 
times the usual factor $R_{0}=3 \sum_{f} q_{f}^{2}$ which account for the color factor and quark charges, and also happens to be the asymptotic value of $R_{\text {had }}(s)$ for these flavors. Then by differentiating Eq. 2.21 once with respect to $s_{0}$, we get

$$
-R_{0} \frac{\alpha}{3 \pi s_{0}}=-\frac{\alpha}{3 \pi} P \int_{4 m_{\pi}^{2}}^{\infty} \frac{R_{h a d}(s)}{\left(s+Q_{0}^{2}\right)^{2}} d s
$$

This is a special case of the ITEP sum rules [35]. If we assume that $R_{\text {had }}(s)$ is in the asymptotic regime $R_{\text {had }}(s) \approx R_{0}$ for $s>\Lambda$ with $\Lambda \ll Q_{0}^{2}$, we can rewrite

$$
\frac{R_{0}}{s_{0}} \approx \int_{4 m_{\pi}^{2}}^{\Lambda} \frac{R_{h a d}(s)}{Q_{0}^{4}} d s+P \int_{\Lambda}^{\infty} \frac{R_{0}}{\left(s+Q_{0}^{2}\right)^{2}} d s
$$

so that

$$
\frac{1}{\Lambda} \int_{4 m_{\pi}^{2}}^{\Lambda} R_{h a d}(s) d s=\frac{s_{0}}{\Lambda+s_{0}} R_{0} \stackrel{s_{0}}{\vec{\approx}^{\infty}} R_{0}
$$

In other words, although the shape of $R_{\text {had }}(s)$ is locally altered by resonant effects the average value of its peaks and dips must be the same as in the parton model case.

\subsubsection{Correction to $R_{\text {had }}(s)$ due to the running of $\alpha_{s}$}

In Section 2.2.2, $R_{\text {had }}$ was given away from thresholds by the parton model value of Eq. 2.10, which only takes into account the electromagnetic interactions of quarks. Since quarks carry color, QCD effects also occur in the final state, as is obvious from the resonance structures near thresholds. In the high- $Q^{2}$ regions however $\alpha_{s}$ is small due to the asymptotic freedom of QCD; this justifies the parton model approximation, but also makes it possible to treat QCD corrections in these regions perturbatively, in the same way as QED corrections. Fig. 2.13 illustrates the leading-order terms in this expansion. As for QED the corrections are given in powers of $\alpha_{s} / \pi$. Corrections to this relation have been calculated to third order in the $\overline{\mathrm{MS}}$ scheme [36], giving

$$
R_{h a d}(s)=R_{h a d}^{0}(s)\left[1+\frac{\alpha_{s}}{\pi}+1.411\left(\frac{\alpha_{s}}{\pi}\right)^{2}-12.8\left(\frac{\alpha_{s}}{\pi}\right)^{3}\right]
$$




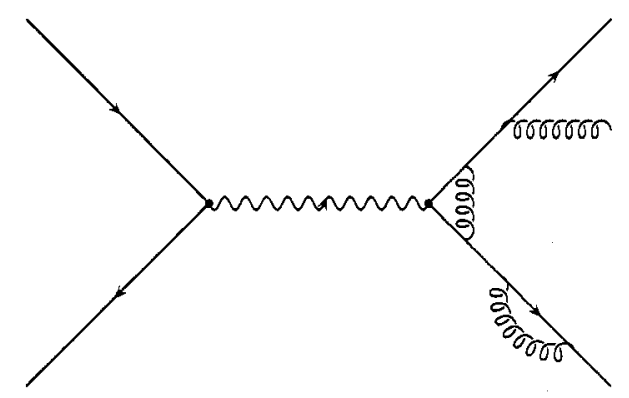

Figure 2.13: Perturbative QCD corrections to $R_{\text {had }}$

These corrections have the effect of making $R_{\text {had }}(s)$ dependent on the local value of $\alpha_{s}$. Since $\alpha_{s}$ decreases as $s$ increases, this results in a gradual decrease in the plateaus of $R_{\text {had }}(s)$ with increasing $s$. (Note that this formula is only valid for massless quarks the generalization to massive quarks has been done [37] but it is not used in this work).

This ingredient happens to be missing from the KKMC parameterization of $R_{\text {had }}$. To correct it and get the correct $s$ dependence for $R_{\text {had }}$ we need to know $\alpha_{s}(s)$. This has been calculated beyond three-loop order in the $\overline{\mathrm{MS}}$ scheme [38]. We use the three-loop expression from Ref. [39], which is given by the perturbation series

$$
\alpha_{s}(s)=\alpha_{s}^{(1)}(s)+\alpha_{s}^{(2)}(s)+\alpha_{s}^{(3)}(s)+\cdots
$$

with the terms

$$
\begin{aligned}
\alpha_{s}^{(1)}(s) & =\frac{\alpha_{s}\left(s_{0}\right)}{1+\alpha_{s}\left(s_{0}\right) \frac{\beta_{0}}{4 \pi} \log \frac{s}{s_{0}}} \\
\alpha_{s}^{(2)}(s) & =-\left(\alpha_{s}^{(1)}(s)\right)^{2} b_{1} \log K(s) \\
\alpha_{s}^{(3)}(s) & =-\left(\alpha_{s}^{(1)}(s)\right)^{3}\left[b_{1} \log K(s)(\log K(s)-1)-\left(b_{1}^{2}-b_{2}\right)(1-K(s))\right],
\end{aligned}
$$


where the $\beta_{i}$ are the $\beta$ functions of QCD in $\overline{\mathrm{MS}}$, given by

$$
\begin{aligned}
& \beta_{0}=11-\frac{2}{3} n_{f} \\
& \beta_{1}=102-\frac{38}{3} n_{f} \\
& \beta_{2}=\frac{1}{2}\left(2857-\frac{5033}{9} n_{f}+\frac{325}{27} n_{f}^{2}\right),
\end{aligned}
$$

$n_{f}$ is the number of quark flavors with $\overline{\mathrm{MS}}$ masses below $\sqrt{s}$ and

$$
\begin{aligned}
b_{1} & =\frac{\beta_{1}}{4 \pi \beta_{0}} \\
b_{2} & =\frac{\beta_{2}}{(4 \pi)^{2} \beta_{0}} \\
K(s) & =\frac{\alpha_{s}\left(s_{0}\right)}{\alpha_{s}^{(1)}(s)} .
\end{aligned}
$$

These expressions give the value of $\alpha_{s}$ at $s$ in terms of a reference value $\alpha_{s}\left(s_{0}\right)$. In the literature $\alpha_{s}\left(m_{Z}^{2}\right)$ is normally used as a reference, with the value [15]:

$$
\alpha_{s}\left(m_{Z}^{2}\right)=0.1187 \pm 0.0020
$$

When evolving $\alpha_{s}$ down from $m_{Z}^{2}$ one must also decrease $n_{f}$ when crossing mass thresholds. In order for the radiative corrections to stay consistent one must then apply a shift to $\alpha_{s}$ at some value $s=s_{t h r}$ close to the threshold, given by [39]:

$$
\begin{aligned}
\alpha_{s}^{n_{f}-1}\left(s_{t h r}\right) & =\alpha_{s}^{n_{f}}\left(s_{t h r}\right)-\frac{\left(\alpha_{s}^{n_{f}}\left(s_{t h r}\right)\right)^{2}}{3 \pi} \log \frac{m_{q}\left(s_{t h r}\right)}{\sqrt{s_{t h r}}} \\
& +\frac{\left(\alpha_{s}^{n_{f}}\left(s_{t h r}\right)\right)^{3}}{9 \pi^{2}}\left[\left(\log \frac{m_{q}\left(s_{t h r}\right)}{\sqrt{s_{t h r}}}\right)^{2}+\frac{33}{4} \log \frac{m_{q}\left(s_{t h r}\right)}{\sqrt{s_{t h r}}}+\frac{7}{8}\right]
\end{aligned}
$$

where $m_{q}\left(s_{t h r}\right)$ is the $\overline{\mathrm{MS}}$ value of the quark mass. In terms of $m_{q}=m_{q}\left(m_{q}^{2}\right)$, the 
quark mass evaluated at its own value,

$$
\begin{aligned}
\alpha_{s}^{n_{f}-1}\left(s_{t h r}\right) & =\alpha_{s}^{n_{f}}\left(s_{t h r}\right)-\frac{\left(\alpha_{s}^{n_{f}}\left(s_{t h r}\right)\right)^{2}}{3 \pi} \log \frac{m_{q}}{\sqrt{s_{t h r}}} \\
& -\frac{\left(\alpha_{s}^{n_{f}}\left(s_{t h r}\right)\right)^{3}}{9 \pi^{2}}\left[\left(\log \frac{m_{q}}{\sqrt{s_{t h r}}}\right)^{2}+\frac{57}{4} \log \frac{m_{q}}{\sqrt{s_{t h r}}}+\frac{7}{8}\right] .
\end{aligned}
$$

It seems sensible to use $s_{t h r}=m_{q}^{2}$ to make the logs vanish, but a better choice is to choose a value $\hat{m}_{q}^{2}$ such that the shift cancels altogether. If not for the $\alpha_{s}^{3}$ term this would occur for $\hat{m}_{q}=m_{q}$, and by expanding to first order in $\alpha_{s}$ we get

$$
\begin{array}{r}
\log \frac{m_{q}}{\hat{m}_{q}}=\frac{7}{24 \pi} \alpha_{s}\left(m_{q}\right) \\
\hat{m}_{q}=m_{q}\left(1-\frac{7}{24 \pi} \alpha_{s}\left(m_{q}\right)\right) .
\end{array}
$$

By using the $\hat{m}_{q}$ as transition points between the $n_{f}$ and $n_{f}-1$ expressions of $\alpha_{s}$ we avoid the shift of Eq. 2.35, and the evolution to low $s$ values can be done as follows:

- Evolve $\alpha_{s}$ from $m_{Z}^{2}$ to $m_{b}^{2}$ using Eqs. 2.26 to 2.32 with $n_{f}=5$

- Use the value of $\alpha_{s}\left(m_{b}^{2}\right)$ to calculate $\hat{m}_{b}^{2}$

- Evolve $\alpha_{s}$ from $m_{Z}^{2}$ to $\hat{m}_{b}^{2}$, again with $n_{f}=5$.

- Evolve $\alpha_{s}$ down from $\hat{m}_{b}^{2}$, using $\alpha_{s}\left(\hat{m}_{b}^{2}\right)$ as reference and $n_{f}=4$.

- Proceed in the same way to cross the charm threshold of necessary.

The procedure is supposed to not depend strongly on the values used for the quark masses. We use [15] $m_{b}=4.7 \pm 0.2 \mathrm{GeV}$ and $m_{c}=1.3 \pm 0.2 \mathrm{GeV}$.

The resulting $\alpha_{s}(s)$ shape is shown in Fig. 2.14. The effects on the $R_{\text {had }}(s)$ spectrum are shown in Fig. 2.15. 


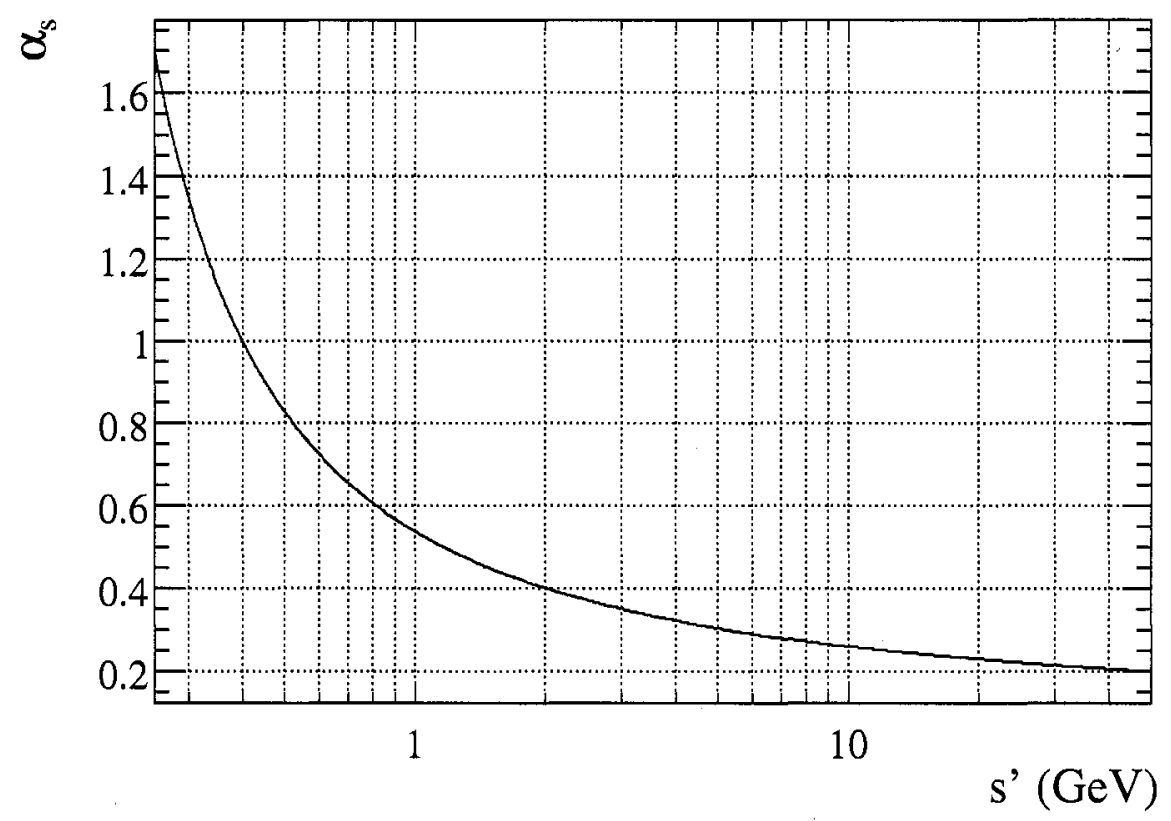

Figure 2.14: Variation of $\alpha_{s}$ as a function of $s=q^{2}$

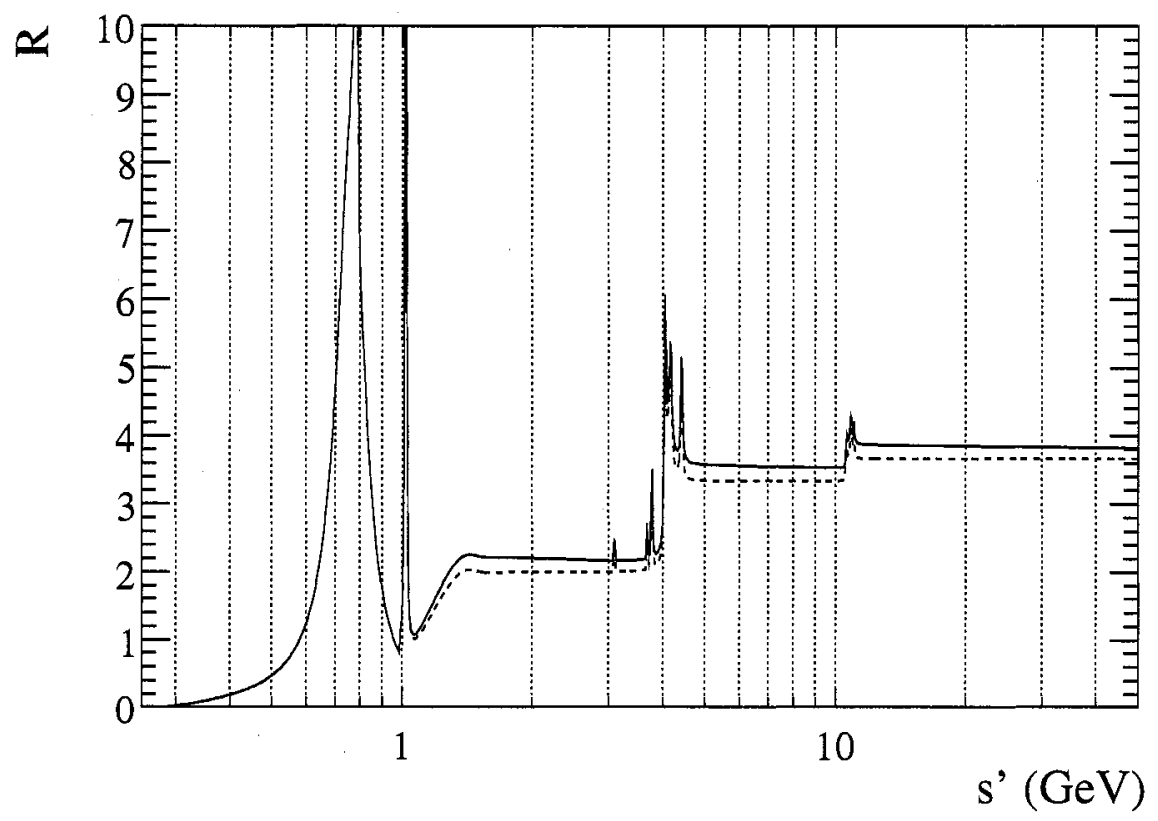

Figure 2.15: $R_{\text {had }}(\sqrt{s})$ shape before the $\alpha_{s}$ correction (dashed line) and after (solid line). 


\subsection{Measuring $\Delta \alpha_{\text {had }}^{(5)}\left(m_{Z}^{2}\right)$ using the Radiative Re- turn}

\subsubsection{Initial-State Radiation}

In $e^{+} e^{-}$annihilation, initial-state radiation (ISR) is the process by which a real photon is emitted by one of the incoming particles before annihilation. The annihilation therefore takes place at a reduced center-of-mass (CM) energy $s^{\prime}$ below the nominal collision CM energy $s$, given by

$$
s^{\prime}=s-2 E_{\gamma}^{*} \sqrt{s}
$$

where $E_{\gamma}^{*}$ is the CM energy of the ISR photon. It should be noted that this process is distinct from bremsstrahlung: in the case of ISR the emission of the photon is part of the interaction process, and the emitting particle is off-shell between the emission the ISR photon and the annihilation. In the case of bremsstrahlung, the photon emission is accompanied by a transfer of momentum to the surrounding material which allows the outgoing particle to remain on-shell (4-momentum conservation forbids the emission of a non-collinear photon by an on-shell particle); in this case the photon emission and $e^{+} e^{-}$annihilation can be considered as separate processes. The leading-order amplitude for the ISR process $e^{+} e^{-} \rightarrow \gamma X$, corresponding to the

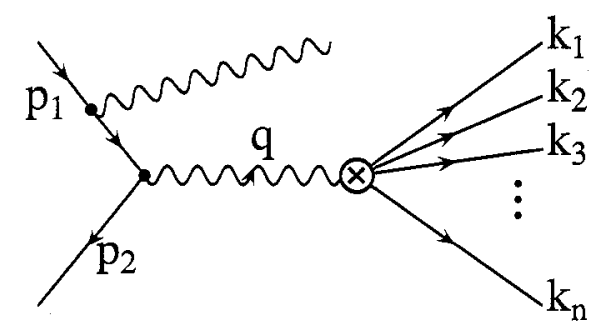

Figure 2.16: Leading-order diagram for ISR emission 
diagram in Fig 2.16, can be written as

$$
i \mathcal{M}_{\gamma X}=-e^{2} \mathcal{J}_{\text {in }}^{\mu} \frac{-g_{\mu \nu}}{s^{\prime}} \mathcal{J}_{\text {out }}^{\nu},
$$

where $\mathcal{J}_{\text {in }}^{\mu}$ is the incoming current involving the $e^{+} e^{-}$system and the ISR photon, and $\mathcal{J}_{\text {out }}^{\mu}$ is the outgoing current involving the final state $X$.

We have

$$
\mathcal{J}_{i n}^{\mu}\left(p_{1}, p_{2}, k, l\right)=-i e \bar{v}\left(p_{1}\right) \gamma^{\alpha} \frac{y-m}{l^{2}-m_{e}^{2}} \gamma^{\mu} u\left(p_{2}\right) \epsilon_{\alpha}(k),
$$

where $\bar{v}\left(p_{1}\right)$ represents the incoming positron, $u\left(p_{2}\right)$ the incoming electron, $\epsilon_{\alpha}(k)$ the polarization vector of the ISR photon, $m_{e}$ the electron mass and $l=p_{2}-k$ is the 4-momentum of the off-shell electron. Using the spin-sum relation

$$
\sum_{s} v_{s}(l) \bar{v}_{s}(l)=\not l-m_{e}
$$

in the helicity basis, we can rewrite

$$
\mathcal{J}_{i n}^{\mu}\left(p_{1}, p_{2}, k, l, s^{+}, s^{-}, \lambda\right)=\frac{-i e}{l^{2}-m_{e}^{2}} \bar{v}_{s^{+}}\left(p_{1}\right) \gamma^{\alpha} \epsilon_{\alpha}(k)\left[\sum_{s} v_{s}(l) \bar{v}_{s}(l)\right] \gamma^{\mu} u_{s^{-}}\left(p_{2}\right),
$$

where $s^{+}$and $s^{-}$are the spin states of the incoming positron and electron. Due to helicity conservation only the $s=s^{+}=s^{-}$term is non-zero, and we can rewrite the full amplitude as

$$
i \mathcal{M}_{\gamma X}=\frac{i e^{3}}{s^{\prime}\left(l^{2}-m_{e}^{2}\right)} \mathcal{J}_{E}^{\nu}\left(p_{1}, k, l\right) \epsilon_{\nu}(k) \mathcal{J}_{A}^{\mu}\left(l, p_{2}\right) \mathcal{J}_{\text {out } \mu},
$$

where $\mathcal{J}_{E}=\bar{v}\left(p_{1}\right) \gamma^{\alpha} v_{s}(l)$ is the current for the photon emission part of the diagram and $\mathcal{J}_{A}^{\mu}=\bar{v}_{s}(l) \gamma^{\mu} u\left(p_{2}\right)$ is the current taking part in the annihilation process. But $-e^{2} / s^{\prime} \mathcal{J}_{A}^{\mu} \mathcal{J}_{\text {out } \mu}$ is just the amplitude $i \mathcal{M}_{X}$ for the annihilation after ISR emission and we have

$$
i \mathcal{M}_{\gamma X}=\left[\frac{-i e}{l^{2}-m_{e}^{2}} \mathcal{J}_{E}^{\nu}\left(p_{1}, k, l\right) \epsilon_{\nu}(k)\right] i \mathcal{M}_{X}\left(p_{2}, l\right)
$$


So the amplitude factorizes into an emission part times the amplitude for $e^{+} e^{-}$ annihilation at a reduced $\mathrm{CM}$ energy. However this is only true in the limit where the fermion in the intermediate state is almost on-shell, so that the annihilation is almost a physical process. This is the case for the emission of soft and collinear photons which is the main interest of this study.

The unpolarized cross-section for $e^{+} e^{-} \rightarrow \gamma X$ is given by

$$
\begin{aligned}
\sigma_{e^{+} e^{-\rightarrow \gamma X}}(s)= & \frac{1}{4 \sqrt{\left(p_{1} \cdot p_{2}\right)^{2}-m_{e}^{4}}} \frac{1}{4} \sum_{s^{+}, \lambda} \int d \Phi_{\gamma} d \Phi_{X}\left|\mathcal{M}_{\gamma X}\left(s^{\prime}(k)\right)\right|^{2} \\
= & \int d \Phi_{\gamma}(k) \frac{1}{2} \sum_{s^{+}, \lambda}\left|\frac{-i e}{l^{2}-m_{e}^{2}} \mathcal{J}_{E}^{\nu}\left(p_{1}, k, l\right) \epsilon_{\nu}(k)\right|^{2} \\
& \times \frac{1}{2 s} \frac{1}{4} \sum_{s^{+}} \int d \Phi_{X}\left|\mathcal{M}_{X}\left(s^{\prime}(k)\right)\right|^{2} \\
= & \int d \Phi_{\gamma}(k) \frac{1}{2} \sum_{s^{+}, \lambda}\left|\frac{e}{l^{2}-m_{e}^{2}} \mathcal{J}_{E}^{\nu}\left(p_{1}, k, l\right) \epsilon_{\nu}(k)\right|^{2} \frac{s^{\prime}}{s} \sigma_{e^{+} e^{-} \rightarrow X}\left(s^{\prime}(k)\right),
\end{aligned}
$$

where $d \Phi_{\gamma}$ and $d \Phi_{X}$ are phase space elements. The factor $s^{\prime} / s$ is due to the $1 / 2 s$ term occurring in cross-section formula, which is actually $1 / 2 s^{\prime}$ for the cross-section on the right-hand side.

We have

$$
d \Phi_{\gamma}=\frac{d E_{\gamma} d^{2} p_{T}}{(2 \pi)^{3} 2 E_{\gamma}}=\frac{d s^{\prime}}{s-s^{\prime}} \frac{d^{2} p_{T}}{16 \pi^{3}}
$$

and thus

$$
\frac{d \sigma_{e^{+} e^{-\rightarrow \gamma X}}}{d s^{\prime}}\left(s, s^{\prime}\right)=\xi\left(s, s^{\prime}\right) \sigma_{e^{+} e^{-} \rightarrow X}\left(s^{\prime}\right)
$$

with

$$
\xi\left(s, s^{\prime}\right)=\frac{s^{\prime}}{2 s\left(s-s^{\prime}\right)} \sum_{s^{+}, \lambda} \int \frac{d p_{T}^{2}}{16 \pi^{3}}\left|\frac{e}{l^{2}-m_{e}^{2}} \mathcal{J}_{E}^{\nu}\left(p_{1}, k, l\right) \epsilon_{\nu}(k)\right|^{2} .
$$

The expression for the radiator function $\xi$ for soft or collinear emission is derived in Appendix B. The result is

$$
\xi\left(s^{\prime}, s\right)=\frac{\alpha}{\pi s} \log \frac{\sqrt{s}}{m} \frac{1+(1-z)^{2}}{z}
$$


The variations of $\xi\left(s^{\prime}, s\right)$ are shown in Fig. 2.17. with $z=1-s^{\prime} / s=2 E_{\gamma} / s$. So

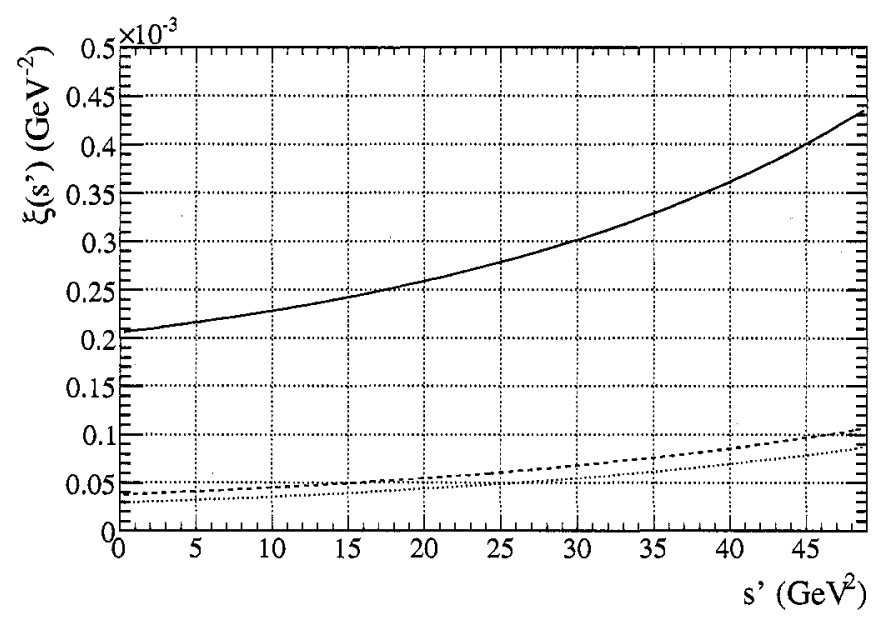

Figure 2.17: The radiative function $\xi\left(s^{\prime}, s\right)$ from Eq. 2.49 with no $\theta$ cut (solid line), and for Eq. 2.49 with $\cos \theta_{\min }=0.87$ (dashed line) and $\cos \theta_{\min }=0.80$ (dotted line).

the cross-section also factorizes. In fact for the total cross-section, integrated over all momenta in the final state $X$ this is the case for any ISR emission due to current conservation properties, as shown in Appendix D.

The factorization can be given in terms of $z$ only as

$$
\frac{d \sigma_{e^{+} e^{-} \rightarrow \gamma X}}{d z}(s, z)=\xi(z) \sigma_{e^{+} e^{-} \rightarrow X}((1-z) s), \quad \xi(z)=\frac{\alpha}{\pi} \log \frac{\sqrt{s}}{m} \frac{1+(1-z)^{2}}{z} .
$$

\subsection{2 $\Delta \alpha_{h a d}^{(5)}\left(m_{Z}^{2}\right)$ from ISR Processes}

The $e^{+} e^{-}$collision occurring after ISR emission is identical to a standard non-radiative collision, apart from the lowering of the CM energy squared from $s$ to $s^{\prime}$. The main process is the production of a final state with $J^{P C}=1^{--}$from annihilation to a single virtual photon, as shown in Fig 2.16. However other processes, such as $\gamma \gamma$ collisions and annihilation to two virtual photons may also occur (see Section 4.4).

The $1 / z$ dependence in $\xi$ gives the cross-section a sharp peak at $z=0$, or $s^{\prime}=s$, corresponding to soft radiation. The form of $\xi$ given in Eq. 2.49 corresponds to the inclusive case where the ISR photon can be emitted in all directions. In the more realistic case where the photon is required to have a polar angle of at least $\theta_{\min }$ with 
respect to the electron beam in the collision CM frame, we have [7]

$$
\xi\left(s^{\prime}, s, \cos \theta_{\text {min }}\right)=\frac{\alpha}{\pi}\left[\frac{1+(1-z)^{2}}{s z} \log \frac{1+\cos \theta_{\text {min }}}{1-\cos \theta_{\text {min }}}-\frac{z}{s} \cos \theta_{\text {min }}\right] .
$$

This expression diverges in the limit of small $\theta_{\text {min }}$, reflecting the fact that the ISR photon is radiated preferentially along the beam direction.

This expression is also not factorizable as a function of $z$ times a function of $\cos \theta_{m} i n$. It is the case for small $z$, in which case the second term on the right of 2.51 can be neglected, but as $z$ increases (i.e. as $s^{\prime}$ decreases) this term leads to lower values of $\xi$ at high $\cos \theta$. The $\cos \theta$ distribution for radiative photons is therefore less peaked at \pm 1 for low $s^{\prime}$ than for high $s^{\prime}$.

We can rewrite $\Delta \alpha_{h a d}^{(5)}\left(m_{Z}^{2}\right)$ as

$$
\Delta \alpha_{h a d}^{(5)}\left(m_{Z}^{2}\right)=\frac{m_{Z}^{2}}{4 \pi^{2} \alpha} \int_{4 m_{\pi}^{2}}^{s_{0}} \frac{d \sigma_{e^{+} e^{-} \rightarrow \gamma \text { hadrons }}\left(s^{\prime}\right)}{\left(m_{Z}^{2}-s^{\prime}\right) \xi\left(s^{\prime}, s\right)}
$$

The weight factor is

$$
w\left(s^{\prime}, s\right)=\frac{m_{Z}^{2}}{4 \pi^{2} \alpha\left(m_{Z}^{2}-s^{\prime}\right) \xi\left(s^{\prime}, s\right)} \sim \frac{1}{4 \pi^{2} \alpha \xi\left(s^{\prime}, s\right)}
$$

Its variations are shown in Figure 2.18.

Since $w\left(s^{\prime}, s\right)$ does not depend strongly on $s^{\prime}$, it is possible to use the ISR photon alone to measure $s^{\prime}$. Energy resolution effects are not negligible, as described in Section 4.3 , but the slow variation of $w\left(s^{\prime}, s\right)$, especially at low $s^{\prime}$, ensure that the repercussions on the value of the integral in Eq. 2.52 are small. Relying on the photon allows greater inclusiveness on the hadronic side, which leads to reduced systematic errors, as discussed in Section 4.2.

\subsubsection{Radiative Corrections}

So far we have been focused on measuring the ISR process $e^{+} e^{-} \rightarrow \gamma$ hadrons. However what nature gives us is the (interfering) sum of this process, the corresponding process with final-state radiation (FSR), and higher-order processes with additional ISR and 


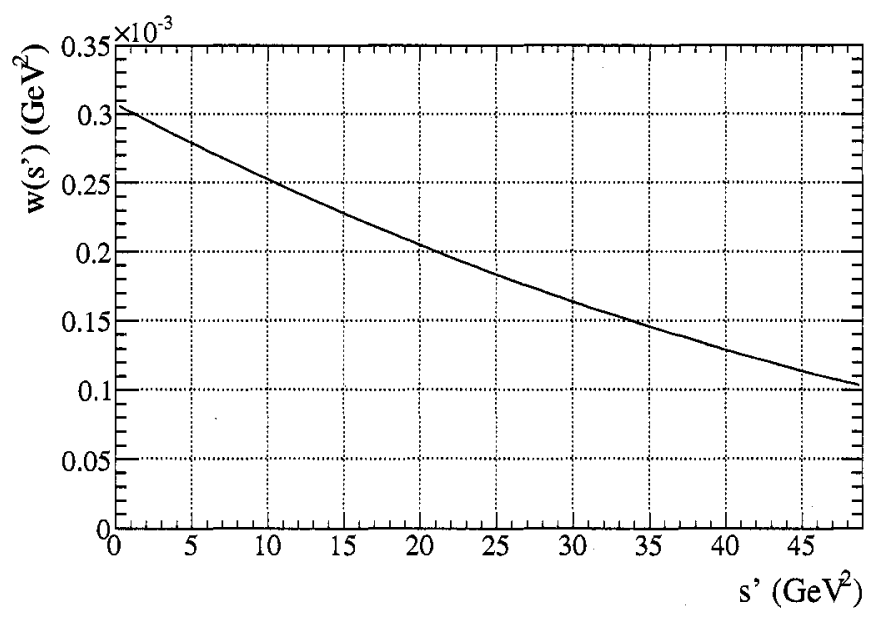

Figure 2.18: Weight function $w\left(s^{\prime}, s\right)$ for the $\Delta \alpha_{\text {had }}^{(5)}\left(m_{Z}^{2}\right)$ integral, for $\cos \theta_{\min }=0.80$

FSR photons. They can be classified as follows:

- Pure FSR: this process is similar to ISR, but with the photon coming from the hadronic final state. It corresponds to annihilation at the nominal collision $\mathrm{CM}$ energy and therefore should not be counted at $s^{\prime}=s-2 E_{\gamma}^{*} \sqrt{s}$ in the $\Delta \alpha_{\text {had }}^{(5)}\left(m_{Z}^{2}\right)$ integral. However,

- In the FSR process, the annihilation occurs at the nominal collision CM energy. The cross-section therefore goes as $\xi\left(s, s^{\prime}\right) \sigma_{X}(s)$ and the FSR/ISR ratio as $\sigma_{X}(s) / \sigma_{X}\left(s^{\prime}\right)=s^{\prime} / s$. The FSR term is therefore suppressed, particularly at low $s^{\prime}$.

- The production cross-section for the low-multiplicity final states that dominate at low $s$ becomes very small for larger $s$ due to the availability of higher-multiplicity channels. This "form factor effect" further suppresses FSR relatively to ISR.

- FSR is preferentially emitted in a narrow cone around the radiating particle, just as ISR peaks around the beam direction. At $10.6 \mathrm{GeV}$ where an $e^{+} e^{-} \rightarrow q \bar{q}$ event looks like back-to-back jets in the CM frame, the FSR photon is mostly collinear to one of the jets, while an ISR photon is 


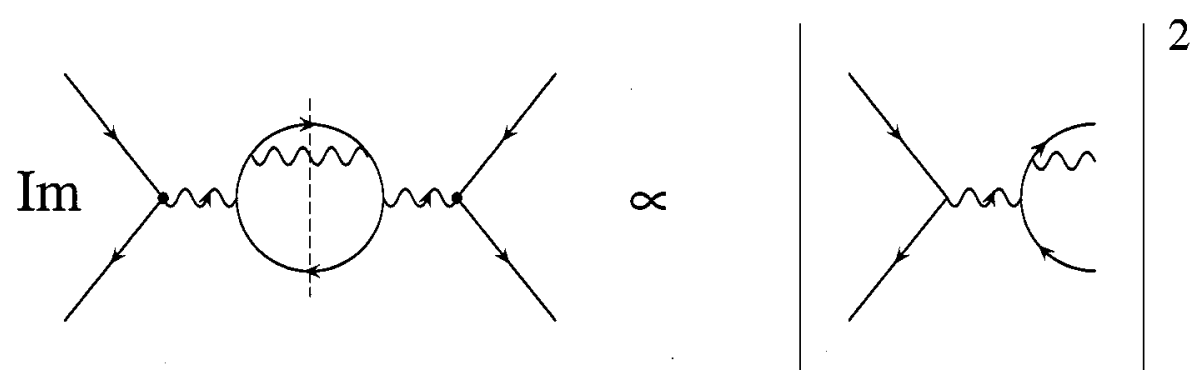

Figure 2.19: The Optical Theorem for hadronic events with final-state photons

isolated, with a single jet recoiling in the back-to-back direction. Eventshape quantities can therefore be used to separate the two, as well as the distribution of the photon polar angle in the CM frame.

- Processes with additional ISR photons: These events can be accounted for by improving the form of the radiator function. This is done for example for the KKMC generator (described in detail in Appendix A), which considers up to three ISR photons.

- Processes with ISR and extra radiation from the hadronic final states: these processes are related through the Optical Theorem to processes with virtual photons in the diagram for $\Pi_{H a d}$, as illustrated in Fig. 2.19. Since $\Pi_{\text {Had }}$ must include all hadronic contributions, these processes must be included in the $\Delta \alpha_{\text {had }}^{(5)}\left(m_{Z}^{2}\right)$ integral.

- Interference between ISR and FSR processes: This term is small for $e^{+} e^{-}$collisions at $10.6 \mathrm{GeV}$ since as shown above ISR and FSR emission operate in different kinematic regimes, with ISR at low polar angles and FSR close to the final-state particles. Furthermore the interference term is C-odd and should therefore vanish for a symmetric $\theta$ acceptance region in the CM frame.

- Additional virtual photons between the leptonic and hadronic systems: these can be either the radiative equivalent of a $e^{+} e^{-}$annihilation to two virtual photons, or box diagrams giving higher-order contributions to the usual ISR process. As described in Appendix C, the former should be negligible compared 
to the one photon annihilation case. The latter is probably of a magnitude similar to that of ISR/FSR interference since it cancels the infrared divergence in the ISR/FSR interference term.

Accounting for FSR effects is difficult since there are no theoretical models at the moment that take into account interference between ISR and radiation from the final-state hadrons, nor the box-diagram contribution to the annihilation process. However $e^{+} e^{-} \rightarrow \mu^{+} \mu^{-}$events can be used to set an upper bound on its magnitude, since muons are expected to generate more FSR than hadrons. The relative amount of ISR/FSR interference between muons and hadrons can also be checked by measuring the forward-backward asymmetry in $e^{+} e^{-} \rightarrow \mu^{+} \mu^{-} \gamma$ and $e^{+} e^{-} \rightarrow h \bar{h} \gamma$, where $h=$ $\pi, K, p$, as described in the next section.

\subsubsection{Forward-Backward Asymmetry in Two-prong Events due to ISR/FSR Interference}

The interference term between ISR and FSR emission is odd under the charge conjugation operator $\mathrm{C}$. Since under $\mathrm{C}$ the electron beam becomes a positron beam and vice versa, this translates into a forward-backward asymmetry in $e^{+} e^{-}$annihilation. In the case of $e^{+} e^{-} \rightarrow \mu^{+} \mu^{-} \gamma$ we have in the soft-photon approximation [40]

$$
\frac{d \sigma_{\mu^{+} \mu^{-} \gamma}^{+}(\cos \theta)}{d \Omega d s^{\prime}}-\frac{d \sigma_{\mu^{+} \mu^{-} \gamma}^{-}(\cos \theta)}{d \Omega d s^{\prime}}=\frac{d \sigma_{\mu^{+} \mu^{-}}(\cos \theta)}{d \Omega} \frac{8 \pi}{\pi} \log \frac{1+\cos \theta}{1-\cos \theta}
$$

where $\theta=\theta_{\mu^{+}}$in $\sigma^{+}$and $\theta=\theta_{\mu^{-}}$in $\sigma^{-}$. The asymmetry is a function of the angle $\phi^{*}$ between the production plane $\left(e^{+}, \gamma\right)$ and the decay plane $\left(\mu^{+}, \gamma\right)$ in the $\mu^{+} \mu^{-}$ center-of-mass frame. The maximum asymmetry occurs at $\left|\cos \phi^{*}\right|=1$, while the $\cos \phi^{*}=0$ configuration corresponds to no asymmetry.

This process can be used to study the final-state radiation properties of hadrons: an open question is to know whether the main contribution to FSR comes from the quarks (before hadronization) of from the hadrons (after hadronization). In the former case, the situation should be similar to the muon case as an asymmetry should be seen; in the latter case the there should be no interference between ISR and FSR, 


\begin{tabular}{lcccc}
\hline \hline$\sqrt{s^{\prime}}$ range $(\mathrm{GeV})$ & $\Delta \alpha_{\text {Had }}\left(\times 10^{-5}\right)$ & Fraction (\%) & Error $(\%)$ & $\delta\left(\Delta \alpha_{\text {Had }}\right)\left(\times 10^{-5}\right)$ \\
\hline$\rho$ & 347 & 12.6 & 0.9 & 3.1 \\
Narrow resonances & 184 & 6.7 & 3.1 & 5.7 \\
$1.05-2$ & 156 & 5.7 & 15.0 & 23.4 \\
$2-5$ & 381 & 13.8 & 5.9 & 22.5 \\
$5-7$ & 183 & 6.6 & 6.0 & 11.0 \\
$7-12$ & 304 & 11.0 & 1.4 & 4.3 \\
$>12$ & 1203 & 43.6 & 0.2 & 2.4 \\
\hline Total & 2758 & 100.0 & 1.3 & 35.2 \\
\hline \hline
\end{tabular}

Table 2.3: Contributions to $\Delta \alpha_{h a d}^{(5)}\left(m_{Z}^{2}\right)$ per energy range, with their relative fraction, relative error and contribution to the total error, taken from Ref. [2].

and therefore no asymmetry. Radiation from the hadrons is expected to be weaker than for quarks and can be simulated for instance using the PHOTOS [41] generator.

\subsubsection{Experimental Status of $\Delta \alpha_{h a d}^{(5)}\left(m_{Z}^{2}\right)$}

The latest determination of $\Delta \alpha_{h a d}^{(5)}\left(m_{Z}^{2}\right)$ is

$$
\Delta \alpha_{\text {had }}^{(5)}\left(m_{Z}^{2}\right)=(2758 \pm 35) \times 10^{-5} .
$$

taken from Ref. [2]. This estimation makes minimal use of perturbative QCD. The more theory-driven approach employed in Ref [42], yields the value

$$
\Delta \alpha_{\text {had }}^{(5)}\left(m_{Z}^{2}\right)=\left(2778 \pm 22_{\exp } \pm 14_{t h}\right) \times 10^{-5}
$$

with a significantly smaller error. Since both values agree The overall error of $1.4 \%$ is relatively small, but some energy ranges in integral 2.21 contribute large errors locally. Table 2.3.5, taken from Ref. [2], gives the contributions of several energy intervals with their errors. The small overall error is driven by the negligible $0.2 \%$ uncertainty on the contribution from the "desert" region between the higher $\Upsilon$ resonances and the $Z$. The very precise determinations of $R_{\text {had }}$ in the $\rho$ region by CMD-2 [3] and KLOE [11] also push down the error, and the region $\sqrt{s}=2-5 \mathrm{GeV}$ has been recently measured 
by $\mathrm{BES}$ [4]. However the region $\sqrt{s}=1.05-7 \mathrm{GeV}$, is measured with relatively low precision, and this is especially true of the region $\sqrt{s}=1.05-2 \mathrm{GeV}$ in which no recent measurements have been made.

The overall error in the region $\sqrt{s}=1.05-7 \mathrm{GeV}$ is currently $4.8 \%$. If this were to be reduced to $3 \%$, the error on the entire energy range would be reduced to $0.8 \%$, which would have a significant impact on the precision of the global EW fits. The goal of this work is to provide a measurement at this level of precision. 


\section{Chapter 3}

\section{The BABAR Detector}

\subsection{Overview}

The BABAR detector [44] and PEP-II follow the "B-factory" design put forward in 1987 [45], with the primary goal of studying CP-violation phenomena in the B meson system. However the breadth of this physics program and the considerable experimental challenges which it poses make BABAR anything but a specialized experiment:

- The B-factory design calls for a high-luminosity collider running at the $\Upsilon(4 S)$ resonance, which provides the best configuration for the study of $\mathrm{B}$ mesons. However this configuration also leads to large production rates for many other physics processes, such as $\tau$ and charm quark pair production and radiative processes, including the ISR modes that constitute the basis of this work.

- B decays typically involve relatively large multiplicities and uniform angular distributions, leading to the requirement of a large geometric acceptance for the detector, and good performance on low-energy particles.

- Tracking:

- Performed in a $1.5 \mathrm{~T}$ magnetic field oriented along the beam direction, provided by a superconducting solenoid coil. 
- Silicon Vertex Detector (SVT): Precise tracking of charged particles near the interaction region, including standalone tracking of low- $p_{t}$ tracks, and measurement of energy loss $(d E / d X)$.

- Drift Chamber (DCH): Excellent momentum resolution and good tracking performance down to very low $p_{t}$, and measurement of energy loss $(d E / d X)$.

- Calorimetry: finely granulated crystal calorimeter (EMC), providing excellent energy resolution and good position resolution for photons and electrons, important for the reconstruction of high-momentum $\pi^{0}$ 's but also ideal for the study of radiative events. Good performance on low-energy photons, important for example for $B \rightarrow J / \psi K_{S}^{0}\left(\pi^{0} \pi^{0}\right)$.

- Particle identification is needed to separate electrons, muon, pions and kaons. over a wide momentum range. Muon chambers (IFR) are incorporated in the magnet flux return. The ionization loss in the drift chamber is used, as well as the energy deposition in the EMC. High-momentum kaon/pion separation is crucial to identify decays such as $B \rightarrow \pi \pi$ and $B \rightarrow K \pi$. This is done using a Cherenkov detector (DIRC), with good separation for the entire momentum range. This also provides proton-kaon separation up to the highest momenta.

The constraints posed by B physics therefore mandate a very general detector design with excellent overall performance that is also well-suited for the study a wide range of physics processes. The excellent calorimeter performance and and large acceptance make it particularly well-suited for the study of $e^{+} e^{-} \rightarrow q \bar{q} \gamma$ events. The detector layout is shown in Fig. 3.1.

\subsection{PEP-II and the Interaction Region}

\subsubsection{Choice of Beam Energies}

The PEP-II accelerator is designed specifically for the goal of producing large numbers of $\mathrm{B}$ mesons to study CP-violation. In the quark model the $e^{+} e^{-} \rightarrow \gamma^{*} \rightarrow q \bar{q}$ cross 


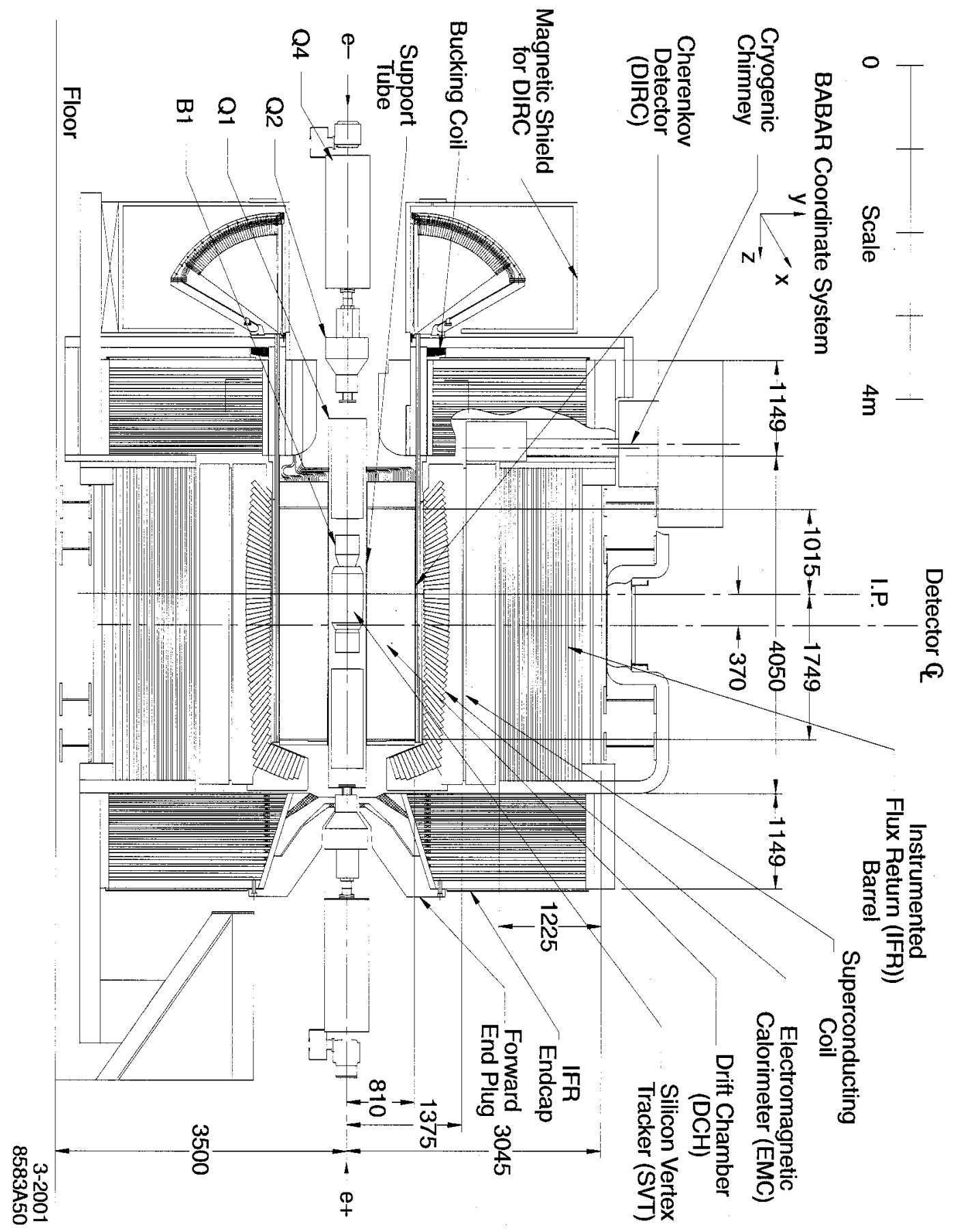

Figure 3.1: Longitudinal cross-section view of the BABAR detector 
sections decrease as the inverse of the center of mass (CM) energy squared, so that the highest cross section for $b \bar{b}$ should a priori be obtained near threshold.

Furthermore, in the threshold region the $e^{+} e^{-} \rightarrow b \bar{b}$ cross section features resonant structures due to $b \bar{b}$ bound states, the $\Upsilon$ resonances shown in Fig. 3.2.

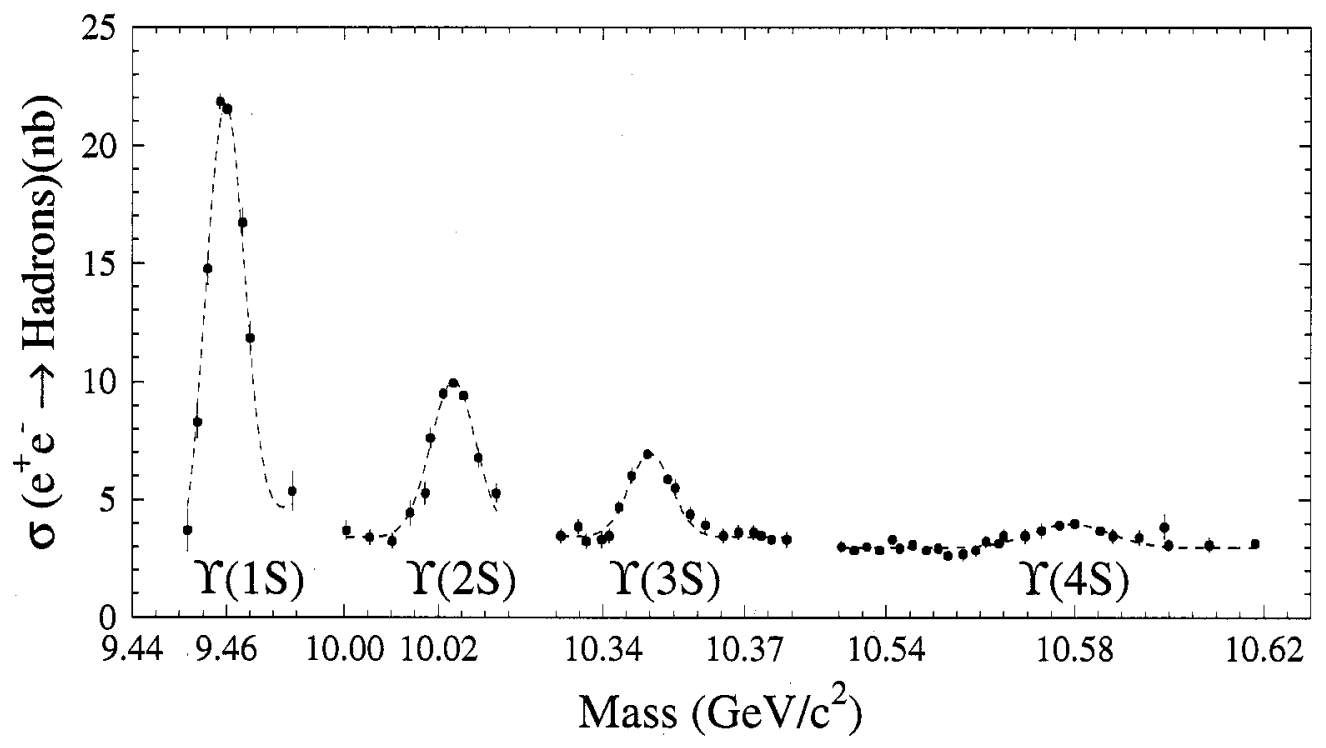

Figure 3.2: $e^{+} e^{-} \rightarrow$ hadrons cross-section in the vicinity of the $\Upsilon(1 S), \Upsilon(2 S), \Upsilon(3 S)$ and $\Upsilon(4 S)$ resonances, as measured by the CUSB and CLEO collaborations [46]

The $\Upsilon(4 S)$ resonance, with a mass of $10.58 \mathrm{GeV}$, is the first $\Upsilon$ resonance above the $B \bar{B}$ production threshold located at $10.56 \mathrm{GeV}$. Since this is the first allowed strong decay channel, it makes the $\Upsilon(4 S)$ much broader than the first three $\Upsilon_{\mathrm{S}}(\sim 20 \mathrm{MeV}$ versus tens of $\mathrm{keV}$ ) and ensures that the branching fraction for $\Upsilon(4 S) \rightarrow b \bar{b}$ is almost $100 \%$.

The increased hadronic cross-section on the resonance peak corresponds almost exclusively to $B \bar{B}$ production. We have $R_{H a d}^{\Upsilon(4, S)} \approx 5$ while $R_{H a d}^{C o n t} \approx 4$ in the continuum directly above. Since $b \bar{b}$ production makes up only about $10 \%$ of the continuum production, even this modest increase of one unit correspond to a factor $\approx 1.4 / 0.4 \approx 3$ increase in the $b \bar{b}$ cross section. The $B \bar{B}$ production cross-section on the resonance was measured by $B A B A R$ [47] to be $\sigma_{b \bar{b}}=1.10 \mathrm{nb}$, in line with expectations. For this 
Table 3.1: Cross-sections on the $\Upsilon(4 S)$ resonance

\begin{tabular}{lcl}
\hline \hline Mode & Cross-section (nb) & Source \\
\hline$\gamma \gamma$ & 2.87 & BkQed $\left(13<\theta_{\gamma}<160^{\circ}\right)$ \\
$e^{+} e^{-}$ & 49.1 & BhWide $\left(13<\theta_{e}<142^{\circ}\right)$ \\
$\mu^{+} \mu^{-}$ & 1.12 & KKMC \\
$\tau^{+} \tau^{-}$ & 0.89 & KKMC \\
$u \bar{u}$ & 1.39 & JETSET 7.4 \\
$d \bar{d}$ & 0.35 & JETSET 7.4 \\
$s \bar{s}$ & 0.35 & JETSET 7.4 \\
$c \bar{c}$ & 1.30 & JETSET 7.4 \\
$b \bar{b}$ & 1.10 & Ref. [47] \\
\hline \hline
\end{tabular}

reason the $\Upsilon(4 S)$ mass is the best location to produce large number of $\mathrm{B}$ mesons in $e^{+} e^{-}$annihilation. The cross-sections for other processes are shown in Table 3.2.1.

A disadvantage of running at $\Upsilon(4 S)$ energies is that due to the proximity to the $B \bar{B}$ threshold the $B$ mesons in a symmetric machine would be produced almost at rest, with $\beta \approx 0.06$. Given the $B$ lifetime of $c \tau \approx 0.5 \mathrm{~mm}$, this translates to a flight distance of $l=\beta \gamma c \tau \approx 30 \mu \mathrm{m}$, too small to be measured using present vertex detector technology. This situation, which occurs at CLEO, is a serious limitation for $\mathrm{CP}$-violation measurements. For this reason, the machine is instead designed to be asymmetric, with an electron beam of $E_{-}=9 \mathrm{GeV}$ colliding with a positron beam of $E_{+}=3.1 \mathrm{GeV}$. The center of mass system is therefore boosted in the detector frame with $\beta \gamma=0.56$, leading to an order-of-magnitude increase of the $B$ fight distance to $l \approx 270 \mu \mathrm{m}$, which is well within reach of vertexing technology.

In the rest of this document the reference frame of the collision will be referred to as the center-of-mass frame or $\mathrm{CM}$ frame, while the frame of the detector components will be called the laboratory frame of lab frame. The boost had the effect of sending the final state of the $e^{+} e^{-}$collisions preferentially in the forward region. This is illustrated by the correspondence between polar angle values in the CM and lab frames shown in Fig. 3.3. This assymetry has a significant influence on the detector design and imposes a coverage down to very low polar angles in the forward region.

In the rest of the document, energies and angles are given in the laboratory frame 


\section{Detector Protractor - $\gamma$ 's}

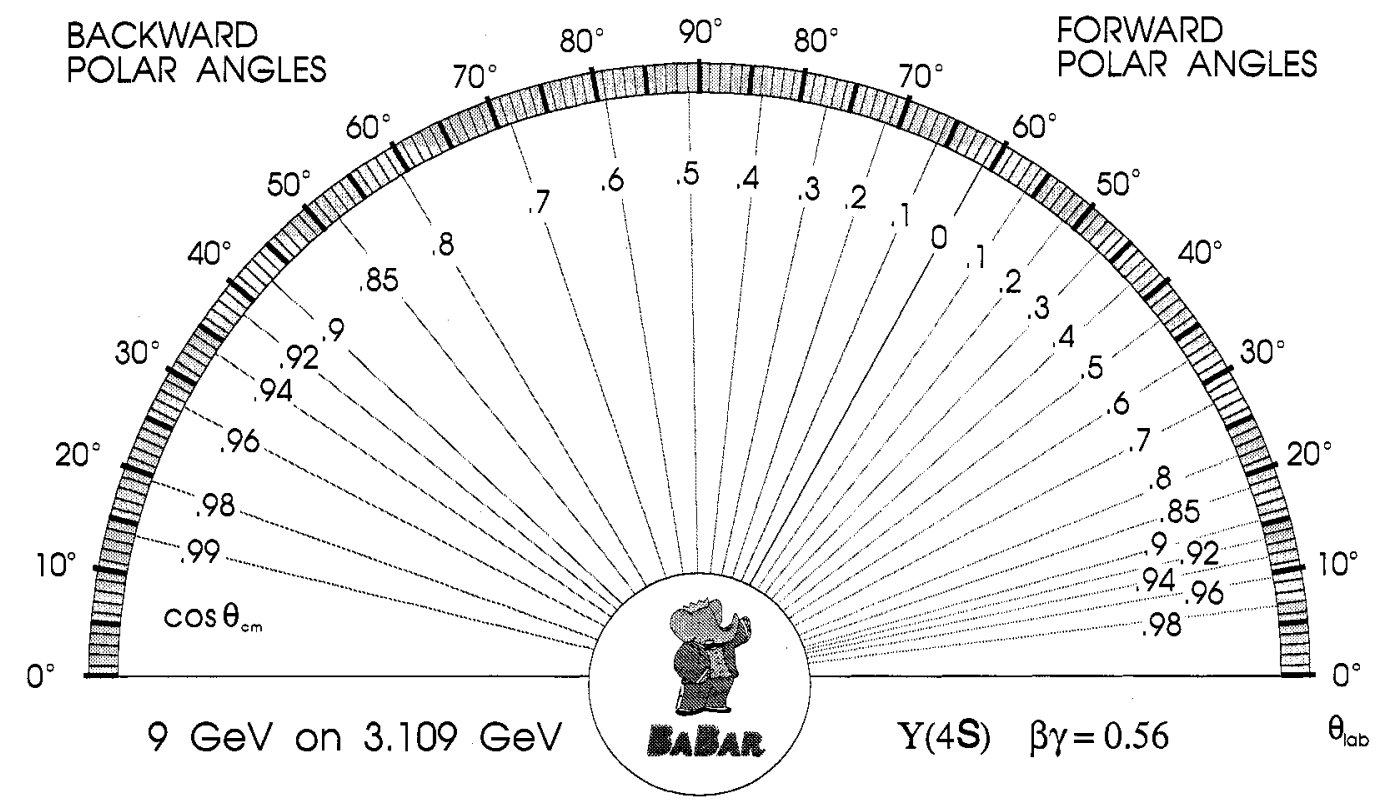

Figure 3.3: Correspondance between the polar angle $\theta_{L a b}$ in the laboratory frame and the cosine of the polar angle $\cos \theta_{c m}$ in the CM frame.

unless stated otherwise, and CM quantities will generally be suffixed with a star $(*)$.

In addition to data taken on the $\Upsilon(4 S)$, a small dataset of about $10 \%$ of the total is taken "off-resonance", $40 \mathrm{MeV}$ below the $\Upsilon(4 S)$ peak. Since this point is located below the $B \bar{B}$ threshold, this sample is composed only of non- $B \bar{B}$ events that form a background to $B \bar{B}$ analyses, and allows to study their properties.

\subsubsection{Luminosity Considerations}

The general definition of luminosity is given by [48]

$$
\mathcal{L}=\left(v_{+}+v_{-}\right) f_{c} \int d x d y d z d t \rho_{+}\left(x, y, z-v_{+} t\right) \rho_{-}\left(x, y, z+v_{+} t\right)
$$

where $v_{ \pm}$and $\rho_{ \pm}$are respectively the velocities and bunch densities of each beam, and $f_{c}$ is the bunch collision frequency. The $x$ and $y$ coordinates are transverse to 
the beam, while $z$ is along it.

In the rest of this section it is assumed that the bunch densities can be described by Gaussian distributions in all variables, and that the Gaussian widths $\sigma_{x}^{*}, \sigma_{y}^{*}$ and $\sigma_{s}^{*}$ at the IP along $x, y$, and $z$ are identical for both beams - conditions that are verified at PEP-II. In this case $\mathcal{L}$ can be written simply as

$$
\mathcal{L}=\frac{1}{4 \pi} \frac{N_{+} N_{-} f_{c}}{\sigma_{x}^{*} \sigma_{y}^{*}}
$$

As the luminosity increases, so do the beam-beam interaction effects that lead to beam instability. The leading effect is the "beam-beam tune spread", which is the perturbation of the transverse phase motion of one beam due to the influence of the other beam. Its magnitude is given [49] by the parameter

$$
\Delta Q_{y}^{+}=\frac{r_{0} m_{e} c^{2}}{2 \pi} \frac{1}{E_{+}} \frac{N_{-}}{\sigma_{y}^{*}\left(\sigma_{x}^{*}+\sigma_{y}^{*}\right)} \beta_{y}^{*}
$$

for the positron beam in the $y$ direction, and an analogous expression in the $x$ direction and for the other beam. $E_{ \pm}$and $N_{ \pm}$refer respectively to the energy and number of particles per bunch of each beam, $m_{e}$ is the mass of the electron and $r_{0}=e^{2} / m_{e} c^{2}$ its classical radius, and $\beta_{y}^{*}$ (the vertical beta function) is a quantity that expresses the amount of focusing applied on the beam in the vertical direction at the IP. $\sigma_{y}^{*}$ can be expressed as $\sigma_{y}^{*}=\sqrt{\epsilon_{y} \beta_{y}^{*}}$, where the emittance $\epsilon_{y}$ is a measure of how much phase space is available to the beam. A similar relation holds between $\sigma_{x}^{*}$, the horizontal emittance $\epsilon_{x}$ and the horizontal beta function $\beta_{x}^{*}$.

For simplicity it is assumed that the tune spread is the same for both beams and in both $x$ and $y$, so that $\Delta Q_{x}^{+}=\Delta Q_{y}^{+}=\Delta Q_{x}^{-}=\Delta Q_{y}^{-}$, an approximation that is verified at PEP-II [50]. Due to the similarity between the expressions of $\mathcal{L}$ and $\Delta Q$, it is difficult to increase the former without also increasing the latter. To show the dependence on $\Delta Q, \mathcal{L}$ can be rewritten as

$$
\mathcal{L}=\frac{2 \pi E_{+} E_{-}}{r_{0}^{2} m_{e}^{2} c^{4}} \Delta Q^{2} \frac{1}{\tau_{b}} \sqrt{\epsilon_{x}^{*} \epsilon_{y}^{*} \frac{\beta_{x}^{*}}{\beta_{y}^{* 3}}}
$$




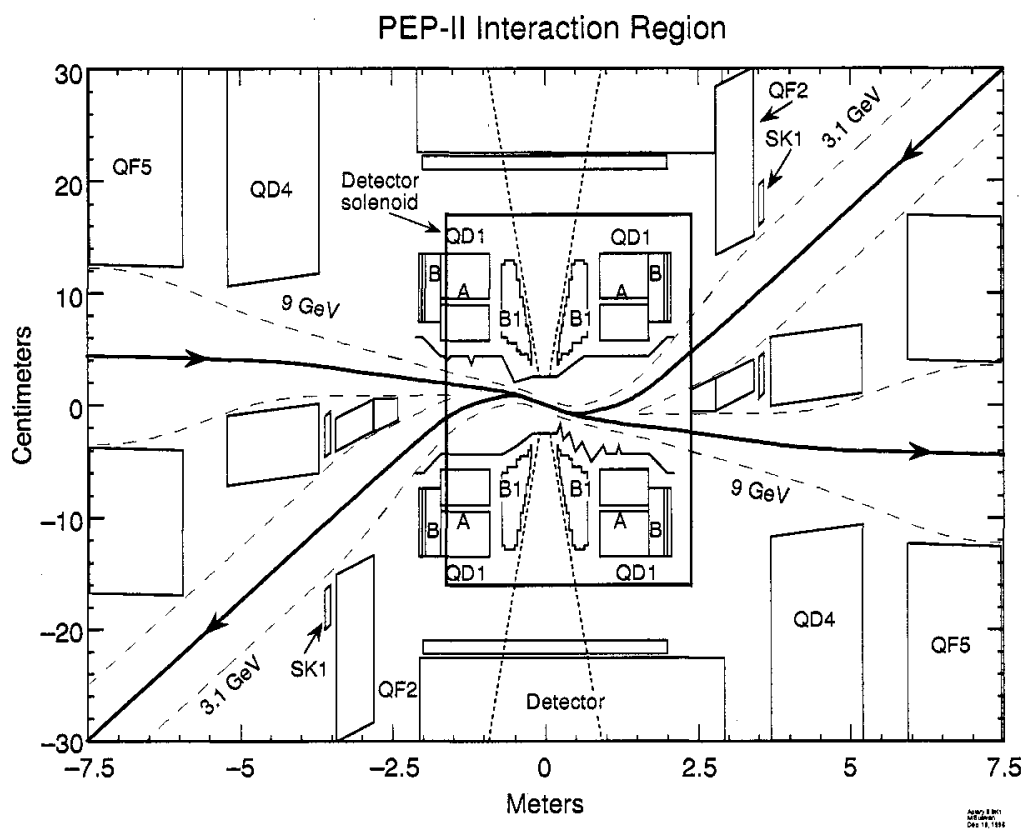

Figure 3.4: Schematic view of the PEP-II interaction region

with $\tau_{b}=2 / f_{c}$ is the time separation between bunches. In $e^{+} e^{-}$storage rings the vertical emittance is naturally much smaller than the horizontal one, a consequence of the fact that bending generally only occurs in the horizontal plane, and it is assumed that $\sigma_{y}^{*} \ll \sigma_{x}^{*}$.

For $E_{+}=9 \mathrm{GeV}, E_{-}=3.1 \mathrm{GeV}, \tau_{b}$ in ns $\Delta Q$ in percent, $\beta_{x}^{*}$ and $\beta_{y}^{*}$ in $\mathrm{cm}$, and $\epsilon_{x}$ and $\epsilon_{y}$ in nm we have

$$
\mathcal{L}=8.44 \times 10^{33} \mathrm{~cm}^{-2} s^{-1} \Delta Q^{2} \frac{1}{\tau_{b}} \sqrt{\epsilon_{x}^{*} \epsilon_{y}^{*} \frac{\beta_{x}^{*}}{\beta_{y}^{* 3}}} .
$$

To increase the luminosity, one has the following possibilities:

- Increase $\Delta Q$ : The effect of the beam-beam tune spread is to increase the "footprint" of the beams in tune space, which makes it harder to find stable beam configurations. The situation is however slightly more favorable for beam tunes near integers of half-integers can help since these regions are less densely populated by resonances. In the PEP-II design a conservative upper bound is set at 
$\Delta Q=3 \%$, although values of up to $7 \%$ could be considered. KEKB uses $3 \%$ in the $y$ direction but $7 \%$ in $x$.

- Increase the emittances: This would also increase the beam size everywhere in the ring; this would require increasing the aperture/acceptance of the beamline, which is technically difficult.

- Increase the beam currents: Since the numbers of particles per bunch is fixed by $\Delta Q$, the only handle is the bunch separation $\tau_{b}$. However small values of $\tau_{b}$ lead to additional beam-beam effects: if bunches are too close together, a bunch coming out of a collision at the IP can "feel" the effects of the next bunch in the opposite beam. To prevent such "parasitic crossings", the beams must be separated quickly. At PEP-II collisions are head-on, and the beams must be separated using $0.75 \mathrm{~T}$ permanent bending magnets (B1) in the horizontal plane located $30 \mathrm{~cm}$ from the IP as shown in Fig 3.4. Additional separation is also provided by the Q1 quadrupoles, which are traversed off-axis by the low-energy beam. The main drawback of this scheme is that the bending produces large synchrotron radiation fluxes that are not present in more conventional $e^{+} e^{-}$ colliders. The PEP-II design [50] specifies $\tau_{b}=4.2 \mathrm{~ns}$, corresponding to every other RF bucket being filled. However due to electron cloud effects a bunch spacing of $6.3-10.5 \mathrm{~ns}$ is achieved in practice. For comparison, the upgraded CESR machine which was used by CLEO-III had a separation of $14 \mathrm{~ns}$, which did not require additional bending magnets. A smaller value for the separation would lead to an increased synchrotron background and the need for magnets closer to the IP, but this can be alleviated by introducing a non-zero crossing angle between the beams at the IP. This is done at the KEKB collider, which has an $22 \mathrm{mrad}$ crossing angle and thus does not need dipole magnets to separate the beams. It allows a much smaller bunch separation: in principle all the RF buckets could be filled at KEKB, leading to a separation of $2.9 \mathrm{~ns}$. However this is spoiled in practice by a combination of beam-beam and electron cloud effects, which limit the separation to about $8 \mathrm{~ns}$.

- Decrease the beta function: A small $\beta_{y}^{*}$ is obtained by strongly focusing of the 
beam at the collision point, and can be done by bringing focusing quadrupoles as close as possible to the IP. This has significant consequences for detector design, as it imposes the presence of a "stay-clear" cone around the beamline at the IP to provide space for the quadrupoles. For BaBar a $300 \mathrm{mrad}$ opening angle is used, with the first quadrupoles (Q1) located $90 \mathrm{~cm}$ from the IP, as shown in Fig 3.4. Since the quadrupoles are inside the detector magnetic field, conventional electromagnets cannot be used. Super-conducting electromagnets are also undesirable since they require a cryostat that would be difficult to fit into the $300 \mathrm{mrad}$ aperture. Permanent magnets made from a samarium-cobalt alloy are therefore used. A similar configuration is also used for the CESR IR. KEKB uses super-conducting magnets with extremely compact cryostats which also fit into a stay-clear region of $300 \mathrm{mrad}$. Another limitation is that $\beta_{y}^{*}$ must be greater that the bunch length $\sigma_{l}$ to avoid beam-beam effect such as the excitation of synchrobetatron resonances and loss of luminosity away from the IP ("Hourglass effect" [48]). A small $\beta_{y}^{*}$ therefore requires short bunches, which in turn require higher RF voltages. For PEP-II the values of $\sigma_{l}=1.1 \mathrm{~cm}$ and $\beta_{y}^{*}=1.5 \mathrm{~cm}$ are used. CESR has similar values, while KEKB manages a bunch length of $3.4 \mathrm{~mm}$ using higher $\mathrm{RF}$ voltages enabling it to use a $\beta_{y}^{*}$ value of $7 \mathrm{~mm}$.

A summary of accelerator parameters as of May 2005 [51] is shown in Table 3.2.2. All the parameters relevant to luminosity are at values either similar or higher well as the corresponding luminosity. The increase from the design luminosity of $3 \times$ $10^{33} \mathrm{~cm}^{-2} \mathrm{~s}^{-1}$ to the achieved value of $9.2 \times 10^{33} \mathrm{~cm}^{-2} \mathrm{~s}^{-1}$ is mostly attributable to the decrease in $\beta_{y}^{*}$ and the increase in the beam-beam tune shift, which permitted an increase in beam currents.

\subsubsection{BABAR Coordinate System}

In the rest of this document, the coordinate system shown in the upper right-hand corner of Fig. 3.1 will be used:

- the origin is located at the center of the detector 
Table 3.2: Operating parameters for the PEP-II accelerator

\begin{tabular}{lcccc}
\hline \hline \multirow{2}{*}{ Parameter } & \multicolumn{2}{c}{ HER } & \multicolumn{2}{c}{ LER } \\
& Design & Typical & Design & Typical \\
\hline Energy (GeV) & 9.0 & 9.0 & 3.1 & 3.1 \\
Current (mA) & 750 & 1550 & 2140 & 2450 \\
Number of bunches & 1658 & 1588 & 1658 & 1588 \\
Bunch spacing (ns) & 4.2 & 4.2 & 4.2 & 4.2 \\
RF Voltage (MV) & 14.0 & 15.5 & 3.4 & 4.04 \\
$\beta_{x}^{*}(\mathrm{~cm})$ & 50.0 & $35-49$ & 50.0 & $35-49$ \\
$\beta_{y}^{*}(\mathrm{~cm})$ & $1.5-2.5$ & 1.1 & $1.5-2.5$ & 1.1 \\
$\epsilon_{x}(\mathrm{~nm})$ & 49 & $31-59$ & 49 & $31-59$ \\
$\epsilon_{y}(\mathrm{~nm})$ & 2.0 & 1.4 & 2.0 & 1.4 \\
$\sigma_{x}^{*}(\mathrm{~mm})$ & 0.16 & $0.10-0.17$ & 0.16 & $0.10-0.17$ \\
$\sigma_{y}^{*}(\mu \mathrm{m})$ & 0.6 & 0.4 & 0.6 & 0.4 \\
$\sigma_{l}^{*}(\mathrm{~mm})$ & 11 & $11-12$ & 11 & $11-12$ \\
$\Delta Q_{x}(\%)$ & 3.0 & 5.5 & 3.0 & 5.3 \\
$\Delta Q_{y}(\%)$ & 3.0 & 4.6 & 3.0 & 6.4 \\
\hline $\mathcal{L}\left(\times 10^{33} \mathrm{~cm}^{-2} s^{-1}\right)$ & 3.0 & 9.2 & 3.0 & 9.2 \\
\hline \hline
\end{tabular}

- the $x$ axis points radially inward.

- the $y$ axis points upward.

- the $z$ axis points along the beam in the direction of the center-of-mass boost, i.e. in the direction of motion of the positron beam.

Spherical coordinates with respect to these axes will frequently be used, with $\theta$ and $\phi$ referring to the polar angle and azimuth respectively. Momentum coordinates in the center-of-mass frame will be denoted by a star $\left({ }^{*}\right)$.

\subsection{Vertex Detector and Interaction Region}

\subsubsection{Interaction region}

The PEP-II beampipe is of a standard design similar to what is used at CESR [52] and KEKB [53]. It is $27.8 \mathrm{~mm}$ in radius, with a structure composed of a double wall 
of beryllium, with respective thicknesses of $830 \mu \mathrm{m}$ and $530 \mu \mathrm{m}$ for the inside and outside walls. Water is circulated in the $1.5 \mathrm{~mm}$ gap between the walls to provide cooling. Beryllium is chosen for its unique properties: it is one of the lightest elements $(Z=4)$ and therefore has a small radiation length, but is also a very stiff metal, with a modulus of elasticity about one third greater than that of steel; these properties make it ideal for structural elements in the inner detector region. The inner surface of the beampipe is coated with a $4 \mu \mathrm{m}$ layer gold to reduce synchrotron radiation at the IP. The material corresponds to 0.011 radiation lengths, half of it coming from the gold.

The beampipe, vertex detector and the permanent magnets are assembled into a common structure, mechanically independent from the rest of the detector, enclosed in a $43 \mathrm{~cm}$ diameter steel support tube spanning the IP. In the detector acceptance region the support tube material is replaced by carbon-fiber epoxy, representing 0.008 radiation lengths of material.

\subsubsection{SVT Layout and Electronics}

The BABAR SVT has been designed to precisely reconstruct charged particle trajectories and decay vertices near the interaction region. It also provides a measurement of ionization loss $(d E / d x)$ which is complementary to that provided by the $\mathrm{DCH}$.

The SVT layout is depicted in Figure 3.5. The detector consists of five layers of double-sided silicon strip sensors, organized into three sets of six modules for the inner three layers, and sixteen and eighteen modules for the outer two layers The silicon sensors are double-sided; on one side, the readout strips run parallel to the beam ( $\phi$ strips), while on the other, they run transverse to the beam axis ( $z$ strips). The readout pitch varies from 50 to $210 \mu \mathrm{m}$; in most cases floating strips (strips that are not read out) lie between two readout strips.

Modules in the inner three layers, which primarily provide position and angle information for measurement of the vertex position, are straight and positioned close to the beam-pipe, in order to minimize the impact of multiple scattering on extrapolation to the vertex. Modules in the fourth and fifth layers are arch-shaped to increase 


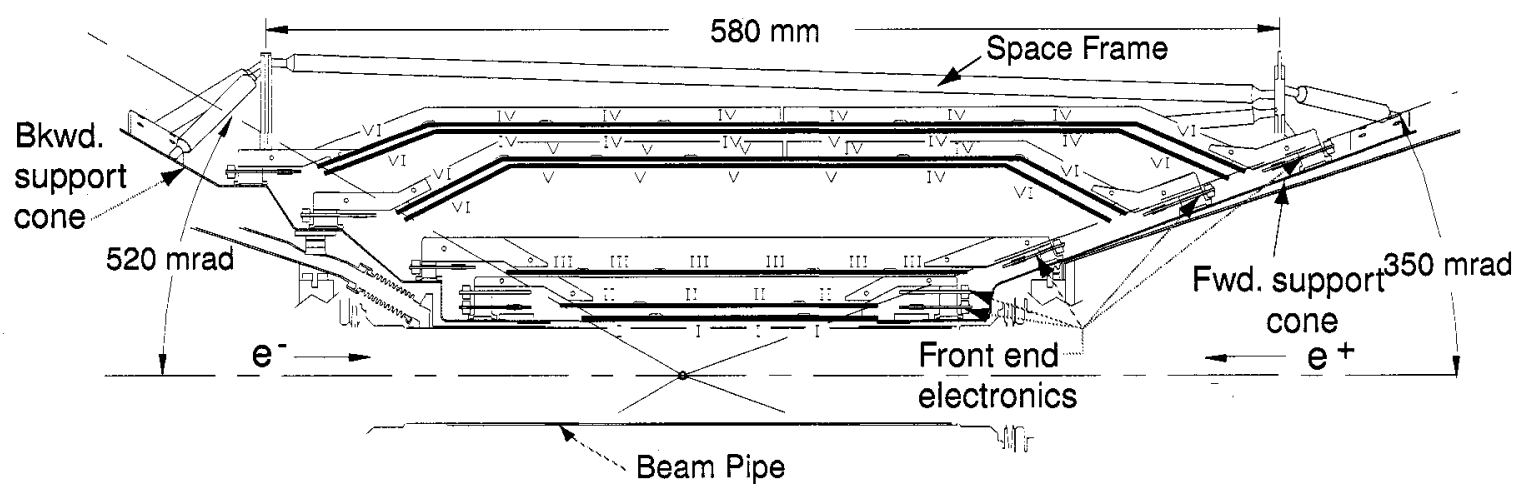

Figure 3.5: Longitudinal cross-section of the SVT.

solid angle coverage and avoid shallow incidence angles for particles near the edges of the module acceptance. The forward acceptance of $350 \mathrm{mrad}$ and the backward acceptance of $520 \mathrm{mrad}$, as well as the $32 \mathrm{~mm}$ radius of the innermost layer relative the interaction point, are determined by the radius of the beam pipe and the size and configuration of the magnets in the interaction region. The total material traversed by particles ranges from $1 \%$ of a radiation length ion the central region to $4 \%$ near the eddges of the acceptance region.

Data from the approximately 140,000 channels are delivered via fanout circuits to a custom integrated chip known as the ATOM (A Time-Over-Threshold-Machine). In the ATOM, the signal is processed by a charge-sensitive preamplifier and shaping circuit, and transformed by a programmable-threshold comparator into a pulse whose width is a quasi-logarithmic function of the collected charge. The comparator output is sampled at $15 \mathrm{MHz}$ onto a 193 bin circular buffer.

\subsubsection{Performance}

To give precise tracking information the relative position of the SVT module with the rest of the detector needs to be accurately known. A "local alignment" procedure is used to calibrate the position of individual modules with respect to the SVT itself 


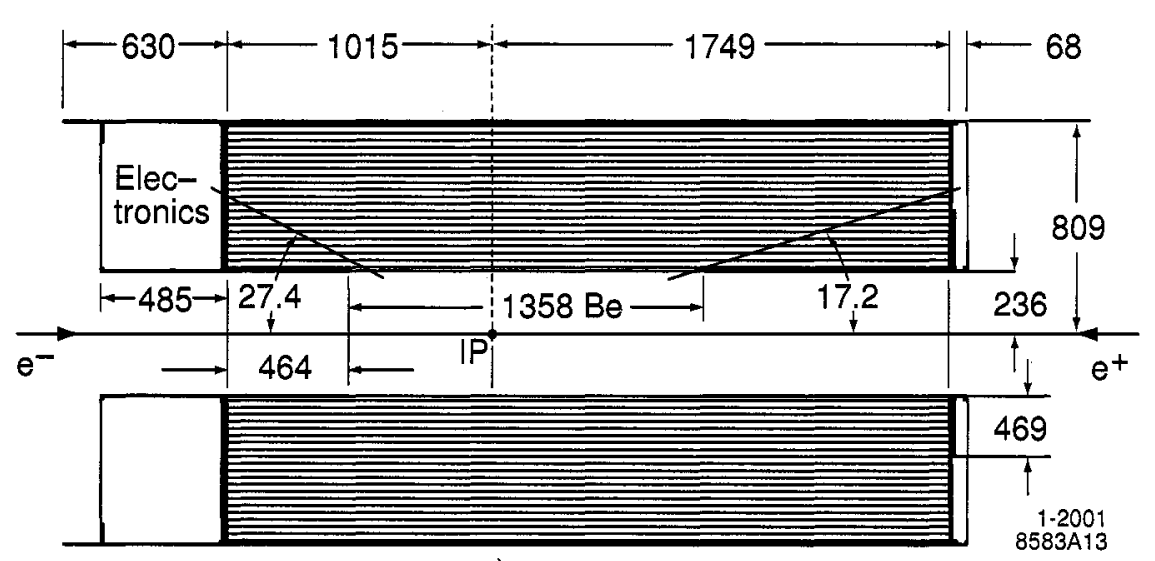

Figure 3.6: Longitudinal cross-section of the DCH.

using a variety of physics processes. It is performed relatively rarely, mainly after magnet quenches or detector access. The "global alignment" determines to overall position of the SVT with respect to the drift chamber using tracks with both SVT and $\mathrm{DCH}$ hits in sufficient number. It is performed approximately every 2-3 hours to correct for the diurnal variations in SVT position.

The achieved spatial resolution for SVT hits, in both $z$ and $\phi$, varies between 10 and $40 \mu m$ depending on the angle of incidence of the track relative to the SVT module, while the mean $d E / d x$ resolution for minimum-ionizing particles sampled over five layers is approximately $14 \%$.

\subsection{Drift Chamber (DCH)}

The BaBar drift chamber ( $\mathrm{DCH})$ is a tracking device which allows for the efficient detection of charged particles and precise measurement of their momenta, as well as the reconstruction of the decay vertices of long-lived particles such as the $K_{s}^{0}$, which may decay outside of the SVT. In addition, the drift chamber measures ionization loss $(d E / d x)$, which provides particle identification information complementary to that provided by the other subsystems. This is particularly critical for low-momentum particles and those in the extreme forward and backward regions of the detector. 


\subsubsection{Design and Geometry}

As shown in Fig. 3.6, the DCH is enclosed by two concentric cylinders with radii of $236 \mathrm{~mm}$ and $809 \mathrm{~mm}$, approximately $3 \mathrm{~m}$ in length, and a pair of aluminum endplates. Gold-coated aluminum field wires form 7,104 densely packed hexagonal drift cells, each with a gold-coated tungsten-rhenium sense wires at the center. The cells are arranged in 40 cylindrical layers. Wires in 24 of the 40 layers are strung at small angles (between $\pm 45 \mathrm{mrad}$ and $\pm 76 \mathrm{mrad}$ ) with respect to the $\mathrm{z}$-axis, allowing the extraction of longitudinal as well as axial position information. The layers are grouped by fours into ten superlayers; each layer of a superlayer has the same wire orientation (stereo angle) and an equal numbers of cells. Sequential layers within a superlayer are staggered by a half a cell. The stereo angles of the superlayers alternate between the axial (A) and stereo ( $\mathrm{U}$ and $\mathrm{V}$ ) pairs, in the order AUVAUVAUVA, as shown in Figure 3.7.

The DCH coverage in azimuth is complete and uniform; the polar acceptance of the $\mathrm{DCH}$ as defined by the most extreme angle at which a particle from the origin crosses at least 20 layers, is $17.2^{\circ}$ in the forward direction and $152.6^{\circ}$ in the backward direction.

The need to minimize multiple scattering, which limits the track resolution below $1 \mathrm{GeV}$, dictates the choice of the physical materials used in the drift chamber construction, as well as the choice of a low-mass gas mixture (an 80:20 helium-isobutane $\mathrm{mix}$ ). The inner cylindrical wall of the DCH is also kept thin to facilitate matching of SVT and DCH tracks and to minimize the background from photon conversions and interactions; the material in the outer wall and in the forward direction is minimized in order not to degrade the performance of the DIRC and EMC.

In order to keep the material in the forward direction to a minimum, the highvoltage (HV) distribution and all $\mathrm{DCH}$ readout electronics are mounted at the rear endplate of the chamber. 


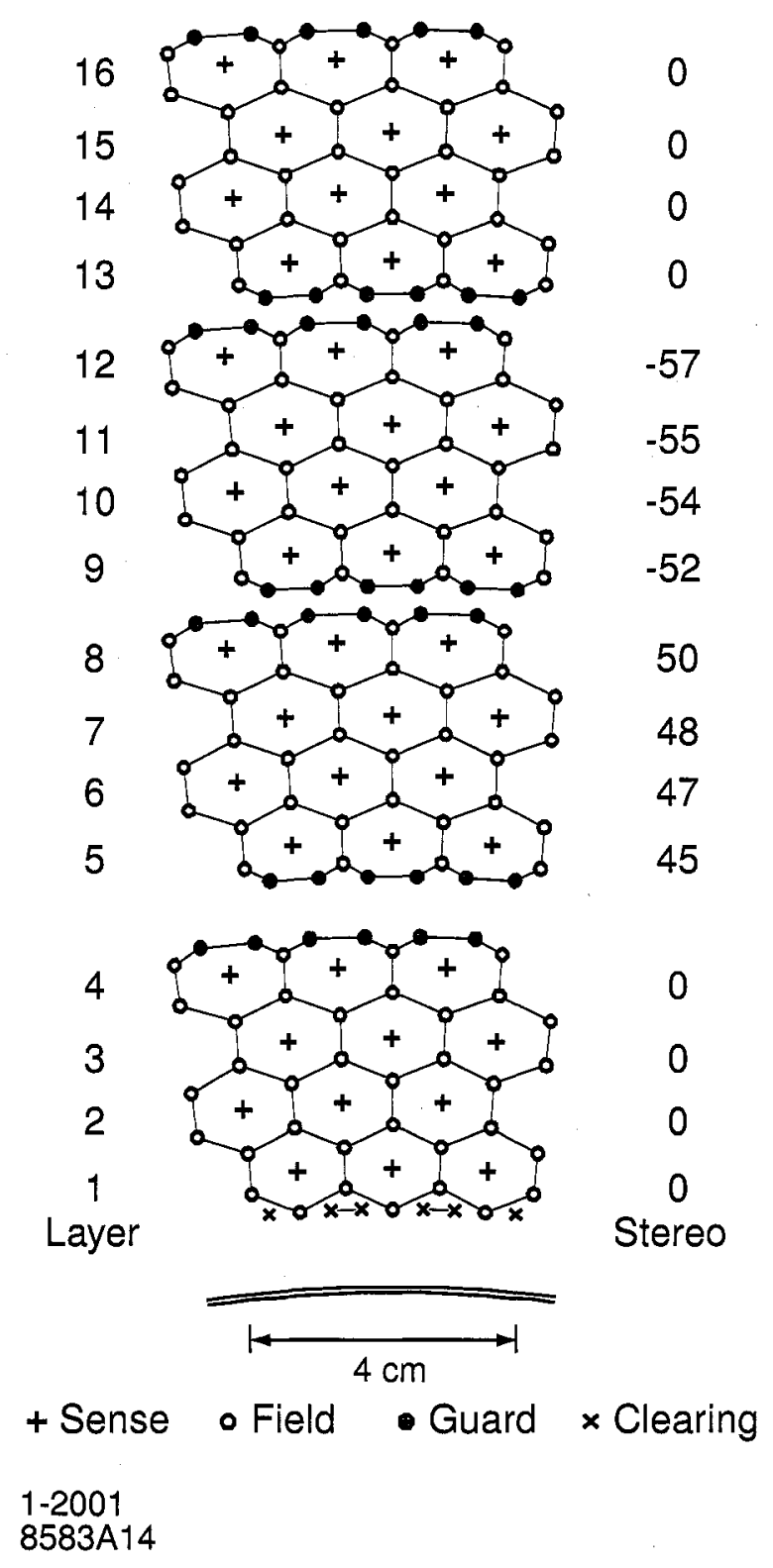

Figure 3.7: Schematic layout of the drift cells in the four inner DCH superlayers. 


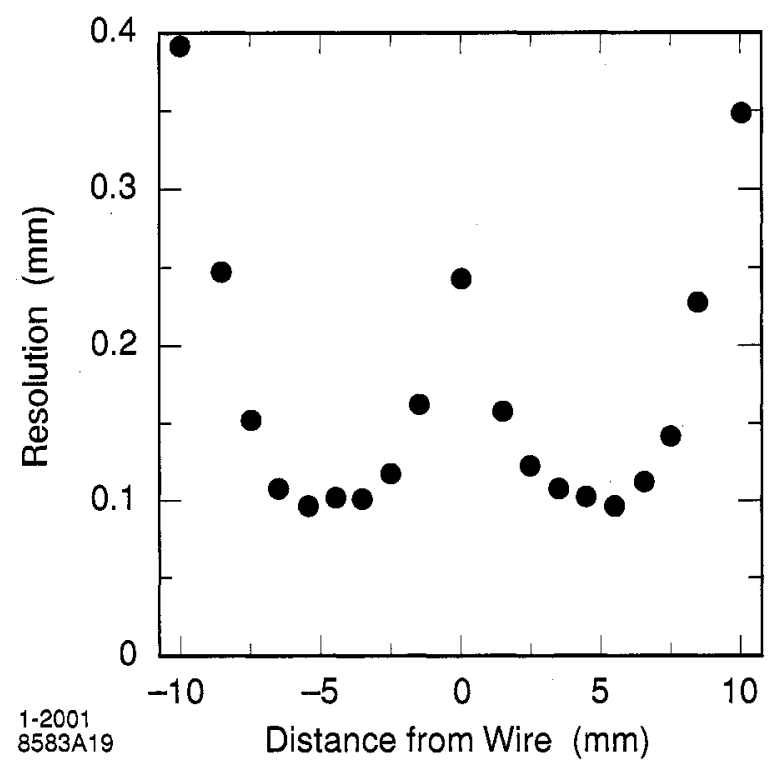

Figure 3.8: DCH single cell resolution.

\subsubsection{Single-Cell Performance}

Knowledge of both the drift time-to-distance relationship and the gas gain are required to determine the drift distance and ionization loss $(d E / d x)$ from the recorded TDC times and accumulated charge. Calibrations for both the time-to-distance relation and $d E / d x$ measurements were developed using cosmic ray data and then implemented for colliding beam data.

The relation between the measured drift time and drift distance is determined using tracks from $e^{+} e^{-}$scattering (Bhabha) and $\mu^{+} \mu^{-}$production. A track trajectory is reconstructed using a set of "hits" (TDC times associated with particular drift cells); an estimated drift distance for a cell along the trajectory is determined by computing the distance of closest approach between the track and the signal wire. An average time-to-distance relation is determined for each layer, but separately for the right and left-hand sides of the sense wire, by fitting a sixth-order Chebyshev polynomial to a set of estimated drift distances and measured drift times. Figure 3.8 shows the single-cell position resolution as a function of the drift distance for layer 18 of the $\mathrm{DCH}$. The resolution is $100 \mu \mathrm{m}$ away from the boundaries of the cell, but worsens close to the sense wire and the outer cell boundary. 
The specific energy loss $\left(\int_{\text {cell }}(d E / d x) d l\right)$ for charged particles traversing the $\mathrm{DCH}$ is derived from the measurement of the total charge deposited in each drift cell, as computed by the feature extraction algorithm. The specific energy loss per track is computed as a truncated mean from the lowest 80 percent of the individual cell $d E / d x$ measurements. Corrections are applied to compensate for changes in gas pressure and temperature, differences in cell geometry and charge collection, signal saturation due to space charge buildup, non-linearities in the most probable energy loss at large dip angles, and variation of charge collection as a function of entrance angle. The typical RMS resolution, which is limited by the number of samples and Landau fluctuations, is about $7.5 \%$.

\subsubsection{Tracking Performance}

Reconstruction of charged tracks relies on data from both the SVT and the DCH. Charged tracks are defined by five parameters: $d_{0}$ and $z_{0}$ (transverse distance and $\mathrm{z}$ coordinate at the point of closest approach of the track helix to the $\mathrm{z}$ axis), $\phi_{0}$ (azimuthal angle), $\lambda$ (dip angle with respect to the transverse plane), and $w=1 / p_{t}$ (track curvature). The track reconstruction builds on information from the Level 3 trigger and tracking algorithms (see Section 3.8.4), first refitting the trigger event time, T0, and then performing helix fits to the hits found by the Level 3 tracking algorithm. A search for additional DCH hits that may belong to a track is performed, and additional track-finding algorithms employed to identify tracks which do not traverse the entire $\mathrm{DCH}$ or do not originate from the interaction point.

Tracks found by this algorithm are refit using a Kalman filter, which accounts for local variations in material and magnetic field along the fitting trajectory. They are extrapolated back into the SVT, where SVT track segments are added. Unassociated SVT hits are passed to a pair of stand-alone SVT track-finding algorithms.

By comparing the number of tracks found in the SVT that extrapolate into the $\mathrm{DCH}$ acceptance to those actually found by the DCH the efficiency for DCH trackfinding has been determined to be $98 \pm 1 \%$. The tracking resolution in the four helix parameters and in transverse momentum $\left(p_{t}\right)$ are determined, in cosmic ray events, 
Table 3.3: Properties of CsI(Tl)

\begin{tabular}{lc}
\hline \hline Parameter & Value(s) \\
\hline Radiation Length & $1.85 \mathrm{~cm}$ \\
Molière Radius & $3.8 \mathrm{~cm}$ \\
Density & $4.53 \mathrm{~g} / \mathrm{cm}^{3}$ \\
Light Yield & $50,000 \mathrm{\gamma} / \mathrm{MeV}$ \\
Peak Emission Wavelength & $565 \mathrm{~nm}$ \\
Signal Decay Time & $680 \mathrm{~ns}(64 \%)$ \\
& $3.34 \mu \mathrm{s}(36 \%)$ \\
\hline \hline
\end{tabular}

to be

$$
\begin{array}{ll}
\sigma_{d_{0}}=23 \mu \mathrm{m} & \sigma_{\phi_{0}}=0.4 \mathrm{mrad} \\
\sigma_{z_{0}}=29 \mu \mathrm{m} & \sigma_{\tan \lambda}=0.53 \cdot 10^{-3}
\end{array}
$$

and

$$
\sigma_{p_{t}} / p_{t}=(0.13 \pm 0.01) \% \cdot p_{t}+(0.45 \pm 0.03) \%
$$

where $p_{t}$ is measured in $\mathrm{GeV} / c$.

\subsection{Electromagnetic Calorimeter (EMC)}

The electromagnetic calorimeter (EMC) is designed to measure electromagnetic showers with excellent efficiency, and provide energy and angular measurements over the energy range from $20 \mathrm{MeV}$ to $9 \mathrm{GeV}$, allowing for the detection of photons from $\pi^{0}$ and $\eta$ decays as well as from electromagnetic and radiative processes. Information about the shape of the electromagnetic showers detected by the EMC also makes it the primary source of information for electron identification. 


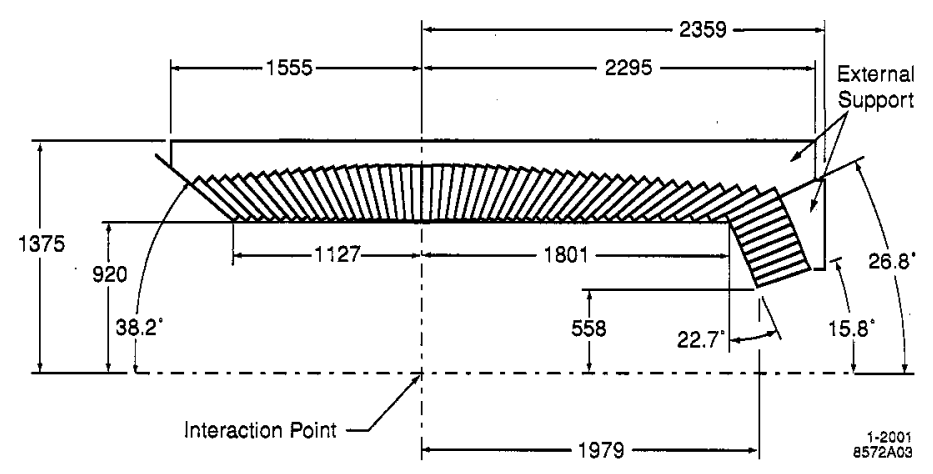

(a)

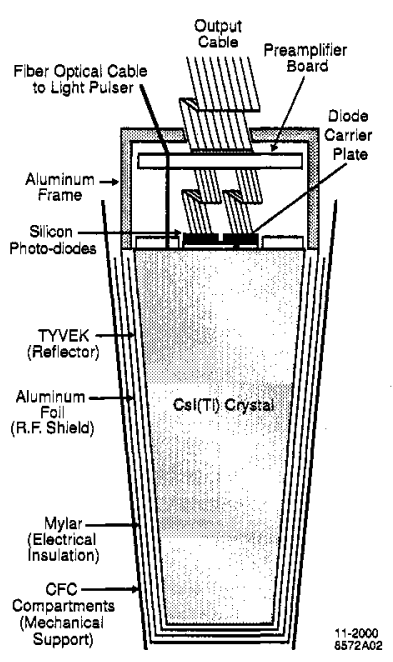

(b)

Figure 3.9: (a) Longitudinal section of EMC. (b) Crystal housing with front-end electronics

\subsubsection{Design}

The EMC is a crystal calorimeter, consisting of 6580 crystals of Thallium-doped Cesium Iodide ( $\mathrm{CsI}(\mathrm{Tl})$ ). Each crystal is approximately trapezoidal, with dimensions of about $4.7 \mathrm{~cm} \times 4.7 \mathrm{~cm}$ for the front face (the one facing the IP), $6.1 \mathrm{~cm} \times 6.1 \mathrm{~cm}$ for the back face and a typical length of $31.5 \mathrm{~cm}$. The exact dimensions of individual crystais vary so as to achieve hermetic coverage of the solid angle.

The properties of $\mathrm{CsI}(\mathrm{Tl})$ are shown in Table 3.5.1. CsI is an inorganic scintillator, with small values of both the radiation length, $X_{0}$, which characterizes the longitudinal size of electromagnetic showers, and the Molière radius $R_{M}$, which characterizes the transverse development of the shower. This allows for a compact calorimeter while ensuring that electromagnetic showers are fully contained, which improves the energy resolution. The EMC provides between 16.0 and 17.4 radiation lengths depending on the polar angle. The small value of the Molière radius enables a fine segmentation of the calorimeter, which improves position resolution.

The CsI is doped with $0.1 \%$ of Thallium, which provides a high light yield per 
$\mathrm{MeV}$ and a spectrum peaking in the region suitable for silicon photodiode readout.

As represented in Figure 3.9, the EMC consists of a cylindrical barrel, containing 48 rings of 120 crystals, and an endcap containing 8 rings rings of 120, 100, and 80 crystals each. It provides full azimuthal coverage and a polar angle acceptance extending from $15.8^{\circ}$ to $141.8^{\circ}$, which for a photon corresponds to a polar angle range of $-0.92<\cos \left(\theta^{*}\right)<0.89$ in the CM frame.

The mechanical support consists in 300 modules of carbon-fiber-epoxy composite which provide a separate compartment for each crystal. The 280 barrel modules consists of $7 \times 3$ (in $\theta \times \phi$ ) blocks of crystals, while the 20 endcap modules are azimuthal wedges, each containing 41 crystals. Crystals are supported on the sides only so as to minimize the amount of material seen by particles coming from the IP. The structure contributes about $300 \mu \mathrm{m}$ of material between crystals, with an additional $300 \mu \mathrm{m}$ at module boundaries. As shown in Figure 3.9, the material between the crystals is further increased by wrappings including $165 \mu \mathrm{m}$ of TYVEK [54], a reflective material that limits stray light losses on the crystal surface, and $25 \mu \mathrm{m}$ of aluminum foil and $13 \mu \mathrm{m}$ of mylar to provide magnetic and electrical insulation.

In order to minimize the amount of material in front of the crystals, the EMC is located inside the solenoid coil that provides the field for the tracking system. The material in front of the EMC varies from 0.3 radiation lengths in the central region to 0.4 radiation lengths, at the backward edge and 0.6 radiation lengths at the forward edge. However the inner three rings of the endcap are partially shadowed by the SVT electronics and B1 dipole and are mainly useful to provide containment for showers in the rest of the endcap.

\subsubsection{Readout}

Each CsI(Tl) crystal is read out by a pair of silicon photodiodes glued at the end of the crystal. Photodiodes are chosen instead of photomultipliers since the EMC is inside the solenoid and the $1.5 \mathrm{~T}$ magnetic field would interfere with their operation. The diodes have a quantum efficiency of $70 \%$ for the CsI $(\mathrm{TI})$ spectrum. The diodes signals consist of pulses of $2-3 \mu \mathrm{s}$. These pulses are processes by a CR-RC-RC 
shaper with shaping times of $0.8 \mu \mathrm{s}, 0.25 \mu \mathrm{s}$ and $0.25 \mu \mathrm{s}$ to reduce noise from beam backgrounds.

The signals are fed to a pair of low-noise preamplifiers. The amplified output is fed into the custom auto-range encoding (CARE) circuit; the total gain of the electronics chain is 256,32 , 4, or 1 for the four energy ranges $0-50 \mathrm{MeV}, 50-400 \mathrm{MeV}, 0.4-3.2$ $\mathrm{GeV}$, and 3.2-13.0 GeV. The two-fold redundancy of photodiodes and preamplifiers ensures reliability, since these components are inaccessible.

When an L1 Accept signal is received, feature extraction is performed in a window of $\pm 2 \mu \mathrm{s}$. Due to the continuous nature of BABARdata-taking (see Section 3.8.1) the event time cannot be determined from beam crossing signal, and prompt timing information is not available from any of the subsystems (unlike at BELLE, for instance, where the time-of-flight system can be used); the peak of the waveform therefore is located by a parabolic fit, which provides both the peak energy and the time. This has the advantage that the waveform peak time is measured independently of the global event time, so that the two can be compared to detect out-of-time events such as the stale Bhabha events described in Section 9.9.

\subsubsection{Reconstruction and Performance}

A typical electromagnetic shower spreads over a number of adjacent crystals, forming a cluster of energy deposits. Pattern recognition algorithms have been developed to not only identify these clusters, but to differentiate clusters with a single energy maximum from those with multiple energy maxima, referred to as bumps. A cluster is required to contain at least one seed crystal with an energy above $10 \mathrm{MeV}$. Surrounding crystals are included in the cluster if their energy exceeds a $1 \mathrm{MeV}$ threshold, or if they abut, in any direction, a crystal with at least $3 \mathrm{MeV}$ of energy. Clusters are split into as many bumps as there are local maxima, and an iterative algorithm is used to determine the bump energies.

At low energies (around 6.13 MeV), a radioactive source calibration measures the fractional EMC energy resolution to be $5.0 \pm 0.8 \%$; at higher energies (between 3 and $9 \mathrm{GeV}$ ), Bhabha scattering events are used to determine the resolution to 
be $1.9 \pm 0.07 \%$. In the intermediate range (below $2 \mathrm{GeV}$ ), the energy resolution is inferred from the mass resolution of reconstructed $\pi^{0} \rightarrow 2 \gamma$ and $\eta \rightarrow 2 \gamma$ decays, with the two photons of approximately equal energies.

The overall energy resolution may be parameterized by:

$$
\frac{\sigma_{E}}{E}=\frac{(2.30 \pm 0.03 \pm 0.30) \%}{\sqrt[4]{E(\mathrm{GeV})}} \oplus(1.35 \pm 0.08 \pm 0.20) \%
$$

The first term is dominated by the statistical fluctuations in scintillation photon yield, beam background, and electronics noise. The constant-energy term, which is dominant at high energies, is associated with light leakage and absorption in front of and between crystals.

The angular resolution is determined solely from symmetric $\pi^{0}$ and $\eta$ decays. A fit to an empirical parameterization of the energy dependence gives:

$$
\sigma_{\theta}=\sigma_{\phi}=\left(\frac{4.16 \pm 0.04}{\sqrt{E(\mathrm{GeV})}} \oplus 0.00 \pm 0.04\right) \mathrm{mrad}
$$

which gives a resolution of about $12 \mathrm{mrad}$ at low energies and $3 \mathrm{mrad}$ at high energies. This slightly exceeds the performance predicted in simulation.

The reconstructed $\pi^{0}$ mass is measured to be $135.1 \mathrm{MeV}$ and is stable to better than a percent over the full photon energy range, with a width of $6.9 \mathrm{MeV}$.

\subsection{Muon Chambers (IFR)}

The Instrumented Flux Return (IFR) is designed to identify muons with high efficiency and good purity, and to detect neutral hadrons (primarily $K_{L}^{0}$ and neutrons) over a wide range of momenta and angles. It uses the steel flux return of the magnet as a hadron absorber. Single gap resistive plate chambers (RPCs) are installed the gaps of the finely segmented steel.

It is composed of a hexagonal barrel region consisting of 6 sextants, and two endcaps, each split vertically into two "doors" that can be opened to access the inner detector components. An illustration is shown in Figure 3.10. There are 19 


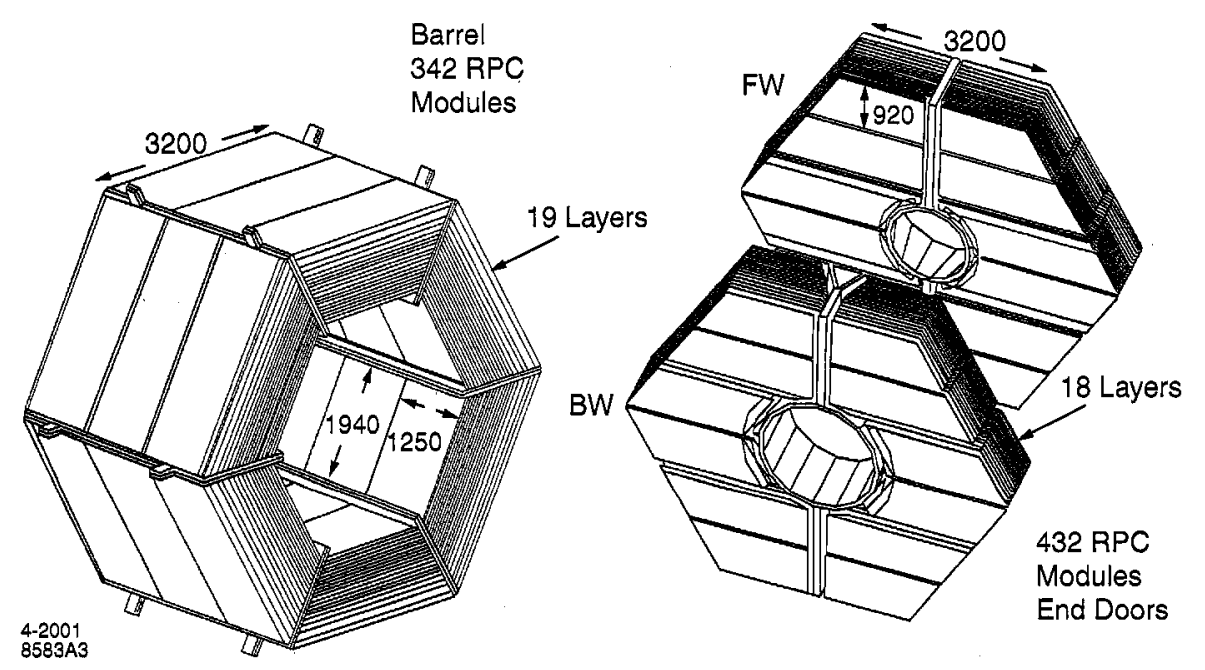

(a)

Figure 3.10: Geometry of the IFRbarrel and endcaps

instrumented gaps (layers) in the barrel, and 18 in the endcap.

\subsubsection{The Resistive Plate Chambers}

The RPCs detect streamers from ionizing particles via capacitive readout strips, and come in two geometric configurations:

- The planar RPCs consist of two Bakelite sheets, $2 \mathrm{~mm}$ thick, separated by a $2 \mathrm{~mm}$ gap, and enclosed at the edge by a $7 \mathrm{~mm}$ wide frame. A uniform gap width is maintained by polycarbonate spacers, glued to the Bakelite and spaced at distances of about $10 \mathrm{~cm}$. The gap is filled with a mixture of $56.7 \%$ argon, $38.8 \%$ freon, and $4.5 \%$ isobutane at about 1500 torr of pressure. The two external surfaces of the Bakelite sheets are coated in graphite; these graphite surfaces are connected to high voltage $(\simeq 8 \mathrm{kV})$ and ground, and protected by an insulating mylar film. The Bakelite surfaces facing the gap are treated with linseed oil. The RPCs are operated in limited streamer mode and the signals read out capacitively, on both sides of the gap, by external electrodes made of 
aluminum strips on a mylar substrate.

- The cylindrical RPCs are identical to the planar RPCs in terms of the gap thickness and spacer configuration, but are made of a set of resistive electrodes composed of conducting polymer and ABS plastic; these electrodes are laminated to fiberglass boards and foam to form a rigid cylindrical structure. Copper readout strips are attached to the fiberglass boards. Unlike the planar RPCs, no surface treatments of any kind have been applied.

The barrel RPCs are constructed in modules of $320 \times 130 \mathrm{~cm}^{2}$, with sets of three modules per gap and hexagonal face. A set of modules extending radially and covering the same region in $z$ and $\phi$ define a sector. The readout strips in each barrel module are arranged with 32 strips perpendicular to the beam axis for $z$ measurement, with another 96 strips orthogonal to the first set, and extending over the three-module set, to measure $\phi$.

\subsubsection{Operational Experience}

Cosmic ray tests determined that during the early period of running, $75 \%$ of the active RPC modules had an efficiency of at least $90 \%$. However, high-temperature conditions during early running, coupled with a problem in the curing of the linseed oil coating, caused a substantial fraction of the RPC modules to experience an ongoing degradation in efficiency.

\subsection{Particle Identification System (DIRC)}

The DIRC (Detector of Internally-Reflected Cherenkov light) is a novel ring-imaging Cherenkov radiation detector used for the identification of charged hadrons. The required momentum coverage of the DIRC is dictated on the one hand by kaon tagging for time-dependent asymmetry measurements, where the typical momentum involved is less than $1 \mathrm{GeV}$, and on the other by $K / \pi$ separation for the $B^{0} \rightarrow$ $\pi^{+} \pi^{-} / K^{+} \pi^{-}$decays, where the relevant momenta lie between 1.7 and $4.2 \mathrm{GeV}$ in 
the laboratory frame. The minimum transverse momentum required for a charged particle to traverse the DCH and reach the DIRC is $280 \mathrm{MeV}$, which means there is no need for the DIRC to have any sensitivity below this threshold.

\subsubsection{Design and Geometry}

The DIRC consists of 144 synthetic fused-silica bars with a refractive index of 1.473, arranged in a 12-sided polygonal barrel around the DCH and an array of 10752 photomultiplier tubes mounted on the stand-off box (SOB) behind the rear IFR doors. The configuration is illustrated in Figure 3.11. Charged particles traversing the bars emit Cherenkov radiation, which propagates by internal reflection to the photomultiplier array in the SOB, allowing reconstruction of the ring and determination of the Cherenkov angle.

The quartz bars are mounted in sets of 12 inside 12 aluminum bar boxes. These bars extend along the entire length of the $\mathrm{DCH}$, covering polar angles down to $25.5^{\circ}$ in the forward direction and up to $141.4^{\circ}$ in the backward direction, and extend back through the IFR doors to the SOB. The water tank of the SOB flares out from the bars in a conical shape, with twelve sets of $89629-\mathrm{mm}$ diameter ETL 8125

photomultiplier tubes (PMTs) mounted on the back wall. At the end of each bar is a silica wedge prism, designed to recover photons at wide angles with respect to the bar axis via total reflection. The typical distance between the end of a bar and the photomultipliers is $1.17 \mathrm{~m}$.

The index of refraction of water is 1.346 , close enough to that of the bars to minimize reflection at the interface, and chromatic dispersion is minimized as well. The typical distance between a bar and the PMTs, along with the size of the bars and PMTs themselves, gives a geometric contribution to the single photon Cherenkov angle resolution of $\simeq 7 \mathrm{mrad}$, a contribution somewhat larger than the approximately $5.4 \mathrm{mrad}$ RMS spread of the photon production and transmission dispersions. The overall single photon resolution is estimated to be about $10 \mathrm{mrad}$. 


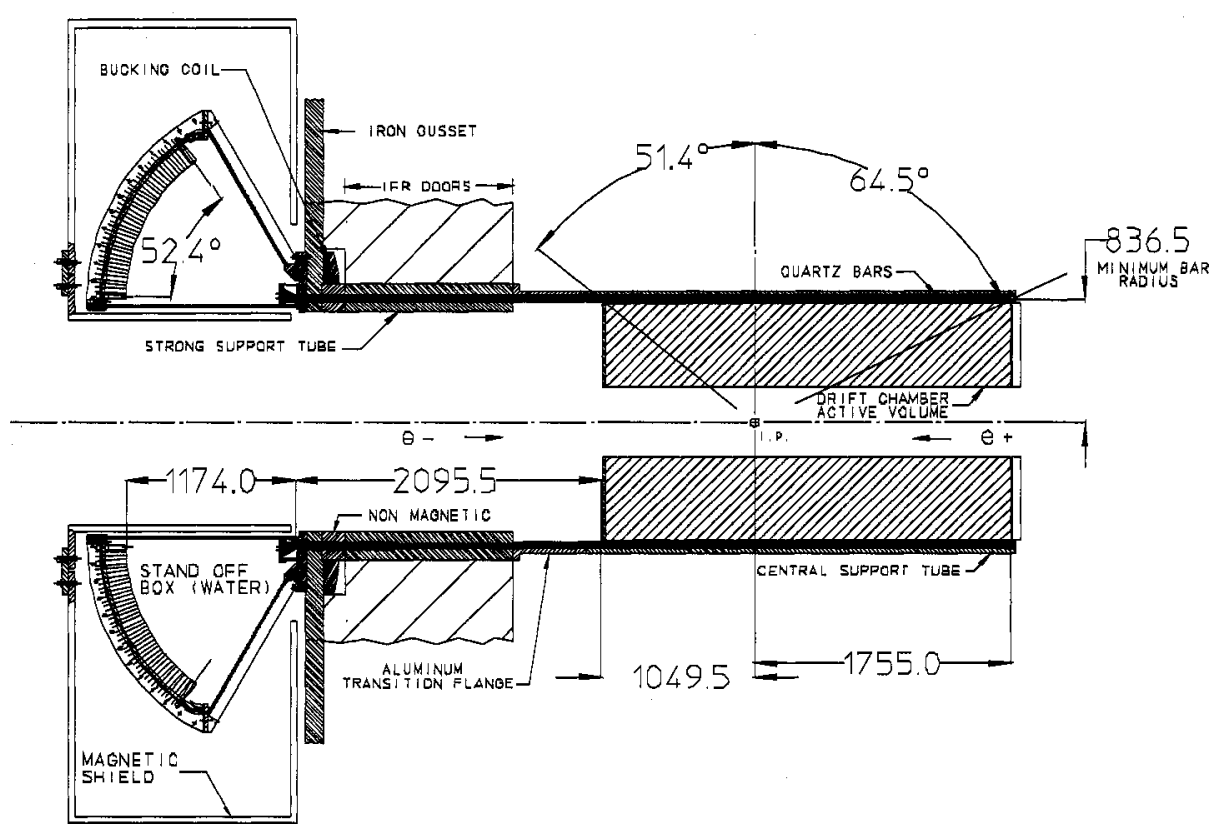

(a)

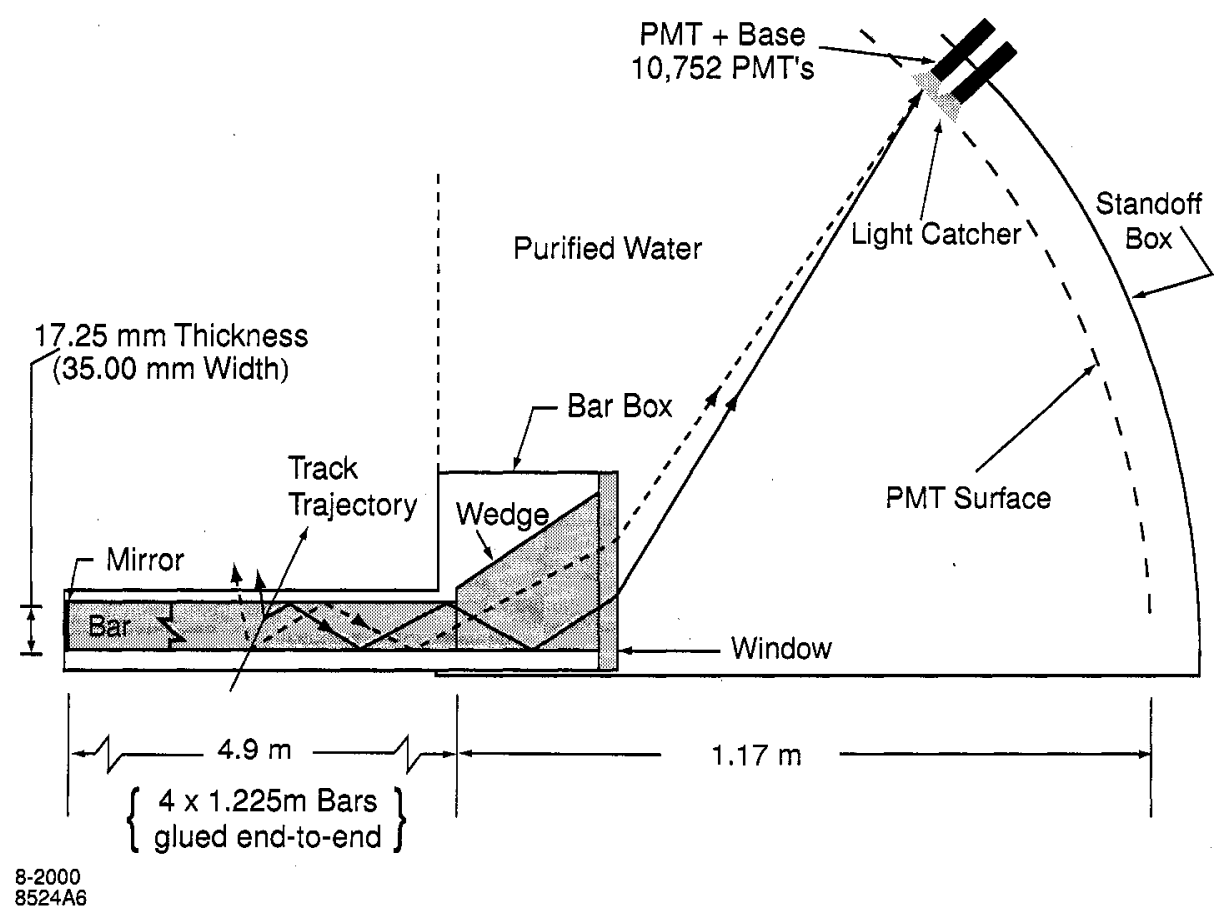

(b)

Figure 3.11: (a) Elevated view of overall DIRC geometry. (b) Bar/SOB transition region. 


\subsubsection{Electronics and Reconstruction}

The DIRC front-end electronics (FEE) are designed to measure the arrival time of each detected Cherenkov photon to an accuracy that is limited by the intrinsic $1.5 \mathrm{~ns}$ transit time spread of the PMTs, and to monitor pulse-height spectra in order to ensure that the PMTs are operating at a voltage which ensures a stable gain (HV plateau) and timing. The DIRC FEE are mounted on the outside of the standoff box, and consist of a set of 168 DIRC Front-end Boards (DFBs), each processing 64 PMT inputs. The four 16-channel custom-IC TDCs allow for an independent timing measurement for each PMT, while a single 8-bit flash ADC (FADC) multiplexes the pulse-height information for all 64 channels. Only the TDC output is used in reconstruction, with the $\mathrm{ADC}$ only used for calibration monitoring purposes. The TDC has $0.5 \mathrm{~ns}$ binning, allowing the photon arrival time to be determined to better than the intrinsic $1.5 \mathrm{~ns}$ accuracy.

Calibration of the DIRC TDCs is achieved using 1 ns pulses from blue LED light pulsers; approximately 65,000 pulses per PMT are used in the calibration, to achieve a statistical accuracy of less than $0.1 \mathrm{~ns}$. A complementary method compares observed and expected light arrival times associated with tracks in actual collision data; this method yields an improved resolution (about $15 \%$ better) and consistent results.

Reconstruction of the emission angle and the arrival time of the Cherenkov photons produced by a charged track in the DIRC is done using observed space-time coordinates of the PMT signals, transformed into the Cherenkov coordinate system $\left(\theta_{c}\right.$ and $\phi_{c}$, the polar and azimuthal angles relative to the cone direction, and $\delta t$, the difference between the observed and expected arrival times). A set of threedimensional vectors, from the end of a radiator bar to the center of each coupled PMT, are extrapolated into the radiator bar using Snell's law and determined up to a 16-fold ambiguity. The uncertainties derive from the last reflection in the bar (forward/backward, left/right), whether the photon scattered off of the coupling wedge, and whether the photon initially propagated forward or backward. The timing resolution cannot provide competitive position information, but is used to suppress beaminduced background by about a factor of 40 , to exclude other tracks in the same event, and to reduce the 16-fold ambiguity to a three-fold ambiguity. The reconstruction 
algorithm then maximizes the likelihood of the entire event, based on the individual track likelihoods for the electron, muon, pion, kaon, and proton hypotheses. When coupled with $d E / d x$ information from the SVT and DCH, the DIRC achieves better than $90 \%$ kaon identification efficiency, with a less than $3 \%$ pion misidentification rate, for tracks which intersect with the radiator bars and have momenta between 0.5 and $3 \mathrm{GeV}$. Protons are identified with more than $80 \%$ efficiency over most of the range of kinematically allowed momenta, with pion and kaon misidentification rates below $4 \%$ for particle momenta up to $6 \mathrm{GeV}$ [55].

\subsection{Trigger}

\subsubsection{Data Acquisition and Trigger requirements}

In previous experiments, such as CLEO or the LEP experiments, the trigger and data acquisition was synchronized with the bunch crossing frequency, with the detector being read out only in a small window around the bunch crossing times. At Bfactories however, high luminosity is achieved in part by a large increase in the number of bunches and a corresponding increase of the bunch frequency: the time between crossings is $4.2 \mathrm{~ns}$ at BABAR compared to $360 \mathrm{~ns}$ at CLEO-III [56] and at $23 \mu \mathrm{s}$ at LEP. Due to the shortness of this time the data is for all intents and purposes be considered as a continuous stream.

$B A B A R$ is therefore read out continuously, with a sampling interval of $67 \mathrm{~ns}$ corresponding to 16 bunch crossings. Most of these samples are empty events, but detector activity alone is not a sufficient criterion to select the event: physics processes, by which we refer to $q \bar{q}, \mu^{+} \mu^{-}$and $\tau^{+} \tau^{-}$events, make up about $6.5 \mathrm{nb}$ of total crosssection, which at the current PEP-II luminosity of about $10^{34} \mathrm{~cm}^{-2} \mathrm{~s}^{-1}$ corresponds to an event rate of $65 \mathrm{~Hz}$. By comparison, Bhabha scattering events contribute a crosssection of about $50 \mathrm{nb}$ in the detector acceptance, corresponding to a rate of $500 \mathrm{~Hz}$, and beam backgrounds produce detectable tracks and clusters at rates greater than $20 \mathrm{kHz}$. Since data processing is limited to about $100-200 \mathrm{~Hz}$, a reduction in the event rate of at least two orders of magnitude is necessary. The role of the trigger 


\begin{tabular}{lcc}
\hline \hline Subdetector & Number of channel & Time granularity (ns) \\
\hline SVT & 140,000 & $67 \mathrm{~ns}$ \\
DCH & 7,104 & $67 \mathrm{~ns}$ \\
DIRC & 10,752 & $0.5 \mathrm{~ns}$ \\
EMC & 6,580 & $250 \mathrm{~ns}$ \\
IFR & 42,000 & $1 \mathrm{~ns}$ \\
\hline \hline
\end{tabular}

Table 3.4: DAQ parameters for the subdetectors

system is to select a subset of events that meets this rate limit and is highly efficient on physics events.

For this purpose, the event is read out and a limited subset of the event data is sent to the trigger for processing. Unlike CLEO, data acquisition at BABAR is fully pipelined: at CLEO the detector is temporarily inhibited while the trigger decision is being reached; at BABAR the trigger processing stages work in parallel, and events are continuously fed into the trigger as they come in, while earlier events move to later stages of processing. While the events are being processed, their data are stored in FIFO (first in, first out) buffers located on the front-end electronics. Since the event data must be stored at least until the trigger decision is returned, the processing time of the trigger is limited by the size of those buffers.

As shown in Table 3.8.1, the SVT is the limiting factor due to its large number of readout channels. The capacity of the FIFO corresponds to 193 events. This adds up to a stored time interval of about $13 \mu \mathrm{s}$, which provides an upper bound on the trigger latency.

\subsubsection{Trigger Design}

The design of the trigger is subject to several requirements:

- Efficiency: The trigger should achieve excellent efficiency in all the physics processes described above. While this is relatively straightforward for $q \bar{q}$ events due to their high multiplicities, other processes such as $\tau^{+} \tau^{-}, \gamma \gamma$ collisions and radiative events pose more difficult challenges. 
- Measurability: The efficiency of the trigger selection should be measurable with a high precision.

- Simplicity: The trigger algorithms should be sufficiently straightforward to be easily reproduced in simulation.

- Stability: The performance of the trigger should be relatively stable over time; in particular, it should not be overly sensitive to variations in beam conditions or changes in the detector local performance. such as the changes in detection efficiencies or the appearance of EMC hot towers.

In order to meet these requirements, the following conceptual guidelines are used:

- Openness: the selection criteria are based on general topologies, not engineered toward specific processes. This ensures that a wide range of processes pass the trigger, including some which were not planned for. Background rates are limited by increasing the momentum and energy thresholds, the required multiplicities, etc.

- Orthogonality: selection criteria for the various trigger lines are chosen to be as orthogonal as possible, thereby producing uncorrelated samples that can be used to cross-calibrate the selections.

- Robustness: selections are made as robust as possible, against both missing and spurious signals. A given process should be passed by multiple trigger lines, using information from multiple subdetectors, and allow for missing hits or particles. Conversely, no one-particle trigger lines are implemented to prevent backgrounds or detector noise to significantly affect the trigger rate.

- Prescaled Events: at all stages in the trigger, a small fraction of events is passed regardless of the trigger decision, to provide a sample for performance studies.

- Storage of Primitives: all the objects ("primitives") used in the trigger decision are stored as part of the event data and can be used for offline performance studies. 
In order to meet the design goals, a two-tier trigger system is implemented: a hardware trigger, Level 1, and a software trigger, confusingly referred to as Level 3 . This is a standard design already used at CLEO [56]. Unlike CLEO and BELLE, however, the trigger is fully pipelined, receiving and processing information continuously.

The read-out of the event buffers of the subdetectors is triggered by the $L 1$ accept signal. The data is then passed to the Online Event Processing (OEP) framework which runs the Level 3 trigger. Events that pass Level 3 are written into the dataset, and the rest is discarded.

The two trigger levels are further required to meet the following rate and latency requirements:

- Rates: The L3 output rate should not exceed $120 \mathrm{~Hz}$ at a luminosity of $3 \times$ $10^{33} \mathrm{~cm}^{-2} \mathrm{~s}^{-1}$. In order to limit the load on $\mathrm{L} 3$, the $\mathrm{L} 1$ rate is limited to $2 \mathrm{kHz}$ at the same reference luminosity.

- Latency and Jitter: As described in the previous section, the latency of the L1 trigger decision should not exceed $12 \mu \mathrm{s}$. Furthermore, the time of the accepted must be determined to better than $1 \mu \mathrm{s}$ to limit the size of the search window that each subsystem must use when collecting event data. The L1 latency should therefore be in the interval $11<L<12 \mu \mathrm{s}$. For L3, the latency limit is set at $10 \mathrm{~ms}$ per event.

In addition to the trigger itself, a further stage of processing, the Background Filter, is applied to the data before they are fully processed for physics use. While this is part of the data processing path it is not part of the trigger per se since it is done offline, and is covered separately in the next section.

Following orthogonality design, The L1 trigger is further split into three separate components, DCT, EMT, and IFT, which process information from the DCH, EMC and IFR respectively. The information from these systems is combined in the GlobalLevel Trigger (GLT) which provides the L1 Accept decision and the event time T0. IFT information is sparingly, primarily to identify cosmics and dimuon events. The SVT and DIRC are not used for triggering purposes, since their data cannot be made available fast enough to meet the latency requirements. 


\subsubsection{Drift Chamber Trigger}

The DCT uses information from the DCH to trigger on events with charged tracks. It implements rudimentary but extremely fast track-finding algorithms that fully process the whole of the $\mathrm{DCH}$ in about $5 \mu \mathrm{s}$. This section describes the version of the hardware that was in place until the end of the year 2004, which used information in the $r-\phi$ plane only and a primitive determination of track $p_{t}$. Since 2005 one of its components (PTD) was replaced by a newer system (ZPD) that uses information along the $z$ axis and performs track fits. Since the data used in this work was not taken before the upgrade, only the old system is described here. More information on the ZPD can be found in Ref. [57]. The rest of the system is described in Refs. $[58,59]$.

\section{Track segment finding}

The Track Segment Finder (TSF) is the first step of DCT processing, using raw DCH hit data to create "TSF segment" objects used at later stages. It processes data from each of the $7104 \mathrm{DCH}$ cells at a rate of $4 \mathrm{MHz}$. For each $269 \mathrm{~ns}$ tick, the hit status of all the wires is read out, and each wire is assigned a two-bit value that is incremented at every clock tick for which a signal is present (that is, the first time the wire reports a hit it is assigned a value 1 , which is increased to 2 if a hit is also recorded in the next clock tick, and so on), providing coarse drift-time information. The data is split between 24 TSF boards. The splitting is designed so that each section contains roughly the same number of bits and goes as follows:

- 16 "type X" boards receive the data from superlayers 1-4 and 8-10 in $1 / 16$ wedges of the drift chamber

- 8 "type $\mathrm{Y}$ " boards receive the data from superlayers 5-7 in neighboring pairs of $1 / 16$ wedges

The 1776 wires in the third layer of each supercell are called pivot wires, and the surrounding 8-wire pattern shown in Fig. 3.12 is the corresponding pivot group. In the case where such cells overlap two 1/16 wedges, neighbor data is shared between TSF modules to reconstitute the full cell information. The TSF electronics boards 


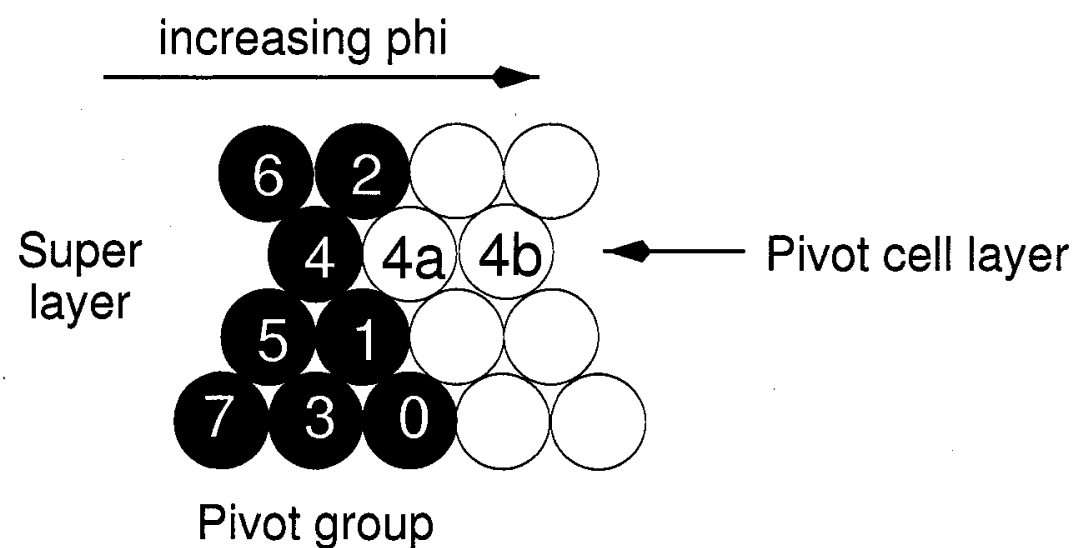

Figure 3.12: Representation of a TSF pivot group. Wire \#4 is the pivot wire.

contain 1776 track segment finder engines, each associated with a pivot group, which check the eight wires of the group at each 269 ns tick. If hits are recorded, the engine identifies the hit pattern from among the 65536 possibilities. Only patterns with hits in at least three layers are considered. The wire pattern is used to address a look-up table, obtained from the study of TSF segments in data, which gives:

- The fine- $\phi$ : for some patterns, the wire hit configuration gives a determination of the segment $\phi$ that is more precise than simply the $\phi$ value of the pivot wire, with a precision of $\sim 800 \mu \mathrm{m}$ in the best cases.

- The pattern weight: a 2-bit word encoding the pattern quality. Its value is 0 if no segment is found, 1 if no fine- $\phi$ value could be determined, and 2 or 3 for patterns with fine- $\phi$ information and hits in respectively two layers and all three layers of the pivot group, in addition to the pivot wire.

- The pattern time: a 2-bit value based on the wire time values.

\section{Binary Link Tracker}

The BLT "strings" together the track segments produced by the TSF to form full tracks. The input data is based on "supercells", each corresponding to a $1 / 32 \phi$ wedge in each superlayer. The full TSF data is reduced to 1 bit per supercell by 
scanning all the pivot groups in the supercell for 6134 - ns ticks. If segments are found, the bit is set at the time of the highest-weight segment; otherwise the bit is set to 0 .

The 320 bits are handled by a single BLT module, which processes information from the entire DCH, every $134 \mathrm{~ns}$. This is twice faster than the TSF clock since the pattern time information allows a finer time granularity.

The algorithm, based on the CLEO-II trigger [60], starts by looking for a "seed" hit on superlayer 1 , then moves radially outward. The stereo angles in the wires create apparent $\phi$ shifts for hits in successive superlayers, except for tracks near the backward DCH endplate, so a rotation is first applied so that the hits from a track passing though the center of the detector at an angle $\theta=\pi / 2$ line up. However hits from tracks with small or large $\theta$ values or not originating from the detector center may not line up. For transitions from an axial superlayer to a stereo superlayer, such as SL $1 \rightarrow$ SL 2, the second hit can therefore be in one of the three supercells with $\phi$ positions closest to the first hit. For transitions from a stereo superlayer to another, where the stereo effect is doubled, the second hit should be in one of the five closest supercells in $\phi$.

A track reaching superlayer 5 is called a "B" track, while a tracks reaching superlayer 10 is called an "A" track. In order to be robust against cell inefficiencies or the loss of a superlayer, the algorithm is implemented so that B tracks can be identified even if one of hits is missing, while A tracks can have one missing hit in superlayers 1-5 and another in superlayers 6-10.

The output of the BLT is a pair of $\phi$ maps, for A and B track information, giving the position of the outermost hit on the track (in superlayer 10 for $\mathrm{A}$ tracks and superlayer 5 for B tracks). This information then goes through a "2-to-1" conversion process which converts the $1 / 32$ wedge maps into $1 / 16$ wedge maps used as input to the GLT.

\section{PT discriminator}

The PTD is designed to process the TSF output to look for high- $p_{t}$ tracks. The TSF data is passed to 8 PTD modules every 269 ns. Each module has access to a $1 / 4$ 
wedge of the drift chamber, but only the central $1 / 8$ wedge is used for the algorithm, the rest being used to supply neighbor data. Only TSF segments in axial superlayers (superlayers 1,4,7 and 10) are used to avoid stereo effects.

The algorithm starts by looking for a "seed" segment in superlayers 7 or 10 (the possibility of a seed either of two superlayers is to ensure robustness against the loss of one superlayer), which must be weight 3 in order to ensure accurate fine- $\phi$ information. If it belongs to a high- $p_{t}$ track, the track should have other hits in the envelopes shown in Fig. 3.13. A look-up table is therefore used to define allowed fine-

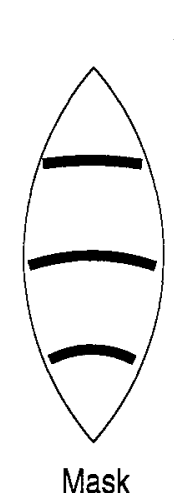

A10 Seed

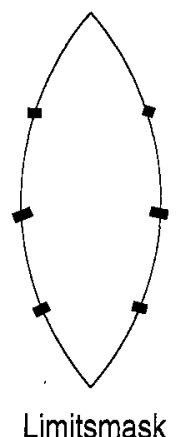

A10

Limitsmask
A7

A4

A1

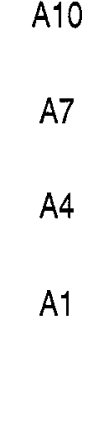

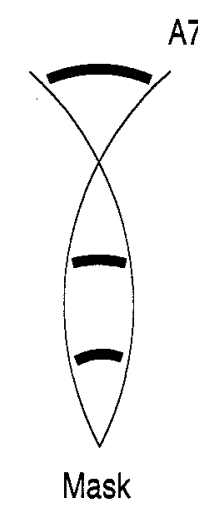

A7 Seed

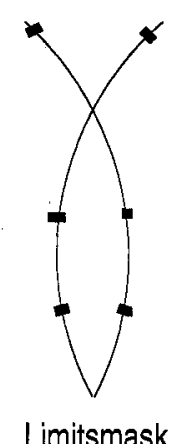

Figure 3.13: Envelopes for high- $p_{t}$ tracks with hits in SL 7 and SL 10.

$\phi$ intervals in the remaining 3 superlayers in which to look for additional segments. These ranges are what determines the effective $p_{t}$ threshold. They are configurable and were set to correspond to a threshold of $800 \mathrm{MeV} / \mathrm{c}$.

If a segment (of any weight) is found in the appropriate range on at least 2 of the 3 remaining superlayers, a high- $p_{t}$ track object, called $\mathrm{A}^{\prime}$, is reported at its supercell position (in units of $1 / 32$ wedge) on superlayer 10.

As for the BLT, as 2-to-1 conversion is performed in the PTD output before it is handed to the GLT, so that the final output is a-16-bit $\phi$ map coding the presence or absence of an $\mathrm{A}^{\prime}$ object.

Fig. 3.14 shows the efficiencies of the BLT and PTD as a function of track $p_{t}$. 


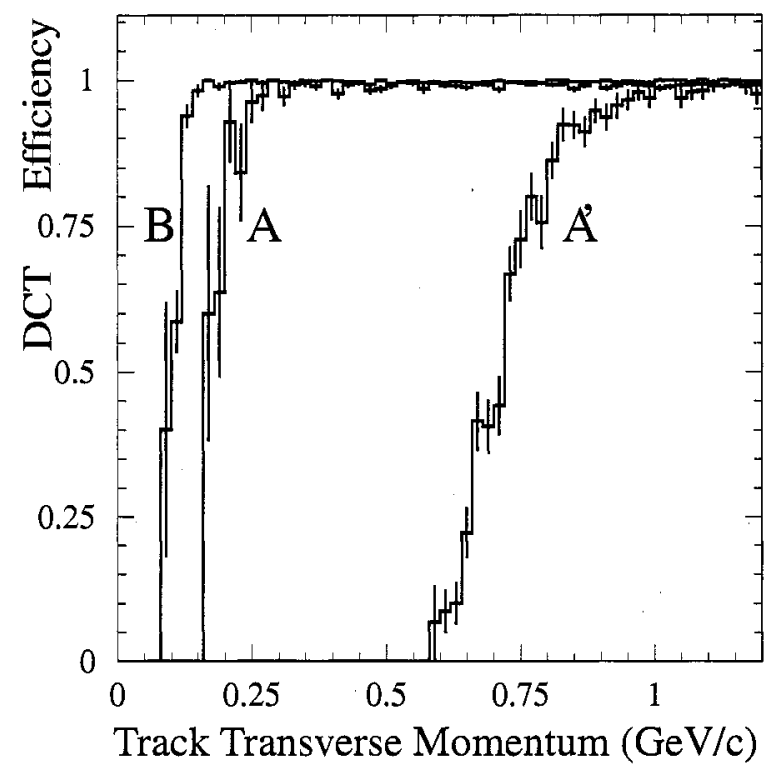

Figure 3.14: Detection efficiencies for the BLT A and B tracks and PTD $\mathrm{A}^{\prime}$ track as a function of track $p_{t}$

\section{The Electromagnetic Calorimeter Trigger}

In parallel to the DCT, the trigger implements an independent set of trigger objects based on EMC information, both to improve the efficiency of event with neutral particles and to provide and orthogonal selection that can be used for cross-calibration.

Unlike the DCT, the EMT uses $\theta$ information as well as $\phi$ : the EMC is divided into 280 towers, with 7 divisions in $\theta$ and 40 in $\phi$. The first $\theta$ bin corresponds to the endcap, and each $\phi$ bin forming a wedge containing either 19 or 22 crystals (rings 3-5 of the endcap contain 100 crystals, which is not divisible by 40). The remaining $\theta$ bins corresponds to the barrel, which is evenly split into 240 blocks of $8 \times 3(\theta \times \phi)$ crystals. In each tower, crystal energies above a $20 \mathrm{MeV}$ threshold are summed and the result, coded as a 16-bit word, is sent to the EMT every $269 \mathrm{~ns}$.

The data is processed by 10 Trigger Processing Board (TPB) modules, each dealing with an EMT $\phi$ sector spanning 4 of the $40 \phi$ divisions and the the whole $\theta$ range. The energies of the towers in each of the $40 \phi$ divisions if performed in three ways:

- Full sum: the energies are summed over the whole $\theta$ range. 
- Y sum: the sum is performed over the last two $\theta$ bins only, corresponding to the backward barrel. This is designed to catch low-angle Bhabha events where the forward electron is outside EMC acceptance.

- X sum: the sum is performed in the first $\theta$ bin (endcap) only, with the purpose of identifying beam background.

In all cases, the summing circuit have access to neighboring $\phi$ sectors in order to identify energy deposits which span adjacent sectors.

This information is used to identify 5 types of EMT primitives:

- M clusters: $\phi$ bin with a full sum of at least $120 \mathrm{MeV}$, used to identify minimumionizing particles

- G clusters: $\phi$ bin with a full sum of at least $300 \mathrm{MeV}$.

- E clusters: $\phi$ bin with a full sum of at least $800 \mathrm{MeV}$, for high-energy clusters

- X clusters: $\phi$ bin with an $\mathrm{X}$ sum of at least $100 \mathrm{MeV}$, to identify clusters from beam background

- Y clusters: $\phi$ bin with an $\mathrm{Y}$ sum of at least $1 \mathrm{GeV}$, to identify high-angle Bhabhas.

The $M$ cluster finding efficiency is shown in Fig. 3.15. A 2-to-1 conversion is applied on to the $\mathrm{M}, \mathrm{G}, \mathrm{E}$ and $\mathrm{X}$ primitives, so that a 20 -bit $\phi$ is passed to the GLT. For the $Y$ primitives, a 4-to- 1 conversion is performed and a 10-bit $\phi$ map is produced. The total processing is done in $184-\mathrm{MHz}$ ticks, or about $5.1 \mu \mathrm{s}$ in total.

\section{The IFR Trigger}

The primary role of the IFR trigger (IFT) is to identify cosmic rays and $\mu^{+} \mu^{-}$events. For this purpose the IFR is segmented into ten regions: the six sextants of the barrel and the four half end doors. In each section, 8 layers are used for triggering. The signals from all $\phi$ strips in this layers are combined to a single bit, and the region is declared active if at least 4 of the 8 layers are active in a given $134 \mathrm{~ns}$ time bin. The 


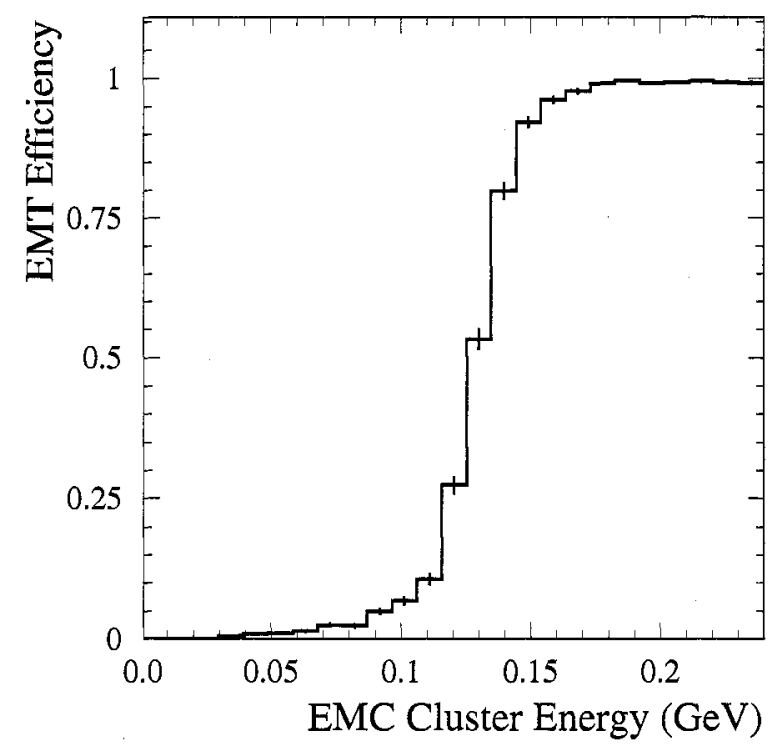

Figure 3.15: Detection efficiency for the EMT M object as a function of cluster energy

signal from all 10 regions is then combined to form 7 trigger primitives, described in Table 3.5.

\section{Global Level Trigger}

The GLT combines the information provided by the DCT, EMT and IFT primitives to make a trigger decision and determine the event time. The GLT receives information from each subsystem every $134 \mathrm{~ns}$.

Table 3.5: IFT trigger objects

\begin{tabular}{ll}
\hline \hline Object & Description \\
\hline U1 & 2 muons, other than U5-U7 \\
U2 & 1 muon in the backward endcap \\
U3 & 1 muon in the forward endcap \\
U4 & 1 muon in the barrel \\
U5 & 2 back-to-back muons in the barrel and 1 forward \\
U6 & 1 muon in the barrel and 1 forward \\
U7 & 2 back-to-back muons in the barrel \\
\hline \hline
\end{tabular}


The first step is to ensure that all sources are synchronized. The BLT and PTD path both have a latency of about $6 \mu$ s (see Table 3.8), including $\mathrm{DCH}$ readout time, while the EMT requires about $7.2 \mu$ s with EMC readout and the IFT only $0.2 \mu \mathrm{s}$. Configurable delays are applied to the inputs to ensure their alignment in time. Some signals are also "stretched" in time to ensure their overlap with others.

The next step is to create composite primitives, as follows:

- track-cluster matching: $\mathrm{AM}, \mathrm{BM}$ and $\mathrm{A}^{\prime} \mathrm{M}$ objects are created by matching $\mathrm{M}$ clusters with the corresponding tracks type. Since for $A$ and $A^{\prime}$ tracks $\phi$ is reported on superlayer 10 this can be done simply by comparing $\phi$ positions, although this is complicated by the fact that tracks are reported in bins of $1 / 16$ wedge and clusters in units of $1 / 20$ wedge. The cluster is required to be either in the EMT $\phi$ bin closest to the track $\phi$ bin or either of its neighbors, which corresponds to an angular tolerance of about $27^{\circ}$. For B tracks the $\phi$ is reported on superlayer 5 , so in principle a larger tolerance should be used, but in fact that is not the case: this imposes that the track $\phi$ at superlayer 10 be not too different from its value at superlayer 5 , which serves as an effective $p_{t}$ cut on BM objects.

- BMX objects: These are a slightly modified form of M clusters in which M clusters which occur in the same $\phi$ bin as an X clusters are vetoed if no B track is matched to the $\mathrm{X}$ cluster. This is designed to veto low-angle beam background but has so far not been used in the trigger decision.

- Back-to-back objects: These objects are obtained from combining two primitives with large angular separation and are denoted by a " $*$ ", referring to the pair. $\mathrm{A}^{*}$ and $\mathrm{B}^{*}$ objects are pairs of tracks with at least $124^{\circ}$ ( 6 bins) of separation, while $\mathrm{E}^{*}$ and $\mathrm{M}^{*}$ are pairs of clusters at least $117^{\circ}\left(7\right.$ bins) apart. $\mathrm{EM}^{*}$ objects are created by reducing the number of bins of the $\mathrm{E}$ and $\mathrm{M} \phi$ maps to 10 and combining objects more than $126^{\circ}$ (4 bins) apart.

- Separation (or skip): A minimal separation is imposed on hits in the $\phi$ maps in order for them to count as separate particles, since a particle may leave signals 
Table 3.6: GLT trigger objects and counting rules.

\begin{tabular}{llcccc}
\hline \hline Object & Description & Threshold & bins & Skip & Skip Angle \\
\hline B & Short track reaching DCH superlayer 5 & - & 16 & 1 & $34^{\circ}$ \\
A & Long track reaching DCH superlayer 10 & - & 16 & 1 & $34^{\circ}$ \\
$\mathrm{A}^{\prime}$ & High $p_{T}$ track & $800 \mathrm{MeV} / c$ & 16 & 1 & $34^{\circ}$ \\
$\mathrm{B}^{*}$ & Large-angle pair of short tracks & - & 16 & 5 & $124^{\circ}$ \\
$\mathrm{A}^{*}$ & Large-angle pair of long tracks & - & 16 & 5 & $124^{\circ}$ \\
\hline $\mathrm{M}$ & All- $\theta$ MIP energy & $120 \mathrm{MeV}$ & 20 & 1 & $27^{\circ}$ \\
$\mathrm{G}$ & All- $\theta$ intermediate energy & $300 \mathrm{MeV}$ & 20 & 1 & $27^{\circ}$ \\
$\mathrm{E}$ & All- $\theta$ high energy & $800 \mathrm{MeV}$ & 20 & 1 & $27^{\circ}$ \\
$\mathrm{Y}$ & Backward barrel high energy & $1 \mathrm{GeV}$ & 10 & 1 & $54^{\circ}$ \\
$\mathrm{M}^{*}$ & Large-angle pair of clusters & - & 20 & 6 & $117^{\circ}$ \\
$\mathrm{G} *$ & Large-angle pair of G clusters & - & 20 & 6 & $117^{\circ}$ \\
\hline $\mathrm{EM}$ & E + M cluster combination & - & $10+10$ & 3 & $126^{\circ}$ \\
$\mathrm{BM}$ & short track matched to a cluster & - & 16 & 1 & $34^{\circ}$ \\
$\mathrm{AM}$ & long track matched to a cluster & - & 16 & 1 & $34^{\circ}$ \\
$\mathrm{A}^{\prime} \mathrm{M}$ & high- $p_{t}$ track matched to a cluster & - & 16 & 1 & $34^{\circ}$ \\
$\mathrm{BMX}$ & MIP cluster with beam background veto & - & 20 & 4 & $27^{\circ}$ \\
\hline $\mathrm{U}$ & Muon IFR sextant hit pattern & - & 8 & - & - \\
\hline \hline
\end{tabular}

in neighboring $\phi$ bins. This separation is set at 1 bin for tracks, clusters and track-cluster matches, 5 (of 16 bins) for $\mathrm{A}^{*}$ and $\mathrm{B}^{*}, 6$ (of 16 bins) for $\mathrm{M}^{*}$ and $\mathrm{G}^{*}$ and 3 (of 10 bins) for $\mathrm{EM}^{*}$.

A summary of primitives and their properties is given in Table 3.6.

The trigger decision is based on 24 trigger lines, which correspond to requirements on the number of primitives present. Their names are usually self-explanatory: for instance $M^{*} \& 1 B$ requires the presence of an $M^{*}$ (two well-separated MIPs) and a B (short track). One should bear in mind that a given particle is often counted twice: for instance $3 \mathrm{M} \& \mathrm{M}^{*}$ can be satisfied with only $3 \mathrm{M}$ clusters if two are well-separated. $\mathrm{A}^{+}$stands for $\mathrm{A} \& \mathrm{~A}^{\prime}$ (a long track and a high- $p_{t}$ track, possibly the same object), D2 for $2 \mathrm{~B} \& 1 \mathrm{~A}$ (two tracks, one of them long), $\mathrm{D} 2^{*}$ for $\mathrm{B} * \& \mathrm{~A}$ (two well-separated tracks, one long) and $\mathrm{D} 2 *+$ for $2 \mathrm{~B} \& \mathrm{~A} \& \mathrm{~A}^{\prime}$ (two tracks, with one long and one (perhaps the same) high- $p_{t}$ ). The configuration used for most of the data in this work is shown in Table 3.7.

No one-particle triggers are defined, to ensure robustness of the trigger rate with 
Table 3.7: L1 trigger configuration for the data used in this analysis.

\begin{tabular}{|c|c|c|c|c|}
\hline$\overline{\text { Line }}$ & "Mask (Hex) & Name & GLT cuts & Prescale \\
\hline 1 & 1 & $3 \mathrm{~B} \& 2 \mathrm{~A} \& 2 \mathrm{M}$ & $n B \geq 3$ and $n A \geq 2$ and $n M \geq 2$ & 1 \\
\hline 2 & 2 & $3 A \& B^{*}$ & $n A \geq 3$ and $n B^{*} \geq 1$ & 1 \\
\hline 3 & 4 & $3 B \& B * \& 1 G$ & $\mathrm{nB} \geq 3$ and $\mathrm{nB}^{*} \geq 1$ and $\mathrm{nG} \geq 1$ & 1 \\
\hline 4 & 8 & $2 \mathrm{E}$ & $\mathrm{nE} \geq 2$ & 1 \\
\hline 5 & 10 & $\mathrm{EM}^{*}$ & $\mathrm{nEM} \geq 1$ & 1 \\
\hline 6 & 20 & $G^{*}$ & $n G^{*} \geq 1$ & 1 \\
\hline 7 & 40 & $\mathrm{D} 2 \& 1 \mathrm{E}$ & $\mathrm{nB} \geq 2$ and $\mathrm{n} A \geq 1$ and $\mathrm{nE} \geq 1$ & 1 \\
\hline 8 & 80 & $1 Y \& 1 B$ & $n Y \geq 1$ and $n B \geq 1$ & 1 \\
\hline 9 & 100 & $\mathrm{D} 2 *+$ & $n B^{*} \geq 1$ and $n A^{\prime} \geq 1$ and $n A \geq 1$ & 1 \\
\hline 10 & 200 & $3 \mathrm{M} \& \mathrm{D} 2$ & $\mathrm{nM} \geq 3$ and $\mathrm{nB} \geq 2$ and $\mathrm{nA} \geq 1$ & 1 \\
\hline 11 & 400 & $4 \mathrm{M}$ & $\mathrm{nM} \geq 4$ & 1 \\
\hline 12 & 800 & $3 \mathrm{M} \& \mathrm{M}^{*}$ & $\mathrm{nM} \geq 3$ and $\mathrm{nM}^{*} \geq 1$ & 1 \\
\hline 13 & 1000 & $2 \mathrm{M} \& \mathrm{~A}+$ & $\mathrm{nM} \geq 2$ and $\mathrm{nA}^{\prime} \geq 1$ and $\mathrm{n} \mathrm{A} \geq 1$ & 1 \\
\hline 14 & 2000 & $\mathrm{M}^{*} \& 5 \mathrm{U}$ & $n M^{*} \geq 1$ and $n U \geq 5$ & 1 \\
\hline 15 & 4000 & 2BM\&2M & $\mathrm{nBM} \geq 2$ and $\mathrm{nM} \geq 2$ & 1 \\
\hline 16 & 8000 & $1 Y$ & $\mathrm{nY} \geq 1$ & 20 \\
\hline 17 & 10000 & $3 \mathrm{M}$ & $\mathrm{nM} \geq 3$ & 4 \\
\hline 18 & 20000 & $3 B \& 2 A$ & $\mathrm{nB} \geq 3$ and $\mathrm{nA} \geq 2$ & 8 \\
\hline 19 & 40000 & $M^{*} \& 1 B$ & $n M^{*} \geq 1$ and $n B \geq 1$ & 4 \\
\hline 20 & 80000 & $\mathrm{D} 2 * \& 1 \mathrm{M}$ & $n B^{*} \geq 1$ and $n A \geq 1$ and $n M \geq 1$ & 16 \\
\hline 21 & 100000 & $2 \mathrm{M}$ & $\mathrm{nM} \geq 2$ & 60 \\
\hline 22 & 200000 & D2 & $n B \geq 2$ and $n A \geq 1$ & 96 \\
\hline 23 & 400000 & 1B & $\mathrm{nB} \geq 1$ & 4096 \\
\hline 24 & 800000 & $1 \mathrm{M}$ & $\mathrm{nM} \geq 1$ & 4096 \\
\hline 25 & 1000000 & daqpulser & & 1 \\
\hline 26 & 2000000 & sourcecalpulse! & & 1 \\
\hline 27 & 4000000 & bunchcross & & 4000 \\
\hline 28 & 8000000 & lightpulser & & 1 \\
\hline 29 & 10000000 & cyclic1 & & 1 \\
\hline
\end{tabular}


respect to spurious signals (e.g. EMC hot towers).

Some lines can be categorized as "pure DCH" or "pure EMC" if they use signals from only one subsystem. These lines are orthogonal and their efficiency can be cross-calibrated. Partial orthogonality occurs if one selection is a subset of the other or if two selections share a common part and the remainders are orthogonal. For instance, EM* could be used to calibrate the efficiency of the DCH part of D2\&1E. The prescaled low-multiplicity lines toward the end of the list are designed to play that role: for instance the efficiency of $3 \mathrm{~B} \& 2 \mathrm{~A} \& 2 \mathrm{M}$ could be calibrated in two steps by using $2 \mathrm{M}$ to measure the $\mathrm{DCH}$ part and $\mathrm{D} 2$ for the EMC part.

The order of the lines in the table defines the priority of the lines, with the priority decreasing from top to bottom, and if multiple lines fire the highest-priority line is used to calculate the event time. Higher-multiplicity triggers are given highest priority for two reasons: first, it ensures that the event time will be closest to the event maximum; second, if a hadronic event and a Bhabha scattering event occur in close succession, it ensures (higher multiplicity) hadronic event sets the event time, avoiding truncated event data.

The event time is determined from the highest-priority line by finding the time interval during which that line stayed active and reporting its center, in bins if $67 \mathrm{~ns}$ to allow for half-ticks. If a higher-priority line fires while the processing is going on, the algorithm switches to the new line. Time offsets and stretches are applied to the other line signals before determining their status at the time of the trigger. The resulting information is encoded in a 24-bit that is delivered to the fast control system (FCTS). The FCTS can optionally mask of prescale any of these triggers. If a valid trigger remains, an "L1 accept" signal is issued which initiates detector readout.

The total latency for the whole Level 1 chain is $11.3 \mu \mathrm{s}$. A breakdown of the latency associated with each step is shown in Table 3.8. The efficiency of the Level 1 trigger for selected processes is shown in Table 3.9. 
Table 3.8: Level 1 latency summary

\begin{tabular}{|c|c|c|c|c|}
\hline \multicolumn{3}{|c|}{ DCT Path } & \multirow{2}{*}{\multicolumn{2}{|c|}{ EMC Path }} \\
\hline & BLT & $\mathrm{PTD}$ & & \\
\hline$\overline{\mathrm{DCH}}$ front-end electronics & \multirow{2}{*}{\multicolumn{2}{|c|}{$\begin{array}{c}1.5 \mu \mathrm{s} \\
0.27 \mu \mathrm{s}\end{array}$}} & EMC front-end electronics & $2.3 \mu \mathrm{s}$ \\
\hline $\mathrm{DCH} \rightarrow \mathrm{TSF}$ transfer & & & \multirow{7}{*}{ - } & \multirow{7}{*}{$4.8 \mu \mathrm{s}$} \\
\hline TSF Processing & $3.26 \mu \mathrm{s}$ & $2.96 \mu \mathrm{s}$ & & \\
\hline TSF $\rightarrow$ BLT/PTD transfer & \multicolumn{2}{|c|}{$0.03 \mu \mathrm{s}$} & & \\
\hline BLT processing & $0.89 \mu \mathrm{s}$ & E & & \\
\hline PTD processing & - & $1.19 \mu \mathrm{s}$ & & \\
\hline PTD delay & - & $1.07 \mu \mathrm{s}$ & & \\
\hline Transfer to GLT & $0.03 \mu \mathrm{s}$ & $0.03 \mu \mathrm{s}$ & & \\
\hline Algorithm Total & & & Algorithm Total & $7.1 \mu \mathrm{s}$ \\
\hline GLT input delay & $1.07 \mu \mathrm{s}$ & $0 \mu \mathrm{s}$ & GLT input delay & $0 \mu \mathrm{s}$ \\
\hline DCT total & \multicolumn{2}{|c|}{$7.05 \mu \mathrm{s}$} & EMT Total & $7.1 \mu \mathrm{s}$ \\
\hline GLT processing & \multicolumn{4}{|c|}{$3.3 \mu \mathrm{s}$} \\
\hline FCT processing & \multicolumn{4}{|c|}{$0.9 \mu \mathrm{s}$} \\
\hline Grand Total & \multicolumn{4}{|c|}{$11.3 \mu \mathrm{s}$} \\
\hline
\end{tabular}

Table 3.9: Level 1 Trigger efficiencies (in \%) for selected triggers applied to various physics processes, as measured in simulation.

\begin{tabular}{lrrrrrrrr}
\hline \hline \multicolumn{1}{c}{ Level 1 Trigger } & $\epsilon_{B \bar{B}}$ & $\epsilon_{B \rightarrow \pi^{0} \pi^{0}}$ & $\epsilon_{B \rightarrow \tau \bar{\nu}}$ & $\epsilon_{c \bar{c}}$ & $\epsilon_{u d s}$ & $\epsilon_{e e}$ & $\epsilon_{\mu \mu}$ & $\epsilon_{\tau \tau}$ \\
\hline 3A\&B* & 97.1 & 66.4 & 81.8 & 88.9 & 81.1 & - & - & 17.7 \\
D2*+ & 95.0 & 63.0 & 83.2 & 89.2 & 85.2 & 98.6 & 99.1 & 79.9 \\
Combined DCT & 99.1 & 79.7 & 92.2 & 95.3 & 90.6 & 98.9 & 99.1 & 80.6 \\
\hline 3M\&M* & 99.7 & 98.6 & 93.7 & 98.5 & 94.7 & - & - & 53.7 \\
EM $^{*}$ & 71.4 & 94.9 & 55.5 & 77.1 & 79.5 & 97.8 & - & 65.8 \\
Combined EMT & 99.8 & 99.2 & 95.5 & 98.8 & 95.6 & 99.2 & - & 77.6 \\
\hline 3B\&2A\&2M & 99.4 & 81.2 & 90.3 & 94.8 & 87.8 & - & - & 19.7 \\
$\mathrm{M}^{*} \& \mathrm{~A} \& \mathrm{~A}^{\prime}$ & 95.1 & 68.8 & 83.7 & 90.1 & 87.0 & 97.8 & 95.9 & 78.2 \\
E\&2B\&A & 72.1 & 92.4 & 60.2 & 77.7 & 79.2 & 99.3 & - & 72.8 \\
$\mathrm{M}^{*} \& 5 \mathrm{U}(\mu$-pair) & - & - & - & - & - & - & 60.3 & - \\
\hline Combined Level 1 & $>99.9$ & 99.8 & 99.7 & 99.9 & 98.2 & $>99.9$ & 99.6 & 94.5 \\
\hline \hline
\end{tabular}




\subsubsection{Software Trigger}

The Level 3 Software trigger (L3) processes the output of the Level 1 trigger for further selection. It runs on a farm of 32 commercial UNIX workstations within the OEP framework.

L3 involves a basic reconstruction of the event in both the DCH and EMC. For the $\mathrm{DCH}$ a basic track-finding algorithm is applied, and a clustering algorithm for the EMC. Level 3 filter algorithms are then applied to the output to determine the Level 3 decision.

The operation of L3 is divided into 3 phases:

- Define "L3 input lines", composed of combinations of L1 lines, to serve as input to the L3 scripts. The two most common are the "Open" input line, which selects all L1 Lines, and the "Physics" input line, which selects all lines except $1 Y$ and $1 Y \& 1 B$, which are designed to select Bhabha events.

- Run L3 Scripts, algorithms processing events that pass their input line and produce a pass/fail decision.

- Define L3 output lines based on logical operation on the output of the scripts.

As for L1, the L3 decision is then defined as an "OR" operation on a set of L3 output lines. If the event passes L3, it is handed to OEP, which may optionally mask or prescale any of the output line. If a valid L3 line remains, the event is passed to the logging manager for storage.

\section{Reconstruction}

The track-finding is divided in two stages. In the first stage, a pattern-recognition algorithm is applied to the TSF segments provided by the Level 1 trigger. This uses a lookup table of hit patterns to identify groups of TSF segments that are likely to form a track. If a pattern of segments matches an entry in the lookup table, the reconstructed track is refined by an iterative fitting algorithm. For added precision, this step uses the DCH hits near the TSF segments in addition to the segments themselves. This produces tracks with 5 -parameter helix fits, with a minimum $p_{t}$ threshold 
of $250 \mathrm{MeV}$. By providing a determination of the track position with respect to the interaction point, this allows a significantly improved separation of $e^{+} e^{-}$collision events and beam background compared to Level 1.

The L3 clustering algorithm forms clusters from adjacent energy depositions that are within $1.3 \mu \mathrm{s}$ of the event time, and have more than $20 \mathrm{MeV}$ of energy. For clusters with at least $100 \mathrm{MeV}$ of energy, the centroid, lateral energy profile, and average cluster time are calculated.

\section{Filter Algorithms}

There two main physics line, each defined as the "OR" of two scripts, as shown below:

- L30utDch output line:

- DCH 1 track script: Require at least 1 track with $p_{t}>600 \mathrm{MeV} / c$ and $\left|d_{0}\right|<1 \mathrm{~cm},\left|z_{0}\right|<7 \mathrm{~cm}$ with respect to the IP

- DCH 2 track script: Require at least 2 tracks with $p_{t}>250 \mathrm{MeV} / c$ and $\left|d_{0}\right|<1.5 \mathrm{~cm},\left|z_{0}\right|<10 \mathrm{~cm}$ with respect to the IP

- L3OutEmc output line:

- EMC high-energy script: Require at least 2 clusters with $E^{*}>350 \mathrm{MeV}$ and an event invariant mass of at least $1.5 \mathrm{GeV}$.

- EMC high-multiplicity script: Require at least 4 clusters (with an implicit $100 \mathrm{MeV}$ energy cut) with an event invariant mass of at least $1.5 \mathrm{GeV}$.

Both selections are highly efficient on physics events, and their orthogonality allows for cross-calibration and stability. The rate of both selections is dominated by Bhabha scattering events. These events are selected by a separate script [61] whose output is used as a veto in both selections. This Bhabha veto selects both two-track Bhabha events and one-track events where the forward track is lost in the forward direction. The selection is designed to be tight, with an emphasis on purity rather than efficiency, in order to avoid rejecting legitimate physics events.

Several samples are defined for detector studies and diagnostics purposes: 
- Bhabha samples: L3 defines four samples of clean Bhabha events that are not subject to the Bhabha veto, L30utBhabha, L30utBhabhaFlat, L30utEmcBhabha and L3OutRadiativeBhabha. L30utBhabha is heavily prescaled, while L30utBhabhaFlat has a $\theta$-dependent prescale factor (going from 1 at the forward end to 13 in the backward end) that is designed to make the rate flat in $\theta$. Unlike the Bhabha veto selection, for these filters the emphasis is on efficiency rather than purity. L30utEmcBhabha is designed to select Bhabha events using only EMC information, making use of the small acolinearity in the cluster positions due to the track curvatures to separate them from $\gamma \gamma$ events. L30utRadiativeBhabha selects radiative events that are used for detector studies, in particular for EMC energy calibration.

- L30utPhiGamma: A dedicated filter was added in 2001 to select $e^{+} e^{-} \rightarrow \phi\left(K_{S}^{0} K_{L}^{0}\right) \gamma$ events. These events are useful to provide an unbiased high-momentum $K_{L}^{0}$ sample to calibrate the IFR, as well as for physics analysis (see Section 5.1). They are not selected by the standard filters since the $K_{S}^{0}$ track may not pass close to the IP and the event may contain only 3 clusters, one of which is high-energy (requiring the $K_{L}^{0}$ to deposit energy in the EMC would bias the sample). A specific algorithm was developed to perform rudimentary vertexing of L3 tracks. The track helices are intersected in the $r-\phi$ plane, and the intersection point where the tracks are closest along the $z$ direction is chosen. The track momentum vectors are then "swum" to the intersection point to get a better estimate of the momentum of the mother particle.

- L30utLumi and L30utCalibHadron: lines used for online diagnostics, as described in the next section.

- Other samples: L3 also implements seletion for a number of other processes. A sample of $\gamma \gamma$ events, used for EMC studies, is provided by L30utGammaGamma. Muon samples are provided by L30utMuPair, which selects $e^{+} e^{-} t o \mu^{+} \mu^{-}$events, and by L30utCosmic and L30utCosmic, which select cosmic events. L30utVirtualCompton provides a sample of virtual Compton scattering events, i.e. events where one of the incoming electrons scatters off a virtual photon 
Table 3.10: L3 trigger configuration used for most of the data in this work. Lines with ${ }^{\dagger}$ are prescaled in OPR, and are strictly speaking OEP input lines rather that L3 output lines.

\begin{tabular}{rccl}
\hline \hline L3 output Line & Input & Prescale & Description \\
\hline L3OutDch & Physics & 1 & Main DCH-based physics line \\
L3OutEmc & Physics & 1 & Main EMC-based physics line \\
L3OutDchEmcPreVeto & Physics & 50 & DCH+EMC sample without Bhabha veto \\
\hline L3OutBhabha & Open & 1000 & Selects 2-track Bhabhas \\
L3OutBhabhaFlat & Open & $1-13$ & Bhabha sample with a flattened $\theta$ distribution \\
L3OutRadiativeBhabha & Open & 1 & Radiative Bhabha sample for detector studies \\
L3OutEmcBhabha & Open & 1 & EMC-only Bhabha sample for detector studies \\
\hline L3OutPhiGamma & Open & 1 & dedicated filter for $\phi\left(K_{S}^{0} K_{L}^{0}\right) \gamma$ \\
\hline L3OutMuPair & Physics & 1 & Sample of $e^{+} e^{-} \rightarrow \mu^{+} \mu^{-}$events for detector studies \\
L3OutCosmic & Physics & 1 & Cosmic event sample for detector studies \\
L3OutWideCosmic & Physics & 1 & Cosmic event sample for detector studies \\
L3OutVirtualCompton & Open & 1 & Sample of Virtual Compton events for detector studies \\
L3OutGammaGamma & Open & 1 & $\gamma \gamma$ sample for detector studies \\
\hline L3OutLumi & Physics & 10 & 2-track sample for online luminosity measurement \\
L3OutCalibHadron & Physics & 1 & Outut of the HadA and HadB diagnostics scripts \\
\hline L3OutL1Open & Open & 200 & Sample of events with no L1 or L3 selection \\
L3OutBunch & Bunch & 80 & Periodic trigger synchronized with bunch crossings \\
L3OutCyclic1 & Cyclic1 & 1 & Cyclic trigger, used for background studies \\
\hline
\end{tabular}

emitted by the other. These events feature an electron and a photon with balanced transverse momenta and colinear in the transverse plane, and are used for EMC calibration.

Table 3.10 shows a summary of L3 Lines configuration for the data analyzed in this work. The efficiency of L3 on selected processes is shown in Table 3.11.

\section{Beam monitoring}

The L3 reconstruction algorithm is sufficiently precise to allow the use of diagnostics tools for data-taking. The loss of precision compared to offline determinations, is compensated by the fact that the result is immediately available and can use a much larger data sample including the full Bhabha rate. The tools are as follows:

- Online Luminosity measurement: This tool uses the rate of two-prong events passing the L30utLumi output line to get an instantaneous luminosity value. 
Table 3.11: Level 3 trigger efficiency (in \%) for various physics processes, derived from simulation.

\begin{tabular}{lrrrrrr}
\hline \hline L3 Trigger & $\epsilon_{B \bar{B}}$ & $\epsilon_{B \rightarrow \pi^{0} \pi^{0}}$ & $\epsilon_{B \rightarrow \tau \nu}$ & $\epsilon_{c \bar{c}}$ & $\epsilon_{u d s}$ & $\epsilon_{\tau \tau}$ \\
\hline 1 track filter & 89.9 & 69.9 & 86.5 & 89.2 & 88.2 & 94.1 \\
2 track filter & 98.9 & 84.1 & 94.5 & 96.1 & 93.2 & 87.6 \\
Combined DCH filters & 99.4 & 89.1 & 96.6 & 97.1 & 95.4 & 95.5 \\
\hline 2 cluster filter & 25.8 & 91.2 & 14.5 & 39.2 & 48.7 & 34.3 \\
4 cluster filter & 93.5 & 95.2 & 62.3 & 87.4 & 85.5 & 37.8 \\
Combined EMC filters & 93.5 & 95.7 & 62.3 & 87.4 & 85.6 & 46.3 \\
\hline Combined DCH+EMC filters & $>99.9$ & 99.3 & 98.1 & 99.0 & 97.6 & 97.3 \\
\hline Combined L1+L3 & $>99.9$ & 99.1 & 97.8 & 98.9 & 95.8 & 92.0 \\
\hline \hline
\end{tabular}

- Monitoring of Hadronic fractions: The HadronicA (HadA) and HadronicB ( HadB) select hadronic events by requiring at least 3 tracks, and HadB includes a cut on the rescaled Fox-Wolfram moment $R_{2}$ (see section 6.5.1) to select $B \bar{B}$ events. Both scripts are passed through the L30utCalibHadron output line. The rate of these scripts is monitored during data taking; the ratio $\mathrm{HadB} / \mathrm{HadA}$ is of particular interest since a decrease may signal a drift in beam energy away from the $\Upsilon(4 S)$ resonance.

- Beam energy monitoring using two-prong events: This tool uses 2-prong event to check for drifts in the beam energy that are too small to be detected using the $\mathrm{HadB} / \mathrm{HadA}$ ratio. The events are boosted in the expected CM frame of the collision, where the two tracks should be back-to-back, and the acolinearity between the track is used to measure the magnitude and direction of the residual boost, whose variations can detect energy drifts in a single beam. Using an electron mass hypothesis and the constraint that the particle energies must be equal to the beam energy in the $\mathrm{CM}$ frame, only the directions fo the track momenta need to be used. Since the momentum direction is measured with considerably higher precision than its magnitude in L3, this leads to added precision, and drifts of less than $1 \mathrm{MeV}$ in magnitude can be observed. By running in L3, the monitor provides a prompt online measurement that can be used during data-taking. Since it runs upstream from the Bhabha veto, 
the monitor also access the full Bhabha cross-section, providing a statistical advantage that offsets the lesser precision of L3 compared to offline tracking.

\subsubsection{Trigger Performance}

\subsubsection{BGFilter}

\section{Description}

Events that are logged after passing OEP correspond to a cross-section of about $28 \mathrm{nb}$, more than half of which is either beam background or Bhabha events. In order to limit the computing power needed to process these events, a "Background Filter" (BGFilter) is applied to the dataset before applying the full reconstruction algorithm. Only events that pass BGFilter are therefore available for physics analyses. This is not a trigger stricto sensu since events rejected in one round of processing may be recovered at a later iteration, but has a similar influence on the physics sample.

The filtering is divided into two stages: the first stage, DigiFilter, applies an optional prescale factor and either passes the event to the next stage or writes it directly to the physics sample, based on which OEP output line the event was passed through. A block diagram showing the possible paths is shown in Fig. 3.16.

The next stage, BGFilter proper, applies a slightly simplified but faster version of the standard event reconstruction procedure and implements a set of filters based on these quantities.

The reconstruction only uses information from the DCH and EMC. The standard offline DCH track-finder algorithms are used, but without the usual step of adding hits from the SVT. The set of produced tracks, referred to as the DchTrackFinder list, is further refined to RecoGoodTracksLoose by applying the cuts $\left|d_{0}\right|<1.5 \mathrm{~cm}$, $\left|z_{0}\right|<10 \mathrm{~cm}$ and $p_{t}>100 \mathrm{MeV} / c$ on the track impact parameters and transverse momentum.

On the EMC side, the standard cluster-finding algorithm is used, but without the final "bumps-finding" stage which separates local energy maxima within a single cluster. A track-cluster matching algorithm is applied using tracks from the 
DchTrackFinder list.

\section{Filters}

Unlike the rest of the trigger, the BGFilter is based on a process-by-process selection philosophy, applying restrictive selections to select a small subset of desirable events. This is imposed by output cross-section requirements, and has little implication for most of BABAR physics since multihadronic events are passed with high efficiency. Inefficiencies are also less problematic than in earlier stages of the trigger since lost events can be recovered by reprocessing the data. Additional filters have been introduced over time to fill gaps in the original selection. The following filters are implemented in the data used in this work:

- BGFMultiHadron: requires at least three RecoGoodTracksLoose track, and that the rescaled 2nd order Fox-Wolfram moment $R_{2}^{c h}$ calculated from these tracks be less than 0.98 , to reject multitrack QED events. Most of the hadronic events in the physics sample are passed through this filter.

- BGFNeutralHadron: this filter complements BGFMultiHadron for certain hadronic modes that feature many neutrals but less than three charged tracks. The selection defines two categories of clusters: low-energy clusters with $E>100 \mathrm{MeV}$ and high-energy clusters with $E^{*}>500 \mathrm{MeV}$. In both cases the clusters are required not to be matched to a charged track. The requirements are as follows:

- Two RecoGoodTracksLoose tracks: At least 3 low-energy clusters, including 2 high-energy clusters.

- One RecoGoodTracksLoose track: At least 4 low-energy clusters, includ- . ing 2 high-energy clusters.

- No RecoGoodTracksLoose tracks: At least 6 low-energy clusters, including 3 high-energy clusters.

In addition, the rescaled 2nd order Fox-Wolfram moment $R_{2}$ (see Section 6.5.1) calculated from all RecoGoodTracksLoose tracks and clusters with $E>100 \mathrm{MeV}$ 
must be less than 0.95. BGFNeutralHadron has a significant impact on some neutral $B$ decay modes. For instance the passing efficiency of $B^{0} \rightarrow \pi^{0} \pi^{0}$ is raised from $77 \%$ to $96 \%$ by the addition of this filter.

- BGFTau, BGFMuMu, BGFTwoProng, BGFPhiGamma: dedicated filters designed to pass the specific event sample after which they are named.

- BGFAliNeutralTwoPhoton: dedicated filter to select all-neutral modes produced in $\gamma \gamma$ collisions.

- BGFIsr: dedicated filter for low-multiplicity ISR events. Most high- $s^{\prime}$ ISR processes are passed by the multihadron filters, but certain low-multiplicity modes such as $\pi \pi \gamma$ do not have sufficient number of tracks or neutrals. Most of them are passed by BGFTau or BGFTwoProng, but this is a side effect that may disappear are these filters are changed to meet their own physics goals. A separate ISR filter is therefore implemented with the following selection:

- Corrected track-cluster matching: The track-cluster match is modified so that matches where the cluster energy is more than twice the track momentum are invalidated. This is to prevent cases when the ISR photon is matched to a random track found nearby, which can happen frequently since bumps are not separated from within clusters. An $E / p$ value is assigned to each track using the sum of energies of matching clusters (after correction).

- ISR photon: An unmatched cluster must be present with CM energy $E^{*}>$ $2.5 \mathrm{GeV}$ and $-0.75<\theta<0.93$ in the lab.

- Track-based selection: The event passes if at least one RecoGoodTracksLoose track verifies $E / p<0.7$ and $p^{*}>600 \mathrm{MeV}$, where $p^{*}$ is the track CM momentum, or if two tracks are found with $E / p<0.8$ and $p^{*}>250 \mathrm{MeV} / c$.

- Cluster-based selection: At least two clusters must be found with $E>$ $100 \mathrm{MeV}$ in the region $-0.75<\theta<0.93$ excluding a cone of opening angle $18^{\circ}$ around the ISR photon direction. In addition, at least one cluster must 
Table 3.12: Efficiencies of BGFilter on ISR events (in \%). The last two samples correspond to generic ISR production, generated with the KKMC event generator (see section A). $m_{H a d}$ is the invariant mass of the hadronic system, obtained from $\mathrm{MC}$ truth.

\begin{tabular}{lccc}
\hline \hline Sample & BGF without BGFIsr & BGFIsr & BGF with BGFIsr \\
\hline$e^{+} e^{-} \rightarrow \rho^{0}\left(\pi^{+} \pi^{-}\right) \gamma$ & 91.5 & 87.6 & 97.5 \\
$1<m_{\text {Had }}<3 \mathrm{GeV}$ & 87.8 & 85.5 & 95.7 \\
$3<m_{\text {Had }}<6 \mathrm{GeV}$ & 95.5 & 88.8 & 98.9 \\
\hline \hline
\end{tabular}

have a direction making an angle of more than $90^{\circ}$ with the ISR photon direction in the $\mathrm{CM}$ frame. To reject $\gamma \gamma$ events, clusters with $E^{*}>2.5 \mathrm{GeV}$ within $|\Delta \phi|<50 \mathrm{mrad}$ and $|\Delta \theta|<50 \mathrm{mrad}$ of the ISR photon back-to-back direction in the CM frame are excluded from the count.

- Bhabha veto: The event is rejected if a track is found with $E / p>0.8$ and $p^{*}>4 \mathrm{GeV}$.

The efficiency of the selection is shown in Table 3.12. The filter adds $0.2 \mathrm{nb}$ to the output cross-section of the BGFilter.

- BGFRadTwoProng: this filter is designed to pass $e^{+} e^{-} \gamma$ and $\mu^{+} \mu^{-} \gamma$ events without any requirements on the photon side, in order to select an unbiased photon sample that can be used for offline photon efficiency determinations.

The filter requires two RecoGoodTracksLoose tracks with $p^{*}>250 \mathrm{MeV}$. The four-momentum of the tracks is calculated with muon mass hypotheses and used to determine the missing four-momentum in the event. The missing threemomentum is required to be at least $2 \mathrm{GeV} / \mathrm{c}$ and point in the region $-0.78<$ $\theta<0.96$. The absolute value of the missing mass is required to be less than $2 \mathrm{GeV} / \mathrm{c}^{2}$.

Tracks are classified as muons if $E / p<0.7$ (with $E / p$ calculated as for BGFIsr), and as electrons if $0.9<E / p<1.4$. Only events with exactly two muons or exactly two electrons are selected; in the case of electrons, a prescale factor of 5 is applied to limit the radiative Bhabha rate. 
The filter has an efficiency of $71 \%$ on $\mu^{+} \mu^{-} \gamma$ events with the photon in the required acceptance. The filter adds $10 \mathrm{pb}$ to the output cross-section of the BGFilter.

The overall BGFilter passing cross-section is $9.8 \mathrm{nb}$, a factor 3 reduction compared to the output of Level 3. 


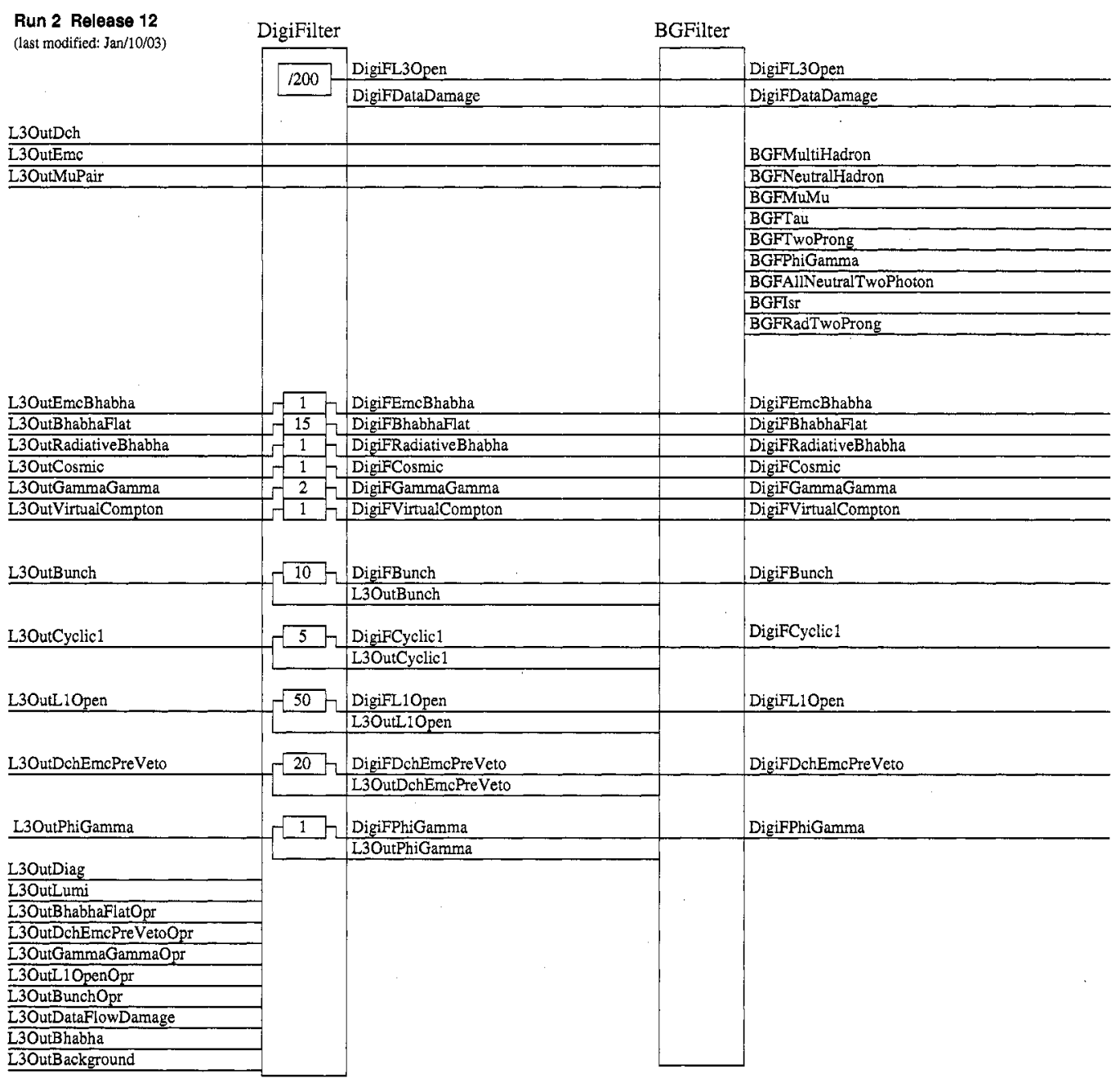

Figure 3.16: BGFilter configuration for the data used in this document 


\section{Chapter 4}

\section{Analysis Overview}

\subsection{ISR process}

As seen in Chapter 3, the ISR photon is radiated preferentially at low energy and along the beam direction, outside the detector acceptance. We are interested in radiating down to $s^{\prime}<49 \mathrm{GeV}^{2}$, which corresponds to high-energy ISR emission with $E_{\gamma}^{*} \approx 3 \mathrm{GeV}$ and above. The production cross-section for this process is about $650 \mathrm{pb}$ for the full photon angular range, which is approximately a factor of five lower than for non-radiative $q \bar{q}$ processes.

In some cases, for instance in low-energy experiments such as KLOE [11] or clean exclusive processes at BaBar [62], it is possible to make use of ISR events without a detected photon in the EMC. This so-called "untagged ISR" technique has the advantage of making use of the entire radiative cross-section. However high background levels make it infeasible in the case of the study of inclusive hadronic final

states. Instead, we use "tagged" ISR processes, in which the ISR photon is detected in the EMC. Typically this corresponds to $\left|\cos \theta^{*}\right|<0.80$, and the corresponding cross-section is about $52 \mathrm{pb}$ (in the single-ISR assumption of section 4.11).

The hadronic final states produced after $e^{+} e^{-}$annihilation are the same as they would be for a non-radiative event at a fixed-energy machine with a collision centerof-mass energy of $\sqrt{s^{\prime}}$.

A typical event is shown in Fig. 4.1. 

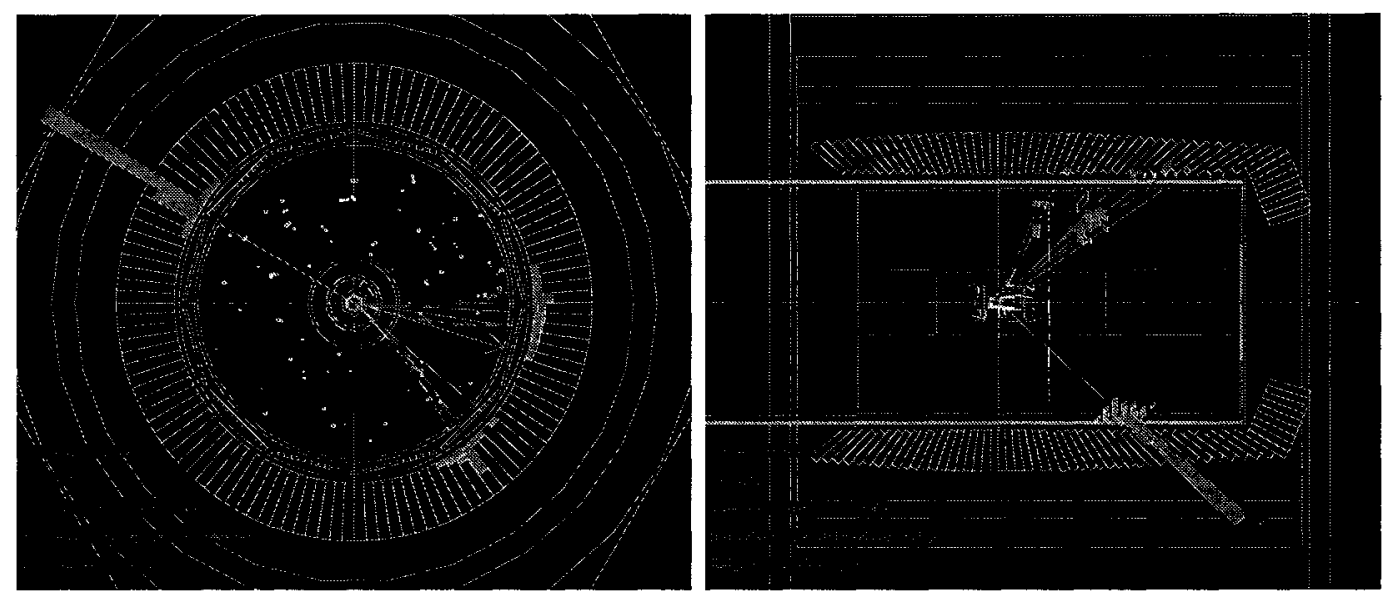

Figure 4.1: Beam (left) and top (right) view of a typical ISR event

\subsection{Goal of the study}

The object of this study is to use ISR to measure the inclusive hadronic cross-section over the range $s^{\prime}=0-40 \mathrm{GeV}^{2}$. Unlike fixed-energy machines, this allows a simultaneous measurement of the hadronic cross-section over the entire $s^{\prime}$ acceptance with a single detector and collider configuration, avoiding "point-to-point" systematic effects.

In order to minimize the dependence on hadronic system modeling the analysis is kept as inclusive as possible on the hadronic side, relying on the photon for background rejection and to determine $s^{\prime}$. These uncertainties are also reduced due to the kinematic properties of ISR events:

- In the laboratory frame, the hadronic system is not spherical but produced as a collimated jet, which can be made to point into the detector acceptance by the choice of an appropriate $\theta$ range for the ISR photon. Due to the asymmetry of the PEP-II energies, hadronic systems in the center of the detector correspond to ISR photons in the forward EMC barrel, where ISR is relatively abundant. This means that it is much more difficult for the hadronic system to lie outside the acceptance, and leads to better detection efficiency and reduced dependence on the hadronization model. 
- As a related benefit, the boost which is imparted to the hadronic system leads to a harder particle spectrum, which further increases detection efficiency and simplifies event reconstruction and particle identification.

- The particle spectrum, and therefore the efficiency, does not depend strongly on $s^{\prime}$. This is particularly important at very low $s^{\prime}$ and near thresholds, where the efficiency at fixed-energy colliders is typically low due to the soft momentum spectrum of the detected particles.

About 10 million hadronic ISR events are available at $B A B A R$, almost two orders of magnitude more than was used in the BES measurement of the range $\sqrt{s^{\prime}}=$ $2-5 \mathrm{GeV}$ [4]. This is due to the fact that ISR is produced as a by-product of the $B \bar{B}$ data that forms the core of the BABAR physics program; ISR data can therefore be collected at no extra effort, whereas a comparable dataset at a fixed-energy machine would require significant periods of dedicated running.

The main disadvantage of the ISR technique is that the event $s^{\prime}$ needs to be determined from the event itself, rather than from the beam energy. For exclusive processes this can be done at a high precision using the hadronic final state, possibly with a kinematic fit constraining the photon to be massless. This technique is used in several $B A B A R$ analyses $[8,9]$, but is incompatible with an inclusive technique in which all the final-state particles may not be detected. In this analysis, the event $s^{\prime}$ is therefore determined from the photon energy.

\subsection{Photon Energy Resolution}

The event $s^{\prime}$ value is obtained from the CM energy of the ISR photon using the relation

$$
s^{\prime}=s-2 \sqrt{s} E_{\gamma}^{*} .
$$

The relative error on $s^{\prime}$ is then given by

$$
\frac{\delta s^{\prime}}{s^{\prime}}=\frac{2 \sqrt{s} \delta E_{\gamma}^{*}}{s^{\prime}}=\frac{s-s^{\prime}}{s^{\prime}} \frac{\delta E_{\gamma}^{*}}{E_{\gamma}^{*}}
$$


In particular, for small $s^{\prime}$ we have

$$
\frac{\delta s^{\prime}}{s^{\prime}} \approx \frac{s}{s^{\prime}} \frac{\delta E_{\gamma}^{*}}{E_{\gamma}^{*}}
$$

Although the errors on the photon energy decrease with increasing energy (and therefore lower $s^{\prime}$ ), for low $s^{\prime}$ they get magnified by the factor $s / s^{\prime}$ which on the $\rho$ peak is $\left(m_{\Upsilon(4 S) / m_{\rho}}\right)^{2} \approx 190$

This issue has however little influence on the measurement of $\Delta \alpha_{h a d}^{(5)}\left(m_{Z}^{2}\right)$ : as reported in section 2.3.2, the quantity that is to be measured is an integral of $d \sigma_{H a d} / d s^{\prime}$, given by Eq. 2.52 .

All events, regardless of their $s^{\prime}$ value, are counted in this integral and the effect of $s^{\prime}$ resolution depends only on the weight factor that is multiplied with $d \sigma_{H a d} / d s^{\prime}$ under the integral: if this weight factor has rapid variations on the scale of the $s^{\prime}$ resolution, $s^{\prime}$ variations will cause large variations in the event weight and therefore of the final value of the integral; conversely, if the weight is constant, the $s^{\prime}$ resolution is irrelevant: the integral is simply the number of events multiplied by a constant, and it is not even necessary to measure $s^{\prime}$.

For $\Delta \alpha_{h a d}^{(5)}\left(m_{Z}^{2}\right)$, the weight factor $w\left(s^{\prime}, s\right)$ is given by Eq. 2.53 , and its variations shown in Fig. 2.18. It follows a gentle upward slope over the whole $s^{\prime}$ range, gaining about $35 \%$ in the interval $s^{\prime}=0-15 \mathrm{GeV}^{2}$ photon resolution effects are maximal. Fig. 4.2 shows the results of a "toy" signal extraction procedure on signal $\mathrm{MC}$, using either the true $s^{\prime}$ value or the one reported by the photon. Since photons hitting the EMC near crystal centers are expected to have slightly better resolution, events with photon front face coordinates below 0.25 in both directions (see Section 7.2.3) are plotted separately. The form of $\xi\left(s, s^{\prime}\right)$ given by the soft-photon formula 2.51 is used for simplicity.

The value $w\left(s^{\prime}=0\right)$ is used for negative $s^{\prime}$. The photon-based $s^{\prime}$ is systematically low, which is expected since the mean of the photon reconstructed energy distribution is low due to the low-side tail and $w\left(s^{\prime}, s\right)$ is a rising function of $s^{\prime}$. Most of the difference occurs at low $s^{\prime}$ and could be corrected for by some modification of $w(s)$. 

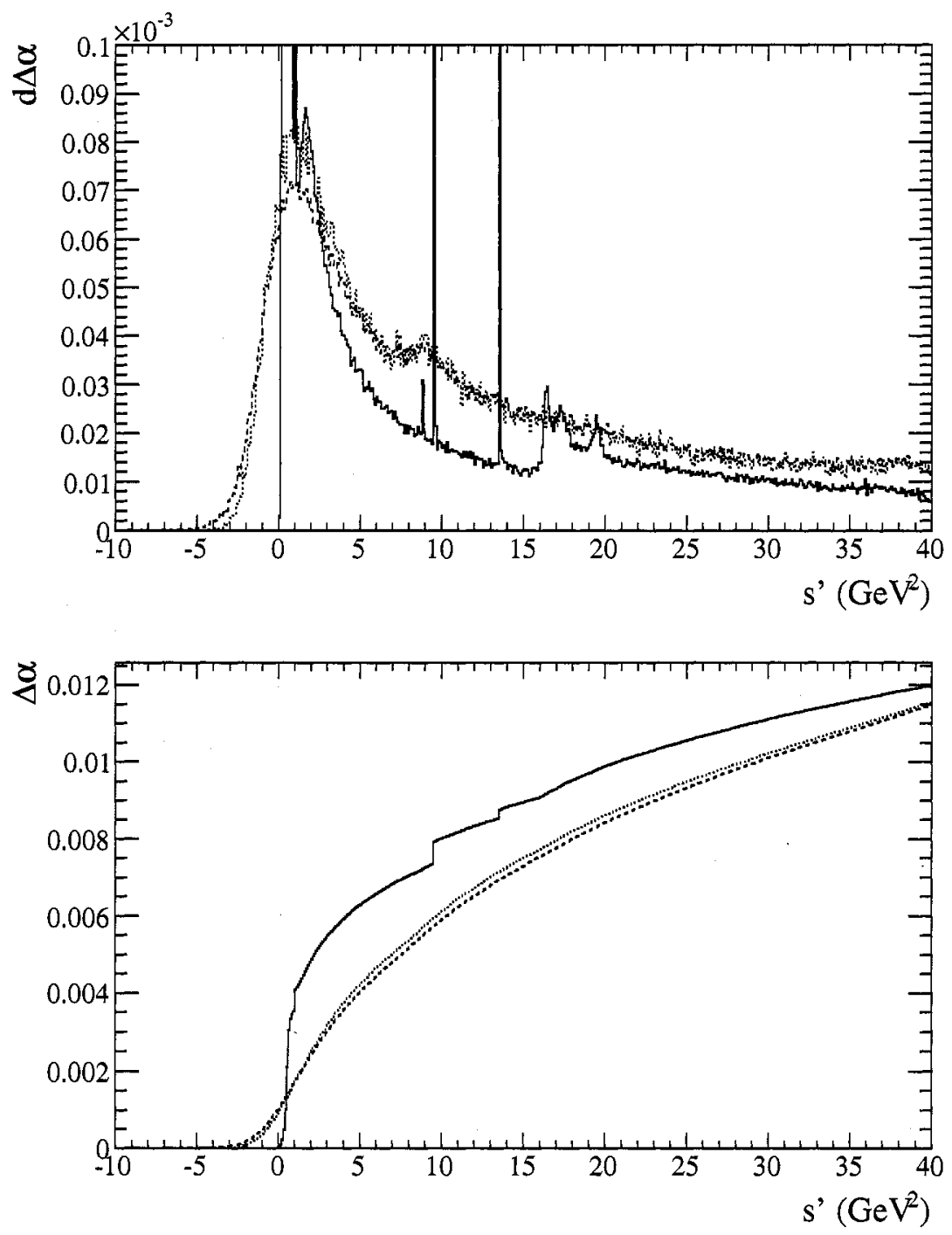

Figure 4.2: Integrand (top) and Cumulative integral (bottom) of Eq. 2.53 in signal $\mathrm{MC}$ using the soft-photon form of the radiative factor, using true $s^{\prime}$ (solid line) and $s^{\prime}$ as given by the ISR photon energy, for all events (dashed line) and events with the photon near a crystal center (dotted line). 


\subsection{Backgrounds}

The main experimental challenge for this analysis is to remove large background while maintaining very high efficiency. Figure 5.3 shows the $s^{\prime}$ distributions of signal and main backgrounds after requiring the presence of a high-energy photon in the calorimeter. The backgrounds are still overwhelming, and can be classified into the following categories:

\section{- QED processes}

These events present a real high-energy photon but can be rejected by hadronicside selections, described in detail in Chapter 5:

- $\gamma \gamma$ Events: Most can be vetoed cleanly, either in the case of two detected photons or the case where one photon converted, but a significant fraction of events with degraded photons can be difficult to identify. To reject these we require events with no tracks to have at least one $\gamma \gamma$ pair with an invariant mass compatible with a $\pi^{0} \rightarrow \gamma \gamma$ decay.

- Radiative Bhabha Events: These are vetoed by identifying electrons on the "hadronic" side of the event. The selection needs to be kept loose in order not to affect the $\pi^{+} \pi^{-} \gamma$ mode which represents about $20 \%$ of the signal: for 2-track events we veto only if both tracks are electron-like.

$-\mu^{+} \mu^{-} \gamma$ events: These are difficult to separate from signal (e.g. $\pi^{+} \pi^{-} \gamma$,). They are instead accepted as part of the signal, and subtracted using Monte-Carlo estimates. The same rule as signal therefore applies: the selection efficiency must be kept high for the subtraction to give adequate errors

- $\tau \tau \gamma$ events : As for $\mu \mu \gamma$, these events are accepted and subsequently subtracted. A further issue is that $\tau \rightarrow e$ decays are rejected by the radiative Bhabha veto, lowering the overall efficiency. However since the branching fraction for $\tau \rightarrow e$ is well-measured this does not affect the overall precision. 
- Photons from hadronic events : this consists mainly of photons from (possibly merged) $\pi^{0} \rightarrow \gamma \gamma$ decays, with contributions from decays of $\eta$ s and other resonances, mainly originating from $e^{+} e^{-} \rightarrow u \bar{u}, d \bar{d}$ and $\tau^{+} \tau^{-}$events. They are rejected by a combination of three selections, described in Chapter 6:

- An explicit veto on $\gamma_{I S R} \gamma$ pairs (in which one of the photons is the ISR photon), with invariant masses compatible with a $\pi^{0}, \eta, \eta^{\prime}, \omega$ or $\phi \rightarrow \gamma \gamma$ decay.

- We require the ISR photon cluster to have a lateral moment compatible with an electromagnetic shower, rejecting merged $\pi^{0} \mathrm{~s}$ and other hadronic sources such as antineutrons and $K_{L}^{0}$.

- We use a Fisher discriminant to separate signal and continuum events based on event-shape quantities.

- Stale Bhabha events: in the case where a hadronic event occurs immediately after a Bhabha event, the Bhabha clusters may still be visible in the EMC and be mistaken for ISR photons. This background is studied in detail in Section 9.9.

- Radiative decays of $b \bar{b}$ resonances: $b \bar{b}$ resonances below the $B \bar{B}$ threshold decay to $\gamma g g$ as well as $g g g$, with a photon CM energy above the ISR energy cut in a significant fraction of events. This is not the case for $\Upsilon(4 S)$ which decays almost exclusively to $B \bar{B}$, but can occur for other $b \bar{b}$ states after $\pi \pi$ or soft ISR transitions. This background is studied in Section 9.12.

- Radiative $\gamma \gamma$ Collisions: by $\gamma \gamma$ collision, we refer to the process in which each of the incoming leptons emits a virtual photon and the hadronic system is produced through $\gamma^{*} \gamma^{*} \rightarrow q \bar{q}$. This process is a well-known [63] background to $e^{+} e^{-} \rightarrow \gamma^{*} \rightarrow q \bar{q}$ and the corresponding radiative process, shown in Fig. 9.67 is therefore expected to be a background to hadronic ISR production. It is studied in detail in Section 9.13.

- $e^{+} e^{-}$Annihilation to two virtual photons: $e^{+} e^{-}$annihilation processes in which the hadronic system is produced by two virtual photons have been recently 
observed [64]. These processes are not contribute to $\Delta \alpha_{\text {had }}^{(5)}\left(m_{Z}^{2}\right)$ and should be removed. They are studied in Section 9.14.

\subsection{Analysis Strategy}

The overall analysis strategy is as follows:

- Events are selected with a neutral particle candidate having a center-of-mass energy $E^{*}>3.3 \mathrm{GeV}$ and $0.386<\theta<2.109$ in the laboratory frame (which corresponds approximately to $\left|\cos \theta^{*}\right|<0.80$ in the center-of-mass).

- Removed QED and hadronic backgrounds using the selection procedure outlined in the previous section.

- $e^{+} e^{-} \rightarrow \mu^{+} \mu^{-} \gamma$ and $e^{+} e^{-} \rightarrow \tau^{+} \tau^{-} \gamma$ events are kept as part of the signal, since these processes are hard to separate from $q \bar{q} \gamma$ events. Since their rates are calculated to high precision we subtract them at the end of the analysis.

- Corrections are applied to the simulation based on data control samples. The photon energy and $\theta$ resolution functions are measured and the MC parameters are corrected and smeared to agree with data. The production rates and detection efficiencies of the leading background processes are also measured and a weighting procedure is used to correct for data/MC discrepancies. Corrections are also applied to the signal detection efficiency.

- We normalize the spectrum based on the integrated luminosity of the data sample reported by BaBar, with an error of about $1.1 \%$, and the cross-sections reported by $\mathrm{KKMC}$, the precision of which is $0.1 \%$. We also use KKMC to evaluate the efficiency of the photon acceptance cuts.

- Radiative corrections based on the KKMC are applied to the signal.

- $\Delta \alpha_{h a d}^{(5)}\left(m_{Z}^{2}\right)$ is calculated by numerically integrating the spectrum as in Eq. 2.52, using the radiator function given by KKMC, with a correction for $\gamma$ energy resolution effects. 


\subsection{Event Generators}

\subsubsection{Signal}

For our signal modes we use the KKMC event generator [65]. This is a generic $e^{+} e^{-} \rightarrow \bar{f} f(\gamma)$ generator, where $f$ is an arbitrary lepton or quark and up to three radiative photons are emitted. In the case of $q \bar{q}$ production the hadronization of the $q \bar{q}$ system is handled by a dedicated package, HADGEN [66]. The generator is described in detail in appendix A.

For the $\tau^{+} \tau^{-} \gamma$ mode, the $\tau$ decays are handled by TAUOLA [67].

One limitation of KKMC is that it is not possible to set limits on the photon energies and polar angles. We therefore use a generator-level filter to select those events with hard photons in the acceptance before reconstruction. We select events with $\sqrt{s^{\prime}}$ in the range $0-7.5 \mathrm{GeV}$ (corresponding to a single-photon energy of $E^{*}=$ $2.6 \mathrm{GeV})$ and $\left|\cos \left(\theta^{*}\right)\right|<0.87 \mid$. This is done in the multi-photon assumption of Section 4.11; that is, all fiducial photons are used to determine $s^{\prime}$. This is a weaker cut than the one used in the rest of the analysis (only the highest-energy photon is used) and therefore has no effects downstream. The filter is applied to the $q \bar{q} \gamma$ and $\tau^{+} \tau^{-} \gamma$ final states. For the $\mu^{+} \mu^{-} \gamma$ final state, a very large sample is used for detector studies and generated with no cuts. Running on this sample, we obtain sufficient statistics in the desired kinematic region.

A separate generator, PHOKHARA, is used for cross-checks. It is described in Section A.4.

\subsubsection{QED modes}

For background studies we use samples of radiative Bhabha and $e^{+} e^{-} \rightarrow \gamma \gamma$ processes. For radiative Bhabhas we use the BHWIDE [68] generator with the following parameters:

- Photon $\mathrm{CM}$ polar angle in the range $\left|\cos \theta^{*}\right|<0.87$

- Photon lab energy above $1.7 \mathrm{GeV}$, corresponding to the energy of a photon with $E^{*}=2.6 \mathrm{GeV}$ at the backward edge of the $\cos \theta^{*}$ range. 
- Electron polar angles in the range $13<\theta<142^{\circ}$.

The $e^{+} e^{-} \rightarrow \gamma \gamma$ events are generated using the BkQed generator, with the photons produced in the polar angle range $13<\theta<160^{\circ}$.

\subsection{3 $B \bar{B}$ and continuum production}

Generic $u d s$ and $c \bar{c}$ event samples are produced using the standard JetSet 7.4 package. For $\tau^{+} \tau^{-}$samples, the KKMC generator is used. $B \bar{B}$ samples are generated using the EvtGen package.

\subsubsection{Detector Simulation}

All simulation events use the GEANT 4 [69] package to model the interaction of particles with the detector. In order to ensure that detector conditions are reproduced in $\mathrm{MC}$, the detector model is changed on a month-by-month basis to reflect the typical detector status for this month. The proportions of Monte-Carlo events generated with each set of conditions is calibrated to reflect the corresponding proportions in data. In order to simulate beam background effects, $\mathrm{MC}$ events are overlaid with background events collected using runs with open L1 and L3 triggers.

\subsection{Data and Simulation Samples}

The data samples used in the ISR analysis consist of the $10 \mathrm{MC}$ modes described in the previous section, along with the real data. The data consists of $189.4 \mathrm{fb}^{-1}$ of on-resonance running and $19.1 \mathrm{pb}^{-1}$ on off-resonance running, but since $B \bar{B}$ events are not relevant to this analysis and represent only a negligible background we do not distinguish between the two.

$B A B A R$ data is divided into four main datasets corresponding to the four main data-taking periods, as shown in Table 4.1.

The Run1 data suffers non-linearities in the energy measurement caused by EMC electronics problems [70], which make the photon energy resolution quite different 
Table 4.1: Datasets used for the analysis

\begin{tabular}{lcc}
\hline \hline Dataset & Integrated Luminosity $\left(\mathrm{fb}^{-1}\right)$ & Run Period \\
\hline Run 1 & 21.6 & Oct. 1999-Oct. 2000 \\
Run 2 & 66.2 & Feb. 2001-Jun. 2002 \\
Run 3 & 33.0 & Dec. 2002 - Jun. 2003 \\
Run 4 & 109.3 & Sep. 2003 - Jul. 2004 \\
Runs 2-4 & 208.6 & - \\
Runs 1-4 & 230.2 & - \\
\hline \hline
\end{tabular}

(and much worse) from the rest of the dataset. Since Run1 represents only about $10 \%$ of the total data, we remove it from the dataset. In the rest of this note, "the data" will refer to the on-resonance and off-resonance Runs 2-4.

The samples are summarized in Table 4.2. The cross-sections are the ones quoted from the generator in the case of exclusive modes, and taken from the BaBar Physics Book [71] in the case of generics. For data the cross-section is obtained from the integrated luminosity value reported for the dataset. The data cross-sections depend on data-taking configurations such as prescale factors for the Bhabha lines and may therefore vary with time.

\subsection{ISR Pre-selection}

In order to reduce the amount of processing in data and generic modes, the processing is done on a subset of the data, the InclusiveIsr skim, with the following selection:

- Photon Energy Cut: We require at least one neutral with $E^{*}>3 \mathrm{GeV}$ and $\left|\cos \theta^{*}\right|<0.95$

- $\gamma \gamma$ Veto: if the event has no tracks, we identify the 3 highest-energy clusters in the event. If the 2 clusters with highest energy both have $E^{*}>2 \mathrm{GeV}$, are back-to-back to within $|\Delta \theta|<0.1$ and $|\Delta \phi|<0.05$, and the 3rd-highest energy cluster has $E^{*}<500 \mathrm{MeV}$, the event is vetoed. 
Table 4.2: Summary of the data samples using in the ISR analysis. $N_{E v t}$ refers to the number of events in the full sample, $\epsilon_{\text {Skim }}$ is the passing efficiency for the preselection described in Secction 4.8 and $\epsilon_{\text {Pre }}$ the fraction passing the preselection of Section 5.1.

\begin{tabular}{lccccc}
\hline \hline Sample & $\sigma(\mathrm{nb})$ & $\mathrm{L}\left(\mathrm{fb}^{-1}\right)$ & $N_{\text {Evt }}$ & $\epsilon_{\text {Skim }}(\%)$ & $\epsilon_{\text {Pre }}(\%)$ \\
\hline KKMC & 0.096 & 118.6 & 11414959 & 77.95 & 71.05 \\
$\gamma \gamma$ & 2.869 & 0.5 & 1422005 & 52.89 & 18.93 \\
$e^{+} e^{-} \gamma$ & 1.002 & 1.3 & 1270673 & 36.61 & 8.99 \\
$\mu^{+} \mu^{-} \gamma$ & 1.122 & 199.1 & 223501941 & 3.34 & 72.85 \\
$\tau^{+} \tau^{-} \gamma$ & 0.013 & 182.0 & 2326199 & 74.92 & 54.17 \\
$\tau^{+} \tau^{-}$ & 0.894 & 366.0 & 327032835 & 7.17 & 34.68 \\
$u d s$ & 2.070 & 323.0 & 668509467 & 2.71 & 49.23 \\
$c \bar{c}$ & 1.300 & 302.3 & 392976230 & 0.89 & 19.25 \\
$B^{0} \bar{B}^{0}$ & 0.525 & 436.9 & 229353682 & 0.08 & 16.85 \\
$B^{+} B^{-}$ & 0.525 & 653.5 & 343098551 & 0.08 & 18.61 \\
\hline Run2 Data & 13.94 & 66.3 & 942720819 & 9.79 & 26.37 \\
Run3 Data & 13.85 & 33.0 & 469498680 & 9.74 & 26.98 \\
Run4 Data & 15.13 & 109.4 & 1583086028 & 10.45 & 24.29 \\
All Data & 14.55 & 208.6 & 2968823195 & 10.22 & 25.33 \\
\hline
\end{tabular}

- QED Veto: The event is rejected if all charged tracks pass the electron selection and no track passes either the muon, pion, kaons or proton selections. These selections are described in Section E.2.

This selection is applied to all data and MC simulation samples. The numbers of events passing the skim is shown in Table 4.2. Overall, the data is reduced by an order of magnitude, as are the $u d s$ and $\tau^{+} \tau^{-}$components. The $c \bar{c}$ component loses 2 orders of magnitude, while the $B \bar{B}$ component is reduced by more than a factor 1000. The effect on the signal modes is small and corresponds mostly to a reduction in the geometric acceptance of the photon.

\subsection{Data Analysis}

The standard BABAR framework is used to process the data and produce N-tuples in ROOT format. We apply the following selection criteria before writing the ntuples: 
- Require at least one neutral with $E^{*}>3 \mathrm{GeV}$ and $\left|\cos \theta^{*}\right|<0.85$

- Apply the $\gamma \gamma$ and radiative Bhabha vetos described in section 5.1 .

The ntuples include kinematic and particle identification (PID) information for all the tracks and clusters in the event, as well as limited detector information. $K_{S}^{0}$ and $\Lambda$ candidates are reconstructed by combining pairs of oppositely charged tracks and applying kinematic and geometric constraints. For $\Lambda \mathrm{s}$, one of the tracks is assigned a proton mass and the track parameters corresponding to the proton hypothesis are used. To speed up processing, we also cut on $m_{\text {Raw }}$, the invariant mass of the tracks before fitting. The selections are as follows:

- $K_{S}^{0}:\left|m_{\pi \pi}^{R a w}-m_{K^{0}}\right|<200 \mathrm{MeV},\left|m_{\pi \pi}-m_{K^{0}}\right|<30 \mathrm{MeV}$ and $\chi^{2}<50$

- $1: 1000<m_{p \pi}^{\text {Raw }}<1300 \mathrm{MeV}, 1100<m_{p \pi}<1130 \mathrm{MeV}$ and $\chi^{2}<50$

\subsection{Trigger/Filter Issues}

Most ISR events pass standard Level 1 and Level 3 trigger selections with high efficiency.

In Level 1 a variety of filters can select ISR events. $\mathrm{EM}^{*}$ and $2 \mathrm{M} \& \mathrm{~A}+$ lines (see Section 3.8.3) are particularly efficient.

In Level 3, most events passed either the L30utDch or L30utEmc selections (see section 3.8.4). One exception is $\phi\left(K_{S}^{0} K_{L}^{0}\right) \gamma$ events, which fails Level 3 if neither of the $K_{S}^{0}$ daughter tracks come close to the IP. However Level 3 includes a specific filter for this mode, L30utPhiGamma.

In the BGFilter stage, a specific filter, BGFIsr, was designed specifically to select ISR events with high efficiency. Its algorithm is described in section 3.8.6.

The triggering efficiencies for the three signal modes are shown in Table 4.3. The denominator is taken to be events containing a reconstructed ISR photon with the requirements of Section 5.2 and passing the radiative Bhabha and $\gamma \gamma$ vetos of Section 5.1. The variations of the efficiency versus $s^{\prime}$ is shown in Fig. 4.3. 

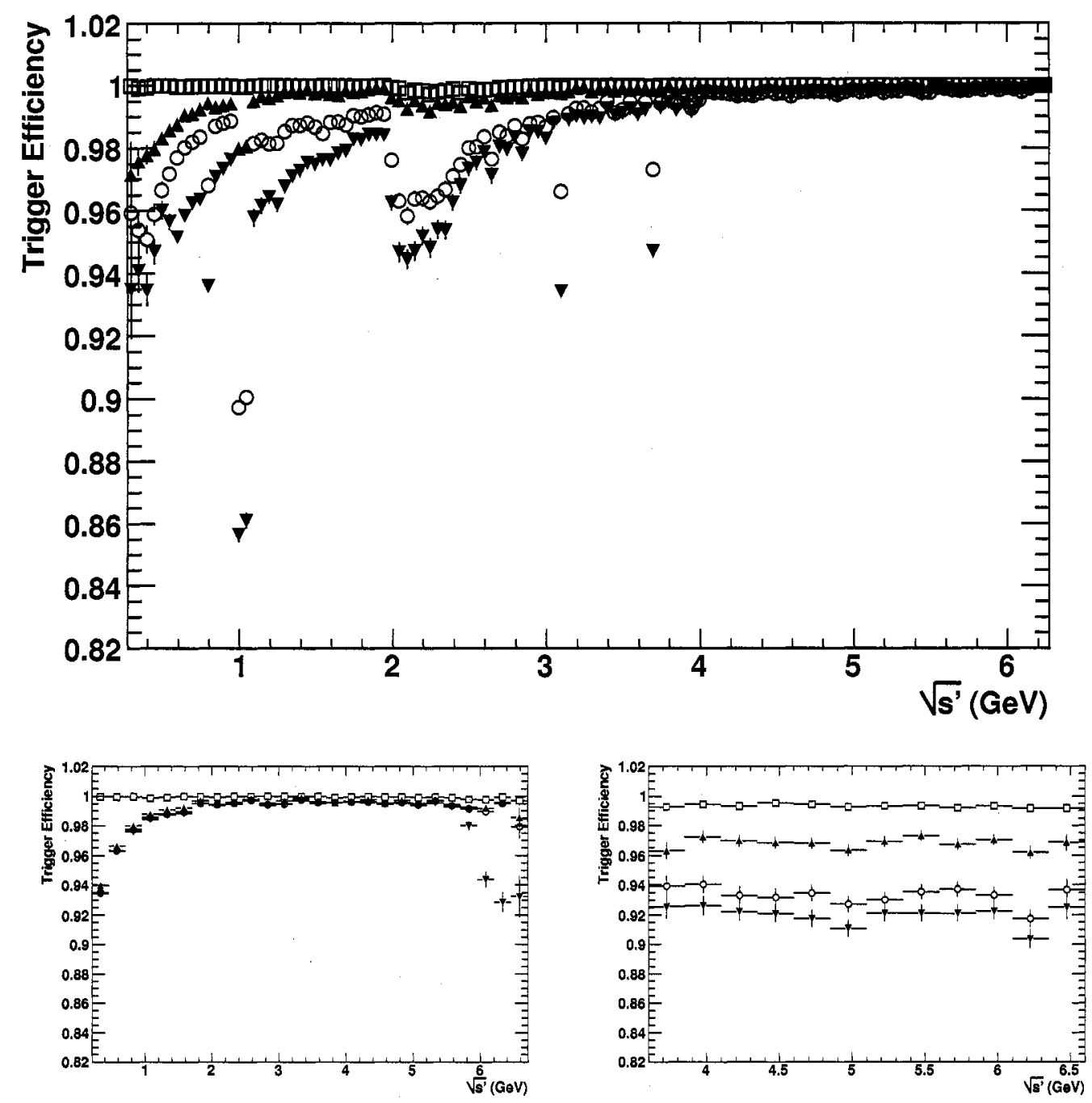

Figure 4.3: Triggering efficiencies versus $s^{\prime}$ for $q \bar{q} \gamma$ (top), $\mu^{+} \mu^{-} \gamma$ (bottom left) and $\tau^{+} \tau^{-} \gamma$ (bottom right). From top to bottom, the curves are the Level 1 (open squares) Level 3 (upward triangles) BGF (open circles) and the "Physics BGF" (downward triangles) efficiencies. 
Table 4.3: Trigger and Filter efficiencies for signal samples

\begin{tabular}{lccc}
\hline \hline & $q \bar{q} \gamma$ & $\mu^{+} \mu^{-} \gamma$ & $\tau^{+} \tau^{-} \gamma$ \\
\hline Level 1 & 100.0 & 99.9 & 99.2 \\
Level 3 & 99.5 & 98.0 & 96.6 \\
All BGFilter & 97.8 & 97.6 & 90.6 \\
Hadron, $\phi \gamma$ and ISR Filters & 95.9 & 96.0 & 88.3 \\
\hline \hline
\end{tabular}

The BGF efficiency is what ultimately matters for the analysis, but we also separate a "Physics BGF" selection corresponding to the combination of BGFIsr, BGFMultiHadron, BGFNeutralHadron and BGFPhiGamma. Since these filters are either generic or dedicated to ISR processes, this corresponds to the fraction of events that is guaranteed to be independent of changes in the BGFilter (e.g. modification of BGFTau).

The efficiency shows dips at the $\phi$, associated with the $K_{S}^{0} K_{L}^{0}$ decay mode and the small opening angle of the tracks in the $K^{+} K^{-}$mode, and at the $\psi$ and $\psi(2 S)$. The drop on the $\psi$ is mostly due to the loss of the $e^{+} e^{-}$decay mode, which is rejected be the BGFIsr Bhabha veto. As reported in section A, KKMC treats the $\psi(2 S)$ in the same way as $\psi$ so the plot shows similar problems, although it may not be so bad in reality, due to the smaller branching fraction for $\psi(2 S) \rightarrow e^{+} e^{-}$. The structure at $2 \mathrm{GeV}$ corresponds to the switch in the KKMC hadronization model from parameterized cross-sections to JETSET fragmentation.

For the $\mu^{+} \mu^{-} \gamma$ mode, the drop at the high $s^{\prime}$ edge is due to events with ISR photons that are very close to tracks, probably because of FSR. In that case there is a significant probability for the cluster to be matched to the track. As described in section 3.8.6, track/cluster matches with $E / p>2$ are vetoed for precisely this reason. However for a track radiating a fraction $z$ of its energy, we have (neglecting the muon mass and assuming collinear radiation) $E / p=z /(1-z)$, so that the veto on track/cluster matching applies for $z>2 / 3$. Since $s^{\prime}=s(1-z)$ this corresponds to $\sqrt{s^{\prime}}>6.1 \mathrm{GeV}$, which is about the position of the drop. 


\subsection{Single and Multiple Radiation}

The simplest situation is the case where a single ISR photon is emitted. In this case, the invariant mass squared of the hadronic system is given from the photon energy in the CM frame by the relation:

$$
s^{\prime}=s-2 \sqrt{s} E_{\gamma}^{*} .
$$

However in some cases additional energetic photons can be produced. This raises two issues:

- In an inclusive ISR analysis, we rely on the detected photons to determine $s^{\prime}$. In the case of multiple energetic photons, should we use all photons to determine $s^{\prime}$, or just the most energetic?

- How do deal with limits in the $\theta_{\gamma}$ range in the case of multiple photons?

The last issue is particularly relevant to $\mathrm{MC}$ event generation: we are only interested in radiation into the detector acceptance so for practical purposes it is necessary to limit the $\theta_{\gamma}$ range at the generator level. There are two ways to deal with these problems:

1. Consider all photons within the given $\theta_{\gamma}$ acceptance, keep the event if the $s^{\prime}$ calculated from the sum of their 4-momenta is within the range of interest (typically $\sqrt{s^{\prime}}<7 \mathrm{GeV}$ ).

2. Consider only the most energetic photon within the $\theta_{\gamma}$ acceptance, and keep the event if the $s^{\prime}$ value calculated using 4.4 is within the range of interest.

In theory the first option seems better, but in practice the second ("single-ISR assumption") is more practical. Since soft photons may also originate from the hadronic system, it would be necessary to consider only additional photons with above a relatively high energy cut, and the gain in cross-section would be too small to justify the complications involved with dealing with multiple photons. One may worry that 
for events with multiple radiation that pass the single-photon $s^{\prime}$ cut, the $s^{\prime}$ value will be calculated incorrectly leading to an error in the $\Delta \alpha_{\text {had }}^{(5)}\left(m_{Z}^{2}\right)$ integral. However as we have seen errors in the $s^{\prime}$ value have only a small impact on the integral value. We therefore consider all events as single-radiation, and use an event generator simulating multiple ISR to evaluate the correction and errors needed to account for this approximation. 


\section{Chapter 5}

\section{Hadronic Event Selection}

The first stage of the event selection is to reject QED processes, mainly $e^{+} e^{-} \gamma$ and $\gamma \gamma$ events. It is described in this chapter, while the rejection of non-QED backgrounds is considered in Chapter 6.

QED backgrounds are the leading sources of background at low $s^{\prime}$, as can be seen on the $s^{\prime}$ spectrum in Fig. 5.3. Their respective cross-sections after the selections of Sections 5.1 and 5.2 are $7 \mathrm{pb}$ and $13 \mathrm{pb}$, compared with $46 \mathrm{pb}$ for signal (see Table 5.7). These processes are sufficiently different from the $e^{+} e^{-} \rightarrow q \bar{q} \gamma$ signal to be efficiently rejected while maintaining an acceptable signal efficiency. In order to achieve the required purity, background efficiencies of $1 \%$ or below should be achieved.

The problem is particularly acute in the cases where there are no good tracks in the event, since neutrals offer less information on the QED vs. hadronic nature of the process. For this reason we explicitly require a $\pi^{0}$ to be present in the event in this case. For the more manageable case where tracks are present we instead apply a positive selection while applying vetoes to particular processes.

\subsection{Preselection cuts}

In order to reduce the amount of data to be processed, the following $\gamma \gamma$ and radiative Bhabha rejections requirements are applied during ntuple production:

- $\gamma \gamma$ Veto: 
$-E_{1}^{*}>4.5 \mathrm{GeV}$

$-E_{2}^{*}>4.0 \mathrm{GeV}$

$-|\Delta \theta|<0.3$ and $|\Delta \phi|<0.2$

- No track in with DCH hits in the event

where $E_{1}^{*}$ and $E_{2}^{*}$ are the $\mathrm{CM}$ energies of the highest energy cluster and secondhighest energy cluster in the event, and $\delta \phi$ and $\delta \theta$ the back-to-back angular differences between the two.

- Radiative Bhabha Veto:

- All tracks in the event to be identified as ELoose (see Appendix E.2).

- At least one track in the event to be identified as ETight (see Appendix E.2).

The output of this selection corresponds to the efficiency column $\epsilon_{\text {Pre }}$ in Table 4.2.

In $B A B A R, M C$ events are not required to pass trigger requirements: the trigger is run only in "flagging" mode, so that the information is available but not used to reject events. The first step to ensure agreement between $\mathrm{MC}$ and data samples is to require identical selections at the trigger level. Events are required to pass "Physics" trigger lines in L3 and the BG Filter, which guarantees a stable triggering efficiency. We require:

- Any FCT algorithm (already the case for data, but not applied by default in $\mathrm{MC})$.

- Either L30utDch, L30utEmc or I30utPhiGamma. The L30utPhiGamma line is not present in some early Run 2 data, so only the other 2 lines are used. Since we use "balanced" MC samples with the correct proportion of Run 2, 3 and 4 this should not be an issue.

- Either BGFMultiHadron, BGFNeutralHadron, BGFMuMu, BGFTau, BGFTwoProng, BGFPhiGamma or BGFIsr. 
We also demand that the total energy visible in the event be less than $17 \mathrm{GeV}$. This is required in order to reject "stale Bhabha" events, as described in detail in section 9.9. The visible energy is calculated by adding the energies of all the tracks with DCH hits, calculated from their momenta using a pion mass hypothesis, and the energies of all the clusters in the EMC.

\subsection{Photon Selection}

The main background-fighting tool in this analysis is the presence of the ISR photon. We require that this photon is detected in the EMC (tagged ISR) and impose the following selection:

- The CM energy of the EMC cluster must verify $E_{\gamma}^{*}>3.3 \mathrm{GeV}$, corresponding to $s^{\prime}<42 \mathrm{GeV}^{2}$ and $\sqrt{s^{\prime}}<6.5 \mathrm{GeV}$.

- Its polar angle must be in the range $0.386<\theta_{\gamma}<2.109$, corresponding to $\left|\cos \theta_{\gamma}^{*}\right|<0.80$ in the CM frame.

- The number of crystals in the cluster must be such that $3<N_{C r y} \leq 100$.

- The cluster lateral moment must be less than 0.95 .

- The cluster must not be matched to any track.

- The EMC "status word" of the cluster should be 0 (no problems flagged).

The value of the $E^{*}$ cut is somewhat arbitrary. The cut needs to be low enough that the charm resonance region is included, which corresponds to $E^{*}>4 \mathrm{GeV}$. It is desirable to go lower if possible, but backgrounds become increasingly problematic at lower energies: as shown in Fig. 5.3 the amount of continuum background increases roughly linearly for $s^{\prime}>20 \mathrm{GeV}^{2}$, limiting the precision of the final measurement. $B \bar{B}$ backgrounds also come into play for $E^{*}<2.5 \mathrm{GeV}$.

The lower cut on the number of crystals ensures that a sensible lateral moment can be calculated for the cluster. The upper cut rejects high-energy beam "accidents" 
such as the one shown in Fig. 5.1. Problematic clusters of this type also tend to have lateral moments very close to 1 , and the lateral moment cut is designed to further remove problematic clusters. As shown in Fig. 5.2, these cuts are extremely loose and designed to remove remote outliers.
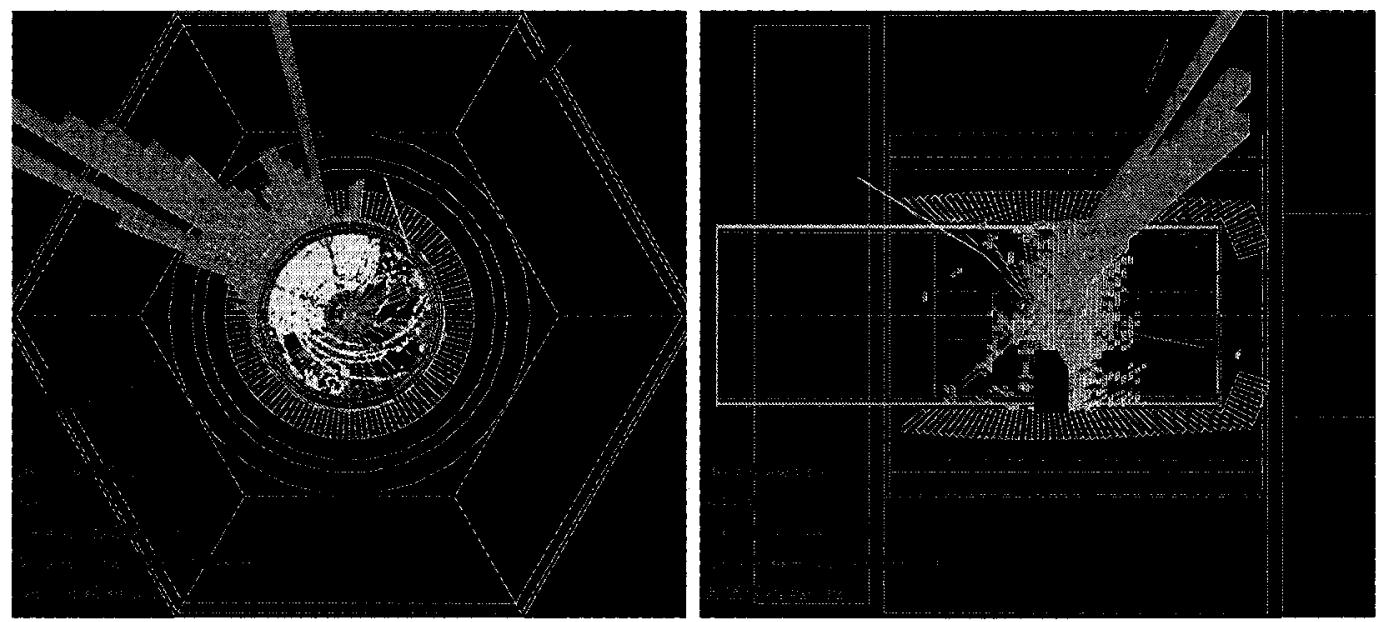

Figure 5.1: Example of a background event resulting from a beam "accident". The cluster has $E=17.6 \mathrm{GeV}, N_{C r y}=726$ and Lat $=0.997$.

Figs 5.3 and 5.4 show the $s^{\prime}$ and $\theta_{\gamma}$ distributions at this level in the selection for data and stacked MC histograms. The MC is normalized to $208.4 \mathrm{fb}^{-1}$ (the integrated luminosity of the data sample) and the data is normalized to equal area with the sum of MC components to avoid experimenter's bias. The color scheme in these plots will be used throughout this document. From top to bottom, we use

- Signal KKMC) : Blue

- $\gamma \gamma$ : Purple

- $e^{+} e^{-} \gamma$ : Gray

- $\mu^{+} \mu^{-} \gamma:$ Green

- $\tau^{+} \tau^{-} \gamma$ : Yellow

- uds: Red 

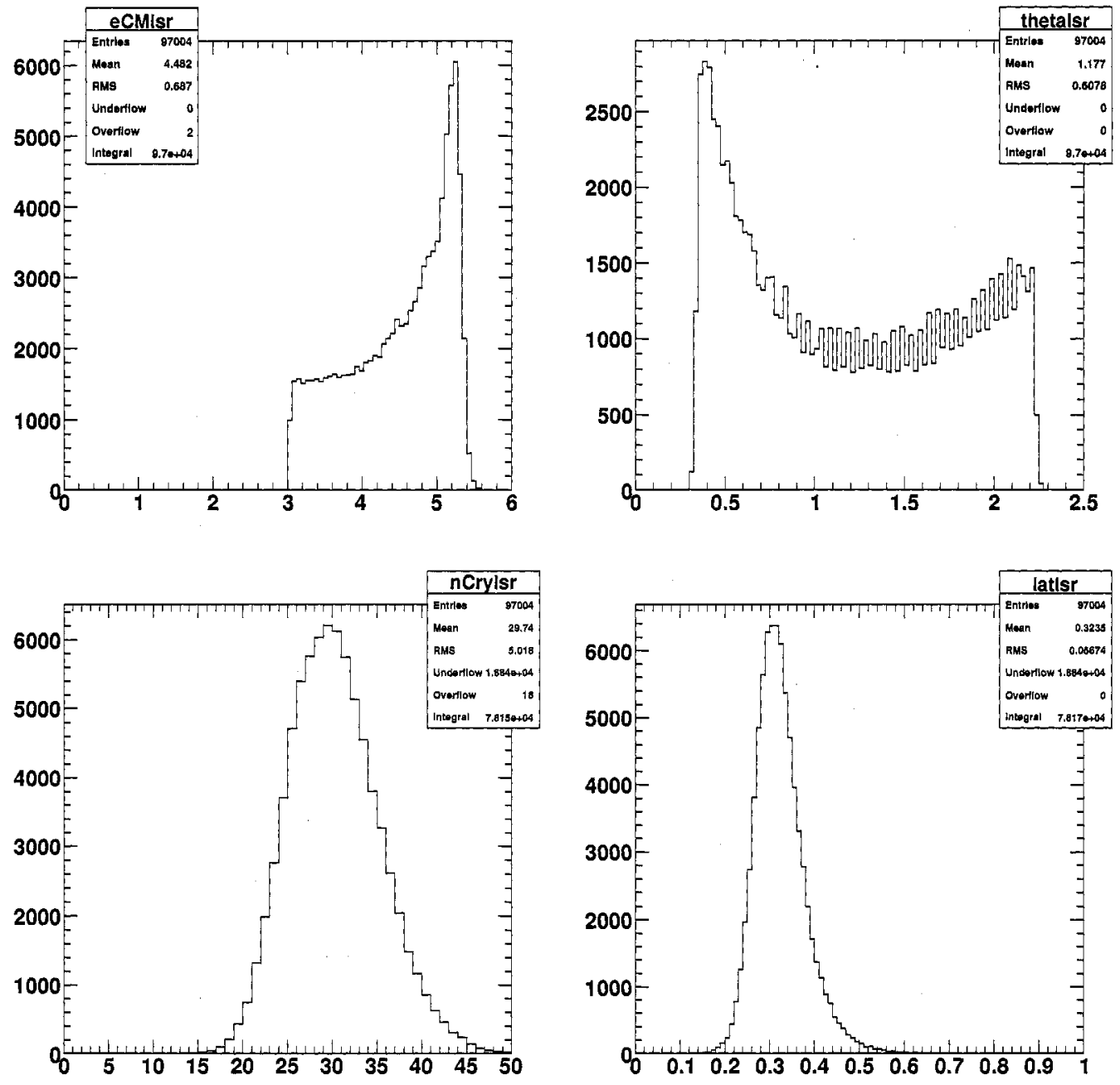

Figure 5.2: $E^{*}, \theta, N_{C r y}$ and Lat distributions for the highest-energy cluster in the event in signal MC. The $N_{C r y}$ and Lat distributions are shown with $E^{*}$ and $\theta$ cuts applied. 
- $c \bar{c}:$ Black

- $\tau^{+} \tau^{-}:$Cyan

- $B^{0} \bar{B}^{0}$ and $B^{+} B^{-}$: white and brown, but too small to be visible anyways.

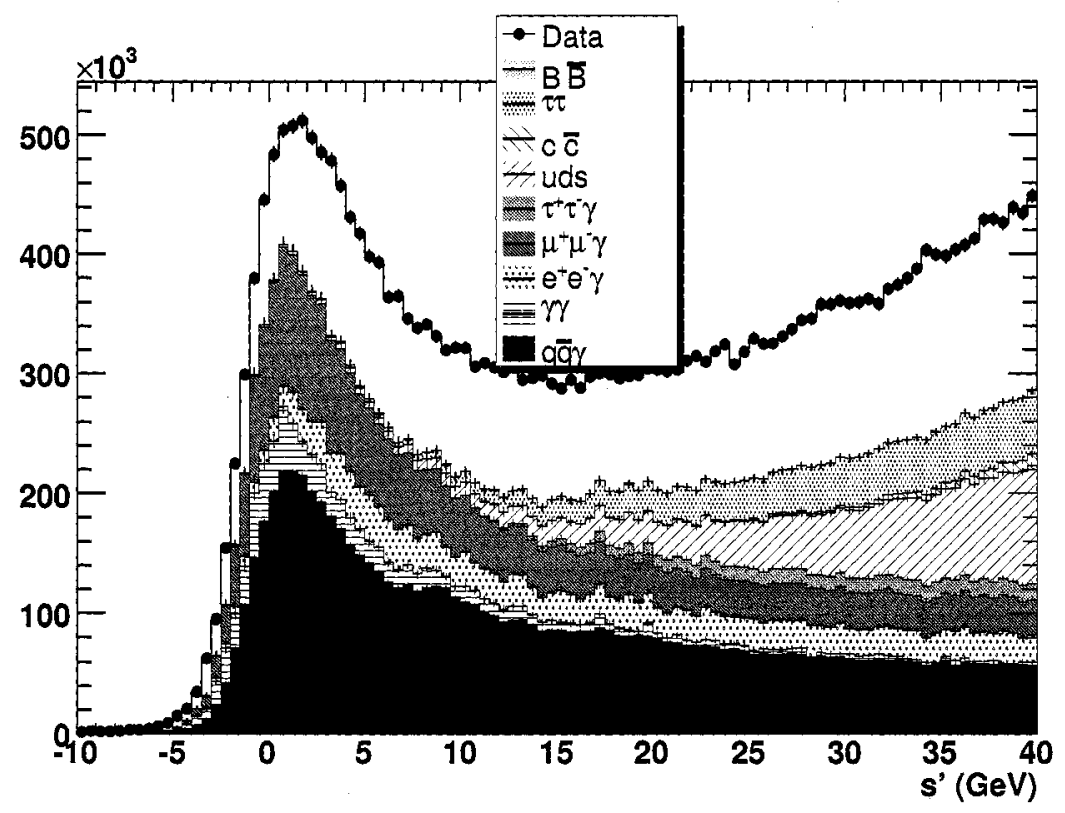

Figure 5.3: $s^{\prime}$ distribution for data (round dots) and stacked MC samples (color scheme is explained in the text), after preselection cuts (trigger, $E_{\text {Total }}$ and ISR photon selections).

\subsection{Track requirements}

One of the characteristics of the QED processes is their low track multiplicity, in general 2 tracks or less. Figure 5.5 shows the distributions of the numbers of charged tracks for events passing the preliminary vetoes described above.

The expected features are present but some there are also several problems, in particular the large number of $\gamma \gamma$ events with tracks and the number of radiative Bhabha events with more than 2 tracks. This is due in part to conversions and 


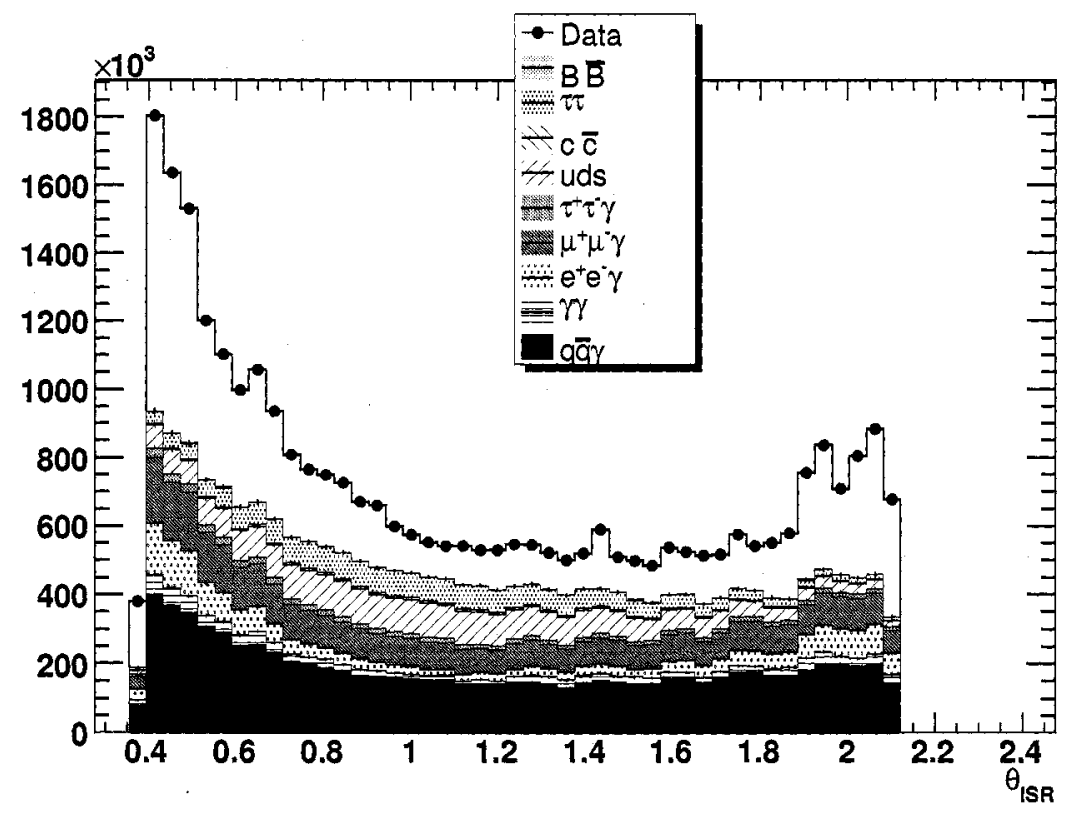

Figure 5.4: $\theta_{\gamma}$ distribution for data (round dots) and stacked MC samples (color scheme is explained in the text), after preselection cuts (trigger, $E_{\text {Total }}$ and ISR photon selections). 

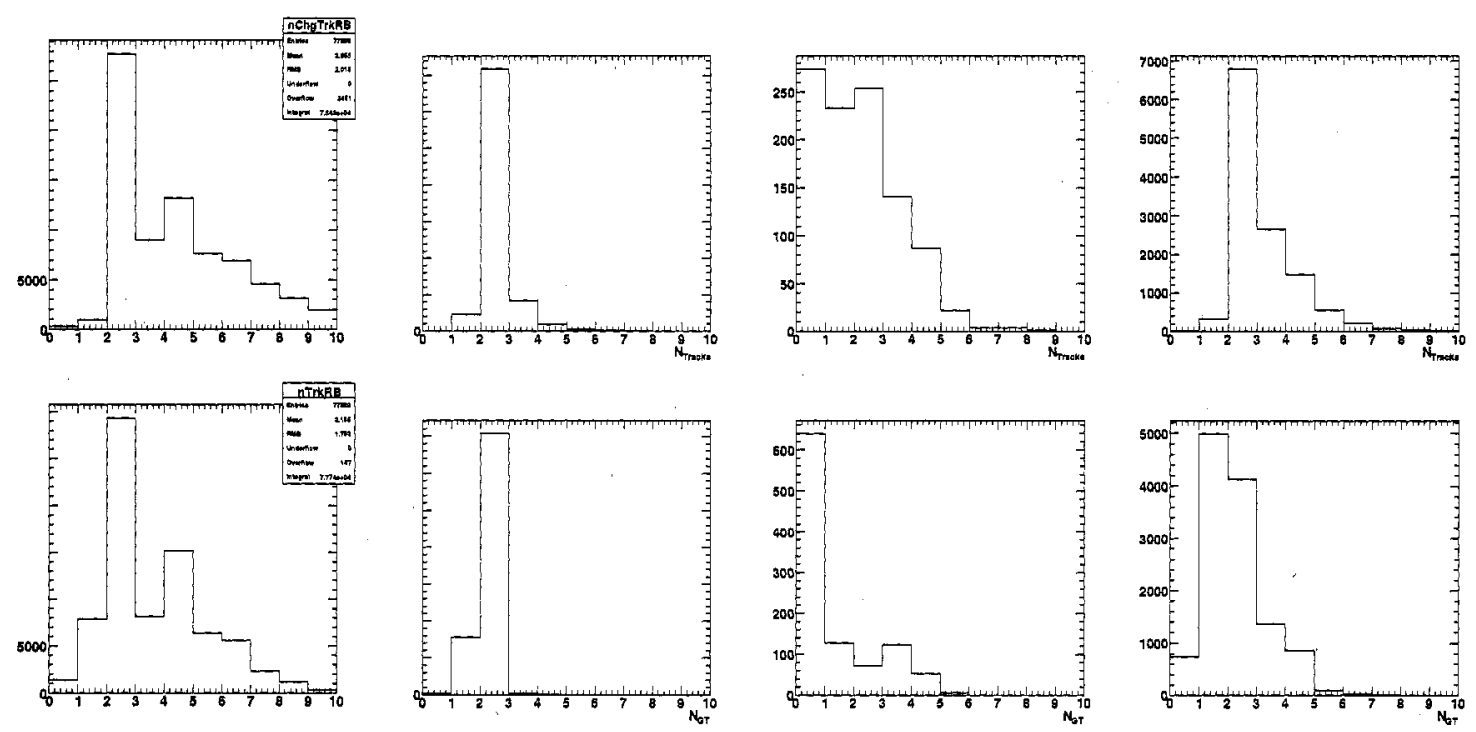

Figure 5.5: Number of tracks in event for simulated samples of signal (far left), $\mu^{+} \mu^{-} \gamma$ (middle left), $\gamma \gamma$ (middle right) and radiative Bhabha (far right). The top row corresponds to all tracks and the bottom row to IsrGoodTracks.

higher-order processes, but most of it comes from either beam-background processes or fake tracks, mainly spurious SVT-only tracks created from background hits in the SVT.

We therefore define track quality criteria, referred to as IsrGoodTrack in the rest of this document, designed to reject fake tracks, background tracks and tracks produced in material interactions. The selection is as follows:

- Require $\left|z_{0}\right|<30 \mathrm{~cm}$ and $\left|d_{0}\right|<20 \mathrm{~cm}$.

- Require $p_{t}>100 \mathrm{MeV}$.

- Require at least $10 \mathrm{DCH}$ hits.

- For tracks with $\left|z_{0}\right|>10 \mathrm{~cm}$, also demand $p_{t}>400 \mathrm{MeV}$ and a matching cluster.

- Real proton tracks from beampipe interactions are removed by requiring $\left|z_{0}\right|>$ $5 \mathrm{~cm}$ and $-1.5<d_{0}<3 \mathrm{~cm}$ for tracks identified as protons. The $d_{0}$ cut is 
widened in the positive $d_{0}$ direction since protons from $\Lambda$ decays tend to have positive $d_{0}$ (i.e. curl away from the IP).

In addition we flag the daughters of reconstructed $K_{S}^{0}$ and $\Lambda$ candidates (see section 4.9) as IsrGoodTracks, provided that the candidates have a CM energy of at least $1 \mathrm{GeV}$ in the case of $K_{S}^{0}$ and $1.3 \mathrm{GeV}$ in the case of $\Lambda$.

We also define a tighter criterion, IsrGoodTrackTight, with the following additional requirements:

- $0.35<\theta<2.35$

- $\left|d_{0}\right|<1.5 \mathrm{~cm}$ and $\left|z_{0}\right|<4 \mathrm{~cm}$

- Demand a matched EMC cluster and require $E_{\text {cluster }} / E_{\max }<1.2$, where $E_{\max }$ is the maximum possible deposited energy in the EMC, which we determine using a mass hypothesis determined using information from the DIRC. If the mass of the particle calculated from its momentum and Cherenkov angle is less that $200 \mathrm{MeV}$ a pion mass hypothesis is used. Between 200 and $700 \mathrm{MeV}$ the kaon mass is used, between 700 and $1200 \mathrm{MeV}$ the proton mass and above $1200 \mathrm{MeV}$ the mass of the deuteron.

Finally, tracks are "demoted" in 2 specific situations:

- For events with two good tracks or less, $p_{t}<600 \mathrm{MeV}$ for at least one good track, and a $\gamma \gamma$-like topology. This topology is defined as 2 EMC clusters with a CM energy greater than $3 \mathrm{GeV}$ and a back-to-back 3D angle less than $0.2 \mathrm{rad}$. In addition, the second cluster must pass the photon selections of Sections 6.2 and 6.4, i.e. its lateral moment must be less than 0.40 and it may not be combined with other photons to for a $\pi^{0}, \eta, \eta^{\prime}$, or $\omega$ candidate. This recovers some signal events with high-momentum $\pi^{0} \mathrm{~S}$ or $\eta \mathrm{s}$ and low track multiplicity in the hadronic system.

- For all events with $N_{\text {IsrGoodTracks }} \leq 2$, which are particularly exposed to QED backgrounds, all IsrGoodTrack tracks are required to also pass the IsrGoodTrackTight criteria or be demoted. 
These requirements ensure that degraded $\gamma \gamma$ events are classified as having no good tracks, so that they are more likely to be vetoed by the filters described in the next sections. In the rest of this document $N_{G T}$ refers to the number of good tracks in the event.

The resulting distributions for $N_{G T}$ are shown in Figure 5.5. The number of tracks per event is reduced for all samples. In particular, QED processes are better confined to the low $N_{G T}$ region and therefore easier to veto.

\subsection{Selection of Electron and Non-Electron tracks}

In order to identify and reject QED events, we rely mainly on the information from the tracks. In particular we define two categories of tracks, IsrElectron and IsrNonElectron based on the particle identification requirements described in Appendix E.2.

- IsrElectron track: an IsrGoodTrack identified as an electron.

- IsrNonElectron track: either

- an IsrGoodTrack identified as a muon, a kaon, a proton or a pion and having $m_{D r c}>100 \mathrm{MeV}$, where $m_{D r c}$ is the mass calculated from the track momentum as measured by the tracking system and the track velocity measured by the DIRC.

- an IsrGoodTrackTight which fails the loose electron selection and its momentum $p$ and matching EMC energy $E_{C a l}$ verify either

* $p>500 \mathrm{MeV}$ and either $E_{C a l}<300 \mathrm{MeV}$ or $E_{C a l} / p<0.3$, or

* $100<d E / d x<500$ or $d E / d x>1000$. 


\subsection{Virtual Compton Veto}

Virtual Compton scattering corresponds to the scattering of an electron or positron against a bremsstrahlung photon produced by the other beam. In the case of largeangle scattering a high-energy cluster is visible in the EMC, along with an electron track. The track and cluster are back-to-back in the transverse plane but not in the longitudinal direction, since the bremsstrahlung photon does not in general carry the full energy of the other beam. We therefore require that

- $N_{G T}=1$

- The good track is identified as IsrElectron.

- The cluster and track be back-to-back to within $|\Delta \phi|<0.2$ in the $r-\phi$ plane.

- The difference in transverse momentum $\left|p_{t}^{\text {Track }}-p_{t}^{\text {Cluster }}\right|$ be less than $500 \mathrm{MeV}$.

\section{$5.6 \gamma \gamma$ Veto}

This veto is designed to reject event with a $\gamma \gamma$ topology in the EMC, and is applied only on the $N_{G T}=0$ sample. As seen above, this however also includes events with soft tracks if a clear $\gamma \gamma$ topology signature is visible in the EMC. We require:

- Exactly 2 EMC clusters with energy $E>250 \mathrm{MeV}$

- $E^{*}>2 \mathrm{GeV}$ for both clusters

- The clusters should be back-to-back to within $|\Delta \theta|<0.3$ and $|\Delta \phi|<0.2$ The distributions of these quantities for KKMC and $\gamma \gamma$ Monte-Carlo samples are shown in Fig 5.6.

\section{$5.7 \gamma \gamma$ Conversion Veto}

In $9.8 \%$ of the $\gamma \gamma$ events that pass the preselection cuts, one of the photon converts and leaves tracks in he $\mathrm{DCH}$, and the $\gamma \gamma$ rejection algorithm of the previous section 

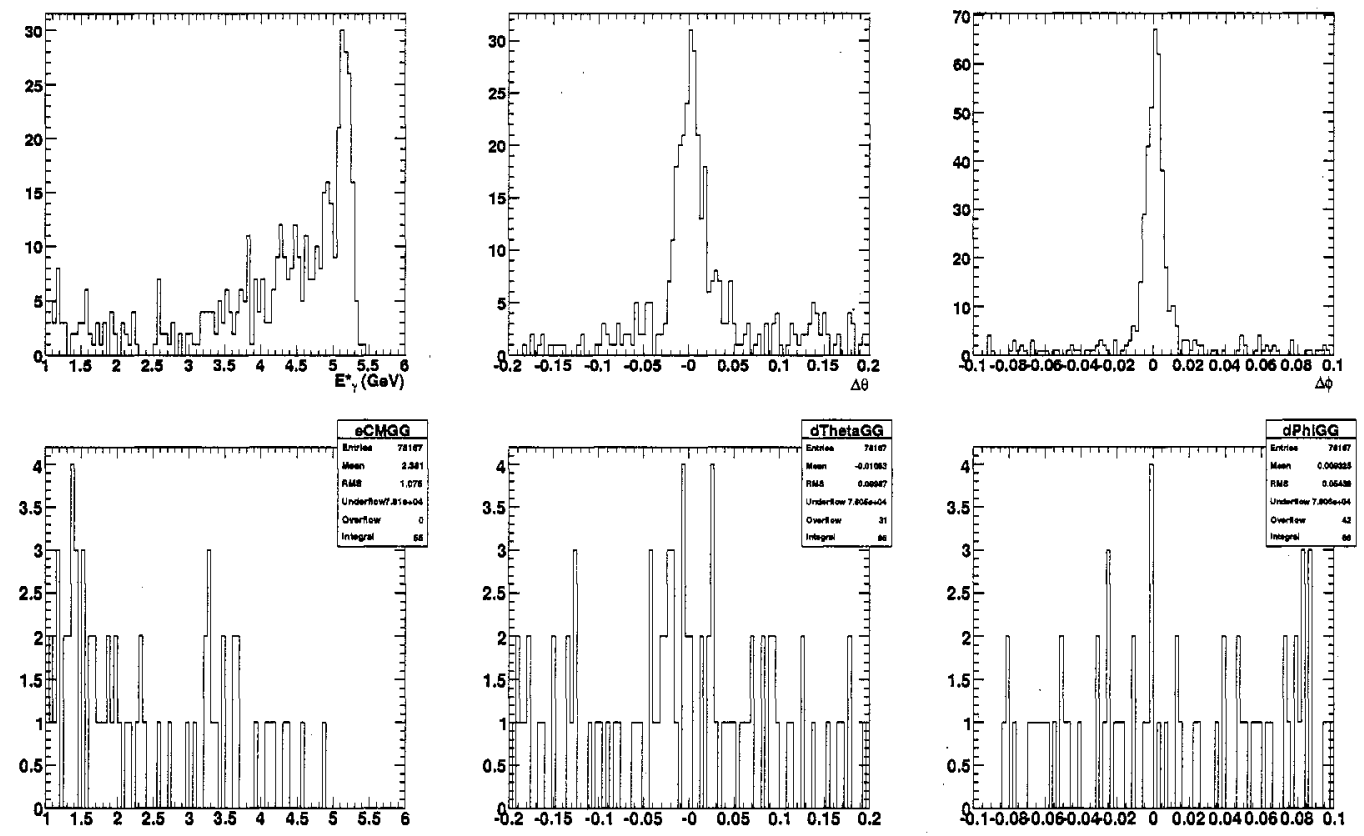

Figure 5.6: Center-of-mass energy of the second-highest energy cluster in the event (left) and $\Delta \theta$ (center), $\Delta \phi$ (right) with respect to the ISR photon back-to-back direction. Top row is non-converted $\gamma \gamma \mathrm{MC}$, bottom is KKMC. 
does not apply. These events are suppressed by the radiative Bhabha veto described in Section 5.8, but a specific algorithm can be implemented if both conversion tracks are detected.

Conversions are reconstructed using the technique described in Section 8.3.1. As described in this section, we call $r_{x y}$ the transverse component of the decay radius, $d_{x y}$ and $d_{z}$ the minimal distances between the tracks in the transverse and $z$ directions, $E_{C o n v}^{*}$ and $m_{C o n v}$ the CM energy and mass of the conversion.

The distributions for these quantities are shown in Figs. 5.7 and 5.8. The selections are as follows:

- $E_{C o n v}^{*}>3 \mathrm{GeV}$

- $m_{\text {Conv }}<200 \mathrm{MeV}$

- $2<r_{x y}<60 \mathrm{~cm}$

- $d_{x y}<2 \mathrm{~cm}$

- $\left|d_{z}\right|<10 \mathrm{~cm}$

Unfortunately this selection also picks up about $20 \%$ of the $e^{+} e^{-} \rightarrow \phi\left(K^{+} K^{-}\right) \gamma$ signal events. Due to the low $Q^{2}$ value of the $\phi$ decay these events have a similar topology; most are saved by the $r_{x y}>2 \mathrm{~cm}$ requirement but there are some losses due to the poor resolution on the conversion radial position.

In order to reduce these losses we further require that at least one conversion track not be identified as a kaon. This increases the number of passing $\gamma \gamma$ events with two visible tracks by $5.4 \%$; on $\phi\left(K^{+} K^{-}\right) \gamma$ events with two visible tracks the efficiency goes from $80.1 \%$ to $\sim 100 \%$.

The selection has an efficiency of $44 \%$ on converted $\gamma \gamma$ events with 2 visible tracks. The signal inefficiency on the sample passing the unconverted $\gamma \gamma$ veto is $0.4 \%$. 

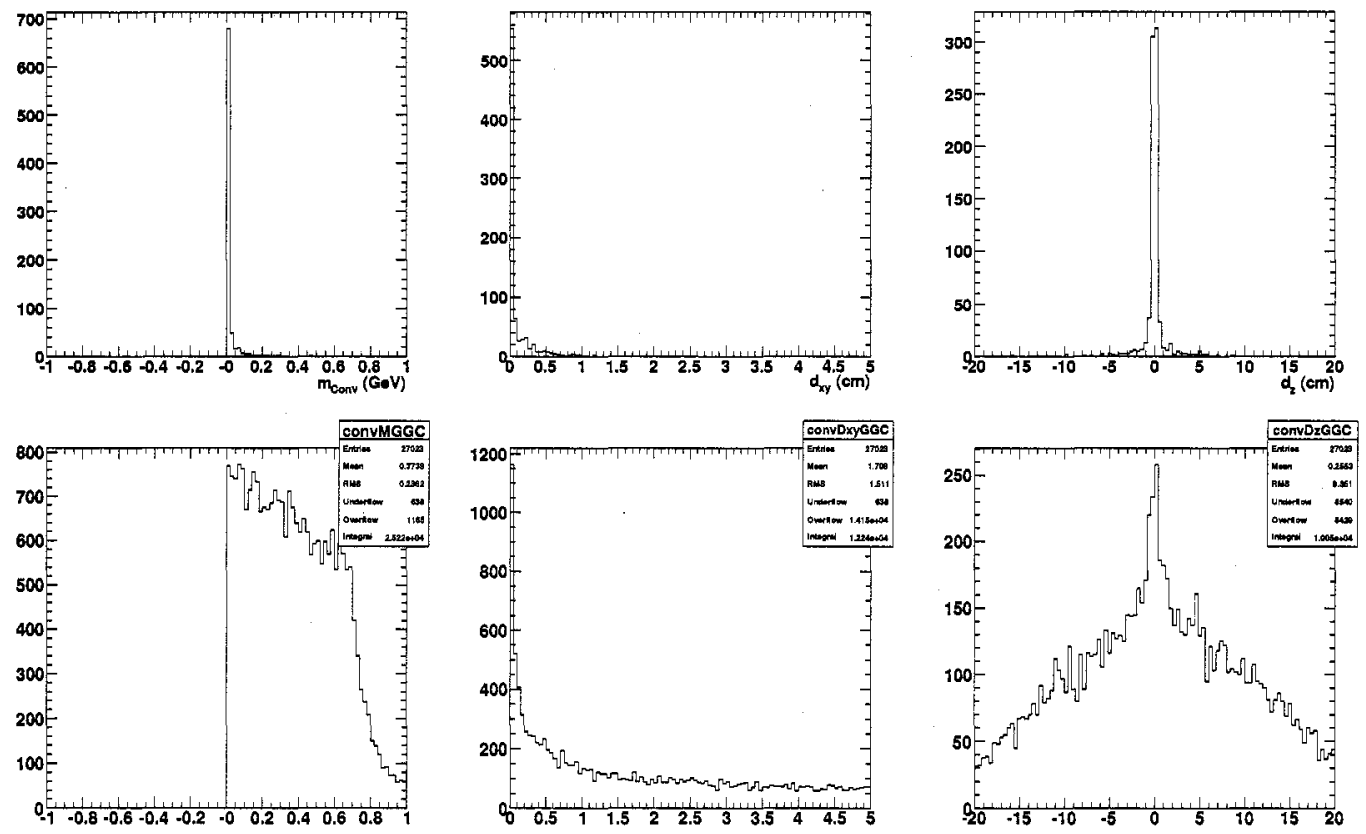

Figure 5.7: Conversion invariant mass (left), $d_{x y}$ (center) and $d_{z}$ (right) distributions. Top row is converted $\gamma \gamma \mathrm{MC}$, bottom is KKMC.
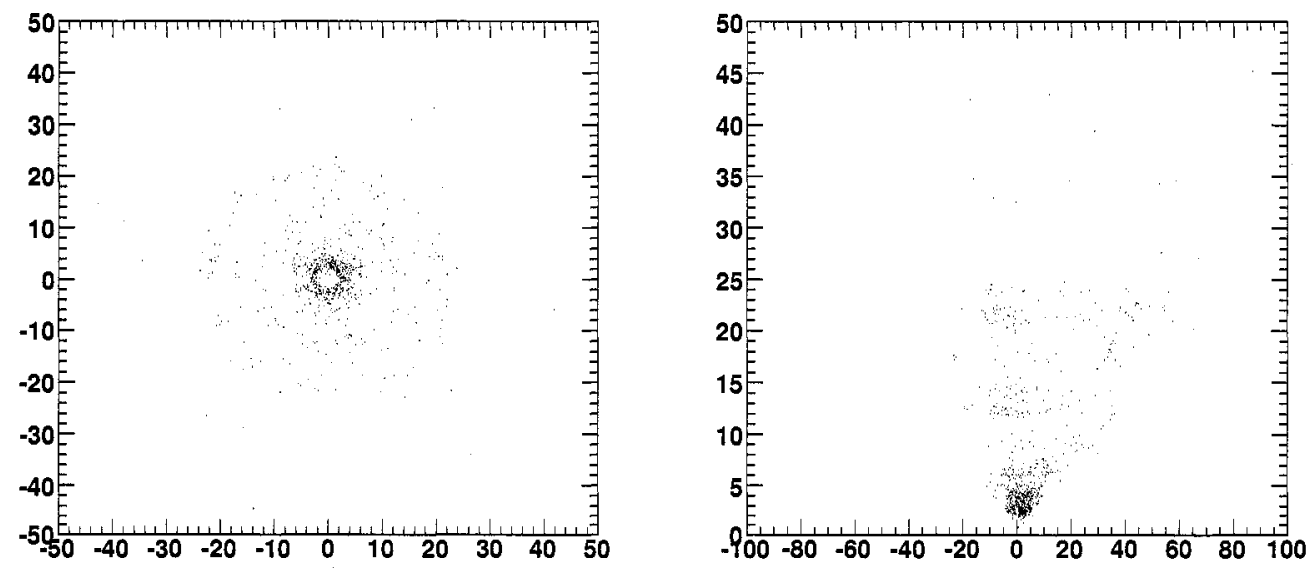

Figure 5.8: Conversion vertex position in $x-y$ (left) and $r-z$ (right) coordinates for converted $\gamma \gamma \mathrm{MC}$. 


\subsection{Radiative Bhabha Veto}

This selection rejects events with many electrons in the final states. It is therefore applicable to generic QED processes, including converted $\gamma \gamma$ events and higherorder processes. The veto is based on the numbers $N_{e}$ and $N_{h}$ of IsrElectrons and IsrNonElectrons respectively. The selection is as follows:

- Require $1 \leq N_{G T} \leq 6$

- Veto the event if $N_{\epsilon}<N_{G T} / 2$

- Veto the event if $N_{e} \geq 1$ and $N_{h}<2$.

In the one-track case, an electron identification on the track lead to a veto. In the two-track case however, the event can survive an electron PID on one of the tracks if both tracks are also flagged as IsrNonElectrons.

\subsection{All-Neutral QED Veto}

For the $N_{G T} \geq 1$ sample, it is possible to apply a positive selection to signal while rejecting a number of specific background signatures. This approach in no longer possible for the $N_{G T}=0$ due to a much smaller ratio of signal to background: from MC samples, the signal cross-section is about $1.3 \mathrm{pb}$ in this sample, compared with $6 \mathrm{pb}$ for $\gamma \gamma$ events and $\sim 1.4 \mathrm{pb}$ for radiative Bhabha events.

Instead we require events to contain at least one $\pi^{0} \rightarrow \gamma \gamma$ decay, reconstructed by combining pairs of clusters with $E>100 \mathrm{MeV}$ to form a $\pi^{0}$ candidate with:

- $100<m_{\gamma \gamma}<160 \mathrm{MeV}$

- $E_{\gamma \gamma}^{*}>500 \mathrm{MeV}$

- $\theta_{\gamma \gamma}>350 \mathrm{mrad}$

- $\left|\cos \left(\mathcal{H}_{\gamma \gamma}\right)\right|<0.67$ 
where $\mathcal{H}_{\gamma \gamma}$ is the helicity angle of the decay. The recoil momentum for the helicity calculation is taken to be half the momentum of the hadronic side of the event, as calculated from the ISR photon momentum. This is the correct value for the process $\phi \rightarrow K_{S}^{0} K_{L}^{0}, K_{S}^{0} \rightarrow \pi^{0} \pi^{0}$ which is of particular interest, and a good estimation for other modes. In the case of multiple $\pi^{0}$ candidates falling within the $E_{\gamma \gamma}^{*}, \theta_{\gamma \gamma}$ and helicity angle cut the candidate with the mass closest to the nominal $\pi^{0}$ mass is chosen.

Fig 5.9 shows the distributions of the quantities above for $\gamma \gamma$ and signal MC events.

\subsection{1-Track QED Veto}

The $N_{G T}=1$ sample also suffers from large levels of QED background, although not as severe as in the $N_{G T}=0$ case. More stringent electron rejection cuts are therefore implemented for this subsample: events are rejected if the lone track satisfies either of the following requirements:

- $E_{C a l}>2 \mathrm{GeV}$

- $p^{*}<1 \mathrm{GeV}$ and either $d E / d x<100$ or $500<d E / d x<1000$

- $\left|\Delta \theta_{\gamma}\right|<50 \mathrm{mrad}$ and $\left|\Delta \phi_{\gamma}\right|<50 \mathrm{mrad}$, where $\Delta \theta_{\gamma}$ and $\Delta \phi_{\gamma}$ are the angular separations in the $\theta$ and $\phi$ directions of the track momentum and the ISR photon back-to-back direction in the CM frame.

- The intersection of the track and the EMC is either between crystals or within the outer $10 \%$ of a crystal in either dimension (in terms of the normalized crystal coordinates of section 7.2.3, this translates into $|x|>0.45$ or $|y|>0.45$ ).

The angular cut is motivated by the fact that the peak in $\Delta \theta_{\gamma}$ and $\Delta \phi_{\gamma}$ is much sharper in the radiative Bhabha sample that in the hadronic sample. This is probably due to the fact that the "ISR photon" is actually a hard bremsstrahlung photon from the other Bhabha leg, which is emitted collinearly and therefore exactly in the direction back-to-back to the other track. 

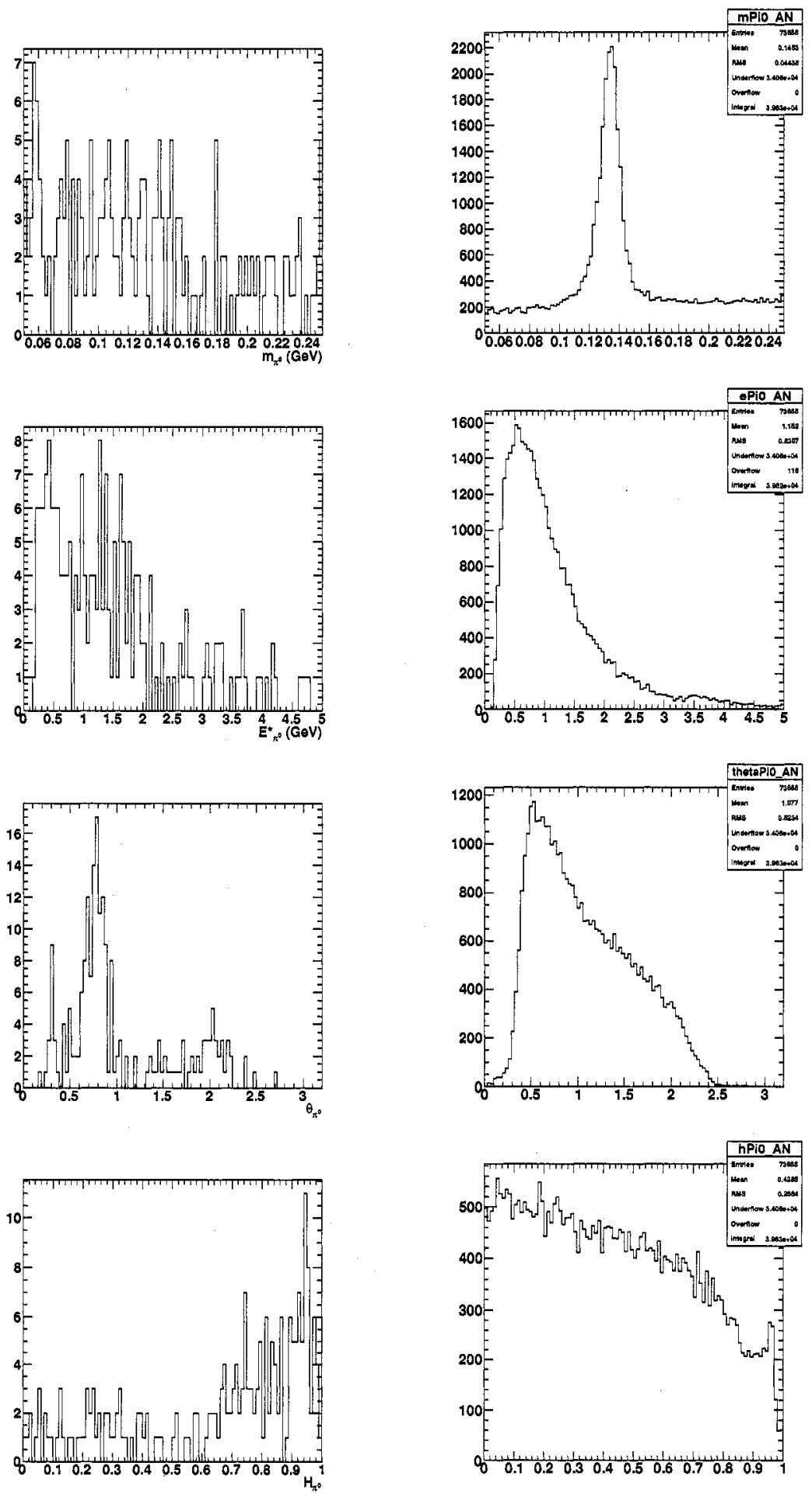

Figure 5.9: Distributions of $\pi^{0} \rightarrow \gamma \gamma$ candidate parameters in non-converted $\gamma \gamma$ MC (left column) and KKMC (right column) MC. From top to bottom, the quantities are the candidate invariant mass, CM energy, $\theta_{L a b}$ and helicity angle cosine 
Table 5.1: Efficiencies for the main ISR event selection

\begin{tabular}{|l|c|c|c|}
\hline & $q \bar{q} \gamma$ & $\mu^{+} \mu^{-} \gamma$ & $\tau^{+} \tau^{-} \gamma$ \\
\hline ISR Photon & $89.99 \pm 0.09 \%$ & $89.92 \pm 0.10 \%$ & $88.14 \pm 0.07 \%$ \\
L1 & $89.96 \pm 0.10 \%$ & $89.87 \pm 0.10 \%$ & $87.53 \pm 0.07 \%$ \\
L3 Physics & $89.58 \pm 0.10 \%$ & $88.18 \pm 0.10 \%$ & $85.26 \pm 0.08 \%$ \\
BGF Physics & $87.64 \pm 0.10 \%$ & $88.07 \pm 0.10 \%$ & $79.96 \pm 0.09 \%$ \\
$E_{\text {Total }}<17 \mathrm{GeV}$ & $87.56 \pm 0.10 \%$ & $88.04 \pm 0.10 \%$ & $79.95 \pm 0.09 \%$ \\
$\gamma \gamma$ Veto & $87.53 \pm 0.10 \%$ & $88.03 \pm 0.10 \%$ & $79.94 \pm 0.09 \%$ \\
Converted $\gamma \gamma$ Veto & $87.23 \pm 0.11 \%$ & $87.34 \pm 0.11 \%$ & $79.94 \pm 0.09 \%$ \\
Virtual Compton Veto & $87.23 \pm 0.11 \%$ & $87.34 \pm 0.11 \%$ & $79.92 \pm 0.09 \%$ \\
Rad. Bhabha Veto & $84.68 \pm 0.11 \%$ & $87.16 \pm 0.11 \%$ & $62.35 \pm 0.11 \%$ \\
1-Track Veto & $82.21 \pm 0.12 \%$ & $81.59 \pm 0.12 \%$ & $59.78 \pm 0.11 \%$ \\
Neutral Veto & $81.59 \pm 0.12 \%$ & $81.30 \pm 0.12 \%$ & $59.14 \pm 0.11 \%$ \\
Lat. Moment & $74.56 \pm 0.14 \%$ & $74.25 \pm 0.14 \%$ & $54.11 \pm 0.11 \%$ \\
$m_{\gamma \gamma \text { Veto }}$ Evt. Shape & $73.05 \pm 0.14 \%$ & $73.88 \pm 0.14 \%$ & $52.55 \pm 0.11 \%$ \\
IsrInc. Tag & $71.55 \pm 0.14 \%$ & $73.79 \pm 0.14 \%$ & $50.19 \pm 0.11 \%$ \\
Tag $\gamma \gamma$ Veto & $71.55 \pm 0.14 \%$ & $73.79 \pm 0.14 \%$ & $50.19 \pm 0.11 \%$ \\
Mini $\gamma \gamma$ Veto & $71.54 \pm 0.14 \%$ & $73.79 \pm 0.14 \%$ & $50.19 \pm 0.11 \%$ \\
Mini $e^{+} e^{-} \gamma$ Veto & $71.54 \pm 0.14 \%$ & $73.79 \pm 0.14 \%$ & $50.19 \pm 0.11 \%$ \\
Events in 208fb ${ }^{-1}$ & $6640249.6 \pm 24837.1$ & $3462138.3 \pm 12745.2$ & $376371.1 \pm 1188.6$ \\
\hline
\end{tabular}

The cut on the crystal front-face position is justified by the fact that energy leakage is typically higher in the EMC near crystal boundaries, so that the track $E / p$ can be significantly below 1 for events is these regions, leading to misidentified electrons.

In order to save signal events with many neutrals, we reconstruct $\pi^{0} \rightarrow \gamma \gamma$ candidates as in the previous section, except for the helicity angle cut which is loosened to 0.9. If such a candidate is found the event is kept.

The distributions of relevant quantities are shown in Fig. 5.10.

\subsection{Summary of QED background rejection}

The cumulative efficiencies for the various selections performed in this section are shown in in Table 5.1 for the signal modes, Table 5.2 for continuum backgrounds, 

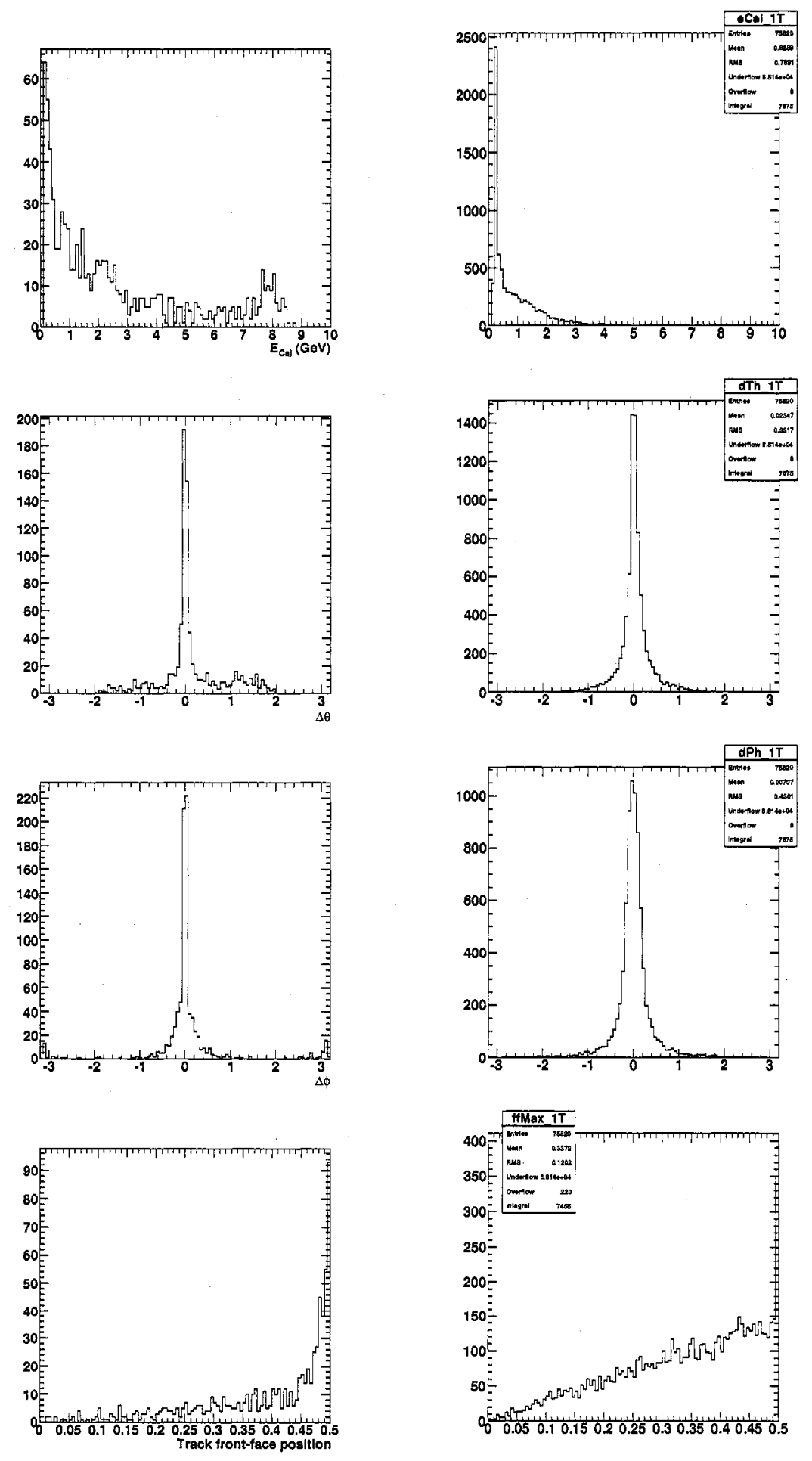

Figure 5.10: Variables used in the one-track veto algorithm, in $e^{+} e^{-} \gamma$ (left column) and $q \bar{q} \gamma \mathrm{MC}$ (right column). From top to bottom, the variables are the track $E_{C a l}$, $\Delta \phi, \Delta \theta$ and front-fact position. 
Table 5.2: Efficiencies for the main ISR event selection

\begin{tabular}{|l|c|c|c|}
\hline & $\tau^{+} \tau^{-}$ & uds & $c \bar{c}$ \\
\hline Precuts & $2.21 \pm 0.01 \%$ & $1.31 \pm 0.00 \%$ & $(1.65 \pm 0.01) \times 10^{-3}$ \\
Isr Photon & $1.55 \pm 0.01 \%$ & $(8.19 \pm 0.03) \times 10^{-3}$ & $(7.38 \pm 0.04) \times 10^{-4}$ \\
$\gamma \gamma$ Veto & $1.55 \pm 0.01 \%$ & $(8.18 \pm 0.03) \times 10^{-3}$ & $(7.38 \pm 0.04) \times 10^{-4}$ \\
Converted $\gamma \gamma$ Veto & $1.55 \pm 0.01 \%$ & $(8.18 \pm 0.03) \times 10^{-3}$ & $(7.38 \pm 0.04) \times 10^{-4}$ \\
Virtual Compton Veto & $1.55 \pm 0.01 \%$ & $(8.18 \pm 0.03) \times 10^{-3}$ & $(7.38 \pm 0.04) \times 10^{-4}$ \\
Rad. Bhabha Veto & $1.35 \pm 0.01 \%$ & $(7.99 \pm 0.03) \times 10^{-3}$ & $(7.17 \pm 0.04) \times 10^{-4}$ \\
1-Track Veto & $1.30 \pm 0.01 \%$ & $(7.93 \pm 0.03) \times 10^{-3}$ & $(7.16 \pm 0.03) \times 10^{-4}$ \\
Neutral Veto & $1.29 \pm 0.01 \%$ & $(7.89 \pm 0.03) \times 10^{-3}$ & $(7.14 \pm 0.03) \times 10^{-4}$ \\
Lat. Moment & $(5.39 \pm 0.04) \times 10^{-3}$ & $(3.59 \pm 0.02) \times 10^{-3}$ & $(2.67 \pm 0.02) \times 10^{-4}$ \\
$m_{\gamma \gamma \text { Veto }}$ & $(2.13 \pm 0.02) \times 10^{-3}$ & $(1.33 \pm 0.01) \times 10^{-3}$ & $(1.15 \pm 0.01) \times 10^{-4}$ \\
Evt. Shape & $(1.31 \pm 0.02) \times 10^{-3}$ & $(6.70 \pm 0.09) \times 10^{-4}$ & $(4.06 \pm 0.08) \times 10^{-5}$ \\
Events in 208fb & $244411.0 \pm 3363.3$ & $288998.9 \pm 4074.9$ & $11008.8 \pm 225.8$ \\
\hline
\end{tabular}

Table 5.3: Efficiencies for the main ISR event selection

\begin{tabular}{|l|c|c|}
\hline & $B^{0} \bar{B}^{0}$ & $B^{+} B^{-}$ \\
\hline Precuts & $(1.17 \pm 0.01) \times 10^{-4}$ & $(1.26 \pm 0.01) \times 10^{-4}$ \\
Isr Photon & $(2.03 \pm 0.04) \times 10^{-5}$ & $(3.36 \pm 0.04) \times 10^{-5}$ \\
$\gamma \gamma$ Veto & $(2.03 \pm 0.04) \times 10^{-5}$ & $(3.36 \pm 0.04) \times 10^{-5}$ \\
Converted $\gamma \gamma$ Veto & $(2.03 \pm 0.04) \times 10^{-5}$ & $(3.36 \pm 0.04) \times 10^{-5}$ \\
Virtual Compton Veto & $(2.03 \pm 0.04) \times 10^{-5}$ & $(3.36 \pm 0.04) \times 10^{-5}$ \\
Rad. Bhabha Veto & $(2.00 \pm 0.04) \times 10^{-5}$ & $(3.32 \pm 0.04) \times 10^{-5}$ \\
1-Track Veto & $(2.00 \pm 0.04) \times 10^{-5}$ & $(3.32 \pm 0.04) \times 10^{-5}$ \\
Neutral Veto & $(2.00 \pm 0.04) \times 10^{-5}$ & $(3.31 \pm 0.04) \times 10^{-5}$ \\
Lat. Moment & $(9.92 \pm 0.28) \times 10^{-6}$ & $(1.86 \pm 0.03) \times 10^{-5}$ \\
$m_{\gamma \gamma}$ Veto & $(9.00 \pm 0.27) \times 10^{-6}$ & $(1.68 \pm 0.03) \times 10^{-5}$ \\
Evt. Shape & $(7.26 \pm 0.24) \times 10^{-6}$ & $(1.24 \pm 0.03) \times 10^{-5}$ \\
Events in 208 $\mathrm{fb}^{-1}$ & $793.8 \pm 26.1$ & $1354.2 \pm 28.2$ \\
\hline
\end{tabular}


Table 5.4: Efficiencies for the main ISR event selection

\begin{tabular}{|l|c|c|}
\hline & $\gamma \gamma$ & $e^{+} e^{-} \gamma$ \\
\hline Precuts & $(2.87 \pm 0.05) \times 10^{-3}$ & $1.63 \pm 0.01 \%$ \\
Isr Photon & $(1.86 \pm 0.04) \times 10^{-3}$ & $1.14 \pm 0.01 \%$ \\
$\gamma \gamma$ Veto & $(1.54 \pm 0.04) \times 10^{-3}$ & $1.12 \pm 0.01 \%$ \\
Converted $\gamma \gamma$ Veto & $(9.27 \pm 0.29) \times 10^{-4}$ & $1.12 \pm 0.01 \%$ \\
Virtual Compton Veto & $(9.17 \pm 0.29) \times 10^{-4}$ & $1.04 \pm 0.01 \%$ \\
Rad. Bhabha Veto & $(6.86 \pm 0.25) \times 10^{-4}$ & $(1.72 \pm 0.04) \times 10^{-3}$ \\
1-Track Veto & $(6.25 \pm 0.24) \times 10^{-4}$ & $(1.09 \pm 0.03) \times 10^{-3}$ \\
Neutral Veto & $(7.09 \pm 0.80) \times 10^{-5}$ & $(4.58 \pm 0.20) \times 10^{-4}$ \\
Lat. Moment & $(6.19 \pm 0.75) \times 10^{-5}$ & $(4.11 \pm 0.19) \times 10^{-4}$ \\
$m_{\gamma \gamma}$ Veto & $(6.19 \pm 0.75) \times 10^{-5}$ & $(4.00 \pm 0.19) \times 10^{-4}$ \\
Evt. Shape & $(6.01 \pm 0.73) \times 10^{-5}$ & $(3.62 \pm 0.18) \times 10^{-4}$ \\
Events in 208 $\mathrm{fb}^{-1}$ & $35943.7 \pm 4391.2$ & $75522.0 \pm 3672.0$ \\
\hline
\end{tabular}

Table 5.5: Efficiencies for the main ISR event selection

\begin{tabular}{|l|c|c|}
\hline & Non-Converted $\gamma \gamma$ & Converted $\gamma \gamma$ \\
\hline Precuts & $(1.37 \pm 0.04) \times 10^{-3}$ & $1.67 \pm 0.04 \%$ \\
Isr Photon & $(9.70 \pm 0.31) \times 10^{-4}$ & $1.00 \pm 0.03 \%$ \\
$\gamma \gamma$ Veto & $(6.27 \pm 0.25) \times 10^{-4}$ & $(9.90 \pm 0.30) \times 10^{-3}$ \\
Converted $\gamma \gamma$ Veto & $(6.27 \pm 0.25) \times 10^{-4}$ & $(3.69 \pm 0.18) \times 10^{-3}$ \\
Virtual Compton Veto & $(6.27 \pm 0.25) \times 10^{-4}$ & $(3.59 \pm 0.18) \times 10^{-3}$ \\
Rad. Bhabha Veto & $(5.91 \pm 0.24) \times 10^{-4}$ & $(1.56 \pm 0.12) \times 10^{-3}$ \\
1-Track Veto & $(5.73 \pm 0.24) \times 10^{-4}$ & $(1.11 \pm 0.10) \times 10^{-3}$ \\
Neutral Veto & $(4.58 \pm 0.67) \times 10^{-5}$ & $(3.02 \pm 0.53) \times 10^{-4}$ \\
Lat. Moment & $(4.08 \pm 0.64) \times 10^{-5}$ & $(2.56 \pm 0.48) \times 10^{-4}$ \\
$m_{\gamma \gamma}$ Veto & $(4.08 \pm 0.64) \times 10^{-5}$ & $(2.56 \pm 0.48) \times 10^{-4}$ \\
Evt. Shape & $(3.88 \pm 0.62) \times 10^{-5}$ & $(2.56 \pm 0.48) \times 10^{-4}$ \\
Events in 208 $\mathrm{fb}^{-1}$ & $20922.4 \pm 3350.3$ & $15021.2 \pm 2838.7$ \\
\hline
\end{tabular}


Table 5.6: Efficiencies for the main ISR event selection broken down by number of tracks

\begin{tabular}{lcccc}
\hline \hline & $q \bar{q} \gamma\left(N_{G C}=0\right)$ & $q \bar{q} \gamma\left(N_{G C}=1\right)$ & $q \bar{q} \gamma\left(N_{G C}=2\right)$ & $q \bar{q} \gamma\left(N_{G C} \geq 3\right)$ \\
\hline ISR Photon & $89.99 \pm 0.56 \%$ & $94.00 \pm 0.23 \%$ & $90.66 \pm 0.15 \%$ & $88.56 \pm 0.15 \%$ \\
L1 & $89.22 \pm 0.58 \%$ & $93.99 \pm 0.23 \%$ & $90.66 \pm 0.15 \%$ & $88.56 \pm 0.15 \%$ \\
L3 Physics & $86.66 \pm 0.64 \%$ & $93.07 \pm 0.25 \%$ & $90.18 \pm 0.15 \%$ & $88.51 \pm 0.15 \%$ \\
BGF Physics & $53.73 \pm 0.93 \%$ & $89.02 \pm 0.31 \%$ & $88.83 \pm 0.16 \%$ & $88.40 \pm 0.15 \%$ \\
$E_{\text {Total }}<17 \mathrm{GeV}$ & $53.73 \pm 0.93 \%$ & $89.00 \pm 0.31 \%$ & $88.81 \pm 0.16 \%$ & $88.26 \pm 0.15 \%$ \\
$\gamma \gamma$ Veto & $52.64 \pm 0.93 \%$ & $89.00 \pm 0.31 \%$ & $88.81 \pm 0.16 \%$ & $88.26 \pm 0.15 \%$ \\
Converted $\gamma \gamma$ Veto & $52.61 \pm 0.93 \%$ & $88.40 \pm 0.31 \%$ & $88.21 \pm 0.16 \%$ & $88.26 \pm 0.15 \%$ \\
Virtual Compton Veto & $52.61 \pm 0.93 \%$ & $88.33 \pm 0.31 \%$ & $88.21 \pm 0.16 \%$ & $88.26 \pm 0.15 \%$ \\
Rad. Bhabha Veto & $52.61 \pm 0.93 \%$ & $85.33 \pm 0.35 \%$ & $86.08 \pm 0.18 \%$ & $85.29 \pm 0.16 \%$ \\
1-Track Veto & $52.61 \pm 0.93 \%$ & $61.59 \pm 0.48 \%$ & $86.08 \pm 0.18 \%$ & $85.29 \pm 0.16 \%$ \\
Neutral Veto & $30.80 \pm 0.86 \%$ & $61.58 \pm 0.48 \%$ & $86.08 \pm 0.18 \%$ & $85.29 \pm 0.16 \%$ \\
Lat. Moment & $28.18 \pm 0.84 \%$ & $55.94 \pm 0.49 \%$ & $78.70 \pm 0.21 \%$ & $77.97 \pm 0.19 \%$ \\
$m_{\gamma \gamma}$ Veto & $27.65 \pm 0.84 \%$ & $55.04 \pm 0.49 \%$ & $78.02 \pm 0.21 \%$ & $75.62 \pm 0.20 \%$ \\
Evt. Shape & $24.92 \pm 0.81 \%$ & $53.17 \pm 0.49 \%$ & $77.74 \pm 0.21 \%$ & $73.28 \pm 0.20 \%$ \\
IsrInc. Tag & $24.92 \pm 0.81 \%$ & $53.17 \pm 0.49 \%$ & $77.74 \pm 0.21 \%$ & $73.28 \pm 0.20 \%$ \\
Tag $\gamma \gamma$ Veto & $24.61 \pm 0.81 \%$ & $53.17 \pm 0.49 \%$ & $77.74 \pm 0.21 \%$ & $73.28 \pm 0.20 \%$ \\
Mini $\gamma \gamma$ Veto & $24.47 \pm 0.80 \%$ & $53.17 \pm 0.49 \%$ & $77.74 \pm 0.21 \%$ & $73.28 \pm 0.20 \%$ \\
Mini $e^{+} e^{-} \gamma$ Veto & $23.98 \pm 0.80 \%$ & $53.10 \pm 0.49 \%$ & $77.66 \pm 0.21 \%$ & $73.27 \pm 0.20 \%$ \\
\hline Events in 208 fb ${ }^{-1}$ & $63636.8 \pm 2431.4$ & $512532.1 \pm 6900.3$ & $2805130.8 \pm 16143.1$ & $3258671.1 \pm 17399.2$ \\
\hline \hline
\end{tabular}

Table 5.3 for $B \bar{B}$ backgrounds and Table 5.4 for QED backgrounds. Table 5.5 shows a breakdown between converted and non-converted $\gamma \gamma$ events.

For background modes the common denominator of the efficiencies is the original number of events in the sample (given in Table 4.2). For signal modes, the denominator is the number of events containing a photon in MC truth with $E_{\text {True }}^{*}>3.5 \mathrm{GeV}$ and $0.396<\theta_{\text {True }}^{*}<2.099$, both slightly wider than the cuts applied to the reconstructed photon. This ensures that the efficiencies are correct, but it also means that the number of events in $208.4 \mathrm{fb}^{-1}$ reported in the last line is slightly underestimated, since it represents only the number which passes the MC truth cuts.

Table 5.6 shows the signal efficiency broken down by $N_{G T}$, and Table 5.7 shows the passing cross-sections at various points in the selection.

The $s^{\prime}$ and $\theta_{\gamma}$ distributions with all QED background rejections applied are shown in Figs. 5.11 and 5.12. The excess of events at $\theta \sim 1.45$ is due to a hot EMC crystal, 
Table 5.7: Summary of passing cross-sections after preselection cuts and after $e^{+} e^{-} \gamma$, $\gamma \gamma$, 1-track, neutral and virtual Compton vetos.

\begin{tabular}{|l|c|c|c|c|c|c|}
\hline Sample & $\sigma_{\text {Presel }}(\mathrm{pb})$ & $\sigma_{e^{+} e^{-} \gamma}(\mathrm{pb})$ & $\sigma_{\gamma \gamma}(\mathrm{pb})$ & $\sigma_{1-\text { track }}(\mathrm{pb})$ & $\sigma_{\text {Neutral }}(\mathrm{pb})$ & $\sigma_{V C}(\mathrm{pb})$ \\
\hline KKMC & 41.64 & 40.52 & 40.36 & 39.24 & 38.95 & 38.95 \\
$\gamma \gamma$ & 5.40 & 3.28 & 1.98 & 1.80 & 0.21 & 0.21 \\
$e^{+} e^{-} \gamma$ & 11.39 & 1.87 & 1.73 & 1.09 & 0.46 & 0.46 \\
$\mu^{+} \mu^{-} \gamma$ & 21.32 & 21.27 & 21.10 & 19.76 & 19.68 & 19.68 \\
$\tau^{+} \tau^{-} \gamma$ & 3.32 & 2.66 & 2.66 & 2.55 & 2.52 & 2.52 \\
$\tau^{+} \tau^{-}$ & 13.74 & 11.90 & 11.89 & 11.44 & 11.35 & 11.35 \\
$u d s$ & 16.52 & 16.13 & 16.12 & 15.98 & 15.90 & 15.90 \\
$c \bar{c}$ & 0.97 & 0.94 & 0.94 & 0.94 & 0.94 & 0.94 \\
$B^{0} \bar{B}^{0}$ & 0.01 & 0.01 & 0.01 & 0.01 & 0.01 & 0.01 \\
$B^{+} B^{-}$ & 0.02 & 0.02 & 0.02 & 0.02 & 0.02 & 0.02 \\
\hline
\end{tabular}

as described in section 9.7 .

It may seem puzzling that the $\tau^{+} \tau^{-} \gamma$ contribution seems so much smaller than the $\mu^{+} \mu^{-} \gamma$ in these plots in the region well above the $\tau^{+} \tau^{-}$threshold. However this can be explained by a combination of two factors: first, the $\tau^{+} \tau^{-} \gamma$ is partially rejected be the radiative Bhabha veto, as shown in Table 5.8, and second the $\mu^{+} \mu^{-} \gamma$ is enhanced with respect to both $\tau^{+} \tau^{-} \gamma$ and $q \bar{q} \gamma$ due to the presence of final-state radiation. 


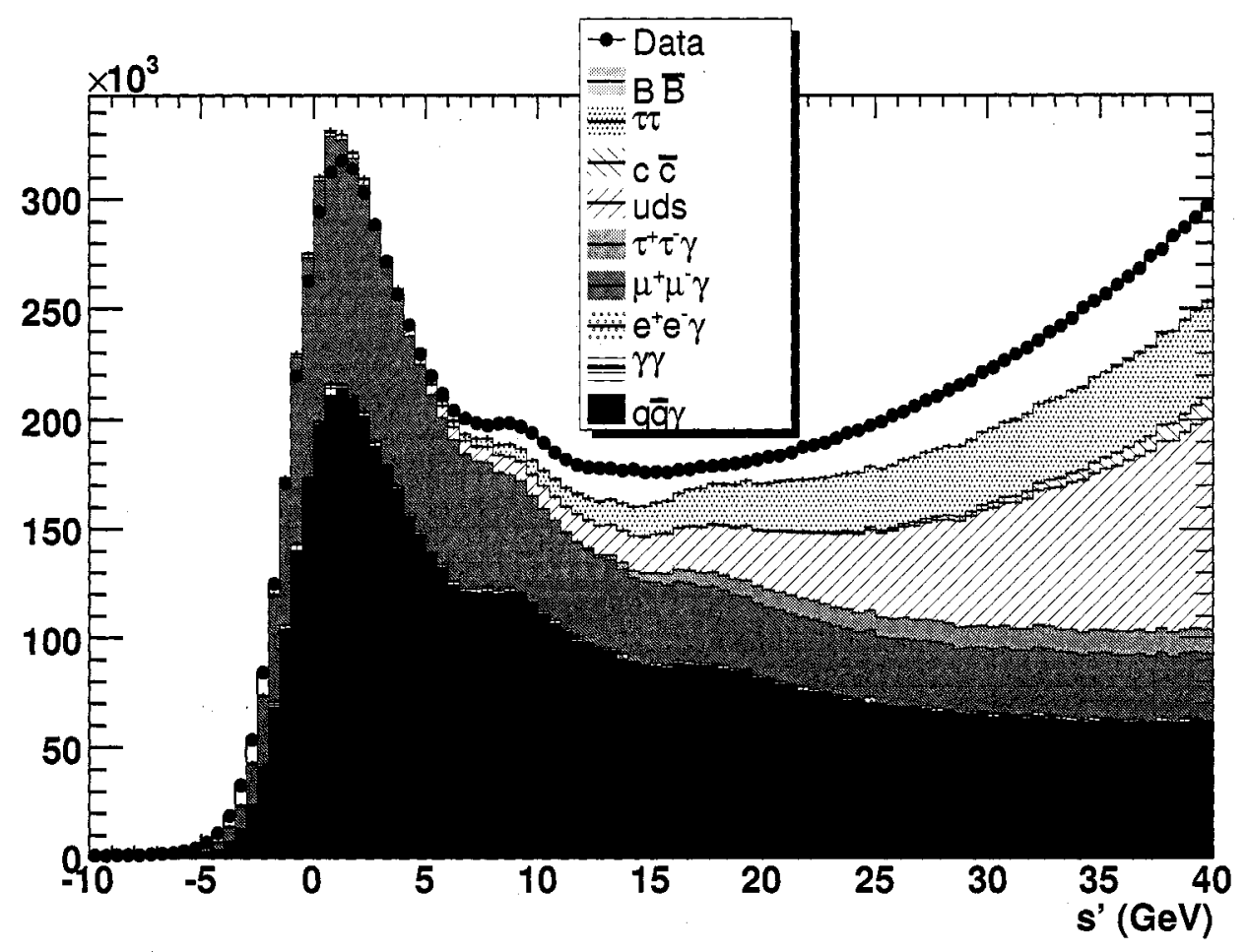

Figure 5.11: $s^{\prime}$ distribution for data (round dots) and stacked MC samples after hadronic selection cuts. 


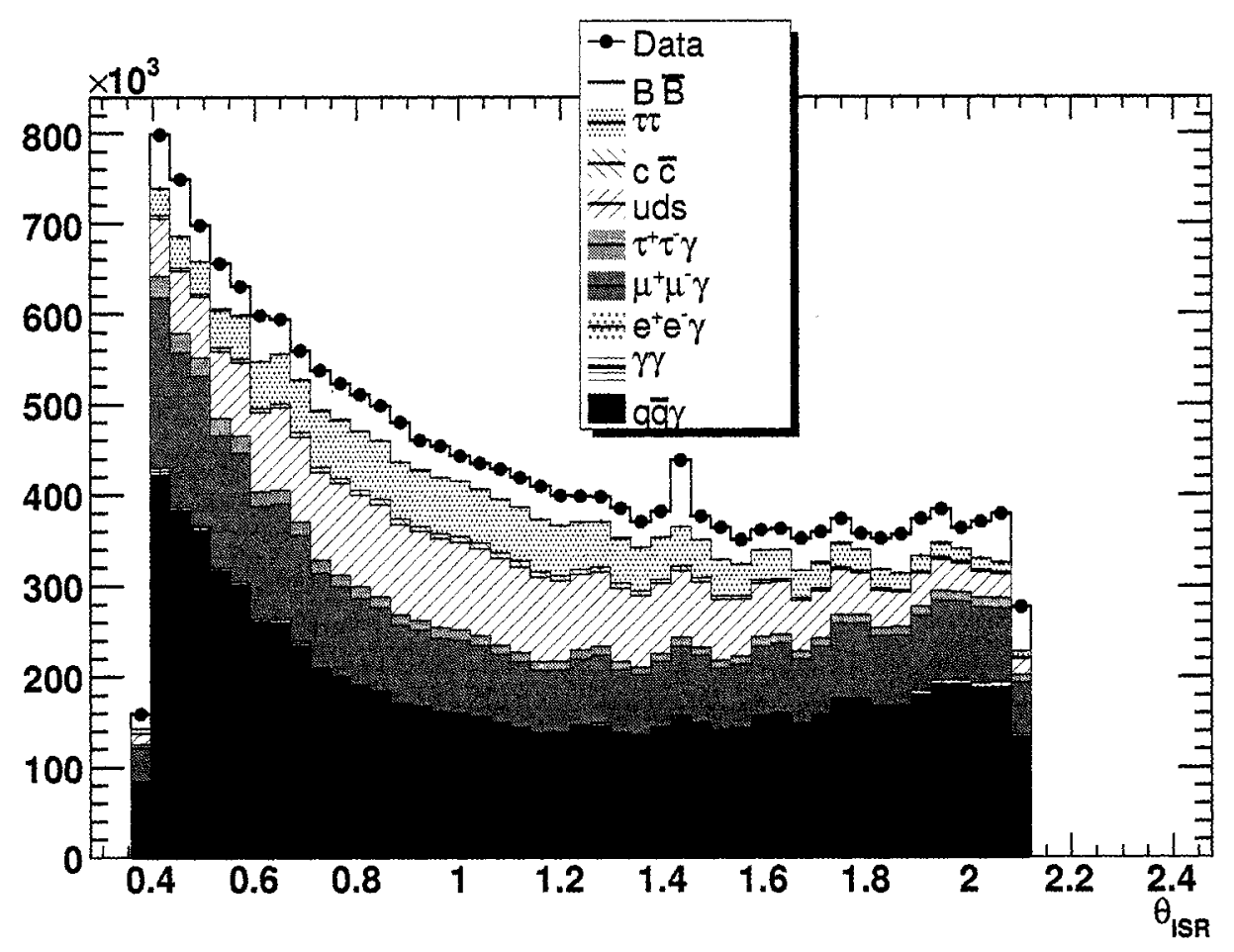

Figure 5.12: $\theta_{\gamma}$ distribution for data (round dots) and stacked MC samples after hadronic selection cuts. 
Table 5.8: Efficiencies for the main ISR event selection

\begin{tabular}{|l|c|c|c|}
\hline & $\tau^{+} \tau^{-} \gamma($ no $\tau \rightarrow e)$ & $\tau^{+} \tau^{-} \gamma(1 \tau \rightarrow e)$ & $\tau^{+} \tau^{-} \gamma(2 \tau \rightarrow e)$ \\
\hline ISR Photon & $87.93 \pm 0.12 \%$ & $88.70 \pm 0.20 \%$ & $89.63 \pm 0.64 \%$ \\
L1 & $87.54 \pm 0.12 \%$ & $87.61 \pm 0.21 \%$ & $87.04 \pm 0.71 \%$ \\
L3 Physics & $85.64 \pm 0.13 \%$ & $84.40 \pm 0.23 \%$ & $82.64 \pm 0.80 \%$ \\
BGF Physics & $83.61 \pm 0.14 \%$ & $74.34 \pm 0.27 \%$ & $24.27 \pm 0.90 \%$ \\
$E_{\text {Total }}<17 \mathrm{GeV}$ & $83.59 \pm 0.14 \%$ & $74.34 \pm 0.27 \%$ & $24.27 \pm 0.90 \%$ \\
$\gamma \gamma$ Veto & $83.58 \pm 0.14 \%$ & $74.33 \pm 0.27 \%$ & $24.27 \pm 0.90 \%$ \\
Converted $\gamma \gamma$ Veto & $83.57 \pm 0.14 \%$ & $74.32 \pm 0.27 \%$ & $24.27 \pm 0.90 \%$ \\
Virtual Compton Veto & $83.57 \pm 0.14 \%$ & $74.28 \pm 0.27 \%$ & $24.27 \pm 0.90 \%$ \\
Rad. Bhabha Veto & $77.85 \pm 0.15 \%$ & $24.25 \pm 0.27 \%$ & $(8.01 \pm 1.88) \times 10^{-3}$ \\
1-Track Veto & $75.00 \pm 0.16 \%$ & $22.00 \pm 0.26 \%$ & $(5.34 \pm 1.54) \times 10^{-3}$ \\
Neutral Veto & $74.28 \pm 0.16 \%$ & $21.45 \pm 0.26 \%$ & $(1.78 \pm 0.89) \times 10^{-3}$ \\
Lat. Moment & $68.04 \pm 0.17 \%$ & $19.69 \pm 0.25 \%$ & $(1.78 \pm 0.89) \times 10^{-3}$ \\
$m_{\gamma \gamma}$ Veto & $66.00 \pm 0.18 \%$ & $19.38 \pm 0.25 \%$ & $(1.78 \pm 0.89) \times 10^{-3}$ \\
Evt. Shape & $62.99 \pm 0.18 \%$ & $18.58 \pm 0.24 \%$ & $(1.78 \pm 0.89) \times 10^{-3}$ \\
IsrInc. Tag & $62.99 \pm 0.18 \%$ & $18.58 \pm 0.24 \%$ & $(1.78 \pm 0.89) \times 10^{-3}$ \\
Tag $\gamma \gamma$ Veto & $62.99 \pm 0.18 \%$ & $18.58 \pm 0.24 \%$ & $(1.78 \pm 0.89) \times 10^{-3}$ \\
Mini $\gamma \gamma$ Veto & $62.99 \pm 0.18 \%$ & $18.58 \pm 0.24 \%$ & $(1.78 \pm 0.89) \times 10^{-3}$ \\
Mini $e^{+} e^{-} \gamma$ Veto & $62.94 \pm 0.18 \%$ & $18.46 \pm 0.24 \%$ & $(1.78 \pm 0.89) \times 10^{-3}$ \\
Events in 208fb fb $^{-1}$ & $340903.2 \pm 1599.8$ & $35486.7 \pm 516.1$ & $30.0 \pm 15.0$ \\
\hline
\end{tabular}




\section{Chapter 6}

\section{Rejection of Hadronic Backgrounds}

\subsection{Nature of backgrounds}

The main source of background after QED processes consists of non-radiative hadronic events in which either a real photon is emitted in a hadronic decay or a hadronic process is misidentified as a photon. The main source of background of this types are photons from high-momentum $\pi^{0}$ decays, either merged or non-merged, and showers created by neutral hadrons in the calorimeter. Due to kinematic constraints such backgrounds appear mostly at the low end of the allowed photon energy range, i.e. the high end of the $s^{\prime}$ spectrum, as can be seen in Fig. 5.11. Table 6.1 shows the main sources of continuum background in $u d s, c \bar{c}$ and $\tau^{+} \tau^{-}$simulation.

\subsection{Non-merged $\pi^{0}$ Veto}

High-momentum $\pi^{0} \rightarrow \gamma \gamma$ can produce high-energy photons which are a background to ISR production. For high $\pi^{0}$ momenta and small values of the helicity parameter $\left|\cos \left(\mathcal{H}_{\gamma \gamma}\right)\right|$, the decay photons form a single bump in the EMC which cannot be resolved into two objects. These so-called merged $\pi^{0}$ are considered Section 6.4. On the other hand, if the $\pi^{0}$ is not merged it is possible to identify it if the second photon from the decay is detected. We therefore loop over all photons with $E>100 \mathrm{MeV}$ and look for combinations with the ISR photon that have an invariant mass $100<m_{\gamma \gamma}<$ 
Table 6.1: Processes in $u d s, c \bar{c}$ and $\tau^{+} \tau^{-}$MC samples contributing to background. The samples correspond to events passing the QED rejection cuts.

\begin{tabular}{lccc}
\hline \hline Category & uds & $\bar{c}$ & $\tau^{+} \tau^{-}$ \\
\hline Unmerged $\pi^{0} \rightarrow \gamma \gamma$ & $20.2 \pm 0.02 \%$ & $20.9 \pm 0.10 \%$ & $23.5 \pm 0.03 \%$ \\
Unmerged $\eta \rightarrow \gamma \gamma$ & $11.1 \pm 0.02 \%$ & $10.5 \pm 0.07 \%$ & $0.0 \pm 0.00 \%$ \\
Unmerged $\eta^{\prime} \rightarrow \gamma \gamma$ & $0.1 \pm 0.00 \%$ & $0.7 \pm 0.02 \%$ & $\mathrm{n} / \mathrm{a}$ \\
Unmerged $\omega \rightarrow \pi^{0} \gamma$ & $0.3 \pm 0.00 \%$ & $0.1 \pm 0.01 \%$ & $\mathrm{n} / \mathrm{a}$ \\
\hline Merged $\pi^{0} \rightarrow \gamma \gamma$ & $60.8 \pm 0.04 \%$ & $56.1 \pm 0.17 \%$ & $73.2 \pm 0.05 \%$ \\
Merged $\eta \rightarrow \gamma \gamma$ & $0.2 \pm 0.00 \%$ & $0.0 \pm 0.00 \%$ & $\mathrm{n} / \mathrm{a}$ \\
Merged $\eta^{\prime} \rightarrow \gamma \gamma$ & $\mathrm{n} / \mathrm{a}$ & $\mathrm{n} / \mathrm{a}$ & $\mathrm{n} / \mathrm{a}$ \\
Merged $\omega \rightarrow \pi^{0} \gamma$ & $0.7 \pm 0.00 \%$ & $0.1 \pm 0.01 \%$ & $\mathrm{n} / \mathrm{a}$ \\
\hline Other $\pi^{0}$ decay & $0.2 \pm 0.00 \%$ & $0.2 \pm 0.01 \%$ & $0.2 \pm 0.00 \%$ \\
Other $\eta$ decay & $0.0 \pm 0.00 \%$ & $0.0 \pm 0.00 \%$ & $0.0 \pm 0.00 \%$ \\
Other $\eta^{\prime}$ decay & $0.0 \pm 0.00 \%$ & $0.0 \pm 0.00 \%$ & $\mathrm{n} / \mathrm{a}$ \\
Other $\omega$ decay & $1.4 \pm 0.01 \%$ & $0.2 \pm 0.01 \%$ & $\mathrm{n} / \mathrm{a}$ \\
Other $\gamma$ & $\mathrm{n} / \mathrm{a}$ & $\mathrm{n} / \mathrm{a}$ & $\mathrm{n} / \mathrm{a}$ \\
\hline $\bar{n}^{0}$ & $2.9 \pm 0.01 \%$ & $6.7 \pm 0.06 \%$ & $\mathrm{n} / \mathrm{a}$ \\
$K_{L}^{0}$ & $0.2 \pm 0.00 \%$ & $0.5 \pm 0.02 \%$ & $0.0 \pm 0.00 \%$ \\
$n^{0}$ & $0.0 \pm 0.00 \%$ & $0.0 \pm 0.00 \%$ & $0.0 \pm 0.00 \%$ \\
$p^{-}$ & $\mathrm{n} / \mathrm{a}$ & $\mathrm{n} / \mathrm{a}$ & $\mathrm{n} / \mathrm{a}$ \\
$p^{+}$ & $0.2 \pm 0.00 \%$ & $0.6 \pm 0.02 \%$ & $0.0 \pm 0.00 \%$ \\
$\pi^{ \pm}$ & $0.3 \pm 0.00 \%$ & $0.4 \pm 0.01 \%$ & $0.7 \pm 0.00 \%$ \\
$K^{ \pm}$ & $0.0 \pm 0.00 \%$ & $0.1 \pm 0.01 \%$ & $0.0 \pm 0.00 \%$ \\
$e^{ \pm}$ & $0.9 \pm 0.00 \%$ & $0.9 \pm 0.02 \%$ & $1.3 \pm 0.01 \%$ \\
Other & $0.2 \pm 0.00 \%$ & $0.4 \pm 0.01 \%$ & $0.7 \pm 0.00 \%$ \\
\hline \hline
\end{tabular}


$170 \mathrm{MeV}$. Figure 6.1 shows the $m_{\gamma \gamma}$ distributions for $u d s$ and signal MC samples.
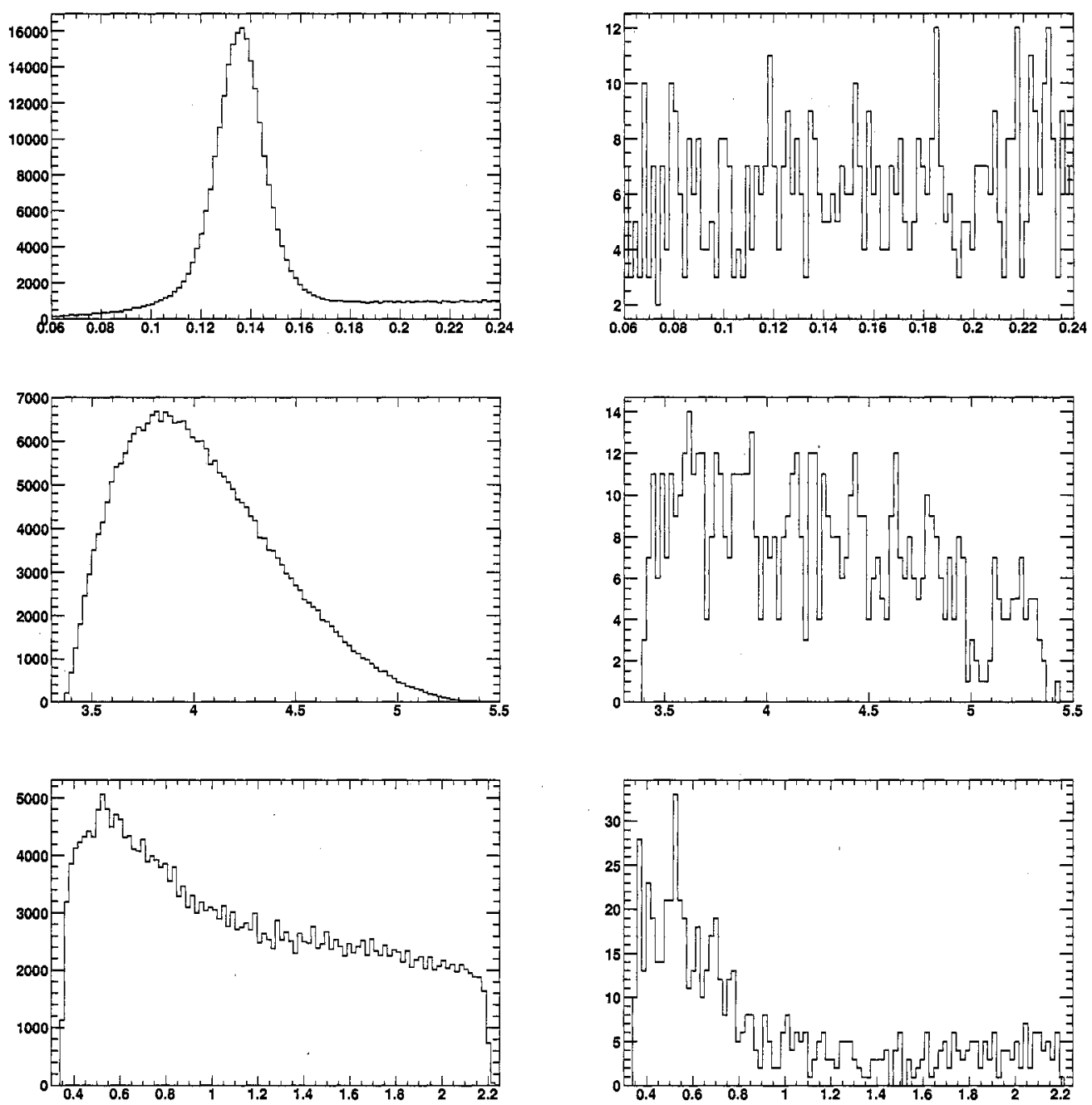

Figure 6.1: Distribution for the invariant mass (top row), CM energy (middle row) and lab polar angle (bottom row) for the $\gamma_{I S R} \gamma$ combination with mass closest to the nominal $\pi^{0}$ mass, where $\gamma_{I S R}$ is the ISR photon candidate and the other $\gamma$ is required to have $E>100 \mathrm{MeV}$. The left-hand and right-hand columns are uds and signal MC respectively.

The efficiency of this selection on events with real $\pi^{0}$ is expected to be welldescribed by simulation, since they mainly depend on $\pi^{0}$ kinematics. However the efficiency on signal is mostly due to random combinations of the ISR photon with 
Table 6.2: Minimum photon energy and $h$ mass interval used for the veto of $h \rightarrow$ $\gamma_{I S R} \gamma(\gamma)$.

\begin{tabular}{lccc}
\hline \hline Veto & $E_{\min }^{\gamma}(\mathrm{MeV})$ & $m_{\text {Min }}^{\text {Veto }}(\mathrm{GeV})$ & $m_{\text {Max }}^{\text {Veto }}(\mathrm{GeV})$ \\
\hline$\pi^{0}$ & 100 & 100.0 & 170.0 \\
$\eta$ & 150 & 497.8 & 597.8 \\
$\eta^{\prime}$ & 200 & 907.8 & 1007.8 \\
\hline \hline
\end{tabular}

a soft photon yielding a mass in the window defined above. The signal efficiency is therefore expected to depend on the number of soft photons in the event, which can vary with beam conditions and is a priori not expected to be well-described by simulation. The number of $\pi^{0}$ candidates in the mass window is therefore crosschecked in data as described in section 9.1.

\subsection{Other resonances}

As shown in Table 6.1, other resonances such as $\eta$ and $\eta^{\prime}$ also contribute small amounts of $\gamma \gamma$ decays that can fake ISR photons, and we therefore also veto the mass ranges corresponding to these resonances. The decay $\omega \rightarrow \pi^{0} \gamma$ can also produce a highenergy photon, and a veto is therefore also applied to this mode.

The mass ranges and minimum photon energies for each resonance are given in Table 6.2.

\subsection{Merged $\pi^{0}$ and Neutral Hadron Rejection}

In the case of a merged $\pi^{0}$ decay the two photon clusters cannot be separated. However the presence of a second photon may be inferred from the shape of the cluster. Electromagnetic showers are typically narrow and cylindrically symmetric, with most of the energy concentrated in the center crystal; on the other hand, in the case of a merged $\pi^{0}$ the cluster is composed of two photons with similar energies and may look elongated if the separation between the photons is not too small compared to 
the crystal size. The same criterion may be used to reject clusters created by neutral hadrons such as anti-neutrons and $K_{L}^{0}$, which produce hadronic showers that typically have a broader transverse profile.

A particularly useful variable to quantify these effects is the so-called lateral moment which measures the relative contribution of the two highest-energy crystals to the shower energy moment. It is calculated as follows: Let $E_{1}>E_{2}>\ldots>E_{N}$ be the energy of the individual crystals in the cluster, let $r_{i}$ the distance from each crystal center to to the shower maximum and $r_{0}$ the intercrystal distance. then

$$
L a t=\frac{\sum_{i=3}^{N} E_{i} r_{i}^{2}}{E_{1} r_{0}^{2}+E_{2} r_{0}^{2}+\sum_{i=3}^{N} E_{i} r_{i}^{2}} .
$$

Lateral moment distributions for photons and merged $\pi^{0} \mathrm{~s}$ in $\mathrm{MC}$ are shown in Figure 6.2. We require Lat $<0.40$.
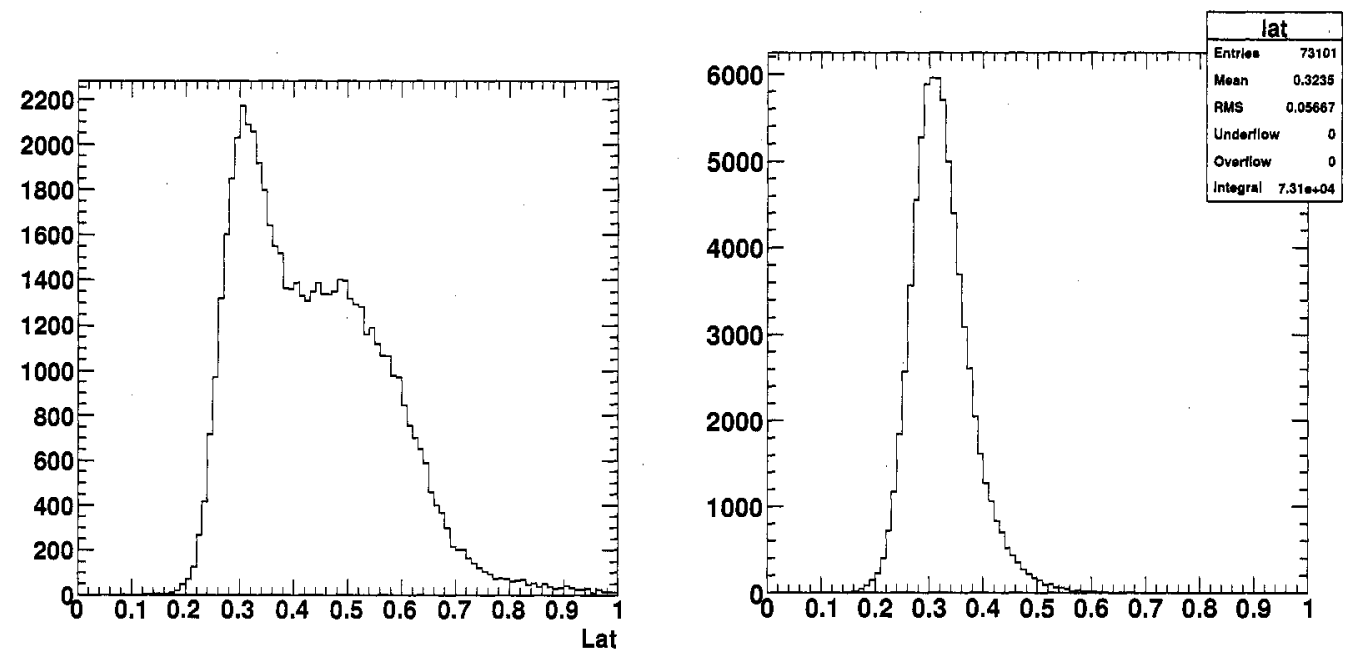

Figure 6.2: Lateral moment distribution for the ISR photon candidate in uds (left) and signal (right) MC.

Since Lat depends on the details of the interactions of photons with the EMC, the simulation is not expected a priori to give a sufficiently accurate determination of the Lat distributions for signal and background. The shapes are therefore cross-checked 
using control samples of photons, merged $\pi^{0}$ and neutral hadrons, as described in sections $9.2 .5,9.3 .4,9.5$ and 10.1 .

\subsection{Event-Shape Selection}

The usual way to reject $e^{+} e^{-} \rightarrow q \bar{q}$ background in BABAR analyses is to use eventshape information: such events are often "jetty", with tracks and clusters aligned near a common axis in the CM frame. In events that mimic ISR, one of the jets consists primarily of a single particle, often a $\pi^{0}$, that is misidentified as the ISR photon. ISR production peaks sharply at low polar angles while $q \bar{q}$ events have a softer $1+\cos ^{2} \theta$ dependence, so the angle between the "ISR photon" direction and the z-axis in the $\mathrm{CM}$ frame can help distinguish the two processes. Also, in the case of ISR the recoil side consist of a 2-quarks system, which is expected to be less collimated than a jet, so the distribution of tracks and cluster around the photon direction can also provide separation.

One can also consider the event in the recoil frame of the ISR photon, where ISR events should now be the ones looking jetty; one can consider the angle between the jets and the original ISR photon direction and the distribution of tracks and cluster around that direction.

\subsubsection{Thrust and Moments}

\section{Thrust}

To quantify the statements above we define the thrust vector $\overrightarrow{\mathcal{T}}$ of a set of particles as follows: consider the the scalar sum

$$
\Sigma(\vec{u})=\sum_{i}\left|\vec{p}_{i} \cdot \vec{u}\right|
$$

of the projections of the particle momenta $\vec{p}_{i}$ along an arbitrary unit vector $\vec{u}$. Then the vector $\vec{u}_{\max }$ along which $\Sigma(\vec{u})$ gets its maximal value is taken to be the direction 
of $\overrightarrow{\mathcal{T}}$, and its magnitude is the maximal value of the sum:

$$
\overrightarrow{\mathcal{T}}=\Sigma\left(\vec{u}_{\max }\right) \vec{u}_{\max }, \quad \Sigma\left(\vec{u}_{\max }\right)=\max _{\vec{u}} \Sigma(\vec{u})
$$

So $\overrightarrow{\mathcal{T}}$ points in the direction of the principal axis of the system, while its magnitude gives an measure of the spread of particle momenta around this direction.

We calculate the thrust using a numerical maximization procedure, using all tracks satisfying IsrGoodTrack and unmatched clusters with $E>250 \mathrm{MeV}$. For low multiplicities the maximization algorithm is unstable, so for the case 1 and 2 input tracks or clusters, we use the explicit formulas $\overrightarrow{\mathcal{T}}=\vec{p}$ and $\overrightarrow{\mathcal{T}}=\overrightarrow{p_{1}} \pm \overrightarrow{p_{2}}$.

\section{Legendre Moments}

A measure of the spread of the event around the thrust axis is obtained from the Legendre moments of the particles around the axis, defined by

$$
\tilde{L}_{n}(\overrightarrow{\mathcal{T}})=\sum_{i} p_{i} \mathcal{L}_{n}\left(\cos \left\langle\overrightarrow{p_{i}}, \overrightarrow{\mathcal{T}}\right\rangle\right)
$$

where $\left\langle\overrightarrow{p_{i}}, \overrightarrow{\mathcal{T}}\right\rangle$ is the angle between $\vec{p}_{i}$ and $\overrightarrow{\mathcal{T}}$ and $\mathcal{L}_{n}$ in the n-th Legendre polynomial. We have

$$
\begin{aligned}
\mathcal{L}_{0}(x) & =1 \\
\mathcal{L}_{1}(x) & =x \\
\mathcal{L}_{2}(x) & =\frac{1}{2}\left(3 x^{2}-1\right) \\
\mathcal{L}_{3}(x) & =\frac{1}{2}\left(5 x^{3}-x\right) \\
\mathcal{L}_{4}(x) & =\frac{1}{8}\left(35 x^{4}-30 x^{2}+3\right)
\end{aligned}
$$

$\tilde{L}_{0}(\overrightarrow{\mathcal{T}})$ is therefore the scalar sum of the particle momenta, $\tilde{L}_{1}(\overrightarrow{\mathcal{T}})$ gives the longitudinal momentum balance along $\overrightarrow{\mathcal{T}}, \tilde{L}_{1}(\overrightarrow{\mathcal{T}})$ includes a $\cos ^{2}\left\langle\overrightarrow{p_{i}}, \overrightarrow{\mathcal{T}}\right\rangle$ term that gives a measure of the spread of the particles around $\overrightarrow{\mathcal{T}}$, and the higher-order $\tilde{L}_{n}$ 's give 
higher moments of the distribution.

For $n \geq 1$, we rescale the moments by $\tilde{L}_{0}$ :

$$
\begin{aligned}
& L_{0}(x)=\tilde{L}_{0}(\overrightarrow{\mathcal{T}}) \\
& L_{n}(x)=\tilde{L}_{n}(\overrightarrow{\mathcal{T}}) / \tilde{L}_{0}(\overrightarrow{\mathcal{T}}), n \geq 1
\end{aligned}
$$

This makes the $n>1$ moments independent of the overall normalization of the $p_{i}$ and therefore better measures of the shape of the system.

\section{Fox-Wolfram Moments}

Another way to compute moments around $\overrightarrow{\mathcal{T}}$ is to use Fox-Wolfram moments, defined as

$$
\tilde{F}_{n}=\sum_{i, j} p_{i} p_{j} \mathcal{L}_{n}\left(\cos \left\langle\vec{p}_{i}, \overrightarrow{p_{j}}\right\rangle\right)
$$

The form is similar to Legendre moments, but uses the angles of the momenta "relative to each other" instead of angles with respect to a fixed direction. Also the moments are quadratic in the $p_{i}$ instead of linear.

Again, it is customary to rescale the moments and instead use

$$
F_{n}=\frac{1}{\left(\sum_{i} E_{i}\right)^{2}} \tilde{F}_{n}, n \geq 0
$$

where $E_{i}$ is the energy of particle $i$. The quantity which is denoted here by $F_{2}$ is also referred to as $R_{2}$ in the literature.

\subsubsection{Event-Shape Variables}

It is not obvious a priori which event-shape variables will give the best separation between ISR and $q \bar{q}$ events. We consider the variables given in the list below. The moments are calculated using all tracks passing the IsrGoodTrack criteria and clusters with $E>250 \mathrm{MeV}$ in the recoil system (that is, excluding the ISR photon candidate.)

- In the CM Frame 
- $\theta_{z}$ : The angle between the ISR photon candidate momentum and the $\mathrm{z}$ axis.

- $L_{n}, 0 \geq n \geq 3:$ The Legendre moments with respect to the ISR candidate momentum.

- $F_{n}, 0 \geq n \geq 3$ : The Fox-Wolfram moments with respect to the ISR candidate momentum.

- In the Hadronic system Frame

- $T$ : The thrust magnitude of the recoil system.

$-\theta_{T}$ : In the photon recoil frame (i.e. the frame of the system recoiling against the photon), the angle between the thrust direction of the hadronic system. and the photon recoil direction (i.e. $\vec{p}_{\text {Collision }}-\vec{p}_{\gamma}$ )

- $\bar{S}_{0}, S_{n}, n=2,3$ : The Legendre moments of the hadronic system around its thrust direction, with $\bar{S}_{0}=S_{0} / L_{0}$. We use $\bar{S}_{0}$ since $S_{0}$ is just an total energy measurement that is strongly correlated with $L_{0}$. Also $S_{1}=0$ since the total momentum of the hadronic system vanishes in its own rest frame.

$-T_{n}, n=2,3:$ The Legendre moments of the hadronic system around the photon recoil direction. We use neither $T_{0}=S_{0}$ (the scalar sum of momenta), nor $T_{1}=0$.

\subsubsection{Fisher Discriminant}

All the variables described above contain some information on signal/background separation, but none of them provide sufficient separation by itself. In order to achieve the best possible discrimination between signal and background, we combine the variables into a Fisher discriminant. For a set of variables $\left\{X_{i}\right\}$, this corresponds to building the linear combination

$$
\tilde{F}=\sum_{i} c_{i} X_{i}
$$


with coefficients $\left\{c_{i}\right\}$ such that the discriminating power of $\tilde{F}$ is largest. It can be shown [72] that this optimal set of coefficients is given by

$$
c_{i}=\sum_{j}\left(\bar{X}_{j}^{S i g}-\bar{X}_{j}^{B k g}\right)\left[V^{-1}\right]_{j i},
$$

where $\left\{\bar{X}_{j}^{S i g}\right\}$ and $\left\{\bar{X}_{j}^{B k g}\right\}$ are the average values of the $\left\{X_{i}\right\}$ on the signal and background samples respectively, and $V^{-1}$ is the inverse of the variance-covariance matrix for the $\left\{X_{i}\right\}$. In principle the formula is valid only if $V$ is the same for signal and background. In practice the shapes of the variable distributions are sometimes quite different in signal and background, leading to $V^{S i g} \neq V^{B k g}$. We therefore take $V=\left(V^{S i g}+V^{B k g}\right) / 2$ for our calculations, so that

$$
c_{i}=\sum_{j}\left(\bar{X}_{j}^{S i g}-\bar{X}_{j}^{B k g}\right)\left[\left(\frac{V^{S i g}+V^{B k g}}{2}\right)^{-1}\right]_{j i} .
$$

Finally, we normalize the expression of the discriminant given above so that the its averages over signal and background are equal to 1 and 0 respectively: we define $F=\alpha \tilde{F}+\beta$ and fix $\alpha$ and $\beta$ to satisfy these relations.

\subsubsection{Variable Selection}

Using a Fisher discriminant technique to combine the 16 variables described in Section 6.5 is both unpractical and inefficient: since correlations between variables are taken into account only at first order (the variance-covariance matrix), the Fisher discriminant method is optimal only in the case of small correlations, for which higherorder correlation terms are negligible. The first step should therefore be to reduce the number of variables, and in particular identify a small subset with small correlations that provides adequate separation.

To achieve this we use the following technique: first we form a Fisher discriminant with all 16 variables. To establish a figure of merit, we consider the background efficiency $\epsilon_{90}$ obtained for a signal efficiency of $90 \%$, which is of the order of what we can tolerate for an efficiency loss. We then start to remove variables in two ways: 
Table 6.3: Boundaries of the $s^{\prime}$ bins used for Fisher discriminants

\begin{tabular}{|l|c|}
\hline Bin & $s^{\prime}$ Range $\left(\mathrm{GeV}^{2}\right)$ \\
\hline 1 & $-10-9$ \\
2 & $9-15$ \\
3 & $15-20$ \\
4 & $20-25$ \\
5 & $25-30$ \\
6 & $30-35$ \\
7 & $35-38.5$ \\
8 & $38.5-42$ \\
\hline
\end{tabular}

- If there are pairs of variables with more than $50 \%$ correlation, we consider the pair with the highest correlation. Then, removing each member of the pair in turn, we make two Fisher discriminants with 15 variables. These discriminants are then optimized and the one with the lowest $\epsilon_{90}$ is retained. The other variable is discarded.

- If no pair of variables has more than $50 \%$ correlation, form all 1615 -variable Fisher with one variable removed and calculate their $\epsilon_{90}$ value. If the separation power of the variable removed is significant we expect $\epsilon_{90}$ to increase, and to stay constant if the separation power of the variable is weak. We then select the 15-variable combination which has the lowest $\epsilon_{90}$ value, and discard the remaining variable

The procedure is then repeated until we reach the desired number of variables.

The technique is complicated by the fact that the situation is quite different at various $s^{\prime}$ values. Hadronic background is absent for $s^{\prime}<10 \mathrm{GeV}^{2}$ and both the signal to background ratio and the distributions of event-shape variable vary with $s^{\prime}$. For this reason, we split the $s^{\prime}$ interval into the bins shown in Table 6.3, and perform a separate optimization in each one.

We apply the method using a signal MC sample and uds $\mathrm{MC}$ for the background, both with the same mix of Run 2, 3 and 4 conditions as data. The samples are selected to pass the preliminary selections, QED vetoes, lateral shape cut and $\pi^{0}$ veto. 
Table 6.3 shows the evolution from 16 to 2 variables in the regions $15 \leq s^{\prime} \leq 25 \mathrm{GeV}^{2}$ and $35 \leq s^{\prime} \leq 40 \mathrm{GeV}^{2}$.

We see that in the first few interactions the $\epsilon_{90}$ actually decreases, probably due to the fact that the variables removed have little separation power but large correlations with others, which makes the discriminant sub-optimal as described above. However below $\sim 10$ variables the $\epsilon_{90}$ rises again as useful variables are removed. The increase is however quite gradual until only very few variables remain.

Determining the number of variables that are kept in the final Fisher discriminant is a trade-off between complexity and performance - keeping 4-8 variables seems reasonable. We choose not to use $\theta_{z}$, since it is very useful for cross-checks at a later stage and better left unbiased. We choose to use the 6-variable combination of $L_{0}, \tilde{R}_{0}, T_{2}, F_{2}$ and $F_{3}$, which is the optimal 6-variable candidate for the high-s' region and close to the optimal one for the low- $s^{\prime}$ region. Fig. 6.4 shows the graphs of signal vs. background efficiency for the various Fisher choices, highlighting that little is lost by the removal of 9 variables.

It should be noted that this method follows only one branch of the tree of possible combinations of variables, implementing a sort of "steepest descent" algorithm. However it has the advantage of being much more economical in computing power: reducing the variable set from 16 to 6 requires the calculation of $\sum_{i=7}^{16} i=115$ Fisher discriminants, compared to $C_{15}^{6}=8008$ for the brute-force investigation of all possible 6 -variable combinations, and $2^{16}=65536$ for all combinations.

Various cross-checks were performed to check the stability of this result. The main issue is to verify that no interesting variable was dropped in the early stages of the optimization, when correlations make the Fisher discriminant sub-optimal. To check this we reduce the number of variables by removing unambiguously bad candidates which are dropped in early stages in both $s^{\prime}$ bins.

The correlation matrices for these variables in signal and background are shown in Table 6.5, showing that strong correlations remain between $\bar{R}_{0}, F_{2}$ and $F_{3}$. 


\begin{tabular}{|l|c|}
\hline Variable Set & $\epsilon_{B k q}^{0.90}(\%)$ \\
\hline All Variables & 29.12 \\
After removing $T\left[C\left(T, F_{2}\right)=0.94\right]$ & 29.13 \\
After removing $L_{2}\left[C\left(L_{2}, L_{3}\right)=0.84\right]$ & 29.15 \\
After removing $\theta_{T}\left[C\left(\theta_{T}, S_{2}\right)=0.73\right]$ & 28.99 \\
After removing $L_{1}\left[C\left(L_{1}, \tilde{R}_{0}\right)=0.65\right]$ & 29.03 \\
After removing $F_{1}\left[C\left(F_{1}, F_{3}\right)=0.62\right]$ & 29.06 \\
After removing $F_{2}\left[C\left(F_{2}, \tilde{R}_{0}\right)=0.59\right]$ & 31.47 \\
After removing $L_{3}\left[C\left(L_{3}, S_{2}\right)=0.51\right]$ & 33.22 \\
$\tilde{R}_{0} R_{2} R_{3} S_{2} S_{3} F_{0} F_{3} \theta_{z}$ & 33.09 \\
$\tilde{R}_{0} R_{2} S_{2} S_{3} F_{0} F_{3} \theta_{z}$ & 33.10 \\
$\tilde{R}_{0} S_{2} S_{3} F_{0} F_{3} \theta_{z}$ & 33.18 \\
$\tilde{R}_{0} S_{2} F_{0} F_{3} \theta_{z}$ & 33.41 \\
$\tilde{R}_{0} F_{0} F_{3} \theta_{z}$ & 34.20 \\
$\tilde{R}_{0} F_{3} \theta_{z}$ & 35.13 \\
\hline
\end{tabular}

\begin{tabular}{|l|c|}
\hline Variable Set & $\epsilon_{B k g}^{0.90}(\%)$ \\
\hline All Variables & 20.08 \\
After removing $T\left[C\left(T, F_{2}\right)=0.87\right]$ & 19.95 \\
After removing $L_{2}\left[C\left(L_{2}, S_{2}\right)=0.78\right]$ & 20.01 \\
After removing $\theta_{T}\left[C\left(\theta_{T}, S_{2}\right)=0.78\right]$ & 19.64 \\
After removing $F_{1}\left[C\left(F_{1}, L_{0}\right)=0.57\right]$ & 20.23 \\
After removing $L_{3}\left[C\left(L_{3}, S_{2}\right)=0.54\right]$ & 20.46 \\
$L_{0} L_{1} \tilde{R}_{0} R_{2} S_{2} S_{3} F_{0} F_{2} F_{3} \theta_{z}$ & 20.32 \\
$L_{0} \tilde{R}_{0} R_{2} S_{2} S_{3} F_{0} F_{2} F_{3} \theta_{z}$ & 20.31 \\
$L_{0} \tilde{R}_{0} R_{2} S_{2} S_{3} F_{2} F_{3} \theta_{z}$ & 20.26 \\
$L_{0} \tilde{R}_{0} R_{2} S_{2} F_{2} F_{3} \theta_{z}$ & 20.30 \\
$\tilde{R}_{0} R_{2} S_{2} F_{2} F_{3} \theta_{z}$ & 20.59 \\
$\tilde{R}_{0} R_{2} F_{2} F_{3} \theta_{z}$ & 21.11 \\
$\tilde{R}_{0} F_{2} F_{3} \theta_{z}$ & 21.59 \\
$\tilde{R}_{0} F_{2} \theta_{z}$ & 24.78 \\
\hline
\end{tabular}

Figure 6.3: Pruning sequence for Fisher discriminants in the regions $s^{\prime}=15-25 \mathrm{GeV}^{2}$ (left) and $s^{\prime}=35-40 \mathrm{GeV}^{2}$. (right), as described in the text. $\epsilon_{B k g}^{0.90}$ is the background efficiency at $90 \%$ signal efficiency, which is the figure of merit used. The initial variable set is $L_{0}, L_{1}, L_{2}, L_{3}, \tilde{S}_{0}, S_{2}, S_{3}, T_{2}, T_{3}, F_{0}, F_{1}, F_{2}, F_{3}, T, \theta_{T}$. 

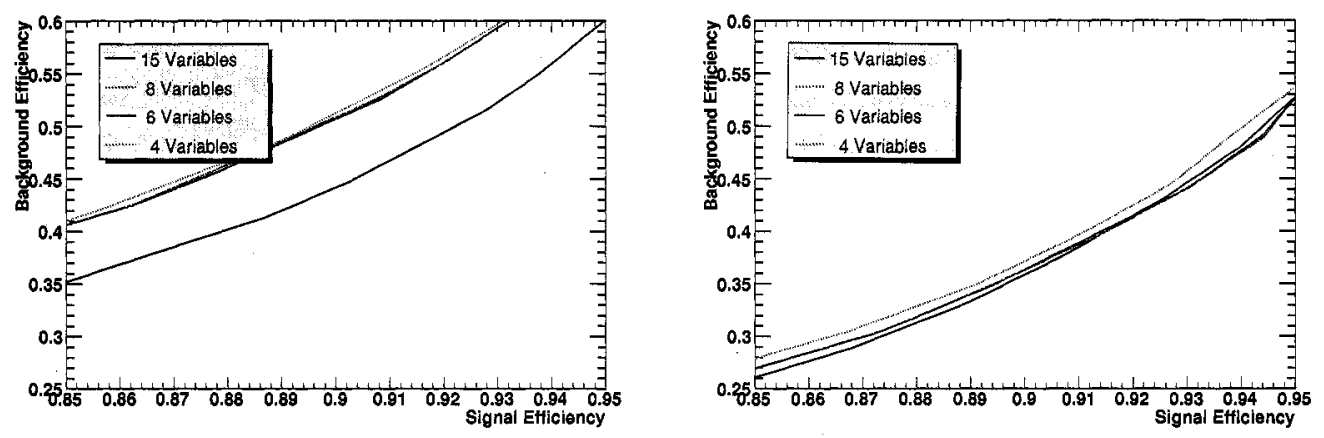

Figure 6.4: Background efficiency versus signal efficiency for pruned Fishers with given numbers of variables, for $s^{\prime}=15-25 \mathrm{GeV}^{2}$ (top) and $s^{\prime}=35-40 \mathrm{GeV}^{2}$ (bottom).

Signal

\begin{tabular}{|l|c|c|c|c|c|c|c|}
\hline & $L_{0}$ & $\tilde{R}_{0}$ & $R_{2}$ & $T_{2}$ & $F_{2}$ & $F_{3}$ & $s^{\prime}$ \\
\hline$L_{0}$ & 1 & 0.0355 & 0.139 & -0.00986 & -0.0384 & -0.283 & 0.0228 \\
$\tilde{R}_{0}$ & 0.0355 & 1 & -0.139 & 0.0264 & -0.905 & 0.578 & 0.794 \\
$R_{2}$ & 0.139 & -0.139 & 1 & -0.00761 & 0.199 & 0.0676 & -0.0988 \\
$T_{2}$ & -0.00986 & 0.0264 & -0.00761 & 1 & 0.123 & -0.24 & -0.0409 \\
$F_{2}$ & -0.0384 & -0.905 & 0.199 & 0.123 & 1 & -0.689 & -0.794 \\
$F_{3}$ & -0.283 & 0.578 & 0.0676 & -0.24 & -0.689 & 1 & 0.546 \\
$s^{\prime}$ & 0.0228 & 0.794 & -0.0988 & -0.0409 & -0.794 & 0.546 & 1 \\
\hline
\end{tabular}

Background

\begin{tabular}{|l|c|c|c|c|c|c|c|}
\hline & $L_{0}$ & $\tilde{R}_{0}$ & $R_{2}$ & $T_{2}$ & $F_{2}$ & $F_{3}$ & $\mathrm{~s}^{\prime}$ \\
\hline$L_{0}$ & 1 & 0.155 & 0.15 & 0.0499 & 0.0119 & -0.431 & 0.0661 \\
$\tilde{R}_{0}$ & 0.155 & 1 & 0.11 & 0.0162 & -0.398 & 0.191 & 0.564 \\
$R_{2}$ & 0.15 & 0.11 & 1 & 0.325 & 0.583 & -0.217 & 0.0675 \\
$T_{2}$ & 0.0499 & 0.0162 & 0.325 & 1 & 0.323 & -0.245 & -0.106 \\
$F_{2}$ & 0.0119 & -0.398 & 0.583 & 0.323 & 1 & -0.458 & -0.263 \\
$F_{3}$ & -0.431 & 0.191 & -0.217 & -0.245 & -0.458 & 1 & 0.0442 \\
$s^{\prime}$ & 0.0661 & 0.564 & 0.0675 & -0.106 & -0.263 & 0.0442 & 1 \\
\hline
\end{tabular}

Figure 6.5: Correlation matrices for the selected Fisher variables and $s^{\prime}$ 


\subsubsection{Fisher coefficients and cut values}

Having determined the variables to use, we build an optimal set of Fisher coefficients (according to the method of 6.5.3) in each of the $s^{\prime}$ bins of Table 6.3. The shapes of the input variables are shown in Fig 6.6. Fig 6.7 shows the values of the coefficients

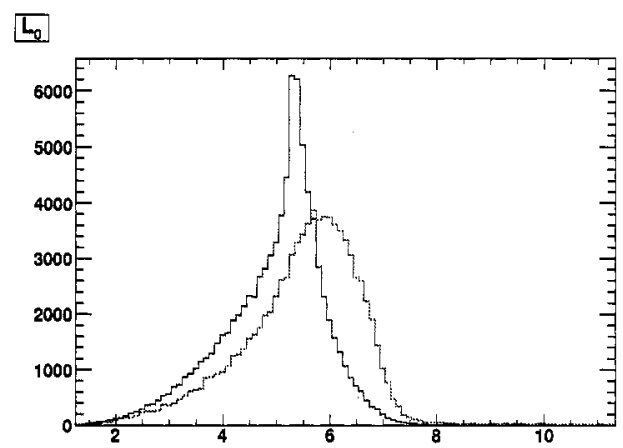

$\mathbf{R}_{2}$

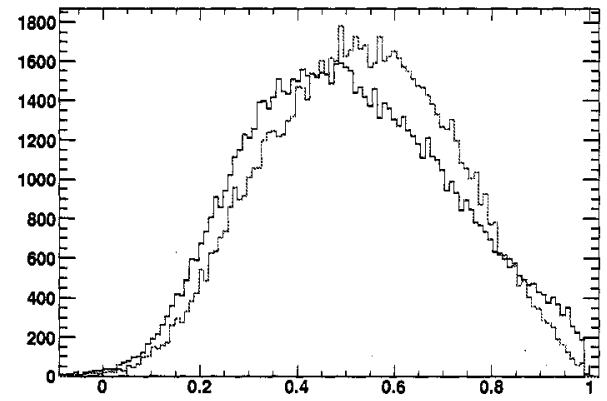

$F_{2}$

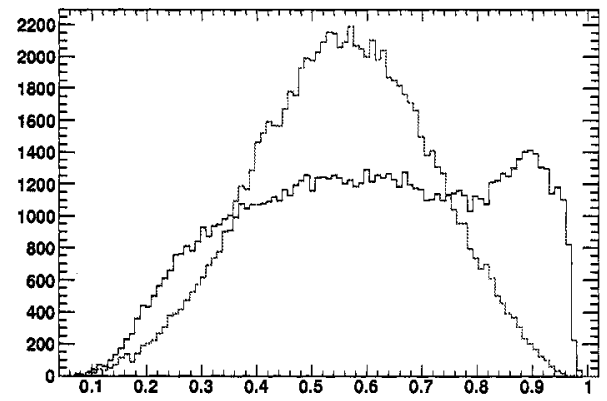

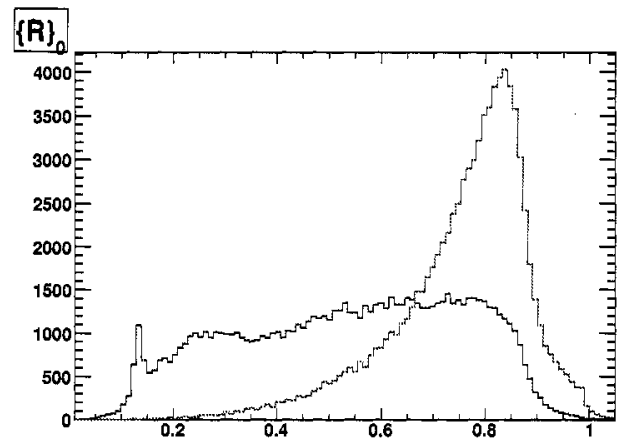

$\mathrm{T}_{3}$

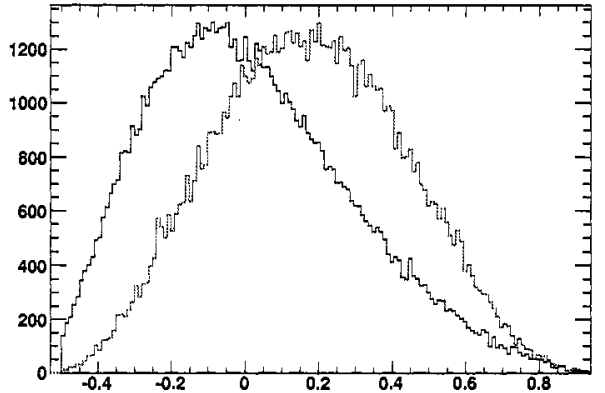

$\bar{F}_{3}$

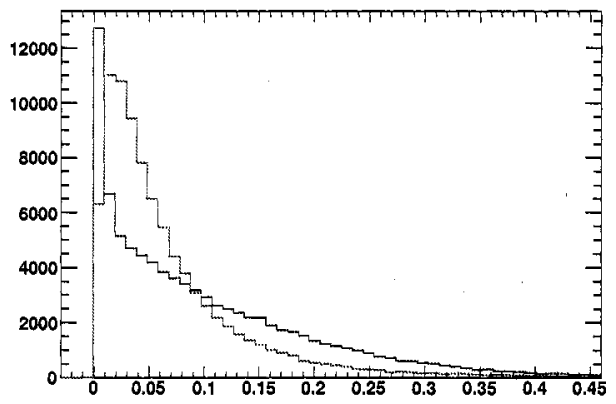

Figure 6.6: Distributions for the variables used in the Fisher discriminant for signal (Blue) and background (Red)

obtained for each $s^{\prime}$ bin, and Fig 6.8 shows the Fisher discriminant distributions for 
signal and background in each bin. The last step is to determine the optimal cut value for the selection. This value should be the one that minimizes the error in the cross-section measurement. We obtain the final result by subtracting the background and correcting for efficiency, so the error has two sources, one being the error from the background subtraction and the other from the efficiency correction. A tight Fisher cut will decrease the background, and therefore the background error, but it will decrease the efficiency and thus increase the efficiency error, and vice versa for a loose Fisher cut.

Call $N^{\text {Pass }}$ the total number of events (signal and background) passing the cut, $N_{B}^{\text {Pass }}$ the expected number of passing background events, and $\epsilon_{S}$ the signal efficiency. Then we get the original number of signal events by

$$
N_{S}=\frac{N^{\text {Pass }}-N_{B}^{\text {Pass }}}{\epsilon_{S}} \text {. }
$$

The error on this is

$$
\frac{\delta N_{S}}{N_{S}}=\frac{\delta \epsilon_{S} N_{S}^{\text {Pass }} \ominus \delta N_{B}^{\text {Pass }} \epsilon_{S}}{N_{S} \epsilon_{S}^{2}}=\frac{1}{\epsilon_{S}}\left(\delta \epsilon_{S} \oplus \delta \epsilon_{B} \frac{N_{B}}{N_{S}}\right)
$$

Where $\epsilon_{B}=N_{B}^{\text {Pass }} / N_{B}$ is the background efficiency and $N_{B}$ is the original number of background events. Since the signal efficiency is presumably high we take its error to be proportional to the inefficiency, $\delta \epsilon_{S}=\eta_{S}\left(1-\delta \epsilon_{S}\right)$, while the error on the background efficiency is taken proportional to its value: $\delta \epsilon_{B}=\eta_{B} \epsilon_{B}$. Thus we have

$$
\frac{\delta N_{S}}{N_{S}}=\sqrt{\eta_{S}^{2}+\eta_{B}^{2}\left(\frac{N_{B}}{N_{S}}\right)^{2}\left(\frac{\epsilon_{B}}{\epsilon_{S}}\right)^{2}} .
$$

The values of the parameters $\eta_{S}$ and $\eta_{B}$ are somewhat arbitrary, reflecting how well we think we know $\epsilon_{S}$ and $\epsilon_{B}$. In what follows we use the values $\eta_{S}=\eta_{B}=25 \%$.

Figs. 6.9 and 6.10 show the variations of the signal and background efficiencies versus the position of the Fisher cut and versus each other.

The calculated values of $\delta N_{S} / N_{S}$ for $\eta_{S}=\eta_{B}=25 \%$ are shown in Fig. 6.11. The shape of these curves is similar in each bin: the leftmost part is a plateau, 

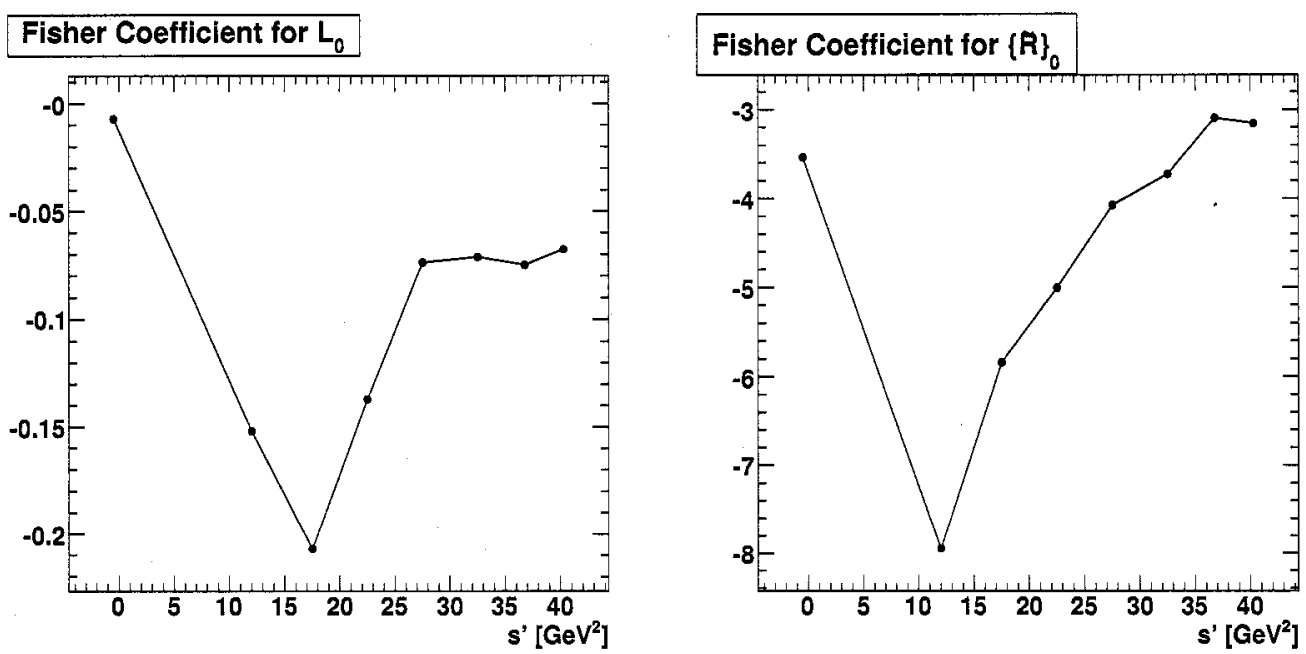

Fisher Coefficient for $\mathrm{R}_{2}$

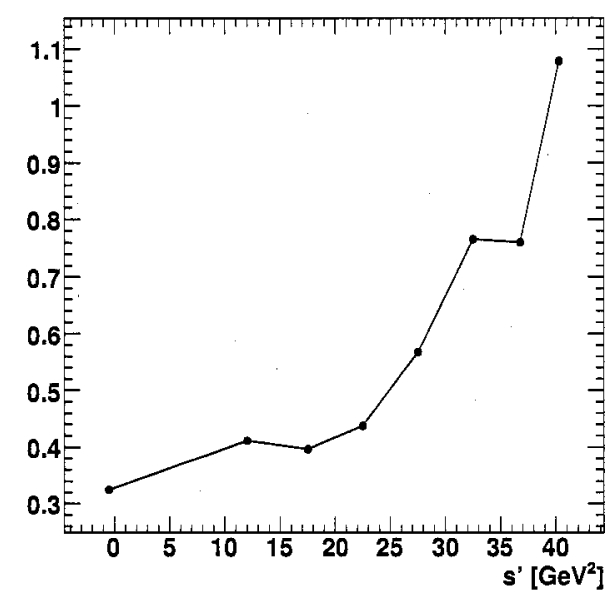

Fisher Coefficient for $\mathrm{T}_{2}$

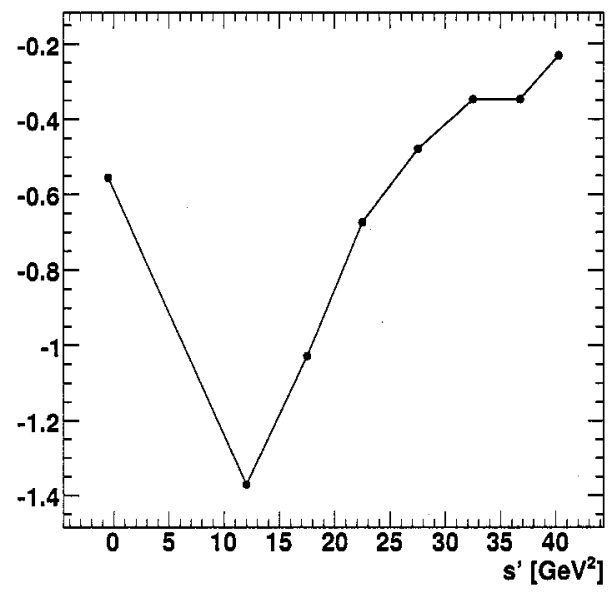

Fisher Coefficient for $F_{2}$

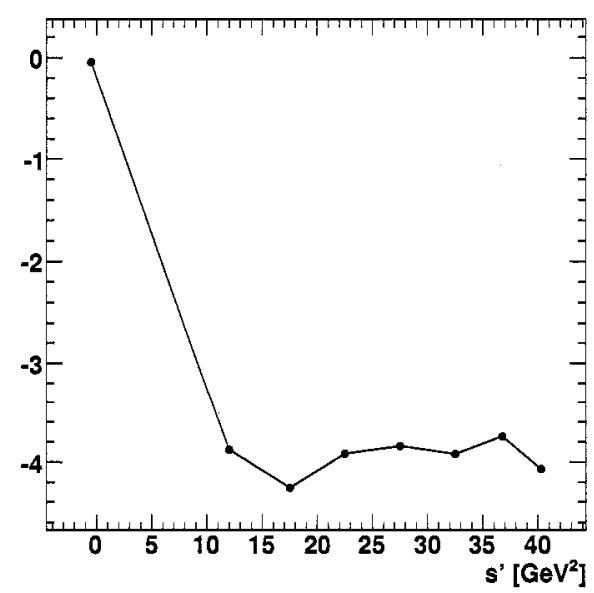

Fisher Coefficient for $\mathrm{F}_{3}$

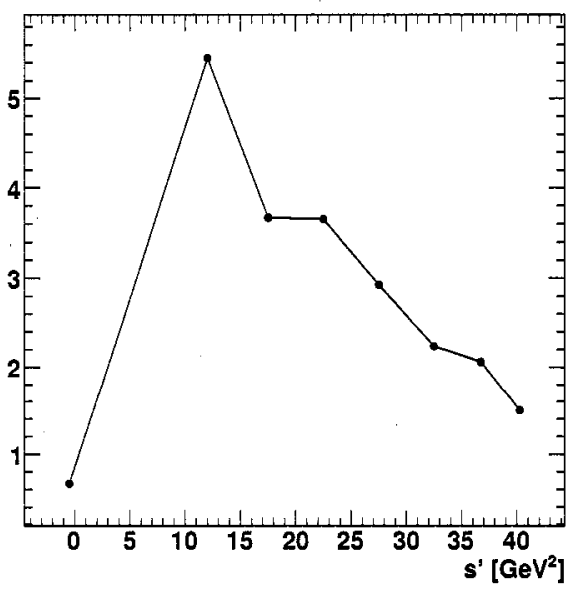

Figure 6.7: Values of the Fisher coefficients for versus $s^{\prime}$. 

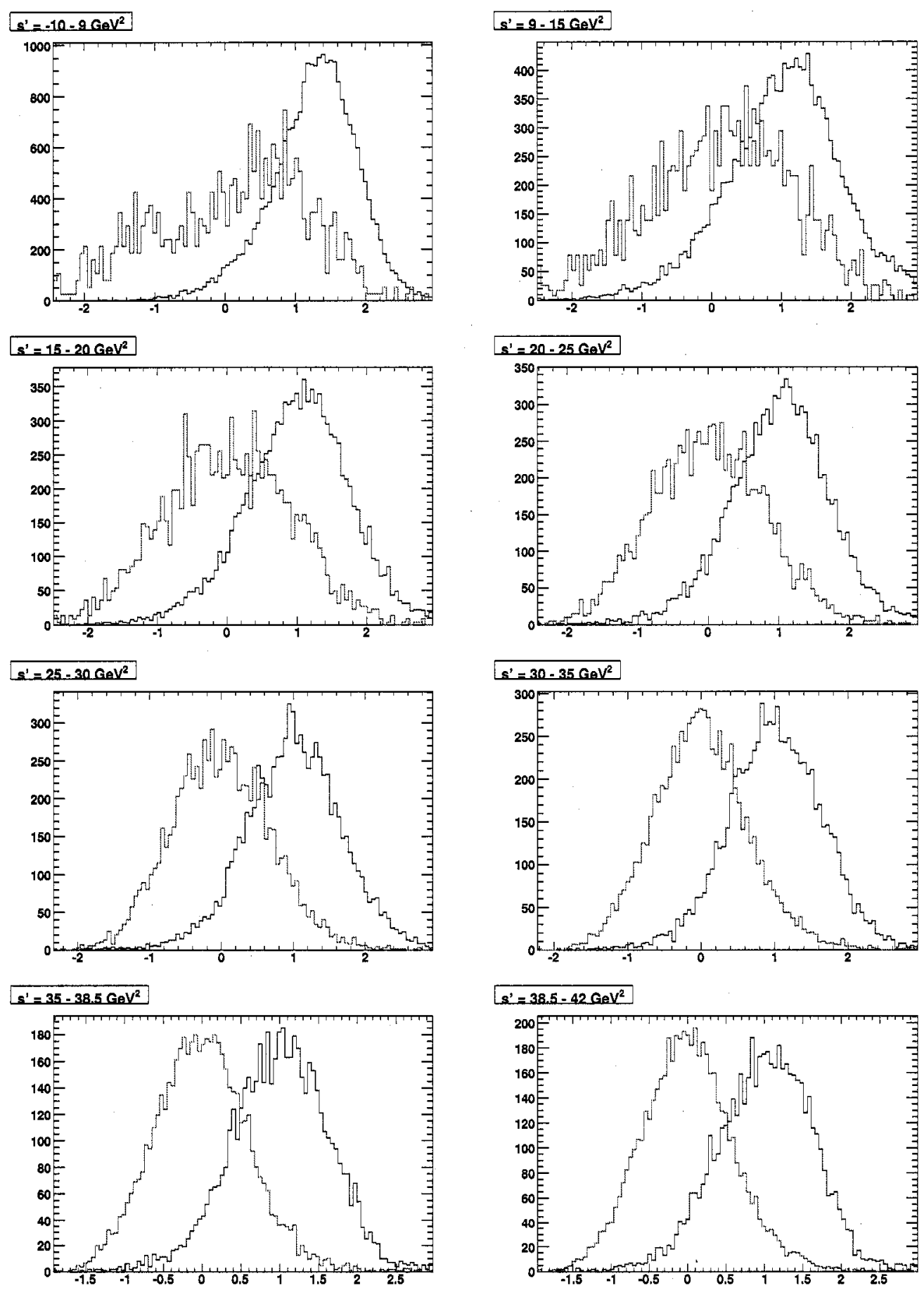

Figure 6.8: Distributions of the Fisher discriminant for signal (Blue) and background (Red) in bins 1 (to left) to 8 (bottom right) 

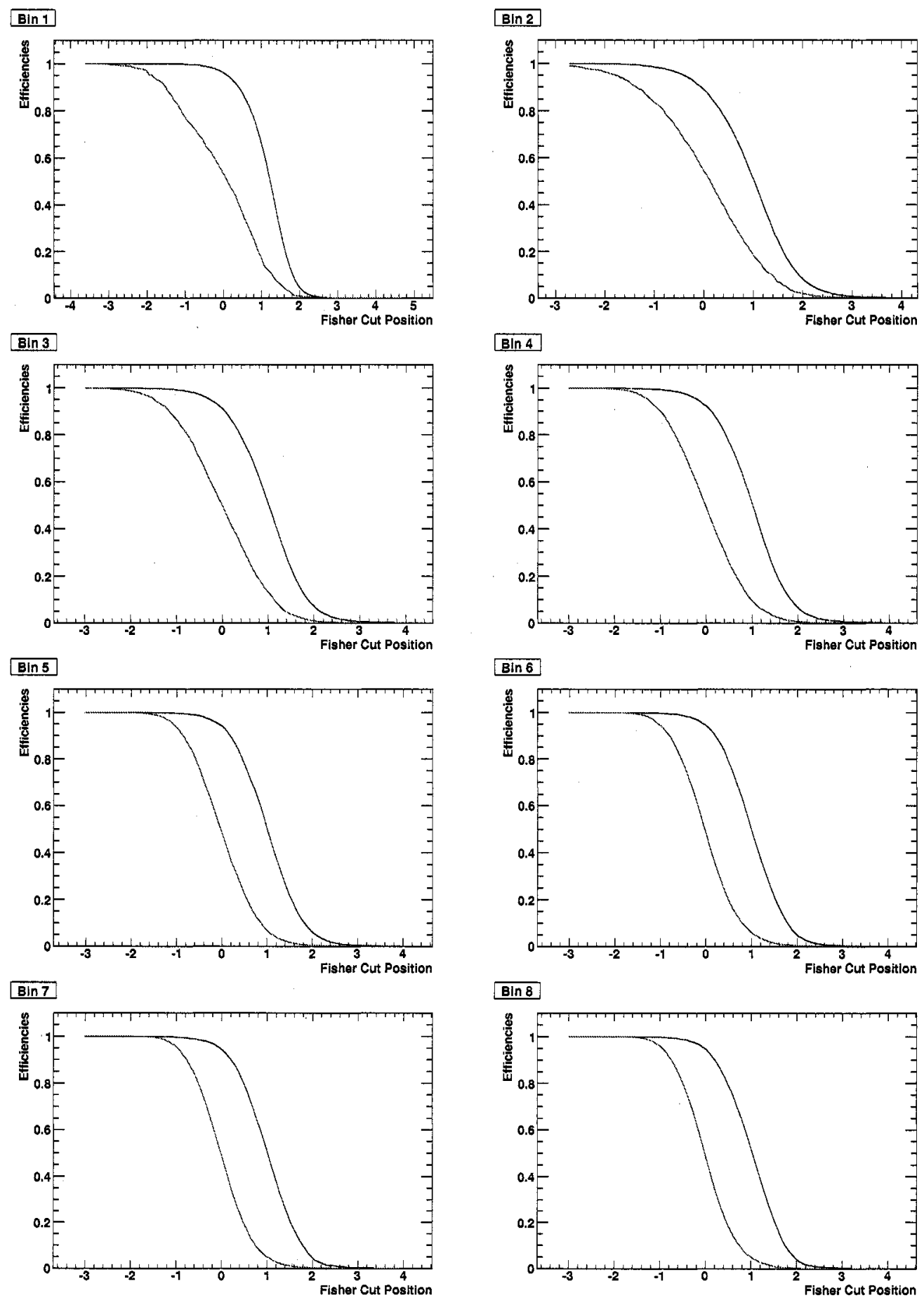

Figure 6.9: Signal (Blue) and background (Red) efficiencies versus Fisher cut position 

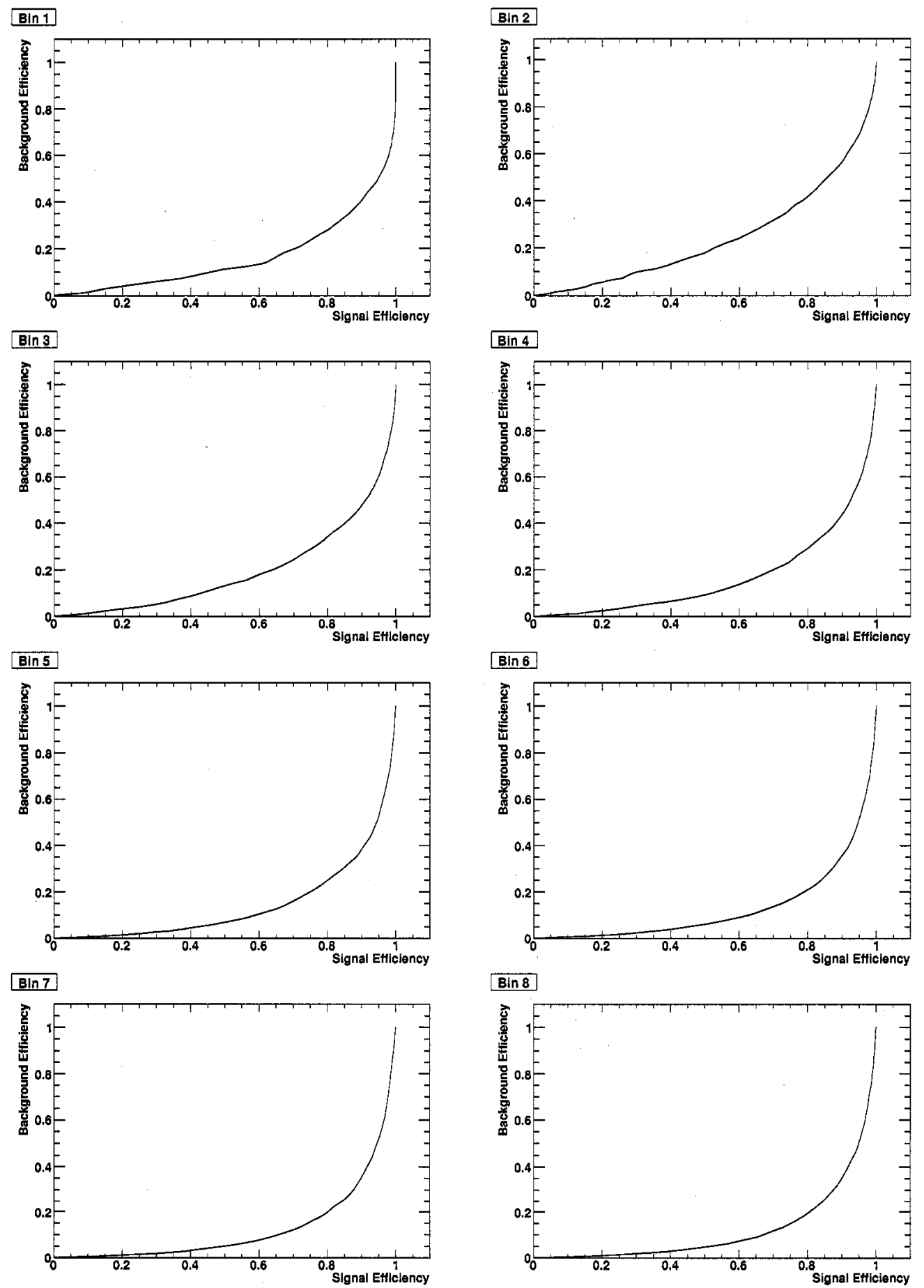

Figure 6.10: Background versus signal efficiency 

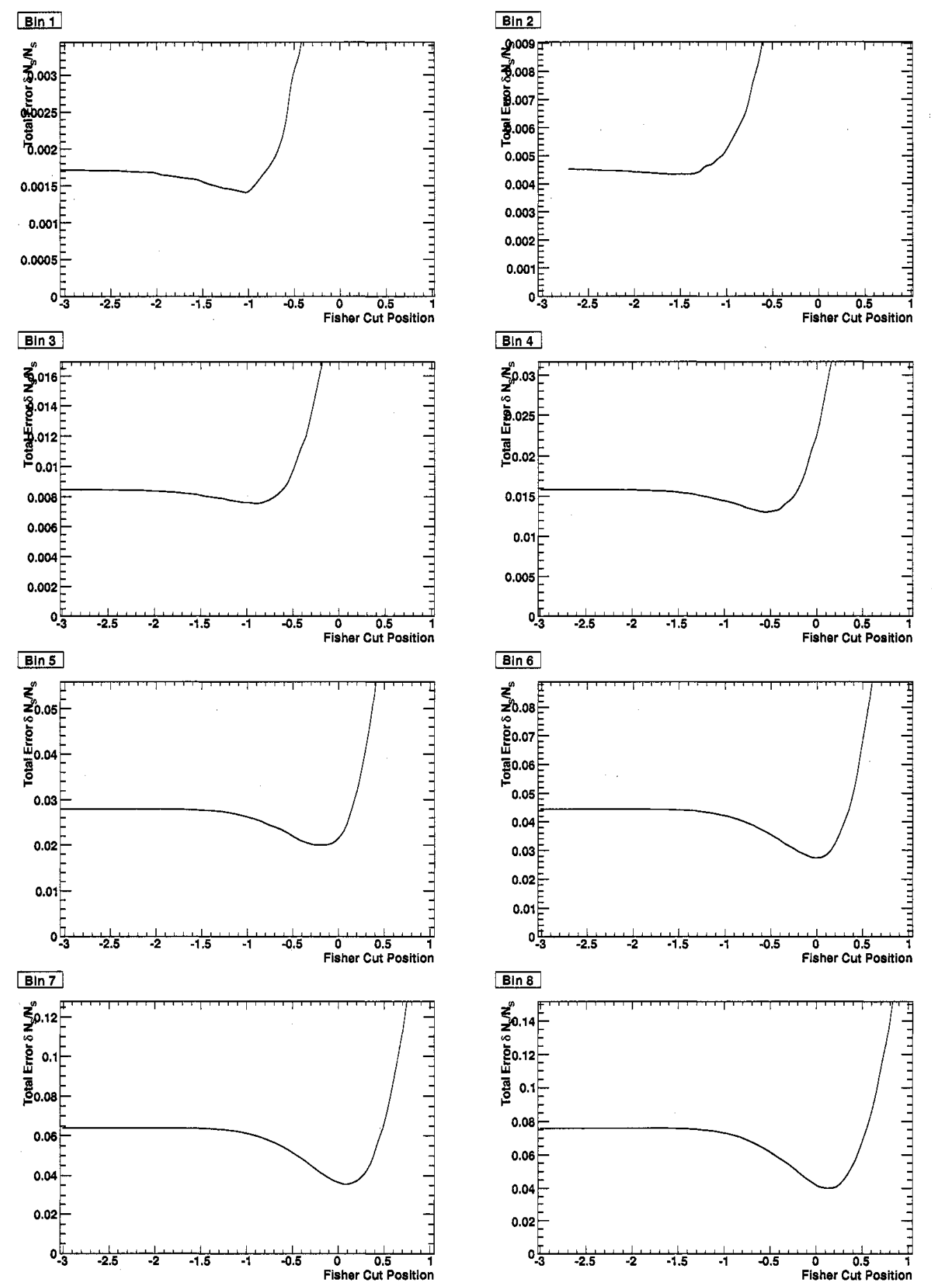

Figure 6.11: Total Error $\delta N_{S} / N_{S}$ versus Fisher cut position for $\eta_{S}=\eta_{B}=25 \%$ 
Table 6.4: Value of the Fisher cut position for each bin, and corresponding values of the signal and background efficiencies and associated error as described in the text

\begin{tabular}{|l|c|c|c|c|}
\hline Bin & Cut Value & $\epsilon_{S}(\%)$ & $\epsilon_{B}(\%)$ & $\delta N_{S} / N_{S}(\%)$ \\
\hline 0 & -1.022 & 99.8 & 77.8 & 0.1 \\
1 & -1.553 & 99.7 & 92.8 & 0.4 \\
2 & -0.905 & 99.0 & 83.6 & 0.8 \\
3 & -0.550 & 98.1 & 75.0 & 1.3 \\
4 & -0.207 & 96.3 & 60.4 & 2.0 \\
5 & 0.006 & 94.3 & 48.3 & 2.7 \\
6 & 0.078 & 93.0 & 44.0 & 3.6 \\
7 & 0.151 & 91.3 & 38.6 & 4.0 \\
\hline
\end{tabular}

corresponding to $\epsilon_{S} \approx \epsilon_{B} \approx 1$ for very loose Fisher cuts, followed by a sharp rise caused by rapidly dropping signal efficiencies. In the transition region a small dip is visible, and we take the lowest point to be the optimum cut value. Results are summarized in Table 6.4.

\subsection{Rejection Summary}

The efficiencies are shown in in Table 5.1 for signal modes, and Tables 5.2 to 5.4 for backgrounds. A summary of efficiencies for various signal MC modes is shown in Tables 6.5 and 6.6 .

For background modes the common denominator of the efficiencies is the original number of events in the sample (given in Table 4.2). For signal modes, the denominator is the number of events containing a photon in $\mathrm{MC}$ truth with $E_{\text {True }}^{*}>3.5 \mathrm{GeV}$ and $0.360<\theta_{\text {True }}^{*}<2.18$, both slightly wider than the cuts applied to the reco photon.

In order to better appreciate the effects on specific background modes, Table 6.8 shows the effects of the selection on the most significant modes listed in 6.1 , combining contributions from the $u d s, c \bar{c}, \tau^{+} \tau^{-}$samples.

The $s^{\prime}$ and $\theta_{\gamma}$ distributions with all selections applied is shown in Figs. 6.12 and 6.13 . 
Table 6.5: Summary of selection efficiencies broken down by signal MC mode. All ratios are given in percent.

\begin{tabular}{lcccccccccc}
\hline \hline Mode & nEvents & Fraction & Trg & BGF & $e^{+} e^{-} \gamma$ & QED & $\pi^{0}$ & Lat & Fisher & Total \\
\hline All Events & 89986 & 100 & 99.6 & 97.8 & 97.1 & 95.7 & 98 & 91.4 & 97.9 & 79.4 \\
\hline$\rho^{0} \rightarrow \pi^{+} \pi^{-}$ & 24714 & 27.1 & 99.2 & 99 & 98 & 92.8 & 99.4 & 91.6 & 99.6 & 81 \\
$\omega \rightarrow \pi^{+} \pi^{-} \pi^{0}$ & 1887 & 2.05 & 100 & 97.1 & 93.3 & 91.2 & 99.5 & 90.7 & 99.4 & 74.2 \\
$\omega \rightarrow \gamma \pi^{0}$ & 197 & 0.216 & 98.5 & 18.6 & 63.9 & 34.8 & 100 & 100 & 100 & 4.06 \\
$\omega \rightarrow$ Other & 52 & 0.055 & 100 & 98.1 & 96.1 & 81.6 & 100 & 92.5 & 100 & 71.2 \\
$\phi \rightarrow K^{+} K^{-}$ & 1880 & 2.08 & 96.7 & 96 & 99.7 & 85.1 & 99.5 & 91.2 & 99.7 & 71.2 \\
$\phi \rightarrow K_{S}^{0} K_{L}^{0}$ & 1283 & 1.38 & 97 & 67.9 & 95.9 & 91.9 & 99.5 & 90.5 & 99.7 & 52.1 \\
$\phi \rightarrow$ Other & 670 & 0.737 & 99.9 & 94.9 & 93.7 & 93.6 & 98.7 & 89.8 & 99 & 73 \\
$\psi \rightarrow e^{+} e^{-}$ & 392 & 0.428 & 99.5 & 14.9 & 8.62 & 20 & 100 & 100 & 0 & 0 \\
$\psi \rightarrow \mu^{+} \mu^{-}$ & 416 & 0.458 & 99.8 & 100 & 100 & 94 & 99.7 & 93.3 & 100 & 87.3 \\
$\psi \rightarrow$ Other & 5619 & 6.2 & 99.8 & 98.9 & 97.8 & 97.8 & 98.7 & 91.3 & 99 & 84.3 \\
\hline$\pi^{+} \pi^{-}$ & 229 & 0.259 & 99.6 & 99.6 & 98.7 & 93.3 & 100 & 90 & 99.5 & 81.7 \\
$K^{+} K^{-}$ & 966 & 1.07 & 98.8 & 99.3 & 99.2 & 91.9 & 99.2 & 90.4 & 99.5 & 79.7 \\
$K_{S}^{0} K_{L}^{0}$ & 109 & 0.114 & 96.3 & 76.2 & 96.2 & 89.6 & 100 & 97.1 & 100 & 61.5 \\
$p^{+} p^{-}$ & 243 & 0.257 & 100 & 99.2 & 99.2 & 88.7 & 99.1 & 93.8 & 99 & 80.2 \\
$n^{0} \bar{n}^{0}$ & 60 & 0.066 & 86.7 & 25 & 100 & 23.1 & 100 & 100 & 100 & 5 \\
$\Lambda \bar{\Lambda}$ & 51 & 0.054 & 100 & 92.2 & 100 & 87.2 & 100 & 90.2 & 100 & 72.5 \\
Other 2-body & 494 & 0.549 & 98.4 & 81.9 & 93.2 & 85.7 & 96.5 & 89.6 & 94.9 & 52.8 \\
\hline \hline
\end{tabular}


Table 6.6: Summary of selection efficiencies broken down by signal MC mode. All ratios are given in percent.

\begin{tabular}{|c|c|c|c|c|c|c|c|c|c|c|}
\hline Mode & nEvents & Fraction & Trg & BGF & $e^{+} e^{-} \gamma$ & QED & $\pi^{0}$ & Lat & Fisher & Total \\
\hline$\pi^{+} \pi^{-} \pi^{0}$ & 1423 & 1.55 & 100 & 97.6 & 94.6 & 93.2 & 98.6 & 92.7 & 97.9 & 77 \\
\hline$\pi^{+} \pi^{-} \eta\left(^{\prime}\right)$ & 129 & 0.14 & 100 & 98.4 & 96.1 & 92.6 & 100 & 91.2 & 98.1 & 78.3 \\
\hline$\pi^{0} \pi^{0} \eta\left(^{\prime \prime}\right)$ & 28 & 0.029 & 100 & 96.4 & 85.2 & 73.9 & 100 & 88.2 & 100 & 53.6 \\
\hline$K^{+} K^{-} \pi^{0}$ & 269 & 0.289 & 100 & 96.7 & 98.1 & 93.7 & 97.9 & 90.2 & 96.7 & 75.8 \\
\hline$K^{+} K^{-} \eta\left({ }^{\prime}\right)$ & 54 & 0.057 & 98.1 & 94.3 & 98 & 93.9 & 97.8 & 93.3 & 97.6 & 75.9 \\
\hline$K_{S}^{0} K^{ \pm} \pi^{\mp}$ & 740 & 0.801 & 99.7 & 98.6 & 98.5 & 96.5 & 99.7 & 90.9 & 99.2 & 84.1 \\
\hline$K_{L}^{0} K^{ \pm} \pi^{\mp}$ & 739 & 0.804 & 98.2 & 97.5 & 99.3 & 89.8 & 99.2 & 92.2 & 98.8 & 77.1 \\
\hline$p^{+} p^{-} \pi^{0}$ & 49 & 0.054 & 100 & 98 & 100 & 91.7 & 97.7 & 88.4 & 94.7 & 73.5 \\
\hline$p^{+} n^{0} \pi^{-}$ & 65 & 0.072 & 100 & 98.5 & 100 & 96.9 & 100 & 95.2 & 96.6 & 87.7 \\
\hline$p^{-} \tilde{n}^{0} \pi^{+}$ & 63 & 0.07 & 100 & 98.4 & 96.8 & 85 & 100 & 90.2 & 100 & 73 \\
\hline$n^{0} \bar{n}^{0} \pi^{0}$ & 16 & 0.017 & 87.5 & 57.1 & 100 & 62.5 & 100 & 100 & 100 & 31.2 \\
\hline Other 3-body & 784 & 0.881 & 99.1 & 94.7 & 95.5 & 92 & 97.4 & 93.2 & 96.1 & 71.9 \\
\hline$\pi^{+} \pi^{-} \pi^{+} \pi^{-}$ & 3702 & 4.07 & 99.9 & 99.8 & 99 & 99.1 & 99.5 & 91.2 & 99.2 & 88.1 \\
\hline$\pi^{+} \pi^{-} \pi^{0} \pi^{0}$ & 5954 & 6.44 & 100 & 96.7 & 92 & 94.9 & 98.9 & 91.1 & 98.8 & 75.2 \\
\hline$K^{+} K^{-} \pi^{+} \pi^{-}$ & 660 & 0.735 & 99.8 & 100 & 99.8 & 99.5 & 99.2 & 94.3 & 98.4 & 91.4 \\
\hline$K^{+} K^{-} \pi^{0} \pi^{0}$ & 110 & 0.117 & 100 & 100 & 100 & 96.4 & 97.2 & 88.3 & 97.8 & 80.9 \\
\hline$K_{S}^{0} K_{L}^{0} \pi^{+} \pi^{-}$ & 180 & 0.196 & 100 & 100 & 97.8 & 98.9 & 100 & 93.1 & 96.9 & 87.2 \\
\hline$K_{S}^{0} K_{L}^{0} \pi^{0} \pi^{0}$ & 15 & 0.019 & 100 & 100 & 93.3 & 85.7 & 100 & 100 & 91.7 & 73.3 \\
\hline$K_{S}^{0} K^{ \pm} \pi^{=} \pi^{0}$ & 374 & 0.416 & 100 & 98.7 & 98.4 & 97.5 & 98.3 & 90.2 & 97.8 & 82.1 \\
\hline$K_{L}^{0} K^{ \pm} \pi^{\mp} \pi^{0}$ & 385 & 0.425 & 99.5 & 97.7 & 96 & 92.2 & 98.8 & 92.4 & 98.3 & 77.1 \\
\hline Other 4-body & 1897 & 2.12 & 99.7 & 98.5 & 95.8 & 94.3 & 97.6 & 91.9 & 96.4 & 76.6 \\
\hline Generic 5-body & 6136 & 6.89 & 99.9 & 99.6 & 96.4 & 98.2 & 98.2 & 91.6 & 97.1 & 82.3 \\
\hline Generic 6-body & 6577 & 7.33 & 100 & 99.8 & 97.8 & 98.8 & 97.3 & 91.4 & 96.6 & 82.8 \\
\hline 7-body and more & 10452 & 11.9 & 100 & 100 & 98 & 99.5 & 95 & 91.1 & 95 & 80.2 \\
\hline
\end{tabular}


Table 6.7: Summary of passing cross-sections after QED vetos, after $m_{\gamma \gamma}$ veto, lateral moment cut and Fisher cut.

\begin{tabular}{|l|c|c|c|c|}
\hline Sample & $\sigma_{Q E D}(\mathrm{pb})$ & $\sigma_{h \rightarrow n \gamma \text { Veto }}(\mathrm{pb})$ & $\sigma_{\text {Lat }}(\mathrm{pb})$ & $\sigma_{\text {Fisher }}(\mathrm{pb})$ \\
\hline KKMC & 38.95 & 38.00 & 34.68 & 33.77 \\
$\gamma \gamma$ & 0.21 & 0.21 & 0.18 & 0.17 \\
$e^{+} e^{-} \gamma$ & 0.46 & 0.45 & 0.40 & 0.36 \\
$\mu^{+} \mu^{-} \gamma$ & 19.68 & 19.56 & 18.26 & 18.23 \\
$\tau^{+} \tau^{-} \gamma$ & 2.52 & 2.44 & 2.22 & 2.11 \\
$\tau^{+} \tau^{-}$ & 11.35 & 7.96 & 2.11 & 1.31 \\
$u d s$ & 15.90 & 9.83 & 2.97 & 1.51 \\
$c \bar{c}$ & 0.94 & 0.60 & 0.16 & 0.05 \\
$B^{0} \bar{B}^{0}$ & 0.01 & 0.01 & 0.01 & 0.00 \\
$B^{+} B^{-}$ & 0.02 & 0.02 & 0.01 & 0.01 \\
\hline
\end{tabular}

Table 6.8: Effects of the rejection cuts on selected background modes, combined across $u d s, c \bar{c}$ and $\tau^{+} \tau^{-}$samples

\begin{tabular}{lccccc}
\hline \hline Mode & $\sigma_{\text {Init }}(\mathrm{pb})$ & $\epsilon_{h \rightarrow \gamma \gamma \text { Veto }}$ & $\epsilon_{\text {Lat Veto }}$ & $\epsilon_{\text {Fisher }}$ & $\sigma_{\text {Final }}(\mathrm{pb})$ \\
\hline Unmerged $\pi^{0} \rightarrow \gamma \gamma$ & 6.19 & $36.6 \%$ & $68.5 \%$ & $52.8 \%$ & 0.82 \\
Unmerged $\eta \rightarrow \gamma \gamma$ & 1.91 & $28.5 \%$ & $87.4 \%$ & $49.4 \%$ & 0.24 \\
Unmerged $\eta^{\prime} \rightarrow \gamma \gamma$ & 0.03 & $34.8 \%$ & $86.2 \%$ & $47.2 \%$ & 0.00 \\
Merged $\pi^{0} \rightarrow \gamma \gamma$ & 18.82 & $68.5 \%$ & $14.2 \%$ & $61.1 \%$ & 1.12 \\
Merged $\eta \rightarrow \gamma \gamma$ & 0.03 & $46.0 \%$ & $90.0 \%$ & $26.7 \%$ & 0.00 \\
Other $\pi^{0}$ decay & 0.06 & $81.4 \%$ & $81.4 \%$ & $30.2 \%$ & 0.01 \\
Other $\omega$ decay & 0.22 & $55.2 \%$ & $88.2 \%$ & $35.1 \%$ & 0.04 \\
$\bar{n}^{0}$ & 0.54 & $63.4 \%$ & $3.1 \%$ & $37.0 \%$ & 0.00 \\
$K_{L}^{0}$ & 0.03 & $65.1 \%$ & $15.7 \%$ & $57.0 \%$ & 0.00 \\
\hline \hline
\end{tabular}




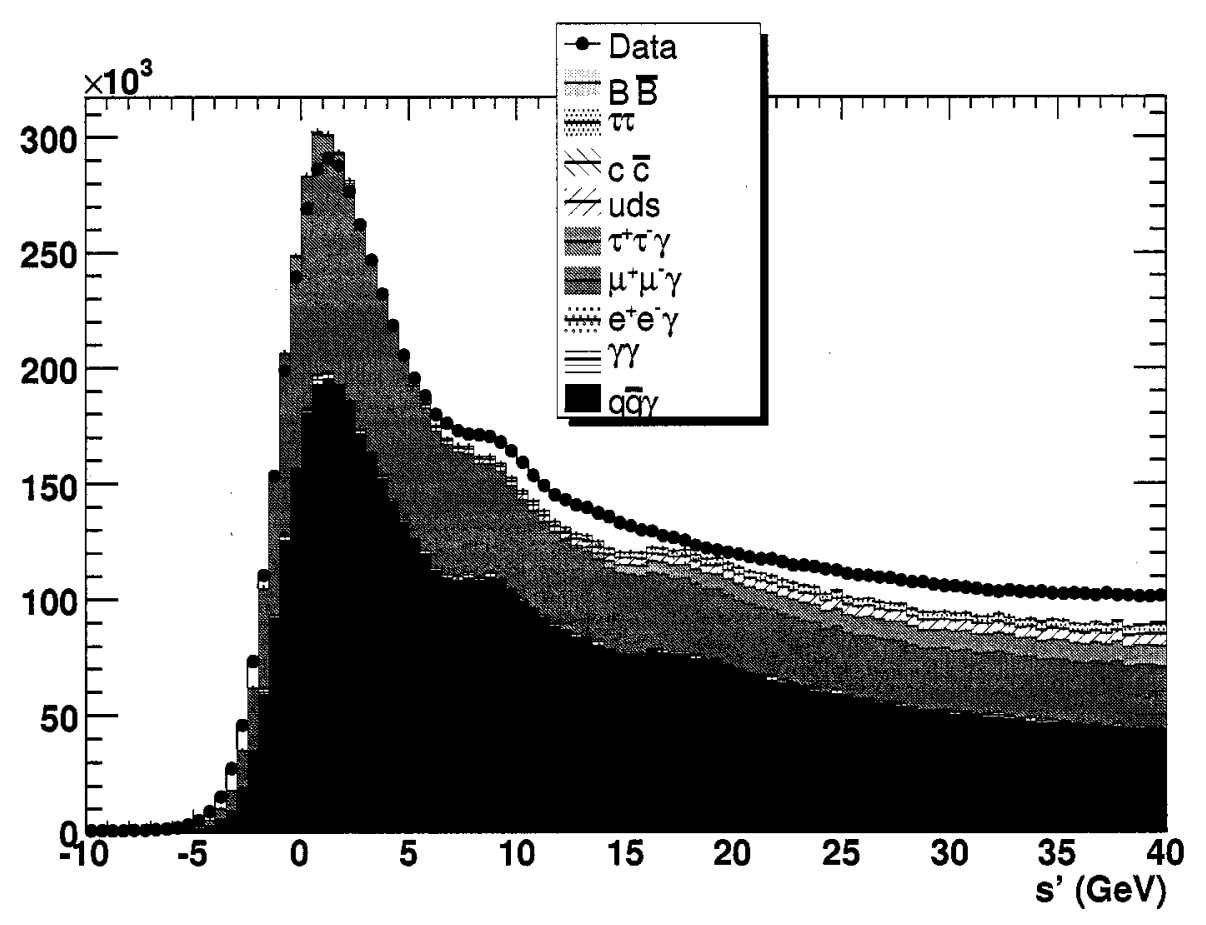

Figure 6.12: $s^{\prime}$ distribution for data (round dots) and stacked MC samples after the full selection. 


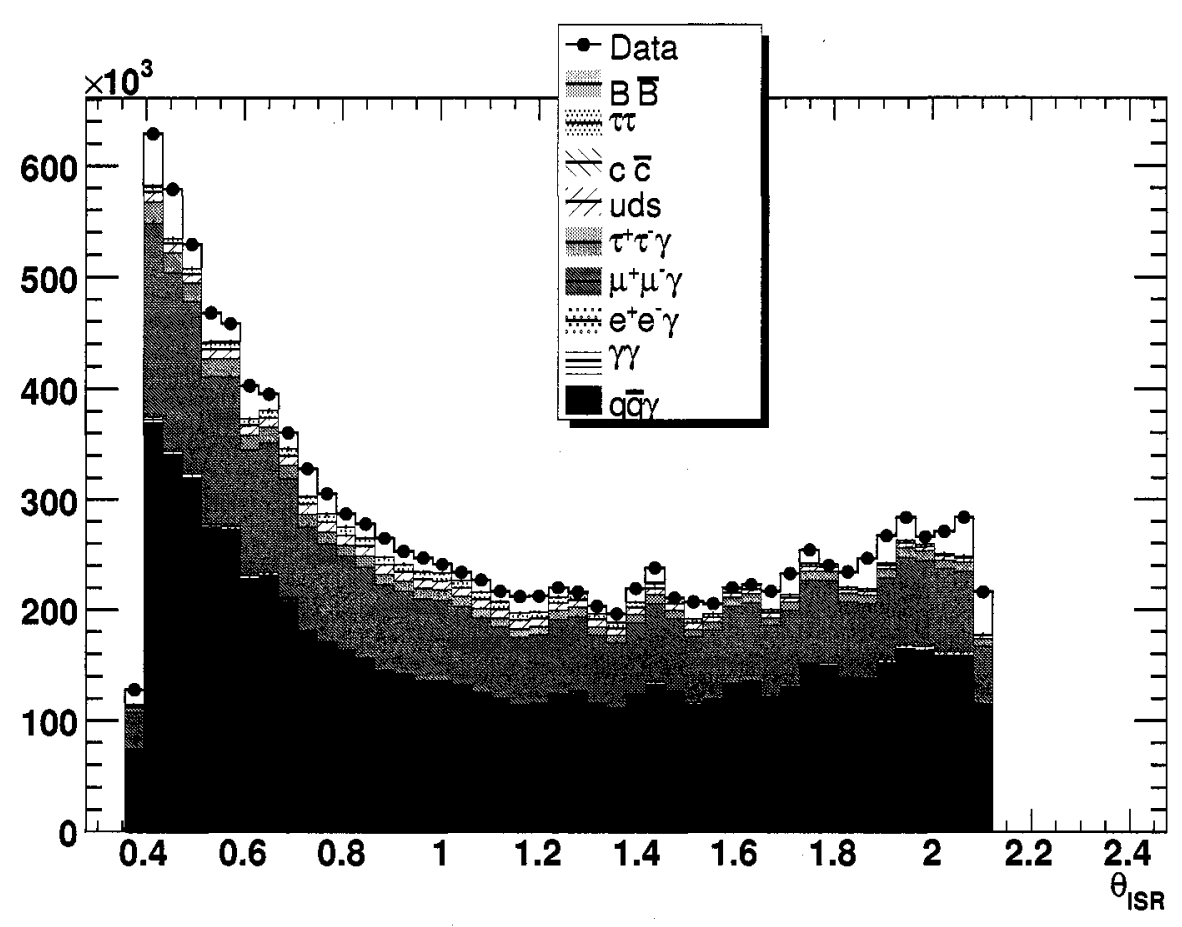

Figure 6.13: $\theta_{\gamma}$ distribution for data (round dots) and stacked MC samples after the full selection. 


\section{Chapter 7}

\section{Photon Calibration}

Although the simulation provides an adequate description of the photon parameters measured in the EMC, some deviations are seen: Fig. 7.1 shows the comparison between data and stacked MC samples (the relative normalization between data and MC is arbitrary). The MC does not reproduce the position of the low-s' peak, which is lower in data, as well as its width and the shape of the tails in the region $s^{\prime}<0$. In fact the shift of the peak corresponds to a shift of only about $1 \%$ in the photon energy scale, but the effect of this small difference magnified at low $s^{\prime}$, as described in Section 4.3.

The main reason for the difference is that the photon energy calibration is applied to the simulation and to real data used in this analysis in different ways [88]. For data the energy scale is set so that the peak of the $\pi^{0} \rightarrow \gamma \gamma$ mass distribution comes out at the PDG value. In simulation the energy scale is obtained from single-photon $\mathrm{MC}$ samples of predefined energies, and is set so that the peak of the reconstructed photon energy distribution coincides with the generated value. Due to the asymmetry of the energy resolution distributions, this calibration makes the $\pi^{0}$ mass come out too low by about $1 \%$.

Good data-MC agreement is important to ascertain that the efficiency of the photon selection is well described by MC. This concerns both the photon energy and polar angle. The polar angle cut in the forward direction is especially important since the ISR cross-section in the forward region is both high and rapidly varying, so that 


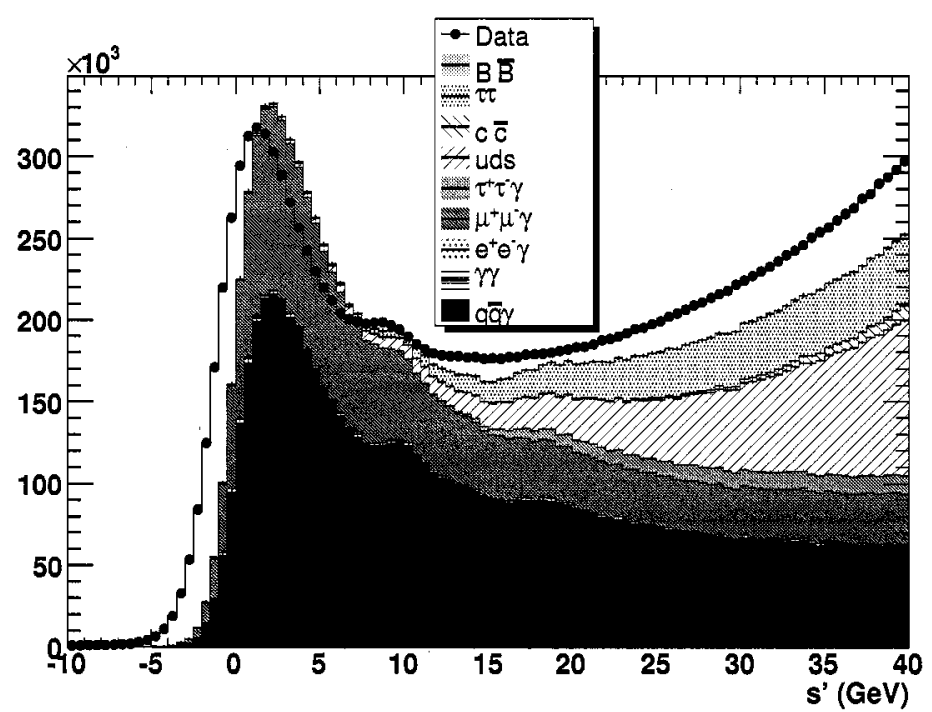

Figure 7.1: s' distribution with no energy correction, for data (points) and MC (stacked histograms

small variations in the cut position can have significant effects on acceptance.

To obtain satisfactory MC/Data agreement it is therefore necessary to introduce additional corrections to the $\mathrm{MC}$ for photon energy and $\theta$ distributions.

This can be done using photon samples for which expected values of the photon parameters can be obtained from the rest of the event. Resolution functions relating the measured photon parameters to their expected values can then be obtained. A correction for the $\mathrm{MC}$ values can then be determined by comparing the resolution parameters in data and simulation.

\subsection{Event Selection}

\subsubsection{Samples}

The photon samples to be used here must be cleanly identified and with a significant cross-section. The obvious candidates are $e^{+} e^{-} \rightarrow \mu^{+} \mu^{-} \gamma$ and $e^{+} e^{-} \rightarrow \gamma \gamma$ processes. The former seems more desirable for several reasons: 
- The $\mu^{+} \mu^{-} \gamma$ final state is more closely related to $q \bar{q} \gamma$.

- The recoil side is made up of charged tracks, so the recoil-side determination of the photon parameters is done in an "orthogonal" manner, without relying on the calorimeter.

- Unlike the case of $e^{+} e^{-} \rightarrow \gamma \gamma$, where the photons are monochromatic, a spectrum of photon energies is produced, with a distribution quite close to that of $e^{+} e^{-} \rightarrow q \bar{q} \gamma$. The $\theta$ distribution is also similar, although this is somewhat spoiled by the presence of FSR.

Statistics are more limited for $e^{+} e^{-} \rightarrow \mu^{+} \mu^{-} \gamma$, but sufficient for this study.

The emphasis here is to provide an extremely clean selection. The requirements of this study are similar to those of the photon detection efficiency study described in Chapter 8, and the selections are made to be as similar as possible.

The photon efficiency study also selects events with converted photons separately, so the selection is structured in groups:

- A general selection, mainly requirements on the recoil-side muons

- Additional cuts for 2-prong events

- Additional cuts for this particular study.

\subsubsection{General Selection}

The common selection applied for both this study and the photon efficiency study of Chapter 8 is as follows:

- Trigger: pass L1, L3 and the BGF Physics lines described in section 3.8.6

- Muons: Require 2 "good muon" tracks, which are required to

- Pass the GoodTracksLoose selection described in Section E.1

- Be identified as muons 
- Not be identified as electrons

- Have an associated EMC cluster

- Missing Momentum: The term "missing momentum" refers to the 4-momentum $p_{\text {Mis }}=p_{\text {Collision }}-p_{\text {Muons }}$. It is required to verify

$$
\begin{aligned}
& -E_{M i s}^{*}>2.8 \mathrm{GeV} \\
& -0.310<\theta_{M i s}<2.430 \\
& -m_{M i s}^{2}<4 \mathrm{GeV}
\end{aligned}
$$

where $E_{M i s}^{*}, \theta_{M i s}$ and $m_{M i s}$ are respectively the CM energy, polar angle in the laboratory frame and invariant mass of $p_{M i s}$. The $E^{*}$ and $\theta$ selections are slightly looser than those applied to the ISR photon in Section 5.2.

The distributions of the number of good muons and missing mass are shown in Fig. 7.2.
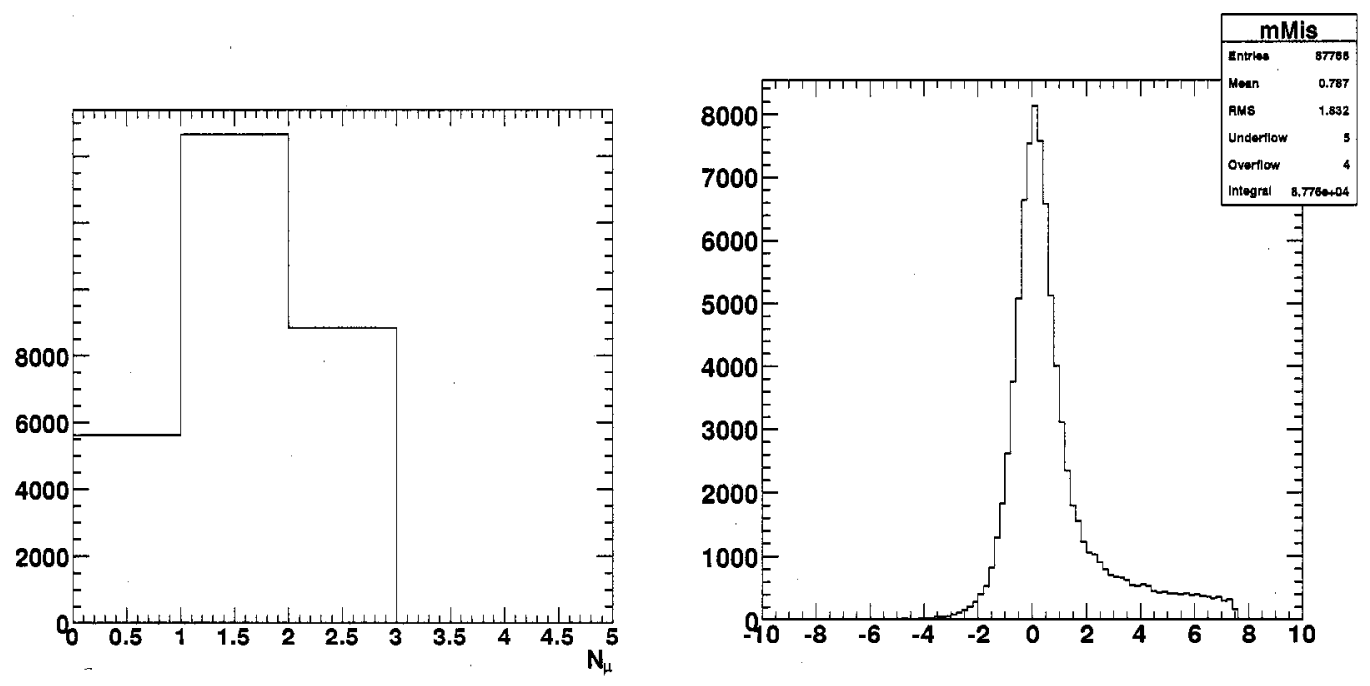

Figure 7.2: Number of good muon candidate (left) and missing mass (right) in $\mu^{+} \mu^{-} \gamma$ MC. 


\subsubsection{Two-prong cuts}

In addition to the selection described in the previous section, the study of this section and Section 8.2.2 requires the following:

- Exactly 2 tracks with $\mathrm{DCH}$ hits (the $\mathrm{DCH}$ hit requirement is to reject background SVT tracks, see Section 5.3). These must coincide with the muons.

- No EMC clusters with energy greater than $100 \mathrm{MeV}$ except in a cone of opening angle $250 \mathrm{mrad}$ centered around the missing momentum direction. This reduces cross-feed from events with multiple radiative photons.

- The angle between the missing momentum and both the tracks to be greater than $250 \mathrm{mrad}$. This is an "isolation cut" designed to prevent the energy deposition of the track in the EMC from interfering with the photon energy measurement. This is a particularly important issue for $\mu^{+} \mu^{-} \gamma$ events since a significant fraction of events involve FSR, in which the radiative photon is within a small angle of one of the tracks.

The distributions for $\mu^{+} \mu^{-} \gamma \mathrm{MC}$ are shown in Fig. 7.3.

\subsubsection{Photon cuts}

For this study, we also demand the presence of a photon within a specified tolerance of the expected values: we require that a photon be present with:

- $\left|\theta_{\gamma}-\theta_{M i s}\right|<0.1$

- $\left|\phi_{\gamma}-\phi_{M i s}\right|<0.05$.

As described in Section 7.2.1, the missing momentum resolution can be improved by a fitting procedure. Fig. 7.4 shows the distributions for the "raw" and fitted missing momentum quantities.

For this study we require the fit $\chi^{2}$ to be less than 10 . This is a fairly loose cut designed to reject pathological fits. 

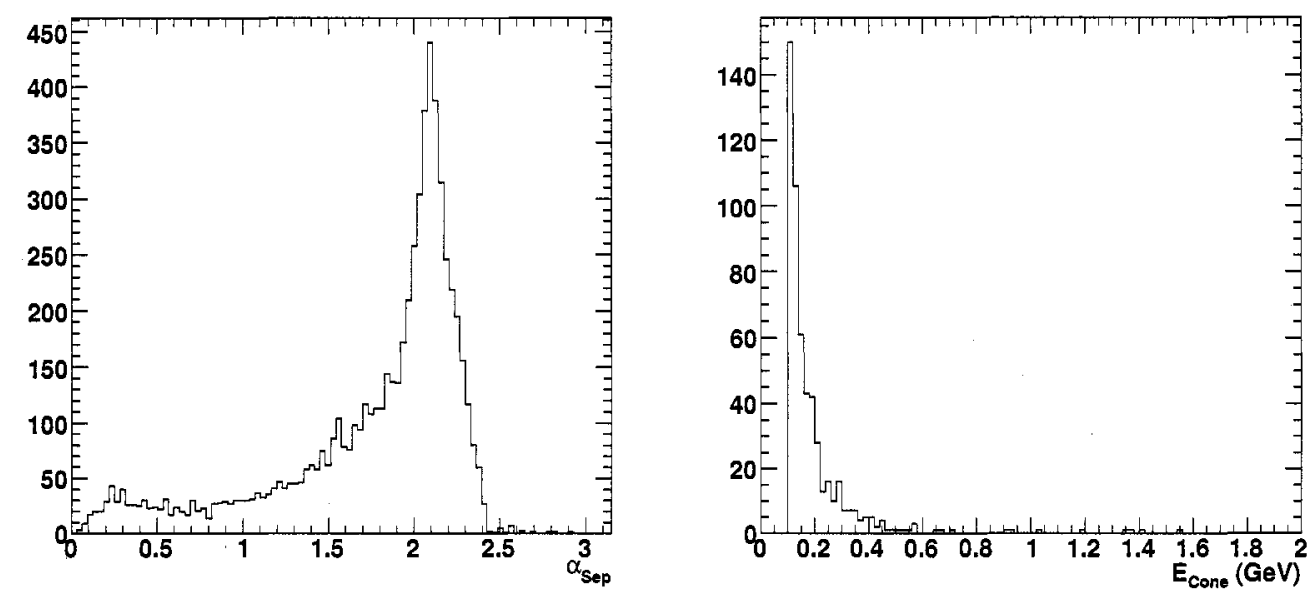

Figure 7.3: Angular separation between the missing momentum and the closest muon track (left) and energy outside a cone of $250 \mathrm{mrad}$ opening angle around the missing momentum (right).
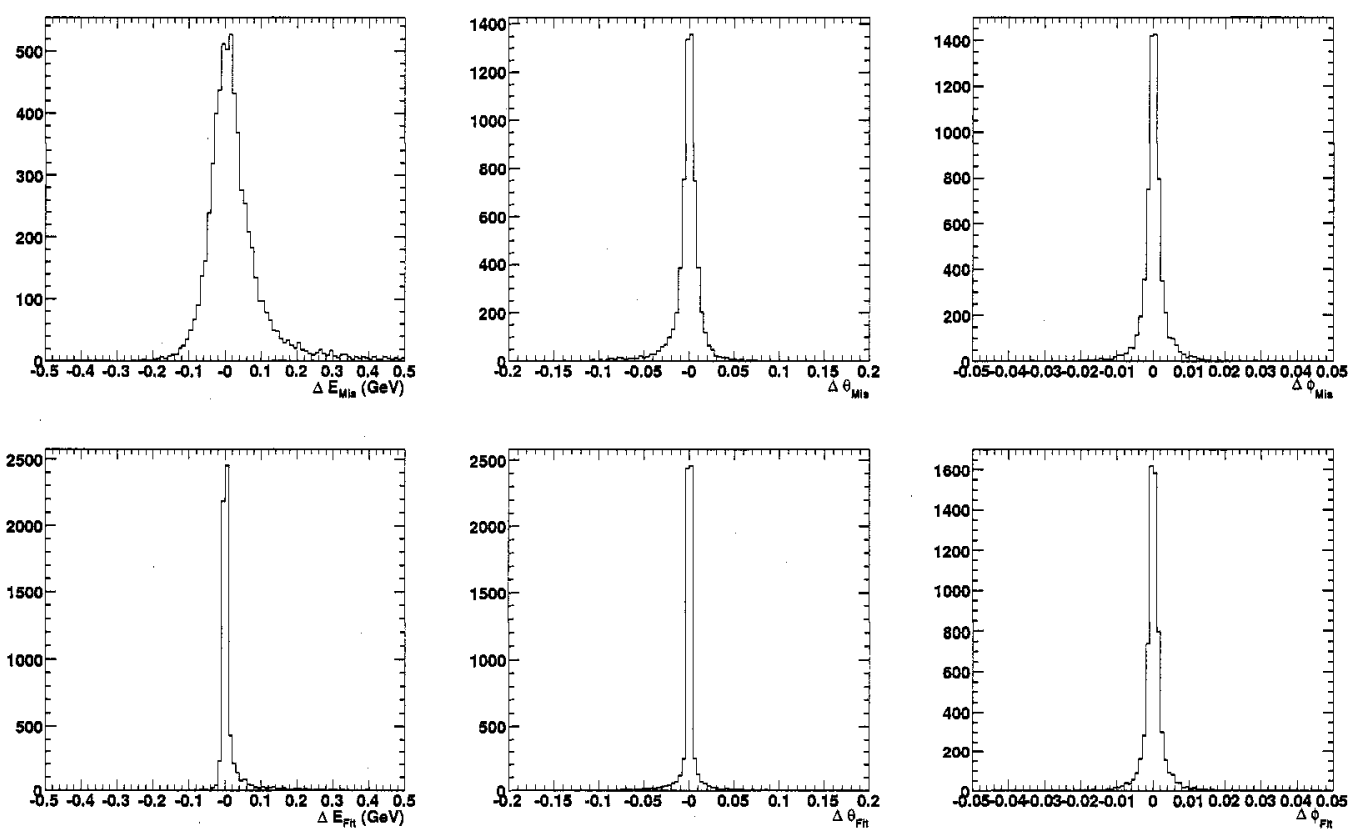

Figure 7.4: $E_{\gamma}^{*}-E_{M i s}^{*}$ (left), $\theta_{\gamma}-\theta_{M i s}$ (center) and $\phi_{\gamma}-\phi_{M i s}$ (right) for Raw (top row) and Fitted (bottom row) missing momenta. 
Table 7.1: Efficiency of the selection of Section 7.1 on $\mu^{+} \mu^{-} \gamma$ MC.

\begin{tabular}{lc}
\hline \hline & $\mu^{+} \mu^{-} \gamma(\%)$ \\
\hline L1 & $99.92 \pm 0.01$ \\
L3 Physics & $98.02 \pm 0.07$ \\
BGF Wide & $97.86 \pm 0.07$ \\
2 Good Muons & $28.59 \pm 0.23$ \\
$E_{\text {Mis }}$ & $18.19 \pm 0.19$ \\
$\theta_{\text {Mis }}$ & $18.17 \pm 0.19$ \\
$M_{\text {Mis }}$ & $18.17 \pm 0.19$ \\
2 DCH Tracks & $18.04=0.19$ \\
EMC Veto & $15.94 \pm 0.18$ \\
Track/Cluster sep. & $14.85 \pm 0.18$ \\
ISR Photon & $13.60 \pm 0.17$ \\
Tag 2 Tracks & $13.60 \pm 0.17$ \\
Tag Missing P4 & $13.55 \pm 0.17$ \\
Mini 2 Muons & $13.55 \pm 0.17$ \\
Events in 250/fb & $746319.7 \pm 10146.7$ \\
\hline \hline
\end{tabular}

The efficiency of the selection on $\mu^{+} \mu^{-} \gamma \mathrm{MC}$ is shown in Table 7.1. The efficiency denominator corresponds to events containing a photon in $\mathrm{MC}$ truth with $E^{*}>$ $3.8 \mathrm{GeV}$ and $0.46<\theta<2.09$.

\subsubsection{Backgrounds}

The efficiency of the selection on the various simulation samples used in the ISR study is shown in Tables 7.2 and 7.3. The only measurable background comes from the $q \bar{q} \gamma$ sample, presumably in the $\pi^{+} \pi^{-} \gamma$ and $J / \psi\left(\mu^{+} \mu^{-}\right) \gamma$ channels, which makes up approximately $1.6 \%$ of the final sample. However these events have kinematic properties similar to $\mu^{+} \mu^{-} \gamma$, with an ISR photon at the same energy and position as would be expected in the $\mu^{+} \mu^{-} \gamma$ case (the mass difference between pions and muons is negligible). No significant backgrounds are therefore expected in the selected sample. 
Table 7.2: Efficiency for the selection of Section 7.1 on background MC samples.

\begin{tabular}{lcccc}
\hline \hline & $q \bar{q} \gamma(\%)$ & $e^{+} e^{-} \gamma(\%)$ & $\tau^{+} \tau^{-} \gamma(\%)$ & $\gamma \gamma(\%)$ \\
\hline Precuts & $(8.35 \pm 0.91) \times 10^{-4}$ & $\mathrm{n} / \mathrm{a}$ & $(7.86 \pm 3.21) \times 10^{-6}$ & $\mathrm{n} / \mathrm{a}$ \\
2 Good Muons & $(7.85 \pm 0.88) \times 10^{-4}$ & $\mathrm{n} / \mathrm{a}$ & $(7.86 \pm 3.21) \times 10^{-6}$ & $\mathrm{n} / \mathrm{a}$ \\
$E_{M i s}$ & $(5.86 \pm 0.76) \times 10^{-4}$ & $\mathrm{n} / \mathrm{a}$ & $(7.86 \pm 3.21) \times 10^{-6}$ & $\mathrm{n} / \mathrm{a}$ \\
$\theta_{M i s}$ & $(5.86 \pm 0.76) \times 10^{-4}$ & $\mathrm{n} / \mathrm{a}$ & $(6.55 \pm 2.93) \times 10^{-6}$ & $\mathrm{n} / \mathrm{a}$ \\
$M_{M i s}$ & $(5.86 \pm 0.76) \times 10^{-4}$ & $\mathrm{n} / \mathrm{a}$ & $(1.31 \pm 1.31) \times 10^{-6}$ & $\mathrm{n} / \mathrm{a}$ \\
2 DCH Tracks & $(5.86 \pm 0.76) \times 10^{-4}$ & $\mathrm{n} / \mathrm{a}$ & $(1.31 \pm 1.31) \times 10^{-6}$ & $\mathrm{n} / \mathrm{a}$ \\
EMC Veto & $(5.37 \pm 0.73) \times 10^{-4}$ & $\mathrm{n} / \mathrm{a}$ & $(1.31 \pm 1.31) \times 10^{-6}$ & $\mathrm{n} / \mathrm{a}$ \\
Track/Cluster sep. & $(5.27 \pm 0.72) \times 10^{-4}$ & $\mathrm{n} / \mathrm{a}$ & $(1.31 \pm 1.31) \times 10^{-6}$ & $\mathrm{n} / \mathrm{a}$ \\
ISR Photon & $(4.87 \pm 0.70) \times 10^{-4}$ & $\mathrm{n} / \mathrm{a}$ & $0.00 \pm 0.00$ & $\mathrm{n} / \mathrm{a}$ \\
Events in 250/fb & $11718.5 \pm 1674.1$ & $0.0 \pm 177.7$ & $0.0 \pm 4.2$ & $0.0 \pm 478.8$ \\
\hline \hline
\end{tabular}

Table 7.3: Efficiency for the selection of Section 7.1 on background MC samples.

\begin{tabular}{lccccc}
\hline \hline & $u d s(\%)$ & $c \bar{c}(\%)$ & $\tau^{+} \tau^{-}(\%)$ & $B^{0} \bar{B}^{0}(\%)$ & $B^{+} B^{-}(\%)$ \\
\hline Precuts & $(3.24 \pm 1.87) \times 10^{-9}$ & $(1.56 \pm 0.59) \times 10^{-8}$ & $(7.43 \pm 1.26) \times 10^{-8}$ & $\mathrm{n} / \mathrm{a}$ & $\mathrm{n} / \mathrm{a}$ \\
2 Good Muons & $(3.24 \pm 1.87) \times 10^{-9}$ & $(1.56 \pm 0.59) \times 10^{-8}$ & $(7.22 \pm 1.24) \times 10^{-8}$ & $\mathrm{n} / \mathrm{a}$ & $\mathrm{n} / \mathrm{a}$ \\
$E_{\text {Mis }}$ & $(3.24 \pm 1.87) \times 10^{-9}$ & $(1.56 \pm 0.59) \times 10^{-8}$ & $(7.00 \pm 1.22) \times 10^{-8}$ & $\mathrm{n} / \mathrm{a}$ & $\mathrm{n} / \mathrm{a}$ \\
$\theta_{M i s}$ & $(3.24 \pm 1.87) \times 10^{-9}$ & $(1.12 \pm 0.50) \times 10^{-8}$ & $(7.00 \pm 1.22) \times 10^{-8}$ & $\mathrm{n} / \mathrm{a}$ & $\mathrm{n} / \mathrm{a}$ \\
$M_{M i s}$ & $(1.08 \pm 1.08) \times 10^{-9}$ & $(4.46 \pm 3.16) \times 10^{-9}$ & $(1.27 \pm 0.52) \times 10^{-8}$ & $\mathrm{n} / \mathrm{a}$ & $\mathrm{n} / \mathrm{a}$ \\
2 DCH Tracks & $(1.08 \pm 1.08) \times 10^{-9}$ & $(4.46 \pm 3.16) \times 10^{-9}$ & $(1.27 \pm 0.52) \times 10^{-8}$ & $\mathrm{n} / \mathrm{a}$ & $\mathrm{n} / \mathrm{a}$ \\
EMC Veto & $0.00 \pm 0.00$ & $(4.46 \pm 3.16) \times 10^{-9}$ & $(4.24 \pm 3.00) \times 10^{-9}$ & $\mathrm{n} / \mathrm{a}$ & $\mathrm{n} / \mathrm{a}$ \\
Track/Cluster sep. & $0.00 \pm 0.00$ & $(4.46 \pm 3.16) \times 10^{-9}$ & $(4.24 \pm 3.00) \times 10^{-9}$ & $\mathrm{n} / \mathrm{a}$ & $\mathrm{n} / \mathrm{a}$ \\
ISR Photon & $0.00 \pm 0.00$ & $(2.23 \pm 2.23) \times 10^{-9}$ & $(2.12 \pm 2.12) \times 10^{-9}$ & $\mathrm{n} / \mathrm{a}$ & $\mathrm{n} / \mathrm{a}$ \\
Events in 250/fb & $0.0 \pm 0.6$ & $0.7 \pm 0.7$ & $0.5 \pm 0.5$ & $0.0 \pm 0.2$ & $0.0 \pm 0.2$ \\
\hline \hline
\end{tabular}




\subsubsection{Data-MC Comparison}

Finally, the selection can be validated by comparing data and MC distributions. Since cross-feed from other modes is negligible, as shown in Section 7.1.5, we compare data with $\mu^{+} \mu^{-} \gamma$ signal MC. Distributions for relevant quantities are shown in Figs. 7.6 and 7.7.

The only significant discrepancy is in the $E_{F i t}^{*}$ distributions in Fig. 7.6, where data shows a peak near $E_{F i t}^{*}=4.8 \mathrm{GeV}$. Since $\sqrt{s^{\prime}}=m_{\psi}$ corresponds to $E_{\gamma}^{*}=4.83 \mathrm{GeV}$ by Eq. 4.1, this is mostly due to $\psi \rightarrow \mu^{+} \mu^{-}$, which is indistinguishable from "QED" $\mu^{+} \mu^{-} \gamma$ events. In the close-up view of Fig. 7.5, the $\psi$ and $\psi^{\prime}$ peaks can be identified at their expected positions pf $4.837 \mathrm{GeV}$ and $4.648 \mathrm{GeV}$ respectively. The secondary peak below the $\psi$ is at the $E_{F i t}^{*}=4.815 \mathrm{GeV}$ position expected for $\psi$ production in off-peak data, where the lower beam energy leads to a slightly decreased radiative photon energy. The discrepancy in the $E_{\gamma}^{*}$ in Fig. 7.6 corresponds to the photon

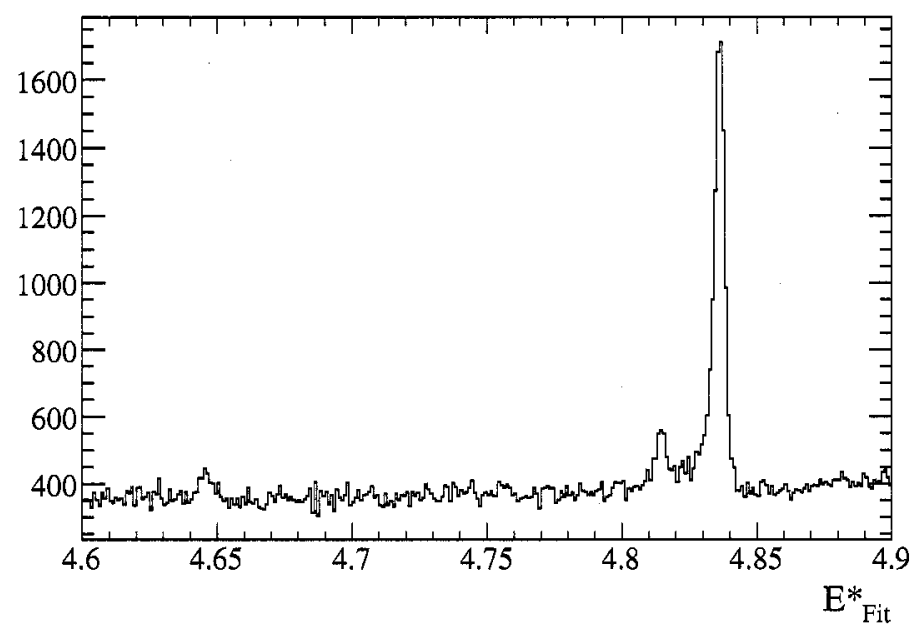

Figure 7.5: Distribution of $E_{F i t}^{*}$ in data, showing from right to left the $\psi, \psi$ in offresonance data and $\psi^{\prime}$ resonances.

resolution effects which this study is designed to correct.

The passing cross-section in data, based on its integrated luminosity value, is $5.877 \pm 0.071 \mathrm{pb}$ (of which about $0.55 \%$ are $\psi$ events). In $\mathrm{MC}$, based on the production 
cross-section reported by the generator we have a produced cross-section of $5.880 \mathrm{pb}$, in good agreement with data.
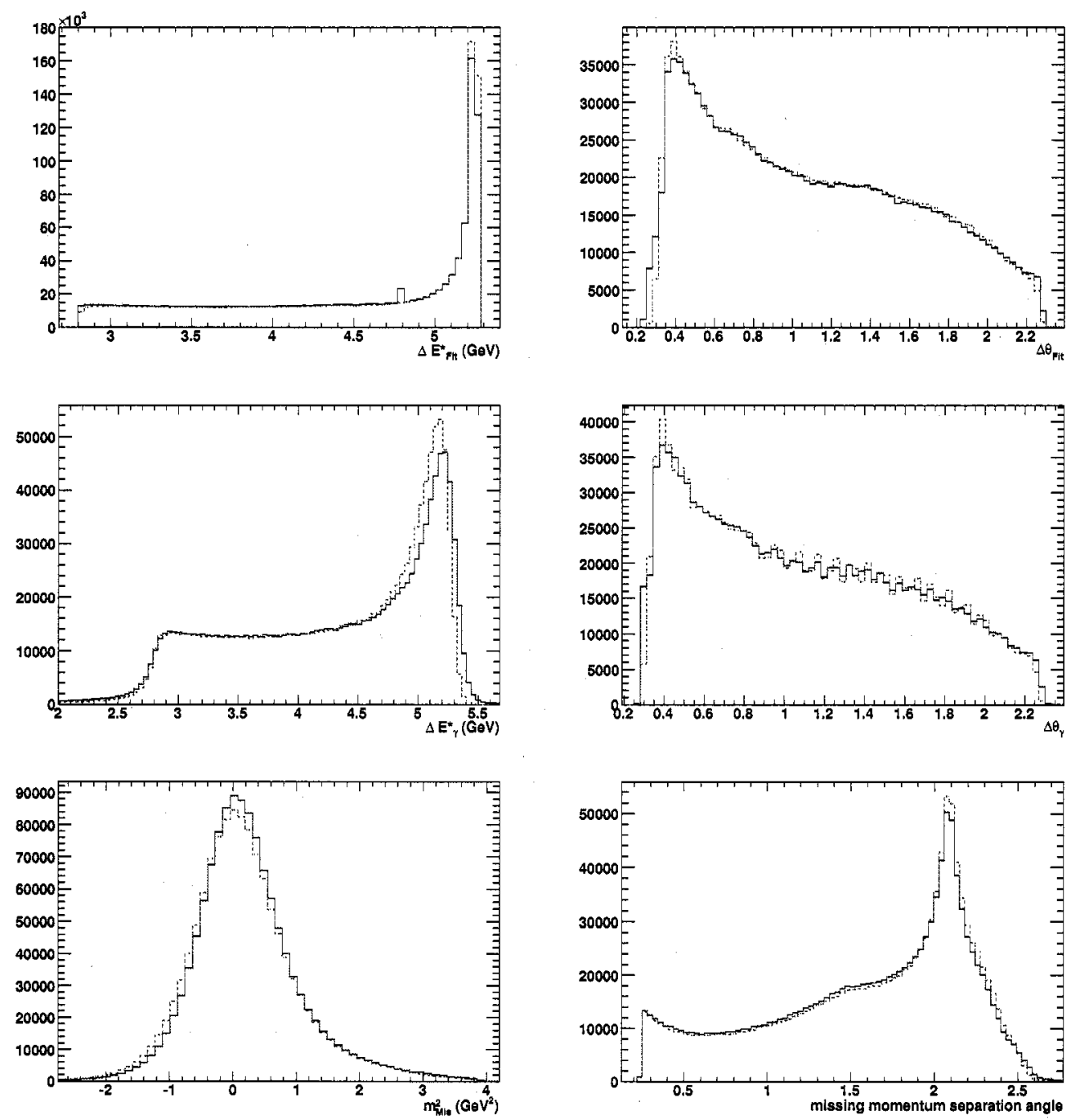

Figure 7.6: Comparison between data (solid blue line) and $\mu^{+} \mu^{-} \gamma \mathrm{MC}$ (dashed red line). From top to bottom, we have $E_{F i t}^{*}$ and $\theta_{F i t}, E_{\gamma}^{*}$ and $\theta_{F i t}, \alpha_{T \gamma}$ and $m_{M i s}^{2}$, where $\alpha_{T \gamma}$ is the angle between the missing momentum direction and the momentum direction of the closest muons. 

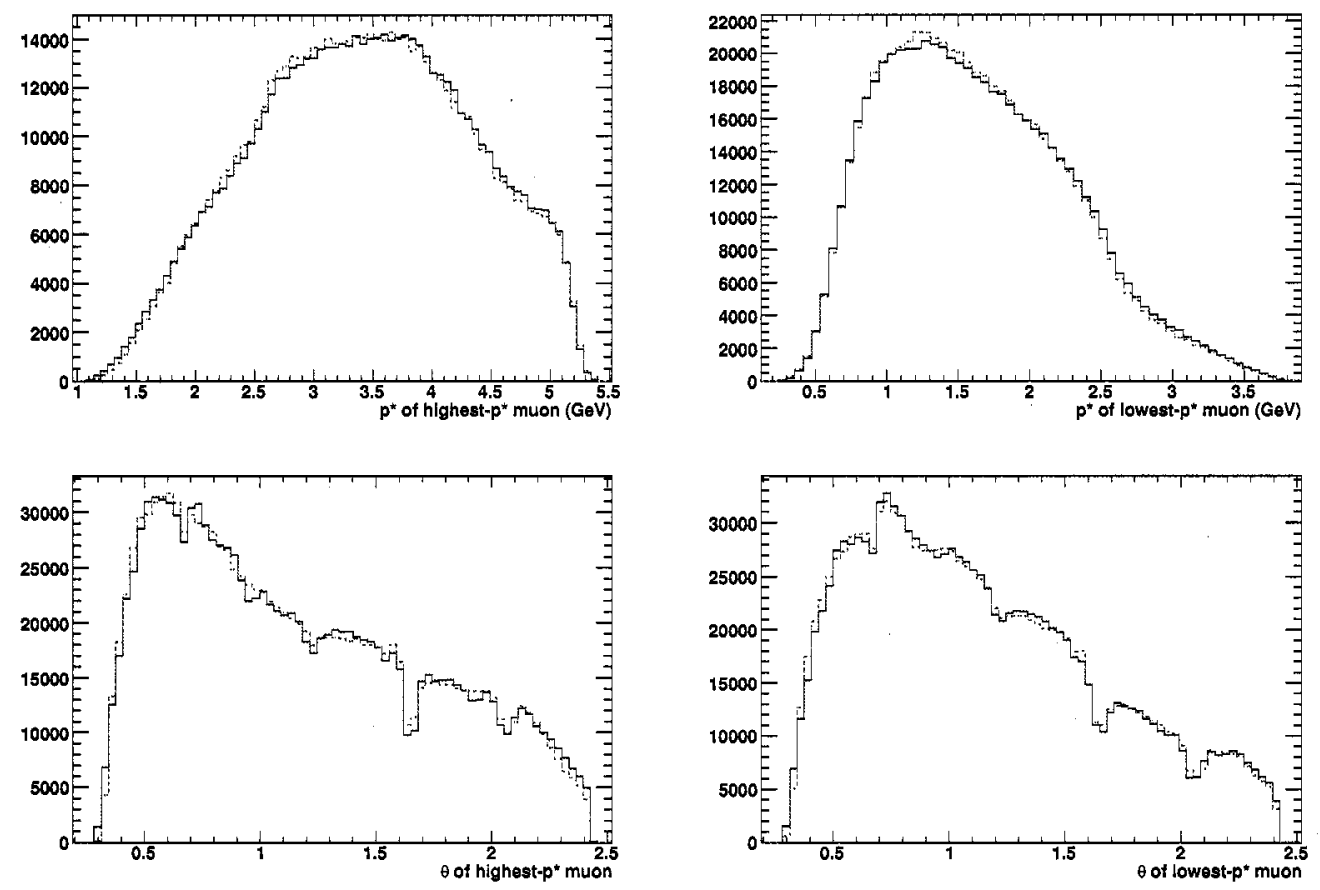

Figure 7.7: Comparison between data (solid blue) and $\mu^{+} \mu^{-} \gamma \mathrm{MC}$ (dashed red). From top to bottom, we have $p_{t}^{\mu 1}$ and $p_{t}^{\mu 2}$, and $\theta^{\mu 1}$ and $\theta^{\mu 2}$, where $\mu 1$ and $\mu 2$ are respectively the muons with highest and lowest $p^{*}$. 


\subsection{Correction Procedure}

The photon response in the EMC is measured by the distribution of $E_{\gamma}^{*} / E_{M i s}^{*}$. This ratio is calculated in the $\mathrm{CM}$ frame since both the photon energy cut and the determination of $s^{\prime}$ involve the CM energy. The goal of the correction is to modify the measured photon parameters in MC so that this distribution is identical in MC and data.

\subsubsection{Fitted Missing Momentum}

The effectiveness of the correction is limited by the resolution that can be achieved for the missing momentum. The difference between the true photon energy and that evaluated from the missing momentum in $\mu^{+} \mu^{-} \gamma \mathrm{MC}$ is shown in Fig 7.4. The resolution in $\left(E_{\text {Mis }}^{*}-E_{\text {True }}^{*}\right) / E_{\text {True }}^{*}$ is about $1 \%$, while $\left(\theta_{\text {Mis }}-\theta_{\text {True }}\right) / \theta_{\text {True }}$ and $\left(\phi_{\text {Mis }}-\right.$ $\left.\phi_{\text {True }}\right) / \phi_{\text {True }}$, are about $1.2 \%$ and $0.2 \%$ respectively. Since the photon energy and positions are measured in the EMC to a precision of about $2 \%$ in $E_{\gamma}^{*}$ and $0.5 \%$ in $\theta_{\gamma}^{*}$, a correction based on the missing momentum would not significantly improve data-MC agreement.

The missing momentum resolution can however be improved by the use of kinematic fits. The method is already in use at BABAR [87]. The following kinematic constraints can be used:

- The missing mass can be constrained to zero

- The missing momentum direction can be constrained to the measured photon direction.

The motivation for the second item is that the position resolution of the EMC is significantly smaller effect than the energy resolution. However in the interest of limiting the correlations between energy and position measurements only the first constraint was implemented. Distributions of the same quantities as previously after applying the kinematic fit are shown in the bottom plots of Fig 7.4. The fitted distributions show marked improvement over the raw ones. The resolution on the fitted $\mathrm{CM}$ energy is $0.25 \%$, significantly lower than the photon energy resolution, so 
that a meaningful correction is possible. Similarly, the resolution in $\theta$ drops to $0.2 \%$, which also improves on the measurement from the EMC.

\subsection{2 "Sculpting" distributions}

Having obtained a precise reference $E_{F i t}^{*}$ for the photon energy, we now need to find a way to modify the photon parameters in $\mathrm{MC}$ so that the distribution of $E_{\gamma}^{*} / E_{F i t}^{*}$ matches the data. The correction is applied as a rescaling of the photon 4-momentum by a quantity $1+\delta\left(E_{\gamma}^{*} / E_{F i t}^{*}\right)$. With this correction,

$$
\frac{E_{\gamma}^{*}}{E_{F i t}^{*}} \rightarrow\left(\frac{E_{\gamma}^{*}}{E_{F i t}^{*}}\right)^{\prime}=\left(1+\delta\left(E_{\gamma}^{*} / E_{F i t}^{*}\right)\right) \frac{E_{\gamma}^{*}}{E_{F i t}^{*}}
$$

The goal is to choose $\delta$ so that the quantity $\left(E_{\gamma}^{*} / E_{F i t}^{*}\right)^{\prime}$ follows the data distribution and not the MC distribution. Let $X$ be a variable, and let $D_{X}(x)$ be the probability density function for $X$. We want to define another variable $\tilde{X}=f(X)$ which is distributed according to a given density function, $\Delta(\tilde{x})$.

By the conservation of probability, we must have

$$
D_{X}(x) d x=\Delta(\tilde{x}) d \tilde{x}
$$

for $\tilde{x}=f(x)$. Defining the distribution functions $F_{X}(x)=\int_{-\infty}^{x} D_{X}\left(x^{\prime}\right) d x^{\prime}$, and $\Phi(x)=\int_{-\infty}^{x} \Delta\left(x^{\prime}\right) d x^{\prime}$ and integrating the equation above we get:

$$
F_{X}(x)=\Phi(\tilde{x})=\Phi(f(x))
$$

So finding $f(x)$ is just a matter of inverting the function $\Phi$, which is always possible since $\Phi$ is a monotonously increasing function of its argument.

In our case, this is implemented by using normalized histograms $H_{D a t a}$ and $H_{M C}$ of $x=E_{\gamma}^{*} / E_{F i t}^{*}$ for data and Monte-Carlo. To find the value $f(x)$ for a given uncorrected value of $x$, one proceeds as follows:

- Calculate the value of $I=\int_{-\infty}^{x} H_{M C}\left(x^{\prime}\right) d x^{\prime}$ by doing a binned integration of the histograms starting at the lower edge. 
- Look up the point $\tilde{x}$ such that the binned integral $\int_{-\infty}^{\tilde{x}} H_{\text {Data }}\left(x^{\prime}\right) d x^{\prime}=I$. This is implemented using a binary search technique, which is both fast and numerically stable due to the fact that the integral is a monotonous function of its upper bound.

To improve precision, a linear interpolation is used between the bin centers when calculating integrals. The correction $\delta(x)$ is then calculated as

$$
\delta(x)=\frac{\tilde{x}-x}{x} .
$$

Clearly $\delta \equiv 0$ if $H_{\text {Data }}$ and $H_{M C}$ are identical, and should be small when they are similar.

The correction is then used to calculate the corrected energy of a photon in MC by

$$
E_{\text {corr }}^{*}=\left[1+\delta\left(\frac{E_{\gamma}^{*}}{E_{\text {Truth }}^{*}}\right)\right] E_{\gamma}^{*}
$$

where $E_{\gamma}^{*}$ is the photon energy, $E_{\text {Truth }}^{*}$ is the energy of its MC Truth partner and $E_{\text {corr }}^{*}$ is the corrected energy.

\subsubsection{Implementation}

The correction is computed using the algorithm described above. Histograms for $E_{\gamma}^{*} / E_{F i t}^{*}$ are plotted for $\mathrm{MC}$ and data over the range $[0.5,1.5]$ with a bin size of $510^{-3}$. The correction $\delta$ is computed for each event in the MC ntuples by lookups in the data and MC histograms, and the correction is presented as a "binned curve" with the same binning as the histograms, where the correction value in each bin is the average of the corrections for the events in that bin.

To achieve the required precision the correction must be separated in bins of $E_{\gamma}^{*}$ and $\theta_{\gamma}$ : the energy resolution is expected to vary significantly, in particular vs. $\theta_{\gamma}$, and this must be taken into account since the $E_{\gamma}^{*}$ and $\theta_{\gamma}$ distributions in $\mu^{+} \mu^{-} \gamma$ events differ significantly from those of $q \bar{q} \gamma$ (due to the presence of FSR). The binning is implemented in terms of $\mathrm{CM}$ energy and lab. $\theta$. This reflects the fact that the fiducial region is defined in terms of these quantities $\left(3.3<E_{\gamma}^{*}<5.29 \mathrm{GeV}\right.$ and 
$\left.0.386<\theta_{\gamma}<2.109\right)$. If the binning were done in laboratory-frame energies, this region would not be a rectangle but a crescent, and the energy bins would need to vary with $\theta$. Binning in $\mathrm{CM}$ energy also allows the use of smaller bins near the cut value, which improves the precision of the photon efficiency determination (see Section 8.1.4). The disadvantage of this method is that the missing energy resolution gets contributions from $\theta_{M i s}$ as well as $E_{M i s}^{*}$, leading to correlated errors. However since the $\theta_{\text {Mis }}$ resolution is significantly smaller than the energy resolution this is not a problem.

We split the energy range in 6 bins, with boundaries at 3.0, 3.3, 3.8, 4.3, 4.8, 5.1 and $6.0 \mathrm{GeV}$. The $\theta$ range is also split into 5 bins, with boundaries at $0.386,0.475$, $0.90,1.40,1.90$ and 2.109. The extreme values are taken to be the same as the limits on photon acceptance since the position resolution is small. The value 0.475 is just above the boundary between the EMC endcap and barrel sections at 0.468. This value ensures that the lower resolution in the boundary region does not affect the first bin of the barrel.

Due to the excellent position resolution of the fitted missing momentum, we can resolve the expected photon position to within about $5 \%$ of an EMC crystal size. Since position on the crystal is expected to play an important role in the description of the energy leakage we also introduce bins in crystal front-face position.

We define front-face position by two coordinates $x$ and $y$. Since crystal frontfaces are generally trapezoidal we do not take $\mathrm{x}$ and $\mathrm{y}$ to form an orthonormal basis, but define them so that the crystal center is at $(0,0)$ and the corners at $(-0.5,0.5)$, $(0.5,0.5),(0.5,-0.5)$ and $(-0.5,-0.5)$. To keep statistics high, we limit ourselves to 3 bins: the "center" bin is the center quarter of the crystal, i.e. $|x|<0.25$ and $|y|<0.25$. The "edge" region is composed of the 4 regions directly above, below, and to the left and right of the center region, defined by $|x|>0.25$ xor $|y|>0.25$. Finally the "corner" bin is defined as the union of the 4 remaining regions near the corners, given by $|x|>0.25$ and $|y|>0.25$.

The correction is applied in $\mathrm{MC}$ by looking up the correction value for $E_{\gamma}^{*} / E_{\text {True }}^{*}$ in the relevant plot and rescaling the photon 4-momentum. The correction is not applied if it is found to be in excess of $20 \%$. 
Also, to avoid boundary effects in $E_{\gamma}^{*}, \theta_{\gamma}$, the correction is interpolated in the $2 \mathrm{D}$ bin lattice by using nearest-neighbor bins and the relative position of the actual $E_{\gamma}^{*}$, $\theta_{\gamma}$ with respect to the bin centers. However since the corrections are much larger in the endcap than in the barrel this leads to an overcorrection in the half-bin closest to the endcap in $\theta$, corresponding to the region $0.475<\theta<0.679$. To avoid this, the interpolation is performed only in the $E_{\gamma}^{*}$ direction in this region, for both corrections.

\subsubsection{Smoothing}

The method described above works well in regions with large numbers of events, but if statistics are limited artifacts may occur. This is the case in particular near the endpoints of the resolution functions: the data endpoints are typically farther away than the $\mathrm{MC}$ endpoints ("outliers" are more frequent in data); the events on the extreme upper and lower edges of the $\mathrm{MC}$ resolution function will therefore be assigned a large correction, which shows up as a spike in the correction function.

To avoid these artifacts, the correction histograms are smoothed before use using a 5-bin running average algorithm: the value of each bin is replaced by an average including two neighboring bins on each side, with the inverse square of the bin error used as weight.

Also, the constant sections in regions with no statistics are set to the last non-zero value, moving away from the center.

Apart from the removal of the large corrections at either end of the correction distribution this operation does not introduce biases into the procedure.

\subsection{Results}

\subsubsection{Energy Correction}

Results for the first and fourth $\theta$ bin (corresponding to the endcap and the middle of the barrel) in Figs. 7.8 and 7.9.

As a cross-check, the correction is re-calculated using the corrected energy. Excellent agreement is seen between data and $\mathrm{MC}$, which validates the procedure. 

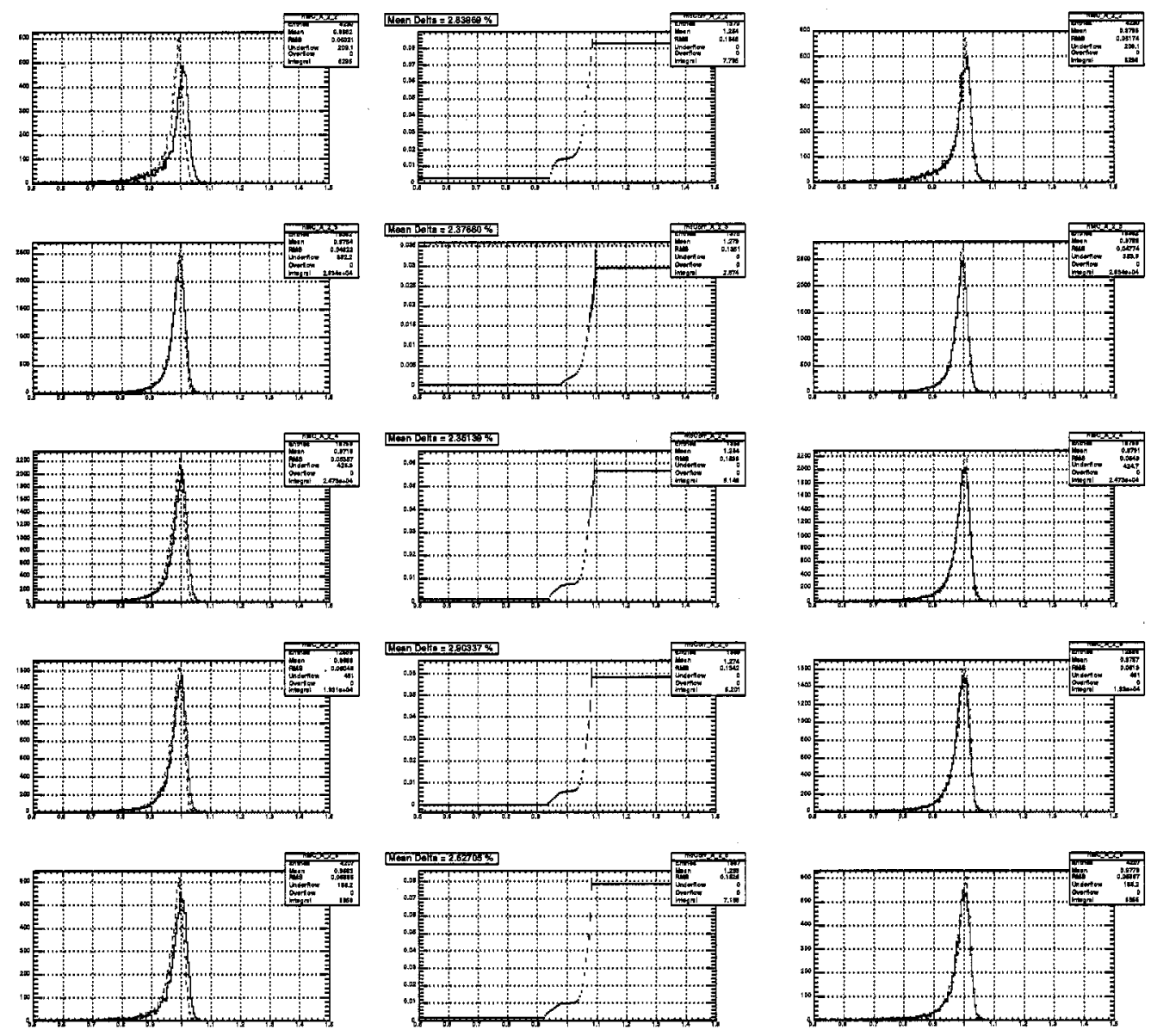

Figure 7.8: Energy correction distributions for the bin $0.386<\theta_{\gamma}<0.468$, in bins of increasing energy. Left column: Photon $E_{\gamma}^{*} / E_{F i t}^{*}$ distributions for data (solid blue line) and MC (dashed red line). Center column: correction factor $\delta$ as a function of $E_{\gamma}^{*} / E_{F i t}^{*}$. Right Column: Photon $E_{\gamma}^{*} / E_{F i t}^{*}$ after energy correction. 

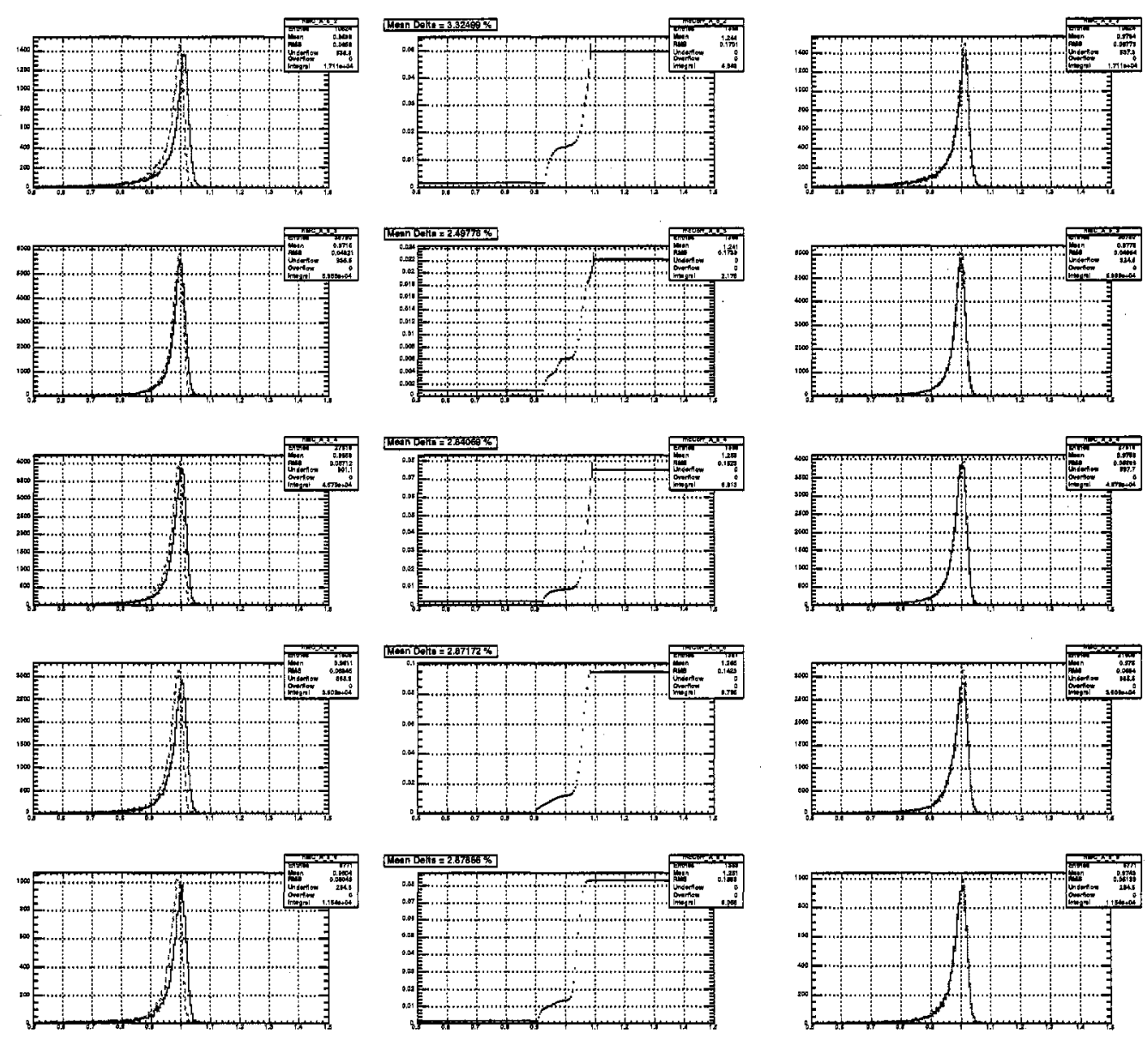

Figure 7.9: Energy resolution parameters for the bin $1.40<\theta_{\gamma}<1.90$, in bins of increasing energy. Left column: Photon $E_{\gamma}^{*} / E_{F i t}^{*}$ distributions for data (solid blue line) and MC (dashed red line). Center column: correction factor $\delta$ as a function of $E_{\gamma}^{*} / E_{F i t}^{*}$. Right Column: Photon $E_{\gamma}^{*} / E_{F i t}^{*}$ after energy correction. 


\subsection{2 $\theta$ Correction}

The same procedure is applied to correct the photon $\theta$ position. A slight complication occurs due to the fact that the photon momentum direction is computed by assuming the origin of the particle to be the BABAR origin; if the IP is displaced relative to the origin, the momenta is therefore biased. Track momenta are reported at their point of closest approach (POCA) to the origin, and therefore do not suffer from this problem: therefore shifts in the IP position introduce discrepancies between the missing momentum and the measured photon momentum.

To remedy this problem, a correction is first applied to the photon so that the primary vertex of the event, as computed from the muon tracks, is used as the photon origin.

The resulting corrected $\theta_{\gamma}$ variable is then compared to $\theta_{M i s}$. Results are shown in Figs 7.10 and 7.11, again corresponding the the first and fourth $\theta$ bins.

We again repeat the procedure with the corrected $\theta_{\gamma}$, and good agreement is seen between $\mathrm{MC}$ and data. 

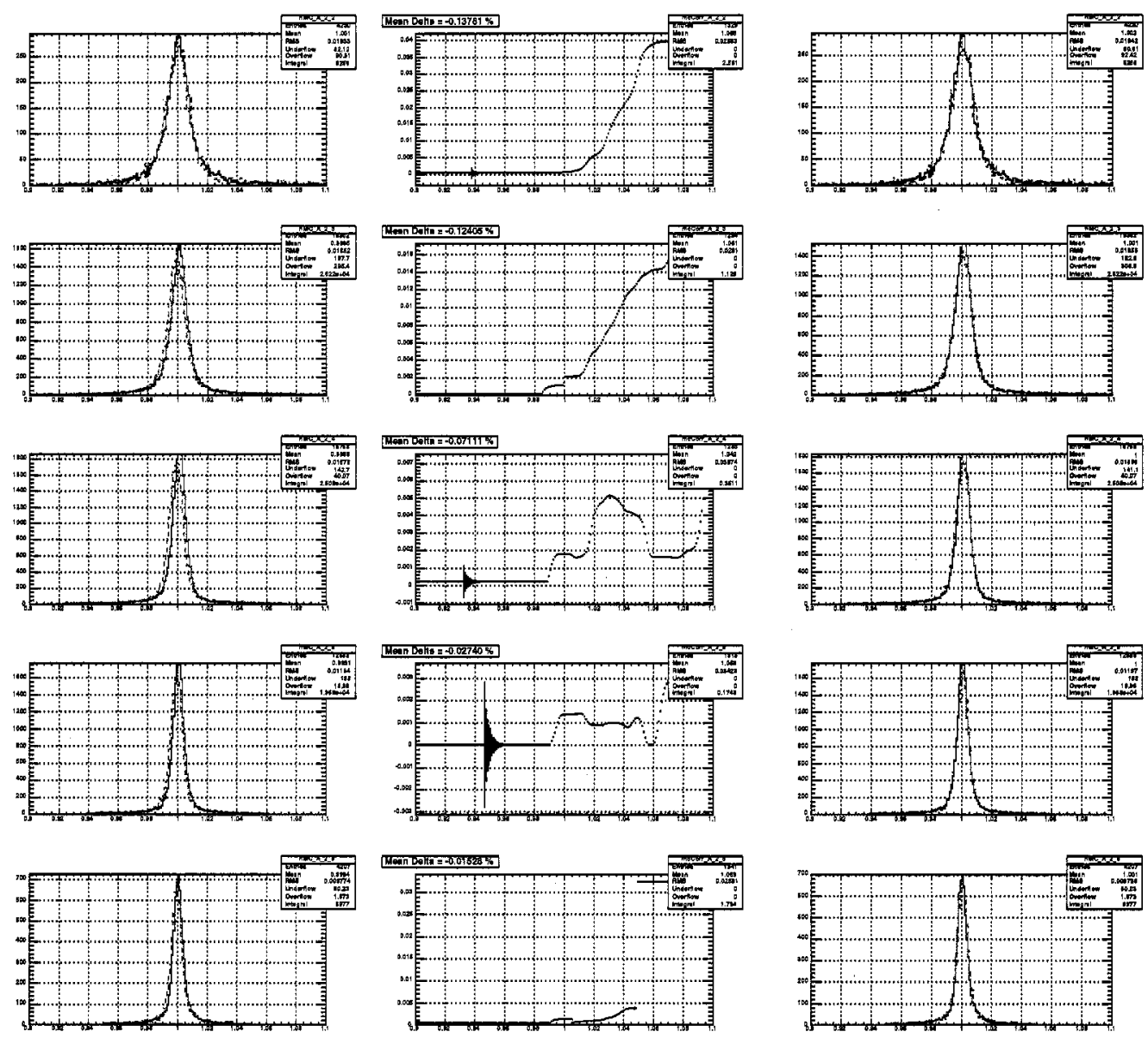

Figure 7.10: $\theta$ correction distributions for the bin $0.386<\theta_{\gamma}<0.468$, in bins of increasing energy. Left column: Photon $\theta_{\gamma} / \theta_{\text {Fit }}$ distributions for data (solid blue line) and MC (dashed red line). Center column: correction factor $\delta$ as a function of $\theta_{\gamma} / \theta_{\text {Fit }}$. Right Column: Photon $\theta_{\gamma} / \theta_{\text {Fit }}$ after $\theta$ correction. 

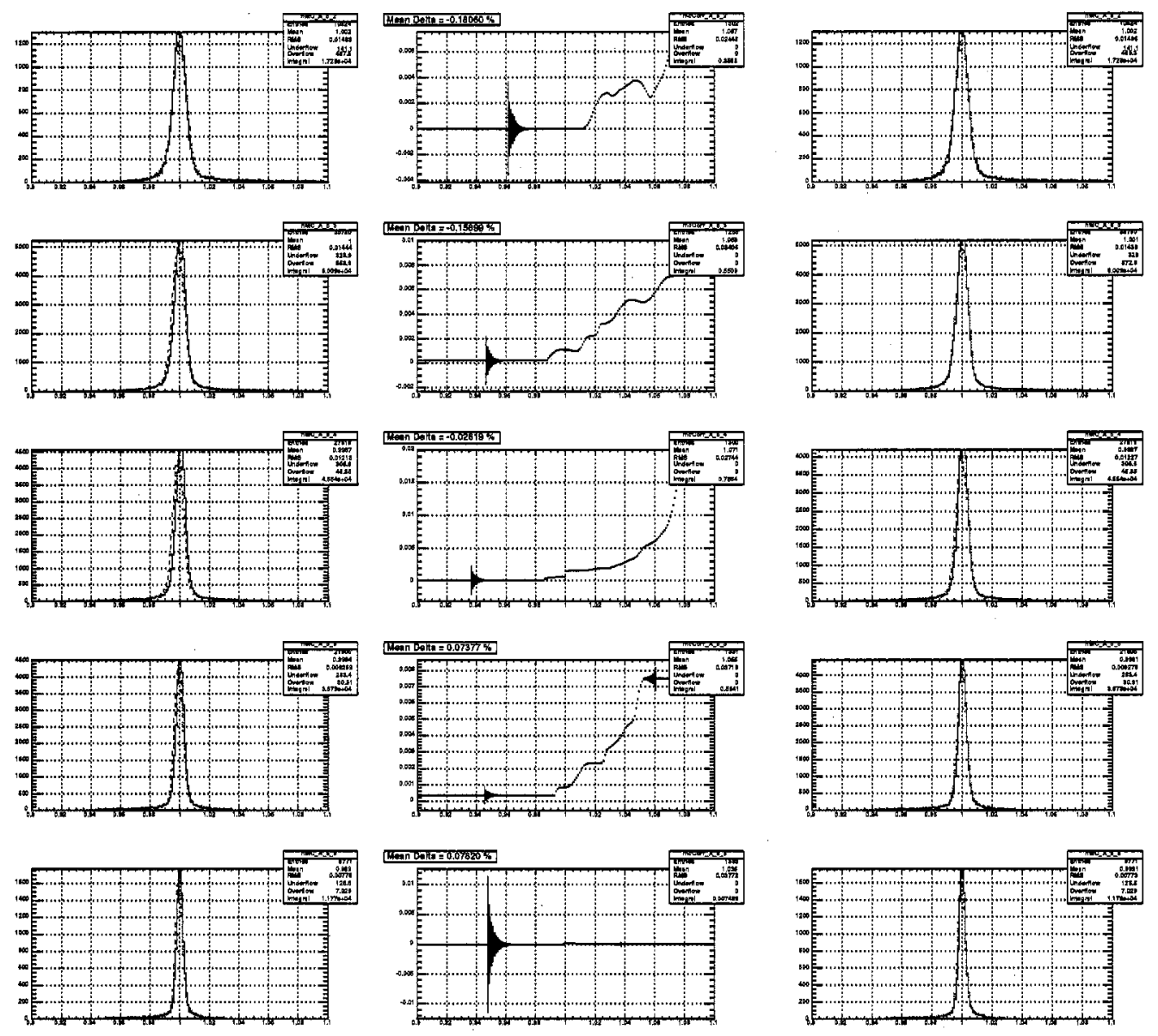

Figure 7.11: $\theta$ resolution parameters for the bin $1.40<\theta_{\gamma}<1.90$, in bins of increasing energy. Left column: Photon $\theta_{\gamma} / \theta_{F i t}$ distributions for data (solid blue line) and MC (dashed red line). Center column: correction factor $\delta$ as a function of $\theta_{\gamma} / \theta_{F i t}$. Right Column: Photon $\theta_{\gamma} / \theta_{\text {Fit }}$ after $\theta$ correction. 


\section{Chapter 8}

\section{Photon Efficiency}

For the purpose of this analysis, the absolute photon reconstruction efficiency must be known to an accuracy below $3 \%$, since this factor is directly multiplied into the absolute cross-section value.

It is useful to separate the detector response to the ISR photon in the following way:

- The photon is detected in the EMC at the expected position and energy.

- The photon converts in the inner detector material and both conversion tracks are reconstructed in the $\mathrm{DCH}$.

- The photon converts but only one track is reconstructed.

- The photon "disappears": no DCH tracks are detected and no EMC activity compatible with the expected signal is seen.

- Other: this includes photons detected away from their expected positions or with incorrect energies, etc.

The rates of occurrence of these different outcomes in simulation are shown in Table 8.1. We use the notations $\epsilon_{\gamma}, \epsilon_{1}, \epsilon_{2}, \epsilon_{0}$ and $\epsilon_{X}$ for the respective proportion of photons in Table 8.1. 


\begin{tabular}{lc}
\multicolumn{1}{c}{ Table 8.1: Fractions of photons by category } \\
\hline \hline Category & Fraction (\%) \\
\hline Good Photons $\left(\epsilon_{\gamma}\right)$ & 92.2 \\
2-track Conversions $\left(\epsilon_{1}\right)$ & 5.2 \\
1-track Conversions $\left(\epsilon_{2}\right)$ & 0.9 \\
No DCH or EMC response $\left(\epsilon_{O}\right)$ & 1.0 \\
Other $\left(\epsilon_{X}\right)$ & 0.7 \\
\hline \hline
\end{tabular}

In the rest of this section we first describe how these numbers are measured in the simulation. In order to achieve the required precision these numbers must be checked using data control samples.

We use two such samples:

- A clean, unbiased photon sample, which can be used to determine the magnitude of $\epsilon_{0}$.

- A converted photon sample, which gives the scale of $\epsilon_{2}$. This will also be used to scale $\epsilon_{1}$.

In Sections 8.2 and 8.3 these samples are used to determine corrections to be applied on the MC numbers.

\subsection{Determination of the Efficiency in MC Truth}

\subsubsection{Naive method}

Our strategy is to first measure the magnitude of the different efficiency terms in MC, and then cross-check and rescale them using comparisons with data. The first step is to measure the efficiencies in $\mathrm{MC}$ in a reliable way. The naive way of doing this is to require that a $\mathrm{MC}$ photon be present in the acceptance $\left(E^{*}>3.3 \mathrm{GeV}, 0.35<\right.$ $\theta<2.2$ ), and determine the proportion of those events that have a reconstructed EMC cluster in the same region. This gives an efficiency of about $89 \%$. However this 
method gives an incorrect treatment of boundary effects. Events with MC photons in acceptance but reconstructed photons outside the acceptance due to energy and $\theta$ smearing are correctly rejected, but the reverse process is ignored: MC photon falling outside the acceptance are not included, although in some cases the reconstructed photon may. feed into the acceptance region. For this reason the number given above is artificially low, since it includes "feed-out" but not "feed-in". This is illustrated in Fig. 8.1, which shows the efficiency versus the true $E^{*}$ : near the cut position the efficiency drops sharply due to feed-out uncompensated by feed-in.
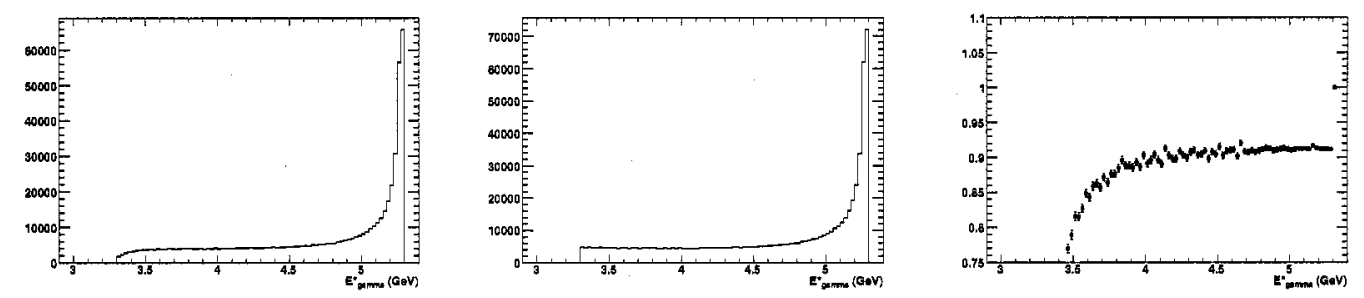

Figure 8.1: $E *$ distributions for the numerator sample (left), denominator sample (center) and their ratio (right), for the range $0.35<\theta<2.2$

\subsubsection{Improved method}

A possible solution to the problem above is to widen the selection on the MC photon sufficiently so that most of the "feed-in" is also included. However as the MC photon cuts are widened the efficiency drops mechanically, not because of photon efficiency effects but because MC photons further and further from the acceptance region have less and less chance of feeding in. The photon efficiency term therefore includes a contribution from photon resolution effects, which we would prefer to isolate.

A way out of this problem is to simulate the effects of photon resolution effects in the denominator. The technique is as follows:

- measure the resolution functions of the reconstructed parameters with respect to the $\mathrm{MC}$ truth parameters in the same way as done in Chapter 7 , in the same bins of $E^{*}$ and $\theta$ and with the same range $|\Delta E / E|<50 \%$ and $|\Delta \theta / \theta|<10 \%$. 
- For each MC photon within the wider acceptance $\left(E^{*}>2.7 \mathrm{GeV}, 0.350<\theta<\right.$ 2.20 ), produce a set of smeared parameters using the resolution functions above (the acceptance is not widened in $\theta$ since $\theta$ smearing effects are small, and events that feed-in due to $\theta$ smearing are close enough to the acceptance region that their resolution is well-described by what we get from the acceptance region).

- If the smeared parameters fall within the acceptance region, count the event into the denominator, otherwise reject it.

- For the numerator, take all events with a reconstructed photon within the acceptance, regardless of MC parameters.

With this technique, the denominator represents what the photon sample should be like if smearing was the only effect occurring. The difference between numerator and denominator is therefore a measure of photon efficiency alone.

It should be noted that near the edges of the acceptance region the denominator and numerator samples are independent: whether or not an $\mathrm{MC}$ photon falls in the denominator depends on a random number and not on the reconstructed parameters. In particular the numerator is not in general a subset of the denominator, so efficiencies greater than unity can occur locally. This is not true away from the edges, where photon efficiency is the dominating effect and photons in the numerator also appear in the denominator.

The method is illustrated in Fig. 8.2: both the numerator the denominator sample exhibit feed-in and feed-out and the efficiency shows reasonably consistent behavior near the cut position. There seems to be a tendency for the efficiency to be low below the cut. This is dependent on the shape of the tails of the energy resolution curves on the high side and in particular on the binning used, and will be discussed in more detail in Section 8.1.3.

It should be noted that the ranges used for the energy and $\theta$ resolution functions ( $\pm 50 \%$ in energy and $\pm 10 \%$ in $\theta$ ) play an important role: they define in effect the boundary between what we categorize as a "mis-measured photon" and a "missing photon". The goal here is to measure the second category. It should be noted that if we had extended the energy range to $\pm 100 \%$ the photon efficiency would be essentially 

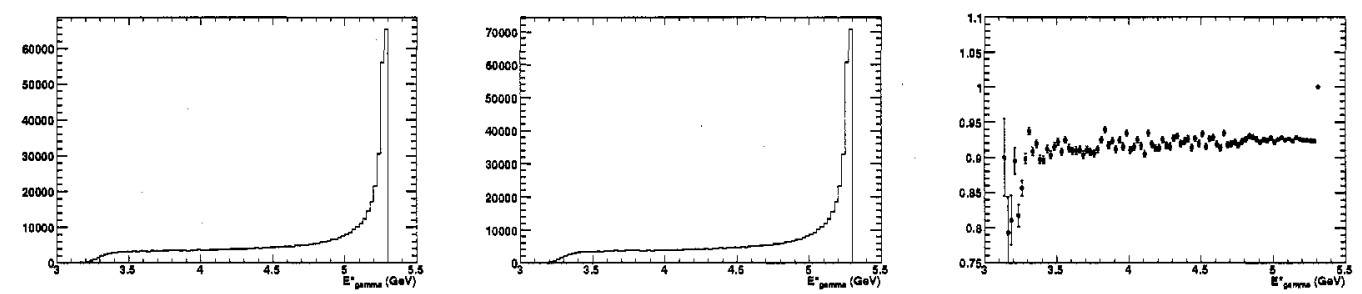

Figure 8.2: $E^{*}$ distributions for the numerator sample (left), denominator sample (center) and their ratio (right), for the range $0.35<\theta<2.2$

$100 \%$. However the challenge would then have been to measure the resolution function in data down to zero energy, a much more difficult task!

\subsubsection{Truth Resolution Functions}

The resolution functions used to smear the $\mathrm{MC}$ truth parameters are obtained using a method similar to the one used in Chapter 7 for energy resolution.

- The numerator of the resolution function is the $E^{*}$ or $\theta$ value of the highestenergy reconstructed cluster in the wide acceptance region $E^{*}>2.7 \mathrm{GeV}$, $0.350<\theta<2.20$, with the energy and $\theta$ corrections of Chapter 7 applied.

- The denominator is the $E^{*}$ or $\theta$ value of the best MC partner for this photon. If a partner is found by truth-matching this is used, otherwise we look the highestenergy $\mathrm{MC}$ photon with $E *$ at least $2.7 \mathrm{GeV}$, first in the ISR acceptance region $0.350<\theta<2.20$, then in the wider region $0.240<\theta<2.31$ and finally in the entire $\theta$ range.

When carrying out this procedure it is necessary to isolate the photon from the muon tracks: in the cases where the photon and MIP cluster from the muon overlap the photon gets an apparent boost of $100-200 \mathrm{MeV}$ which creates a tail on the high side of the energy resolution function, which is precisely the part that is crucial to describe the feed-in from the region $E^{*}<3.3 \mathrm{GeV}$. The effect is shown in Fig. 8.3 

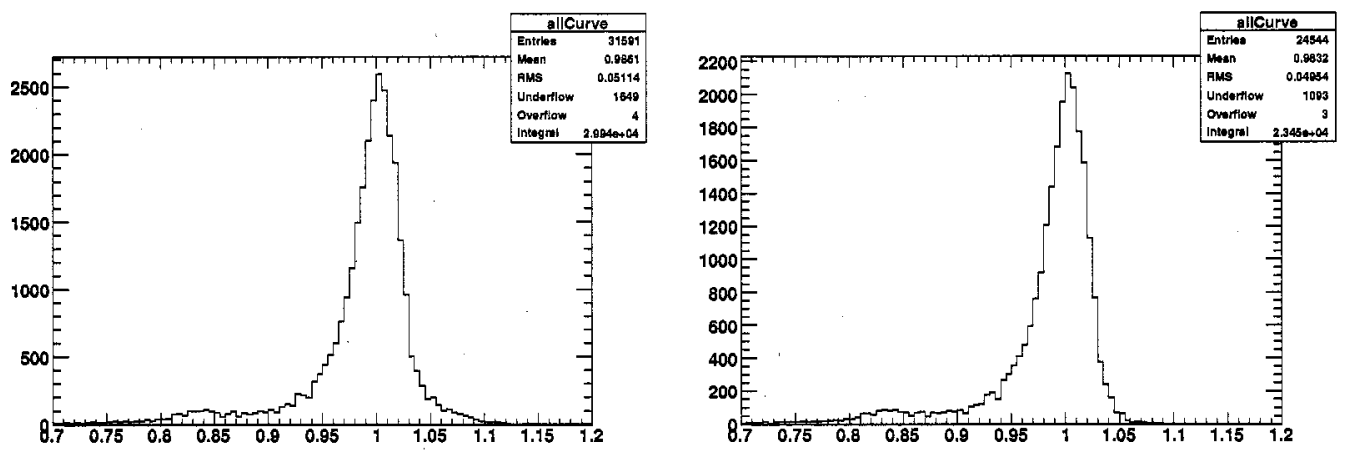

Figure 8.3: $E_{\text {reco }}^{*} / E_{\text {true }}^{*}$ distributions without (left) and with (right) a photon separation cut (left) for the range $0.35<\theta<2.2$

The main problem is that the size of the high-side tail varies sharply in $E^{*}$ and $\theta$. The top row of Fig 8.4 shows the size of the tails relative to the peak region as a function of $E^{*}$ for different $\theta$ bins, which show strong variations.

The reason for these variations is somewhat puzzling. For $\mu^{+} \mu^{-} \gamma$ events, a significant fraction of the radiation comes from FSR, particularly for radiation away from the beam axes, which corresponds to the forward barrel region of the EMC in the lab. The turn-on at $3 \mathrm{GeV}$ may be an effect of our cuts.

In order to remove this effect we compute the angle between the photon and the closest other particle. For charged particles we use reconstructed tracks with associated clusters. For neutrals we use MC truth particles (since neutrals would not be visible when overlapping with the photon) with zero charge of at least $30 \mathrm{MeV}$ (corresponding to a $1 \%$ shift in the photon $E_{\text {reco }}^{*} / E_{\text {true }}^{*}$ due to the overlap at $3 \mathrm{GeV}$ ) that do not decay inside the tracking volume. In both cases we require that the particle or its MC partner not have the photon as one of their ancestors in the MC decay tree, since we do not want to veto particles coming from the photon itself.

We require this angle to be at least $0.25 \mathrm{rad}$ in the determination of truth resolution functions.

The efficiency extraction method relies on the fact that the smeared truth parameters reproduce exactly the distribution of reconstructed parameters. This is in principle achieved by construction (up to binning effects) if the cuts that are applied in 

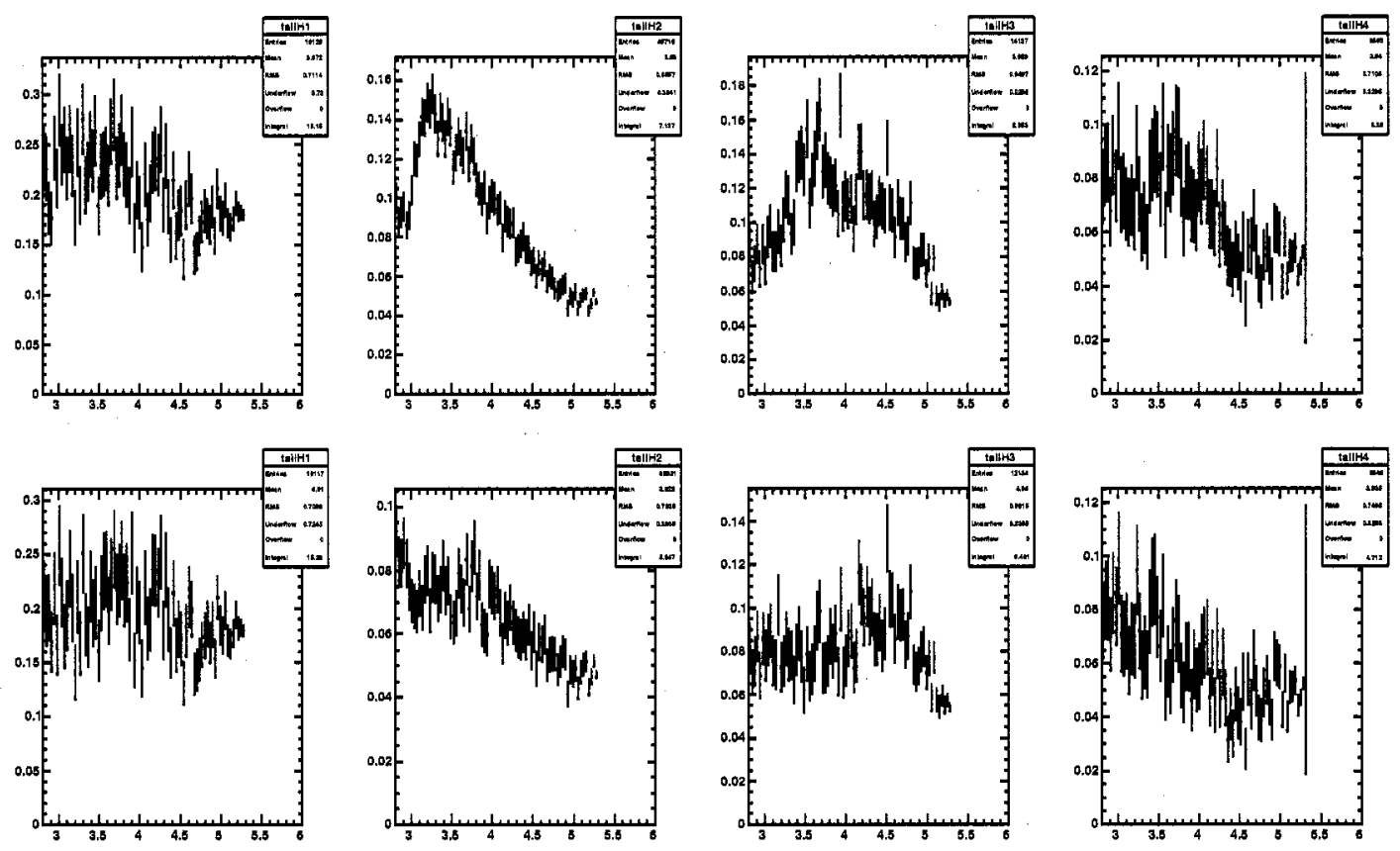

Figure 8.4: Ratio between the fraction of the $E_{\text {reco }}^{*} E_{\text {true }}^{*}$ curve in the interval $[1.03,1.10]$ to the fraction in the interval $[0.98,1.02]$, as a function of $E_{\text {true }}^{*}$, without (top row) and with (bottom row) a photon separation cut. In both rows, the plots correspond from left to right to the $\theta$ bins $0.35<\theta<0.468,0.468<\theta<1.4$, $1.4<\theta<1.9$ and $1.9<\theta<2.2$. 
the analysis are also applied when extracting the resolution functions. The minimalangle cut is therefore applied on both the nominator and denominator samples when calculating the photon efficiency.

The results are shown in Figs 8.5 and 8.6.
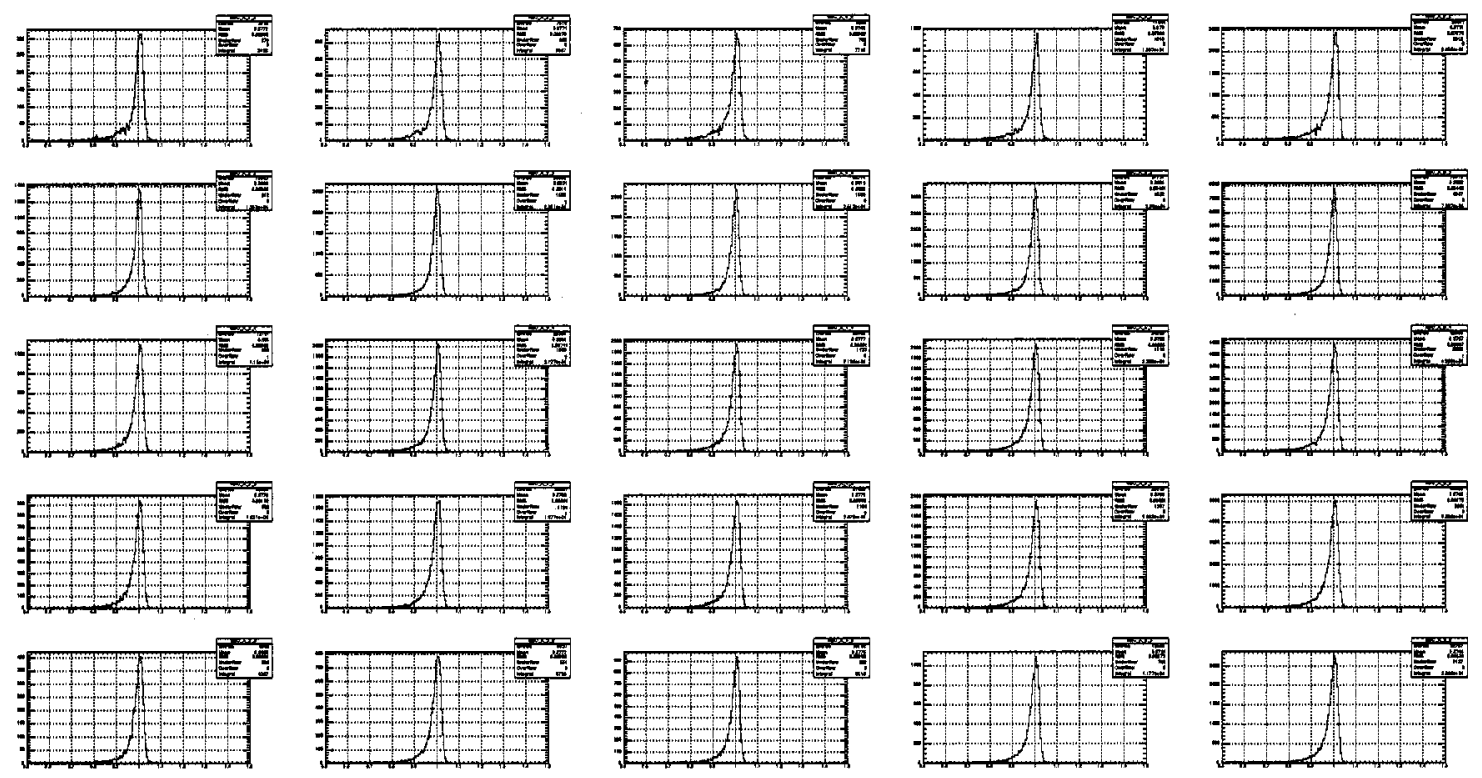

Figure 8.5: Resolution functions for $E^{*}$ in MC Truth, in bins of energy (increasing from left to right) and $\theta$ (increasing from top to bottom).

\subsubsection{True Photon Efficiency}

With all the machinery in place, the efficiency can be obtained as the ratio of the nominator and denominator samples defined above, both with a separation cut applied. Since this cut is based on properties of the recoil system rather than the photon, it is not expected to introduce any biases in the efficiency value. It should be noted that in the case of multiple radiation "the" photon can be a different object in the nominator, denominator and resolution samples, and the separation angle cut can therefore have a slightly different effect in each sample, particularly near the edges of the acceptance region. These effects are expected to be small. 

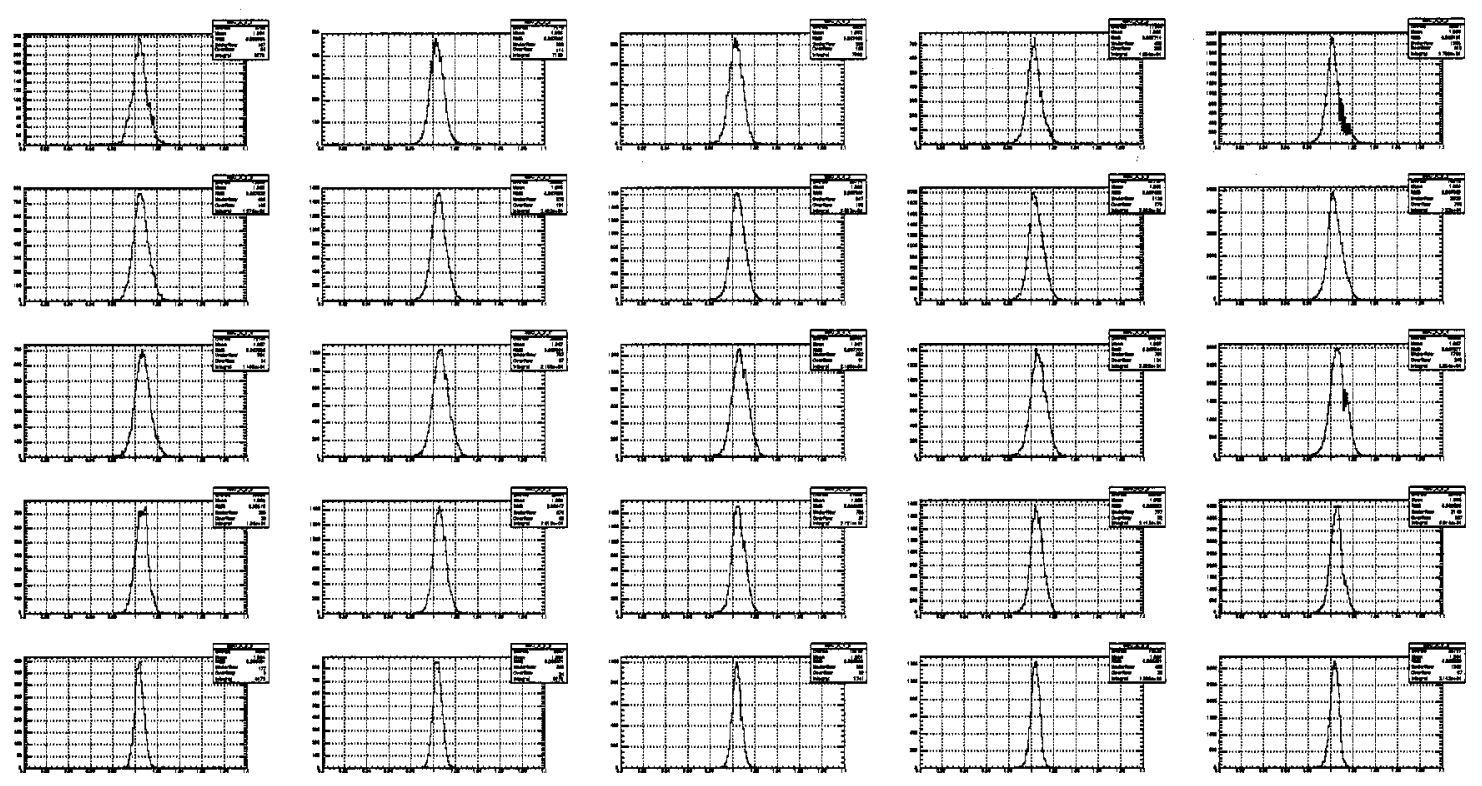

Figure 8.6: Resolution functions for $\theta$ in MC Truth, in bins of energy (increasing from left to right) and $\theta$ (increasing from top to bottom).

The efficiency is calculated in bins of $E^{*}$ and $\theta$. They are identical to the bins used in the photon parameter resolution studies. Fig 8.7 shows the measured efficiency and the acceptance fraction that gets multiplied into the denominator.

We can also compute the efficiencies $\epsilon_{1}, \epsilon_{2}$ and $\epsilon_{0}$ of the other processes given in Table 8.1:

- Conversion events $\left(\epsilon_{1}, \epsilon_{2}\right)$ with one or two visible tracks are selected by requiring either one or two of the daughters of a MC photon in the acceptance to be matched to reconstructed tracks with at least $12 \mathrm{DCH}$ hits. The parameters of the event are taken to be those of the MC photon with track daughters.

- Events with no EMC activity $\left(\epsilon_{0}\right)$ are defined as follows:

- None of the reconstructed tracks with $\mathrm{DCH}$ hits have MC partners that are descendants of $\mathrm{MC}$ photons with $E^{*}>2.7 \mathrm{GeV}$.

- There is at most one EMC Cluster with $E>100 \mathrm{MeV}$, and its parameters $E, \theta, \phi$ are not compatible with those of its MC partner $E_{M C}, \theta_{M C}, \phi_{M C}$ in 

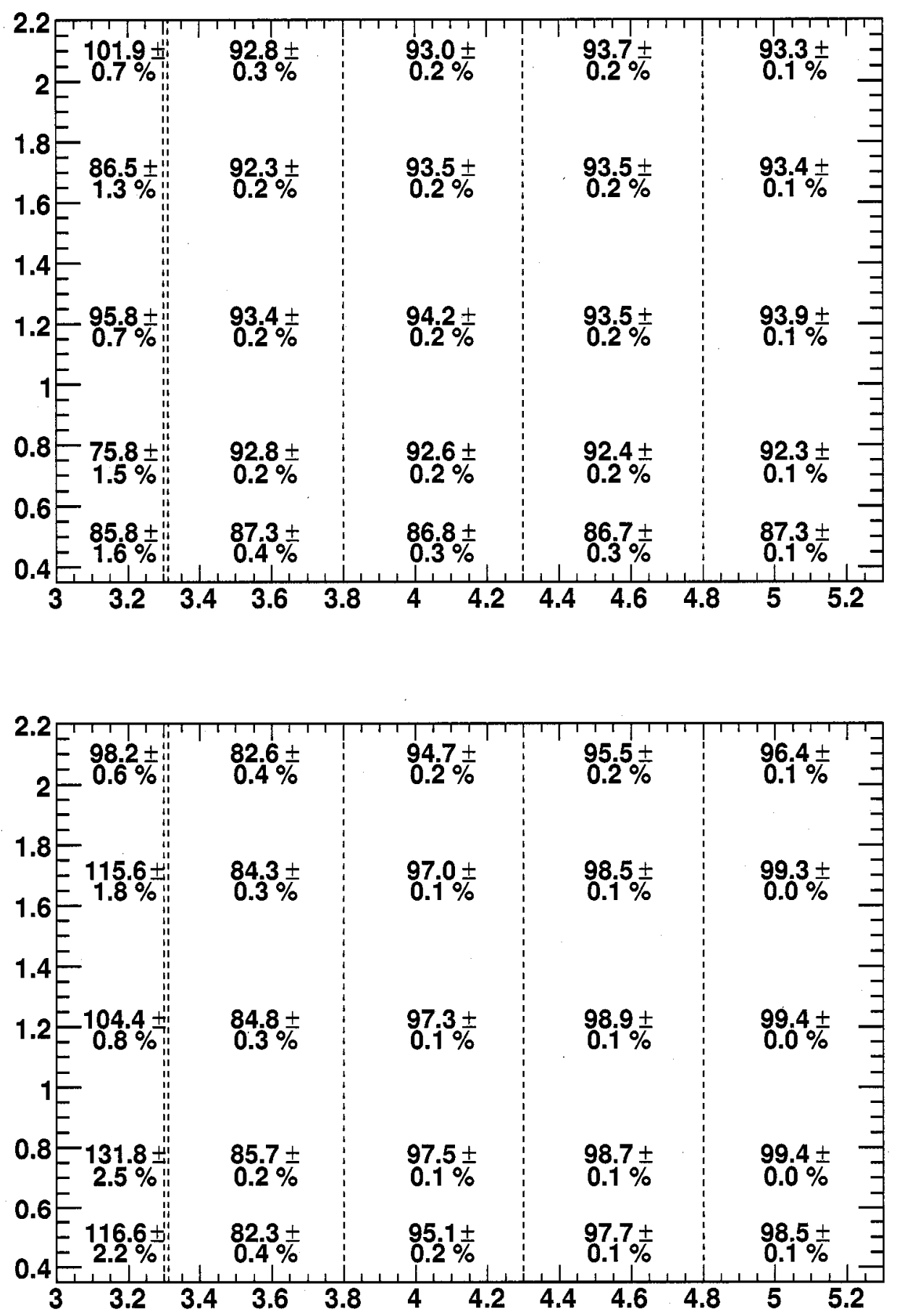

Figure 8.7: Photon Efficiency (left) and acceptance fraction (right) in MC Truth, in bins of $E^{*}$ and $\theta$. The dotted line shows the lower limit of the $E^{*}$ acceptance. 
the sense that $\left|E-E_{M C}\right| / E_{M C}>50 \%$ or $\left|\theta-\theta_{M C}\right|>0.1$ or $\left|\phi-\phi_{M C}\right|>$ 0.05. Apart from the track requirement, this almost coincides with the events left out of the resolution functions, which is our definition of lost photons.

The parameters of the event are taken to be those of the MC partner of the EMC cluster if there is one, otherwise the best MC photon (the mother of the conversion tracks for conversion events and the highest-energy $\mathrm{MC}$ cluster the $\theta$ acceptance or the wider region $0.240<\theta<2.31$ for $\epsilon_{0}$ ).

- Other events: events that do not fall in either the good photon category or the ones above. As explained above very few events should end up there. Lost photon events with tracks should show up as conversions and almost all the fall in the $\epsilon_{0}$ sample.

There is a small overlap between the samples above and the good photon sample due to multiple radiation. To avoid this, events flagged as having a good photons are excluded from the samples above.

The corresponding plots are shown in Fig 8.8. The numbers in Table 8.1 correspond to the average of each plot over the acceptance region.

In the rest of this study we use data/MC comparisons to rescale some contributions: unbiased photon samples measure $\epsilon_{0}$ in data and are used to rescale this value, while converted $\mu^{+} \mu^{-} \gamma$ events with 2 visible tracks are used to rescale both $\epsilon_{1}$ and $\epsilon_{2}$.

\subsection{Correction of $\epsilon_{0}$ using an Unbiased Photon Sample}

\subsubsection{Preselection}

This study is performed using the process $e^{-} e^{-} \rightarrow \mu^{+} \mu^{-} \gamma$. By using only the muon tracks to tag the event, an unbiased photon sample can be obtained.

The Monte-Carlo sample used for this study is produced using the KKMC generator. We require that the polar angle of the total photon momentum (the beam 

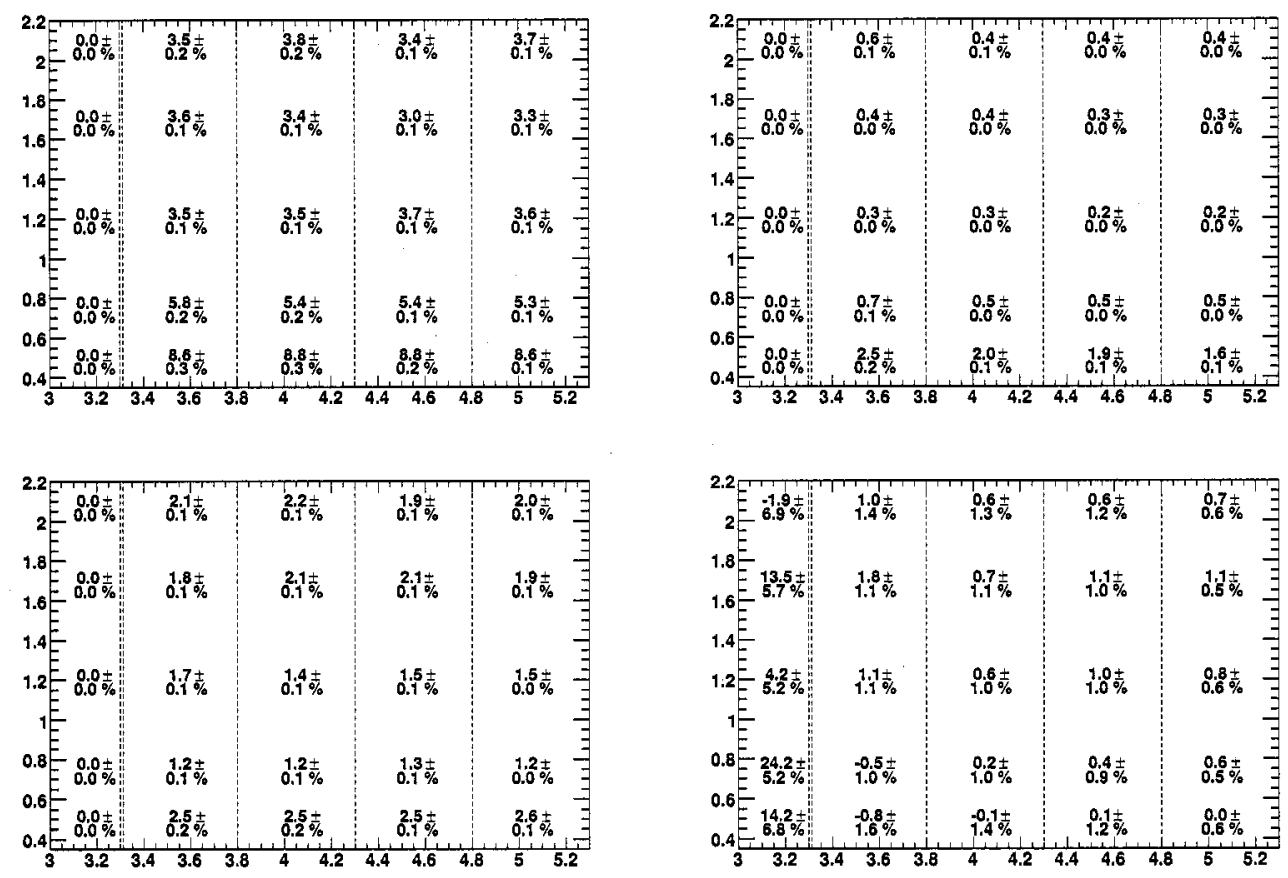

Figure 8.8: Efficiency in MC Truth in bins of $E^{*}$ and $\theta$ for events with 2 visible conversion tracks (top left), 1 visible conversion track (top right), no DCH or EMC activity (bottom left) and events with no reconstructed photon which do not fall into any of these categories (bottom right). The dotted line shows the lower limit of the $E^{*}$ acceptance. 
3-momentum minus the 3-momentum of the dimuon system) be in the range $0.25<$ $\theta_{M i s}<2.75$, and the total energy of the photons to be $E_{M i s}>1 \mathrm{GeV}$.

A possible problem is that the trigger and filter data path can introduce biases into the sample, for instance by making an event with a detected photon more likely to pass the trigger selections. We therefore only select events which are triggered by the dimuon system alone.

- L1: The L1 trigger is highly efficient and therefore unlikely to introduce large biases. However it is possible to remove this bias entirely by selecting events passed by trigger lines which rely on the muons alone. This is the case for the $2 \mathrm{M} \& \mathrm{~A}+$ line which requires $2 \mathrm{EMT}$ clusters and a long, high- $p_{T} \mathrm{~A}+$ track. This is automatically satisfied by the dimuon system as long as the clusters associated with the muons are well-separated: for the clusters to be distinct in L1, they must fall into non-contiguous $\phi$ sectors, which represent $1 / 20$ th of the $\mathrm{DCH}$ (see Section 3.8.3). This can be an issue for low-mass dimuon systems. However the occupancy of the M sectors in the EMT is available in analysis data (in the L1GltMini object), and this information can be used to select $\mu^{+} \mu^{-} \gamma$ events with the correct topologies.

- L3: The L3 physics trigger consists of orthogonal DCH and EMC selections, so any bias with respect to the photon is removed by requiring the event to pass L3Dch.

- BG Filter: The BG Filter presents a particular difficulty since 2-track events such as $\mu^{+} \mu^{-} \gamma$ are rejected by the main physics filters and have to be positively selected by dedicated filters, which have higher efficiency on events with a detected photon. To remove this inefficiency and bias, the dedicated filter BGFRadTwoProng was introduced for the data used in this analysis in order to select those events using only the muon system. The filter is described in Section 3.8.6; it provides an unbiased and efficient selection of the $\mu^{+} \mu^{-}(\gamma)$ events used in this study, as shown in Table 8.2. 


\subsubsection{Event Selection}

A set of selections, similar to the one used in Chapter 7, is applied to isolate a clean sample of $\mu^{+} \mu^{-} \gamma$ events. After selections are applied, we measure the proportion $\epsilon^{\prime}$ of events having a reconstructed photon at the expected energy and position in the EMC.

For the denominator sample, we require:

- The "General" selection from Section 7.1 (muons, missing momentum)

- The "2-prong" selection from Section 7.1.

- In addition, we require the data path to be unbiased by the following:

- At Level 1, pass 2M\&A+

- At Level 3, pass L30utDch

- At BGFilter, pass BGFRadTwoProng

For the nominator, we require the presence of a detected photon matching the missing momentum information by the following cuts:

- $\left|\theta_{\gamma}-\theta_{M i s}\right|<0.1$

- $\left|\phi_{\gamma}-\phi_{M i s}\right|<0.05$

- $\left|E_{\gamma}-E_{M i s}\right| / E_{M i s}<50 \%$,

where $E_{\gamma}, \theta_{\gamma}$ and $\phi_{\gamma}$ are respectively the energy, polar angle and azimuthal angle of the photon in the lab, and $\phi_{M i s}$ is the azimuthal angle of the missing momentum, again in the lab. We call $\epsilon^{\prime}$ the probability to find a photon passing the cuts above.

The selection is looser than that of Chapter 7 since the presence of the photon is not required. However as shown in Tables 8.3 and 8.3 backgrounds remain small. Furthermore they originate mostly from $\pi^{+} \pi^{-} \gamma$ events whose presence does not affect the results of this study.

The efficiency of the selection on $\mu^{+} \mu^{-} \gamma \mathrm{MC}$ is shown in Table 8.2. About 870,000 events pass the selection. There are significant biases associated with this selection. 
Table 8.2: Efficiency and photon-finding bias in $\mu^{+} \mu^{-} \gamma \mathrm{MC}$ for the selection of Section 8.2 .2

\begin{tabular}{lccc}
\hline \hline Cut & Cut Eff. & Photon Eff. & Bias \\
\hline 2M\&A+ & $95.37 \pm 0.11$ & $93.38 \pm 0.14$ & $0.84 \pm 0.18$ \\
Unbiased 2M\&A+ & $68.98 \pm 0.25$ & $92.84 \pm 0.17$ & $0.30 \pm 0.21$ \\
L3Dch & $68.06 \pm 0.25$ & $92.81 \pm 0.17$ & $0.27 \pm 0.21$ \\
2 Good Muons & $18.49 \pm 0.21$ & $93.08 \pm 0.32$ & $0.54 \pm 0.35$ \\
$E_{\text {Mis }}$ & $18.47 \pm 0.21$ & $92.99 \pm 0.33$ & $0.45 \pm 0.35$ \\
$\theta_{\text {Mis }}$ & $18.44 \pm 0.21$ & $93.09 \pm 0.32$ & $0.55 \pm 0.35$ \\
$M_{\text {Mis }}$ & $18.15 \pm 0.21$ & $93.34 \pm 0.32$ & $0.80 \pm 0.34$ \\
2 DCH Tracks & $17.08 \pm 0.21$ & $97.68 \pm 0.20$ & $5.14 \pm 0.23$ \\
EMC Veto & $13.59 \pm 0.19$ & $97.96 \pm 0.21$ & $5.42 \pm 0.24$ \\
Track/Cluster sep. & $13.55 \pm 0.19$ & $98.24 \pm 0.20$ & $5.70 \pm 0.23$ \\
ISR Photon & $13.45 \pm 0.19$ & $98.95 \pm 0.15$ & $6.41 \pm 0.19$ \\
BGFRad2Prong & $12.41 \pm 0.18$ & $98.69 \pm 0.18$ & $6.15 \pm 0.21$ \\
Tag 2 Tracks & $12.41 \pm 0.18$ & $99.17 \pm 0.14$ & $6.63 \pm 0.19$ \\
Tag Missing P4 & $12.39 \pm 0.18$ & $99.00 \pm 0.16$ & $6.46 \pm 0.20$ \\
Mini 2 Muons & $12.39 \pm 0.18$ & $98.81 \pm 0.17$ & $6.27 \pm 0.21$ \\
\hline \hline
\end{tabular}

Table 8.3: Efficiency for the selection of Section 8.2.2 on background MC samples.

\begin{tabular}{lcccc}
\hline \hline & $q \bar{q} \gamma(\%)$ & $e^{+} e^{-} \gamma(\%)$ & $\tau^{+} \tau^{-} \gamma(\%)$ & $\gamma \gamma(\%)$ \\
\hline Precuts & $(5.96 \pm 0.77) \times 10^{-4}$ & $\mathrm{n} / \mathrm{a}$ & $(1.31 \pm 1.31) \times 10^{-6}$ & $\mathrm{n} / \mathrm{a}$ \\
2 Good Muons & $(5.96 \pm 0.77) \times 10^{-4}$ & $\mathrm{n} / \mathrm{a}$ & $(1.31 \pm 1.31) \times 10^{-6}$ & $\mathrm{n} / \mathrm{a}$ \\
$E_{M i s}$ & $(4.77 \pm 0.69) \times 10^{-4}$ & $\mathrm{n} / \mathrm{a}$ & $(1.31 \pm 1.31) \times 10^{-6}$ & $\mathrm{n} / \mathrm{a}$ \\
$\theta_{\text {Mis }}$ & $(4.77 \pm 0.69) \times 10^{-4}$ & $\mathrm{n} / \mathrm{a}$ & $(1.31 \pm 1.31) \times 10^{-6}$ & $\mathrm{n} / \mathrm{a}$ \\
$M_{\text {Mis }}$ & $(4.77 \pm 0.69) \times 10^{-4}$ & $\mathrm{n} / \mathrm{a}$ & $(1.31 \pm 1.31) \times 10^{-6}$ & $\mathrm{n} / \mathrm{a}$ \\
2 DCH Tracks & $(4.77 \pm 0.69) \times 10^{-4}$ & $\mathrm{n} / \mathrm{a}$ & $(1.31 \pm 1.31) \times 10^{-6}$ & $\mathrm{n} / \mathrm{a}$ \\
EMC Veto & $(4.57 \pm 0.67) \times 10^{-4}$ & $\mathrm{n} / \mathrm{a}$ & $(1.31 \pm 1.31) \times 10^{-6}$ & $\mathrm{n} / \mathrm{a}$ \\
Track/Cluster sep. & $(4.57 \pm 0.67) \times 10^{-4}$ & $\mathrm{n} / \mathrm{a}$ & $(1.31 \pm 1.31) \times 10^{-6}$ & $\mathrm{n} / \mathrm{a}$ \\
Events in 250/fb & $11001.0 \pm 1622.0$ & $0.0 \pm 177.7$ & $4.2 \pm 4.2$ & $0.0 \pm 478.8$ \\
\hline \hline
\end{tabular}


Table 8.4: Efficiency for the selection of Section 8.2.2 on background MC samples.

\begin{tabular}{lccccc}
\hline \hline & $u d s(\%)$ & $c \bar{c}(\%)$ & $\tau^{+} \tau^{-}(\%)$ & $B^{0} \bar{B}^{0}(\%)$ & $\bar{B}^{+} B^{-}(\%)$ \\
\hline Precuts & $0.00 \pm 0.00$ & $(4.46 \pm 3.16) \times 10^{-9}$ & $(8.49 \pm 4.24) \times 10^{-9}$ & $\mathrm{n} / \mathrm{a}$ & $\mathrm{n} / \mathrm{a}$ \\
2 Good Muons & $0.00 \pm 0.00$ & $(4.46 \pm 3.16) \times 10^{-9}$ & $(8.49 \pm 4.24) \times 10^{-9}$ & $\mathrm{n} / \mathrm{a}$ & $\mathrm{n} / \mathrm{a}$ \\
$E_{\text {Mis }}$ & $0.00 \pm 0.00$ & $(4.46 \pm 3.16) \times 10^{-9}$ & $(8.49 \pm 4.24) \times 10^{-9}$ & $\mathrm{n} / \mathrm{a}$ & $\mathrm{n} / \mathrm{a}$ \\
$\theta_{\text {Mis }}$ & $0.00 \pm 0.00$ & $(4.46 \pm 3.16) \times 10^{-9}$ & $(8.49 \pm 4.24) \times 10^{-9}$ & $\mathrm{n} / \mathrm{a}$ & $\mathrm{n} / \mathrm{a}$ \\
$M_{\text {Mis }}$ & $0.00 \pm 0.00$ & $(4.46 \pm 3.16) \times 10^{-9}$ & $(4.24 \pm 3.00) \times 10^{-9}$ & $\mathrm{n} / \mathrm{a}$ & $\mathrm{n} / \mathrm{a}$ \\
2 DCH Tracks & $0.00 \pm 0.00$ & $(4.46 \pm 3.16) \times 10^{-9}$ & $(4.24 \pm 3.00) \times 10^{-9}$ & $\mathrm{n} / \mathrm{a}$ & $\mathrm{n} / \mathrm{a}$ \\
EMC Veto & $0.00 \pm 0.00$ & $(4.46 \pm 3.16) \times 10^{-9}$ & $(2.12 \pm 2.12) \times 10^{-9}$ & $\mathrm{n} / \mathrm{a}$ & $\mathrm{n} / \mathrm{a}$ \\
Track/Cluster sep. & $0.00 \pm 0.00$ & $(4.46 \pm 3.16) \times 10^{-9}$ & $(2.12 \pm 2.12) \times 10^{-9}$ & $\mathrm{n} / \mathrm{a}$ & $\mathrm{n} / \mathrm{a}$ \\
Events in 250/fb & $0.0 \pm 0.6$ & $1.5 \pm 1.0$ & $0.5 \pm 0.5$ & $0.0 \pm 0.2$ & $0.0 \pm 0.2$ \\
\hline \hline
\end{tabular}

This is easily explained by the fact that the 2-track requirement and EMC veto remove a large fraction of the events with bad photons. In particular events with visible conversion tracks are removed, as well as events where photon interaction cause deposition of neutral energy in the EMC outside the signal cone. In the categories of the previous sections, the only remaining samples are almost those corresponding to $\epsilon_{\gamma}$ and $\epsilon_{0}$.

Since the sample is unbiased with respect to the presence or absence of the photon, the selection efficiencies are identical in both categories. The "efficiency" $\epsilon$ ' is therefore actually a measure of $\epsilon_{0} /\left(\epsilon_{\gamma}+\epsilon_{0}\right)$, which can be used to calibrate $\epsilon_{0}$.

\subsubsection{MC/Data Comparisons}

Since the backgrounds are negligible, we can again compare data and $\mu^{+} \mu^{-} \gamma$ signal MC. Distributions for relevant quantities are shown in Figs. 8.9 and 8.10.

The passing cross-section in data, based on the integrated luminosity value, is calculated to be $6.01 \mathrm{pb}$. In MC, the production cross-section reported by the generator yields a passing cross-section of $5.92 \mathrm{pb}$, in good agreement with the above value. 

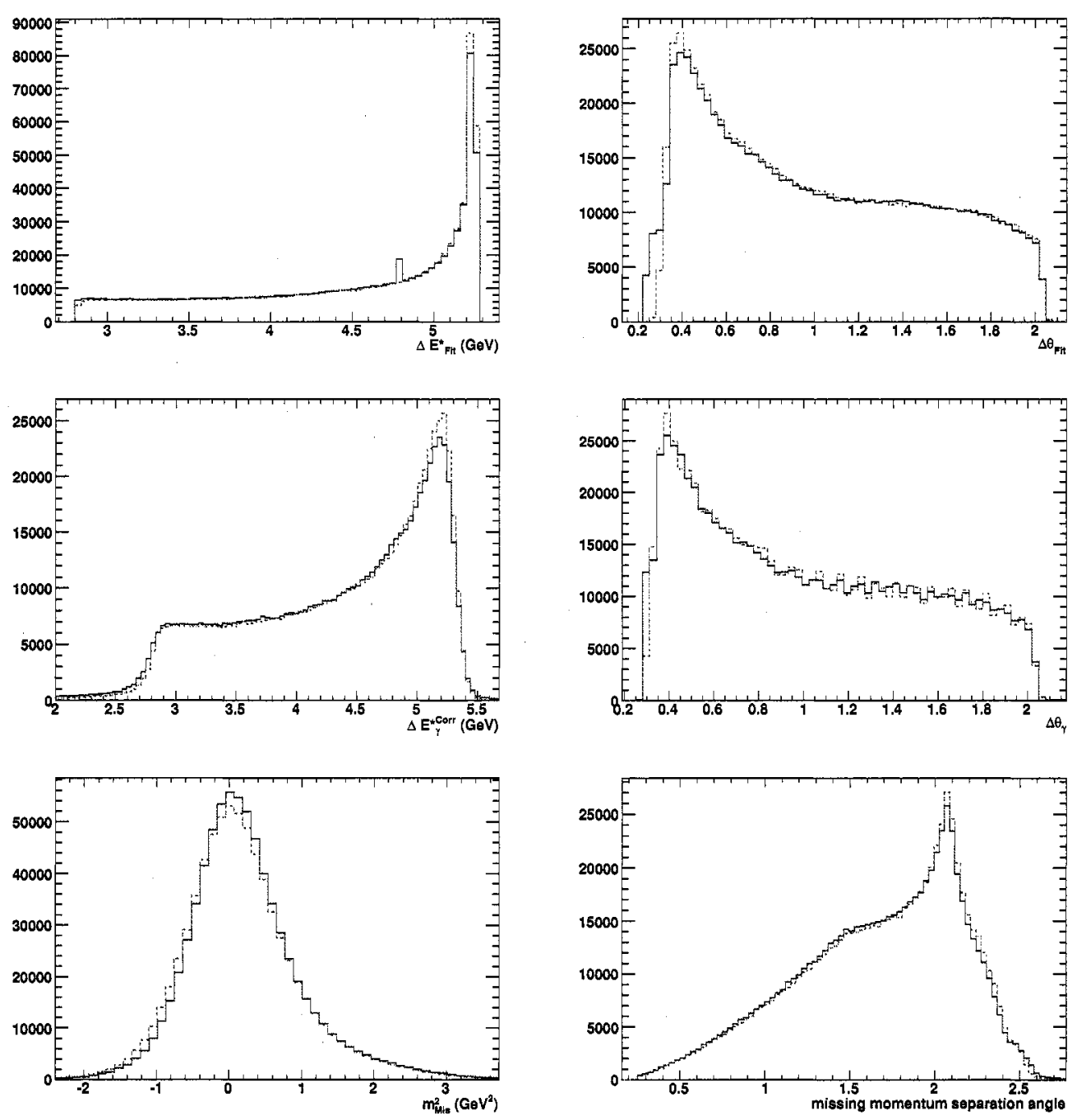

Figure 8.9: Comparison between data (solid blue line) and $\mu^{+} \mu^{-} \gamma \mathrm{MC}$ (dashed red line) quantities. From top to bottom, we have $E_{F i t}^{*}$ and $\theta_{F i t}, E_{\gamma}^{*}$ and $\theta_{F i t}, \alpha_{T \gamma}$ and $m_{M i s}^{2}$, where $\alpha_{T \gamma}$ is the angle between the missing momentum direction and the momentum direction of the closest muons. 

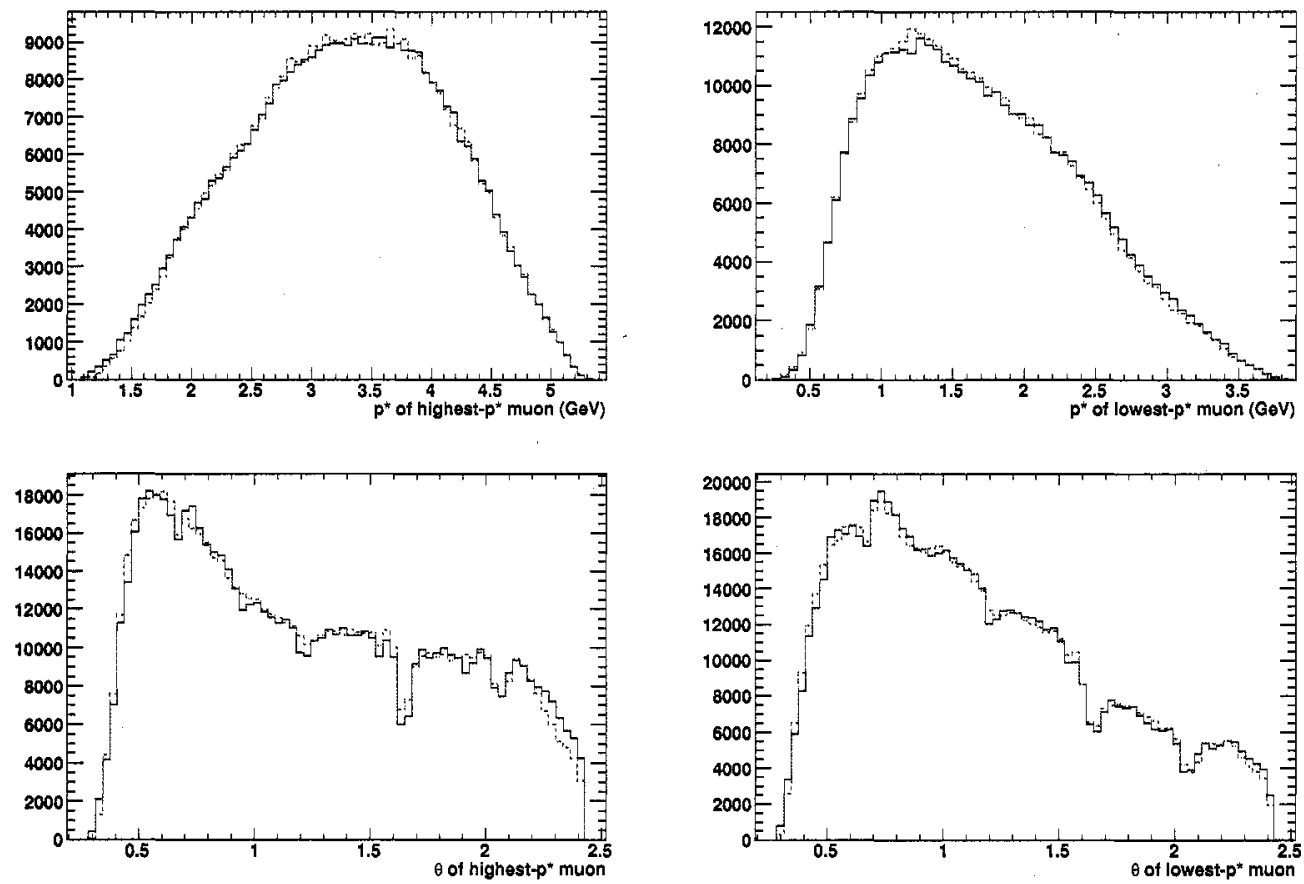

Figure 8.10: Comparison between data (solid blue line) and $\mu^{+} \mu^{-} \gamma \mathrm{MC}$ (dashed red line) quantities. From top to bottom, we have $p_{t}^{\mu 1}$ and $p_{t}^{\mu 2}$, and $\theta^{\mu 1}$ and $\theta^{\mu 2}$, where $\mu 1$ and $\mu 2$ are respectively the muons with highest and lowest $p^{*}$. 


\subsubsection{Correction to $\epsilon_{0}$}

We obtain from this study a measurement of $\epsilon^{\prime}=\epsilon_{0} /\left(\epsilon_{\gamma}+\epsilon_{0}\right)$ in both data and MC. We therefore determine a correction factor $c_{0}$ such that

$$
\left(\frac{\epsilon_{0}}{\epsilon_{\gamma}+\epsilon_{0}}\right)_{\text {Data }}=\frac{1}{c_{0}}\left(\frac{\epsilon_{0}}{\epsilon_{\gamma}+\epsilon_{0}}\right)_{M C}
$$

Results are shown in Fig 8.11.
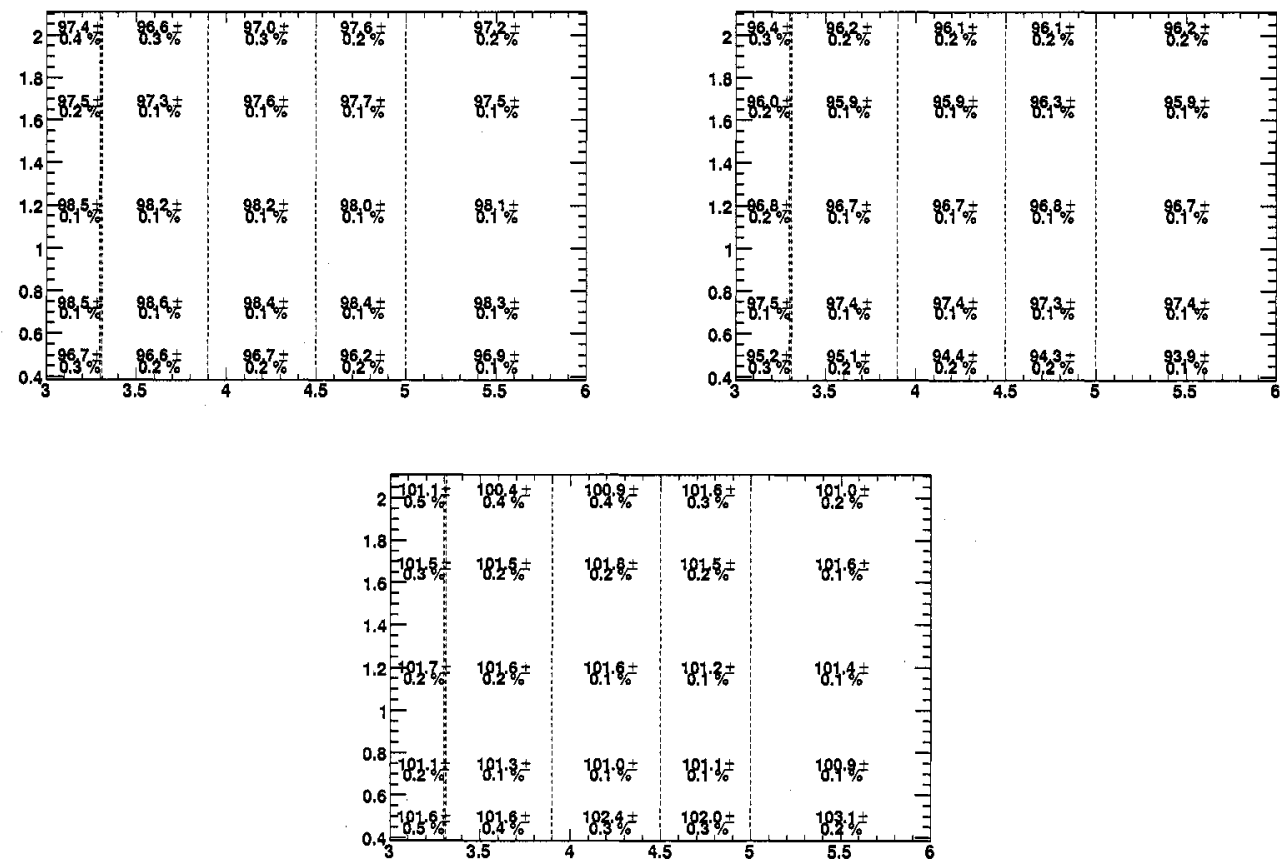

Figure 8.11: Values of $\epsilon^{\prime}$ in $\mathrm{MC}$ (left) and data (right), and their ratio $c_{0}$

The value of $\epsilon^{\prime}$ in MC should also be compatible with the values of $\epsilon_{0}$ and $\epsilon_{\gamma}$ from Section 8.1.4. Fig 8.12 shows the value of $\epsilon_{0}$ extracted from $\epsilon^{\prime}$ in MC using the value of $\epsilon_{\gamma}$ from the previous section, in good agreement with the previous value. 


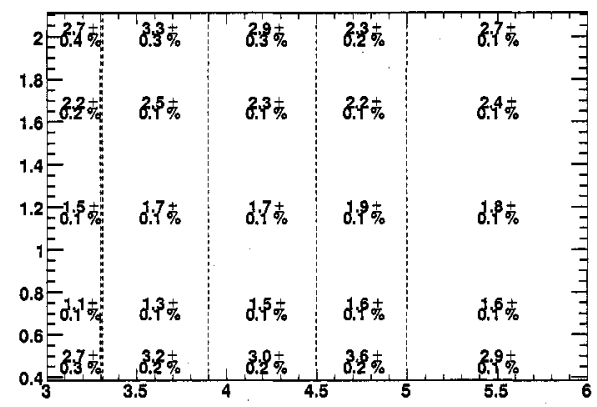

Figure 8.12: Value $\epsilon_{0}$ extracted from $\epsilon^{\prime}$. Good agreement is seen with the values from the bottom left plot of Fig. 8.8.

\subsection{Correction of $\epsilon_{2}$ using Conversion Events}

The goal of this section is to measure the value of $\epsilon_{2}$ from data in order to calibrate the $\mathrm{MC}$ value.

We again use $\mu^{+} \mu^{-} \gamma$ events and measure the ratio $N_{2} / N_{\gamma}$ of 2 -track conversion events to events with good photons. Introducing the selection efficiencies $\epsilon_{2}^{S e l}$ and $\epsilon_{\gamma}^{\text {Sel }}$ for conversion and good-photon events, we have

$$
\frac{\epsilon_{2}}{\epsilon_{0}}=\frac{N_{2}}{N_{\gamma}}=\frac{N_{2}^{S e l}}{N_{\gamma}^{S e l}} \frac{\epsilon_{\gamma}^{S e l}}{\epsilon_{2}^{S e l}}
$$

The advantage of measuring the ratio is that the part of the efficiency relative to the muon side cancels out, reducing the systematics.

\subsubsection{Reconstruction of Conversions}

In $B A B A R$ track three-momenta are reported at the point of closest approach (POCA) of the track to the $z$ axis. In the case where conversions occur far from the IP, the reported track three-momenta can be significantly different from their three-momenta at the conversion point. A vertexing procedure is therefore implemented to estimate the track momenta at the conversion point and improve the conversion mass resolution. 
Since conversions tracks have a very small opening angle at the conversion point, standard kinematic fitting procedures are not optimal and we instead use the method illustrated in Fig. 8.13: in the $x-y$ plane, we calculate the intersection of the track circles with the line segment connecting the centers of curvature. The track three momenta at these points are calculated by rotating the POCA three-momenta by the appropriate angle and their sum is used to reconstruct the conversion threemomentum. The conversion point is taken to be the center of the line segment linking the intersection points.

In order to estimate the quality of the reconstruction, the variables $d_{x y}$ and $d_{z}$ are defined as the separation between the intersection points in the $x-y$ plane and in $z$.

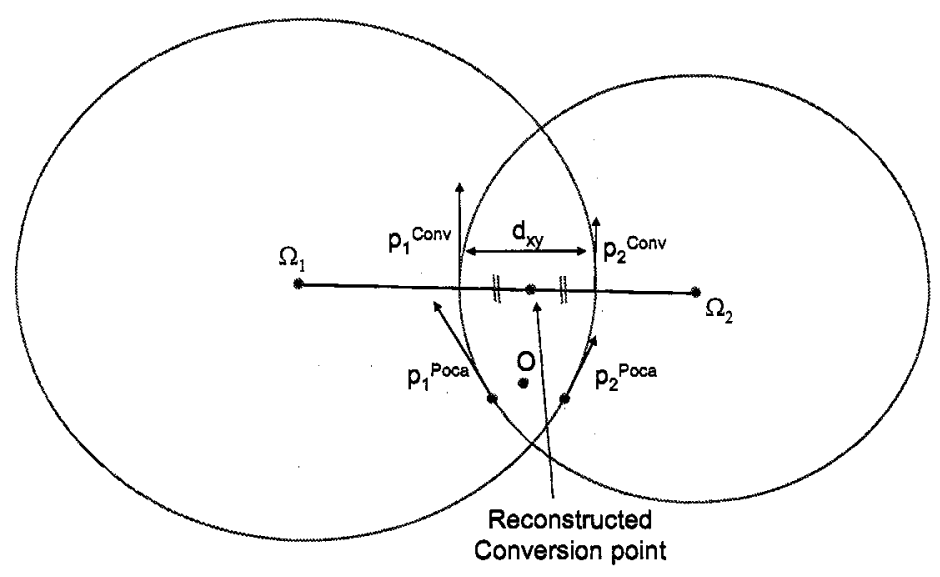

Figure 8.13: Diagram illustrating the method used to reconstruct conversions.

\subsubsection{Event Selection}

The selection is similar to the one of Section 8.2.2; we require: 
- the "General" selection of Section 7.1 (muons, missing momentum). In this case the "missing momentum" is calculated using only the muon tracks, and extra conversion tracks are excluded.

- 4 or 5 charged tracks

- the $\mathrm{CM}$ momenta of one muon to be greater than $2 \mathrm{GeV}$ and greater than $1 \mathrm{GeV}$ for the other. These are tag-level cuts intended to speed up processing without a significant efficiency hit.

- The conversion missing distance in the $x-y$ plane and along the $z$ axis to verify $d_{x y}<1.0 \mathrm{~cm},\left|d_{z}\right|<4.0 \mathrm{~cm}$ and the transverse radius of the conversion point to satisfy $\mid r_{x y}>2 \mathrm{~cm}$ to rejects conversion candidates occurring inside the beampipe.

- the difference in polar and azimuthal angles between the missing momentum and the detected photon or conversion to verify $|\Delta \theta|<0.2$ and $|\Delta \phi|<0.1$.

The efficiencies of these cuts are shown in Table 8.5 below. For conversions the denominator is composed of events with a converted "good photon" with 2 visible tracks from the conversion.

A factor of about $30 \%$ in the efficiency is in common for both this section and the non-converted photon study of Section 8.2.2, with an additional factor of about 0.6 in both cases for the non-common parts. The efficiency of the conversion channel is slightly lower than that of the non-converted photons, mainly due to BGFilter inefficiencies. Background efficiencies are shown in Tables 8.6 and 8.7. As in Section 8.2.2, all backgrounds are negligible except for $e^{+} e^{-} \rightarrow \pi^{+} \pi^{-} \gamma$.

\subsubsection{Data-MC comparison}

Since the backgrounds are negligible, we can again compare data and signal MC, $\mu^{+} \mu^{-} \gamma$ events with a converted photon selected in MC truth. Distributions for relevant quantities are shown in Figs. 8.14-8.16. 
Table 8.5: Efficiency for the selection of Section 8.3.2.

\begin{tabular}{lc}
\hline \hline & $\mu^{+} \mu^{-} \gamma$ \\
\hline Trigger & $81.01 \pm 0.29 \%$ \\
NTracks=4-5 & $74.65 \pm 0.33 \%$ \\
2 Good Muons & $23.25 \pm 0.32 \%$ \\
$E_{\text {Mis }}$ & $23.25 \pm 0.32 \%$ \\
$\theta_{\text {Mis }}$ & $23.24 \pm 0.32 \%$ \\
$M_{\text {Mis }}$ & $19.64 \pm 0.30 \%$ \\
Conv $\Delta \theta, \Delta \phi$ & $19.00 \pm 0.29 \%$ \\
Conv. Quality Cuts & $18.80 \pm 0.29 \%$ \\
Tag ECM Cut & $18.80 \pm 0.29 \%$ \\
Beta Conv. Sel. & $18.80 \pm 0.29 \%$ \\
Beta Rad. Bhabha Veto & $18.80 \pm 0.29 \%$ \\
Beta $\gamma \gamma$ Veto & $18.80 \pm 0.29 \%$ \\
Events in 208 fb & $30011.2 \pm 517.4$ \\
\hline \hline
\end{tabular}

Table 8.6: Efficiency for the event selection of Section 8.3.2.

\begin{tabular}{lcccc}
\hline \hline & \multicolumn{1}{c}{$\bar{q} \gamma$} & $e^{-} e^{-\gamma}$ & $\tau^{+} \tau^{-} \gamma$ & $\gamma \gamma$ \\
\hline Precuts & $1.42 \pm 0.03 \%$ & $(3.23 \pm 0.10) \times 10^{-3}$ & $1.69 \pm 0.05 \%$ & $(3.86 \pm 0.18) \times 10^{-4}$ \\
2 Good Muons & $(5.62 \pm 2.12) \times 10^{-5}$ & $0.00 \pm 0.00 \%$ & $(1.82 \pm 0.52) \times 10^{-4}$ & $0.00 \pm 0.00 \%$ \\
$E_{M i s}$ & $(5.62 \pm 2.12) \times 10^{-5}$ & $0.00 \pm 0.00 \%$ & $(1.82 \pm 0.52) \times 10^{-4}$ & $0.00 \pm 0.00 \%$ \\
$\theta_{\text {Mis }}$ & $(5.62 \pm 2.12) \times 10^{-5}$ & $0.00 \pm 0.00 \%$ & $(1.21 \pm 0.43) \times 10^{-4}$ & $0.00 \pm 0.00 \%$ \\
$M_{\text {Mis }}$ & $(4.82 \pm 1.97) \times 10^{-5}$ & $0.00 \pm 0.00 \%$ & $0.00 \pm 0.00 \%$ & $0.00 \pm 0.00 \%$ \\
Conv $\Delta \theta, \Delta \phi$ & $(4.82 \pm 1.97) \times 10^{-5}$ & $0.00 \pm 0.00 \%$ & $0.00 \pm 0.00 \%$ & $0.00 \pm 0.00 \%$ \\
Conv. Quality Cuts & $(4.82 \pm 1.97) \times 10^{-5}$ & $0.00 \pm 0.00 \%$ & $0.00 \pm 0.00 \%$ & $0.00 \pm 0.00 \%$ \\
Events in 208 fb & $966.5 \pm 394.6$ & $0.0 \pm 650.2$ & $0.0 \pm 40.3$ & $0.0 \pm 508.8$ \\
\hline \hline
\end{tabular}

Table 8.7: Efficiency for the event selection of Section 8.3.2.

\begin{tabular}{lcccc}
\hline \hline & uds & $c \bar{c}$ & $\tau^{+} \tau^{-}$ & $B^{0} \bar{B}^{0}$ \\
\hline Precuts & $(1.59 \pm 0.01) \times 10^{-4}$ & $(2.13 \pm 0.03) \times 10^{-5}$ & $(2.37 \pm 0.01) \times 10^{-4}$ & $\mathrm{n} / \mathrm{a}$ \\
2 Good Muons & $(2.12 \pm 1.23) \times 10^{-8}$ & $(2.44 \pm 1.00) \times 10^{-8}$ & $(2.07 \pm 0.11) \times 10^{-6}$ & $\mathrm{n} / \mathrm{a}$ \\
$E_{\text {Mis }}$ & $(2.12 \pm 1.23) \times 10^{-8}$ & $(2.44 \pm 1.00) \times 10^{-8}$ & $(2.07 \pm 0.11) \times 10^{-6}$ & $\mathrm{n} / \mathrm{a}$ \\
$\theta_{\text {Mis }}$ & $(1.42 \pm 1.00) \times 10^{-8}$ & $(2.44 \pm 1.00) \times 10^{-8}$ & $(1.49 \pm 0.10) \times 10^{-6}$ & $\mathrm{n} / \mathrm{a}$ \\
$M_{\text {Mis }}$ & $0.00 \pm 0.00 \%$ & $(1.63 \pm 0.81) \times 10^{-8}$ & $0.00 \pm 0.00 \%$ & $\mathrm{n} / \mathrm{a}$ \\
Conv $\Delta \theta, \Delta \phi$ & $0.00 \pm 0.00 \%$ & $(1.63 \pm 0.81) \times 10^{-8}$ & $0.00 \pm 0.00 \%$ & $\mathrm{n} / \mathrm{a}$ \\
Conv. Quality Cuts & $0.00 \pm 0.00 \%$ & $(1.63 \pm 0.81) \times 10^{-8}$ & $0.00 \pm 0.00 \%$ & $\mathrm{n} / \mathrm{a}$ \\
Events in 208fb & $0.0 \pm 3.1$ & $4.4 \pm 2.2$ & $0.0 \pm 1.1$ & $0.0 \pm 0.2$ \\
\hline \hline
\end{tabular}



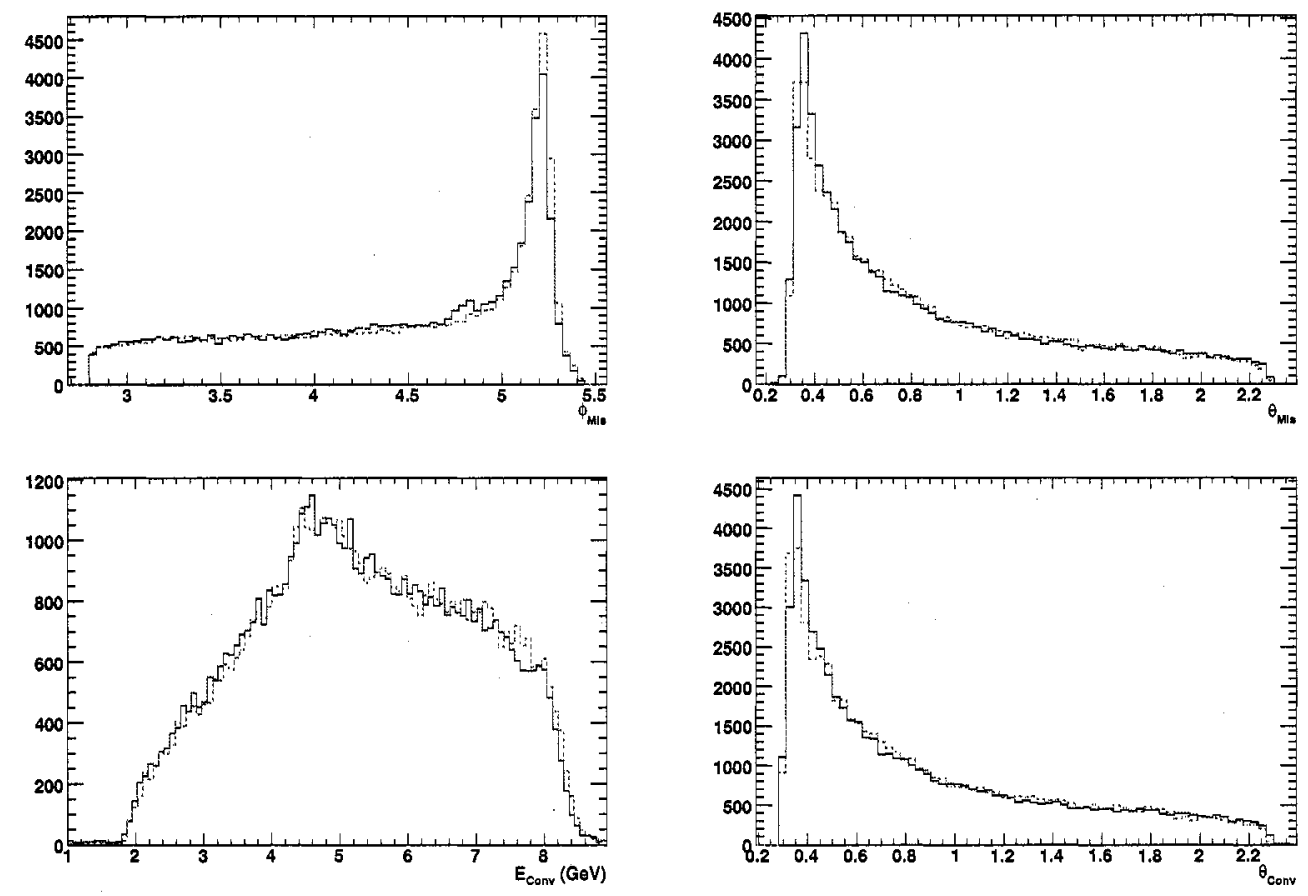

Figure 8.14: Comparison between data (solid blue line) and $\mu^{+} \mu^{-} \gamma \mathrm{MC}$ (dashed red line) for quantities. From top to bottom, we have $E_{F i t}^{*}$ and $\theta_{F i t}, E_{C o n v}^{*}$ and $\theta_{C o n v}$.
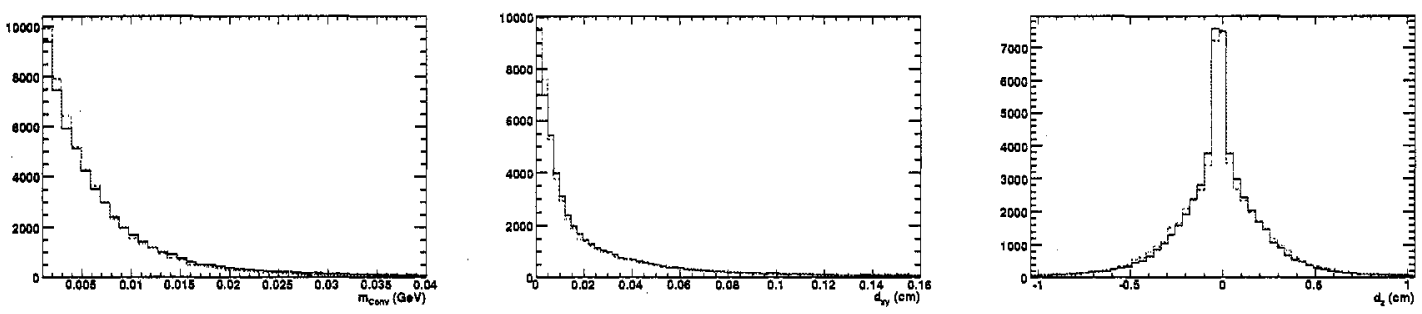

Figure 8.15: Comparison between data (solid blue line) and $\mu^{+} \mu^{-} \gamma \mathrm{MC}$ (dashed red line) for quantities. From left to right, we have $m_{C o n v}$ and $\delta_{x y}^{C o n v}$, and $\delta_{Z}^{\text {Conv }}$. 

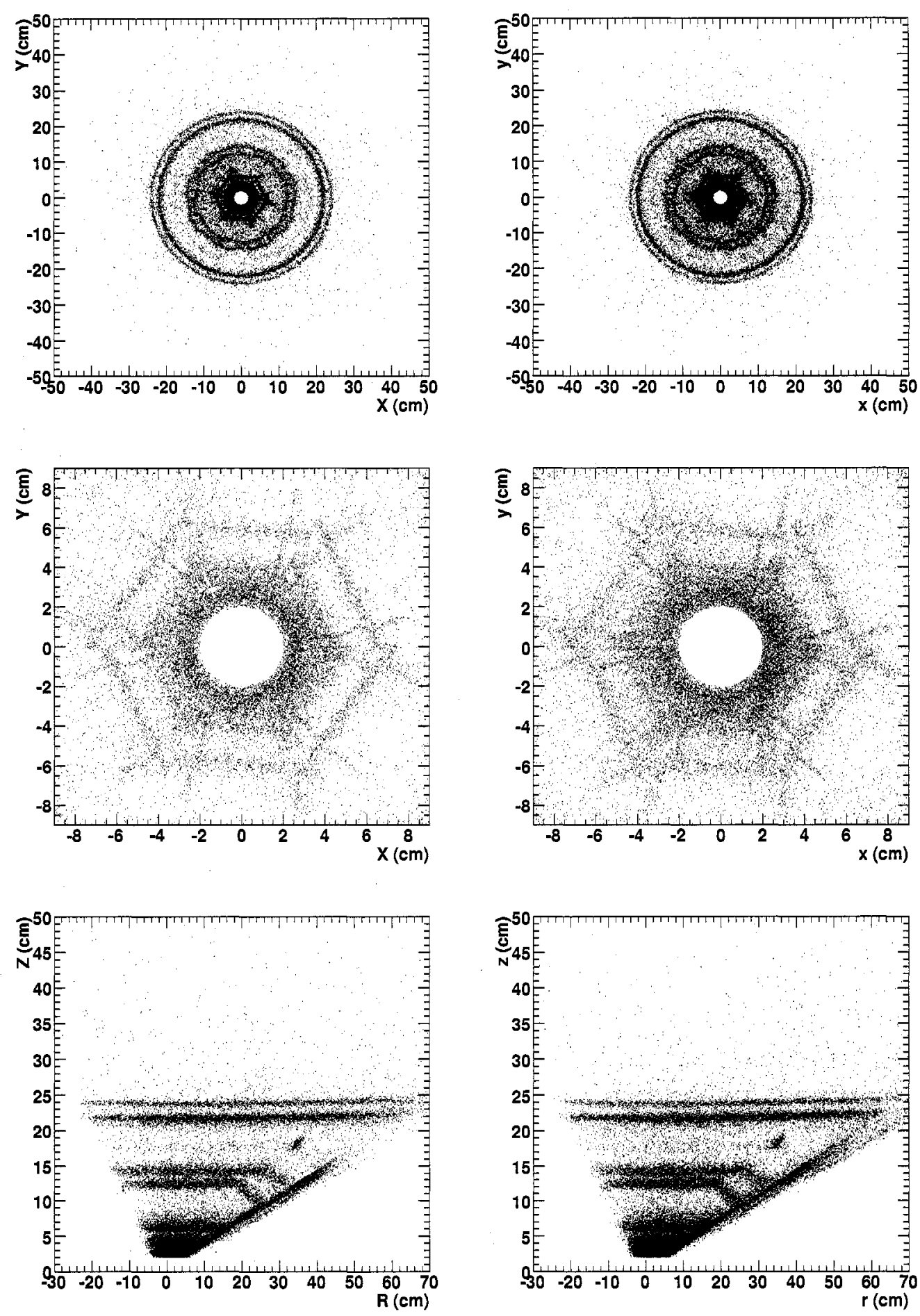

Figure 8.16: Comparison of conversion positions in the $x-y$ plane (top row, zoom on middle row) and $r-\phi$ plane (bottom row) in $\mu^{+} \mu^{-} \gamma \mathrm{MC}$ (left column) and data (right column). 
The passing cross-section in data, based on the integrated luminosity value, is calculated to be $0.281 \mathrm{pb}$. In MC, based on the production cross-section reported by the generator we have $0.283 \mathrm{pb}$, in good agreement.

\subsubsection{Correction}

Values of the selection efficiencies for photons and conversions and of $N_{2}^{\text {Sel }} / N_{\gamma}^{\text {Sel }}$ are obtained in data and MC.

From these values one can extract a correction factor $c_{12}$ on the conversion rate defined as:

$$
\left(\frac{\epsilon_{2}}{\epsilon_{0}}\right)_{\text {Data }}=c_{12}\left(\frac{\epsilon_{2}}{\epsilon_{0}}\right)_{M C} .
$$

The reconstruction efficiencies are not needed since they drop out in the double ratio. Results are shown in Fig. 8.17.

As a cross-check, we can verify that the value of $\epsilon_{2} / \epsilon_{0}$ obtained from MC is consistent with the one from the previous section. Fig. 8.18 shows the reconstruction efficiencies, the efficiency-corrected values for $N_{2} / N_{\gamma}$ and the value of $\epsilon_{2}$ obtained by multiplying this last quantity with the values of $\epsilon_{0}$ from the previous section. The value of $\epsilon_{2}$ obtained here is in good agreement with the one from Fig. 8.8.

\subsection{Corrected photon efficiency}

In section 8.1 we have categorized the possible outcomes so that

$$
\epsilon_{\gamma}+\epsilon_{0}+\epsilon_{1}+\epsilon_{2}+\epsilon_{X}=1
$$

with $\epsilon_{X}$ chosen so that the sum ends up to be 1 .

In the preceding two sections we have described methods to evaluate several of these terms from data. In section 8.2 we have measured a correction $c_{0}$ for the ratio $\epsilon_{\gamma} /\left(\epsilon_{\gamma}+\epsilon_{0}\right)$, such that 

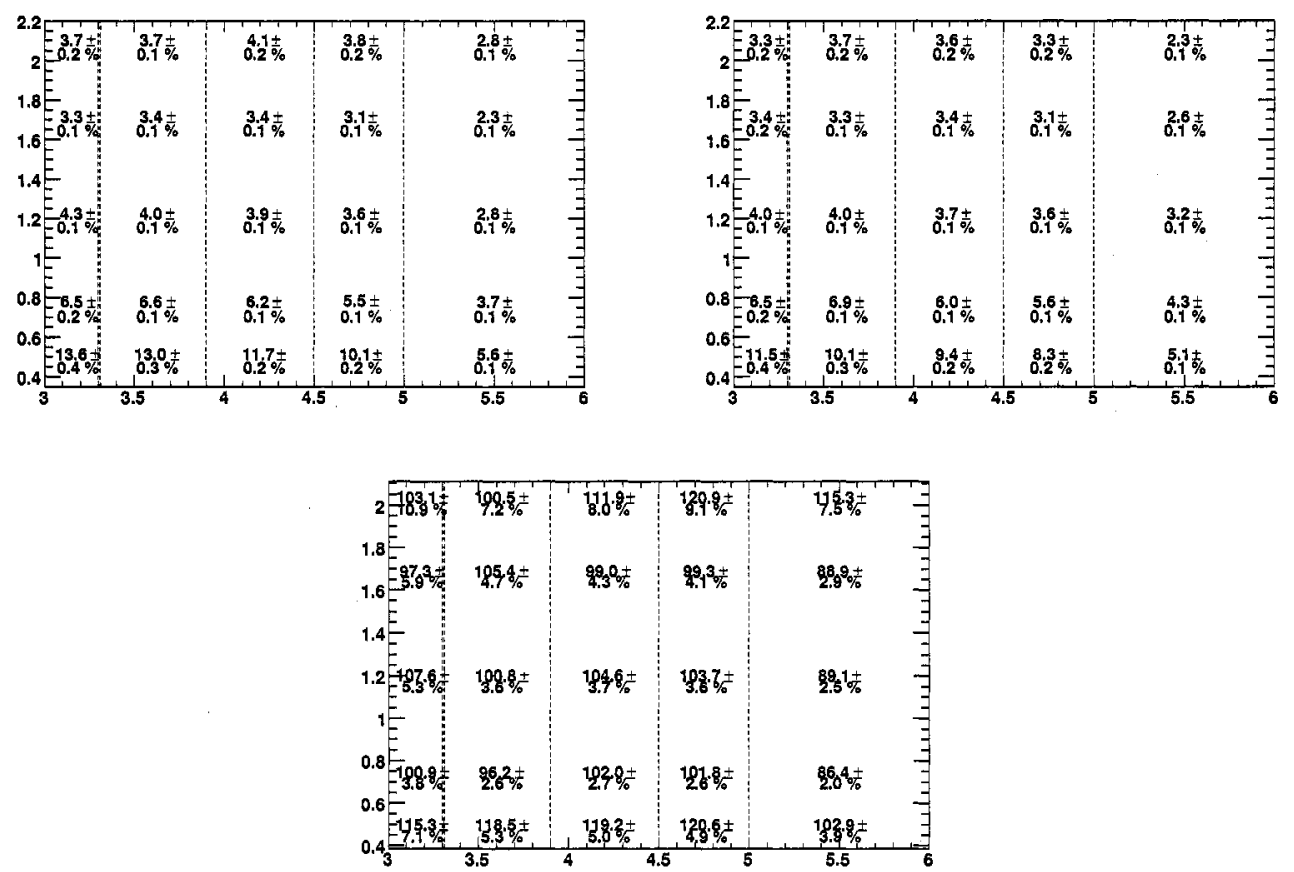

Figure 8.17: Histograms for $N_{2}^{\text {Sel }} / N_{\gamma}^{\text {Sel }}$ in MC (left) and data (right) and the ratio $c_{12}$ of data to MC. 


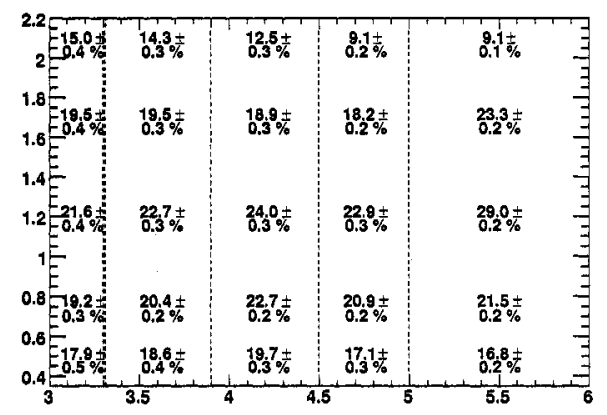

\begin{tabular}{|c|c|c|c|c|}
\hline & 13.8\% & $\begin{array}{l}124 \pm \\
1.4 \%\end{array}$ & $\begin{array}{l}9.9+9+ \\
1.2 \%\end{array}$ & $\begin{array}{l}4.8 \mathrm{~s} \\
0.5 \%\end{array}$ \\
\hline & $17.9 \pm$ & $17.9 \pm$ & $\begin{array}{l}19.6 \pm \pm \\
1.4 \%\end{array}$ & $\begin{array}{l}16.5 \pm \\
0.9 \% \%\end{array}$ \\
\hline & $\begin{array}{l}24.3 \pm \\
.4 \% \%\end{array}$ & $\begin{array}{l}\frac{229 \pm}{1.4 \%} \\
1.9 \%\end{array}$ & $\begin{array}{l}23.9 .1 \\
1.4 \%\end{array}$ & $\begin{array}{l}23.7 \% \\
1.1 .7 \%\end{array}$ \\
\hline & $\begin{array}{l}24.00 \% \\
.00 \%\end{array}$ & $\begin{array}{l}22.8 \pm \pm \\
17,0 \%\end{array}$ & $\begin{array}{l}22.4 \pm t \\
1.0 \% \%\end{array}$ & $\begin{array}{l}16.9 .9 \\
0.6 \% \%\end{array}$ \\
\hline & $\begin{array}{l}18.6 \% \\
1.1 . \% \%\end{array}$ & $\begin{array}{l}18,7 \neq \neq \\
1 ., 0 \%\end{array}$ & $\begin{array}{l}18.5 \pm \pm \\
0.9 \% \%\end{array}$ & $11.9 \%$ \\
\hline
\end{tabular}

\begin{tabular}{|c|c|c|c|c|}
\hline 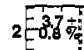 & T.5\% & $3.5 \%$ & $2.7 \%$ & $3.5 \%$ \\
\hline & $3.3 \%$ & $3.3 \%$ & Z.Z. & $3.5 \%$ \\
\hline 9.4. & $\begin{array}{l}3.5 \% \\
0.2 \%\end{array}$ & $3.8 \%$ & $3.2 \%$ & $8.2 \%$ \\
\hline $4 \pm$ & 5.5.5 & 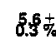 & 2.8. & $8.2 \%$ \\
\hline & $71 \pm$ & 7.5 .5 & $8,8 \%$ & $8.5 \%$ \\
\hline
\end{tabular}

Figure 8.18: Histograms for the reconstruction efficiencies of events with a photon (top) and a conversion (middle), and the extracted value $\epsilon_{2}$ (bottom). 


$$
\left(\frac{\epsilon_{\gamma}}{\epsilon_{\gamma}+\epsilon_{0}}\right)_{\text {Data }}=\frac{1}{c_{0}}\left(\frac{\epsilon_{\gamma}}{\epsilon_{\gamma}+\epsilon_{0}}\right)_{M C}
$$

and in section 8.3 we have determined a correction $c_{12}$ on the ratio $\epsilon_{2} / \epsilon_{0}$. We assume that this correction applies to all corrections regardless of their observed track multiplicity, and can also be applied to the $\epsilon_{1}$ term. We therefore group together $\epsilon_{1}$ and $\epsilon_{2}$ as $\epsilon_{12}$ and write

$$
\left(\frac{\epsilon_{12}}{\epsilon_{\gamma}}\right)_{\text {Data }}=c_{12}\left(\frac{\epsilon_{12}}{\epsilon_{\gamma}}\right)_{M C}
$$

We are left with three independent unknowns, the values of $\epsilon_{\gamma}, \epsilon_{0}$ and $\epsilon_{12}$ in data, and only two equations given by the expressions of $c_{0}$ and $c_{12}$. We therefore make the further assumption that $\epsilon_{X}$ is the same in data as in MC. This is justified by the small magnitude of this term.

The equations can now be solved for $\epsilon_{\gamma}$ and we get

$$
\epsilon_{\gamma}^{D a t a}=\epsilon_{\gamma}^{M C} c_{\gamma}
$$

with

$$
c_{\gamma}=\frac{\epsilon_{\gamma}^{M C}+\epsilon_{0}^{M C}+\epsilon_{12}^{M C}}{c_{0}\left(\epsilon_{\gamma}^{M C}+\epsilon_{0}^{M C}\right)+c_{12} \epsilon_{12}^{M C}}
$$

Results for $c_{\gamma}$ and $\epsilon_{\gamma}^{\text {Data }}$ are shown in Fig 8.19. 

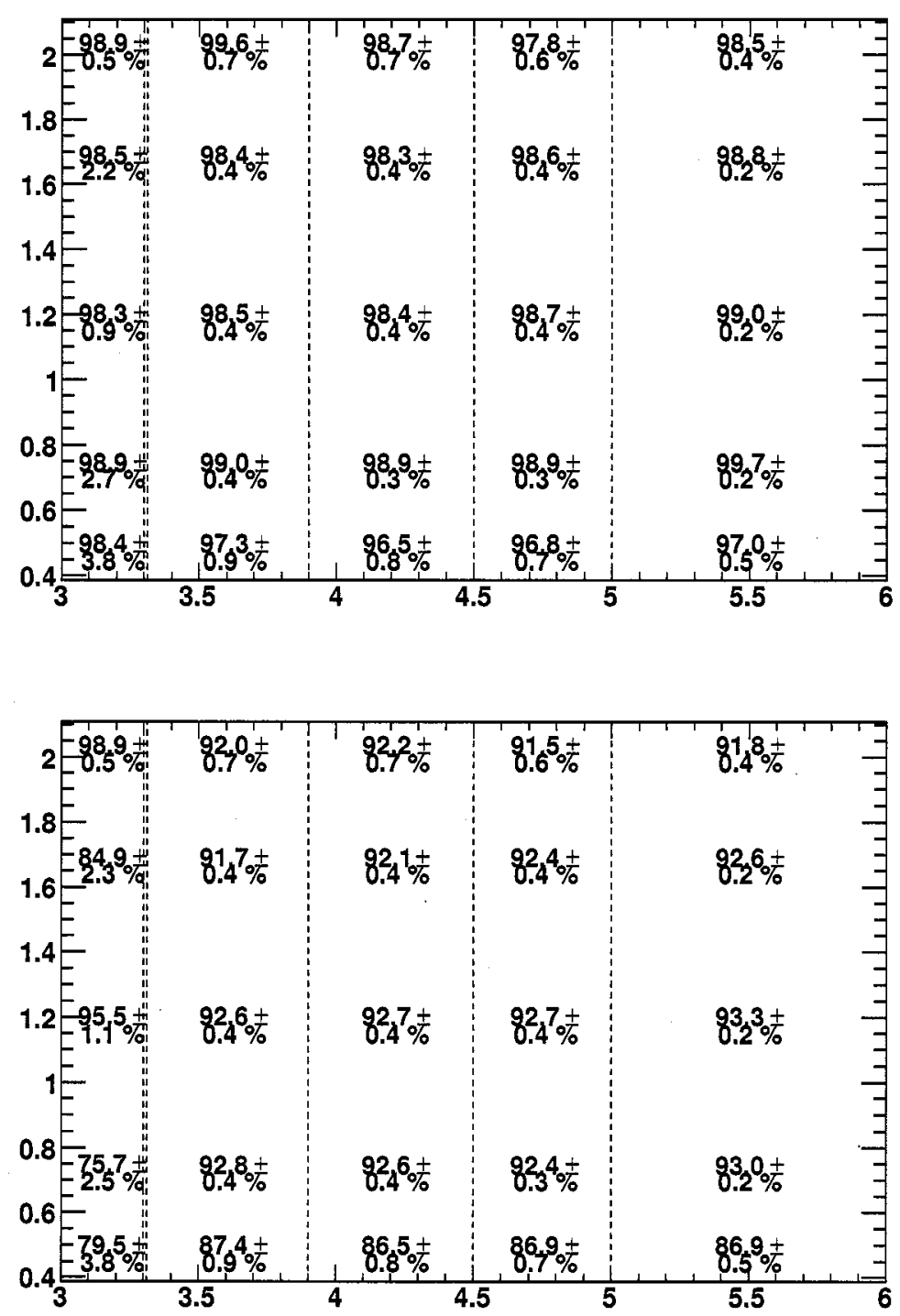

Figure 8.19: Histograms for the correction factor $c_{\gamma}$ applied on the photon efficiency (left) and the corrected efficiency (right). 


\section{Chapter 9}

\section{Cross-checks of Background Production and Rejection Rates}

\subsection{Calibration of the Production Rates of Reso- nances Decaying into Photons}

As described in Section 6.1, photons from decays of $\pi^{0}$ 's, and to a lesser extent other mesons, form the majority of background at high $s^{\prime}$. Even after applying the rejection techniques described in the previous sections significant backgrounds remain, with $S / B \approx 2 / 1$ at the upper end of the $s^{\prime}$ spectrum. This residual background needs to be subtracted, and in order to limit uncertainties several corrections are applied. The most problematic is the overall normalization: a large fraction of the background consists of high-momentum $\pi^{0}$ 's produced in $q \bar{q}$ fragmentation, the rate of which is not expected to be well reproduced in simulation. In this section we cross-check the production rate of $\pi^{0}$ 's and other mesons using reconstructed $h \rightarrow n \gamma(n=2,3)$ combinations.

\subsubsection{Technique}

In Sections 6.2 and 6.3, $\gamma \gamma$ and $\pi^{0} \gamma$ combinations were reconstructed in order to identify $h \rightarrow \gamma \gamma$ and $h \rightarrow \pi^{0} \gamma$ decays, where $h=\pi^{0}, \eta, \eta^{\prime}$ and $\omega$. A veto is then 
applied in the corresponding mass ranges, given in Table 6.2. The veto misses two other categories of backgrounds: cases where a soft photon is not reconstructed, and cases where two photons are merged.

In the case of $h \rightarrow \gamma \gamma$ the outcome mainly depends on acceptance effects and on the value of the decay (or helicity) angle, with soft photons for values near 0 or $\pi$, merged photons for values near $\pi / 2$ and reconstructible $\gamma \gamma$ combinations for intermediate values; Since both effects are expected to be well simulated to first approximation, we can use the rate of vetoed $h \rightarrow \gamma \gamma$ to normalize those that pass the cuts.

For each $h$ we therefore plot invariant mass histograms for $m_{\gamma \gamma(\gamma)}$ in data and in the sum of all $\mathrm{MC}$ samples for the corresponding mass range. The data and MC histograms are then fitted to a sum of a peaking component and a second order polynomial. A single Gaussian is used for the peaking component, but if the $\chi^{2} / n_{\text {dof }}$ of the fit is greater than 1.5 a double Gaussian is used instead.

As described below, for $\pi^{0}$ and $\eta$ decays we bin the corrections in both the CM energy $E^{*}$ and the polar angle $\theta$ of the resonance, which leads to a large number of fits which is impractical to deal with "by hand". An automatic procedure to fit a generic histogram is therefore used. We start by fitting a single Gaussian plus quadratic background, using the histogram mean and RMS as initial values for the Gaussian mean and width and the contents of the leftmost, rightmost and maximum bin to initialize the normalization of Gaussian and the polynomial background. The values obtained from this fit are then copied over as the narrow component of the double Gaussian shape, while the wide component is taken to have initially a width 3 times higher and a $10 \%$ relative normalization. With this procedure we get convergent first for all bins in the study. Two corrections are obtained from the comparison of these fits:

- A peak correction, defined as the ratio of the integrals of the peaking component in data and MC.

- A combinatorics correction, defined as the ratio of the integrals of the polynomial component in the $m_{h}$ veto regions defined in Table 6.2. 
The peak correction is the scale factor that should be applied to the $h \rightarrow n \gamma$ events in $\mathrm{MC}$ before subtracting from data. The combinatorics correction is used to correct the efficiency of the $h \rightarrow n \gamma$ veto on the signal sample: in signal MC there is no peak for $h \rightarrow n \gamma$ and the amount of signal rejected by the veto is determined by the amount of combinatoric background in the veto region. If the EMC cluster multiplicity is incorrectly simulated in MC (for example if beam background is not correctly reproduced), the signal inefficiency is not correctly estimated. When determining signal efficiency from MC, we therefore scale the $h$ veto inefficiency up by the magnitude of the combinatorics correction.

To obtain the uncertainties on the correction values, we proceed as follows:

- Calculate the error on the integral of the polynomial by varying each polynomial coefficient by the fitting uncertainty given by MINUIT, measuring the corresponding variation in the integral value and adding the variations for each coefficient in quadrature.

- Perform the same calculation for full double Gaussian + polynomial PDF, varying all parameters.

- Calculate the error in the peak alone by subtracting the integral of the polynomial component from that of the full PDF.

- Calculate the error on the corrections by the standard formula for the error of a ratio.

\subsection{2 $\pi^{0} \rightarrow \gamma \gamma$}

Since $\pi^{0}$ decays represent a large fraction of the backgrounds, we divide the correction. in bins of $\theta_{\pi^{0}}$ and $E_{\pi^{0}}^{*}$ for more precision. We use 4 bins in $\theta_{\pi^{0}}$, with boundaries at $0.350,0.475,1.0,1.57$ and 2.25 , and 4 bins in $E_{\pi^{0}}^{*}$, with boundaries at 3.3, 3.8, 4.3, 4.8 and $5.3 \mathrm{GeV}$. Fig 9.1 shows the comparison between data and stacked MC samples, both scaled to $208.4 \mathrm{fb}^{-1}$.

Apart from minor differences in the shape of the $\pi^{0}$ peak which are of no importance for this analysis (we only care about the fraction of events inside the mass 

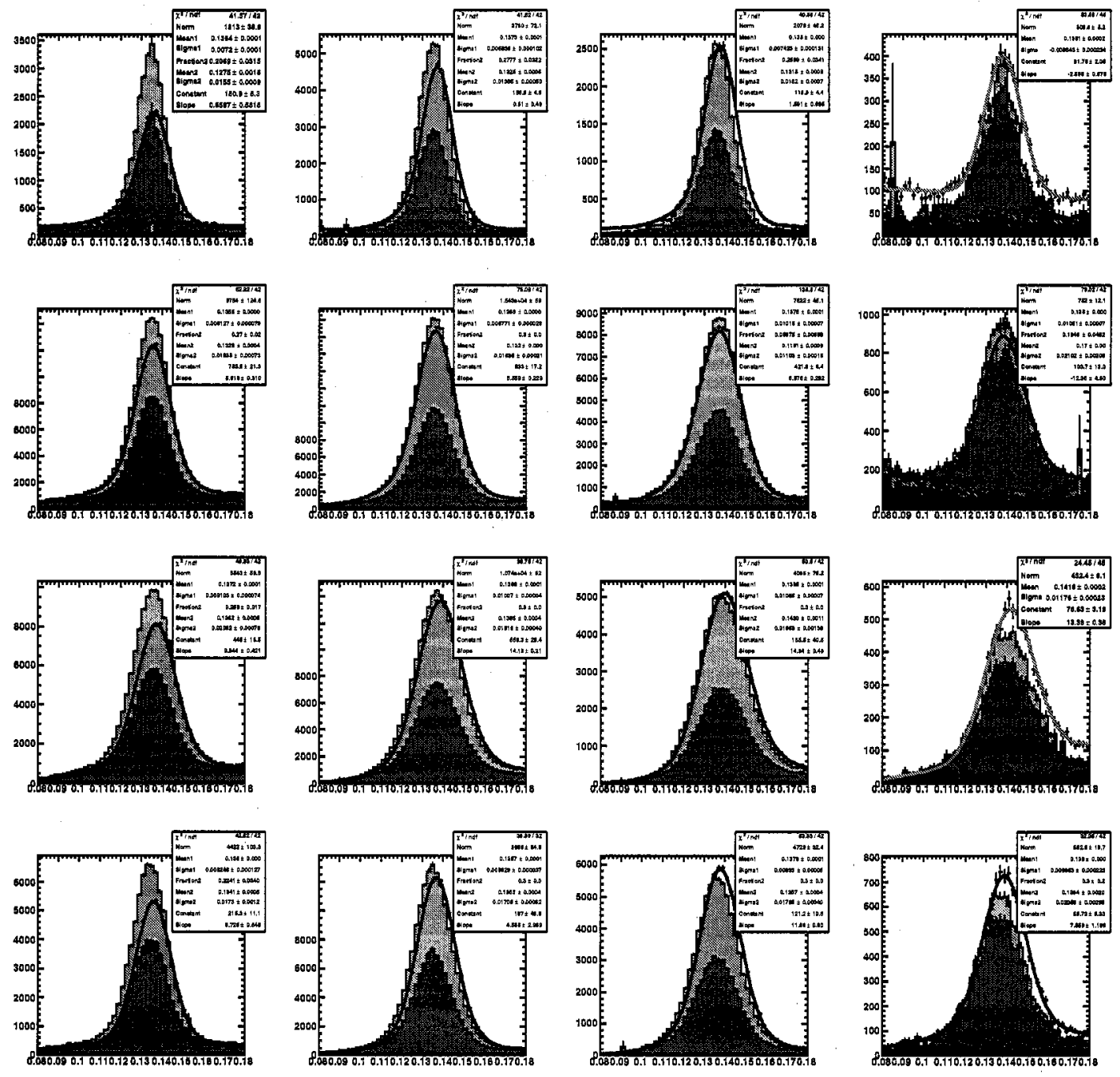

Figure 9.1: Distributions of $m_{\pi^{0}}$ in data (points and blue line) and MC (stacked histograms) in bins of $E_{\pi^{0}}^{*}$ (increasing from left to right) and $\theta_{\pi^{0}}$ (increasing from top to bottom). All histograms are scaled to $250 \mathrm{fb}^{-1}$. The color-coding of the MC samples is the same as for spectrum plots such as Fig. 5.3, with light blue for $\tau^{+} \tau^{-}$ $\mathrm{MC}$ and red for uds $\mathrm{MC}$. 
window) the agreement between $\mathrm{MC}$ and data is rather good, except in the backward region at low energy. Figures 9.2 and 9.3 show the fits to $\mathrm{MC}$ and data respectively.
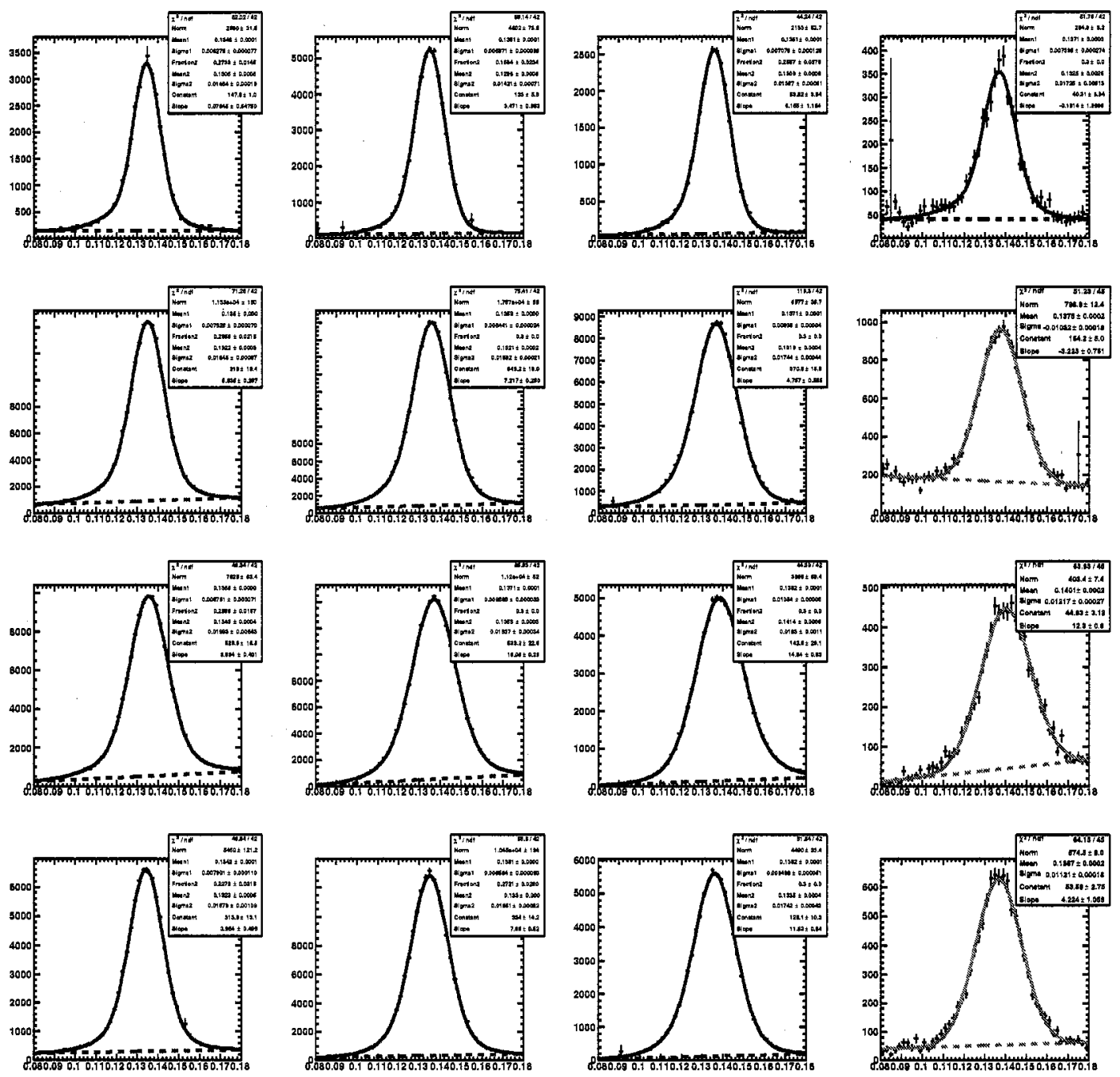

Figure 9.2: Fits of the $m_{\pi^{0}}$ distribution for the sum of all $\mathrm{MC}$ samples, in bins of $E_{\pi^{0}}^{*}$ (increasing from left to right) and $\theta_{\pi^{0}}$ (increasing from top to bottom). Histograms are scaled to $250 \mathrm{fb}^{-1}$.

The double Gaussian plus quadratic function form that we use describes the $\mathrm{MC}$ and data well. The peak and combinatorics corrections are calculated as described in the previous section, and the results are shown in Fig. 9.4. 

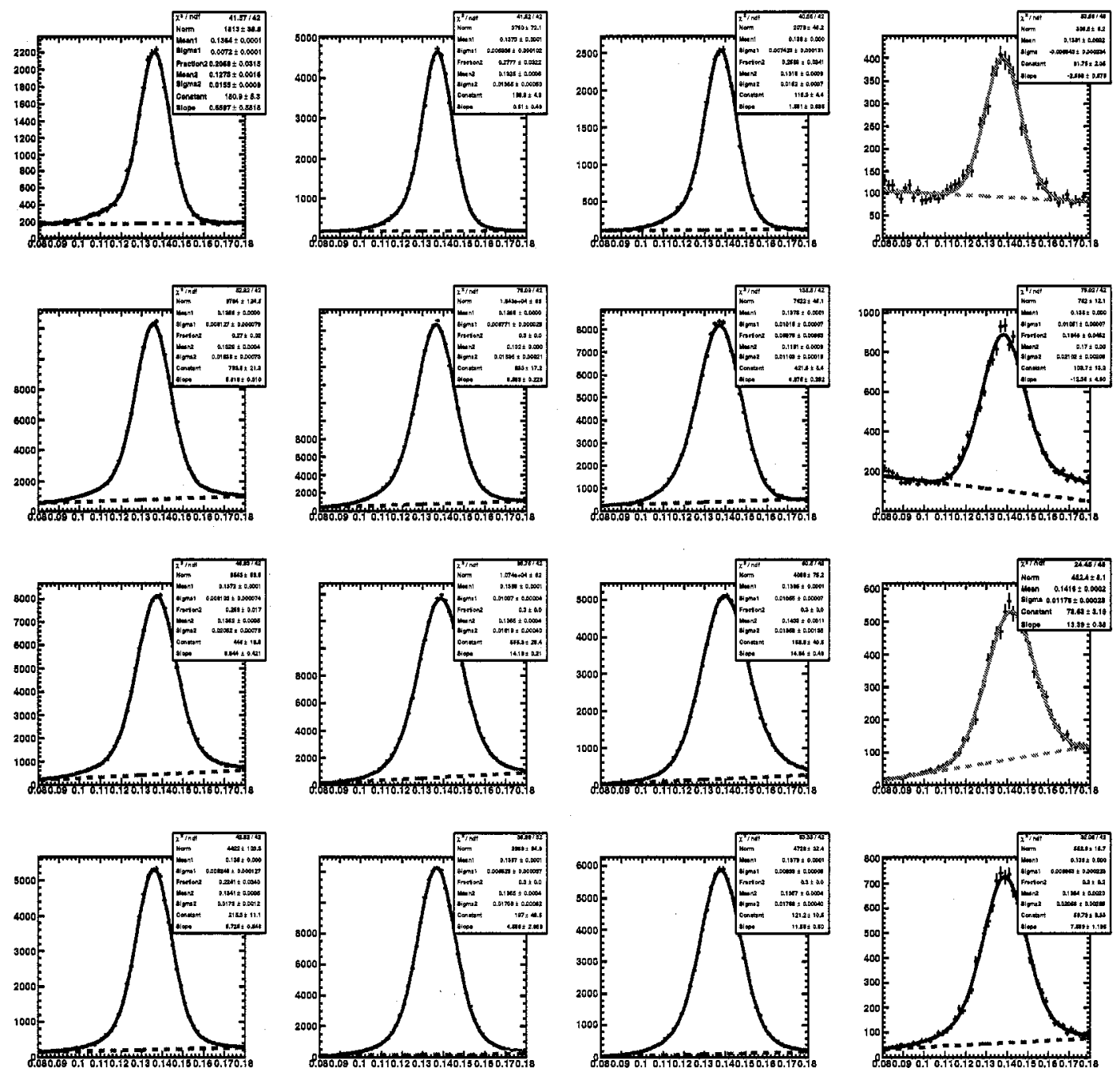

Figure 9.3: Fits of the $m_{\pi^{0}}$ distribution in data, in bins of $E_{\pi^{0}}^{*}$ (increasing from left to right) and $\theta_{\pi^{0}}$ (increasing from top to bottom). Histograms are scaled to $250 \mathrm{fb}^{-1}$. 

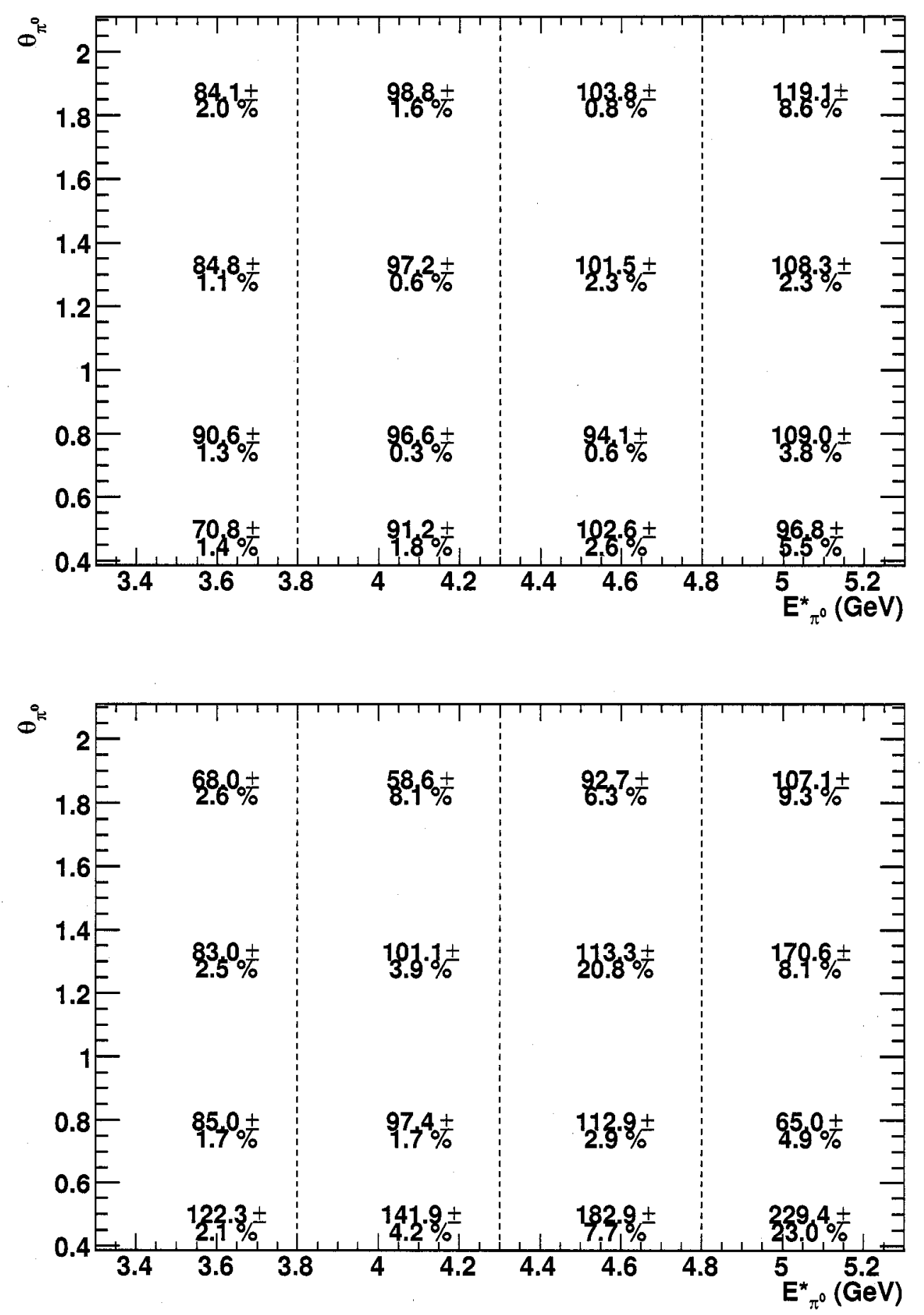

Figure 9.4: Peak (top) and combinatorics (bottom) corrections for the $\pi^{0}$ region 


\subsection{3 $\eta \rightarrow \gamma \gamma$}

The $\eta \rightarrow \gamma \gamma$ case is done in exactly the same way as for $\pi^{0} \rightarrow \gamma \gamma:$ we use the same $\theta$ and $E^{*}$ bins; the only change is the mass range under study and the minimum cluster energy for the other photon.

Fig 9.5 shows the comparison between data and stacked MC samples, Figs 9.6 and 9.7 show the fits to $\mathrm{MC}$ and data and the corrections are given in Fig. 9.8.

\subsection{4 $\eta^{\prime} \rightarrow \gamma \gamma$}

Since the rate of $\eta^{\prime} \rightarrow \gamma \gamma$ is much lower than the corresponding $\pi^{0}$ and $\eta$ processes, the $\eta^{\prime}$ study is performed without $\theta_{\eta^{\prime}}$ and $E_{\eta^{\prime}}^{*}$ binning. This is also justified by the fact that in both the $\pi^{0}$ and $\eta$ case there seemed to be no obvious discrepancies between data and $\mathrm{MC}$ in the $\theta$ and $E^{*}$ dependences.

Due to the smallness of the $\eta^{\prime} \rightarrow \gamma \gamma$ signal in the fitted samples it is impossible to fit a peaking component directly. Instead we determine the peak parameters from in $u d s \mathrm{MC}$ by requiring the presence of a true $\eta^{\prime} \rightarrow \gamma \gamma$ decay. The fit is shown in Fig. 9.9. The values of the peak parameters are then fixed to the obtained values for the fit to the full $\mathrm{MC}$ and data samples. A quadratic background shape is used.

Fig 9.10 shows the comparison between data and stacked MC samples and the fits to MC and data. The corrections are shown in Table 9.1.

\subsection{5 $\omega \rightarrow \pi^{0} \gamma$}

The $\omega \rightarrow \pi^{0} \gamma$ case is slightly different from the previous cases since a three-photon state is reconstructed, with one pair required to have an invariant mass in the range $100<m_{\gamma \gamma}<170 \mathrm{MeV}$. As for the $\eta^{\prime}$, we first obtain the signal shape (shown in Fig. 9.11) from truth-matched $u d s \mathrm{MC}$ events. The signal shape is then fixed during the fits to data and MC, with the background and relative normalization of the signal the floating parameters. A quadratic background shape is used. Fig. 9.12 shows the comparison between data and stacked MC samples and the fits to MC and data. The combinatorics correction is shown in Table 9.1. 

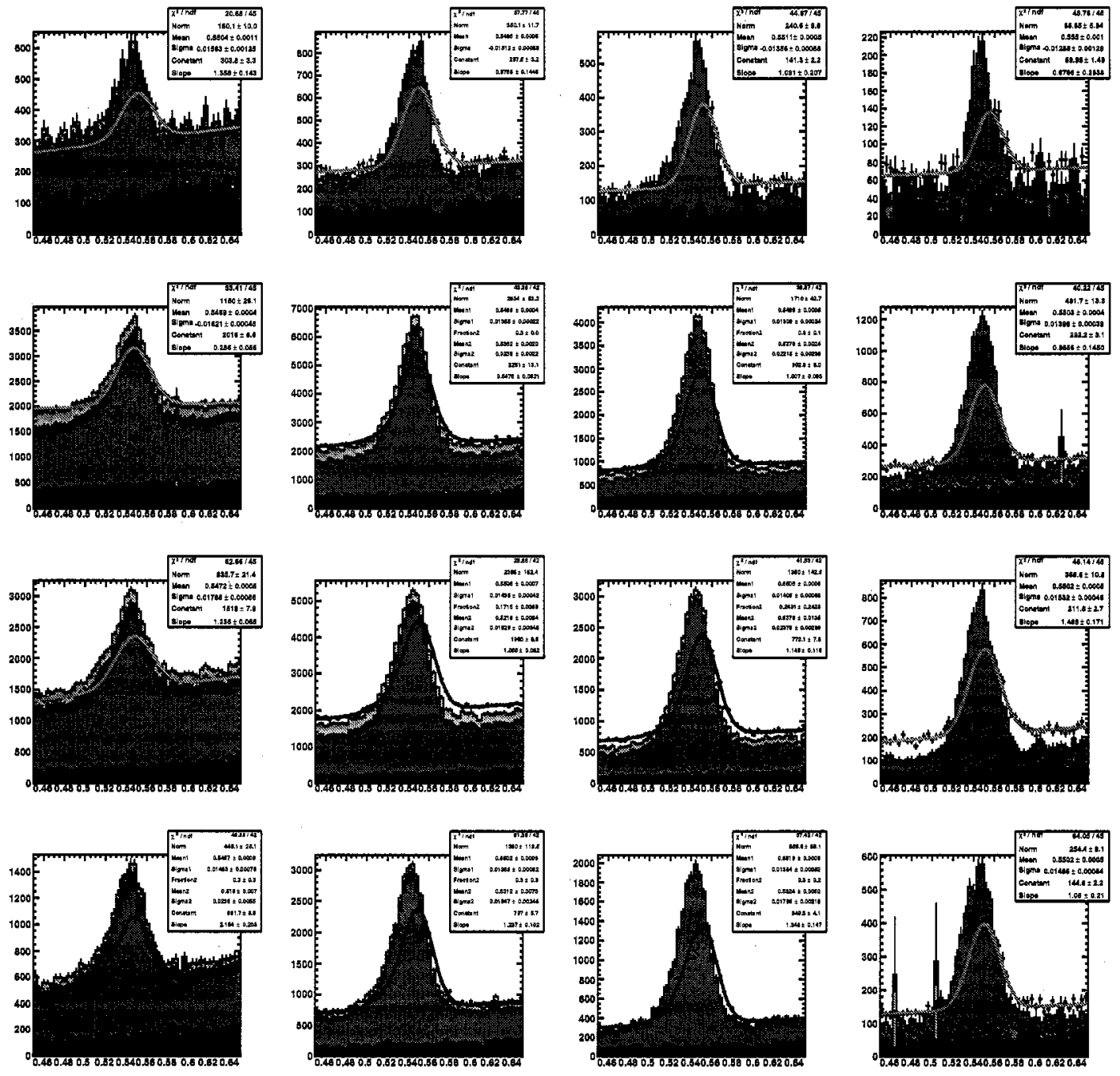

Figure 9.5: Distributions of $m_{\eta}$ in data (points and blue line) and MC (stacked histograms) in bins of $E_{\eta}^{*}$ (increasing from left to right) and $\theta_{\eta}$ (increasing from top to bottom). All histograms are scaled to $250 \mathrm{fb}^{-1}$. The color-coding of the MC samples is the same as for spectrum plots such as Fig. 5.3, with light blue for $\tau^{+} \tau^{-}$ $\mathrm{MC}$ and red for uds $\mathrm{MC}$. 

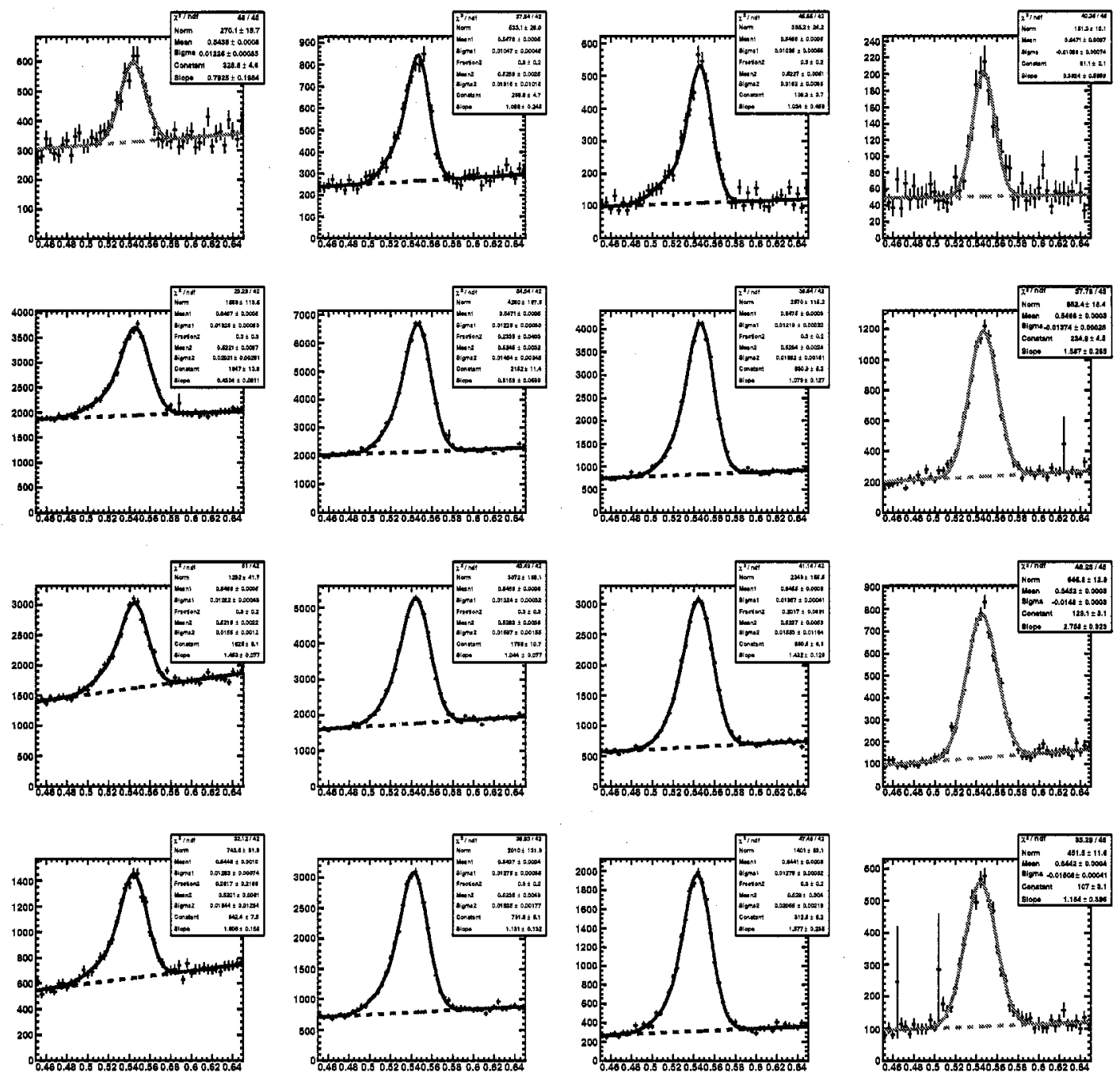

Figure 9.6: Fits of the $m_{\eta}$ distribution for the sum of all $\mathrm{MC}$ samples, in bins of $E_{\eta}^{*}$ (increasing from left to right) and $\theta_{\eta}$ (increasing from top to bottom). Histograms are scaled to $250 \mathrm{fb}^{-1}$.

Table 9.1: Peak and combinatorics corrections for higher resonances

\begin{tabular}{lcc}
\hline \hline Resonance & Peak Corr. & Comb. Corr. \\
\hline$\eta^{\prime}$ & $0.64 \pm 0.25$ & $1.11 \pm 0.00$ \\
$\omega$ & $n / a \pm n / a$ & $1.10 \pm 0.01$ \\
$\psi$ & $n / a \pm n / a$ & $0.17 \pm 0.02$ \\
\hline \hline
\end{tabular}



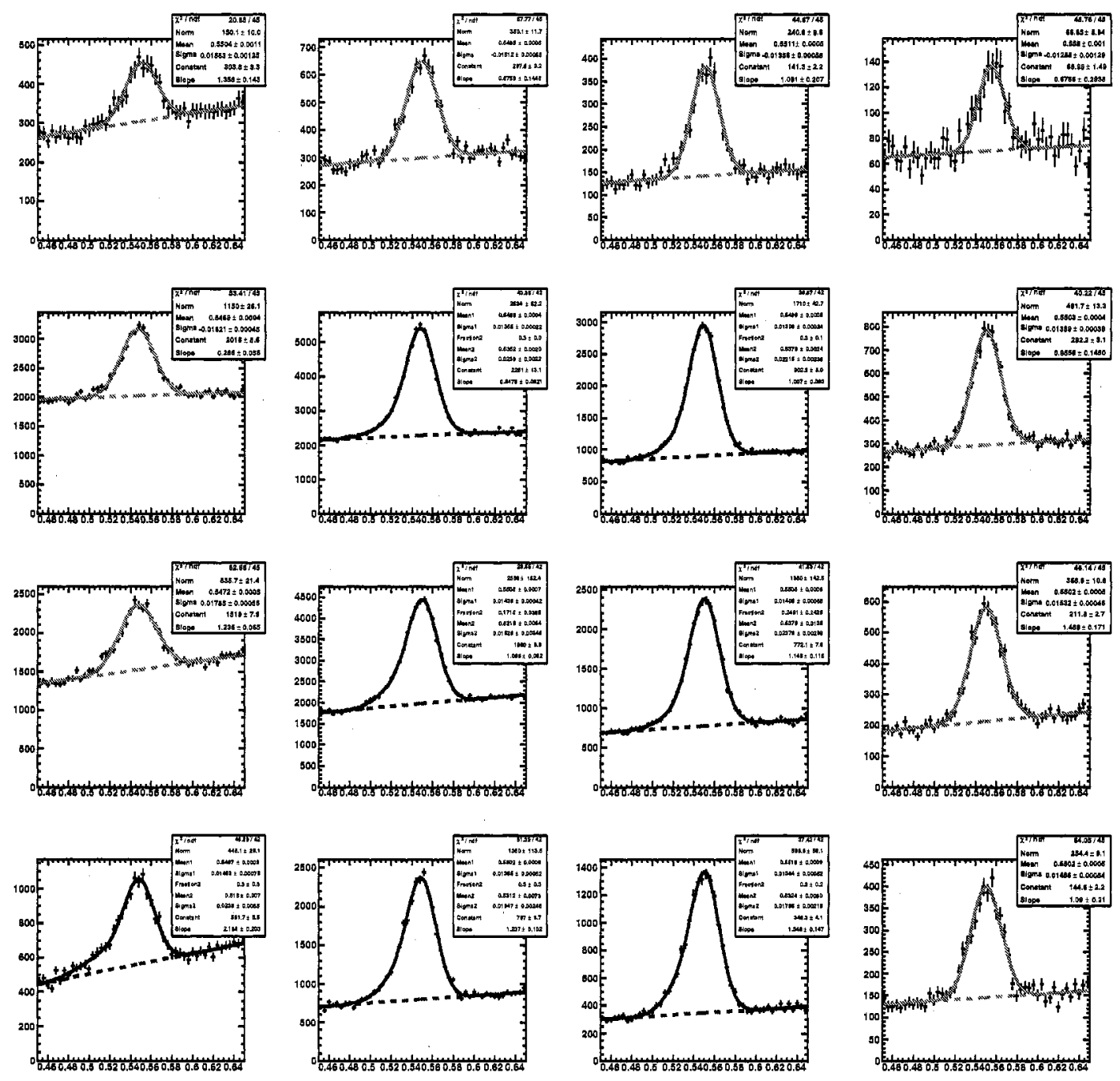

Figure 9.7: Fits of the $m_{\eta}$ distribution in data, in bins of $E_{\eta}^{*}$ (increasing from left to right) and $\theta_{\eta}$ (increasing from top to bottom). Histograms are scaled to $250 \mathrm{fb}^{-1}$. 

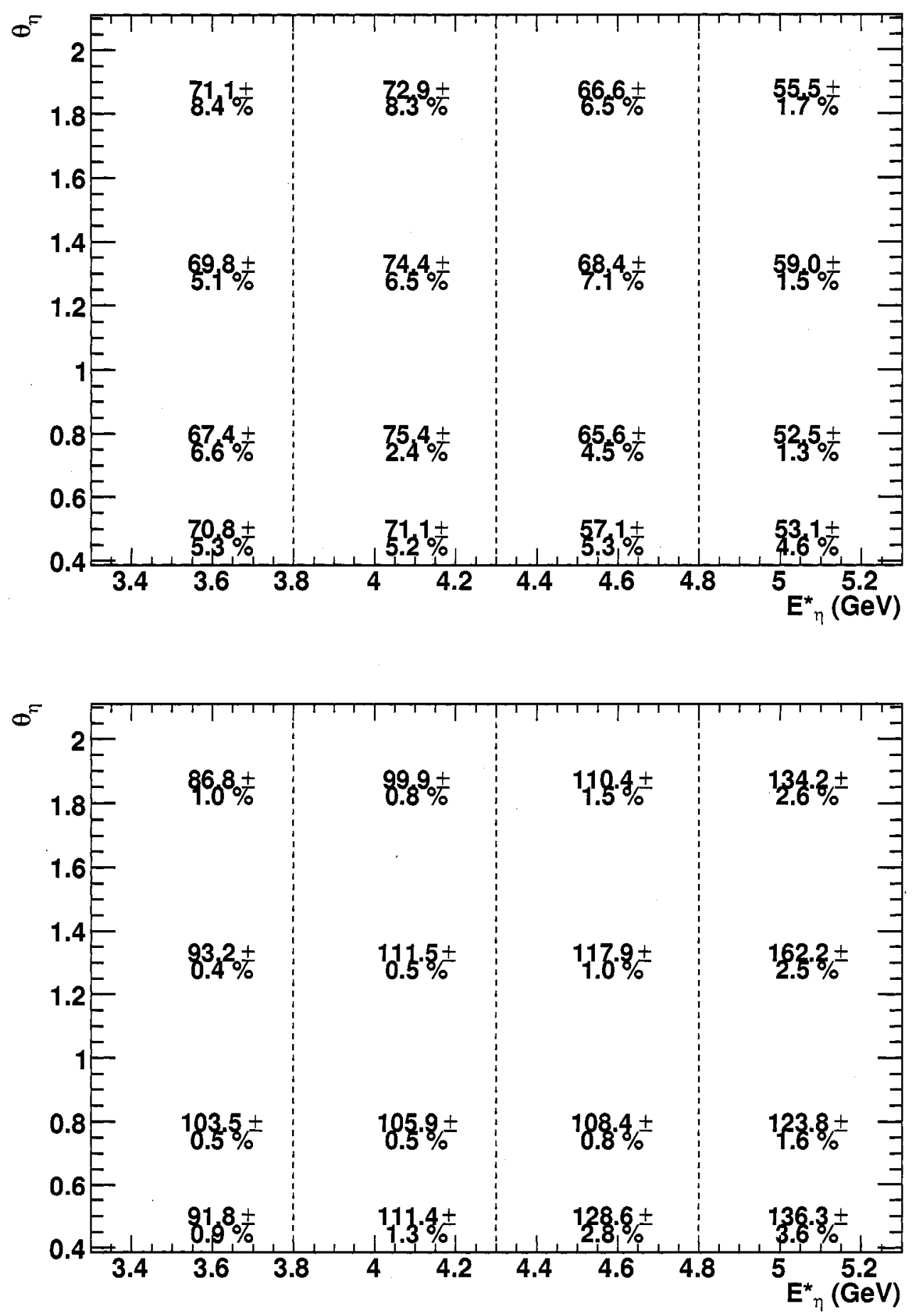

Figure 9.8: Peak (top) and combinatorics (bottom) corrections for the $\eta$ region 


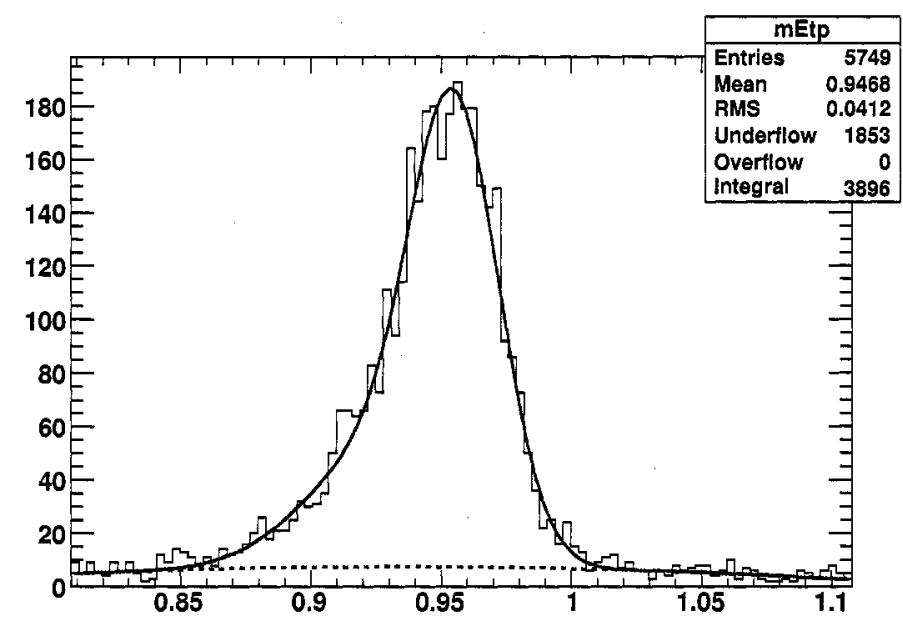

Figure 9.9: Fit of $m_{\eta^{\prime}}$ from $u d s \mathrm{MC}$ events with a true $\eta^{\prime} \rightarrow \gamma \gamma$ decay.
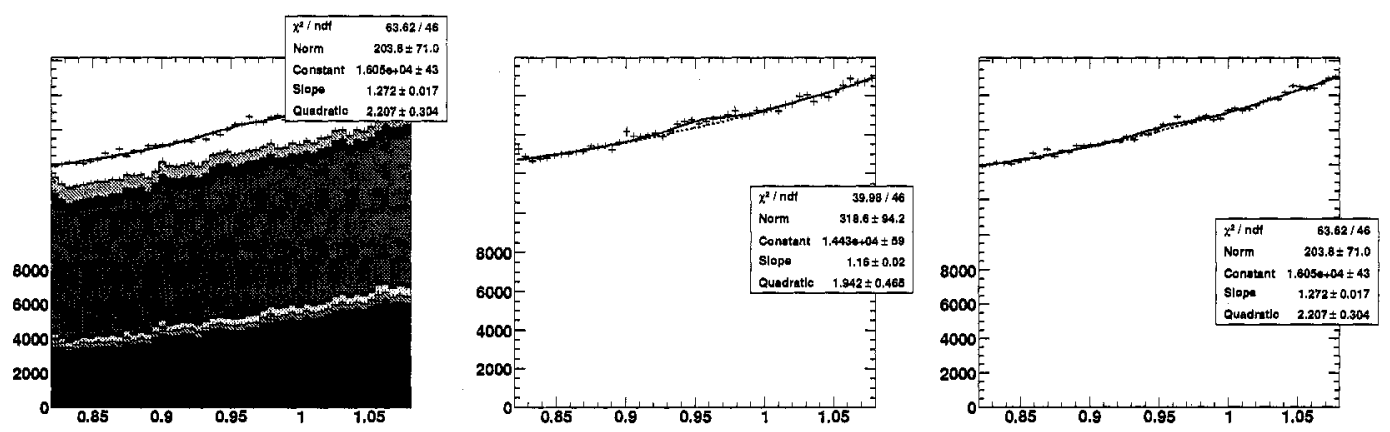

Figure 9.10: Left:Distribution of $m_{\eta^{\prime}}$ in data (points and blue line) and MC (stacked histograms). The color-coding of the $\mathrm{MC}$ sample is the same as for spectrum plots such as Fig. 5.3, with light blue for $\tau^{+} \tau^{-} \mathrm{MC}$ and red for $u d s$ MC. Middle: Fit of the $m_{\eta^{\prime}}$ distribution for the sum of all MC samples. Right: Fit of the $m_{\eta^{\prime}}$ distribution in data. 


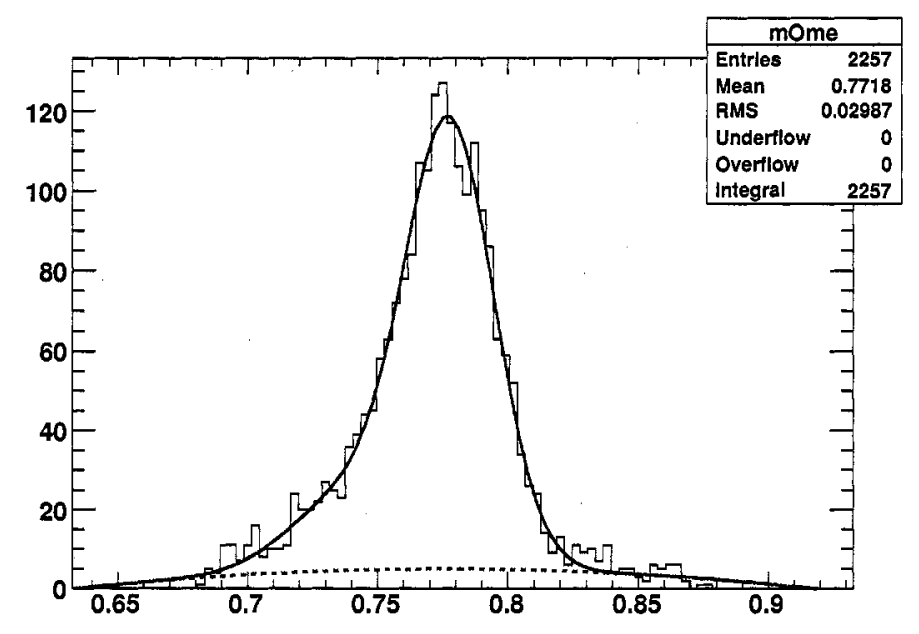

Figure 9.11: Fit of $m_{\omega}$ from $u d s$ MC events with a true $\omega \rightarrow \pi^{0} \gamma$ decay.
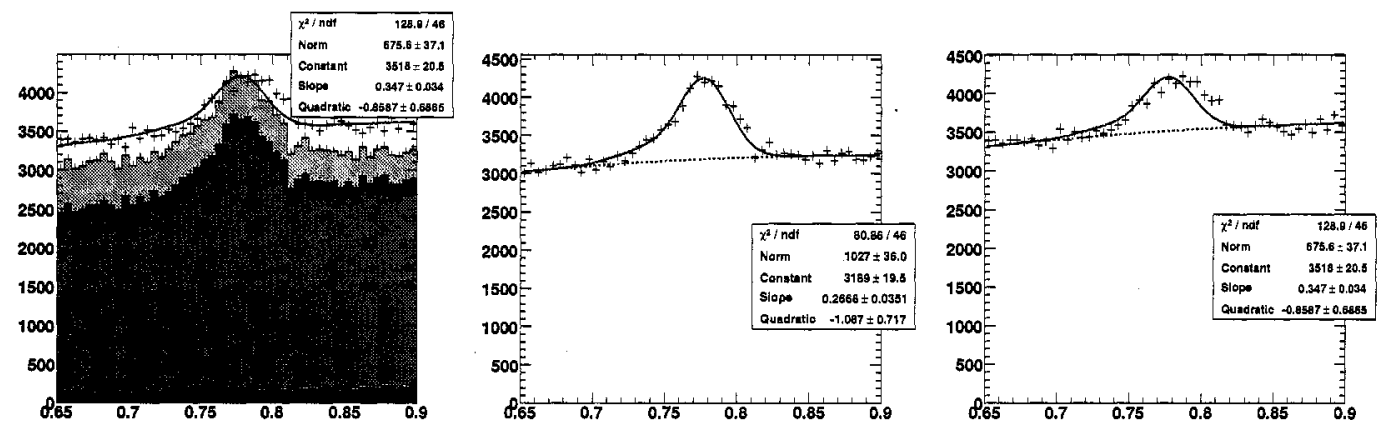

Figure 9.12: Left:Distribution of $m_{\omega}$ in data (points and blue line) and MC (stacked histograms). The color-coding of the MC sample is the same as for spectrum plots such as Fig. 5.3, with light blue for $\tau^{+} \tau^{-}$MC and red for $u d s$ MC. Middle: Fit of the $m_{\omega}$ distribution for the sum of all MC samples. Right: Fit of the $m_{\omega}$ distribution in data 
Table 9.2: Breakdown of of the generic $\tau^{+} \tau^{-}$sample according to $\tau$ decay modes

\begin{tabular}{|l|c|}
\hline Mode & Fraction \\
\hline$\tau \rightarrow \pi \pi^{0}\left(\pi^{0}\right) \nu, \tau \rightarrow \pi \pi^{0}\left(\pi^{0}\right) \nu$ & $24.1 \%$ \\
$\tau \rightarrow \pi \pi^{0}\left(\pi^{0}\right) \nu, \tau \rightarrow$ Other 1-prong & $47.9 \%$ \\
$\tau \rightarrow \pi \pi^{0}\left(\pi^{0}\right) \nu, \tau \rightarrow$ Other & $18.9 \%$ \\
$\tau \rightarrow$ Other 1-prong, $\tau \rightarrow$ Other 1-prong & $5.9 \%$ \\
$\tau \rightarrow$ Other 1-prong, $\tau \rightarrow$ Other & $3.1 \%$ \\
$\tau \rightarrow$ Other, $\tau \rightarrow$ Other & $0.1 \%$ \\
\hline
\end{tabular}

\subsection{Cross-check of the $\pi^{0} \rightarrow \gamma \gamma$ yield in $\tau^{+} \tau^{-}$events}

We have shown in Section 6.1 that the the $\pi^{0} \rightarrow \gamma \gamma$ background, which is the leading source of continuum background to ISR processes, comes mainly from two sources: $u d s$ (mainly primary $\pi^{0}$ production from $u \bar{u}$ or $d \bar{d}$ pairs) and $\tau^{+} \tau^{-}$processes.

In Section 9.1 we determined an overall correction for the $\pi^{0} \rightarrow \gamma \gamma$ yield. However due to the precision of our knowledge of $\tau$ branching fractions and dynamics, we expect the $\tau^{+} \tau^{-}$component to be known with significantly higher precision that the $u d s$ component: the latter depends the correct modeling of hadronization to highmomentum $\pi^{0} \mathrm{~s}$ which is not expected to be known as precisely. For this reason, in this section we cross-check the $\tau^{+} \tau^{-}$component alone, so that independent corrections on the $u d s$ and $\tau^{+} \tau^{-}$samples can be obtained.

\subsubsection{ISR Backgrounds from $\tau^{+} \tau^{-}$}

We first investigate the nature of $\tau^{+} \tau^{-}$backgrounds in $\mathrm{MC}$ truth. The breakdown is shown in Table 9.2.

\subsubsection{Selection of $\tau \rightarrow \rho \nu$ events}

As shown in the previous section the main contributor to ISR backgrounds is the decay $\tau \rightarrow \rho\left(\pi^{0} \pi\right) \nu$. We therefore investigate those events, considering both the case of merged and unmerged $\pi^{0} \mathrm{~s}$. 
In order to achieve a pure selection of such events we also tag the other $\tau$ decay. $\tau \rightarrow \mu$ and $\tau \rightarrow e$ tags are not used since background from $\mu \mu \gamma$ and ee $\gamma$ give a significant background, and $\tau \rightarrow 3 h$ decays get background from continuum production. We therefore use $\tau \rightarrow \rho\left(\pi^{0} \pi\right) \nu$ on the tag side as well, requiring the $\pi^{0}$ to be unmerged on the tag side and cutting on the masses of the $\pi^{0}$ and $\rho$

The selection is based on the output of the QED veto phase and consists in the following cuts:

- Tracks: 2 Good tracks. The vector $\overrightarrow{p_{1}}-\overrightarrow{p_{2}}$ defines the event decay axis, which separates the event into 2 hemispheres. We call the hemisphere containing the ISR photon the "signal side", while the other hemisphere is the "tag side".

- Clusters: We require 2 clusters with $E>100 \mathrm{MeV}$ on the tag side, and 1 or 2 clusters with $E>100 \mathrm{MeV}$ on the signal side (merged or unmerged $\pi^{0}$ case).

- Tag $\pi^{0}$ and $\rho$ : On the tag side, we require $110<m_{\gamma \gamma}<150 \mathrm{MeV}$ and $670<$ $m_{\gamma \gamma \pi}<870 \mathrm{MeV}$.

- Signal $\rho$ : On the signal side, we require $670<m_{\gamma(\gamma) \pi}<870 \mathrm{MeV}$, with either 1 or 2 photons used depending on how many were found above.

Distributions of the relevant quantities are shown in Fig. 9.13.
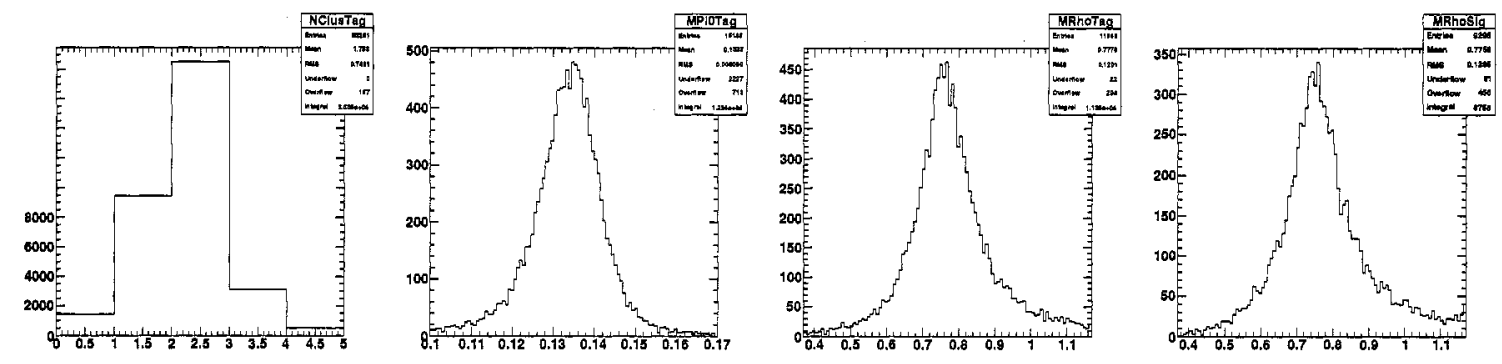

Figure 9.13: Distributions of number of clusters on tag side (top left), tag $m_{\gamma \gamma}$ (top right), tag $m_{\gamma \gamma \pi}$ (bottom left) and signal $m_{\gamma \gamma \pi}$ (bottom right).

The efficiency of the selection on $\tau^{+} \tau^{-} \mathrm{MC}$ and other samples is shown in Tables 9.3, 9.4 and 9.5. An efficiency of $15 \%$ is achieved on signal $\tau$ modes, while backgrounds occur at the per-mil level. 
Table 9.3: Efficiencies for the $\tau \rightarrow \pi^{0} \pi \nu$ event selection

\begin{tabular}{|l|c|c|}
\hline & $\tau^{+} \tau^{-}(\%)$ & $\tau^{+} \tau^{-} \gamma(\%)$ \\
\hline 2 Good Tracks & $80.29 \pm 0.19$ & $53.25 \pm 0.12$ \\
Track PID Cuts & $72.41 \pm 0.21$ & $31.57 \pm 0.11$ \\
Tag-side Clusters & $39.01 \pm 0.23$ & $7.52 \pm 0.06$ \\
ISR-side clusters & $33.06 \pm 0.22$ & $4.63 \pm 0.05$ \\
Tag-side $m_{\gamma \gamma}$ & $25.40 \pm 0.20$ & $1.78 \pm 0.03$ \\
Tag-side $m_{\gamma \gamma \pi}$ & $20.24 \pm 0.19$ & $1.18 \pm 0.03$ \\
ISR-side $m_{\gamma(\gamma) \pi}$ & $14.99 \pm 0.17$ & $(4.37 \pm 0.50) \times 10^{-4}$ \\
Events in 250/fb & $101237.2 \pm 1220.3$ & $318.3 \pm 36.5$ \\
\hline
\end{tabular}

Table 9.4: Efficiencies for the $\tau \rightarrow \pi^{0} \pi \nu$ event selection

\begin{tabular}{|l|c|c|c|c|}
\hline & $q \bar{q} \gamma(\%)$ & $e^{+} e^{-\gamma(\%)}$ & $\mu^{+} \mu^{-} \gamma(\%)$ & $\gamma \gamma(\%)$ \\
\hline 2 Good Tracks & $43.35 \pm 0.11$ & $23.58 \pm 1.42$ & $58.30 \pm 0.11$ & $20.44 \pm 3.45$ \\
Track PID Cuts & $32.08 \pm 0.11$ & $10.57 \pm 1.03$ & $8.40 \pm 0.06$ & $8.03 \pm 2.32$ \\
Tag-side Clusters & $3.82 \pm 0.04$ & $1.33 \pm 0.38$ & $(2.28 \pm 0.11) \times 10^{-3}$ & $2.19 \pm 1.25$ \\
ISR-side clusters & $2.43 \pm 0.04$ & $(4.45 \pm 2.22) \times 10^{-3}$ & $(4.00 \pm 0.45) \times 10^{-4}$ & $1.46 \pm 1.02$ \\
Tag-side $m_{\gamma \gamma}$ & $(7.63 \pm 0.20) \times 10^{-3}$ & $0.00 \pm 0.00$ & $(5.01 \pm 5.00) \times 10^{-6}$ & $0.00 \pm 0.00$ \\
Tag-side $m_{\gamma \gamma \pi}$ & $(2.00 \pm 0.10) \times 10^{-3}$ & $0.00 \pm 0.00$ & $0.00 \pm 0.00$ & $0.00 \pm 0.00$ \\
ISR-side $m_{\gamma(\gamma) \pi}$ & $(5.35 \pm 5.35) \times 10^{-6}$ & $0.00 \pm 0.00$ & $0.00 \pm 0.00$ & $0.00 \pm 0.00$ \\
Events in $250 / \mathrm{fb}$ & nan \pm nan & $0.0 \pm 197.1$ & $0.0 \pm 27.7$ & $0.0 \pm 504.3$ \\
\hline
\end{tabular}

Table 9.5: Efficiencies for the $\tau \rightarrow \pi^{0} \pi \nu$ event selection

\begin{tabular}{|l|c|c|c|c|}
\hline & $u d s(\%)$ & $c \bar{c}(\%)$ & $B^{0} \bar{B}^{0}(\%)$ & $B^{+} B^{-}(\%)$ \\
\hline 2 Good Tracks & $15.70 \pm 0.08$ & $7.12 \pm 0.06$ & $(3.33 \pm 0.33) \times 10^{-3}$ & $(2.91 \pm 0.32) \times 10^{-3}$ \\
Track PID Cuts & $10.54 \pm 0.07$ & $4.26 \pm 0.05$ & $(1.54 \pm 0.22) \times 10^{-3}$ & $(1.38 \pm 0.22) \times 10^{-3}$ \\
Tag-side Clusters & $2.21 \pm 0.03$ & $(4.91 \pm 0.16) \times 10^{-3}$ & $(1.28 \pm 0.64) \times 10^{-4}$ & $(3.54 \pm 3.54) \times 10^{-5}$ \\
ISR-side clusters & $(9.64 \pm 0.22) \times 10^{-3}$ & $(1.15 \pm 0.08) \times 10^{-3}$ & $(3.20 \pm 3.20) \times 10^{-5}$ & $0.00 \pm 0.00$ \\
Tag-side $m_{\gamma \gamma}$ & $(3.34 \pm 0.13) \times 10^{-3}$ & $(1.94 \pm 0.32) \times 10^{-4}$ & $0.00 \pm 0.00$ & $0.00 \pm 0.00$ \\
Tag-side $m_{\gamma \gamma \pi}$ & $(9.86 \pm 0.70) \times 10^{-4}$ & $(6.28 \pm 1.81) \times 10^{-5}$ & $0.00 \pm 0.00$ & $0.00 \pm 0.00$ \\
ISR-side $m_{\gamma(\gamma) \pi}$ & $(6.51 \pm 1.80) \times 10^{-5}$ & $(1.57 \pm 0.91) \times 10^{-5}$ & $0.00 \pm 0.00$ & $0.00 \pm 0.00$ \\
Events in $250 / \mathrm{fb}$ & $260.1 \pm 72.1$ & $3.5 \pm 2.0$ & $0.0 \pm 0.8$ & $0.0 \pm 0.7$ \\
\hline
\end{tabular}




\subsubsection{Production rate}

Figures 9.14 and 9.16 show the fits to $\mathrm{MC}$ and data respectively.
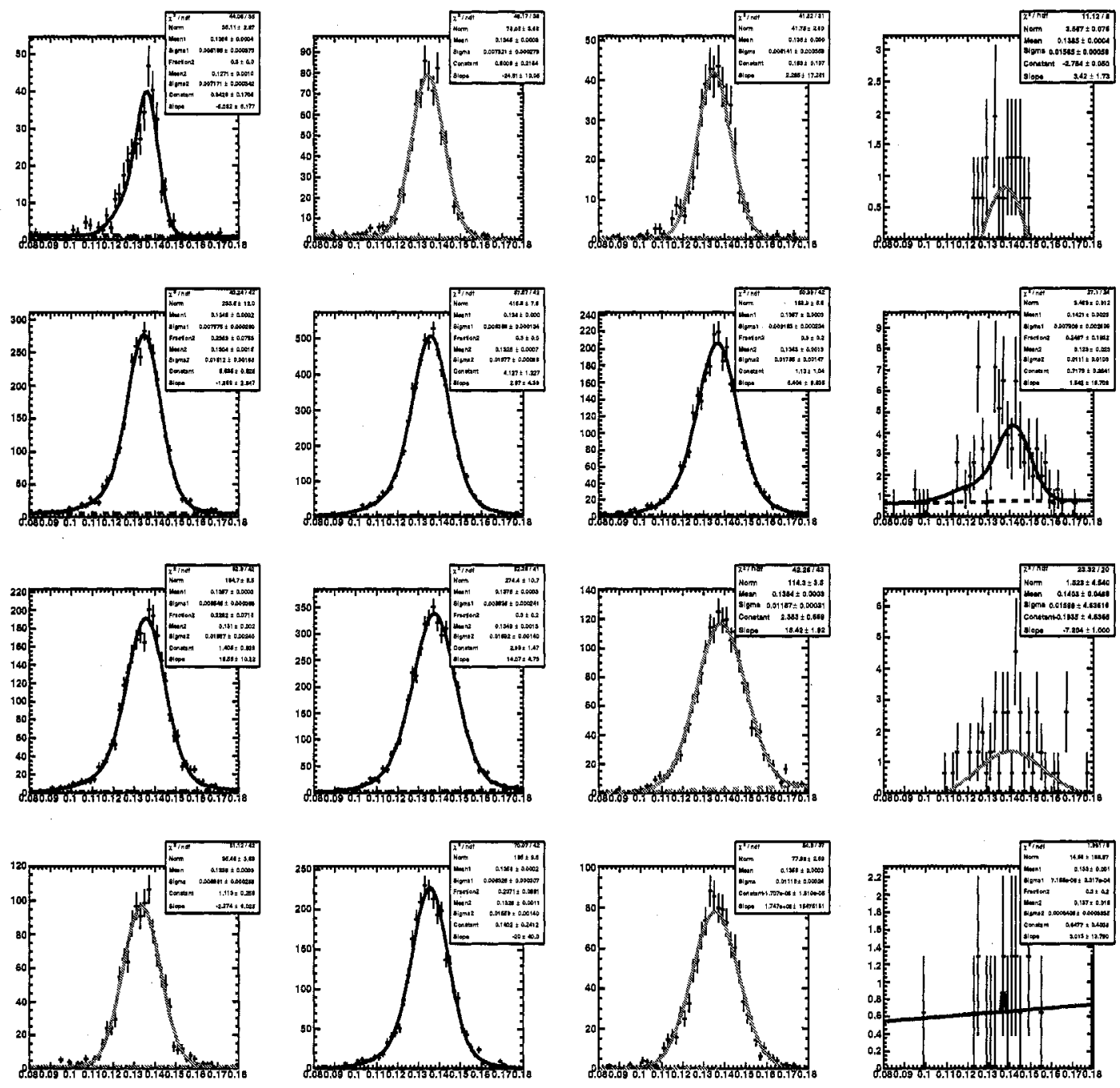

Figure 9.14: Fits of the $m_{\pi^{\circ}}$ distribution with double $\tau \rightarrow \pi \pi^{0} \nu$ selection for $\tau^{+} \tau^{-}$ $\mathrm{MC}$, in bins of $E_{\pi^{0}}^{*}$ (increasing from left to right) and $\theta_{\pi^{\circ}}$ (increasing from top to bottom). Histograms are scaled to $250 \mathrm{fb}^{-1}$.

The Gaussian(s) plus quadratic function form that we use describes the $\mathrm{MC}$ and data well. The peak correction is calculated as described in the previous section, and the results are shown in Fig. 9.17. 

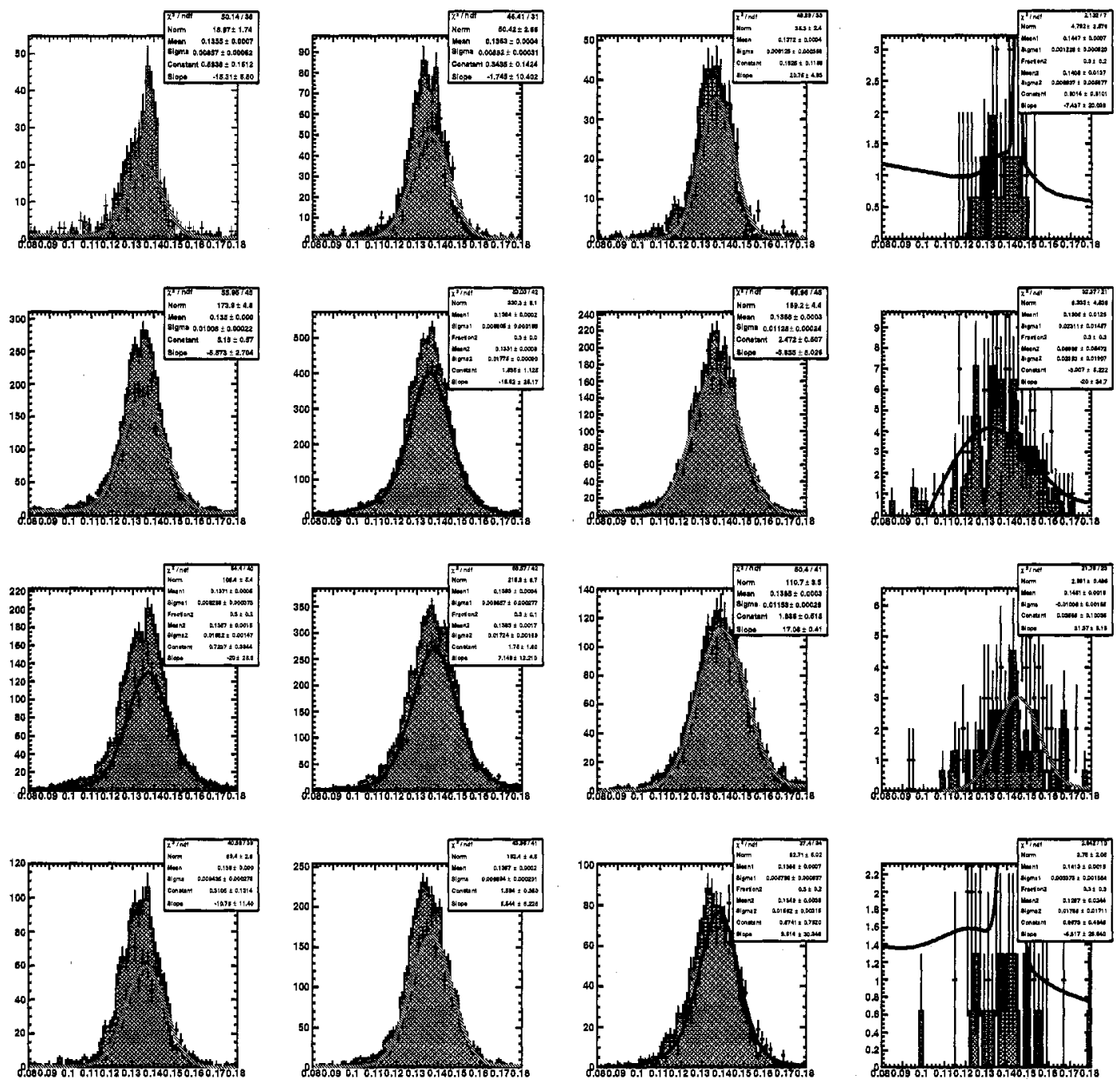

Figure 9.15: Fits of the $m_{\pi^{0}}$ distribution with double $\tau \rightarrow \pi \pi^{0} \nu$ selection in MC (shaded histogram) and data, in bins of $E_{\pi^{0}}^{*}$ (increasing from left to right) and $\theta_{\pi^{0}}$ (increasing from top to bottom). Histograms are scaled to $250 \mathrm{fb}^{-1}$. 

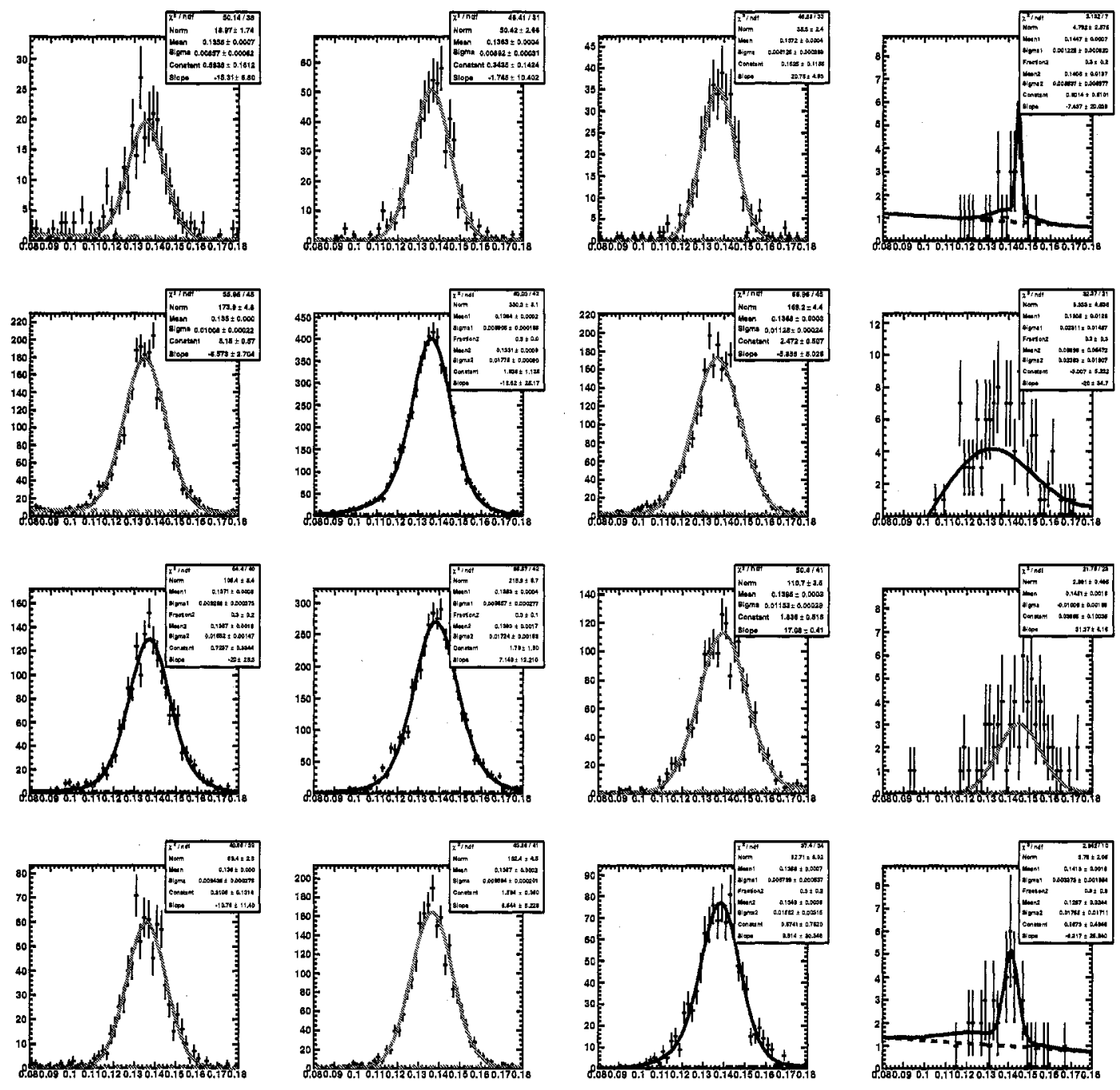

Figure 9.16: Fits of the $m_{\pi^{0}}$ distribution with double $\tau \rightarrow \pi \pi^{0} \nu$ selection in data, in bins of $E_{\pi^{0}}^{*}$ (increasing from left to right) and $\theta_{\pi^{0}}$ (increasing from top to bottom). Histograms are scaled to $250 \mathrm{fb}^{-1}$. 


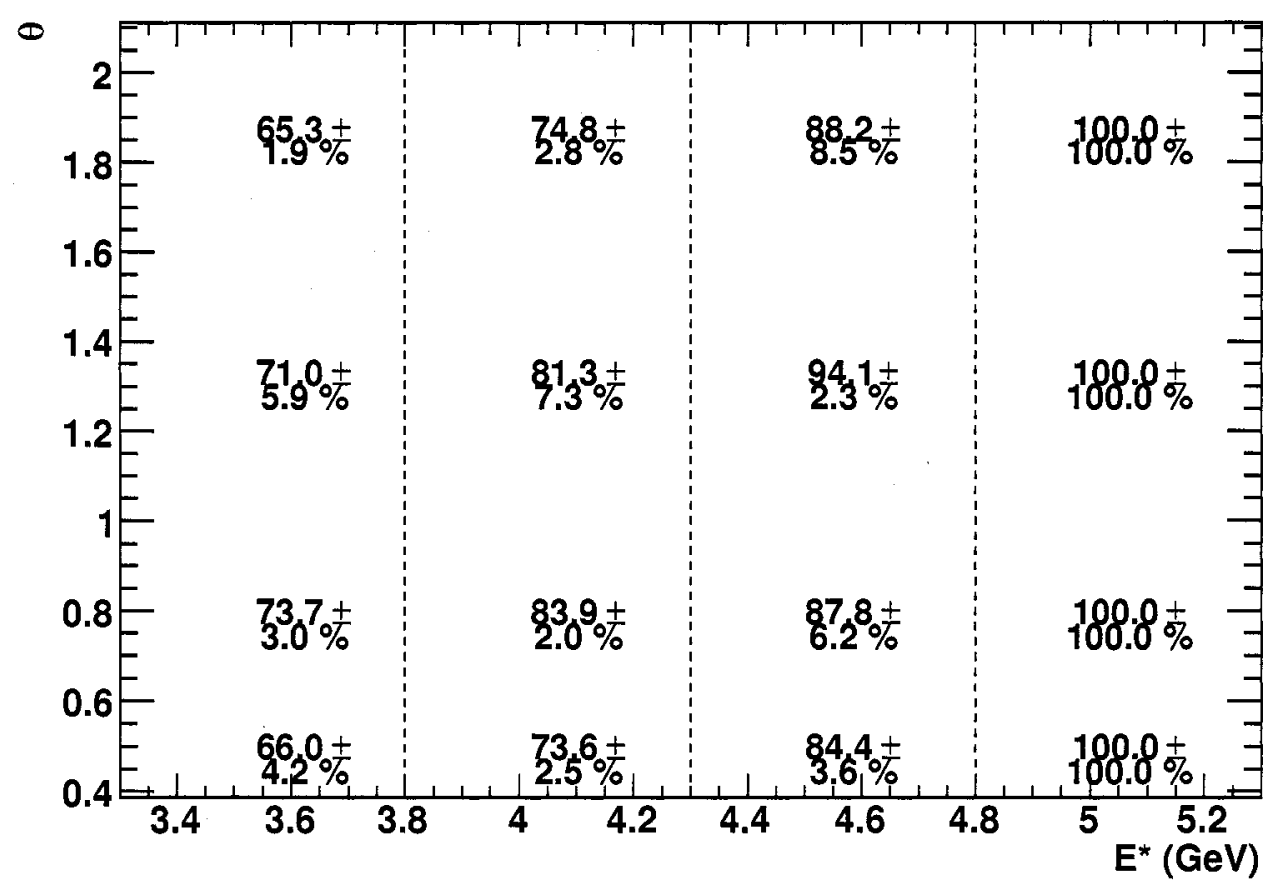

Figure 9.17: Peak correction for the $\pi^{0}$ region in double $\tau \rightarrow \pi \pi^{0} \nu$ events 
The combinatorics correction is not calculated due to the smallness of the backgrounds. Contrary to expectations, the peak correction is of the same order as the one from the inclusive $\pi^{0}$ sample shown in Fig. 9.4. We therefore apply separate corrections to $\tau^{+} \tau^{-}$events and other events. In order to disentangle the "other events" within the inclusive $\pi^{0}$ sample, we measure the fraction $\alpha_{\tau}$ of $\tau^{+} \tau^{-}$events within this sample. This is obtained by fitting the $\tau^{+} \tau^{-}$component of the inclusive sample separately and extracting the integral of the peak, which is then divided by the integral of the full MC sample. The numbers are shown in the left-hand plot of Fig. 9.18. Calling $c_{\pi^{0}}, c_{\tau}$ and $c_{\text {other }}$ the values of the corrections in the inclusive sample, the $\tau^{+} \tau^{-}$events and the other events, we have

$$
\begin{aligned}
c_{\pi^{0}} & =\alpha_{\tau} c_{\tau}+\left(1-\alpha_{\tau}\right) c_{\text {other }} \\
c_{\text {other }} & =\frac{1}{1-\alpha_{\tau}} c_{\pi^{0}}+\frac{\alpha_{\tau}}{1-\alpha_{\tau}} c_{\tau}
\end{aligned}
$$

This is done bin-by-bin, and the results are shown in the right-hand plot of Fig. 9.18.
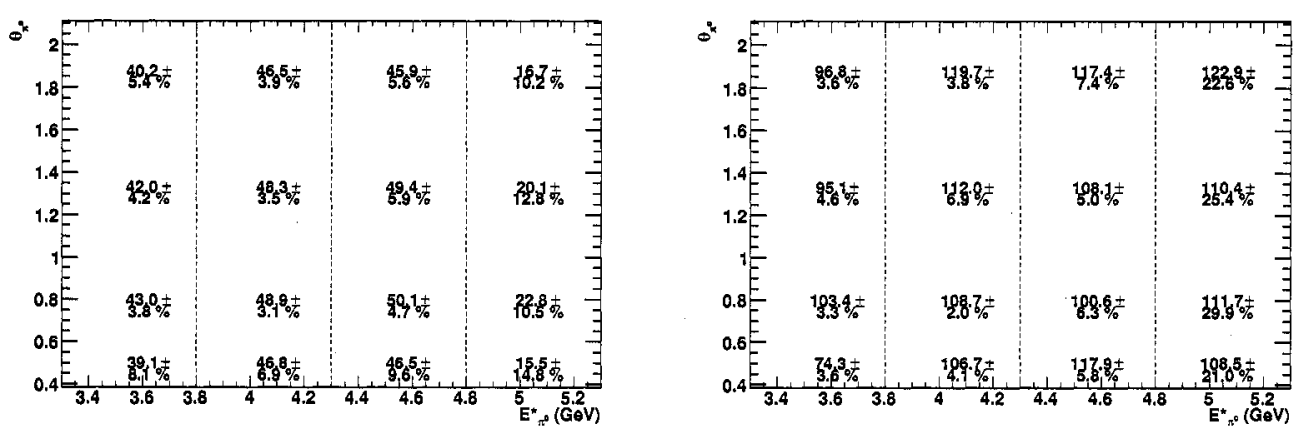

Figure 9.18: Left: Fraction of $\tau^{+} \tau^{-}$events in the $\pi^{0}$ mass peak of the inclusive $\pi^{0}$ sample. Right: Correction for the non- $\tau^{+} \tau^{-}$events in the inclusive $\pi^{0}$ sample. 


\subsubsection{Cross-check of Helicity distributions}

In the previous section, we have used reconstructed $\pi^{0} \rightarrow \gamma \gamma$ to normalize MC using data. An implicit assumption in this study is that the reconstruction efficiency is identical in MC and data. Since photon detection efficiency is reasonably well simulated this should be an adequate approximation. However this also depends on the MC's ability to reproduce the transition from unmerged to merged $\pi^{0} \mathrm{~s}$, corresponding to the fact that the EMC "bumps" deposited by the $\pi^{0}$ daughters can be separated or not. Whether this is correctly simulated or not is a priori non-trivial, but can be studied using the helicity distributions of the $\pi^{0} \mathrm{~s}$. This distribution is initially flat, but it is sculpted by 2 effects:

- The center of the $\cos \theta_{\text {hel }}$ distribution (decay axis perpendicular to the the $\pi^{0}$ direction of motion) is depleted due to $\pi^{0}$ merging

- The edges of the $\cos \theta_{\text {hel }}$ distribution (decay axis along the $\pi^{0}$ direction of motion) is depleted due to the soft photon dropping below the $100 \mathrm{MeV}$ lab energy cut.

By studying the $\cos \theta_{h e l}$ distributions, particularly in the center region, we can therefore estimate whether the MC simulates the fraction of merged $\pi^{0} \mathrm{~s}$ correctly.

The first step is to reweigh the MC using the correction from the previous section, so that the effect of the different $E_{\pi^{0}}^{*}$ and $\theta_{\pi^{0}}$ distributions in $\mathrm{MC}$ are removed. Fig 9.19 shows the $E_{\pi^{0}}^{*}$ and $\theta_{\pi^{0}}$ distributions for data, MC, and corrected MC. The difference between raw MC and data is mainly due to overall normalization and this is corrected by the reweighing.

From this point on, all MC distributions are assumed to be reweighted. Fig. 9.20 shows the comparison of the helicity distributions between data and MC. The bin at $\cos \theta_{\text {hel }}=-1.1$ corresponds to the merged $\pi^{0} \mathrm{~s}$.

The overall normalization of the $\cos \theta_{\text {hel }} \geq-1$ part is identical in data and MC by construction, since the reweighting of the $\mathrm{MC}$ has precisely the effect of making it match data. However as discussed above the merged $\pi^{0}$ part is normalized independently, and the agreement between data and $\mathrm{MC}$ in the $\cos \theta_{\text {hel }}=-1.1$ is indicative 

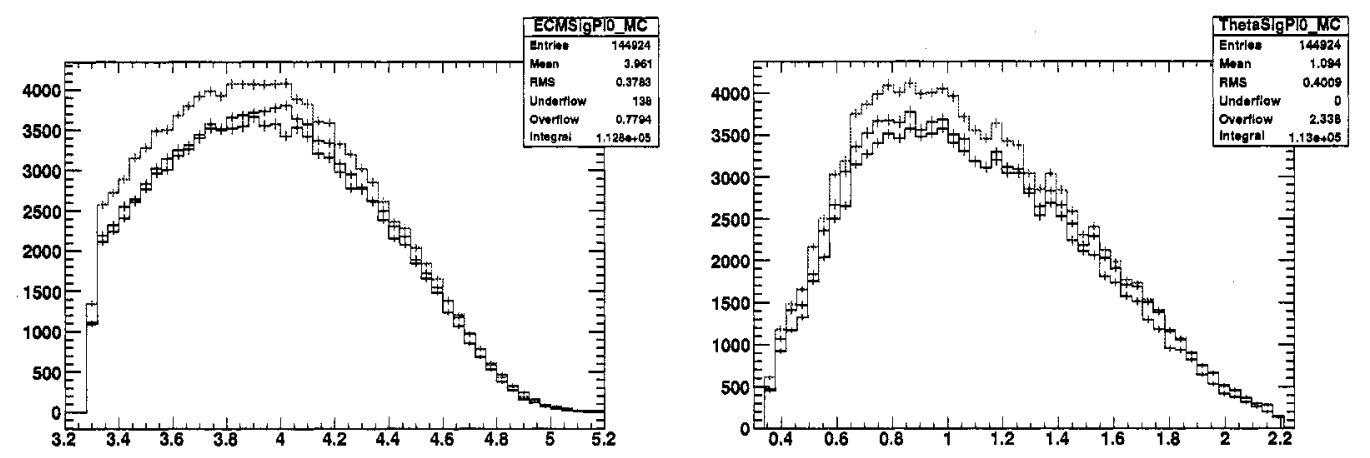

Figure 9.19: $E_{\gamma}^{*}$ (left) and $\theta$ (right) distributions for the highest-energy cluster in the event, in data (blue), $\mathrm{MC}$ (red) and reweighed MC (black), normalized by luminosity

of the accuracy of the simulation. The left plot of Fig. 9.21 shows the ratio of the occupancy of these bins for different values of $E_{\pi^{0}}^{*}$. Another presentation is given in the right plot of Fig. 9.21, which shows the ratio of merged $\pi^{0} \mathrm{~s}$ to total for $\mathrm{MC}$ and data. The agreement is very good overall, and no correction is necessary.

Fig. 9.22 shows cross-checks of the helicity angle calculation in MC. Their purpose is to verify that the helicity distributions shown above are not biased by reconstruction effects. The left-hand plot shows the difference between the reco and true values of the helicity versus the energy of the softer photon. The reconstructed value is close to the MC value, except for very soft photons. The right-hand plot shows the distributions for all events in $\mathrm{MC}$ and reco. The distributions are very similar, except for the fact that there is low-level occupancy in the central region in MC reco which is not seen in truth, where the central region is almost empty. This probably is due to the combinatorial background seen in the previous figure.

\subsubsection{Validation of the $\pi^{0}$ Lateral Shape}

Validation of the Cluster Lateral Shape Distributions for $\pi^{0} \mathrm{~s}$

The $\tau \rightarrow \pi \pi^{0} \nu$ sample can also be used to check the distribution of the cluster lateral moment. Since we cut on this variable as part of the hadronic selection, this allows the validation of this cut and if needed a correction on the efficiency. 

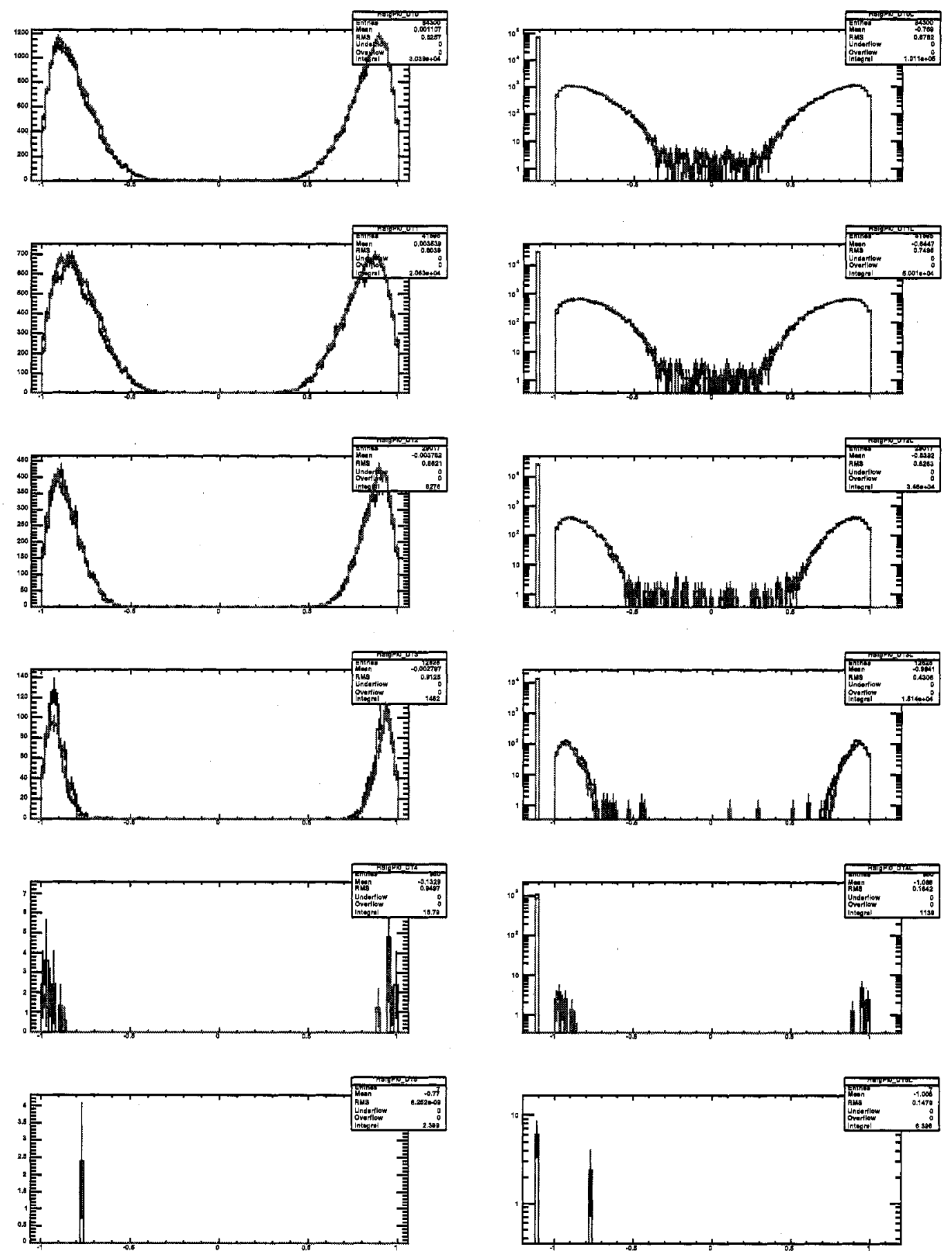

Figure 9.20: $\cos \theta_{\text {hel }}$ distributions in data (red) and reweighted MC (blue) in bins of $E_{\pi^{0}}^{*}$. The bin at $\cos \theta_{h e l}=-1.1$ corresponds to merged $\pi^{0} \mathrm{~s}$. This is shown in a log scale in the right column, while the left column shows the plots in linear scale with this bin truncated off The top row corresponds to all events, while subsequent rows correspond to the usual $E_{\pi^{0}}^{*}$ bins with boundaries at $3.3,3.8,4.3,4.8$ and $5.3 \mathrm{GeV}$. 

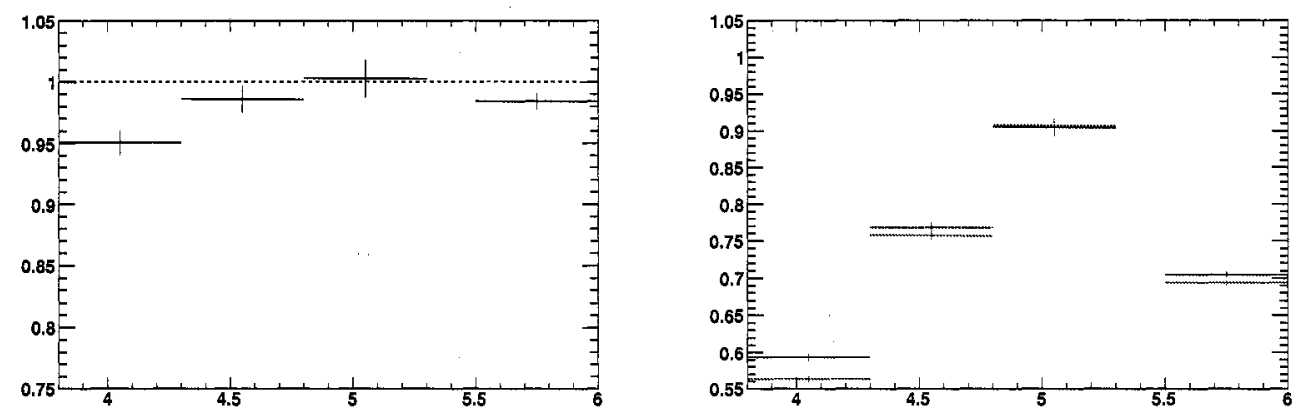

Figure 9.21: Left: ratio of merged $\pi^{0}$ yields in $\mathrm{MC}$ and data in bins of $E_{\pi^{0}}^{*}$, with the rightmost bins representing all events. Right: ratio of merged $\pi^{0} \mathrm{~s}$ to total $\pi^{0} \mathrm{~s}$ in MC (red) and data (blue).
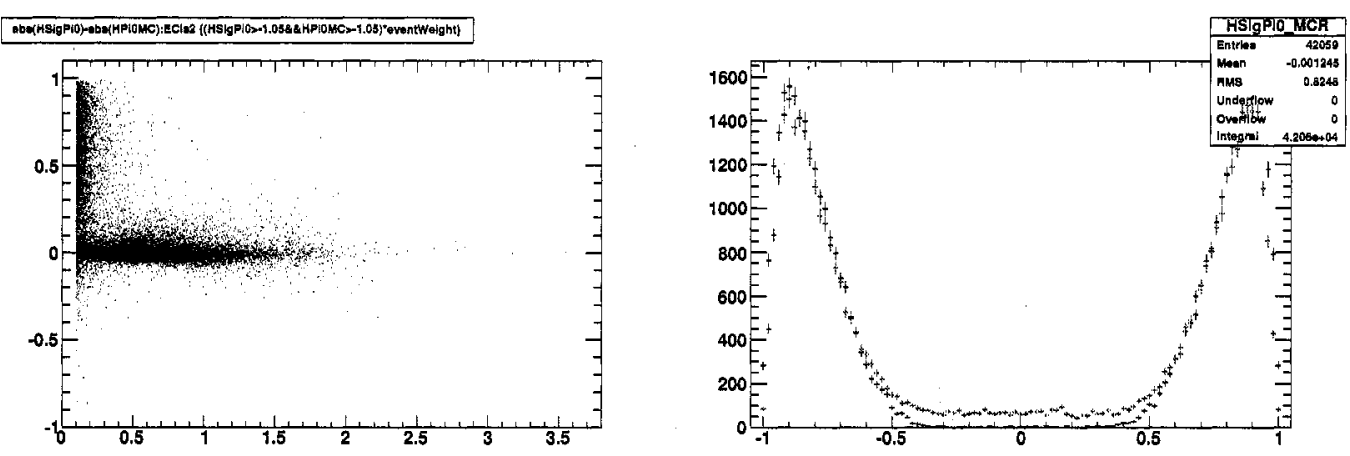

Figure 9.22: Left: difference between true and reco $\pi^{0}$ helicities in MC versus the lab energy of the softer photon. Right: helicity distributions in MC truth (blue) and MC reco (red). 
Fig. 9.23 (left column) shows the lateral moment distributions for the leading photon in the usual bins of its $E_{\pi^{0}}^{*}$. In the case of a merged $\pi^{0}$ this corresponds to the single cluster, and the cluster with the highest $E_{\pi^{0}}^{*}$ in the case of an unmerged $\pi^{0}$. As shown in the figure the shapes match rather well, but there is a clear shift upward for the data. The same figure also shows the distribution of the number of crystals in the cluster: again the MC describes the data well, apart from a small upward shift. Since this variable is not used in the selection it is not investigated further.

Fig 9.24 shows the lateral shape distributions separately for merged and unmerged $\pi^{0} \mathrm{~s}$. The samples show very different distributions, with the photons from unmerged $\pi^{0}$ s peaking at around 0.30 like usual photons, and the merged $\pi^{0} \mathrm{~S}$ appearing as distorted showers around 0.50. However there seems to be significant cross-feed between the samples: the "unmerged" sample includes a long tail toward higher lat values, while the "merged" sample shows a secondary peak around 0.30 , especially at lower $E_{\pi^{0}}^{*}$ values where the proportion of unmerged $\pi^{0} \mathrm{~s}$ are higher.

These effects are explained in Fig. 9.25: the left-hand plot shows the distribution of the lateral moment versus the energy of the softer photon for reconstructed $\pi^{0} \mathrm{~s}$ in data. The tail corresponds almost exclusively to very soft photons, and can be interpreted as combinatorics background, where a merged $\pi^{0}$ clusters is paired with a random soft photon into a combination that fakes a $\pi^{0}$. The right-hand plots shows the distribution of the lateral moment versus the true $\cos \theta_{h e l}$ in MC. This shows two population, one in the center of the $\cos \theta_{\text {hel }}$ with high lat values and the other on the edges of the $\cos \theta_{\text {hel }}$ with low lat values. The first are probably real merged $\pi^{0} \mathrm{~s}$ while the second are unmerged $\pi^{0} \mathrm{~s}$ in which the second photon has been lost. This also explains why the secondary peak at 0.30 is higher in the lower $E_{\pi^{0}}^{*}$ bins, since the $\pi^{0}$ decay products are then softer and thus more likely to fall below the cluster energy cutoff.

In the ISR analysis the lateral moment cut is applied together with a reconstructed $\pi^{0}$ veto that excludes the range $100<m_{\gamma \gamma}<170 \mathrm{MeV}$, so we are only interested in the cut efficiency on the other part of the sample, i.e. merged $\pi^{0} \mathrm{~S}$ and unmerged $\pi^{0}$ with reconstructed mass outside the range given above. Since most of the unmerged $\pi^{0}$ s fall within the $m_{\gamma \gamma}$ range, this subsample is almost identical to the merged $\pi^{0}$ 

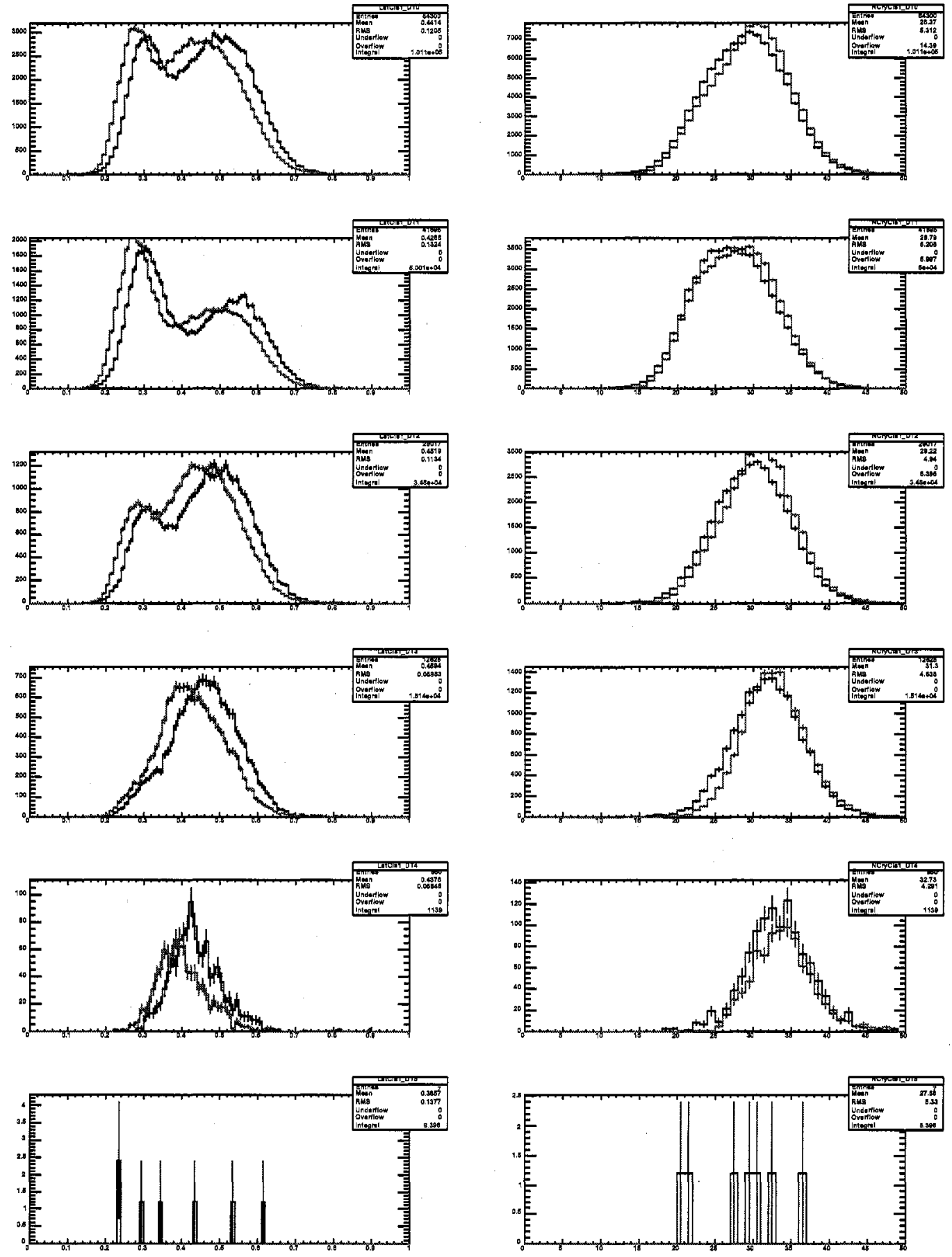

Figure 9.23: Cluster lateral shape distributions (left column) and number of crystals (right column) for the hard photons in unmerged $\pi^{0}$ decays and merged $\pi^{0} \mathrm{~s}$, for data (blue) and reweighted MC (red). The top row corresponds to all events, while subsequent rows correspond to the usual $E_{\pi^{0}}^{*}$ bins with boundaries at 3.3, 3.8, 4.3, 4.8 and $5.3 \mathrm{GeV}$. 

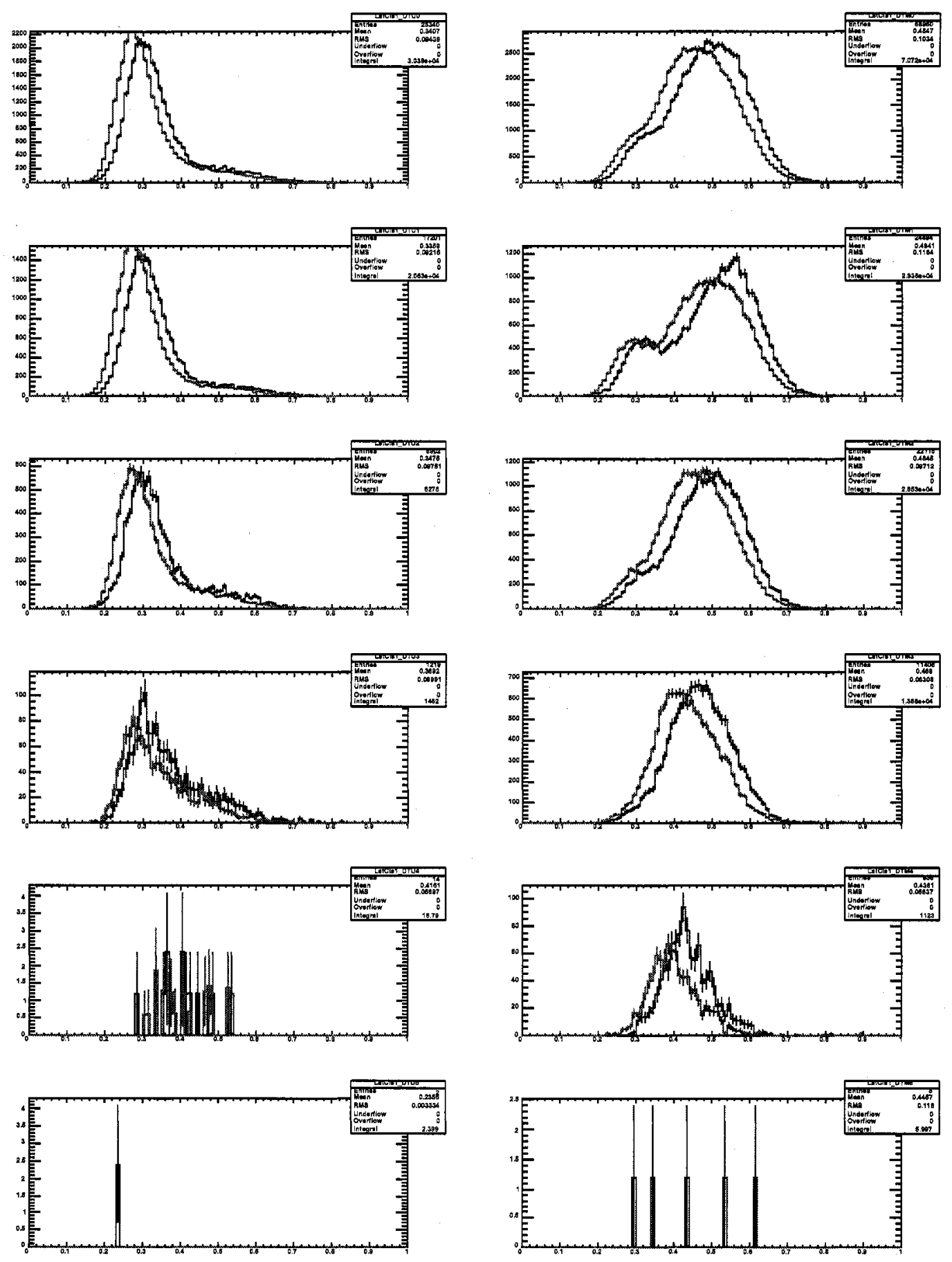

Figure 9.24: Cluster lateral shape distributions for the hard photons in unmerged $\pi^{0}$ decays (left) and merged $\pi^{0} \mathrm{~s}$ (right). The histograms are data (blue) and reweighted $\mathrm{MC}$ (red). The top row corresponds to all events, while subsequent rows correspond to the usual $E_{\pi^{0}}^{*}$ bins with boundaries at $3.3,3.8,4.3,4.8$ and $5.3 \mathrm{GeV}$. 

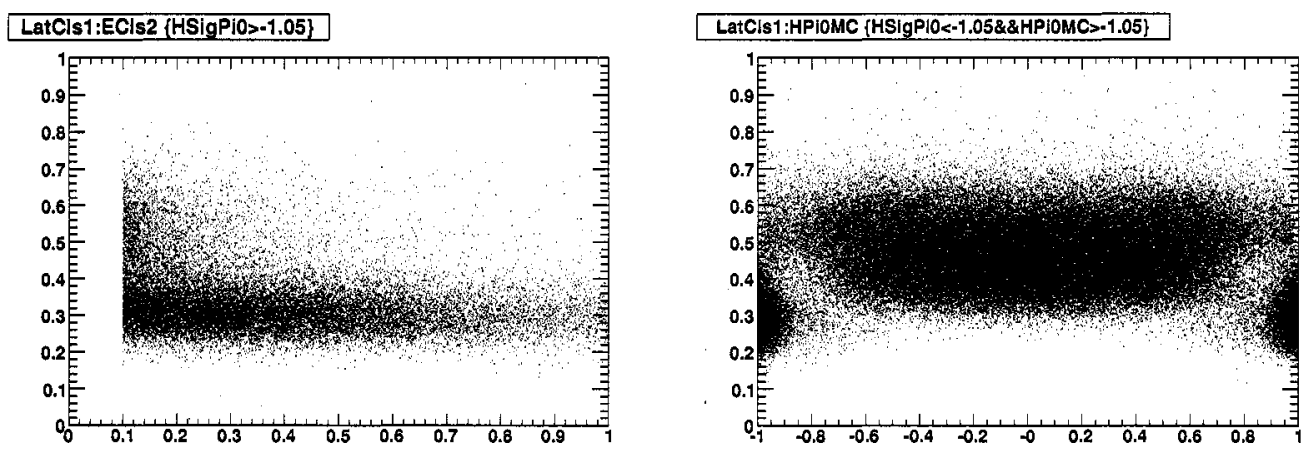

Figure 9.25: Left: lateral moment of the hard photon versus energy of the soft photon for reconstructed $\pi^{0}$ s in data Right: lateral moment of the cluster versus true $\cos \theta_{\text {hel }}$ for merged $\pi^{0} \mathrm{~s}$ in data.

one. Its lateral moment distribution is shown in the left-hand column of Fig. 9.26

There is a clear overall shift between the MC and data distributions. The middle column of Fig. 9.26 shows the data together with an MC distribution that is shifted by the difference of the means of the data and MC histograms. The right-hand column shows the same procedure but with the MC scaled up by the ratio of the histogram means instead. The shifting procedure appears to give the best agreement, and excellent agreement is obtained between the data and shifted MC distributions. The magnitude of the shift is shown in the left plot of Fig. 9.27.

A correction for the residual difference in shape can be determined by calculating the ratio of the integrals of the data and $\mathrm{MC}$ distributions over the interval $0-0.40$, since Lat $<0.40$ is the cut used in the ISR analysis. Fig. 9.27 shows the values for each of the $E_{\pi^{0}}^{*}$ bins. There is insufficient statistics in the last bin, $4.8-5.3 \mathrm{GeV}$, to determine a meaningful number, so the value from the preceding bin is used instead. In any case, only a very small fraction of $\pi^{0}$ events are expected to be found in this bin. 

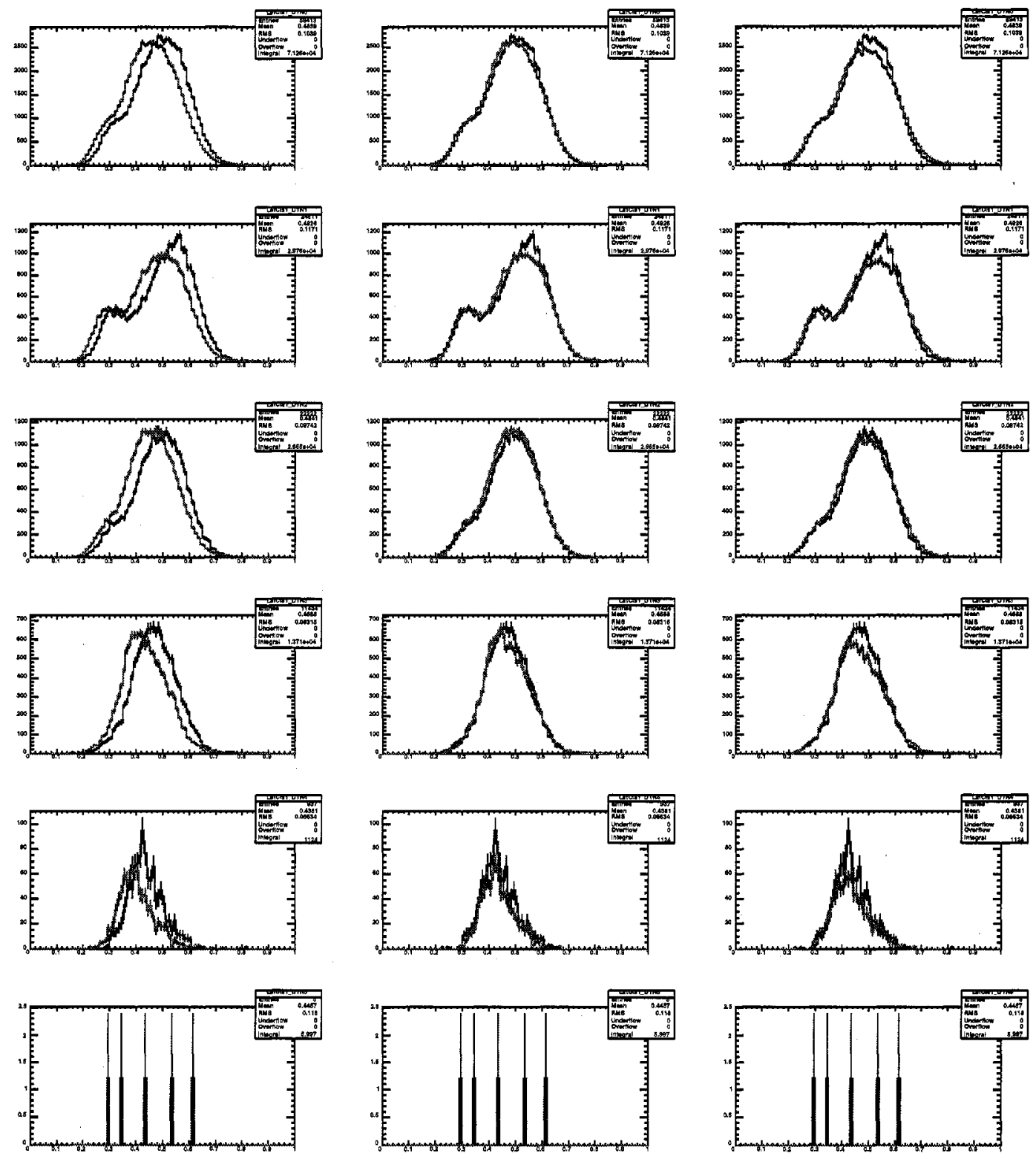

Figure 9.26: Cluster lateral shape distributions for the hard photons in merged $\pi^{0} \mathrm{~S}$ and unmerged with an invariant mass outside the interval $100<m_{\gamma \gamma}<170 \mathrm{MeV}$. The left column shows the data and raw MC distribution, the middle column shows the same with the MC shifted up and the right column the same with the MC scaled up The histograms are data (blue) and reweighted MC (red). The top row corresponds to all events, while subsequent rows correspond to the usual $E_{\pi^{0}}^{*}$ bins with boundaries at $3.3,3.8,4.3,4.8$ and $5.3 \mathrm{GeV}$. 

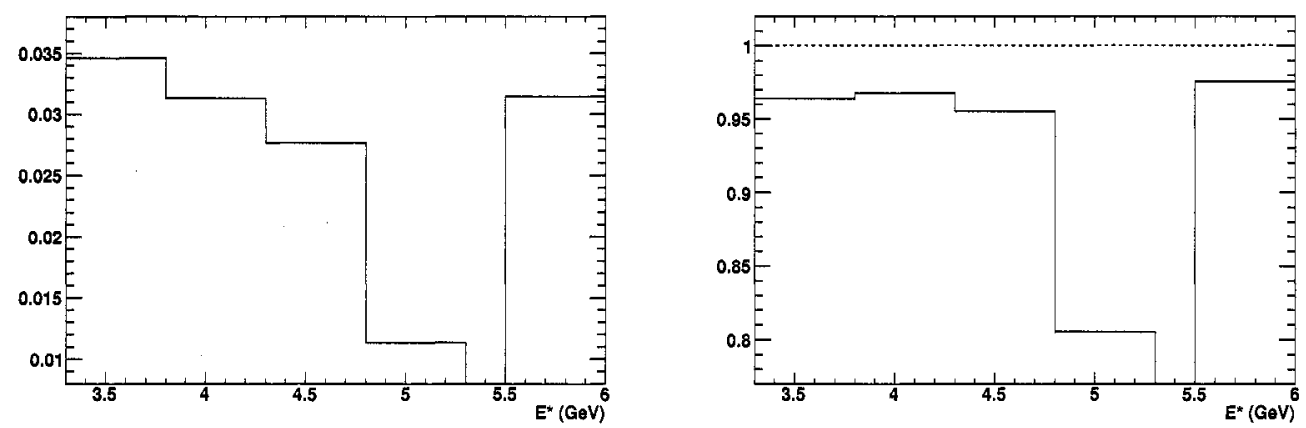

Figure 9.27: Left: magnitude of the shift for the MC lateral moment distribution. The last bin corresponds to all events. Right:Efficiency correction for the lateral moment cut on $\pi^{0}$ s passing the mass veto.

\subsection{Cross-check of ISR cross-feed from antineu- trons}

\subsubsection{Strategy}

As shown in Table 6.1, antineutrons represent about $5 \%$ of all backgrounds. They are suppressed by the cluster lateral moment cut, as shown in Table 6.8 , but this quantity is notoriously badly simulated. To cross-check the value of the rejection rate it is therefore necessary to validate the EMC parameters of antineutrons using a data control sample.

Obtaining an antineutron sample with sufficient purity and large statistics is extremely difficult. However at high energy antiprotons are expected to have the same behavior as antineutrons in the calorimeter:

- There is little difference between the $\bar{p} p$ and $\bar{p} n$ cross-sections for $p_{\bar{p}}>1.5 \mathrm{GeV}[15]$.

- The calorimeter material (CsI) has roughly the same amount of protons and neutrons.

A pure sample of protons and antiprotons with large statistics can be obtained from high-momentum $\Lambda \rightarrow p \pi$ decays. 


\subsubsection{Selection of $\Lambda \rightarrow p^{+} \pi^{-}$events}

The selection is as follows:

- Require the proton to pass the loose proton selection of Section E. ${ }^{1}$.

- Require the pion no to be identified as an electron (to reject suppress conversion background)

- Perform geometric and kinematic fits of $p \pi$ combinations

- $1105.8<m_{\Lambda}<1125.6 \mathrm{MeV}$

- $E_{\Lambda}^{*}>2 \mathrm{GeV}$

- $\alpha_{\Lambda}<30 \mathrm{mrad}$

where $\alpha_{\Lambda}$ is the angle between the $\Lambda$-momentum and its flight direction from the event primary vertex.

Significant amounts of background, mainly for non-hadronic sources, remain after this selection. To purify the sample, we apply the following further cuts:

- $d_{x y}^{\Lambda}>5 \mathrm{~mm}$

- $m_{p}^{D r c}>650 \mathrm{MeV}$

where $d_{x y}^{\Lambda}$ is the transverse flight distance of the $\Lambda$ from the primary vertex and $m_{p}^{D r c}$ is a measure of the mass of the proton derived from its measured momentum in the tracking system and the value of $\cos \theta_{c}=1 /(\beta n)$ measured in the DIRC.

Fig 9.28 shows the $\Lambda$ mass peak before and after these extra cuts.

For efficiency studies we use a $\Lambda_{c}^{+} \rightarrow \Lambda(p \pi) \pi^{+}$sample that is available in SP and should ensure a hard proton spectrum. For the study itself we compare data with with the uds MC sample. The efficiency of the selection is shown in table 9.6.

\footnotetext{
${ }^{1}$ This criterion relies only on tracking and DIRC information, so this requirement does not bias the EMC observables.
} 

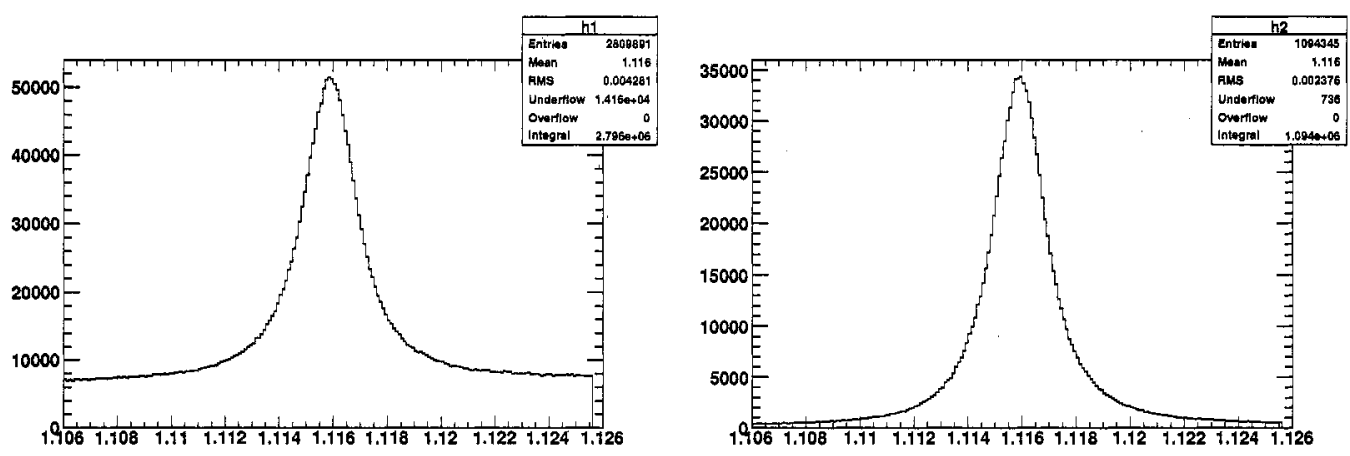

Figure 9.28: $\Lambda$ mass distribution before (left) and after (right) extra selection cuts

Table 9.6: Efficiencies for the $\Lambda \rightarrow p^{ \pm} \pi^{\mp}$ event selection

\begin{tabular}{|l|c|}
\hline & $\Lambda_{c}^{+} \rightarrow \Lambda\left(p^{ \pm} \pi^{\mp}\right) \pi^{+}(\%)$ \\
\hline$\Lambda$ mass cut & $62.00 \pm 0.34$ \\
$\Lambda$ flight angle cut & $52.68 \pm 0.35$ \\
$\Lambda$ mass cut & $52.68 \pm 0.35$ \\
$\Lambda$ flight angle cut & $45.98 \pm 0.35$ \\
$\Lambda d_{X Y}$ cut & $44.87 \pm 0.35$ \\
$m_{p}^{D r c}<650 \mathrm{MeV}$ & $42.56 \pm 0.34$ \\
Lambda Tagbit & $42.54 \pm 0.34$ \\
Proton energy & $42.54 \pm 0.34$ \\
Events in $250 / \mathrm{fb}$ & $16510183.3 \pm 175600.5$ \\
\hline
\end{tabular}




\subsubsection{Comparison of Antiprotons and Antineutrons in MC}

The MC can also be used to test the assumption that antiprotons and antineutrons behave similarly: in the same sample of $\Lambda_{c}^{+} \rightarrow \Lambda(p \pi) \pi^{+}$we isolate antiprotons and antineutrons from $\Lambda$ decays and compare their interactions with the detector. The distributions of deposited energy and lateral moments are shown in Fig 9.29. The energy distribution shows differences at low energy, but there is excellent agreement in the higher-energy region which produces background to ISR.
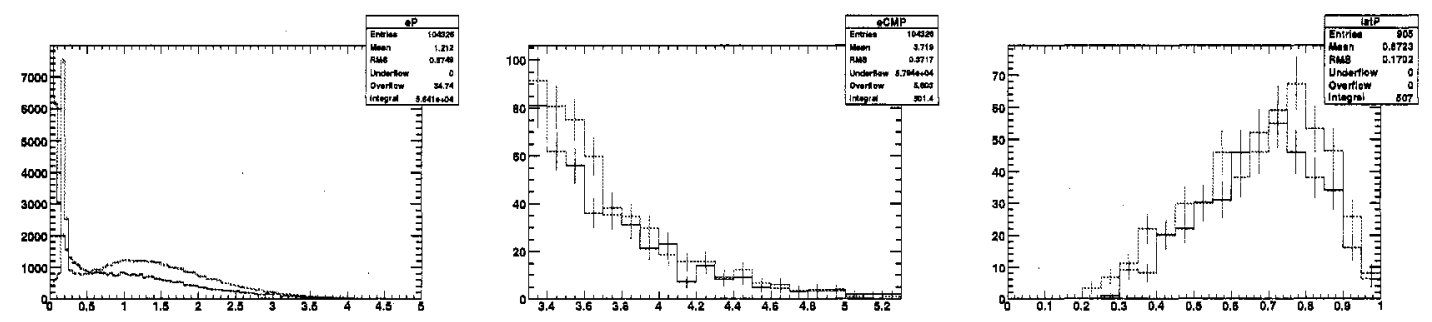

Figure 9.29: Distributions for the lab energy deposition (left), CM energy deposition (center) and cluster lateral moment (right) for antiprotons (red) and antineutrons (blue) produced in simulated $\Lambda$ decays. The antiproton curves are shown rescaled by a factor $35.8 / 63.9$ to account for the difference in $\Lambda$ branching fractions.

\subsubsection{Validation of Antiproton Energy Deposition and Lat- eral Moment Distributions}

We now compare data and MC for the antiprotons. Since some background remains under the $\Lambda$ peak, a background subtraction is performed to remove it. We use the region $1113.6<m_{\Lambda}<1118 \mathrm{MeV}$ around the peak and a symmetric sideband defined by $1105.8<m_{A}<1111.4 \mathrm{MeV}$ and $1120.2<m_{\Lambda}<1125.6 \mathrm{MeV}$. To determine the relative amount of signal and background in each region the $m_{A}$ distribution is fitted to the sum of a linear background term and a Gaussian peak. If the fit $\chi^{2} / n_{d o f}$ exceeds 1.5, a double Gaussian shape for the peak is used instead. The amounts of signal and background in the peak and sideband regions are obtained by integrating the PDFs over their mass ranges. This is performed in 5 bins of the proton lab 
momentum, with boundaries at $1.9,3,4,5$ and $6 \mathrm{GeV}$. Figs 9.30 and 9.31 show the fitted $m_{\Lambda}$ distributions in $\mathrm{MC}$ and data respectively. The distribution of signed proton momenta is shown in Fig. 9.32 Fig 9.33 shows the energy $E_{D e p}$ deposited in the calorimeter by the proton or antiproton. Fig 9.34 shows the ratio of $E_{D e p}$ to the maximum value $m_{p}+\sqrt{p_{p}^{2}+m_{p}^{2}}$, where $m_{p}$ and $p_{p}$ are the proton mass and momentum. The $\mathrm{MC}$ and data differ widely in shape, particularly for antiprotons. In fact it should be noted that the high tail of the ratio distribution in MC extends beyond 1 , which is unphysical.

Fig. 9.35 shows the "deposited CM energy" $E_{D e p}^{*}$ calculated by boosting the cluster in the CM frame, assuming its mass to be 0 as it would be if it were a misidentified ISR photon, for all values of proton momentum and antiprotons only. The closeup on the region $E_{D e p}^{*}>3.3 \mathrm{GeV}$ which contributes background to the ISR analysis shows a clear discrepancy between data and $\mathrm{MC}$, with the data dying off much faster than $\mathrm{MC}$ at high energy. The correction shown on the right-hand plot of Fig. 9.35 is applied to antineutron events in ISR MC.

Finally, this sample can be used to check the distribution of cluster lateral moment shape in data. Fig. 9.36 shows the lateral moment distributions in data and MC in bins of $p_{p}$ as defined above. There seems to be some discrepancy between data and MC, with data shifted toward higher values. This is not completely independent from the previous section since the lateral shape and shower energy may be correlated. However as shown in Fig. 9.37, the lateral shape seem to have little dependence on shower energy in MC.

However in order to remove possible bias we reweigh the MC with the correction function given in Fig. 9.37 for the lateral shape studies. The correction values are interpolated between the $E_{D e p}^{*}$ bins in order to keep smooth distributions. To validate the method the $E_{D e p}^{*}$. distribution after the correction is shown in the left and center plots in Fig. 9.38 : the agreement between corrected MC and data is very good, as it should be, with residual discrepancies probably attributable to binning effects.

The left-hand plot in Fig. 9.39 shows the distributions of the cluster lateral shape in data and MC. The data seems shifted toward higher values compared to MC, similarly to what was already observed in the case of $\tau \rightarrow \pi^{0}$ events. We apply the 

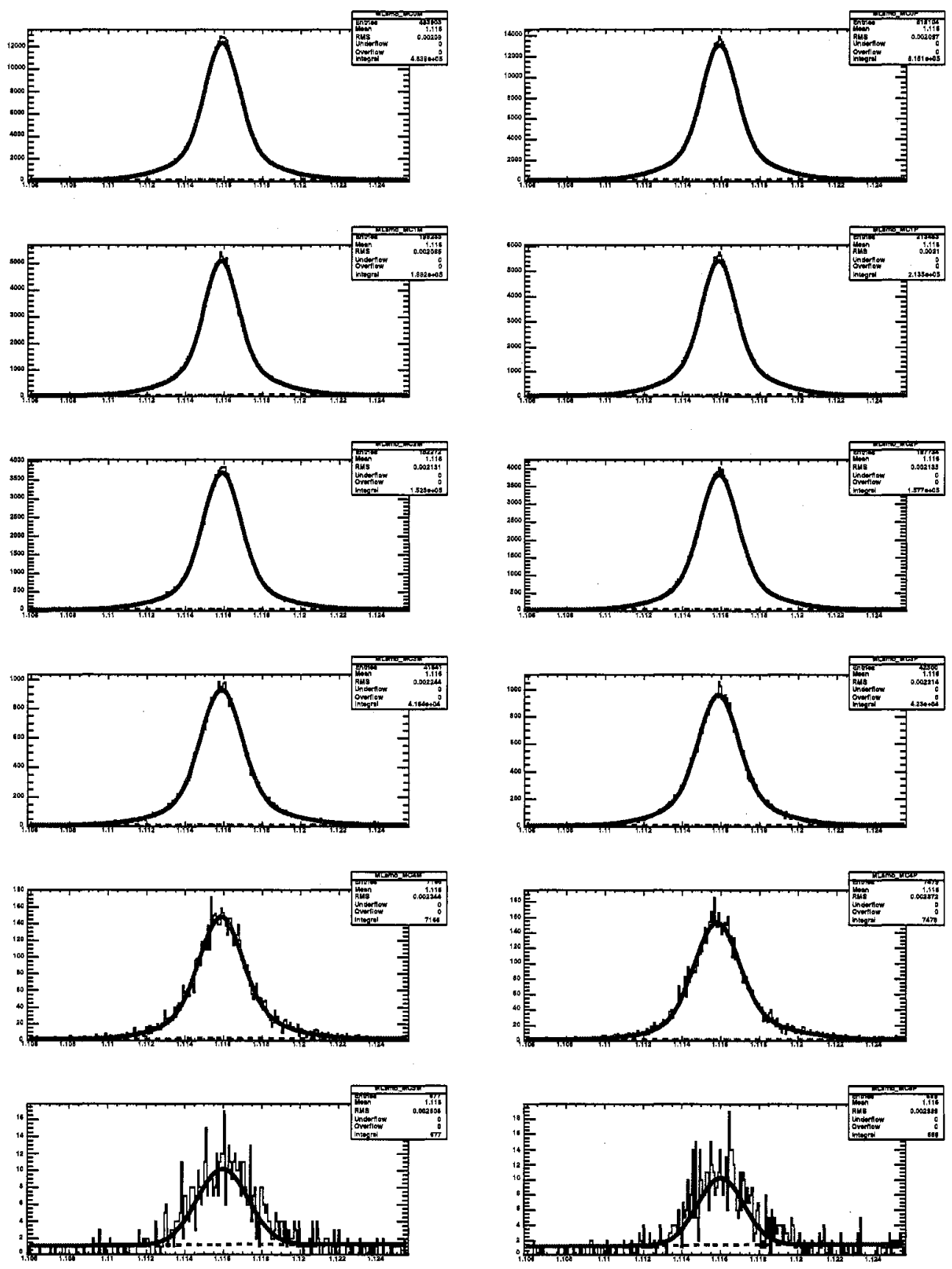

Figure 9.30: Fits of the $m_{\Lambda}$ distributions in MC. The left column in for antiprotons, right for protons, and rows show bins of $p_{\text {proton, }}$ increasing downward. 

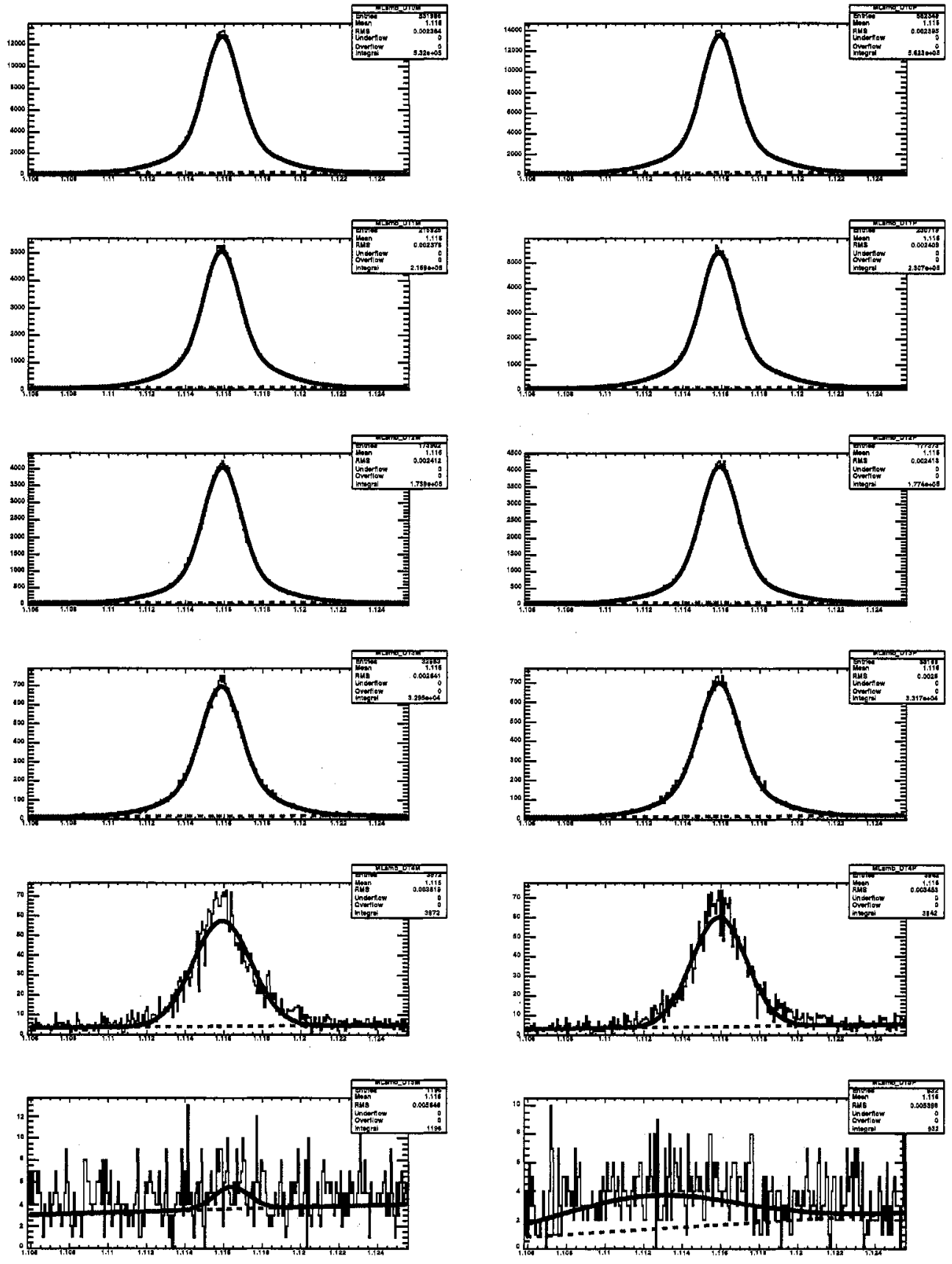

Figure 9.31: Fits of the $m_{\Lambda}$ distributions in data. The left column in for antiprotons, right for protons, and rows show bins of the proton momentum $p_{p}$, increasing downward. 


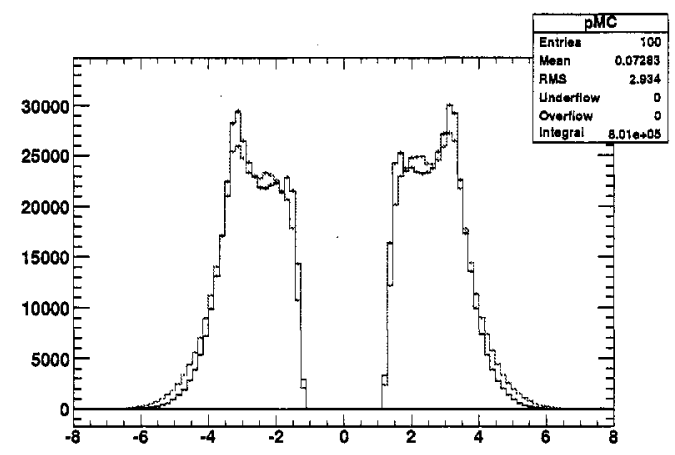

Figure 9.32: Distribution of the proton momentum $p_{p}$, signed with the charge of the particle.

same correction as in this latter case, first shifting the MC distribution so that the means agree (see right-hand plot in Fig. 9.39, which corresponds to a shift of +0.06 in the lat values. A residual correction due to the difference in shapes in then calculated by integrating the curves from 0 to 0.40 . This gives passing efficiencies of $2.9 \%$ for $\mathrm{MC}$ but only $2.2 \%$ in data. An additional suppression of $-24 \%$ corresponding to the ratio of these values is therefore applied to the antineutron MC for the ISR analysis.

\subsection{Study of $K_{L}^{0}$ background using $\phi \rightarrow K_{S}^{0} K_{L}^{0}$ events}

\subsubsection{Event Selection}

As shown in Table 6.1, $K_{L}^{0}$ showers represent about $4.5 \%$ of all backgrounds. Due to approximate CP invariance the inclusive $K_{L}^{0}$ multiplicity should be identical to that of $K_{S}^{0}$, which is expected to be well reproduced in MC. The main uncertainty concerns the characteristics of the showers created by $K_{L}^{0}$ in the EMC. As with antineutrons, two quantities need to be studied in particular: first the deposited energy in the EMC, and in particular the fraction of $K_{L}^{0}$ with a shower above the $3.3 \mathrm{GeV}$ threshold, and second the lateral moment of the shower.

In order to perform these checks we need a pure $K_{L}^{0}$ sample, and as it turns out the best sample for this purpose is the ISR mode $e^{+} e^{-} \rightarrow \phi\left(K_{S}^{0} K_{L}^{0}\right) \gamma$. These events 

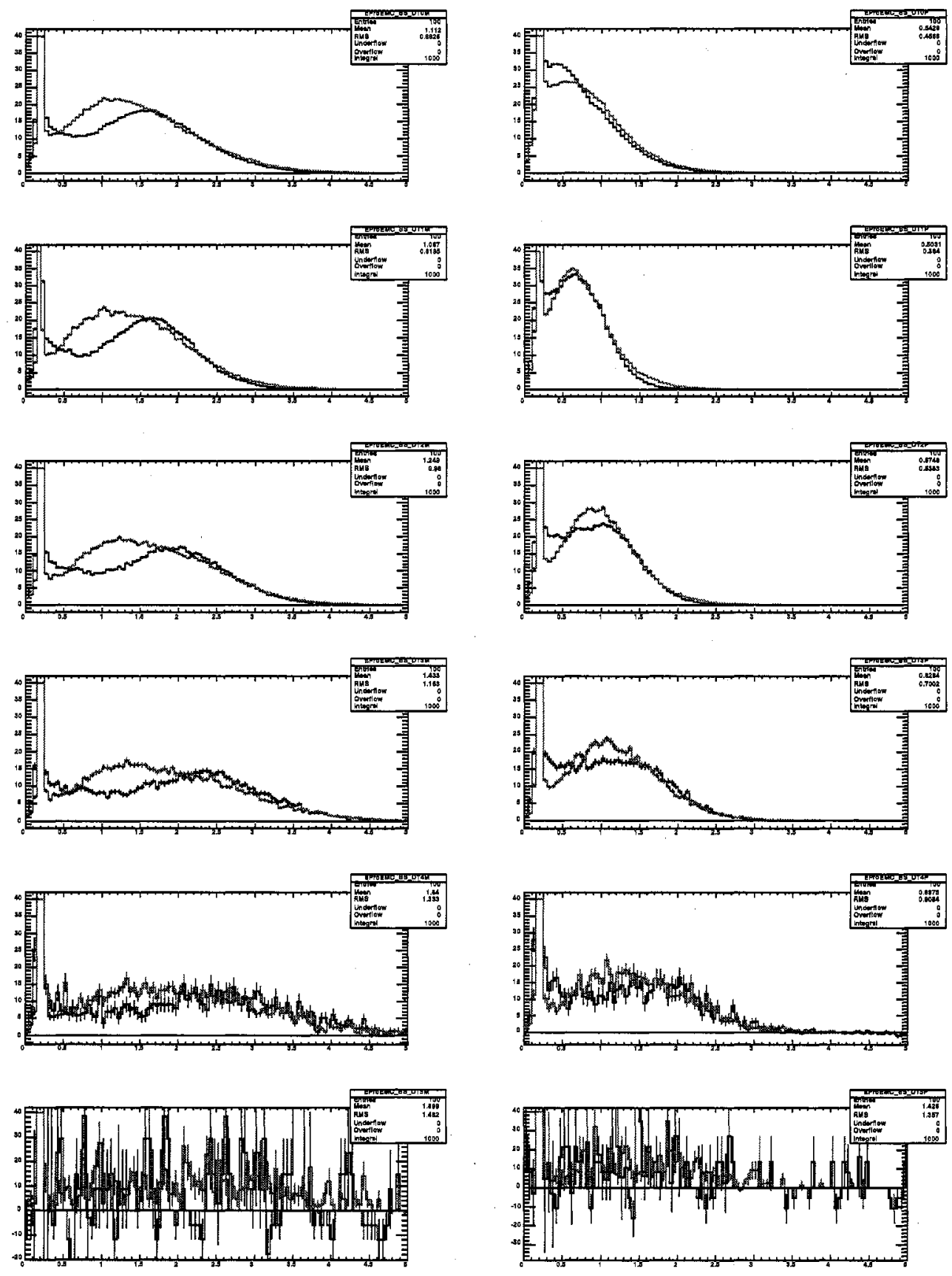

Figure 9.33: Distributions of the energy deposited by protons and antiprotons in the calorimeter, for MC (red) and data (blue). The left column in for antiprotons, right for protons. The first row shows all momenta, and rows below show bins of the proton momentum $p_{p}$, increasing downward. 

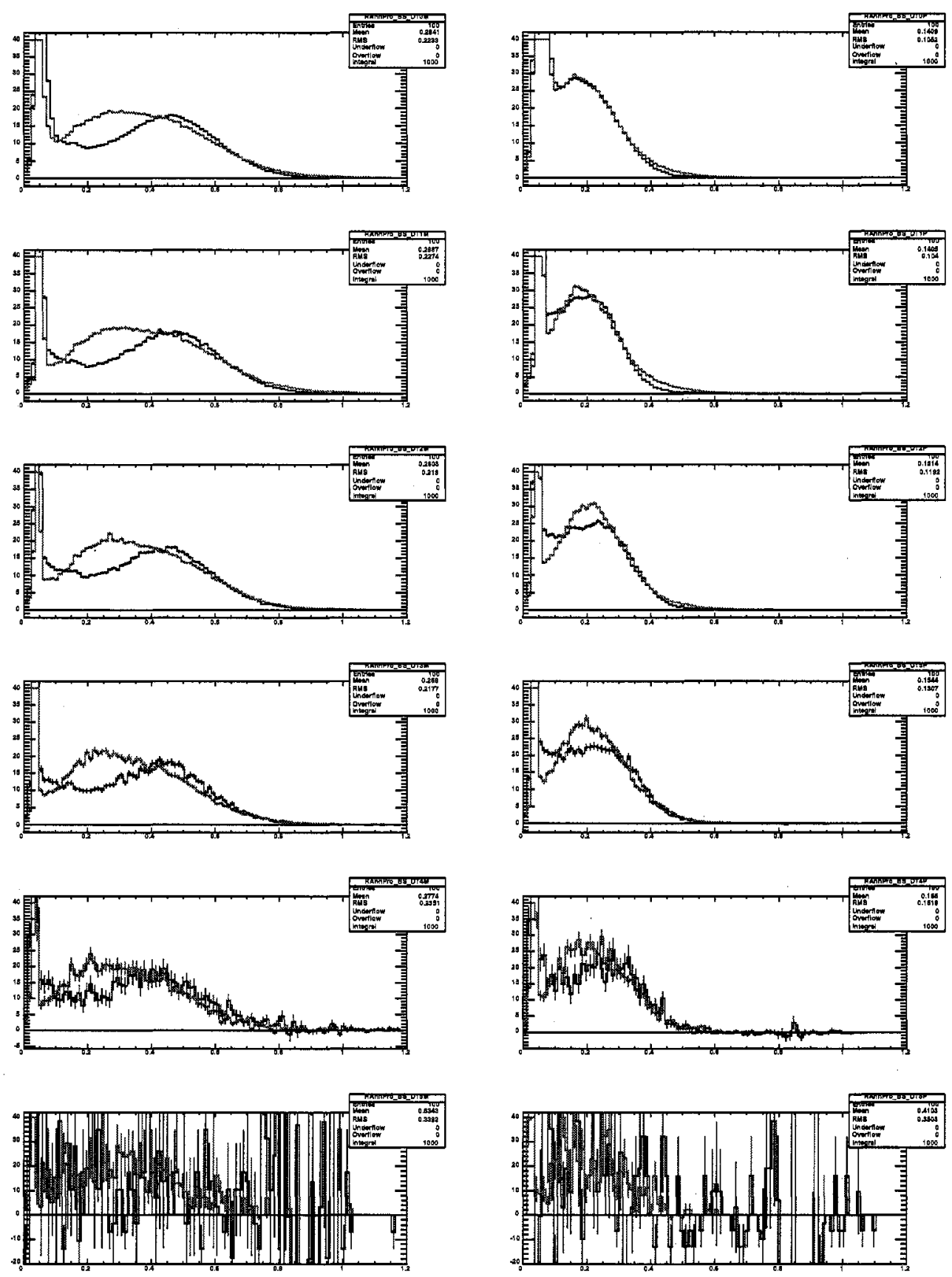

Figure 9.34: Distributions of the ratio of the energy deposited by protons and antiprotons in the calorimeter to the maximum value $m_{p}+\sqrt{p_{p}^{2}+m_{p}^{2}}$ for $\mathrm{MC}$ (red) and data (blue). The left column in for antiprotons, right for protons. The first row shows all momenta, and rows below show bins of the proton momentum $p_{p}$, increasing downward. 

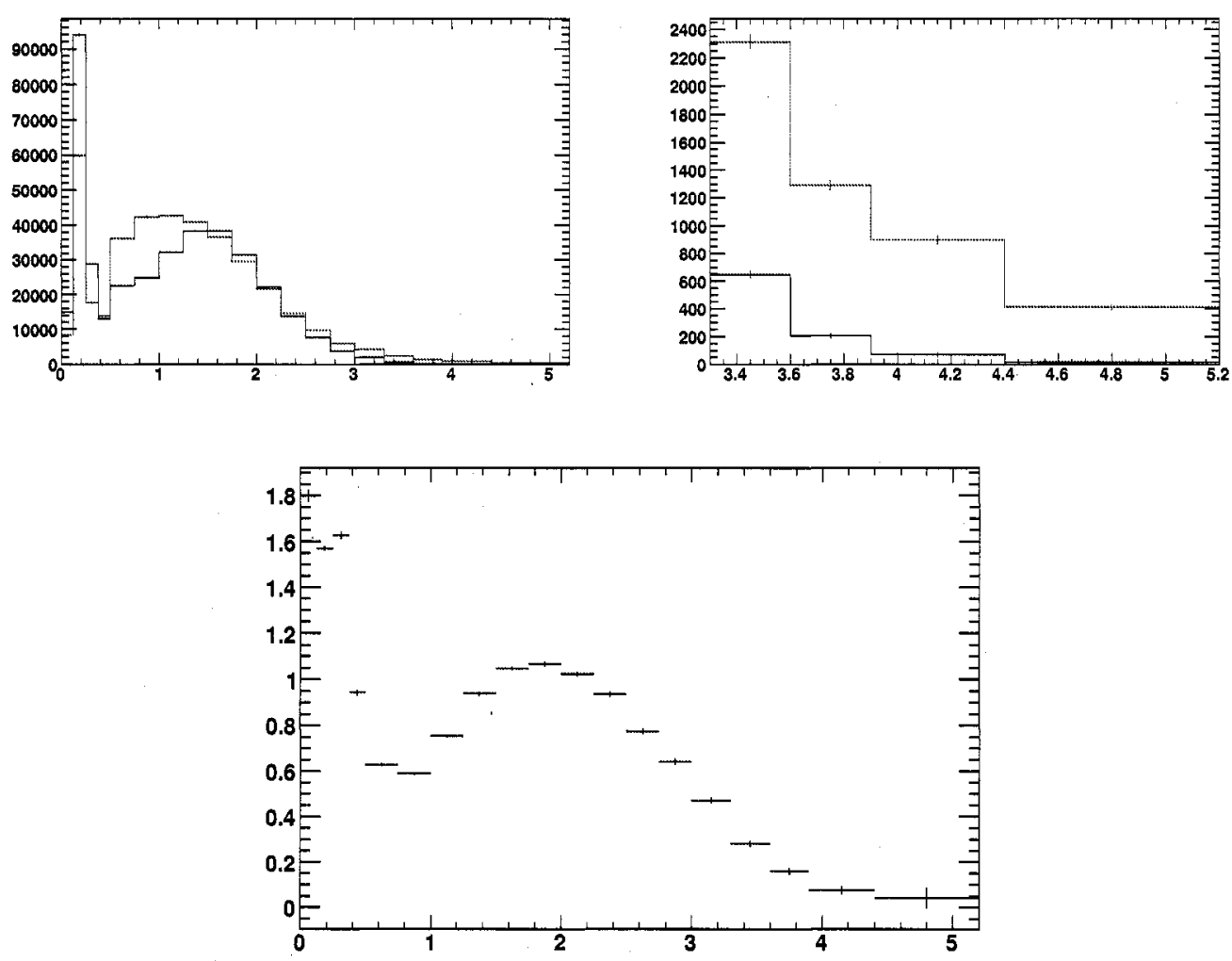

Figure 9.35: Top Left: Distributions of the CM energy deposited by antiprotons of all momenta in the calorimeter, for MC (red) and data (blue). Top Right: closeup of the region above $3.3 \mathrm{GeV}$ which is relevant for the ISR analysis. Bottom: ratio of data to $\mathrm{MC}$ distribution. 

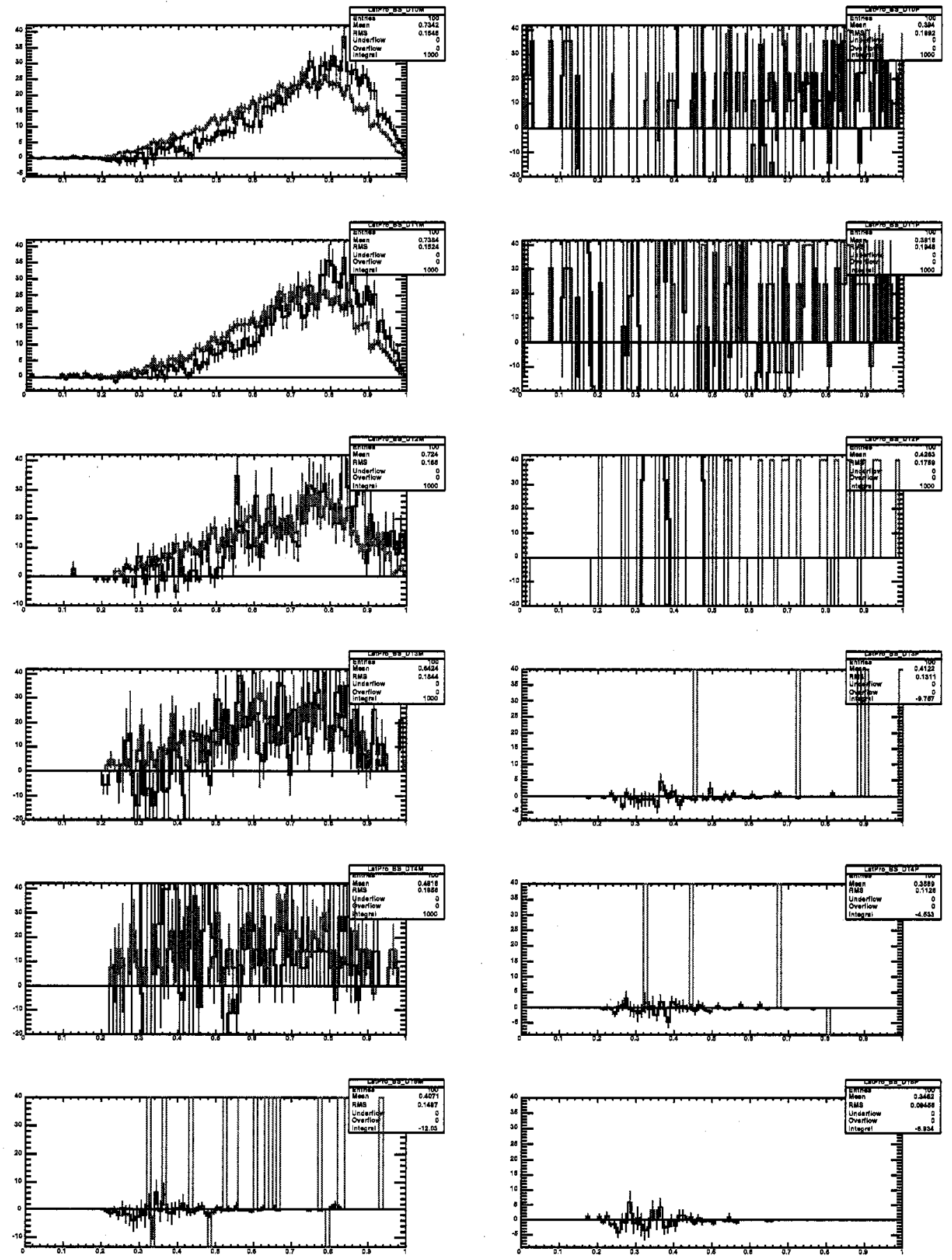

Figure 9.36: Distributions of the cluster lateral moment for MC (red) and data (blue). The left column in for antiprotons, right for protons. The first row shows all momenta, and rows below show bins of the proton momentum $p_{p}$, increasing downward. 


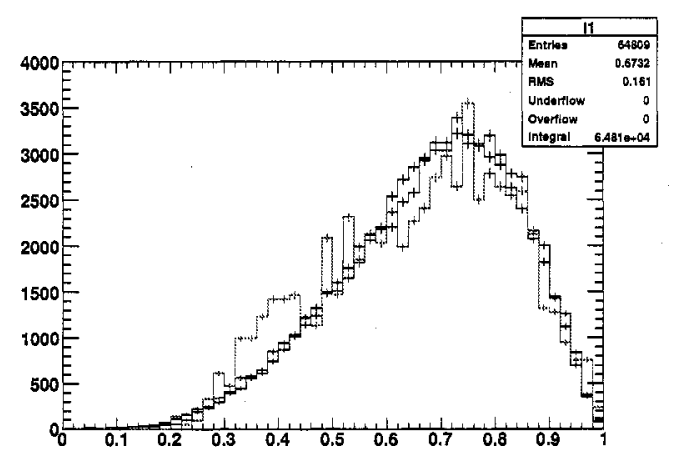

Figure 9.37: Distribution of cluster lateral moment for cluster CM energies $E_{D e p}^{*}$ in the range $2<E_{D e p}^{*}<3 \mathrm{GeV}$ (black), $3<E_{D e p}^{*}<4 \mathrm{GeV}$ (blue) and $E_{D e p}^{*}>4 \mathrm{GeV}$ (red).
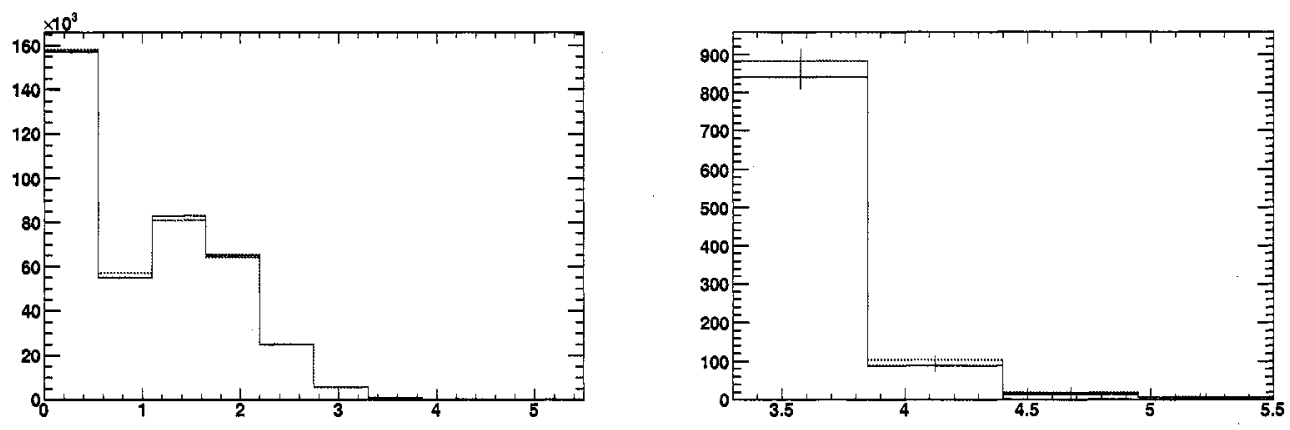

Figure 9.38: Left: distributions of the cluster CM energy $E_{D e p}^{*}$ for data (blue) and reweighted $\mathrm{MC}$ (red). Right: close-up of the region $E_{D e p}^{*}>3.3 \mathrm{GeV}$. 

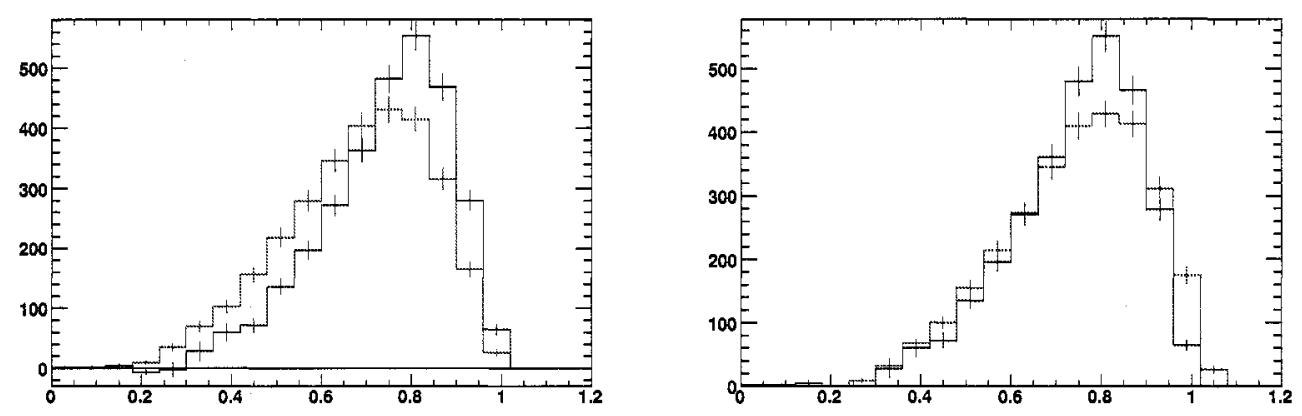

Figure 9.39: Left: distributions of cluster lateral moment for antiprotons with $E_{D e p}^{*}>$ $3.3 \mathrm{GeV}$ in data (blue) and reweighted MC (red). Left: Same plot with a shifted MC distribution.

can therefore be isolated directly from the ISR ntuples.

The selection is facilitated by the distinctive kinematics of the process: The $\phi$ is monochromatic at $E^{*}=5.24 \mathrm{GeV}$ in the CM frame, and the $\phi \rightarrow K_{S}^{0} K_{L}^{0}$ decay has $\sqrt{Q^{2}}=220 \mathrm{MeV}$, so that the kaons are produced with almost collinear momenta along the photon recoil direction, with an energy of roughly $2.6 \pm 1.0 \mathrm{GeV}$ in the $\mathrm{CM}$ frame. This signature can be used to select the event and also allows to locate the $K_{L}^{0}$. We therefore use the following selection:

- Require an ISR photon as in the ISR analysis, with $E_{\gamma}^{*}>4.5 \mathrm{GeV}$

- Require exactly $2 \mathrm{DCH}$ tracks in the event.

- Require the event to pass the QED rejection cuts of the ISR analysis

- Require the invariant mass of the 2 tracks to be within $488<m_{\pi \pi}<508 \mathrm{MeV}$

- Require the angle between the reconstructed $K_{S}^{0}$ and the photon recoil direction to be less than $100 \mathrm{mrad}$ in the CM frame.

- Require the difference $\left|E_{\gamma}^{*}-2 E_{K}^{*}\right|$ between the photon energy and twice the $K_{S}^{0}$ in the $\mathrm{CM}$ frame to be less than $1.5 \mathrm{GeV}$.

- Require no clusters in the EMC with $E>500 \mathrm{MeV}$ outside a cone of opening angle $200 \mathrm{mrad}$ around the ISR photon recoil direction. 
- Require the $K_{S}^{0}$ flight distance to be at least $0.25 \times \beta \gamma c \tau$.

The next-to-last cut in the list is justified by the presence of residual backgrounds in the uds sample, mainly due to the processes $e^{+} e^{-} \rightarrow K_{S}^{0} K_{L}^{0}$ neutrals, $e^{+} e^{-} \rightarrow$ $\bar{K}^{* 0}\left(K_{S}^{0} \pi^{0}\right) K_{L}^{0}$ neutrals and $e^{+} e^{-} \rightarrow \omega\left(\pi^{+} \pi^{-} \pi^{0}\right)$ neutrals where "neutrals" refers to some number of additional $\pi^{0} \mathrm{~s}$ and $\eta \mathrm{s}$.

The distributions of the relevant quantities are shown in Fig. 9.40 and 9.41. The efficiency of the selection is evaluated on a signal sample consisting of the subset KKMC $e^{+} e^{-} \rightarrow q \bar{q} \gamma$ events with the decay mode $\gamma \phi\left(K_{S}^{0}\left(\pi^{+} \pi^{-}\right) K_{L}^{0}\right)$. To study potential cross-feeds the efficiency of the selection is also studied in the usual background channels, as well as on the rest of the KKMC signal.
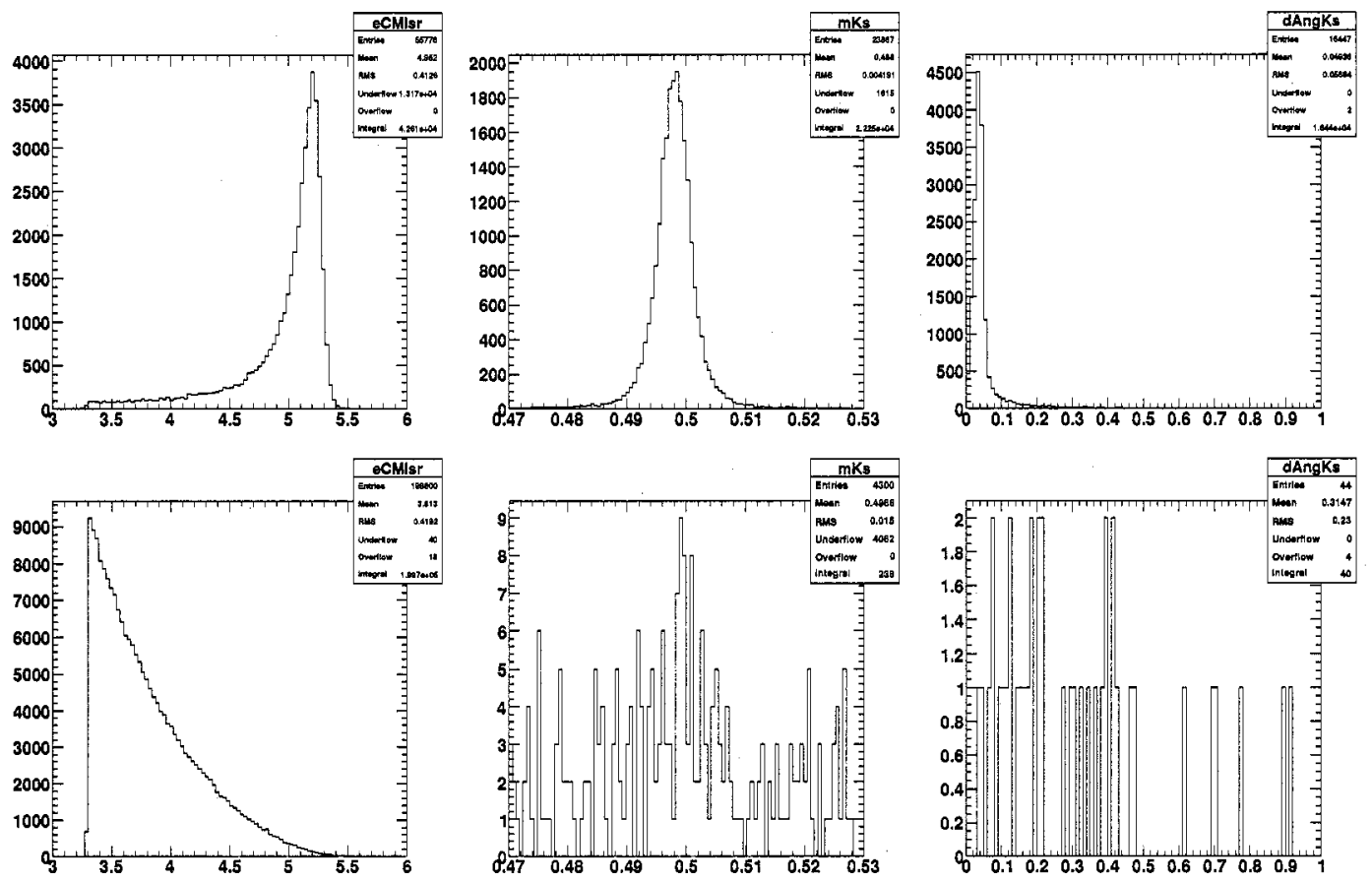

Figure 9.40: Distributions of the cut variables for signal signal (top row) and uds background (bottom row). From left to right the variables are ISR photon $E^{*}, K_{S}^{0}$ mass and angle between the $K_{S}^{0}$ and the ISR photon recoil direction in the CM frame.

Efficiencies as shown in Tables 9.7, 9.8, 9.9 and 9.10. 

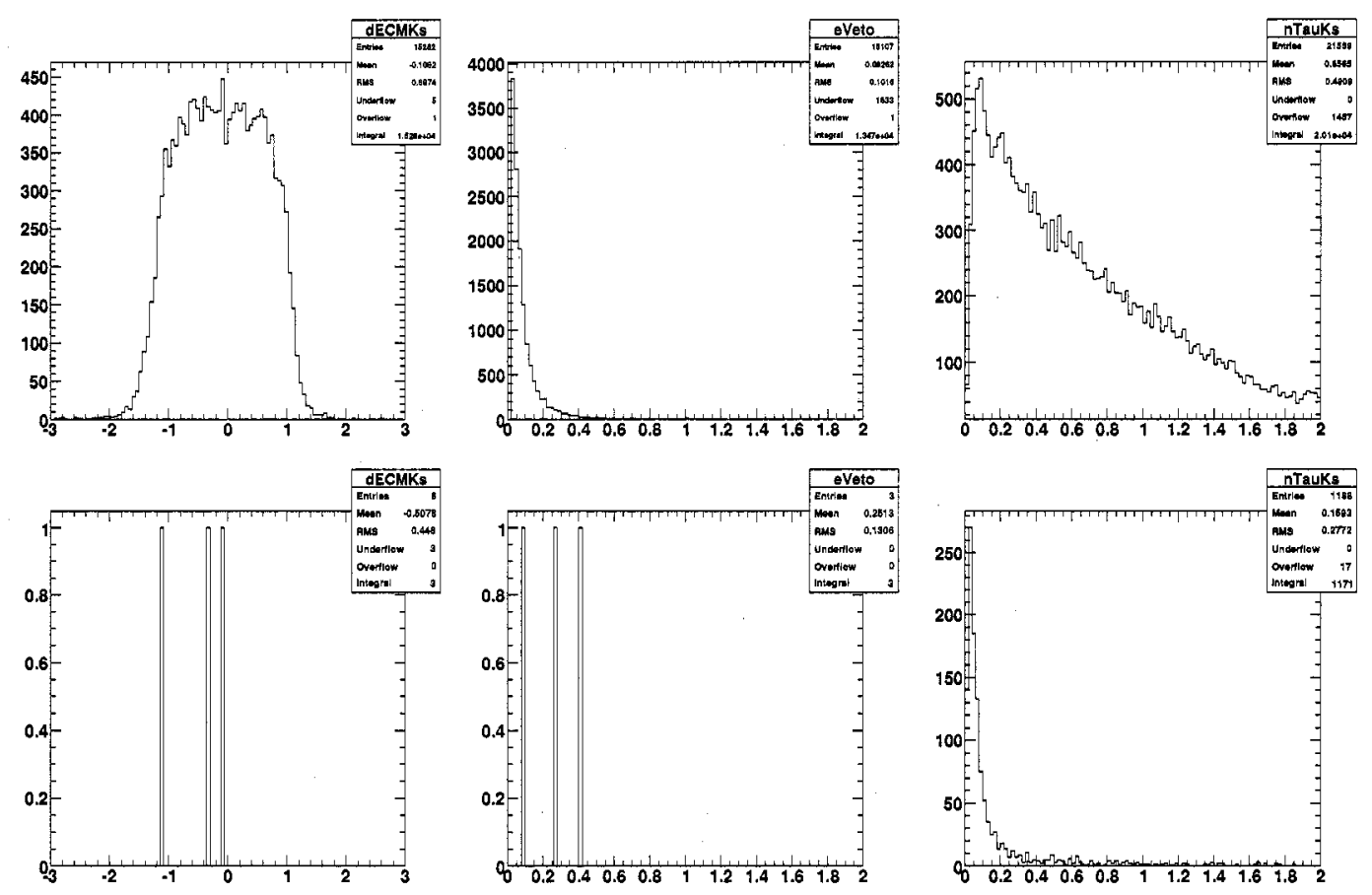

Figure 9.41: Distributions of the cut variables for signal signal (top row) and background (bottom row). From left to right the variables are $\left|E_{\gamma}^{*}-2 E_{K}^{*}\right|$; the energy of the highest-energy cluster outside a $150 \mathrm{mrad}$ cone around the $K_{S}^{0}$ direction and the $K_{S}^{0}$ flight distance in units of $c \tau$. The background sample is uds MC for the first two variables and non- $\gamma K_{S}^{0} K_{L}^{0}$ ISR events for the last one.

Table 9.7: Efficiencies for the $\phi \rightarrow K_{S}^{0} K_{L}^{0}$ event selection

\begin{tabular}{|l|c|}
\hline & $q \bar{q} \gamma(\%)$ \\
\hline$E_{I S R}^{*}>4.5$ & $67.05 \pm 0.20$ \\
2 Good Tracks & $53.86 \pm 0.21$ \\
Hadronic preselection & $42.79 \pm 0.21$ \\
$487<m_{\pi \pi}<507 \mathrm{MeV}$ & $38.65 \pm 0.21$ \\
$d>0.25 \times c \tau$ & $29.49 \pm 0.19$ \\
$\left\langle\vec{p}_{K S},-\vec{p}_{I S R}\right\rangle<0.1$ & $27.40 \pm 0.19$ \\
$\left|E_{\phi}-E_{\phi}^{\text {expec. }}\right|<1.5 \mathrm{GeV}$ & $27.09 \pm 0.19$ \\
EMC Veto & $26.81 \pm 0.19$ \\
Events in $250 / \mathrm{fb}$ & $38660.7 \pm 316.2$ \\
\hline
\end{tabular}


Table 9.8: Efficiencies for the $\phi \rightarrow K_{S}^{0} K_{L}^{0}$ event selection

\begin{tabular}{|l|c|c|c|c|}
\hline & $q \bar{q} \gamma(\%)$ & $e^{+} e^{-} \gamma(\%)$ & $\mu^{+} \mu^{-} \gamma(\%)$ & $\gamma \gamma(\%)$ \\
\hline$E_{I S R}^{*}>4.5$ & $57.59 \pm 0.11$ & $47.94 \pm 1.67$ & $60.04 \pm 0.11$ & $88.32 \pm 2.74$ \\
2 Good Tracks & $33.64 \pm 0.11$ & $32.26 \pm 1.56$ & $55.45 \pm 0.11$ & $28.47 \pm 3.86$ \\
Hadronic preselection & $33.64 \pm 0.11$ & $32.26 \pm 1.56$ & $55.45 \pm 0.11$ & $27.74 \pm 3.82$ \\
$487<m_{\pi \pi}<507 \mathrm{MeV}$ & $(5.98 \pm 0.17) \times 10^{-3}$ & $0.00 \pm 0.00$ & $(8.74 \pm 0.21) \times 10^{-3}$ & $0.00 \pm 0.00$ \\
$d>0.25 \times c \tau$ & $(9.82 \pm 0.70) \times 10^{-4}$ & $0.00 \pm 0.00$ & $(9.51 \pm 2.18) \times 10^{-5}$ & $0.00 \pm 0.00$ \\
$\left\langle\vec{p}_{K S},-\vec{p}_{I S R}\right\rangle<0.1$ & $(1.56 \pm 0.28) \times 10^{-4}$ & $0.00 \pm 0.00$ & $(9.51 \pm 2.18) \times 10^{-5}$ & $0.00 \pm 0.00$ \\
$\left|E_{\phi}-E_{\phi}^{\text {expec. }}\right|<1.5 \mathrm{GeV}$ & $(5.04 \pm 1.59) \times 10^{-5}$ & $0.00 \pm 0.00$ & $(5.01 \pm 5.00) \times 10^{-6}$ & $0.00 \pm 0.00$ \\
EMC Veto & $(3.02 \pm 1.23) \times 10^{-5}$ & $0.00 \pm 0.00$ & $(5.01 \pm 5.00) \times 10^{-6}$ & $0.00 \pm 0.00$ \\
Events in 250/fb & $318.2 \pm 129.9$ & $0.0 \pm 197.1$ & $27.7 \pm 27.7$ & $0.0 \pm 504.3$ \\
\hline
\end{tabular}

Table 9.9: Efficiencies for the $\phi \rightarrow K_{S}^{0} K_{L}^{0}$ event selection

\begin{tabular}{|l|c|c|c|}
\hline & $u d s(\%)$ & $c \bar{c}(\%)$ & $\tau^{+} \tau^{-}(\%)$ \\
\hline$E_{I S R}^{*}>4.5$ & $8.23 \pm 0.06$ & $2.83 \pm 0.04$ & $7.36 \pm 0.06$ \\
2 Good Tracks & $2.15 \pm 0.03$ & $(5.76 \pm 0.55) \times 10^{-4}$ & $4.39 \pm 0.05$ \\
Hadronic preselection & $2.15 \pm 0.03$ & $(5.76 \pm 0.55) \times 10^{-4}$ & $4.39 \pm 0.05$ \\
$487<m_{\pi \pi}<507 \mathrm{MeV}$ & $(6.06 \pm 0.55) \times 10^{-4}$ & $(1.57 \pm 0.91) \times 10^{-5}$ & $(2.00 \pm 1.00) \times 10^{-5}$ \\
$d>0.25 \times c \tau$ & $(2.20 \pm 0.33) \times 10^{-4}$ & $(1.05 \pm 0.74) \times 10^{-5}$ & $(2.00 \pm 1.00) \times 10^{-5}$ \\
$\left\langle\vec{p}_{K S},-\vec{p}_{I S R}\right\rangle<0.1$ & $(3.00 \pm 1.23) \times 10^{-5}$ & $(5.23 \pm 5.23) \times 10^{-6}$ & $0.00 \pm 0.00$ \\
$\left|E_{\phi}-E_{\phi}^{\text {expec. }}\right|<1.5 \mathrm{GeV}$ & $(1.50 \pm 0.87) \times 10^{-5}$ & $0.00 \pm 0.00$ & $0.00 \pm 0.00$ \\
EMC Veto & $(1.50 \pm 0.87) \times 10^{-5}$ & $0.00 \pm 0.00$ & $0.00 \pm 0.00$ \\
Events in $250 / \mathrm{fb}$ & $60.0 \pm 34.7$ & $0.0 \pm 1.2$ & $0.0 \pm 14.7$ \\
\hline
\end{tabular}


Table 9.10: Efficiencies for the $\phi \rightarrow K_{S}^{0} K_{L}^{0}$ event selection

\begin{tabular}{|l|c|c|}
\hline & $B^{0} \bar{B}^{0}(\%)$ & $B^{+} B^{-}(\%)$ \\
\hline$E_{I S R}^{*}>4.5$ & $15.47 \pm 0.20$ & $21.03 \pm 0.24$ \\
2 Good Tracks & $(3.20 \pm 1.01) \times 10^{-4}$ & $(7.09 \pm 5.01) \times 10^{-5}$ \\
Hadronic preselection & $(1.28 \pm 0.64) \times 10^{-4}$ & $(3.54 \pm 3.54) \times 10^{-5}$ \\
$487<m_{\pi \pi}<507 \mathrm{MeV}$ & $0.00 \pm 0.00$ & $0.00 \pm 0.00$ \\
$d>0.25 \times c \tau$ & $0.00 \pm 0.00$ & $0.00 \pm 0.00$ \\
$\left\langle\vec{p}_{K S},-\vec{p}_{I S R}\right\rangle<0.1$ & $0.00 \pm 0.00$ & $0.00 \pm 0.00$ \\
$\left|E_{\phi}-E_{\phi}^{\text {expec. }}\right|<1.5 \mathrm{GeV}$ & $0.00 \pm 0.00$ & $0.00 \pm 0.00$ \\
EMC Veto & $0.00 \pm 0.00$ & $0.00 \pm 0.00$ \\
Events in $250 / \mathrm{fb}$ & $0.0 \pm 0.8$ & $0.0 \pm 0.7$ \\
\hline
\end{tabular}

The leading background contributions are the ones described above, at a level of about $1 \%$ of signal.

\subsection{Study of $K_{L}^{0}$ shower parameters}

This section follows closely the study of antineutrons showers, which present similar issues. Since the $\phi \gamma$ events are fully reconstructed the $K_{L}^{0}$ kinematics are completely determined, and we split the $K_{L}^{0}$ sample in bins in the expected $K_{L}^{0}$ lab energy $E_{K L}^{E x p}$, with bin boundaries at $1,2,3,4$ and $6 \mathrm{GeV}$. The $K_{L}^{0}$ energy spectrum, shown in Fig. 9.42, only covers the region $1-5 \mathrm{GeV}$, by studying of the ratio $E_{K L}^{E M C} / E_{K L}^{E x p}$ of deposited to total energy one can draw conclusions that should also apply to higher energies

Fig. 9.43 shows the center-of-mass EMC energy $E_{K L}^{* E M C}$, the ratio $E_{K L}^{E M C} / E_{K L}^{E x p}$ and the lateral moment of the shower in bins of $E_{K L}^{E x p}$.

The lateral moment distribution is shown for $E_{K L}^{* E M C}>1 \mathrm{GeV}$. Ideally this cut should be set at $3.3 \mathrm{GeV}$ to reflect the situation for the ISR analysis, but this is beyond the reach of the kinematics of this decay.

The same correction procedure as in the case of antineutrons is applied here: first a weight factor is introduced in the $\mathrm{MC}$ so that the $E_{K L}^{E M C}$ distribution matches the one from data. Fig. 9.44 shows the weight function and the corrected energy spectrum. 


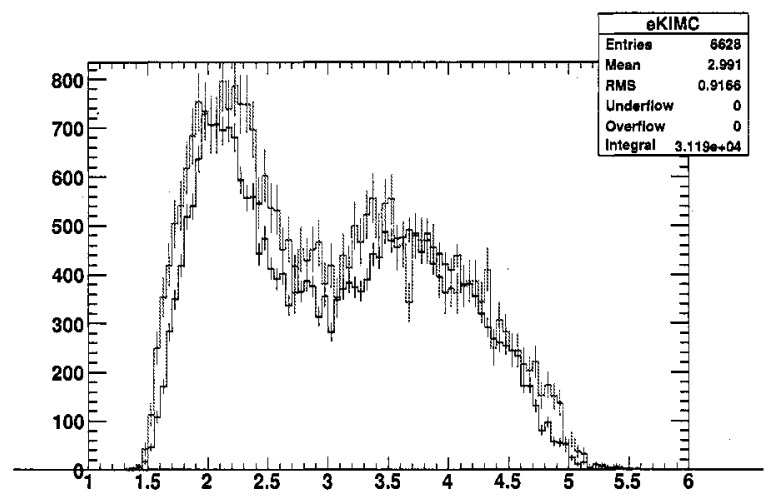

Figure 9.42: Expected lab energy spectrum of $K_{L}^{0}$ from $e^{+} e^{-} \rightarrow \gamma \phi\left(K_{S}^{0} K_{L}^{0}\right)$ based on the reconstructed $\gamma$ and $K_{S}^{0}$ momenta.

A feature of the background processes described in the previous section is that they involve $\pi^{0} \mathrm{~s}$ and $\eta \mathrm{s}$ instead of the $K_{L}^{0}$. They are therefore expected to deposit more energy into the EMC and be especially abundant at the higher end of the $E_{K L}^{E M C}$ spectrum, where the $K_{L}^{0}$ signal is suppressed. For this reason the rise in the weight function which is visible for $E_{K L}^{E M C}>1 \mathrm{GeV}$ is likely due to increasing background levels in data rather than $K_{L}^{0}$ shower characteristics.

We then plot the lateral moment in data and the reweighted MC, as shown in Fig. 9.45. As described above this should be shown for $E_{K L}^{E M C}>3.3 \mathrm{GeV}$ but due to the limited available kinematic region we instead require $E_{K L}^{E M C}>1 \mathrm{GeV}$. The means of the histogram are shifted by only 0.007 units, but we apply the same procedure of shifting the MC histogram, shown on the right-hand side of Fig. 9.45, and calculating a residual correction due to the difference in the relative integral over the interval $0-0.40$, which turns out to be $29 \%$.

\subsection{Validation of the Fisher Discriminant Distri- butions for Continuum Background}

Distributions for the Fisher discriminant variable for continuum background events with a $\pi^{0}$ can be obtained in the same way as we obtained $\pi^{0}$ parameters in previous 

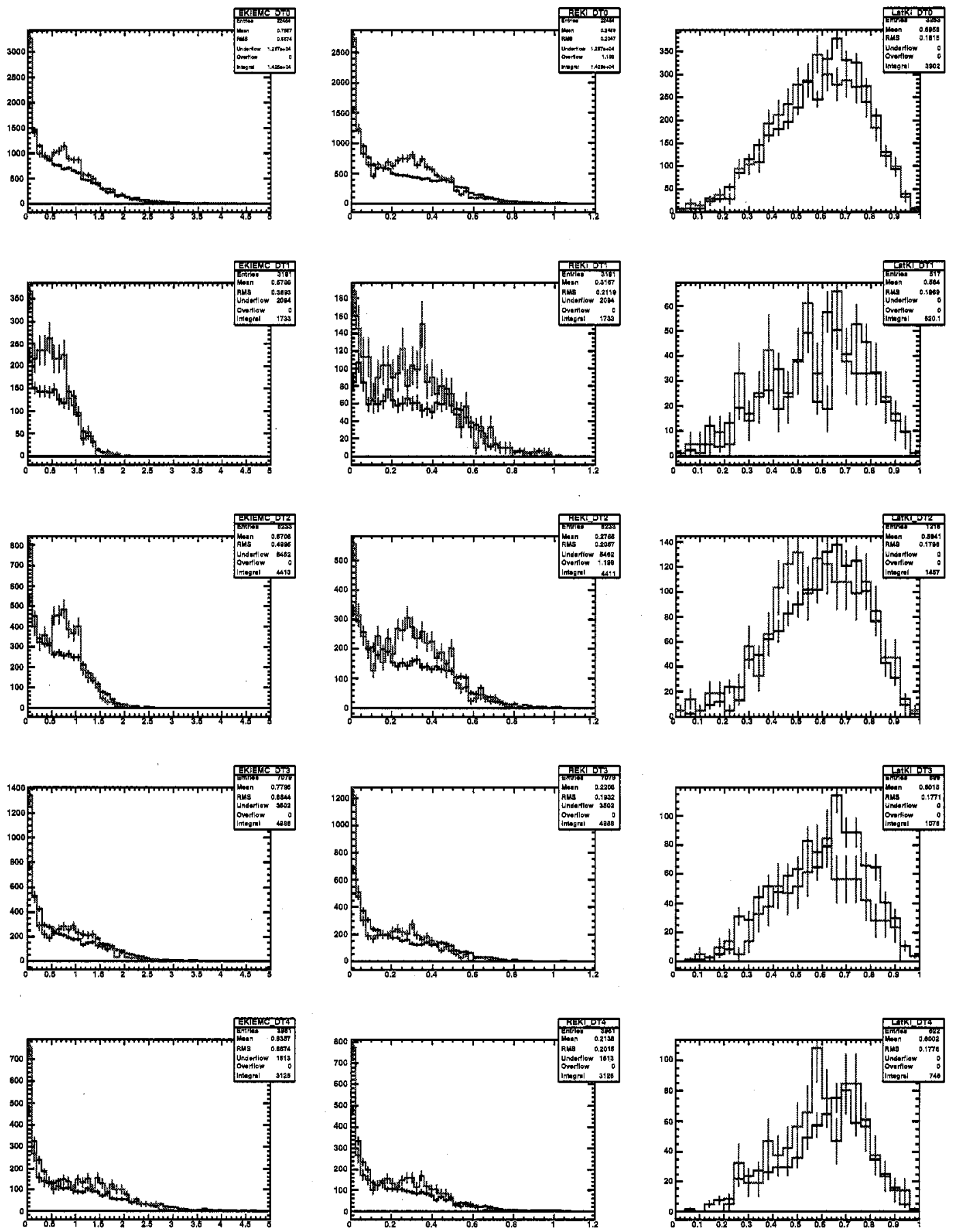

Figure 9.43: Left: $K_{L}^{0}$ center-of-mass deposited energy $E_{K L}^{* E M C}$. Center: ratio $E_{K L}^{E M C} / E_{K L}^{E x p}$ of deposited to expected energy in the lab frame. Right: lateral moment of the $K_{L}^{0}$ cluster for $E_{K L}^{* E M C}>1 \mathrm{GeV}$. The top row includes all events, and the next rows corresponds from top to bottom to the $E_{K L}^{E x p}$ intervals $1-2,2-3,3-4$ and $4-6 \mathrm{GeV}$. 

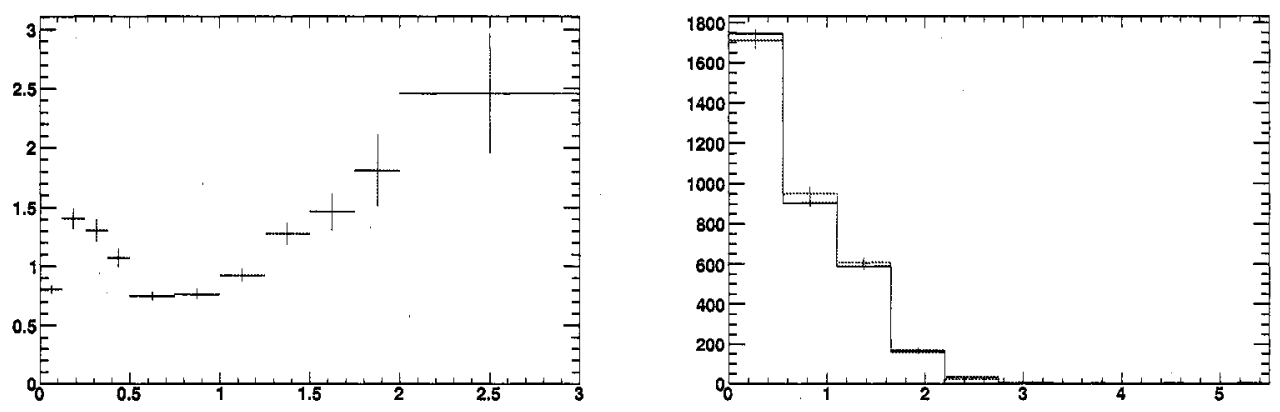

Figure 9.44: Left: Weight function applied to the MC so that the $E_{K L}^{E M C}$ distribution matched that of data. Right: $E_{K L}^{E M C}$ distributions in data (blue) and reweighted MC (red).
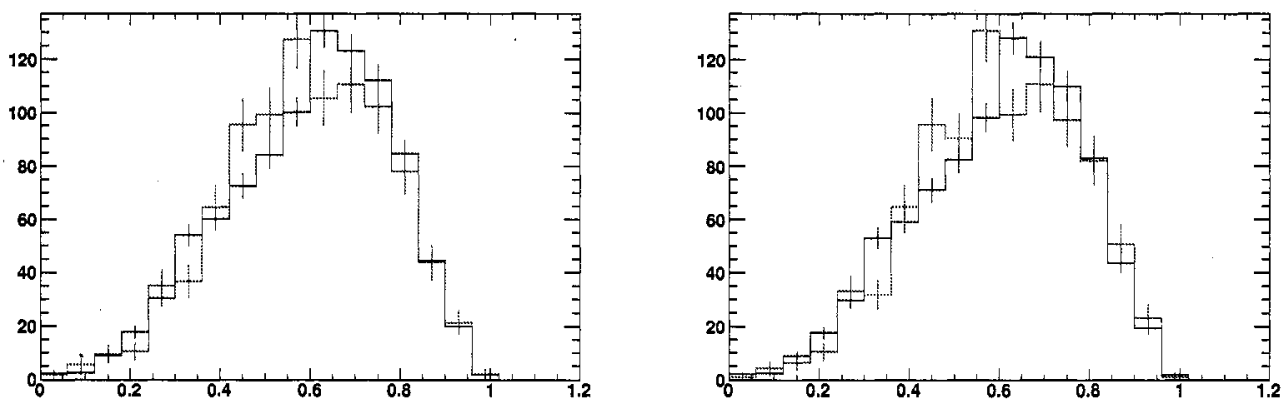

Figure 9.45: Lateral moment distribution in data (blue) and reweighted MC (red) without (left) and with (right) a shift in the MC corresponding to the difference in the histogram means. 
sections. We select events in which the mass $m_{\pi^{0}}$ of the best $\pi^{0}$ candidate is in the interval $120-150 \mathrm{MeV}$ and subtract the non-peaking component using the sideband corresponding to the intervals $80-110 \mathrm{MeV}$ and $160-180 \mathrm{MeV}$. Fig. 9.47 shows the results on data and the usual sum of MC components in bins of $s^{\prime}$. The bins are the usual bins used in Fisher studies, with boundaries at 9, 15, 20, 25, 30, 25, 38 and $42 \mathrm{GeV}$.

The agreement between MC and data is reasonable but not perfect. Since the event-shape variables are expected to be better simulated for events with high multiplicity, we split the sample into those with 2 IsrGoodTracks tracks or less and those with 3 or more. The corresponding plots are shown in Figs. 9.48 and 9.49.

The selection actually splits the sample into one containing mostly $u d s$ events $\left(N_{G T} \geq 3\right)$ and the other mostly $\tau^{+} \tau^{-}$events. The $\tau^{+} \tau^{-}$sample seems to have somewhat better agreement with data, especially at higher $s^{\prime}$, but the difference does not seem significant enough to warrant correcting the two independently. Therefore we determine a single correction in each $s^{\prime}$ bin corresponding to the ratio of the data to $\mathrm{MC}$ efficiencies of the Fisher cut in that bin. The Fisher cut values are shown in table 6.4. The correction is shown in Fig. 9.46.

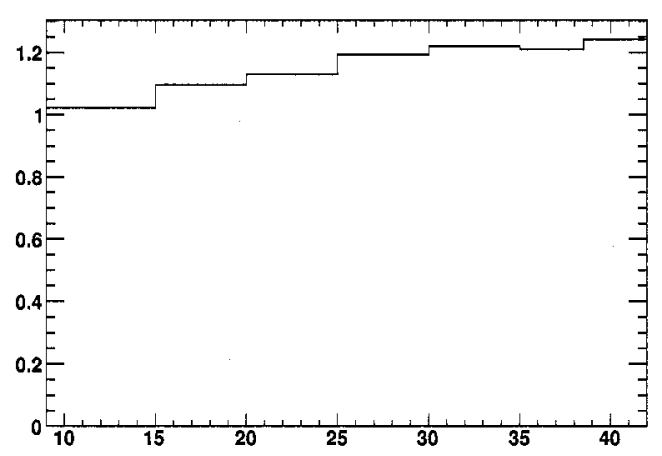

Figure 9.46: Fisher cut correction for continuum background

This study covers only the case of events with a reconstructed $\pi^{0}$. By extension, it should also apply to events with a merged $\pi^{0}$, since the extra photon should not modify the event shape distributions significantly. We further assume that the same 
correction can also be applied to the remaining $20 \%$ of non- $\pi^{0}$ continuum background events: the event-shape distributions should not depend strongly on the presence of specific high-momentum particles, be they $\pi^{0} \mathrm{~s}, \eta \mathrm{s}$, antineutrons or other.

\subsection{EMC Hot Towers}

A potential detector issue is the occurrence of electronics noise in the EMC. This can lead to so called "hot towers" or "hot crystals" which can have a high enough energy to fake an ISR photon. These regions are supposed to be masked out during the $B A B A R$ reconstruction phase, and any problems should also lead to the status word of the EMC cluster to be non-zero, which we reject during the photon selection (see Section 5.2).

However there seems to be circumstantial evidence for the fact that some problems remain. In order to study this, we produce EMC hitmaps with one bin per crystal and try to look for abnormally high occupancies. The problem is intrinsically timedependent and the time granularity must be chosen carefully: if too fine, low statistics limits the significance of the study; if too coarse a granularity the effect problematic crystals is diluted, and any problems lead to the rejection of large swathes of data.

We choose to group the data in blocks of about 10 million events in the original dataset: we combine neighboring runs and add the number of events until the total reaches 10 million. The blocks are also broken at the boundaries between Run2, Run3 and Run4. We obtain 298 such blocks, with about 60000 to 70000 events passing the QED selection. We could obtain even more statistics by looking at the data before QED selection, but due to the size of this dataset no ntuples were made at this stage.

We look for hot crystals in the hitmaps by computing the average occupancy over each EMC phi ring and then looking at the occupancies of individual crystals in the ring. If this occupancy is $3 \sigma$ over the ring average, the crystal is flagged as "hot". We find 61 such crystals, which are listed in Table 9.11.

Fig. 9.50 shows typical hitmaps for blocks with one or more hot crystals, and Fig. 9.51 shows the distribution of hot crystals in the EMC.

For each crystal we then loop over the blocks and flag as bad each block where 

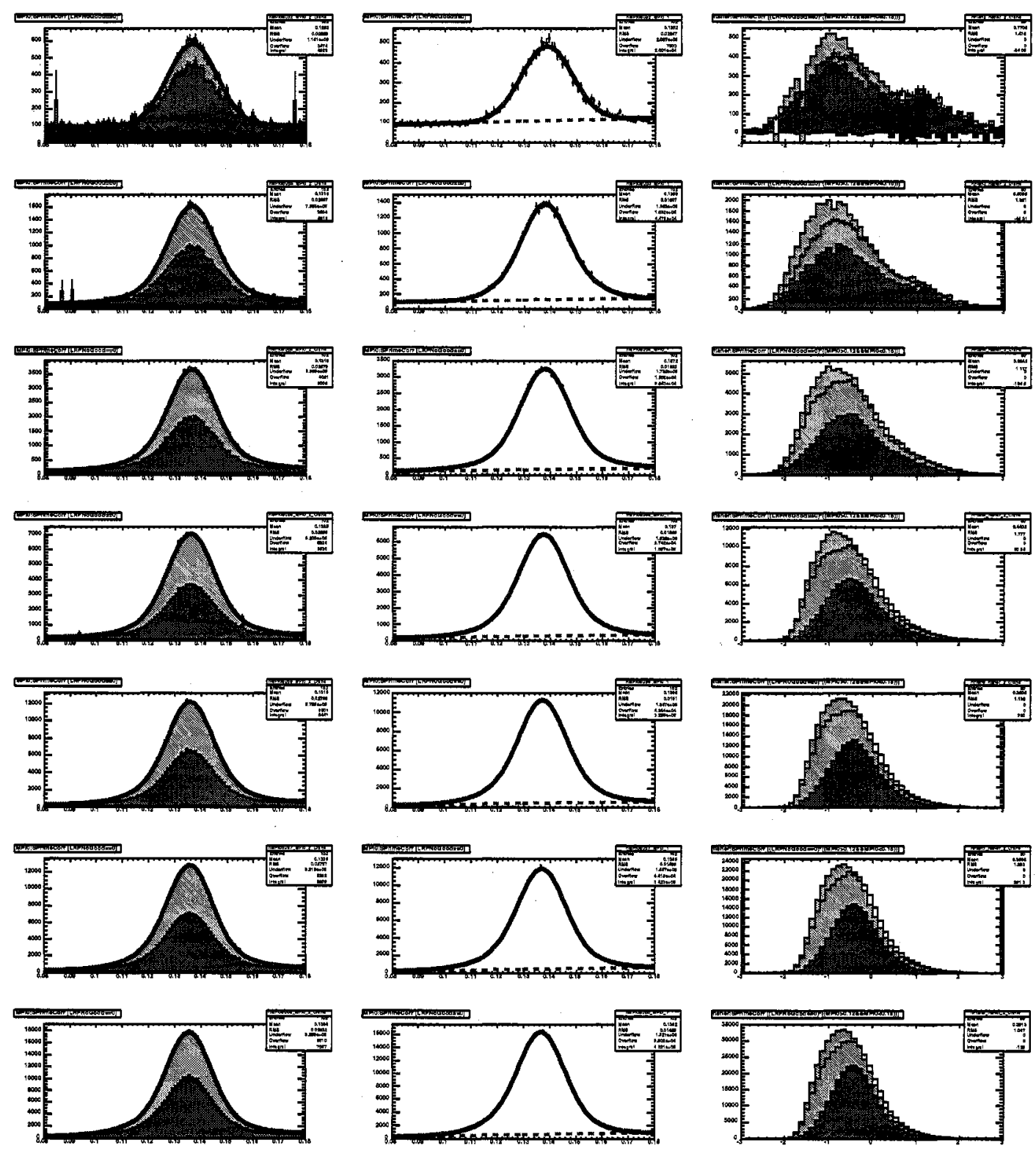

Figure 9.47: Left, Center: Fits of the $m_{\pi^{0}}$ distribution in MC (left) and data (right). Right: background-subtracted Fisher distributions in MC (shaded histograms) and data (points with errors). Rows correspond to $s^{\prime}$ bins, increasing from top to bottom 

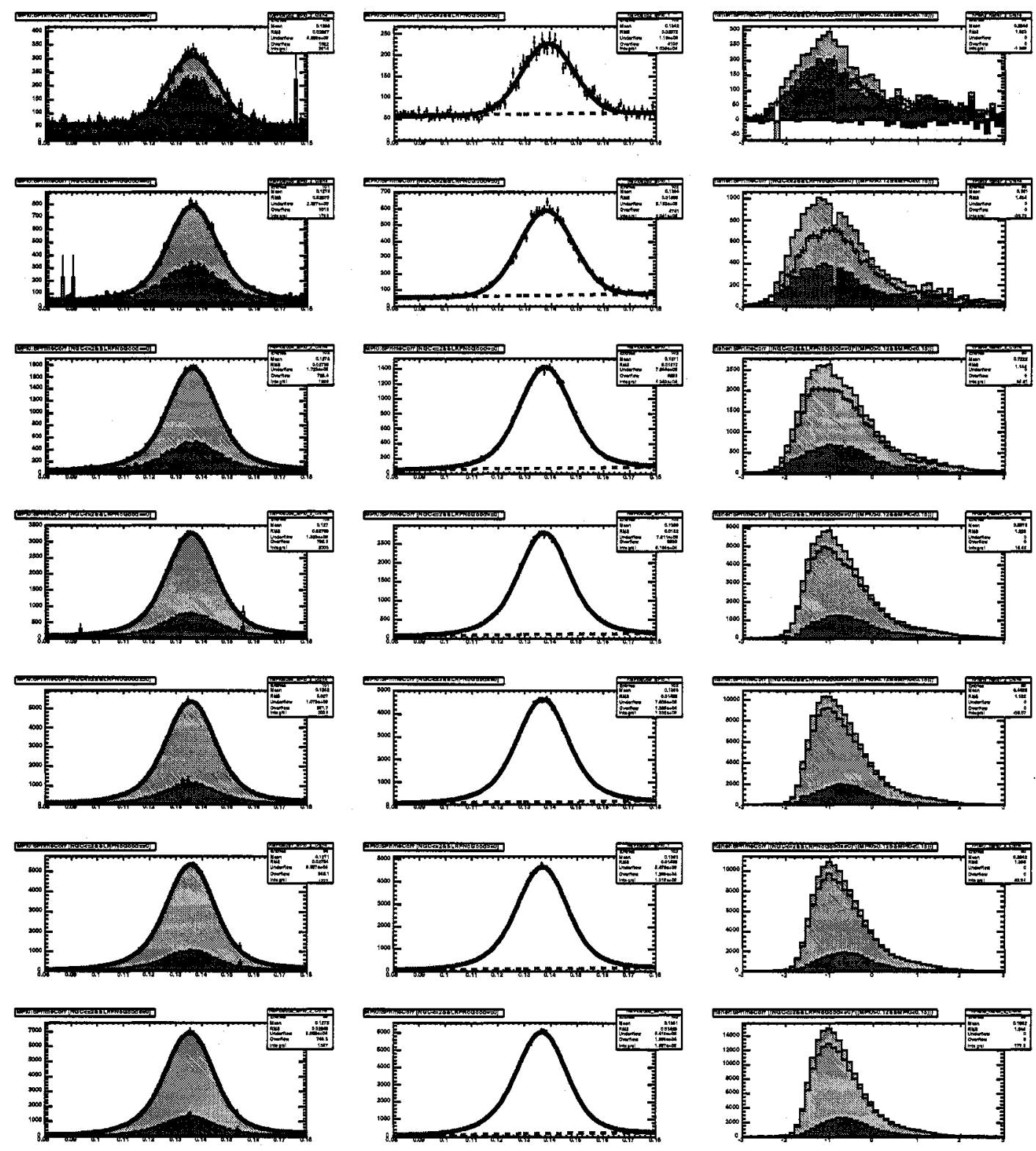

Figure 9.48: Left, Center: Fits of the $m_{\pi^{0}}$ distribution in MC (left) and data (right). Right: background-subtracted Fisher distributions in MC (shaded histograms) and data (points with errors). Rows correspond to $s^{\prime}$ bins, increasing from top to bottom. All plots have the selection $N_{G T} \leq 2$ applied. 

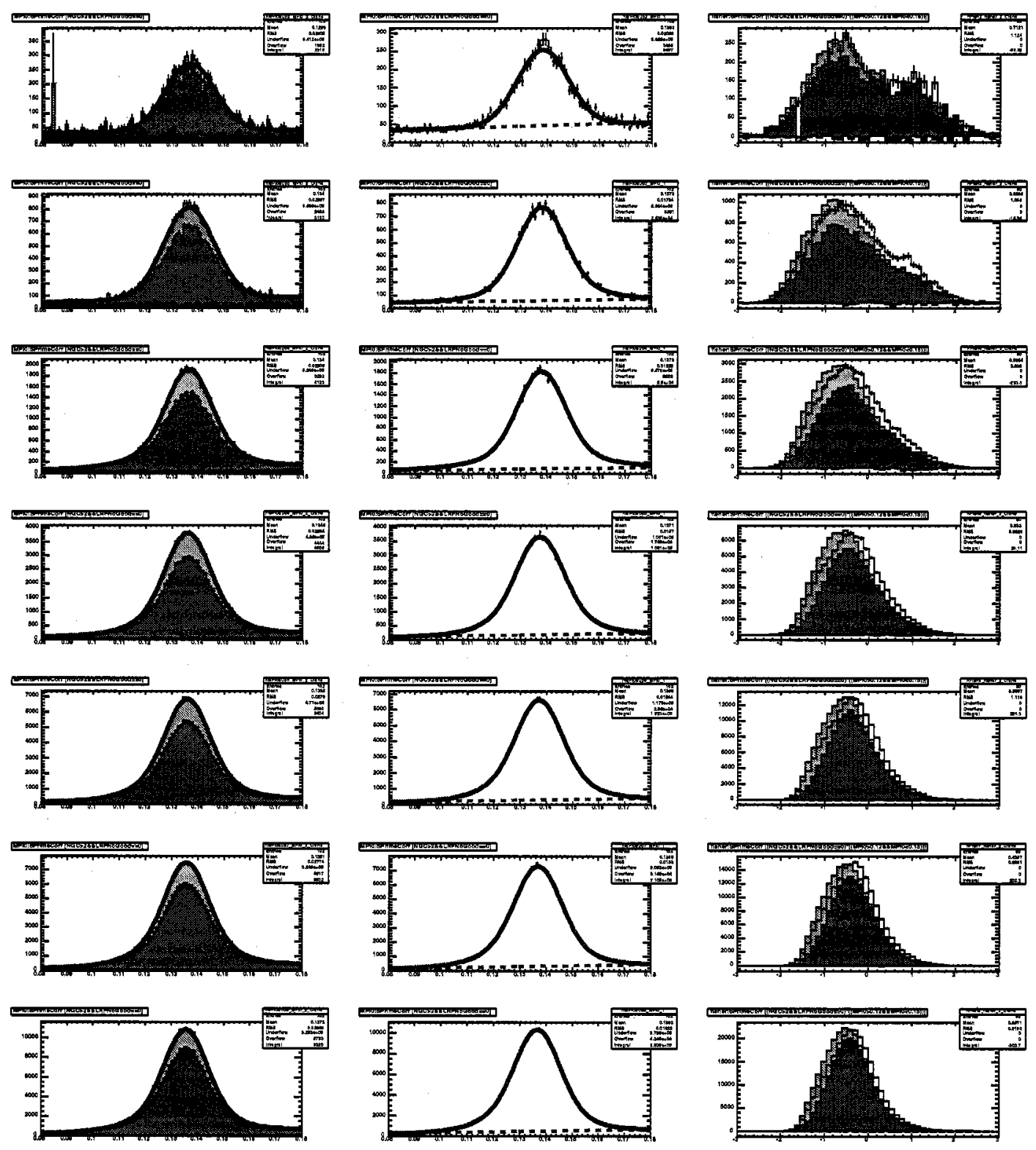

Figure 9.49: Left, Center: Fits of the $m_{\pi^{\circ}}$ distribution in MC (left) and data (right). Right: background-subtracted Fisher distributions in MC (shaded histograms) and data (points with errors). Rows correspond to $s^{\prime}$ bins, increasing from top to bottom. All plots have the selection $N_{G T} \geq 3$ applied. 
Table 9.11: List of EMC $\phi$ and $\theta$ bin indices for crystals flagged as "hot", fraction of fake events caused in the sample $(\mathcal{E})$ and fraction of events in the sample rejected by the veto $(\mathcal{F})$

\begin{tabular}{|l|c|c|c|c|c|c|c|c|c|c|c|}
\hline$\theta$ & $\phi$ & $\mathcal{E} \times 10^{5}$ & $\mathcal{F} \times 10^{5}$ & $\theta$ & $\phi$ & $\mathcal{E} \times 10^{5}$ & $\mathcal{F} \times 10^{5}$ & $\theta$ & $\phi$ & $\mathcal{E} \times 10^{5}$ & $\mathcal{F} \times 10^{5}$ \\
\hline 2 & 19 & 1.41 & 0.44 & 2 & 19 & 2.20 & 0.47 & 3 & 17 & 5.75 & 3.41 \\
3 & 18 & 7.23 & 3.29 & 3 & 22 & 0.92 & 1.11 & 3 & 78 & 7.87 & 3.29 \\
3 & 78 & 9.09 & 3.43 & 3 & 79 & 5.23 & 2.81 & 3 & 81 & 1.24 & 1.53 \\
3 & 83 & 0.32 & 0.38 & 4 & 16 & 0.20 & 0.23 & 4 & 17 & 1.52 & 1.91 \\
4 & 18 & 0.74 & 0.91 & 4 & 19 & 9.91 & 10.03 & 4 & 22 & 0.45 & 0.36 \\
4 & 23 & 0.42 & 0.49 & 4 & 78 & 6.44 & 3.75 & 4 & 78 & 5.53 & 3.22 \\
4 & 79 & 3.40 & 3.33 & 4 & 88 & 0.13 & 0.12 & 5 & 53 & 0.58 & 0.62 \\
5 & 53 & 0.37 & 0.37 & 5 & 78 & 1.94 & 1.99 & 6 & 1 & 1.27 & 1.15 \\
6 & 120 & 1.92 & 1.58 & 7 & 1 & 0.51 & 0.51 & 7 & 120 & 0.68 & 0.65 \\
8 & 32 & 0.39 & 0.32 & 24 & 107 & 0.13 & 0.05 & 29 & 48 & 0.63 & 0.44 \\
30 & 48 & 189.56 & 5.98 & 31 & 48 & 110.95 & 5.20 & 32 & 37 & 0.16 & 0.10 \\
34 & 51 & 9.72 & 3.81 & 34 & 57 & 0.11 & 0.05 & 35 & 51 & 4.60 & 2.75 \\
36 & 51 & 0.82 & 0.59 & 36 & 56 & 0.35 & 0.15 & 37 & 51 & 8.07 & 3.86 \\
38 & 51 & 7.62 & 3.79 & 42 & 59 & 21.37 & 4.72 & 43 & 59 & 2.28 & 1.42 \\
44 & 24 & 0.54 & 0.38 & 45 & 23 & 0.15 & 0.10 & 45 & 82 & 0.64 & 0.45 \\
46 & 25 & 0.57 & 0.36 & 46 & 80 & 0.42 & 0.30 & 47 & 23 & 0.72 & 0.51 \\
47 & 78 & 0.24 & 0.13 & 47 & 82 & 0.63 & 0.43 & 48 & 23 & 1.00 & 0.71 \\
48 & 24 & 0.89 & 0.61 & 48 & 47 & 0.28 & 0.20 & 48 & 64 & 0.17 & 0.10 \\
48 & 79 & 0.89 & 0.57 & 49 & 22 & 0.11 & 0.06 & 49 & 23 & 0.97 & 0.60 \\
49 & 24 & 1.20 & 0.76 & 49 & 25 & 1.01 & 0.70 & 49 & 45 & 0.27 & 0.21 \\
49 & 64 & 0.39 & 0.27 & - & - & - & - & - & - & - & - \\
\hline
\end{tabular}



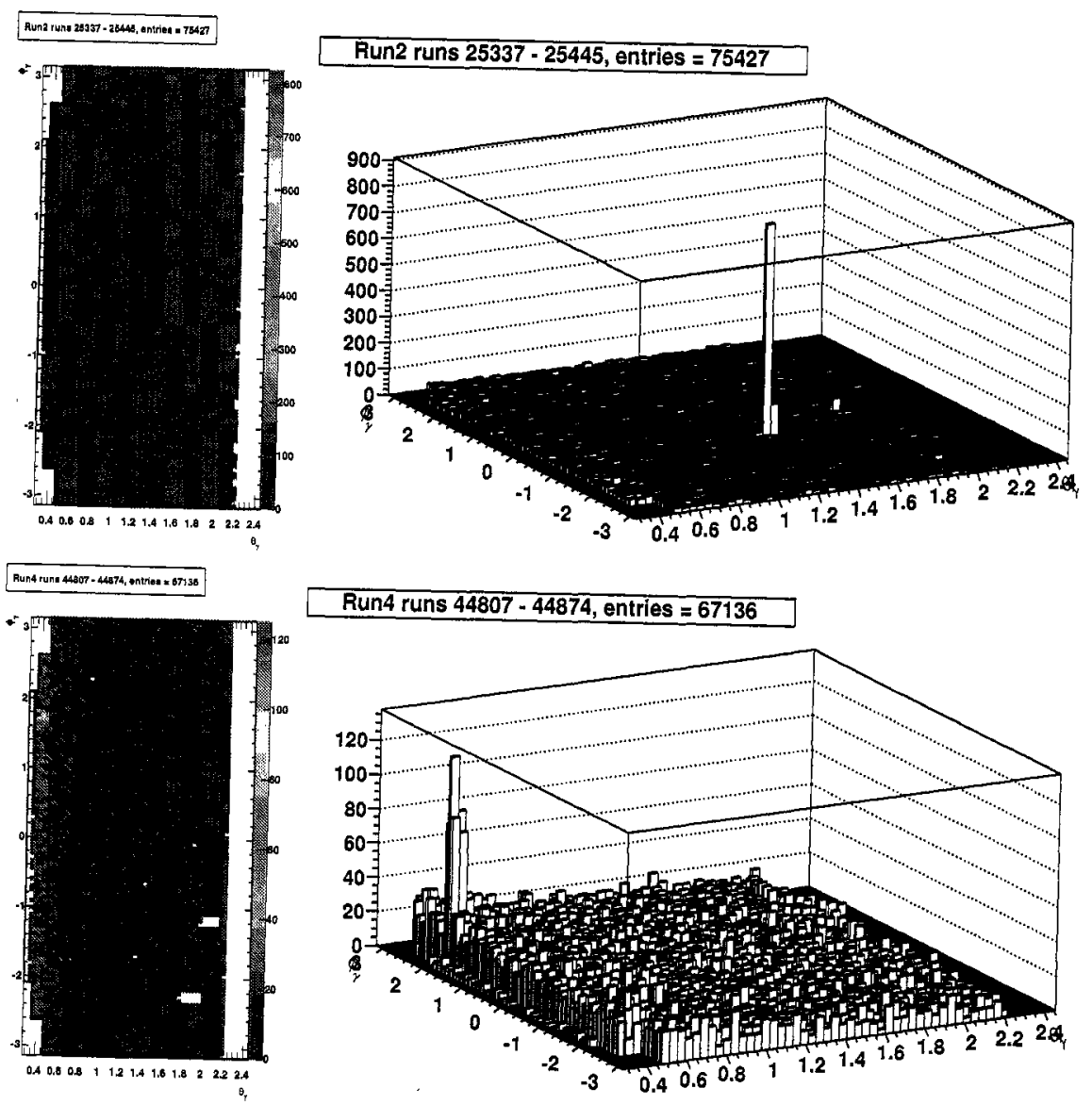

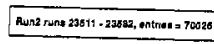

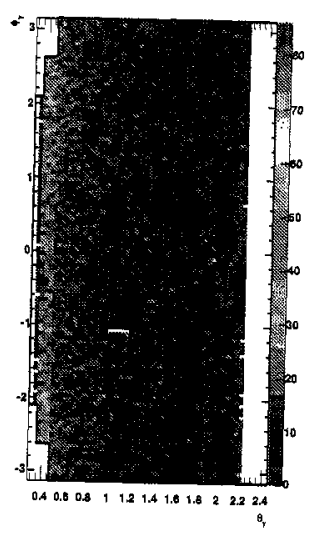

Run2 runs $23511-23582$, entries $=70026$

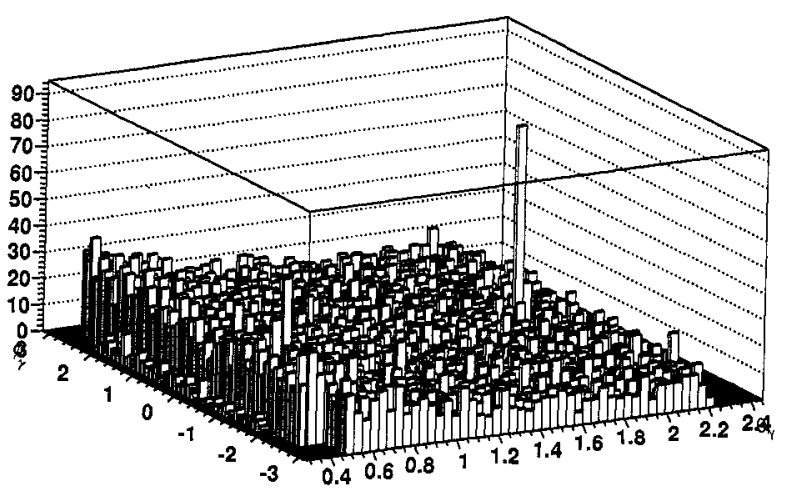

Figure 9.50: Typical hitmaps for blocks with one or more hot crystals 

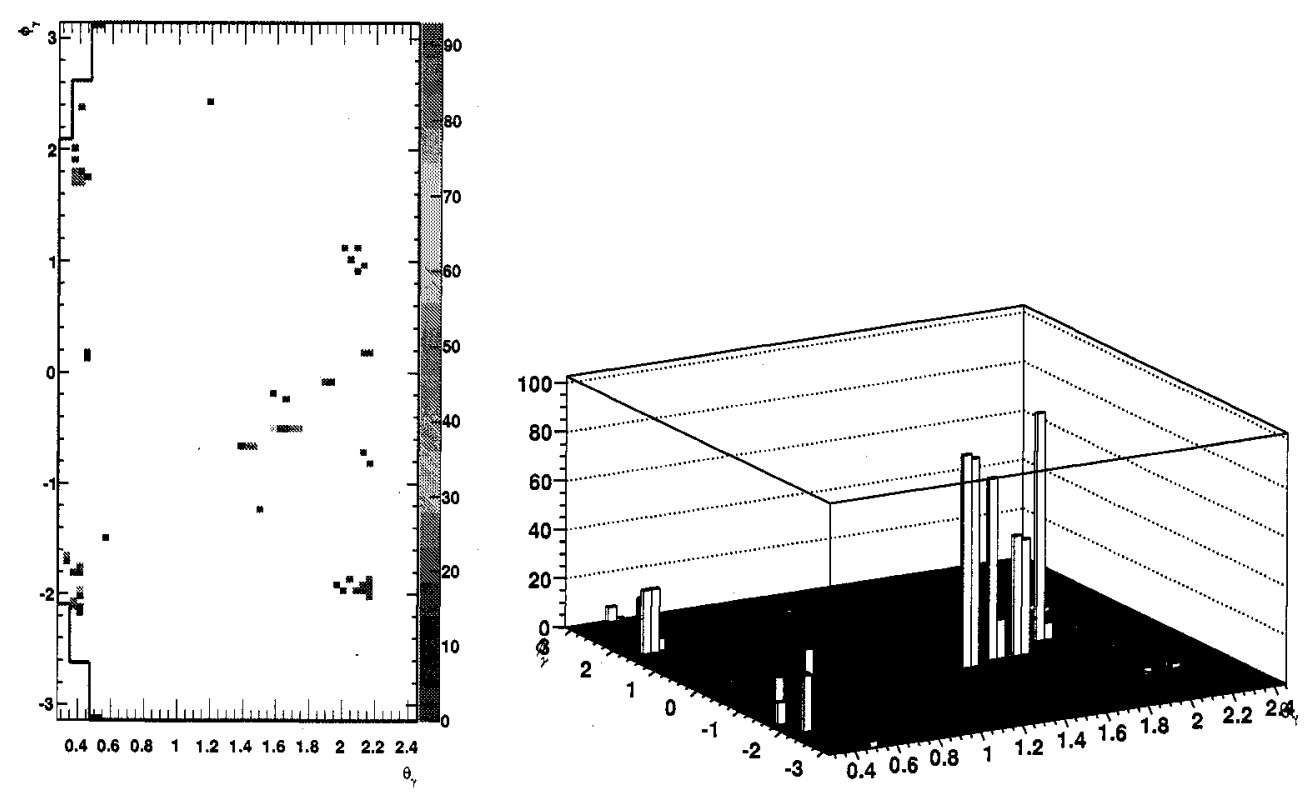

Figure 9.51: Distribution of hot crystals in the EMC

the occupancy of the crystal is $2 \sigma$ over the ring average. The difference between the crystal occupancy and the ring average also gives the amount of events in the sample that is due to this crystal being noisy. The values for each crystal are listed as $\mathcal{E}$ in Table 9.11 , and dominated by two crystals, $(30,48)$ and $(31,48)$. For each crystals we also compute the estimated loss of EMC acceptance as the sum of the average occupancy of the phi ring where this crystal is located over all the runs that are flagged bad. This quantity, divided by the total number of events in the sample, is shown as $\mathcal{F}$ in Table. 9.11. Overall, the acceptance loss is $0.09 \%$ of the sample passing QED veto selections.

The veto is applied on data in a straightforward manner, rejecting events which have the ISR photon centered on a hot crystal within the determined run range. To simulate the same effect on MC, we randomly reject events which have the ISR photon centered on one of the hot crystals using the rejection fraction value $\mathcal{F}$ described above. This respects the spatial distribution of hot crystals in the EMC but not their 
time variation. However due to the smallness of the correction this is probably not a bad approximation. The veto is incorporated in the weighting procedure that is already used to correct the MC, as described in Section 9, with vetoed events assigned a weight of 0 .

Although the correction is small in the final sample, it has significant effects before hadronic background rejection: the peak which is visible in Fig. 5.12 at $\theta \sim 1.45$ is caused by the hot crystal at $(\theta, \phi)=(30,48)$ which is responsible for most of this background. The same plot with the weighting described above included is shown in Fig. 9.52; the peak is not present.

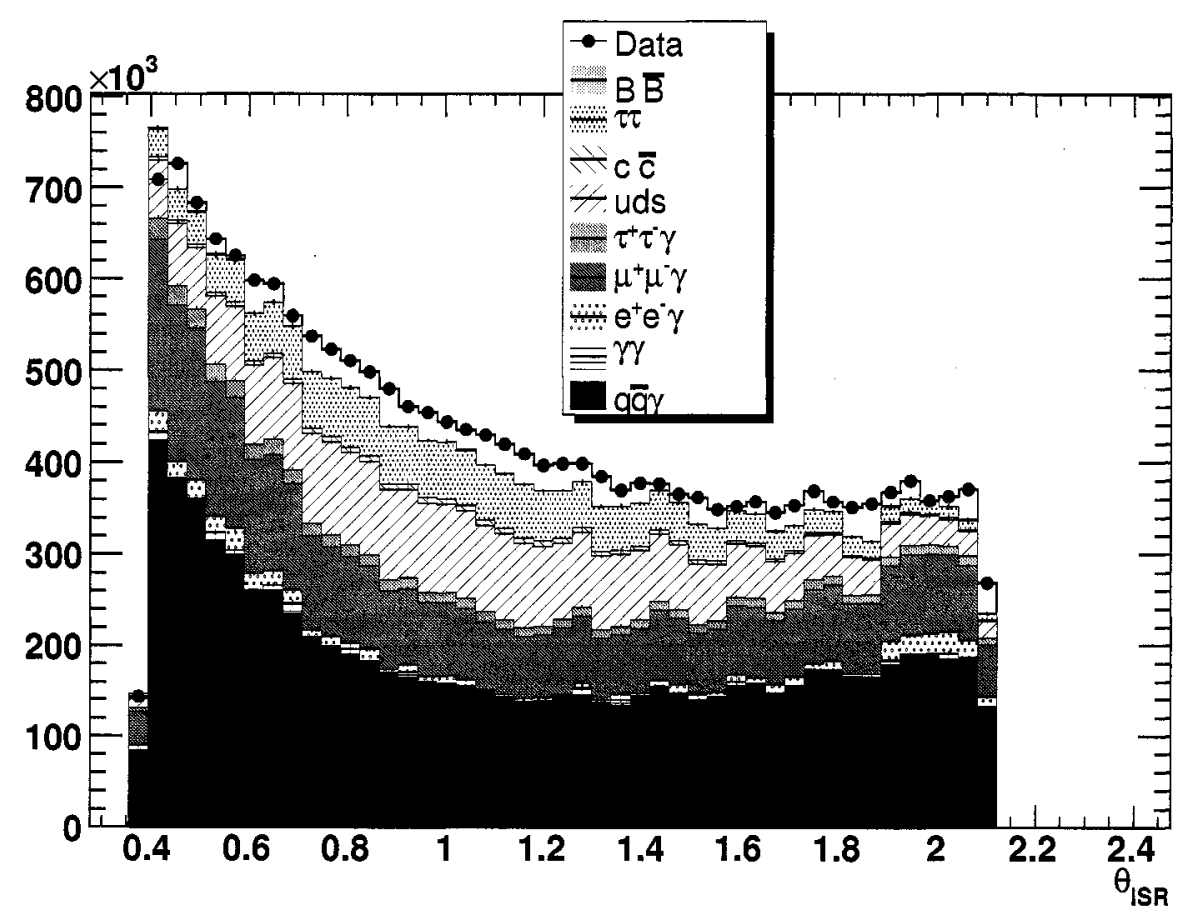

Figure 9.52: $\theta_{\gamma}$ distribution for the ISR photon in data (points with error bars) and MC (filled histograms) after applying the weighting procedure of Section 9.7. 


\subsection{Time Distribution of events}

The aim of this section is to study the distribution of signal events over the data-taking period, in order to identify possible problems. These may be of two kinds: first timedependent detector or collider conditions may lead to increased background levels (for instance the hot tower problem of Section 9.7), and second detector-related problems may cause inefficiencies. The data blocks defined in Section 9.7 are particularly wellsuited to this task since they have the right granularity (298 blocks in total) and contain roughly equivalent number of events. Fig. 9.53 shows the fraction of signal events in each block of the final sample. Although there are curious variations in the yield they do no exceed $10 \%$ and are limited in time.

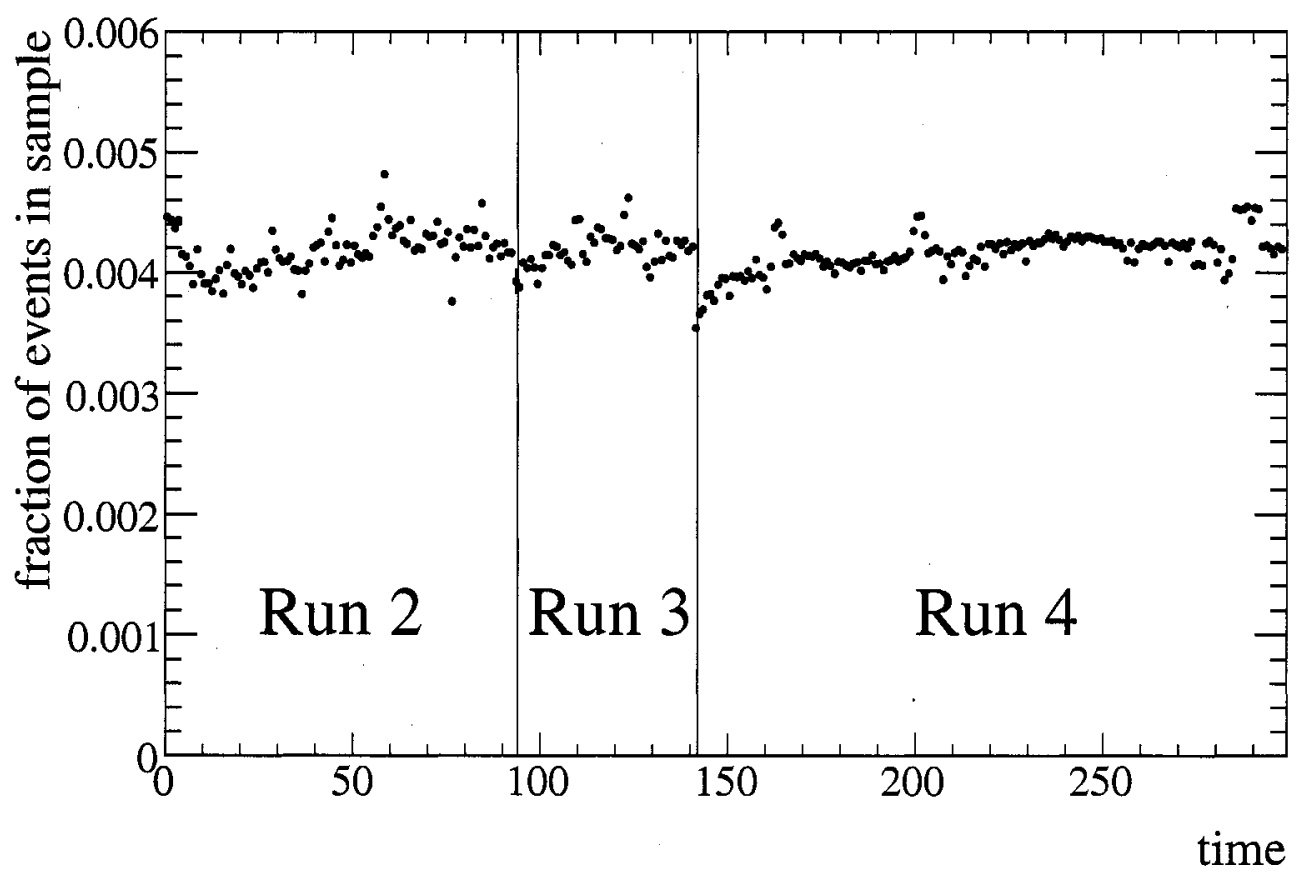

Figure 9.53: Fraction of signal event in each data block after hot tower removal. 


\subsection{Overlapping Bhabha Events}

A potential problem for the analysis is the case where a Bhabha event overlaps with a hadronic event. A particular concern are so-called "Stale" Bhabha events in which a hadronic event is closely preceded by a Bhabha event.

The EMC waveforms have a slow damping time, of the order of $2 \mu \mathrm{s}$, while the drift time in the $\mathrm{DCH}$ is typically less than $1 \mu \mathrm{s}$. Therefore if a high-angle Bhabha event occurs between $1 \mu$ s and $2 \mu$ s before the hadronic event, its clusters may still be visible (although with only a fraction of their original energy) while the tracks had disappeared: the resulting event, with a hadronic system and at least one high-energy cluster is therefore similar to an ISR event. A typical event is shown in Fig. 9.54.
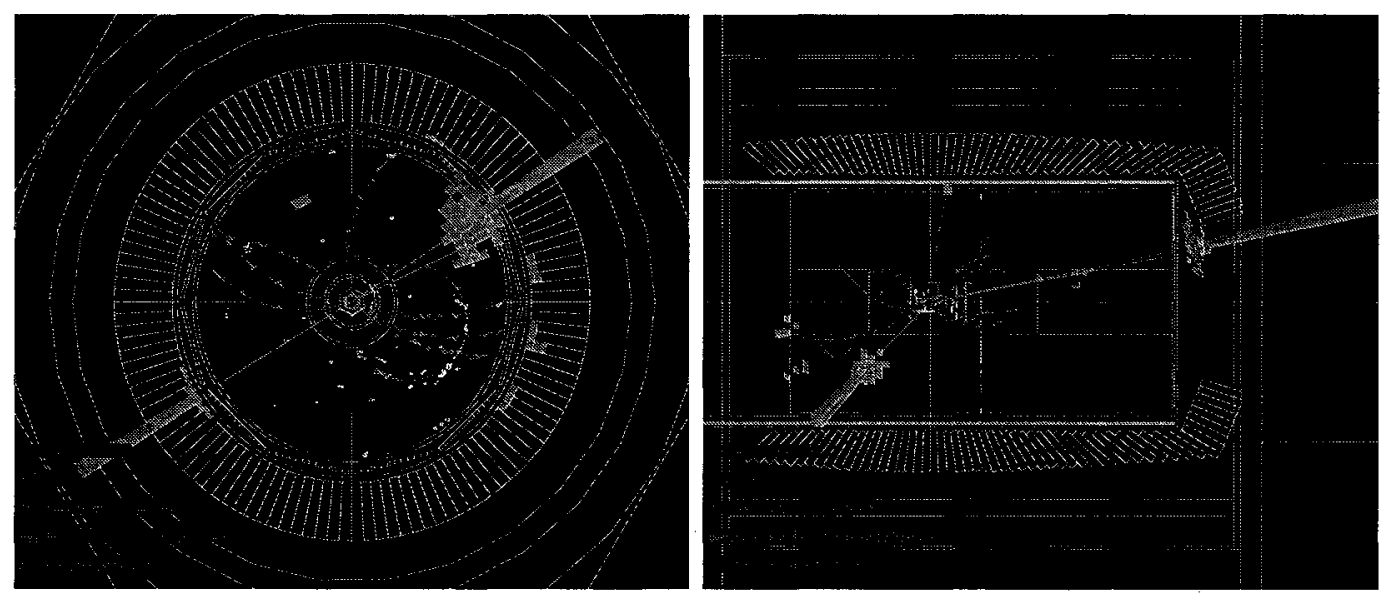

Figure 9.54: Beam (left) and top (right) view of a probable stale Bhabha event

Since the cross-section for Bhabha evens in the EMC acceptance is about $50 \mathrm{nb}$, at a luminosity of $8 \cdot 10^{33} \mathrm{~cm}^{-2} \mathrm{~s}^{-1}$ the probability to have a Bhabha event in a given $1 \mu \mathrm{s}$ interval is $4 \cdot 10^{-4}$. Given that the hadronic cross-section is about $10 \mathrm{nb}$, the cross-section for hadronic events with an overlapping Bhabha is therefore about $4 \mathrm{pb}$. This is not quit negligible compared to the $96 \mathrm{pb}$ of ISR hadronic cross-section (in a slightly smaller $\theta$ acceptance). The fraction of events in the final sample is probably not that high since these events are suppressed by the $\gamma \gamma$ veto (see Section 5.6) and the cut on the total energy in the event of Section 5.1.

Three types of criteria can be used to identify the remaining events: 
- The presence of the other Bhabha cluster, back-to-back to the ISR photon in the CM frame.

- The angle between the ISR photon momentum and the thrust direction of the hadronic system in the CM frame: for an ISR event the hadronic system behaves like a jet recoiling from the ISR photon and the angle is typically small; for a stale Bhabha event there should be no angular correlation.

- Timing information for the ISR cluster: for stale Bhabha events the ISR cluster appears out of time with the rest of the events, which provides an unambiguous determination for this process.

- GLT trigger line information: the GLT trigger provides $3215-\mathrm{MHz}$ ticks of history for the L1 trigger, corresponding to $2.1 \mu \mathrm{s}$. Checking for early $2 \mathrm{E}$ triggers in the history could provide a clear-cut way of identifying these events. However we do not use this method here since the time history is not available in the ntuples.

Fig. 9.55 shows the $E^{*}$ vs. time distributions for the ISR photon for data and signal MC.

The data plot shows a band for $6.2<t<6.4 \mu$ s that corresponds to in-time events, which is also seen in signal MC. However the data also shows a band at $E^{*}=5.3 \mathrm{GeV}$ throughout the time window, peaking at the early edge of the window. This is what is expected for the stale Bhabha events; to check this one can look for the other Bhabha cluster as described above. Fig. 9.56 shows the energy of the cluster with the second-highest $E^{*}$ in the event, with the requirement that it should be back-to-back to the ISR photon in the CM frame to within $|\Delta \theta|<0.3$ and $|\Delta \phi|<0.2$.

As expected, the out-of-time events again show up near $E^{*}=5.3 \mathrm{GeV}$, which shows that they are composed of two clusters at the beam energy. The signal by contrast peaks at low $E^{*}$.

In what follows we define signal and sideband regions in time to be $6.15<t<$ $6.45 \mu$ s and $6.5<t<6.8 \mu$ s respectively. Fig. 9.57 show the distributions of relevant quantities for the signal and sideband regions in data. 

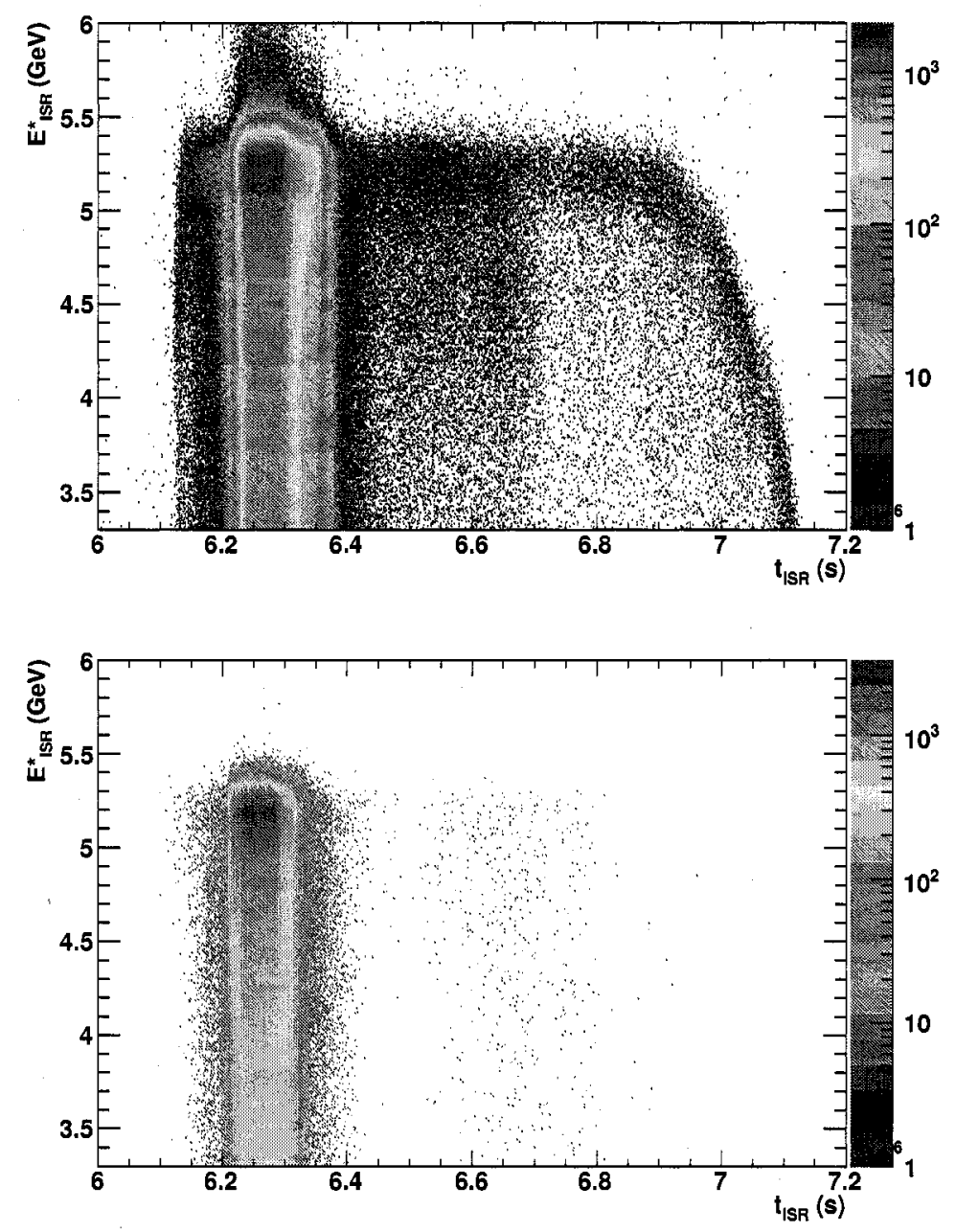

Figure 9.55: $E^{*}$ versus time for the ISR photon in data (top) and signal MC (bottom). 

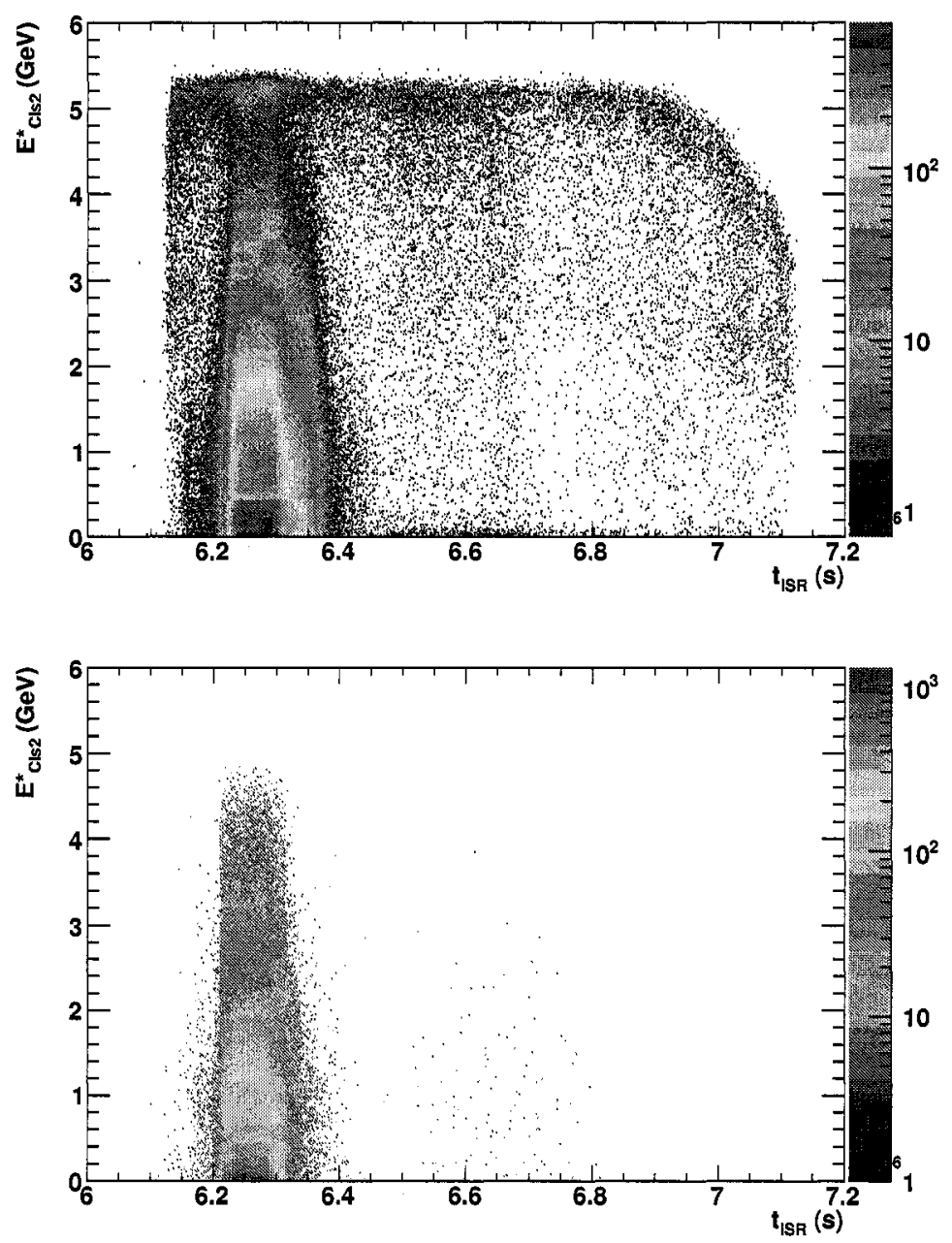

Figure 9.56: $E^{*}$ versus time for the second-highest- $E^{*}$ cluster in the event in data (top) and signal $\mathrm{MC}$ (bottom). 

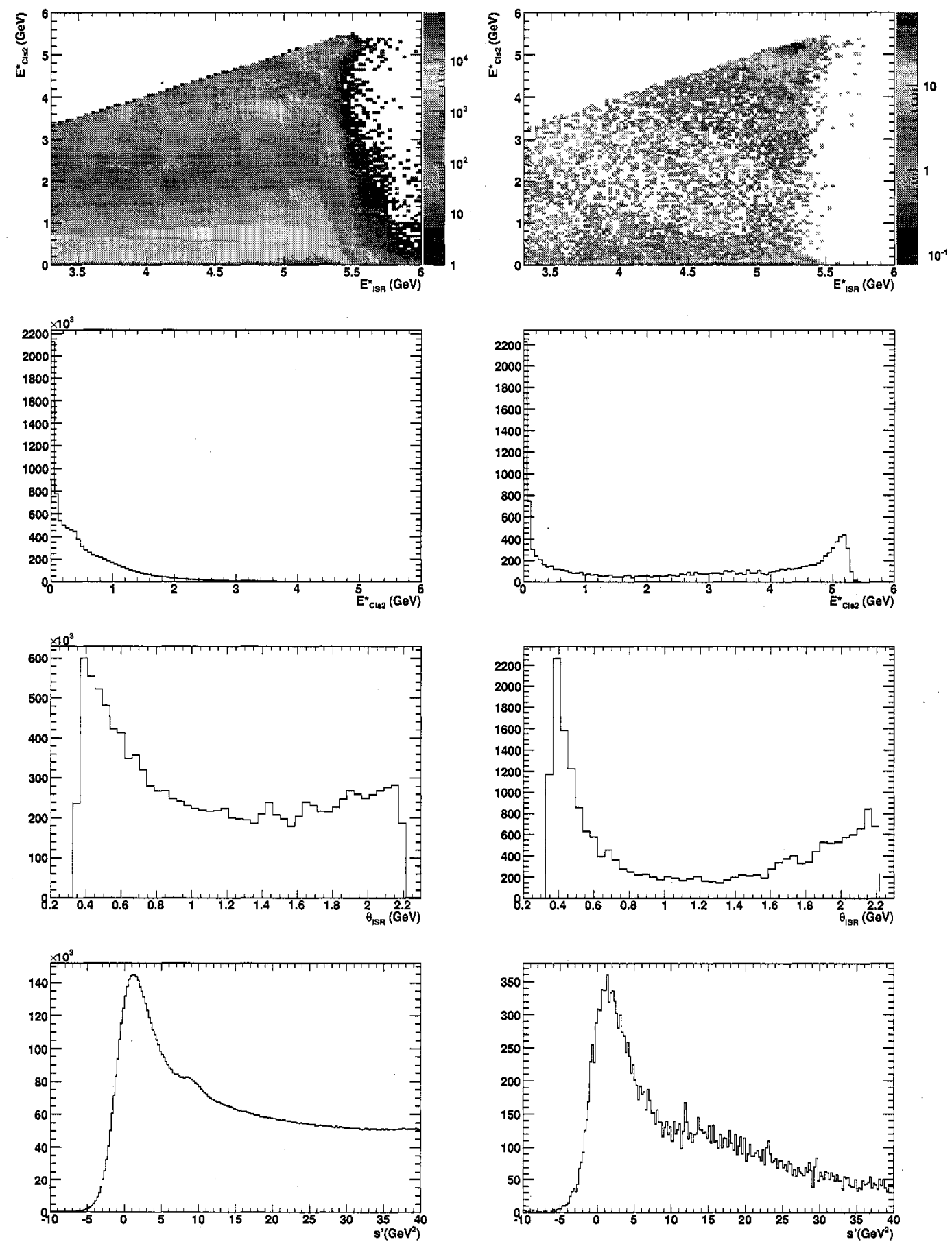

Figure 9.57: $E^{*}$ of the second-highest $\mathrm{CM}$ energy cluster in the event (top), same versus the ISR photon $E^{*}$ (top center), polar angle distribution for the ISR photon (bottom center) and $s^{\prime}$ (bottom). The left and right columns correspond to the signal and sideband regions respectively. 
In order to remove this background, we require the ISR photon time to fall within the signal window. According to the simulation this rejects $0.38 \%$ of the signal, with reasonable agreement from data.

In order to evaluate the remaining background level in the signal region, we extrapolate from the sideband in two different ways:

- Fit the ISR photon time distribution outside the signal region to the sum of a flat background and a decreasing exponential shape. The fit adopts a linear shape, shown in Fig. 9.58. By integrating the fit function in the signal region, we obtain a background level of $0.39 \%$.

- Assume that the time distribution under the peak is a constant with the same average value as in in the sideband region, corresponding to a background level of $0.17 \%$.

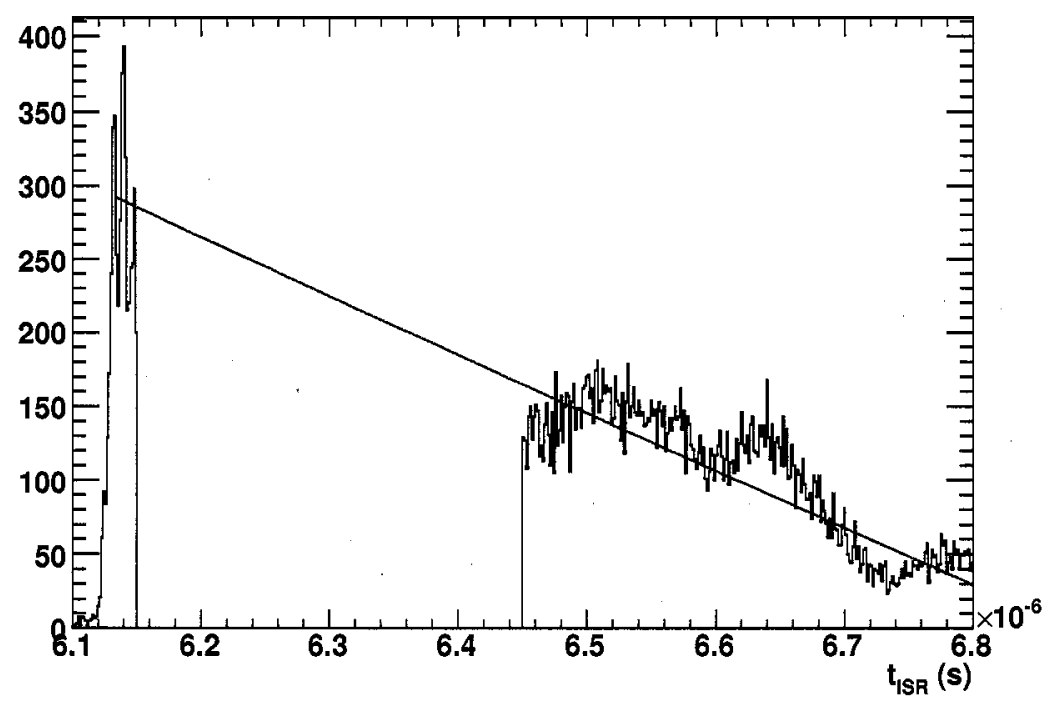

Figure 9.58: Fit of the time distribution to an exponential distribution plus a constant outside the signal region. The fit favors a linear shape

The correct value is probably somewhere in between these two. We use $0.30 \pm$ $0.15 \%$. 
In order to account for this background we subtract the $s^{\prime}$ distribution obtained from the sideband, normalized to $0.30 \%$ of the total sample.

\subsection{Residual $e^{+} e^{-} \gamma$ cross-feed}

The residual cross-feed from radiative Bhabha events is difficult to estimate since it consists of "pathological" events which do not have clear electron signature. Since these events most likely involve interaction with the detector material or an unusual detector response, the simulation is also not expected to reproduce these events correctly.

A possibility is to select these events using kinematics properties only. Fig 9.59 shows the CM momentum $p_{1}^{*}$ of the highest-CM-momentum track in the events in data and simulation.

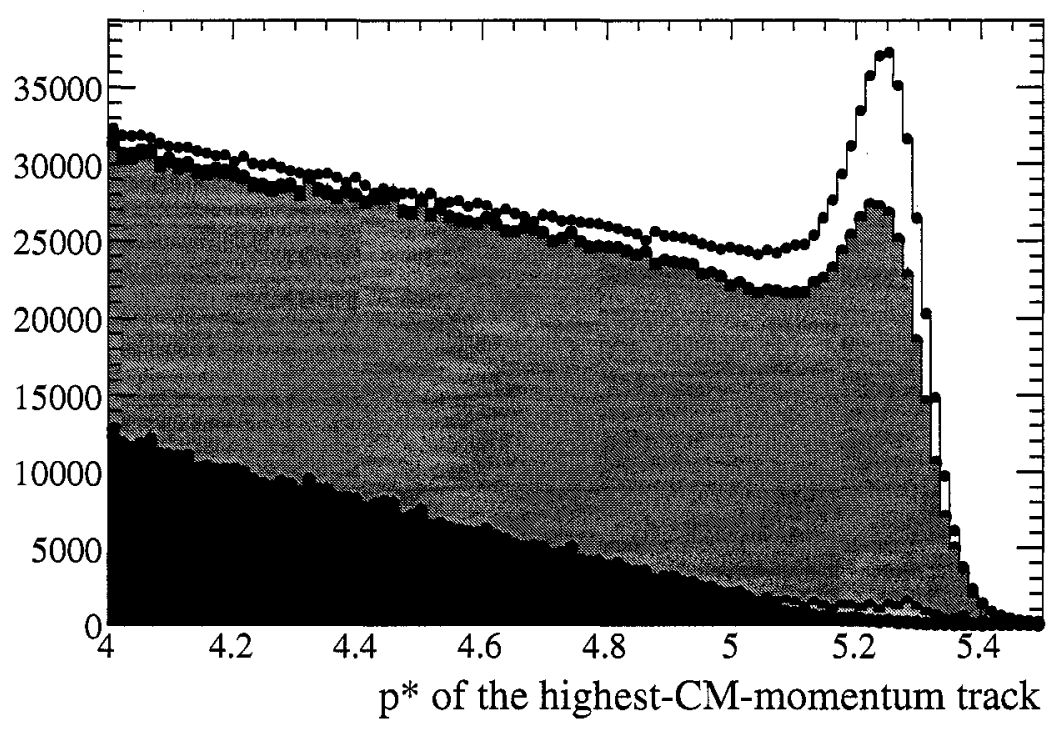

Figure 9.59: $p^{*}$ of the highest- $p^{*}$ track in the event.

The peak at the beam energy can only occur for a two-body final state in which the particle masses vanish, and the "ISR" photon is actually produced by final-state 
radiation. The only possibilities are $e^{+} e^{-} \rightarrow \mu^{+} \mu^{-} \gamma$ and $e^{+} e^{-} \rightarrow e^{+} e^{-} \gamma$; the contribution from $e^{+} e^{-} \rightarrow \pi^{+} \pi^{-} \gamma$ vanishes at the endpoint since it is produced by ISR only. Since the $\mu^{+} \mu^{-} \gamma$ rate is expected to be accurate, the excess can be attributed to residual $e^{+} e^{-} \gamma$ events. This is verified by the distribution shown in Fig. 9.60: the excess events mostly consist of two tracks, the highest-CM-momentum track has large $E / p$ (the edge at $E / p=0.7$ is caused by the radiative Bhabha veto algorithm) and its matching cluster is located near a crystal boundary. As explained in Section 5.10, these regions are expected to contain more electron background due to energy leakage in the EMC.
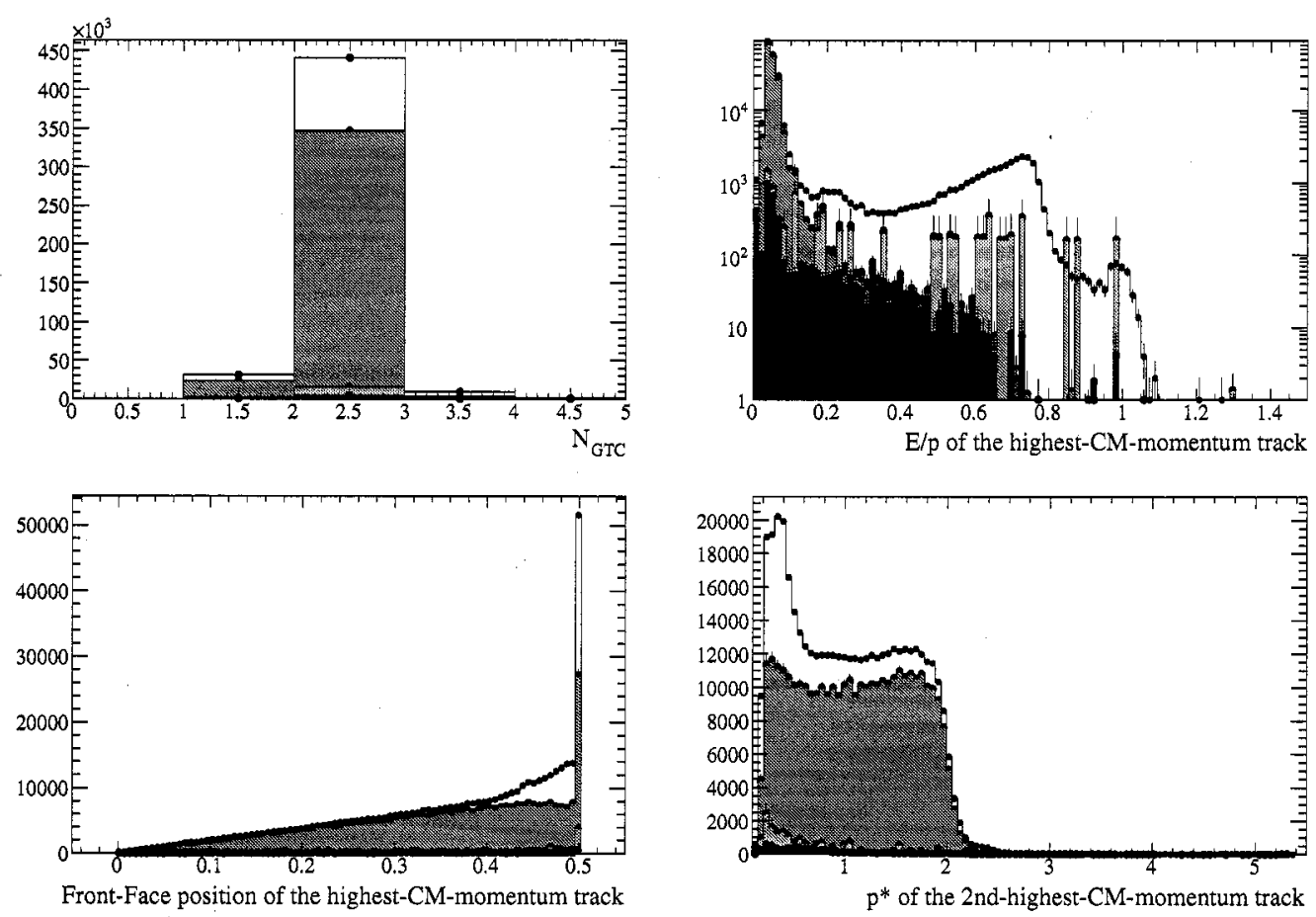

Figure 9.60: Number of IsrGoodTracks (top left), $E / p$ (top right) and crystal frontface position (bottom left) for the highest- $p^{*}$ track, and $p^{*}$ of the second-highest- $p^{*}$ track, in events where the highest- $p^{*}$ track has $p^{*}>5.1 \mathrm{GeV}$.

The goal of this study is to use the peak at the $p_{1}^{*}$ endpoint to reweigh the radiative Bhabha simulation to accurately reproduce the residual cross-feed. We assume that the identification of the highest- $p^{*}$ track is independent of the rest of the event. 
Then one-track events can be used to determine the efficiency of the highest- $p^{*}$ track identification, and multi-track events can be used to get the efficiency for the rest of the event.

Since one-track events are suppressed by a specific veto (see Section 5.10), this part of the study is performed on a small sample of events in which the QED selections are not performed. This sample corresponds to about 1 million data events and equivalent quantities of simulation events, picked to reproduce the mix of detector conditions in the full sample. Studies on multi-track samples are done on the full dataset. We proceed as follows:

- Compare data and the sum of all MC for one-track events where the track is not identified as an electron. The distribution is shown in the top left plot of Fig. 9.61. A multiplicative correction $c_{1}^{\prime}$ is applied to the radiative Bhabha simulation to match the data.

- Do the same comparison with multi-track events, where the highest- $p^{*}$ track is not identified as an electron and the event as a whole passes the radiative Bhabha veto. The distribution is shown in the top right plot of Fig. $9.61 \mathrm{~A}$ multiplicative correction is again applied to the $e^{+} e^{-} \gamma \mathrm{MC}$ to match the data. Since the two sides of the event are independent, we can write this correction as $c_{1}^{\prime} c_{2}$, where $c_{1}^{\prime}$ is given above, and $c_{2}$ represents the additional scaling required to account for misidentification in the rest of the event.

- Since one-track event are subject to an additional veto (the 1-track veto of Section 5.10), perform the same correction as in the first item, but for events passing the veto in addition to the track being identified as an electron. The distribution is shown in the bottom plot of Fig. 9.61. A correction $c_{1}$ is determined as before.

A possible reason for the two sides of the event not being independent occurs when the event has two "non-electron tracks", as defined in Section 5.8: if the highest-p" track is identified as a non-electron, the event has a priori more chance of passing the veto. We therefore compute a correction $c_{2}^{N E}$ identical to $c_{2}$ but for events with 

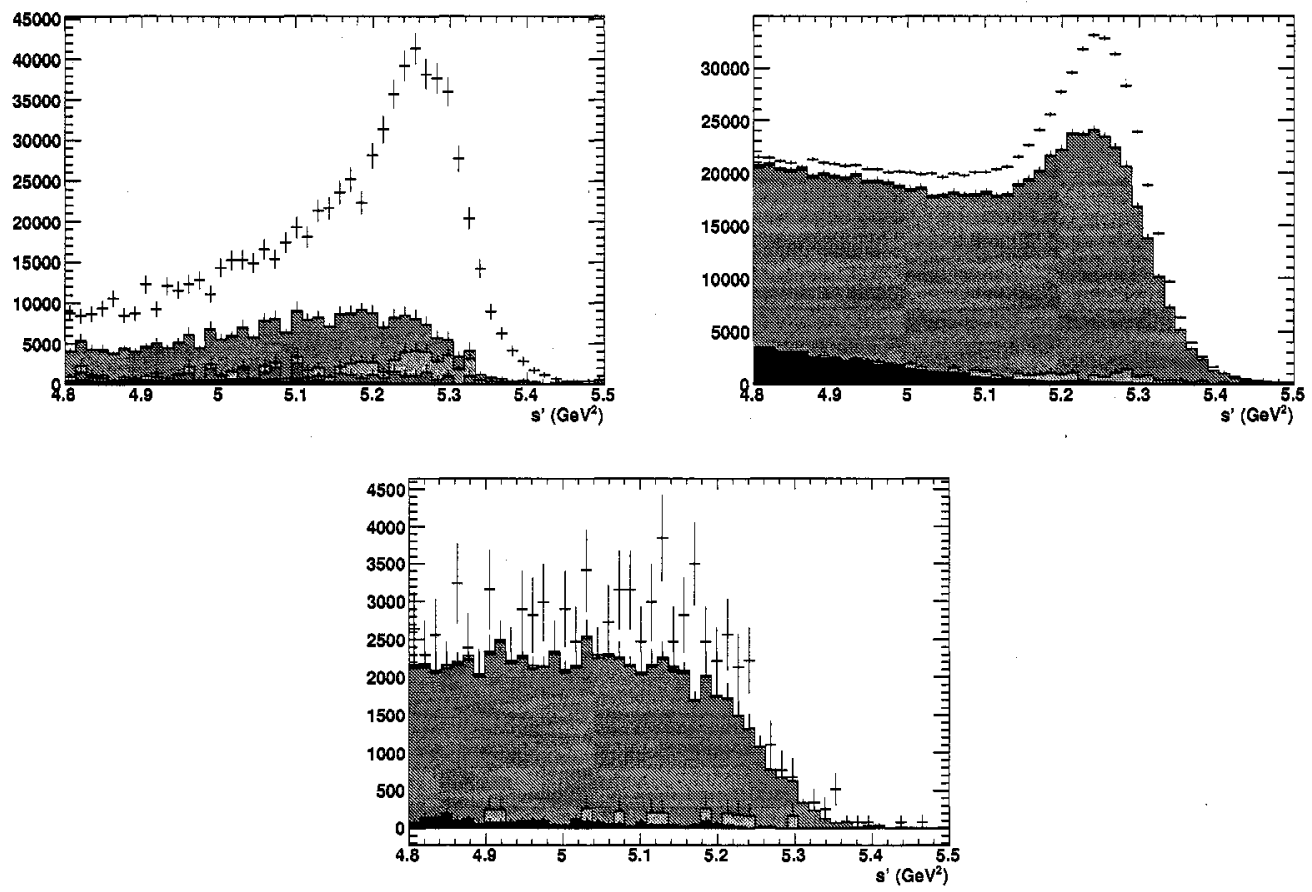

Figure 9.61: $p^{*}$ of the highest- $p^{*}$ track in the event for events with one track, not identified as an electron (top left), with multiple tracks, and the highest- $p^{*}$ track not identified as an electron (top right) and for events with one track, not identified as an electron and passing the single-track veto of Section 5.10 (bottom) 
Table 9.12: Scale factors applied to $e^{+} e^{-} \gamma \mathrm{MC}$ as described in the text

\begin{tabular}{|l|c|c|c|c|c|}
\hline$\theta$ region & $c_{1}$ & $c_{2}$ & $c_{1}^{\prime}$ & $c_{1}^{\prime N E}$ & $c_{2}^{N E}$ \\
\hline All $\theta$ & 13.4 & 0.59 & 10.5 & 12.0 & 0.75 \\
$0.5<\theta<1.8$ & 12.9 & 0.57 & 8.9 & 12.3 & 0.81 \\
$\theta<0.5, \theta>1.8$ & 13.6 & 0.61 & 11.1 & 11.9 & 0.69 \\
\hline
\end{tabular}

a non-electron highest- $p^{*}$ track. We also compute a correction $c_{1}^{\prime N E}$ in the same way as $c_{1}^{\prime}$ but for non-electron highest- $p^{*}$ tracks.

Another issue is that the highest- $p^{*}$ track is typically nearer to the edges of the $\theta$ acceptance as the other side of the event, due to the angular distribution of the $e^{+} e^{-} \gamma$ events. This is expected to have significant effects since the amount of material seen by particles is higher at extreme polar angles. The corrections listed above are therefore calculated in three $\theta$ regions: the entire range, the region $0.5<\theta<1.8$ and the region $(\theta<0.5$ or $\theta>1.8)$.

The results are shown in Table 9.12. There seems to be little polar angle dependence on the correction values, and since $c_{2}^{N E} \approx c_{2}, c_{1}^{\prime N E} \approx c_{1}^{\prime}$ the non-electron identifications seem to play a minor role. So the factor 2-3 difference between the highest- $p^{*}$ track and the rest of the event seems attributable to momentum differences only. The second-highest- $p^{*}$ track typically has $p^{*} \sim 1 \mathrm{GeV}$, as shown in Fig. 9.60. We use the measured values of $c_{1}^{\prime}$ and $c_{2}$ to reweigh the $e^{+} e^{-} \gamma \mathrm{MC}$ events as follows:

- If the event has only one track, apply a weight of $c_{1}$.

- If the event has multiple tracks, apply a weight of $c_{1}^{\prime} c_{2}$ if the highest- $p^{*}$ track has $p^{*}>4 \mathrm{GeV}$, and $c_{2}^{2}$ otherwise.

Fig. 9.62 shows the same distributions as Fig. 9.62 after correction. The agreement is improved, although not perfect.

After the correction is applied, we require $p^{*}<5.0 \mathrm{GeV}$ in order to limit the amount of Bhabha cross-feed in the sample. The cut has a negligible effect on all samples except $\mu^{+} \mu^{-} \gamma$, for which the efficiency is $85 \%$. 

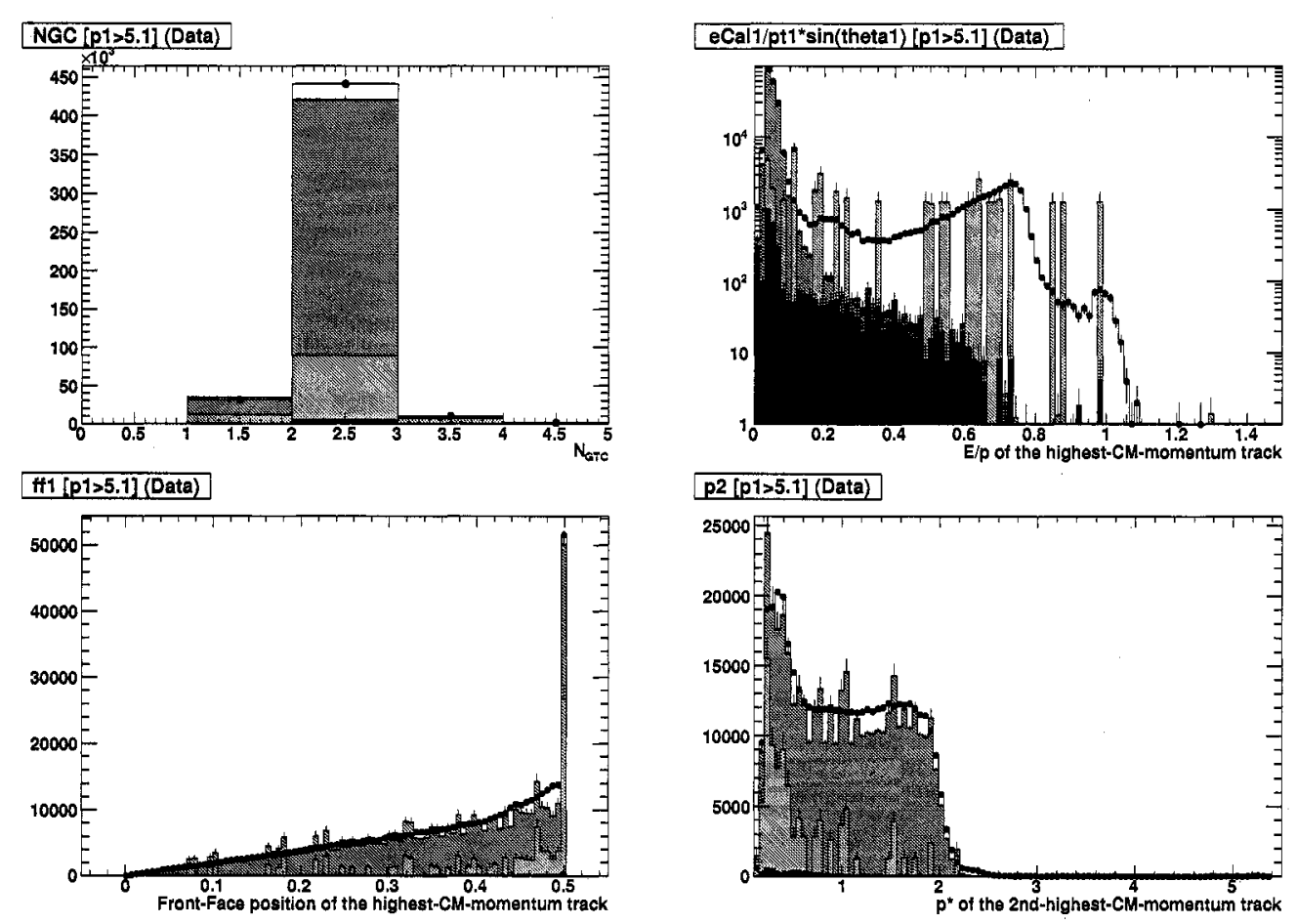

Figure 9.62: Number of IsrGoodTracks (top left), $E / p$ (top right) and crystal frontface position (bottom left) for the highest- $p^{*}$ track, and $p^{*}$ of the second-highest- $p^{*}$ track, in events where the highest- $p^{*}$ track has $p^{*}>5.1 \mathrm{GeV}$. 


\subsection{Residual $\gamma \gamma$ cross-feed}

The $\gamma \gamma$ events feed into the signal sample are either all-neutral or contain electron tracks. In the latter case the same issues apply as for the $e^{+} e^{-} \gamma$ background in the previous section, and the same weighting procedure is applied. All-neutral events can be checked by comparing data and MC in the 0 -track subsample. The $s^{\prime}$ distribution is shown in Fig. 9.63. Simulation is not expected to give a sufficiently precise model

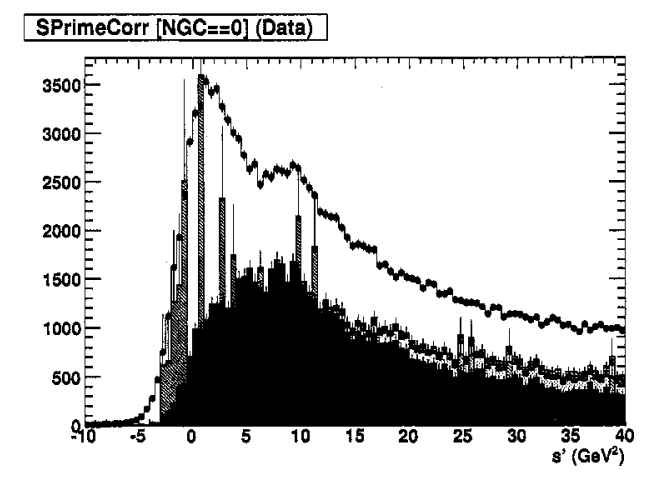

Figure 9.63: $s^{\prime}$ distribution for $N_{G T}=0$

of the fragmentation process to predict the $s^{\prime}$ distribution of the 0-track sample, so the excess can be signal as well as background. Neutral $\gamma \gamma$ events are expected to populate the lower end of the distribution, so the fact that the excess is relatively uniformly distributed seems to indicate that it is mainly signal. This is also suggested by the presence of the bump at $s^{\prime} \sim 10 \mathrm{GeV}^{2}$ in the data which is associated with the $\psi$ and $\psi(2 S)$ resonances.

In order to verify this, we study the event shape quantity $F_{2}$ defined in Section 6.5.1. Its distribution is shown in Fig. 9.64 The $\gamma \gamma$ sample concentrates in the region $F_{2} \approx 1$, but the excess is again spread over the entire spectrum. If we assume that the $q \bar{q} \gamma$ signal MC does not produce enough all-neutral events, the normalization can be adjusted by comparing the yields in the regions $s^{\prime}=5-40 \mathrm{GeV}^{2}$ and $F_{2}=0-0.75$ which contain little $\gamma \gamma$ events. In the first case, a scaling factor of 1.83 needs to be applied to $q \bar{q} \gamma$ to match data, while in the second case a factor of 1.91 should be applied. The plots with rescaled $q \bar{q} \gamma$ component are shown in Fig. 9.65. 


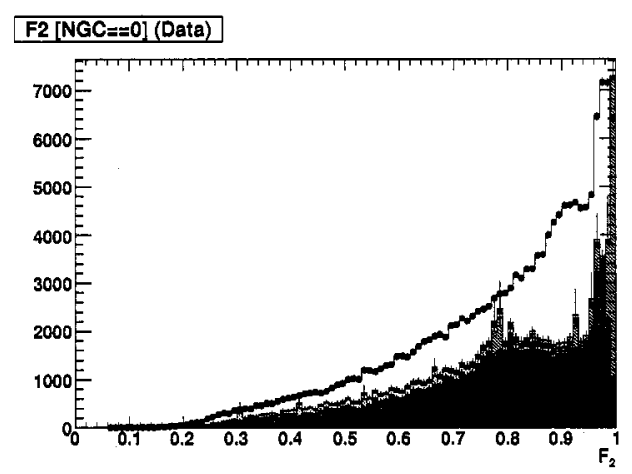

Figure 9.64: Distribution of $F_{2}$ for $N_{G T}=0$.

The agreement is rather good, except for the bump at $F_{2} \sim 0.9$. Since the scaling fac-
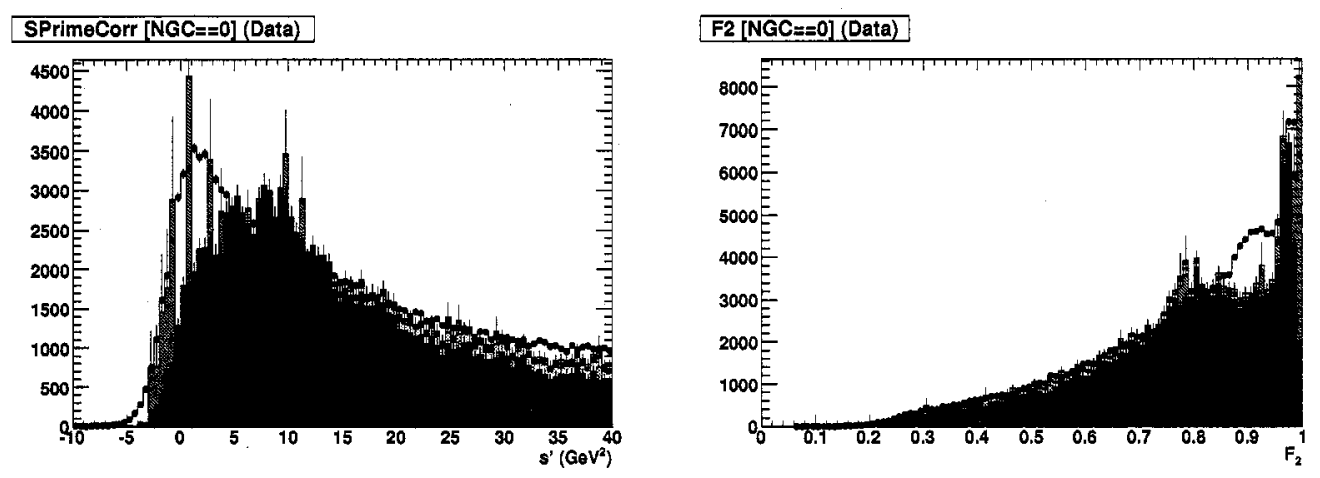

Figure 9.65: Distribution of $s^{\prime}$ (left) and $F_{2}$ (right) for $N_{G T}=0$ after rescaling the $q \bar{q} \gamma$ component as described in the text.

tors in both distributions agree well, we assume that most of the excess is attributable to signal. The residual excess in data after rescaling the $q \bar{q} \gamma$ component represents $39 \%$ and $82 \%$ of the $\gamma \gamma$ componenet in the $F_{2}$ and $s^{\prime}$ distributions respectively. A $60 \%$ systematic error is therefore asssigned to the normaliztion of the $\gamma \gamma$ component. 
Table 9.13: Cross-section for the ISR production of $\Upsilon$ resonances

\begin{tabular}{lcccccc}
\hline \hline State & $m(\mathrm{GeV})$ & $\mathrm{z}$ & $\xi\left(m^{2}, s\right)$ & $\Gamma_{e e}(\mathrm{keV})$ & $\sigma_{\operatorname{Exp}}(\mathrm{pb})$ & $\sigma_{O b s}(\mathrm{pb})$ \\
\hline$\Upsilon(1 S)$ & 9.460 & 0.200 & 0.378 & 1.32 & 21.7 & - \\
$\Upsilon(2 S)$ & 10.002 & 0.106 & 0.784 & 0.52 & 16.8 & $15.5 \pm 1.3 \pm 1.3$ \\
$\Upsilon(3 S)$ & 10.353 & 0.042 & 2.10 & 0.45 & 37.6 & $17.5 \pm 3.0 \pm 1.6$ \\
$\Upsilon(4 S)$ & 10.580 & - & - & - & - & - \\
\hline \hline
\end{tabular}

\section{$9.12 \Upsilon$ resonance decays}

\subsubsection{Order of magnitude estimate}

A potential background comes from the ISR process $e^{+} e^{-} \rightarrow \gamma \Upsilon(n S)$ followed by an $\Upsilon(n S) \rightarrow \gamma g g$ decay, for $n=1,2,3$. The ISR $\gamma$ is rather soft, $\approx 1 \mathrm{GeV}$ for the $\Upsilon(1 S)$ and lower for the other two, but the photon from the radiative decay can be hard. Since the ISR photon is mostly lost down the beampipe these decays are essentially indistinguishable from signal and therefore need to be evaluated.

The production cross-sections for the resonances can be obtained using Eqs. 2.49 and the following formula for the integrated cross-section to produce a narrow resonance:

$$
\sigma_{R}=\frac{12 \pi^{2} \Gamma_{e e}}{m s} \xi\left(m^{2}, s\right) .
$$

The corresponding cross-sections are given in Table 9.13.

Rates have been measured by CLEO and found to agree well with somewhat more precise theoretical estimates [73].

The rate for $\Upsilon$ decays to 3 gauge bosons can be obtained from the standard formula for the decay width of positronium into 2 photons $[77,76]$

$$
\Gamma\left({ }^{3} S_{1} \rightarrow \gamma \gamma \gamma\right)=\frac{16}{9 \pi}\left(\pi^{2}-9\right) \frac{\alpha^{3}}{M^{2}}|R(0)|^{2} .
$$

The corresponding formula for $\Upsilon \rightarrow g g g$ can be obtained by the replacement [76]

$$
\alpha^{3} \rightarrow \frac{5}{18} \alpha_{s}^{3}
$$


where the factor $5 / 18$ is a "color factor". For $\Upsilon \rightarrow \gamma g g$, the replacement is

$$
\alpha^{3} \rightarrow 9 \frac{2}{9} \alpha_{s}^{2} q^{2} \alpha
$$

where the factor of $2 / 9$ is a color factor, the 9 comes from the 3 possible choices for the photon position in the emission diagram (before, between or after the gluons), and $q^{2}$ is the square of the charge of the bottom quark.

We therefore get

$$
R_{\gamma}=\frac{\Gamma(\Upsilon \rightarrow \gamma g g)}{\Gamma(\Upsilon \rightarrow g g g)}=\frac{36}{5} q_{b}^{2} \frac{\alpha}{\alpha_{s}} \approx 3 \%
$$

This is the same for all $\Upsilon(n S)$ resonances $(n=1,2,3)$ except for the variation in $\alpha_{s}$ which is negligible. We use $\alpha_{s} \approx 0.18 . \Upsilon \rightarrow$ hadrons accounts for practically all the $\Upsilon$ decay rate since $\mathcal{B}\left(\Upsilon \rightarrow l^{+} l^{-}\right) \approx 5 \%$. Transitions to other $b \bar{b}$ states can be ignored: at the end of the transition chain the final decay is still hadronic most of the time, and it doesn't matter from which bottomonium state is occurs from.

The ratios $R_{\gamma}$ have been measured by CLEO and agree well with the $3 \%$ estimation [74]. The same reference also shows the photon spectra expected in some theoretical models. The peak of the energy distribution is around $x_{\gamma}=0.70$, where $x_{\gamma}=2 E_{\gamma}^{*} / m_{\Upsilon}$. The energy cut at $E_{\gamma}^{*}=3.3 \mathrm{GeV}$, corresponding to $x_{s} \approx 0.65$, selects about $30-40 \%$ of the spectrum.

The expected cross-section for this background is therefore

$$
\sigma=(21.7+15.5+17.5 \mathrm{pb})_{\Upsilon} \times(3 \%)_{g \gamma \gamma} \times(40 \%)_{\text {spectrum }}=0.76 \mathrm{pb}
$$

The $\cos \left(\theta_{\gamma}^{*}\right)$ distribution for these events seems rather flat $[74,75]$ so a significant fraction of the cross-section may feed into our sample.

\subsubsection{Measurement}

An interesting feature of the soft ISR transitions to lower $\Upsilon$ resonances is that the transition rate varies rapidly with the beam energy. In particular, the rates are 
Table 9.14: Cross-section for the ISR production of $\Upsilon$ resonances

\begin{tabular}{lcccc}
\hline \hline State & $z_{\text {Off }}$ & $\xi_{\text {Off }}\left(m^{2}, s\right)$ & $\sigma_{E x p}^{\text {Off }}(\mathrm{pb})$ & Relative change (\%) \\
\hline$\Upsilon(1 S)$ & 0.194 & 0.391 & 22.4 & $+3.4 \%$ \\
$\Upsilon(2 S)$ & 0.099 & 0.841 & 18.0 & $+7.2 \%$ \\
$\Upsilon(3 S)$ & 0.035 & 2.53 & 45.2 & $+20.2 \%$ \\
Total & - & - & 85.6 & $+12.5 \%$ \\
\hline \hline
\end{tabular}

measurably larger for off-peak data as for on-peak data, as seen by CLEO [73]. In the case of CLEO off-peak data was taken $60 \mathrm{MeV}$ below the nominal energy, whereas for $B A B A R$ the difference is only $40 \mathrm{MeV}$. The corresponding change in production cross-sections are shown in Table 9.14.

We therefore compute the difference in the $s^{\prime}$ spectrum between on-peak and offpeak data. The off-peak data is rescaled beforehand to account for the difference in integrated luminosities between the samples, $\mathcal{L}=189.3 \mathrm{pb}^{-1}$ for on-peak and $\mathcal{L}=19.1 \mathrm{pb}^{-1}$ for on-peak. As shown in Ref. [74], most of the events are expected at high $s^{\prime}$ and we therefore limit the $s^{\prime}$ range to $10<s^{\prime}<41 \mathrm{GeV}^{2}$, which should contain at least $80 \%$ of the total cross-section.

Fig 9.66 shows the comparison between the two and the difference divided by the total integrated luminosity of $208.4 \mathrm{fb}^{-1}$. We observe an excess of $0.18 \pm 0.03 p b$,
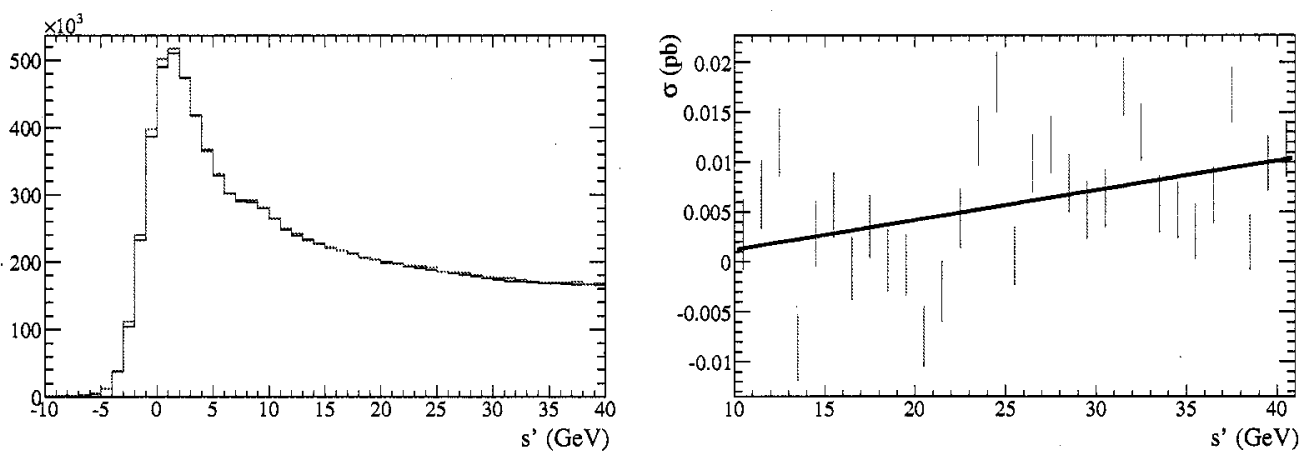

Figure 9.66: Left: $s^{\prime}$ distribution for on-peak (blue curve) and rescaled off-peak (red curve) data. Right: Difference in production cross-section between the two, with a linear fit superimposed. 
corresponding to $0.30 \pm 0.02 \%$ of the total sample. The fit shows that the excess increases toward high $s^{\prime}$, as expected for this process, with a fitted slope of $0.30 \pm$ $0.06 \mathrm{fb} \mathrm{GeV}^{-2}$.

\subsection{Two-photon collisions}

Two photon collisions [84] are a well-known [85] process occurring in $e^{+} e^{-}$collisions, in which the final state is produced by the fusion of two virtual photons, each emitted by one of the incoming leptons. As for the one photon exchange mechanism there is a large cross-section for hadronic production, although the spectrum is different since only resonant structures with positive C-parity are allowed (this has been used at $B A B A R$ to study the $\eta_{c}[86]$ ).

As for the one photon exchange case, there is a corresponding ISR process, shown in Fig. 9.67, which forms a background to the standard ISR hadronic production. As for ISR, the photons are preferentially soft and emitted along the beam direction. In the soft/collinear photon approximation, the differential luminosity at a given value of $z=1-s^{\prime} / s$ is given by [63]

$$
\zeta(z)=\frac{\mathcal{L}}{d z}=\left(\frac{2 \alpha}{\pi}\right)^{2} \log ^{2} \frac{\sqrt{s}}{m_{e}} \frac{(2+z)^{2} \log \frac{1}{z}-\left(1-z^{2}\right)\left(3+z^{2}\right)}{z} .
$$

\subsubsection{Events with less than two detected electrons}

We first study the case most analog to the non-radiative situation, where one or both electrons have go undetected along the beam direction. In this case the observable particles are expected to have good momentum balance in the direction transverse to 


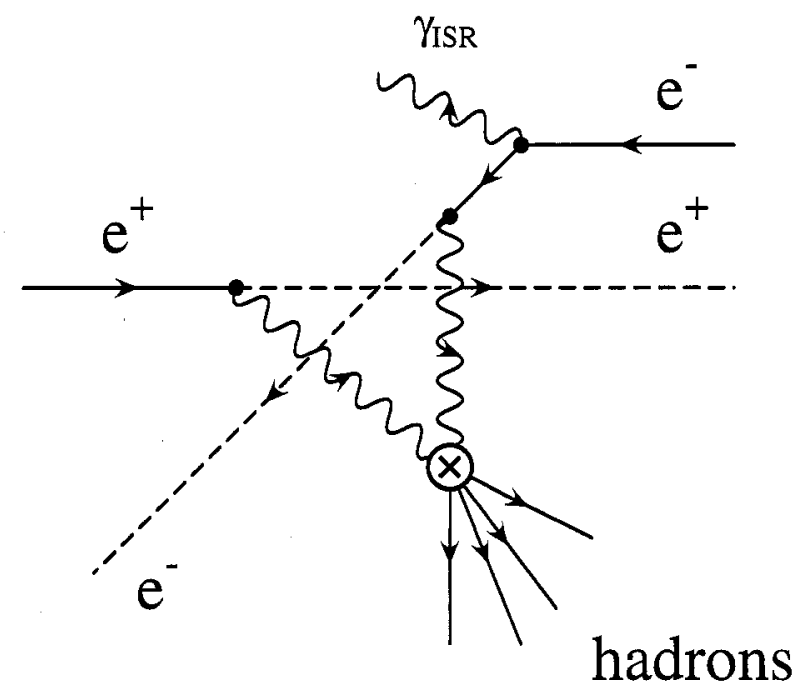

Figure 9.67: Diagram for the radiative two-photon collision process described in Section 9.13.

the beams but missing longitudinal momentum. We define the rescaled variables

$$
\begin{aligned}
x_{t}^{M i s} & =\frac{2 P_{t}^{M i s}}{\sqrt{s}} \\
x_{l}^{M i s} & =\frac{2 P_{l}^{M i s}}{\sqrt{s}},
\end{aligned}
$$

where $P_{t}^{M i s}$ and $P_{l}^{M i s}$ are the transverse and longitudinal components of the missing momentum. The $x_{t}$ distribution is shown in Fig 9.68 for both $\mathrm{MC}$ and data. We require $x_{t}^{\mathrm{Mis}}<0.05$ in the rest of this section. Events with a single electron track are rejected by the radiative Bhabha veto, so we consider the following two samples:

- No electrons: $N_{e}=0, x_{t}^{\text {Mis }}<0.05$,

- one electron: $N_{e}=1, N_{G T C} \geq 2, x_{t}^{M i s}<0.05$.

The $x_{l}^{\text {Mis }}$ distributions both both samples are shown in Fig. 9.69. They are asymmetric due to the asymmetry of the $e^{+} e^{-}$collision, which causes particles lost in the forward direction to be on average more energetic than particles lost in the backward 


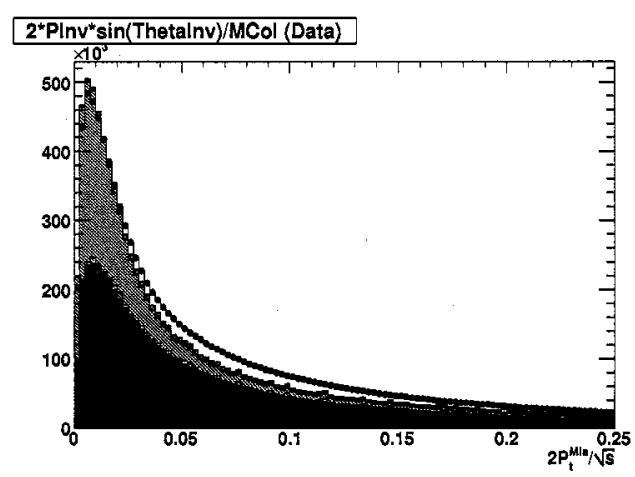

Figure 9.68: $x_{t}$ distribution for data (points) and MC (histograms).

direction. The excess at the high end of the $x_{l}^{M i s}$ spectrum in the no-electron sample is composed mainly of one-track events, and probably correspond to low-angle Bhabha events where the forward electron is lost down the beampipe while the other leg radiates and is misidentified as a non-electron. For simplicity we still include these events in our count of two-photon collisions. In order to evaluate the excess of events
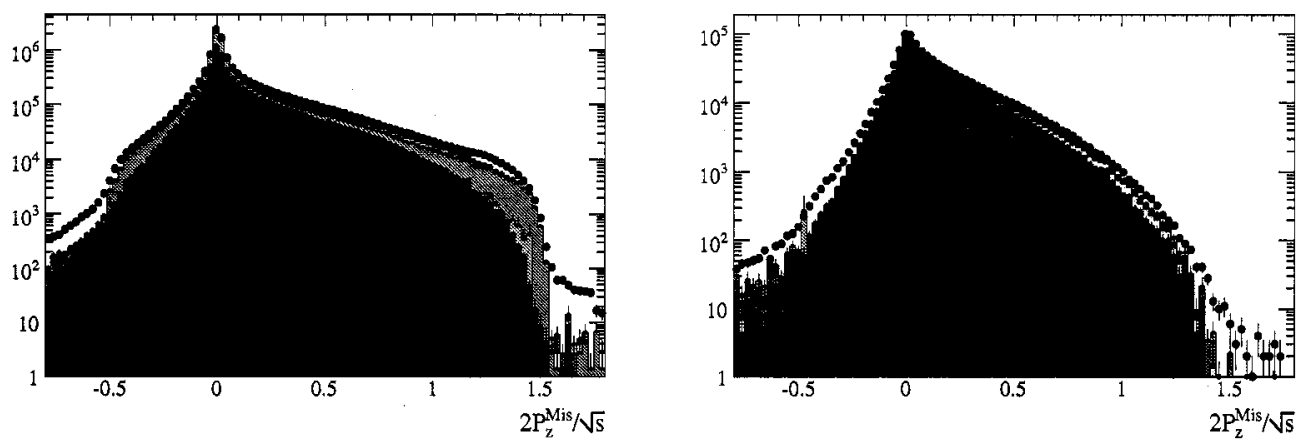

Figure 9.69: $x_{l}^{\text {Mis }}$ distributions for data (points) and MC (histograms) for no-electron sample (left) and the one-electron sample (right).

at high $\left|x_{l}^{\text {Mis }}\right|$ the MC distribution is subtracted from data after applying a scaling factor to ensure the $\mathrm{MC}$ and data distributions have equal area in the central region $\left|x_{l}^{\text {Mis }}\right|<0.2$. The results are shown in Table 9.15. 


\subsubsection{Events with two detected electrons}

It is possible that in some kinematic configurations, both initial electrons be visible in the detector. In the case of a hadronic system with less than two tracks such events would be rejected by the radiative Bhabha veto filters, but in other cases the events may pass the selection cuts. We therefore select events with at least four tracks, including exactly two electrons. Since the hadronic system is created preferentially from soft and collinear photons, we require that the electrons be the highest-momentum particles in the ISR recoil frame.

Fig. 9.70 shows the distribution of the back-to-back angular differences $\Delta \theta_{e}$ and $\Delta \phi_{e}$ along $\theta$ and $\phi$ for the electrons in such events. In the soft/collinear photon limit the electrons should be back-to-back. However the radiative Bhabha background also tends to occupy this kinematic region.
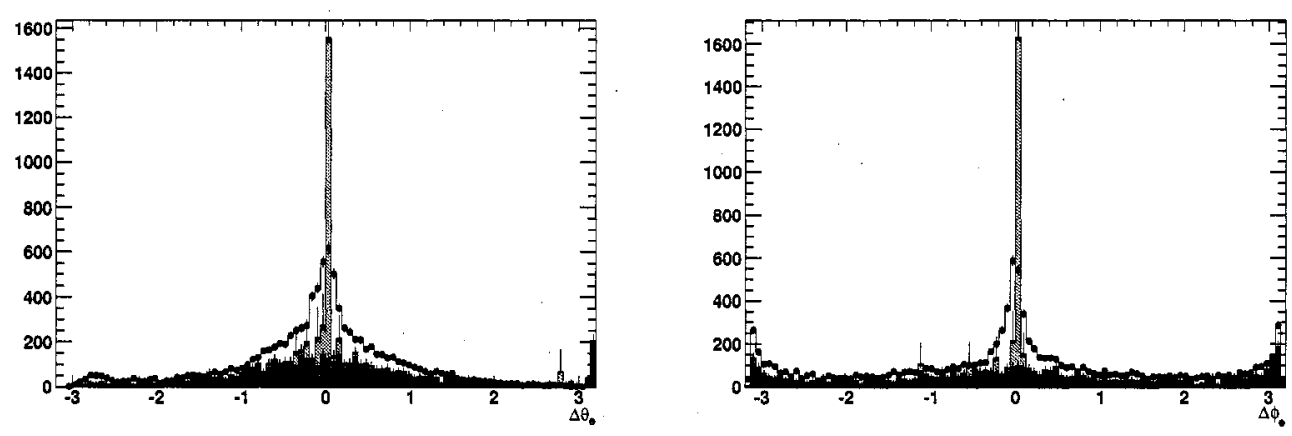

Figure 9.70: Back-to-back $\Delta \theta$ (left) and $\Delta \phi$ (right) distributions for electrons in data and $\mathrm{MC}$ for events with exactly two electrons where the electrons are the highestmomentum particles in the recoil frame.

We evaluate the two-photon background in this case to the difference between MC and data in the region $\left|\Delta \phi_{e}\right|<1,\left|\Delta \theta_{e}\right|<1$. Results are shown in Table 9.15.

A significant number of events is found in each category but it is unclear how much is produced in $\gamma \gamma$ collisions and how much is QED background. It is however not crucial to this analysis, as both types of background are treated similarly. 
Table 9.15: Cross-feed from two-photon collision events

\begin{tabular}{lcc}
\hline \hline Sample & Excess events & Fraction of total $\left(\times 10^{-4}\right)$ \\
\hline 0 electrons & $333002.3 \pm 2401.86$ & $263.7 \pm 1.9$ \\
1 electron & $8118.7 \pm 803.87$ & $6.4 \pm 0.6$ \\
2 electrons & $158555.0 \pm 398.19$ & $125.6 \pm 0.3$ \\
\hline \hline
\end{tabular}

\subsection{4 $e^{+} e^{-}$Annihilation to Two Virtual photons}

The usual $e^{+} e^{-}$annihilation to one virtual photon is the leading scattering process at $e^{+} e^{-}$colliders, but other processes also contribute, such as $\gamma-\gamma$ collisions (see Section 9.13). Recently, a new kind of process, which we call annihilation to two virtual photons has been observed [64]. Its diagram is shown in Fig. C.2: it has some similarity with ISR emission, but instead of a real photon a virtual photon is emitted. As for $\gamma-\gamma$ collisions, there exists a corresponding radiative process, which produces radiative events with hadrons and thus can feed into our sample. This process is however not relevant for $\Delta \alpha_{h a d}^{(5)}\left(m_{Z}^{2}\right)$ and therefore constitutes a background. It is however quite small compared to the one-photon annihilation process: experimental signals are observed at the level of $\sim 100 \mathrm{fb}$ or less, and consistent results are found in a calculation which is presented in Appendix C. This suggests that the two-virtualphoton process is suppressed by at least three orders of magnitude compared to the one-photon-case. It is therefore neglected in the rest of this document. 


\section{Chapter 10}

\section{Signal Efficiency Determination}

\subsection{Lateral Moment}

As described in Section 7, $\mu^{+} \mu^{-} \gamma$ events provide a clean $\gamma$ sample that can be used to compare photon reconstruction parameters in $\mathrm{MC}$ and data. In Section 7 this is used to measure the energy and position resolution, and in this section we use it to compare the lateral shape distributions in data and MC. The selection applied for this study is the same as for the photon resolution studies, described in Section 7.1. As discussed in Section 7.1.6, the sample is pure enough so that the distributions directly with no need for background subtraction.

The study is performed in bins of $E_{\gamma}^{*}$, identical to the ones used for the resolution studies. The $E_{\gamma}^{*}$ distribution for this sample is shown in Fig. 10.1. In order to improve $\mathrm{MC} /$ data agreement the energy and $\theta$ corrections determined in Section 7 are applied.

Figure 10.3 shows in each bin the energy resolution function and the lateral moment distributions for $\mathrm{MC}$ and data. As expected the energy resolutions are identical in $\mathrm{MC}$ and data after correction.

The lateral moment shapes in MC again exhibit a shift, already seen in all background samples. This is corrected for by shifting the MC distribution so that its mean coincides with that of the data distributions. After this procedure, extremely good agreement is seen between data and MC. Fig. 10.2 shows the values of the shift and the correction in efficiency values for the selection Lat $<0.35$. Both values do 
not vary significantly with $E_{\gamma}^{*}$, with the shift varying between 0.025 and 0.028 and the correction between $-1.5 \%$ and $-2.5 \%$.

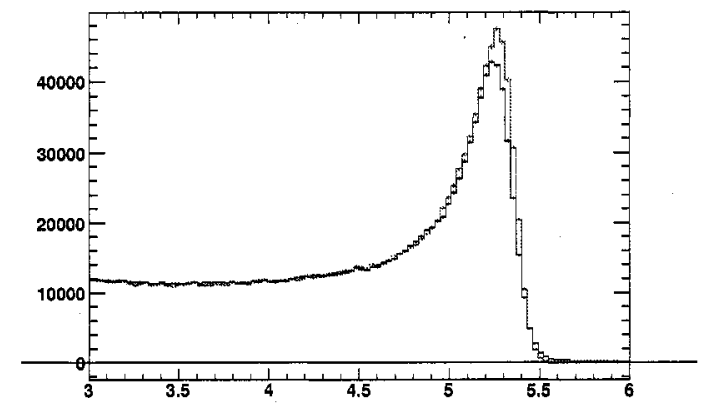

Figure 10.1: $E_{\gamma}^{*}$ distribution for the $\mu \mu \gamma$ sample used in this study
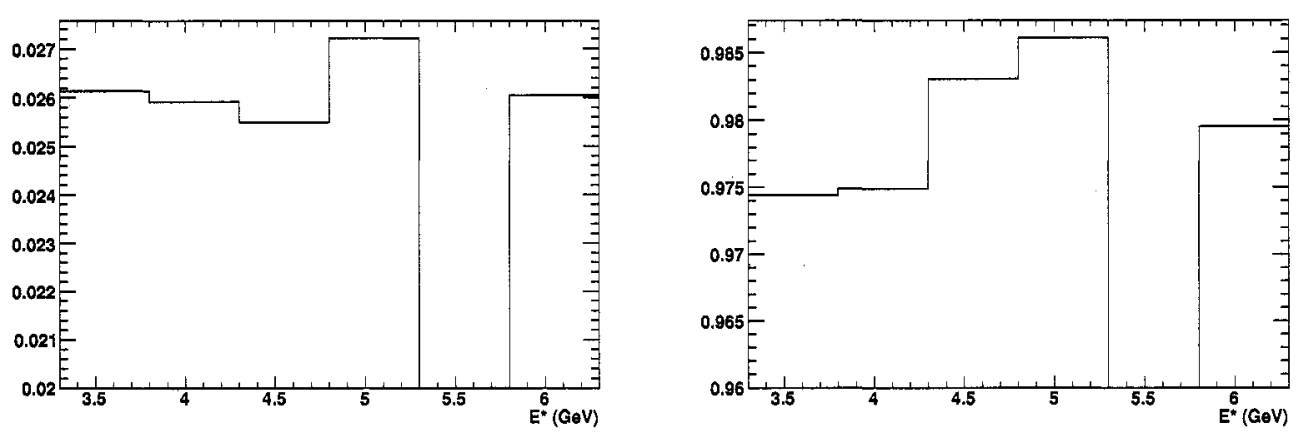

Figure 10.2: Left: shift applied to the $\mathrm{MC}$ lateral moment distribution. Right: weighting to be applied to $\mathrm{MC}$ to correct for the difference in the efficiency of the lateral moment shape.

\subsection{Fisher Selection}

Unlike the case of the background Fisher cross-check there is no way to get a distribution of the pure signal Fisher shape. We instead rely on the other two continuum background rejection cuts to get a reasonably pure signal sample. We reject events with: 

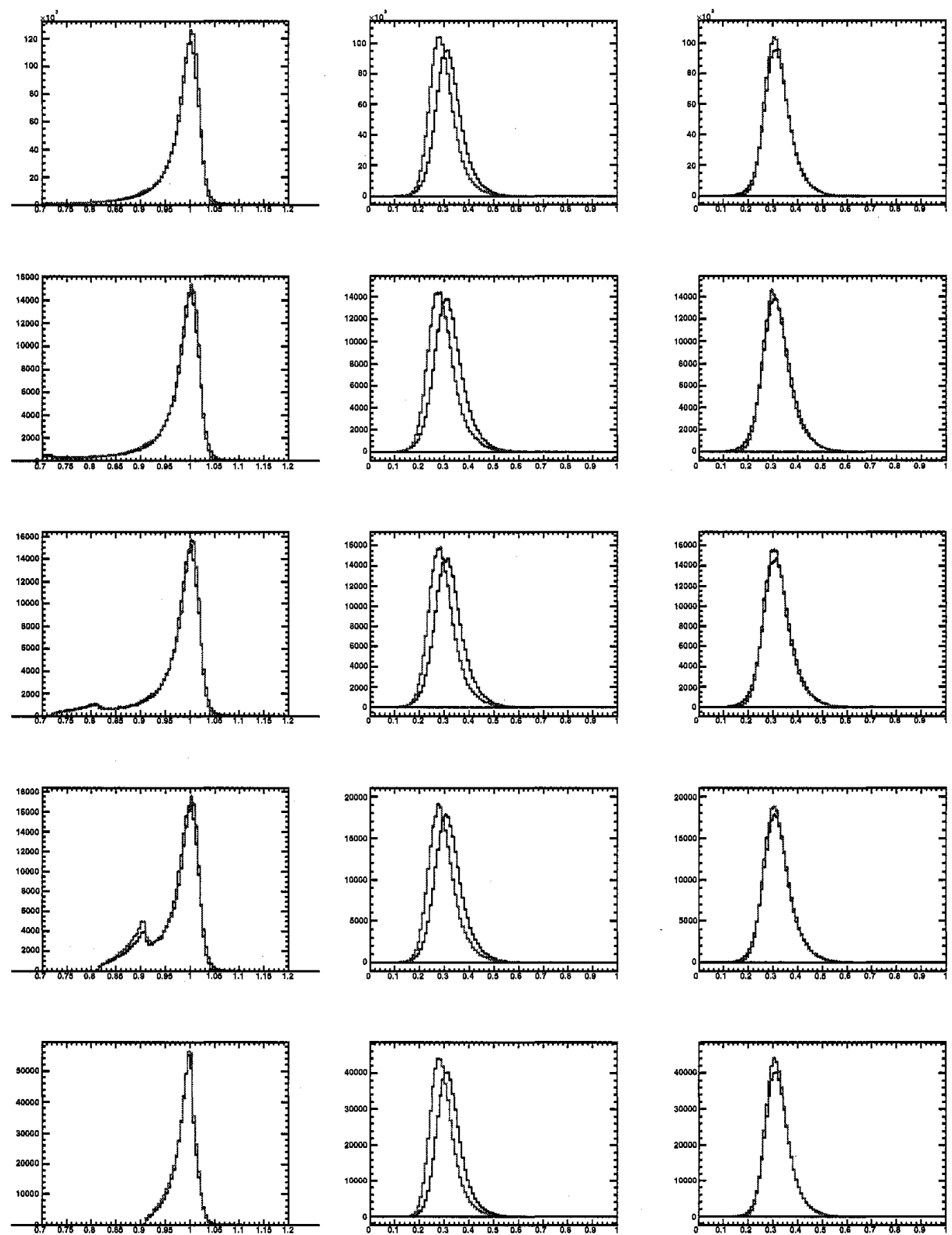

Figure 10.3: Left: photon resolution function in MC (red) and data (blue). Center: lateral moment distributions for $E_{\gamma}^{*}>3.3 \mathrm{GeV}$. Right: same with shifted MC 
- Lat $>0.30$

- $100<m_{\gamma \gamma}<170 \mathrm{MeV}$ or

- $497<m_{\gamma \gamma}<597 \mathrm{MeV}$ or

- $732<m_{\gamma \gamma}<832 \mathrm{MeV}$ or

- $908<m_{\gamma \gamma}<1008 \mathrm{MeV}$ or

- $497<m_{\gamma \gamma}<597 \mathrm{MeV}$ or

- $2997<m_{\gamma \gamma}<3197 \mathrm{MeV}$.

These cuts depend on the photon interaction with the EMC and the presence of random soft photon and are therefore not expected to bias the event-shape distributions. The distributions for all events, $N_{G T} \leq 2$ and $N_{G T} \geq 3$ are shown in Fig. 10.5 .

If one neglects the cross-feed from continuum background events, a correction function can be derived as before to account for the difference in the efficiency of the Fisher cut in data and MC. This function is shown in Fig. 10.4.

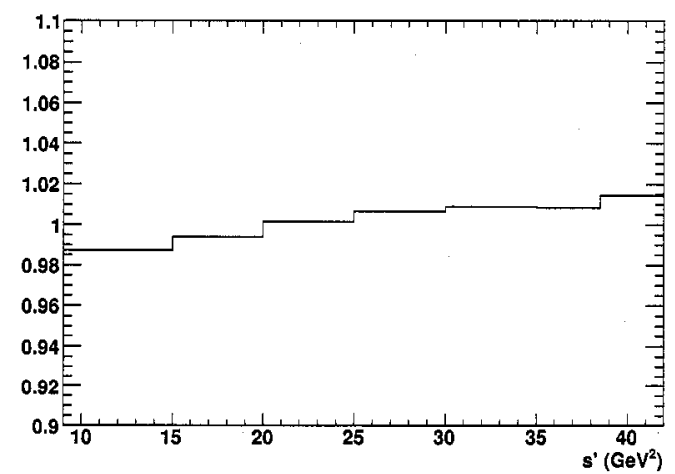

Figure 10.4: Fisher cut correction for signal 

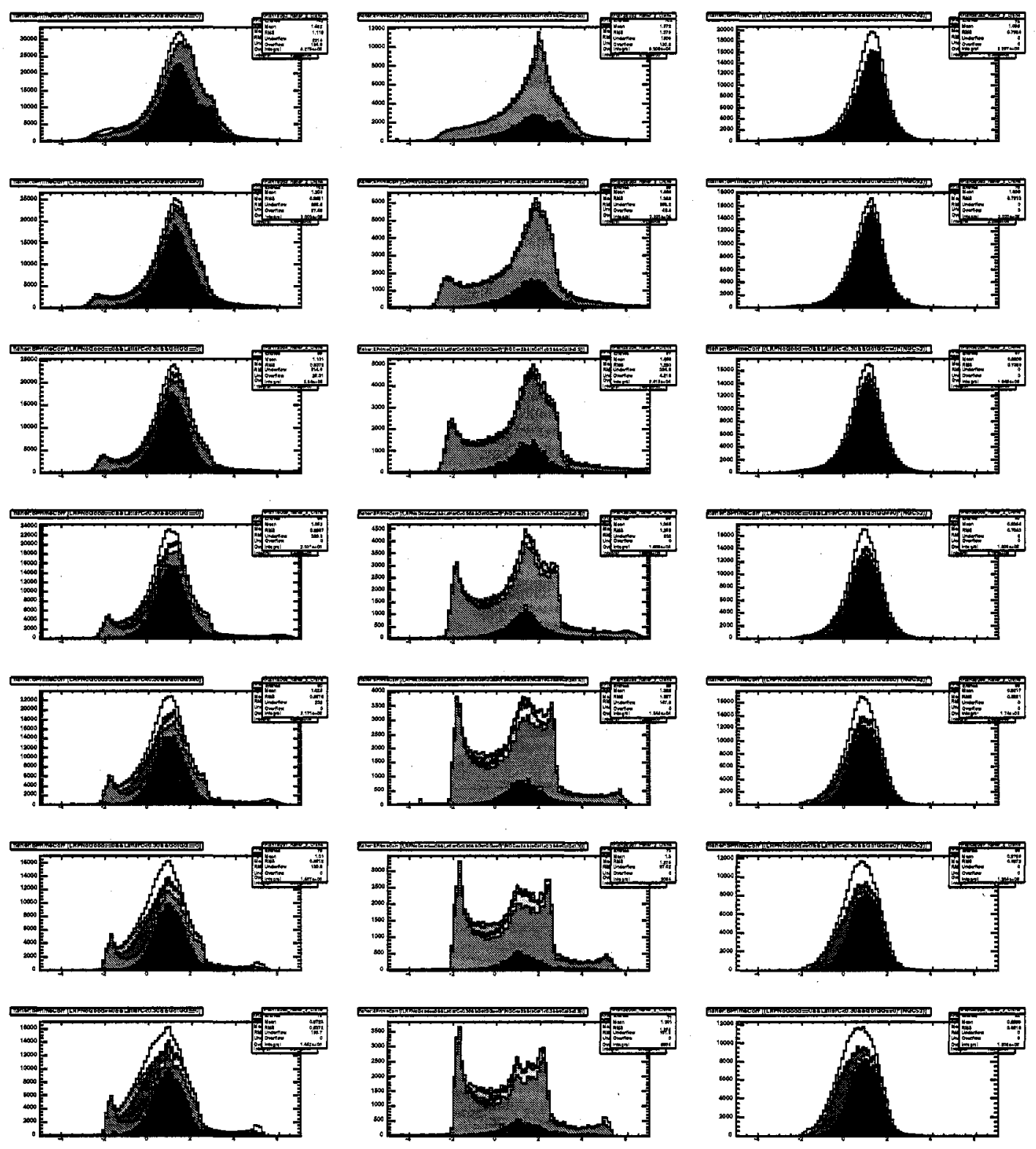

Figure 10.5: Fisher distributions after continuum-background rejection cuts in MC (stacked shaded histograms) and data (points with errors), for all events (left column), events with $N_{G T} \leq 2$ (middle column) and events with $N_{G T} \geq 3$ (right column). Rows correspond to $s^{\prime}$ bins, increasing from top to bottom 


\subsection{Calibration of Particle Identification (PID) rates}

A standard correction procedure is applied to the $\mathrm{MC}$ samples in order to correct for potential differences in PID efficiencies and misidentification rates between data and simulation. Control samples are used to evaluate the efficiencies $\epsilon_{D T}$ in data, and the MC efficiencies $\epsilon_{M C}$ are corrected by the following procedure:

- if $\epsilon_{M C}<\epsilon_{D T}$, tracks that pass the selection are randomly failed with probability

$$
p=1-\frac{\epsilon_{M C}}{\epsilon_{D T}} .
$$

- if $\epsilon_{M C}>\epsilon_{D T}$, tracks that fail the selection are recovered with probability

$$
p=1-\frac{1-\epsilon_{M C}}{1-\epsilon_{D T}} .
$$

The control samples used are radiative and non-radiative Bhabha scattering events for electrons, $\mu^{+} \mu^{-} \gamma$ events for muons, $\Lambda \rightarrow p \pi$ for protons, $D^{*+} \rightarrow D^{0}\left(K^{ \pm} \pi^{\mp}\right) \pi^{+}$ for kaons and $K_{S}^{0} \rightarrow \pi^{+} \pi^{-}$and $\tau^{+} \rightarrow \pi^{+} \pi^{-} \pi^{+} \nu$ for pions. This correction is applied for all the selections described in this document, except for the BG Filter selection of Section 4.10 and the preselection cuts of Section 4.8.

The main PID issue is $\pi \rightarrow e$ misidentification rate, since it is larger than for muons, kaons and protons and a high value can lead to an efficiency drop for $e^{+} e^{-} \rightarrow$ $\pi^{+} \pi^{-} \gamma$ events and similar events with additional neutrals, which make up about $30 \%$ of the signal sample. The main factor affecting this rate is the proportion of charged pions that shower in the EMC and leave large energy deposits, which may not be simulated accurately in MC.

In order to calibrate this efficiency, we use a sample of high-momentum pions from $e^{+} e^{-} \rightarrow \phi\left(K_{S}^{0} K_{L}^{0}\right) \gamma$ events where $K_{S}^{0} \rightarrow \pi^{+} \pi^{-}$. These events are selected directly from the ISR sample by requiring that the event contains exactly two tracks with $\mathrm{DCH}$ hits and that their invariant mass (assuming pion mass hypotheses) is within $30 \mathrm{MeV}$ of the nominal $K_{S}^{0}$ mass. 
Results are shown in Fig. 10.6. Residual backgrounds under the $K_{S}^{0}$ peak are small and can be eliminated by performing a background subtraction. The PID category of the highest- $p^{*}$ track under the criteria of Section 5.4 is shown in the right column of Fig. 10.6, with the value 1 for an identified non-electron, 2 for an identified electron, 3 for both and 0 for no identification. Since efficiency is expected to vary with track $p^{*}$, the sample is split into $4 p^{*}$ bins as described in the figure.

Good agreement is observed between data and MC. The difference between data and $\mathrm{MC}$ is used to tweak the selection of Section 5.4 on pions in the same way as described above, so that the selection efficiencies in MC agree with those in data.

The correction is small, affecting the efficiency at the per-mil level. Figure 10.7 shows the difference in total $q \bar{q} \gamma$ efficiency (see Section 10.5) between the case where the correction is not applied and the case where it is.

\subsection{Tracking Efficiency Correction}

The Monte Carlo efficiency for track reconstruction and the track quality requirements described in Section E.1 are cross-checked in data. Since the stand-alone tracking capability of the SVT allows tracks to be reconstructed independently of DCH information, it is possible to measure the tracking efficiency in data by requiring a track in either the DCH or SVT and looking for its continuation in the other system. The samples used are three-prong $\tau$ decays and $D^{*+} \rightarrow D^{0} \pi^{+}$decays with four prong $D^{0}$ decays. They are reconstructed requiring all tracks save one, and the efficiency is then obtained by requiring the final (excluded) track to satisfy the track requirements.

The results are tabulated into an efficiency correction binned by momentum, polar angle, azimuthal angle, and overall event multiplicity. An average track correction is obtained on a track-by-track basis. The correction suggests that the track reconstruction is somewhat less efficient in the data relative to the Monte Carlo simulation.

Since this analysis does not select events according to track multiplicity track efficiency is not as important an issue as for exclusive analyses. However since the $N_{G T}=0$ and $N_{G T}=1$ samples have lower efficiencies than the rest some effects 

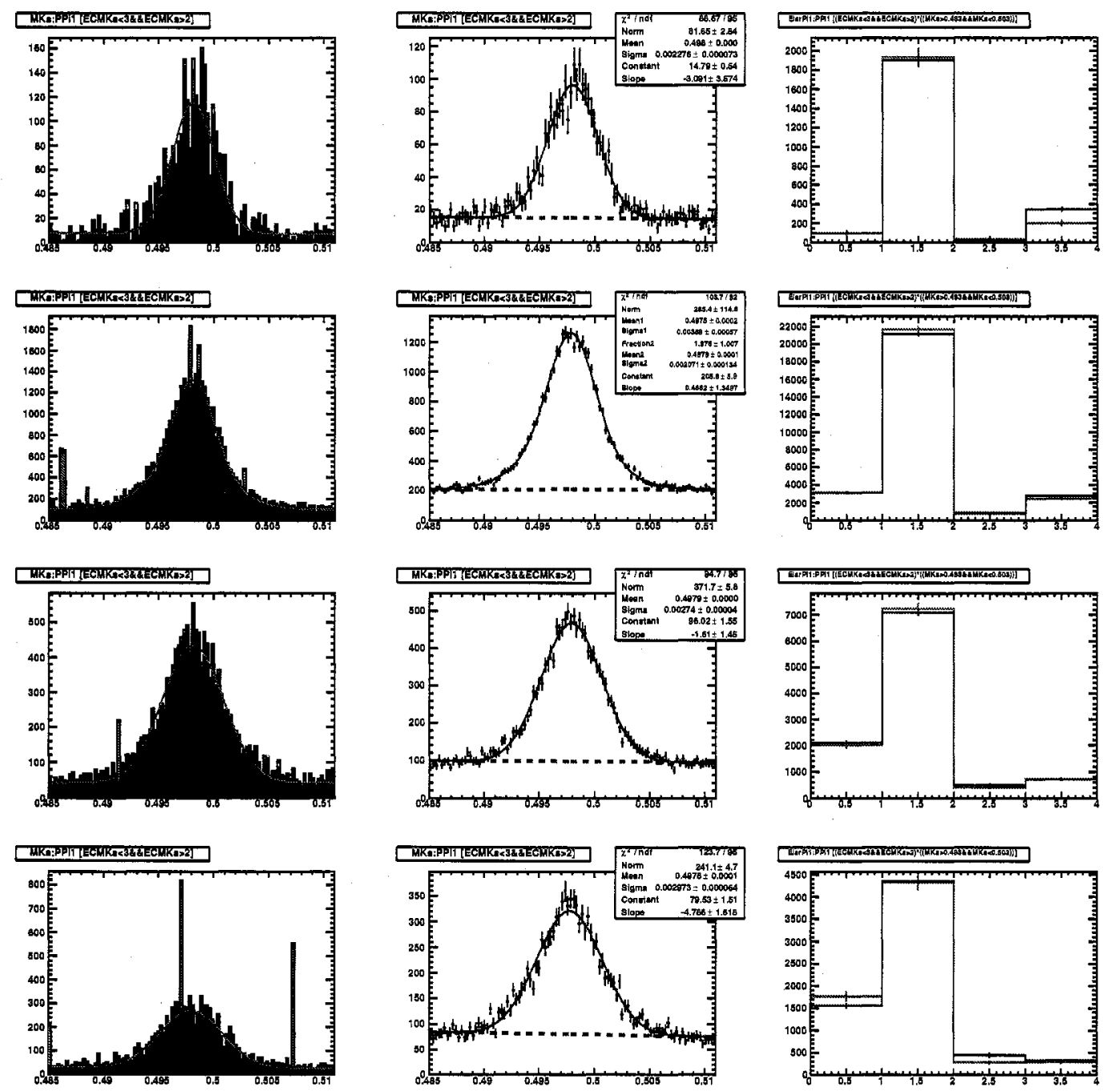

Figure 10.6: $K_{S}^{0}$ mass distributions for $K_{S}^{0} \rightarrow \pi^{+} \pi^{-}$decays in $e^{+} e^{-} \rightarrow \phi\left(K_{S}^{0} K_{L}^{0}\right) \gamma$ events, in MC (left column) and data (center column), and PID category of the highest- $p^{*} K_{S}^{0}$ daughter as described in the text (right column). The vertical bins corresponds to $0<p^{*}<1 \mathrm{GeV}, 1<p^{*}<2 \mathrm{GeV}, 2<p^{*}<2.5 \mathrm{GeV}$, and $2.5<$ $p^{*}<3 \mathrm{GeV}$ from top to bottom, where $p^{*}$ id the CM momentum of the highest- $p^{*} K_{S}^{0}$ daughter. 


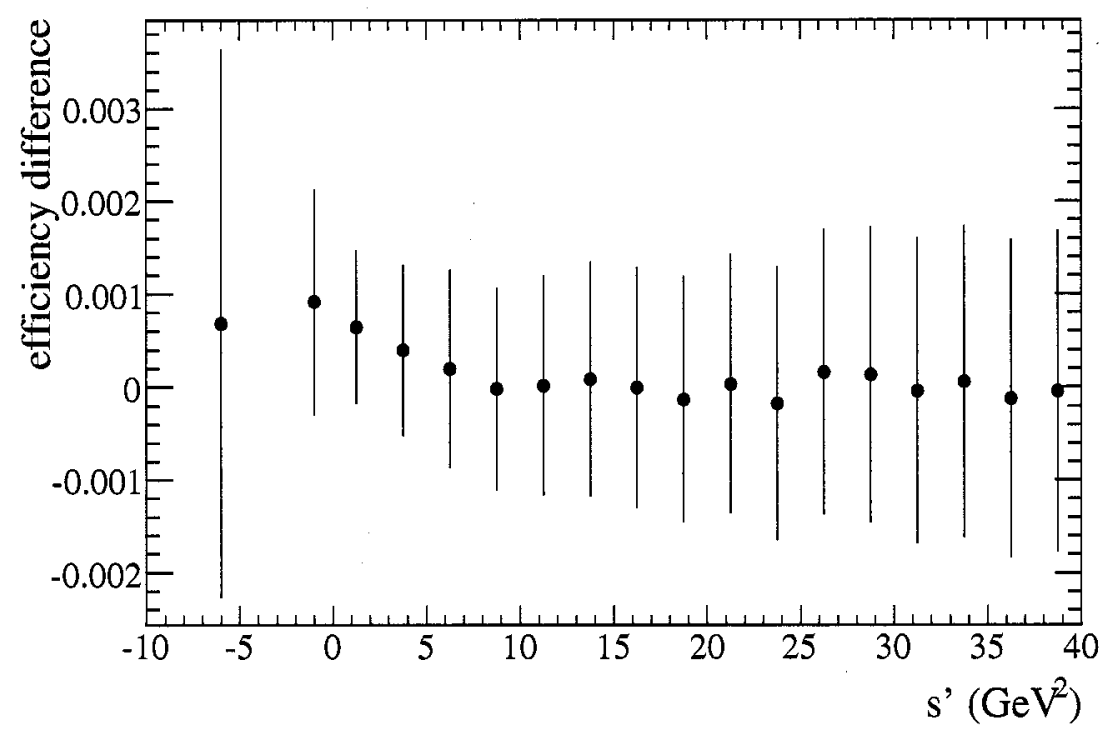

Figure 10.7: Difference between the $q \bar{q} \gamma$ efficiency with the PID correction not applied and PID correction applied

should occur.

In order to evaluate the correction, the analysis selections are modified so that for each event the following procedure is followed:

- for each track in the event, the track efficiency is looked up in the efficiency correction tables.

- For tracks with efficiency lower than unity, the selection is run with the track removed. The results of the selection algorithm ( 0 or 1$)$ with and without the tracks are then averaged to get a passing weight that accounts for the inefficiency of this particular track. The weight is 1 for events that pass the selection with and without the track, 0 for those who fail in both cases and between 0 and 1 for those cases where the removal of the track changed the result.

- The algorithm is applied recursively to all tracks, so that all $2^{n}$ combinations of tracks removed or included ( $n$ being the number of tracks with efficiency lower than unity) are considered and the outcomes of the selection procedure in each 
case averaged with the proper products of tracking (in)efficiencies.

The resulting correction in the final signal sample is shown in Fig. 10.8. The average value is $0.03 \%$.

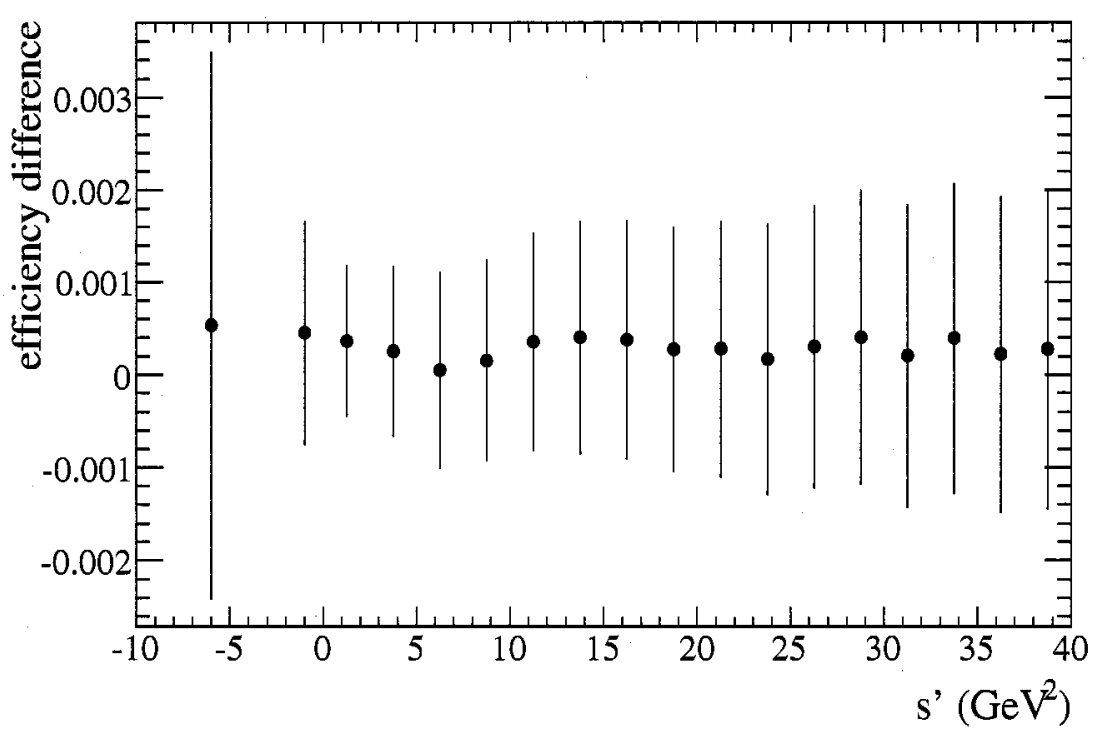

Figure 10.8: Tracking efficiency correction vs. $s^{\prime}$ for $q \bar{q} \gamma \mathrm{MC}$.

For exclusive analyses, the standard systematic uncertainty is $0.8 \%$ per track. However since tracking effects have much lower impact on this analysis we instead use an uncertainty equal to the total correction.

Fig. 10.7 shows the variation in $q \bar{q} \gamma$ efficiency (see Section 10.5) between the case where the correction is not applied and the case where it is.

\subsection{Efficiency Spectrum}

In order to obtain a corrected $s^{\prime}$ spectrum, the signal efficiency must be obtained as a function of $s^{\prime}$. The efficiency at different stages in the selection is shown in shown in Figs. 10.9, 10.10 and 10.11 for $q \bar{q} \gamma, \mu^{+} \mu^{-} \gamma$ and $\tau^{+} \tau^{-} \gamma$ respectively. The denominator is evaluated by counting the number of events with photons satisfying photon selection 
Table 10.1: Average efficiencies for signal modes.

\begin{tabular}{lccc}
\hline \hline & $q \bar{q} \gamma$ & $\mu^{+} \mu^{-} \gamma$ & $\tau^{+} \tau^{-} \gamma$ \\
\hline$I S R \gamma$ & 91.28 & 91.32 & 91.15 \\
$h \rightarrow n \gamma V$ eto & 89.27 & 90.83 & 88.62 \\
Lateral Moment & 81.00 & 82.58 & 80.33 \\
Event Shape & 79.06 & 82.36 & 77.12 \\
$e^{+} e^{-} \gamma V$ eto & 76.44 & 82.17 & 57.76 \\
Other QED Vetos & 72.67 & 75.41 & 52.87 \\
$p_{1}^{*}$ Cut & 72.44 & 64.88 & 52.87 \\
Total & 71.61 & 63.83 & 51.31 \\
Total, No Trk. corr & 71.63 & 63.87 & 51.32 \\
Total, No PID Corr. & 71.63 & 63.87 & 51.30 \\
\hline \hline
\end{tabular}

cuts and rescaling by the MC photon efficiency determined in Chapter 8. The PID correction of Section 10.3 is applied, as well as the tracking correction of Chapter 10.4. The efficiencies averaged over $s^{\prime}$ are shown in Table 10.1. 


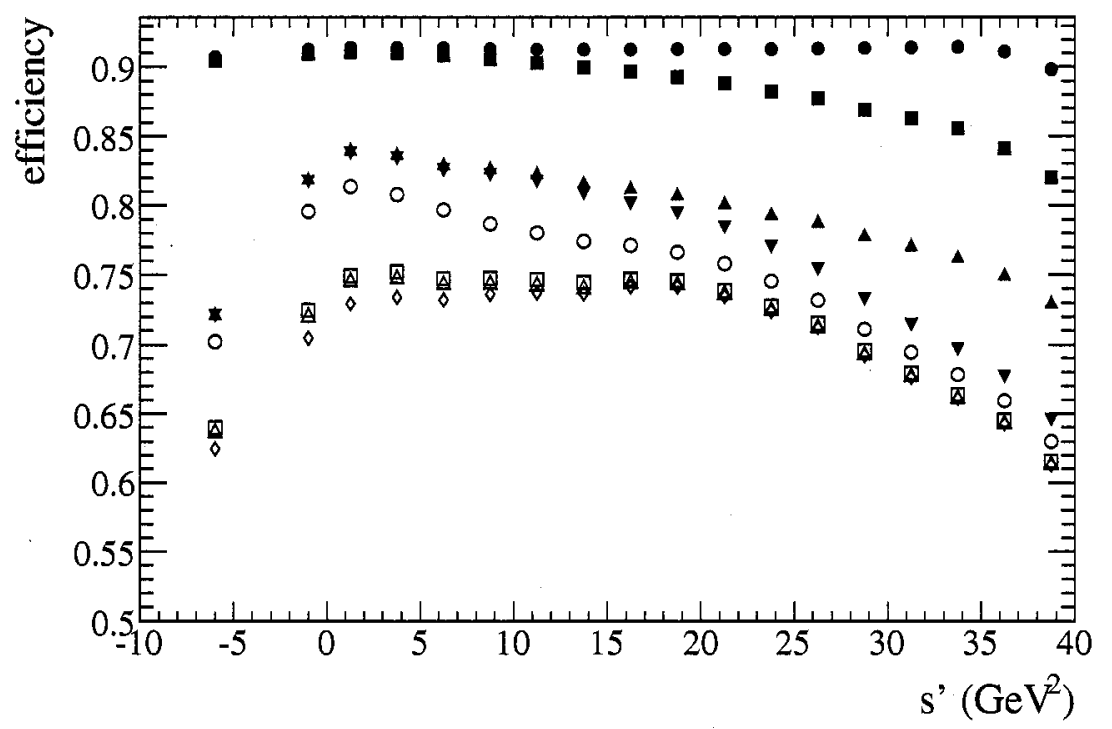

Figure 10.9: Efficiency in $q \bar{q} \gamma$ signal MC after all corrections. From top to bottom the cuts are photon-finding (closed circles) $h \rightarrow n \gamma$ Veto (closed squares), Lat Veto (upward-pointing closed triangles) Event shape (down-pointing closed triangles), Rad. Bhabha veto (open circles), Other QED vetos (open squares), $p_{1}^{*}$ Cut (open triangles) and trigger and precuts (open lozenges). 


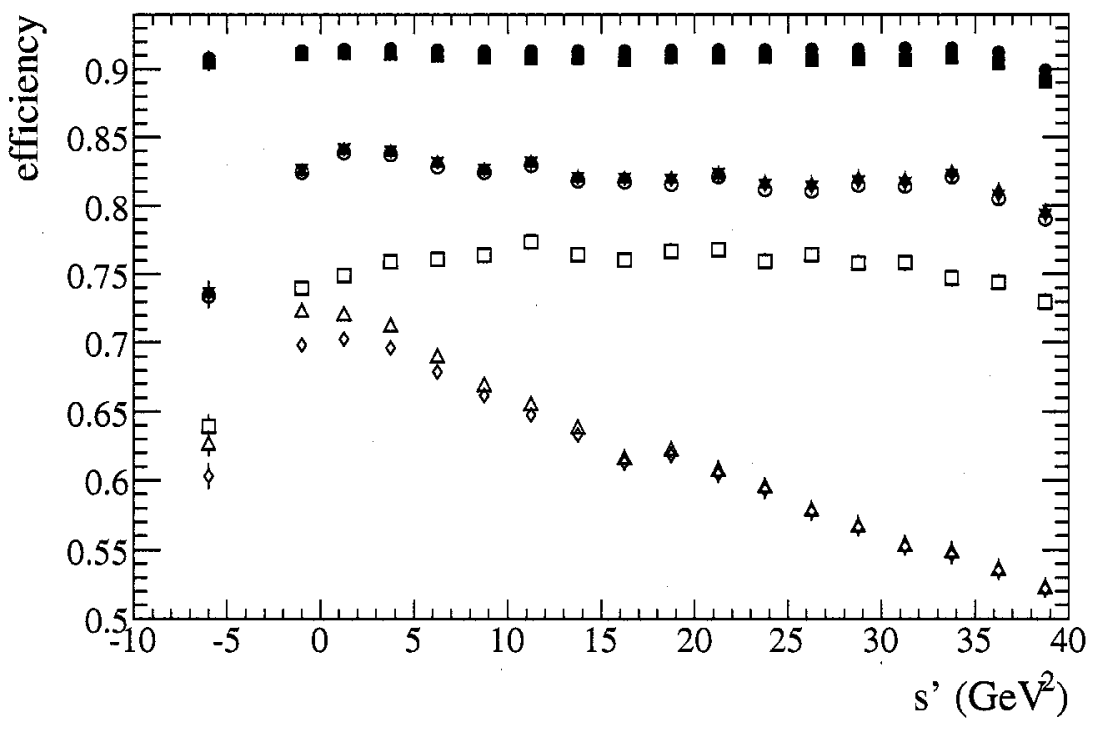

Figure 10.10: Efficiency in $\mu^{+} \mu^{-} \gamma$ signal MC after all corrections.From top to bottom the cuts are photon-finding (closed circles) $h \rightarrow n \gamma$ Veto (closed squares), Lat Veto (upward-pointing closed triangles) Event shape (down-pointing closed triangles), Rad. Bhabha veto (open circles), Other QED vetos (open squares), $p_{1}^{*}$ Cut (open triangles) and trigger and precuts (open lozenges). 


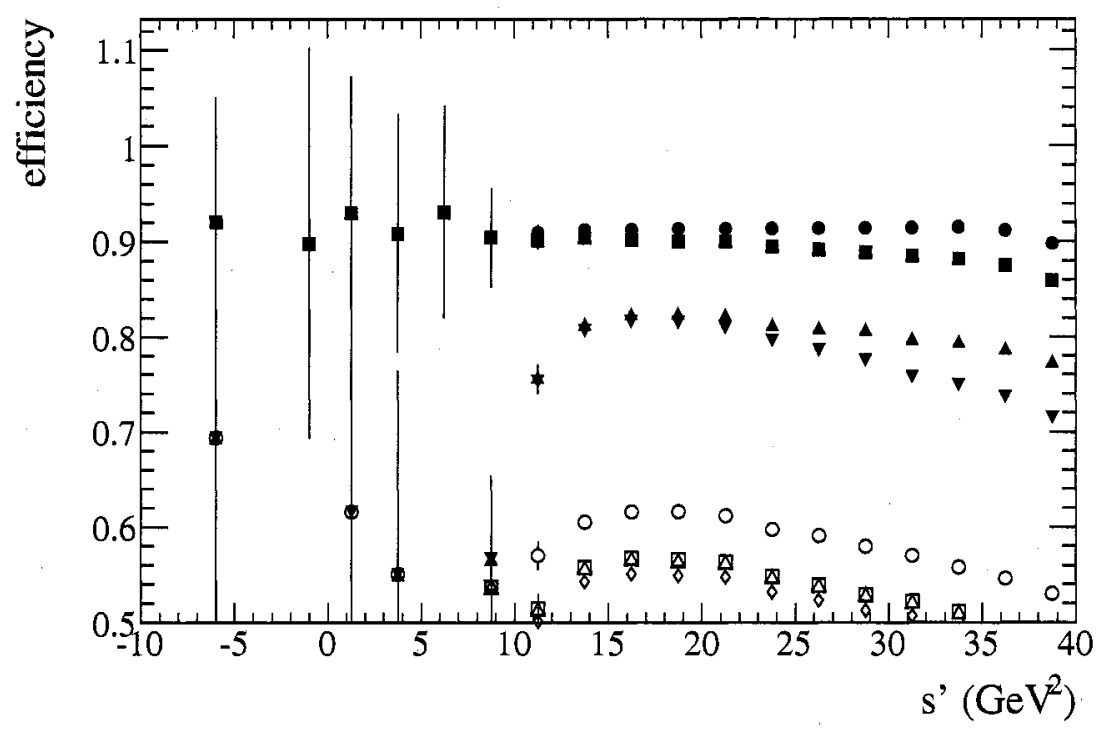

Figure 10.11: Efficiency in $\tau^{+} \tau^{-} \gamma$ signal MC after all corrections.From top to bottom the cuts are photon-finding (closed circles) $h \rightarrow n \gamma$ Veto (closed squares), Lat Veto (upward-pointing closed triangles) Event shape (down-pointing closed triangles), Rad. Bhabha veto (open circles), Other QED vetos (open squares), $p_{1}^{*}$ Cut (open triangles) and trigger and precuts (open lozenges). 


\section{Chapter 11}

\section{Results}

After applying the event selection described Chapters 5 and $6, s^{\prime}$ spectra are obtained in data and signal and background simulation samples. In Chapters 7 and 9 , a procedure to cross-check and correct the background samples is described. In Chapters 8 and 10, corrections are determined for the signal sample and the signal efficiency is determined. Having thus put all the pieces in place, the $s^{\prime}$ spectrum can be obtained by a standard background subtraction procedure.

Results are extracted in the range for $s^{\prime}$ from -10 to $40 \mathrm{GeV}^{2}$ over the polar angle range $0.386<\theta_{\gamma}<2.109$.

\subsection{Data-MC comparisons in the final sample}

In order to check the consistency of the data sample with expectations from MC, several quantities are shown in Figs. 11.1-11.5 for comparison. Fig. 11.1 shows the $s^{\prime}$ and $\theta_{\gamma}$ distributions after all corrections are applied. The $s^{\prime}$ shape is slightly different in data, with more events at higher $s^{\prime}$ and less in the bump at low $s^{\prime}$. The $\theta_{\gamma}$ is a particularly useful quantity to separate signal and continuum since signal is more concentrated at the edges of the acceptance due to the collinear enhancement of the radiative cross-section. The extra events indeed seem to concentrate in these edge regions, suggesting that they might be signal events (or some other kind of radiative events). Fig. 11.2 shows the comparison of track and cluster multiplicities. The 

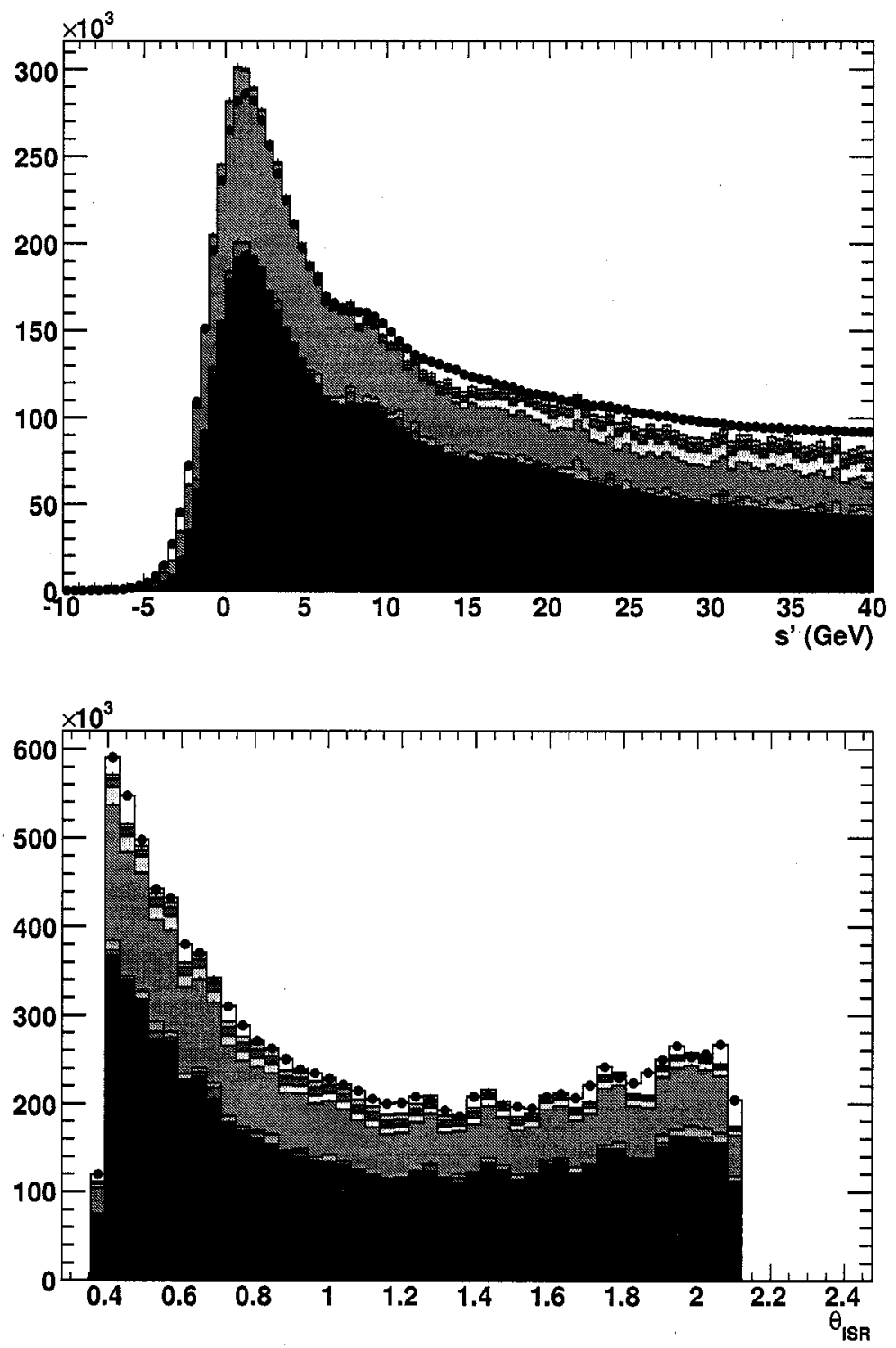

Figure 11.1: $s^{\prime}$ (top) and $\theta_{\gamma}$ (bottom) distributions in the final sample. Both MC and data samples are absolutely normalized and all the corrections are applied. 

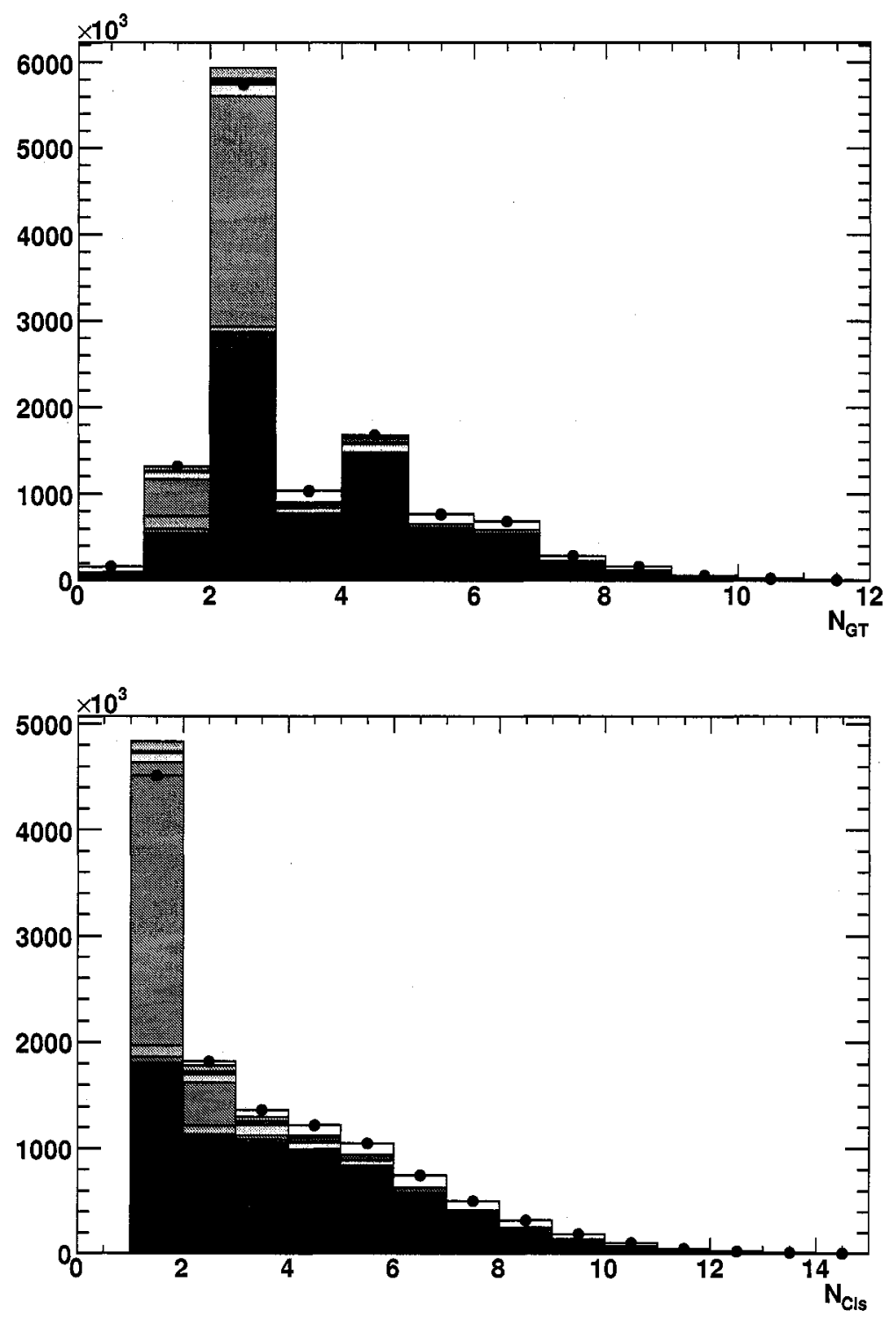

Figure 11.2: $N_{G T}$ (top) and number of unmatched clusters with $E>100 \mathrm{MeV}$ (bottom) in the final sample, with all corrections applied and all samples absolutely normalized. 
extra events seem to have high multiplicities in both categories, which is associated with signal in the plots. Further discrimination is obtained from the event-shape quantities shown in Fig. 11.3. The Fisher variable is shown for $N_{G T} \geq 3$ to ensure that there are enough particles to give meaningful values for event-shape variables. The excess seems to concentrate at high values, associated with signal. The excess is more pronounced in this plot since as shown in Fig. 11.2 the excess visible in the previous plots concentrates at higher $N_{G T}$ values. In the $F_{2}$ distribution, the excess is located at low values, corresponding to multihadronic events. It seems therefore that this excess is hadronic is nature, either from signal or some $q \bar{q}$ background. The identification with signal is suggested by the $\theta_{\gamma}$ and Fisher distributions; therefore no correction is applied and the excess is counted as signal in the final spectrum.

The angular separation between the ISR photon and the closest neighboring track, shown in Fig. 11.3, can be used both to separate the signal and to study radiative effects. The ISR topology favors high-angle configurations, although this becomes less true at higher $s^{\prime}$. By contrast, hadronic backgrounds tend to produce highenergy clusters as parts of a jet, favoring low-angle configuration. This is again not completely true, since events that feed into our sample must contain a very highenergy particle and this can cause the surrounding jet to be depleted, leading to higher separations. However the figure shows the expected trend, with the excess covering the plot uniformly, except for the high-angle region. It is therefore not clear whether to associate the excess with high- $s^{\prime}$ signal or continuum. The shape of the high- $s^{\prime}$ region is determined be radiative effects, and we see good agreement between $\mathrm{MC}$ and data. A secondary peak should have been visible at low angles in $\mu^{+} \mu^{-} \gamma \mathrm{MC}$ due to final-state radiation but this is suppressed by the $p_{1}^{*}$ cut (see Section 9.10). Fig. 11.4 shows views of the $c \bar{c}$ resonance and threshold regions. In $s^{\prime}$ the only visible feature is the broad peak corresponding to the $\psi$. However one can use the hadronic side to get a somewhat finer picture. The invariant mass $M_{\text {Had }}$ of the hadronic system is calculated by summing the four-momenta of all the tracks and clusters in the event (apart from the ISR photon), with a zero-mass hypothesis of for the clusters and a "best guess" hypothesis for the track: the track is checked for proton, kaon, muon and electron identification (in this order) and the corresponding mass is used if one 

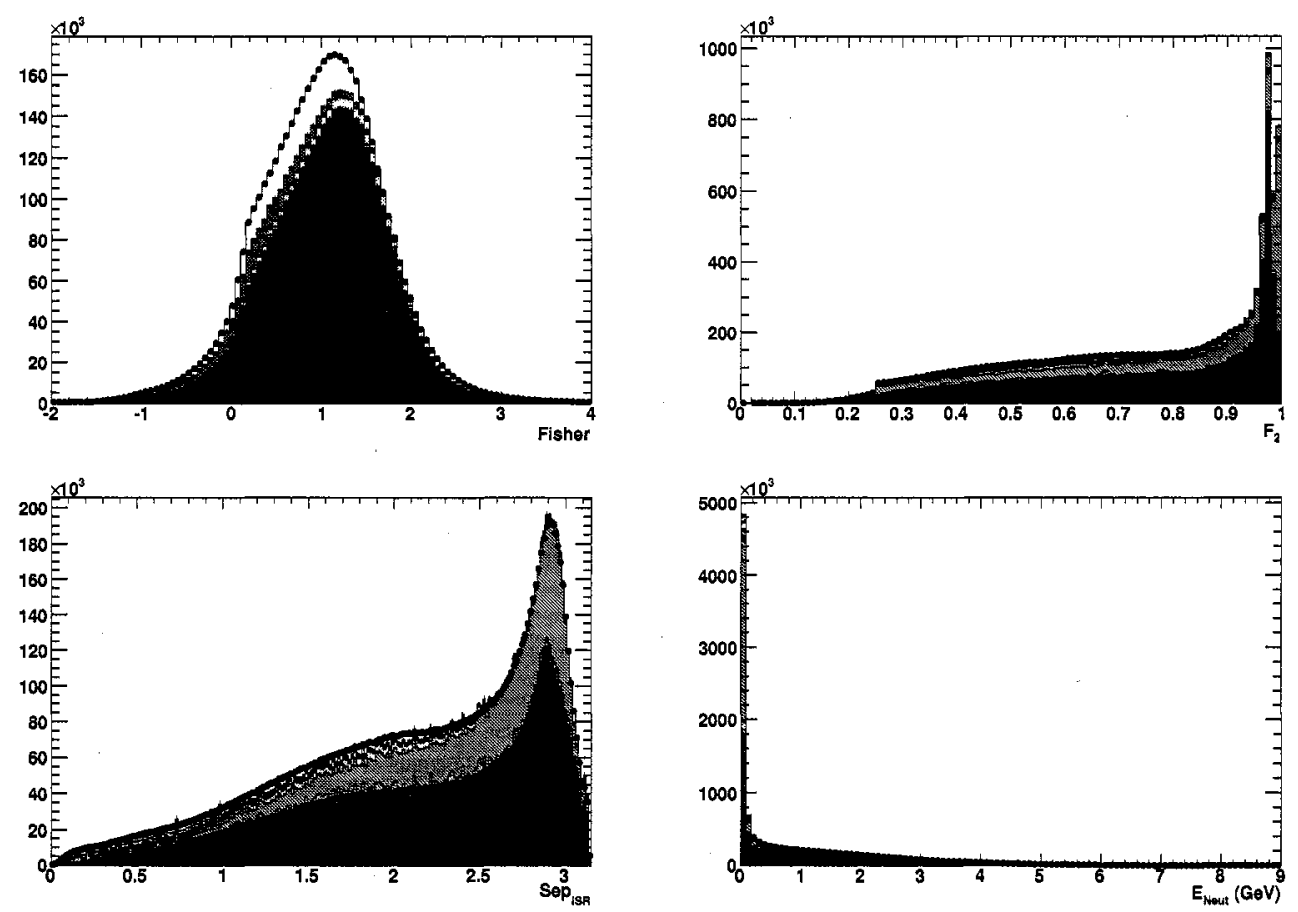

Figure 11.3: Fisher (top left) and $F_{2}$ (top right), angle between the ISR photon and the nearest track (bottom left) and the total neutral energy in the event (bottom right) in the final sample, with all corrections applied and all samples absolutely normalized. The Fisher distribution is represented for $N_{G T} \geq 3$. 
is found, otherwise the pion mass is used. Only IsrGoodTracks and clusters with $E>100 \mathrm{MeV}$ are used to avoid beam background products. Fig. 11.4 shows this quantity in two-track and four-track events in the $\psi$ and $c \bar{c}$ threshold regions. A clear peak is visible for the $\psi$ in the two-track sample, as well as for the $\psi(2 S)$ in the four-track sample. The MC behavior for $\psi(2 S)$ events is a known problem with KKMC, discussed in Appendix A. Fig. 11.5 shows the $M_{\text {Had }}$ distributions for events
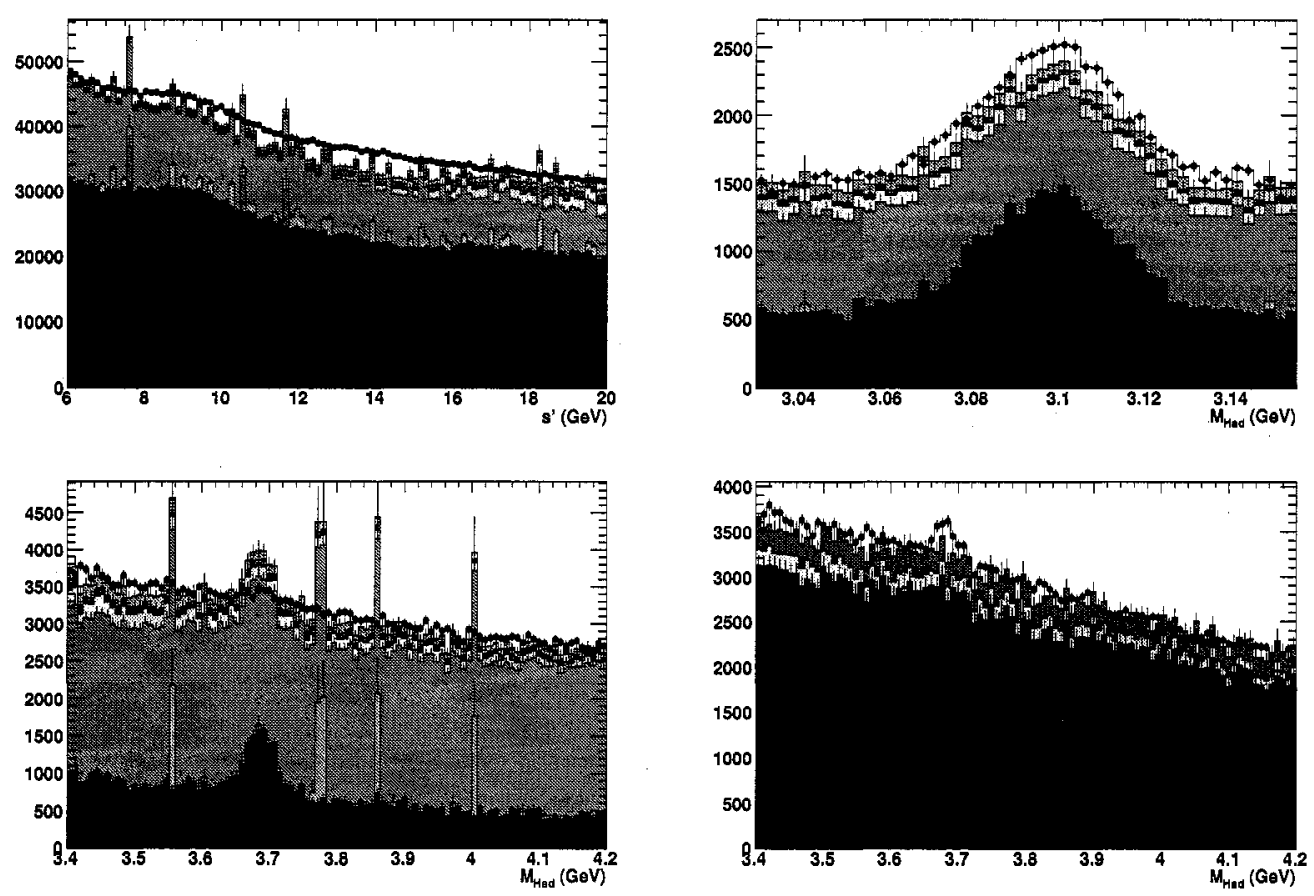

Figure 11.4: Distributions of $s^{\prime}$ (top left) and the invariant mass of the hadronic system (top right) in the $\psi$ region, and hadronic invariant mass for 2 (bottom left) and 4 -track (bottom right) events in the $c \bar{c}$ threshold region.

with $N_{G T}=2$ in the $\rho-\phi$ region. The top plot is obtained using the same procedure as described above, using all selected tracks and clusters, and shows a depletion on the $\rho$ and $\phi$ peaks. However, if the invariant mass is calculated using only the tracks, the distribution $M_{T r k}$ shown in the bottom plot is obtained, with good agreement between $\mathrm{MC}$ and data. The discrepancy in the first plot originates from the fact that pions tend to produce "satellite" clusters in addition to the primary shower when they 
enter the EMC. These clusters are then counted in the hadronic four-momentum and can significantly alter the mass even for small cluster energies. Since this effect is not well simulated the $\rho$ mass peak looks narrower in $\mathrm{MC}$, while in data it is not uncommon for the masses to be shifted to the $2-3 \mathrm{GeV}$ range. The second peak on the right of the $M_{T r k}$ distribution is the $K_{S}^{0}$, which seems more abundant is data than in MC.

\subsection{Systematic errors on Signal Efficiency}

The sources of systematic uncertainties on the signal efficiency are listed below:

- Integrated Luminosity: The integrated luminosity of the dataset used in this analysis is determined by counting $\Upsilon(4 S) \rightarrow B \bar{B}, \mu^{+} \mu^{-}$and Bhabha scattering events. The final results is a combination of these results, and a systematic uncertainty of $1.1 \%$ is reported based on the discrepancies between the different determinations.

- MC Generator: KKMC reports an accuracy of about $0.1 \%$ [65] for the radiative part of the cross-section. However even this has limited effects since it affects the distribution of events in $s^{\prime}$, and the detection efficiency depends weakly on $s^{\prime}$. The systematic uncertainty is therefore set at $1 \%$ of the maximum variation of the efficiency in $s^{\prime}$.

- Photon Efficiency: The uncertainty on each event is taken to be half the value of the photon efficiency correction applied to this event.

- $h \rightarrow n \gamma$ Veto: The combinatorics correction determined in Section 9.1 is applied to correct the signal efficiency for this cut. We use half the correction as the systematic error.

- Lateral Shape: Lateral shape distributions of both signal and background processes are shown to be shifted in MC compared to data, with an additional smaller correction to account for the difference in shapes (see Section 10.1 and 

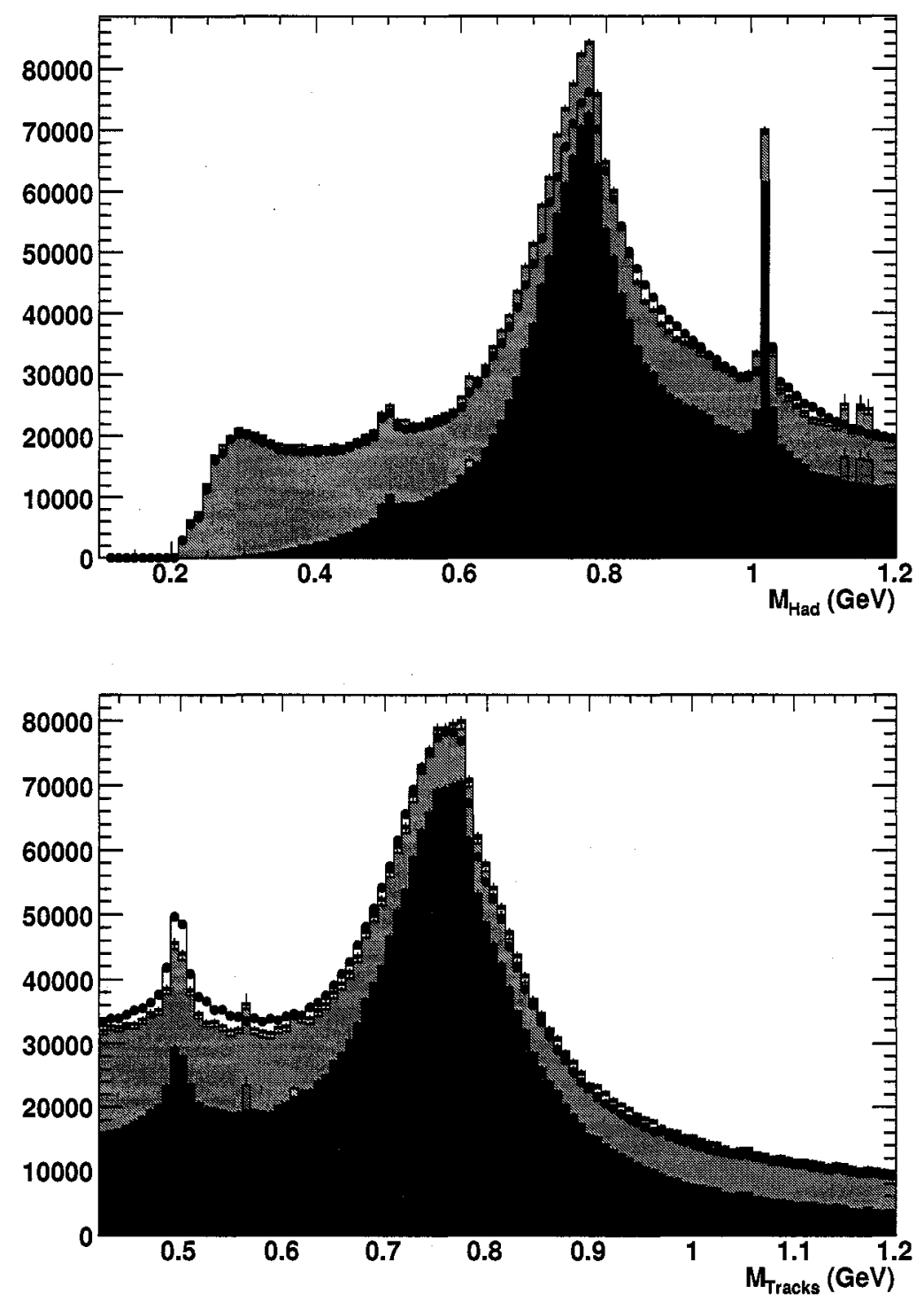

Figure 11.5: Hadronic system invariant mass (top) and two-track invariant mass with a pion mass hypothesis in the $\rho-\phi$ region with the requirement $N_{G T}=2$ (bottom). 
several sections in Chapter 9). Due to the very good agreement seen after applying the shift, the systematic error is chosen to cover only the latter, again using half the correction for the error.

- Event shape: A correction for the Fisher efficiency is determined in Section 10.2. Since significant levels of background remained in the sample used in the study, the correction is not applied to the signal. However half its magnitude is used as a systematic error.

- Tracking Efficiency: As discussed in Section 10.4, the value of the correction is used as systematic error.

- PID correction: As discussed in Section 10.3, misidentification of hadrons as electrons is the main effect. Since the effect of $\pi \rightarrow e$ misidentification on $\rho \rightarrow \pi^{+} \pi^{-}$events is the most problematic configuration, we use the value of the correction as systematic error.

- QED selection: The radiative Bhabha veto is based exclusively on PID information and therefore covered in the previous item. The rest of the QED vetos have however not been extensively cross-checked and the systematic error is taken to be half the inefficiency.

- Hot Towers: We use half the inefficiency as the systematic error.

- $p_{1}^{*}$ cut: In the $\mu^{+} \mu^{-} \gamma$ sample, events rejected by the $p_{1}^{*}$ selection are mainly FSR events where the non-radiative track is at the beam energy. Since FSR is well-described for $\mu^{+} \mu^{-} \gamma$ we assigns a systematic error equal to $10 \%$ of the inefficiency. For $q \bar{q} \gamma$ and $\tau^{+} \tau^{-} \gamma$, the inefficiency cannot be assigned to a single cause and a $50 \%$ systematic is assigned on the inefficiency.

- MC Statistics: The statistical error on the final efficiency in each bin is counted as a systematic error.

Figs. 11.6, 11.7 and 11.8 show the variations of systematic errors versus $s^{\prime}$ for $q \bar{q} \gamma$, $\mu^{+} \mu^{-} \gamma$ and $\tau^{+} \tau^{-} \gamma$ respectively. The average values of the errors over the entire $s^{\prime}$ range are shown in Table 11.1. 

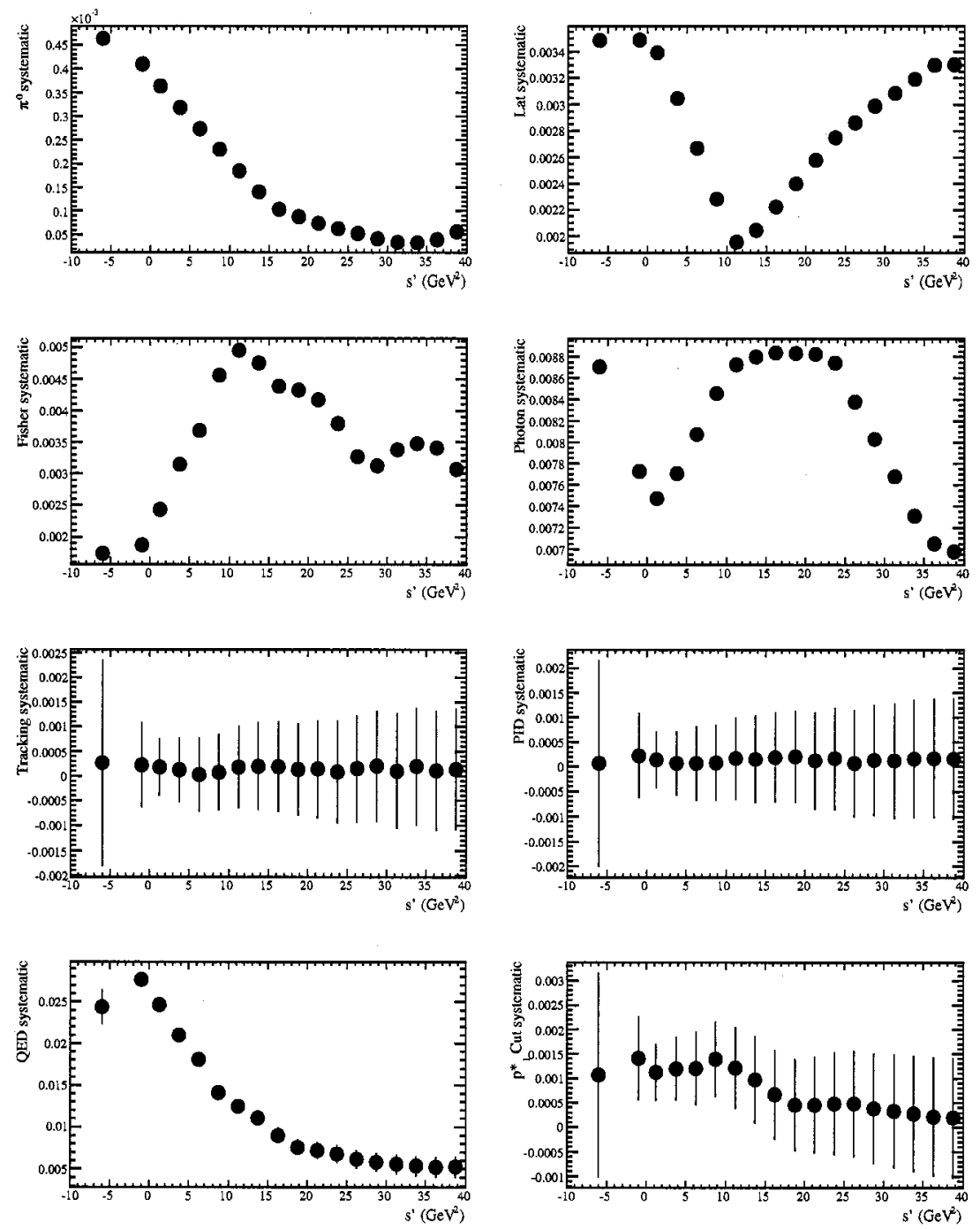

Figure 11.6: Systematic uncertainties versus $s^{\prime}$ for $q \bar{q} \gamma$ signal. From top to bottom, the quantities are: Left column: $h \rightarrow n \gamma$ Veto, Event Shape, Tracking and QED systematics Right Column: Lateral Moment, Photon, PID and $p_{1}^{*}$ cut systematics 

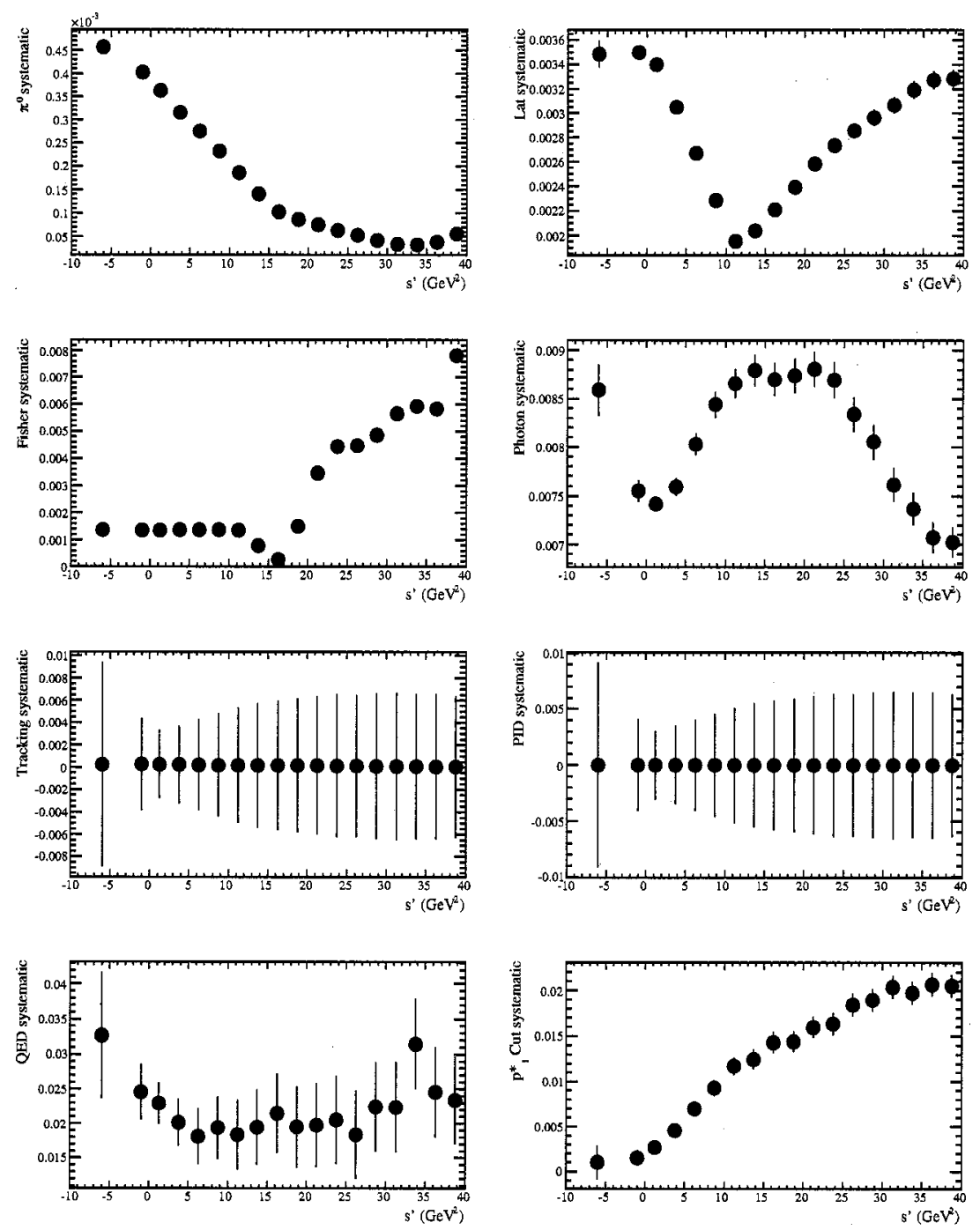

Figure 11.7: Systematic uncertainties versus $s^{\prime}$ for $\mu^{+} \mu^{-} \gamma$ signal. From top to bottom, the quantities are: Left column: $h \rightarrow n \gamma$ Veto, Event Shape, Tracking and QED systematics Right Column: Lateral Moment, Photon, PID and $p_{1}^{*}$ cut systematics 

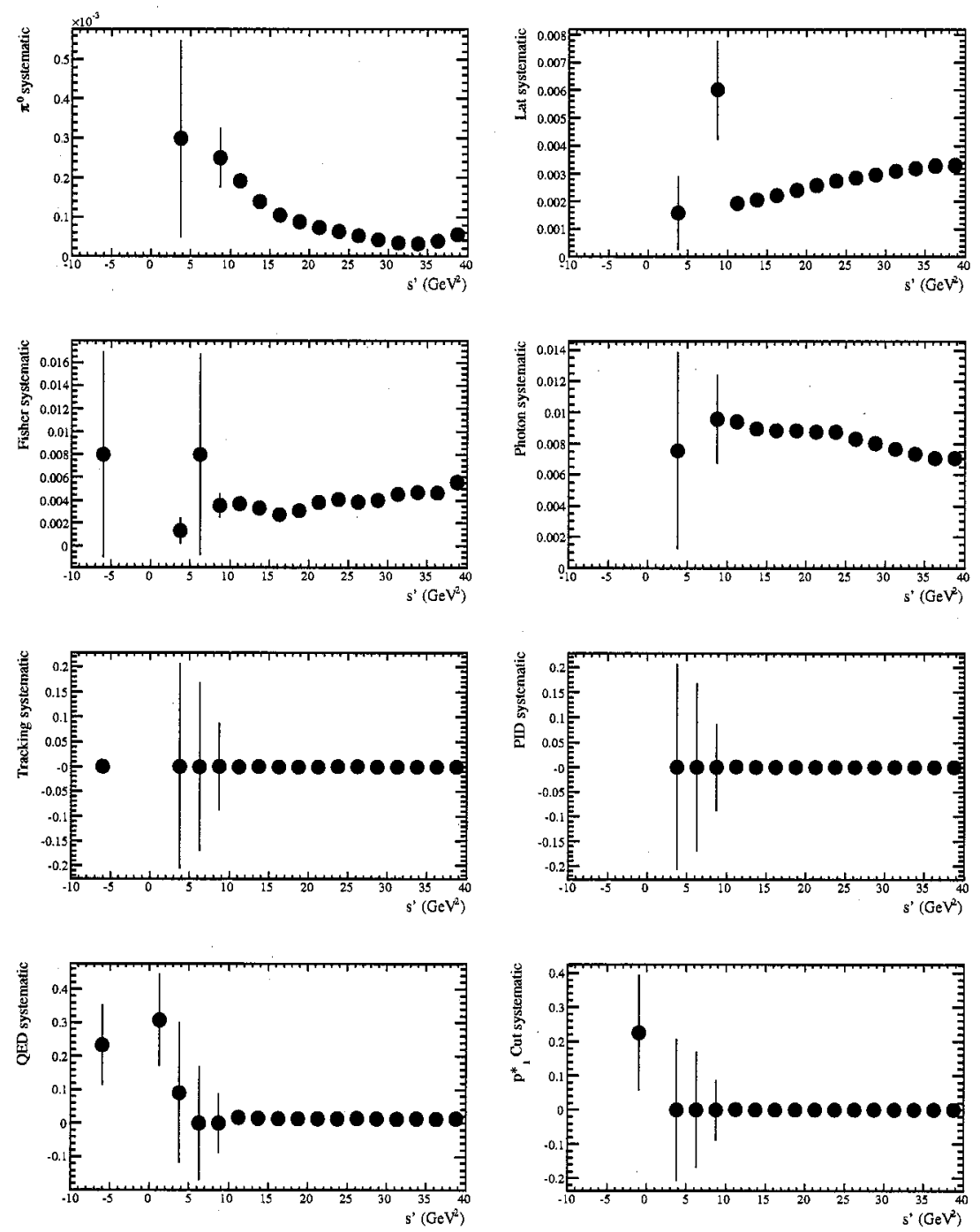

Figure 11.8: Systematic uncertainties versus $s^{\prime}$ for $\tau^{+} \tau^{-} \gamma$ signal. From top to bottom, the quantities are: Left column: $h \rightarrow n \gamma$ Veto, Event Shape, Tracking and QED systematics Right Column: Lateral Moment, Photon, PID and $p_{1}^{*}$ cut systematics 
Table 11.1: Systematic errors on the signal efficiency.

\begin{tabular}{lccc}
\hline \hline & $q \bar{q} \gamma$ & $\mu^{+} \mu^{-} \gamma$ & $\tau^{+} \tau^{-} \gamma$ \\
\hline MC Generator & 0.13 & 0.18 & 0.55 \\
$\gamma$ efficiency & 0.81 & 0.80 & 0.80 \\
$h \rightarrow n \gamma$ veto & 0.02 & 0.02 & 0.01 \\
Lateral moment & 0.28 & 0.29 & 0.29 \\
Event Shape & 0.35 & 0.23 & 0.42 \\
Tracking Eff. & 0.01 & 0.02 & 0.01 \\
PID & 0.00 & 0.00 & 0.01 \\
QED veto & 1.42 & 2.16 & 1.35 \\
$p_{1}^{*}$ Cut & 0.09 & 1.05 & 0.00 \\
Hot Tower Veto & 0.00 & 0.00 & 0.00 \\
MC Statistics & 0.02 & 0.12 & 0.10 \\
Total & 1.70 & 2.57 & 1.75 \\
\hline \hline
\end{tabular}

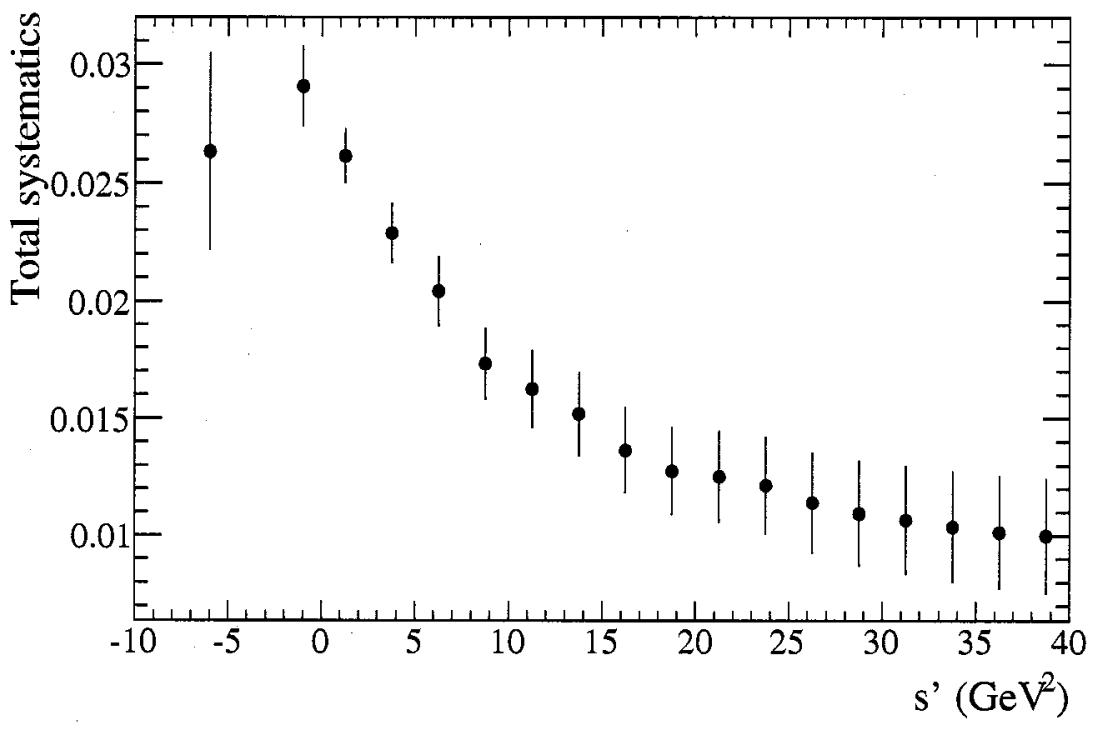

Figure 11.9: Total systematic uncertainties versus $s^{\prime}$ for $q \bar{q} \gamma$ signal. 


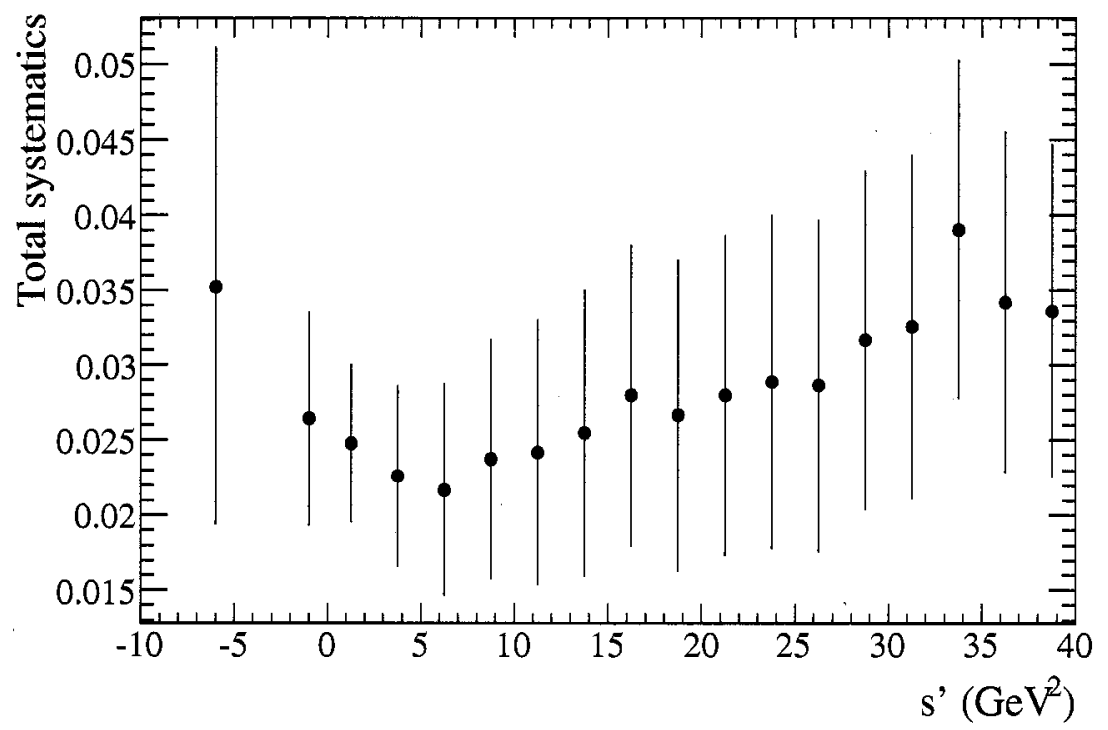

Figure 11.10: Total systematic uncertainties versus $s^{\prime}$ for $\mu^{+} \mu^{-} \gamma$ signal, as fractions of the $\mu^{+} \mu^{-} \gamma$ yield.

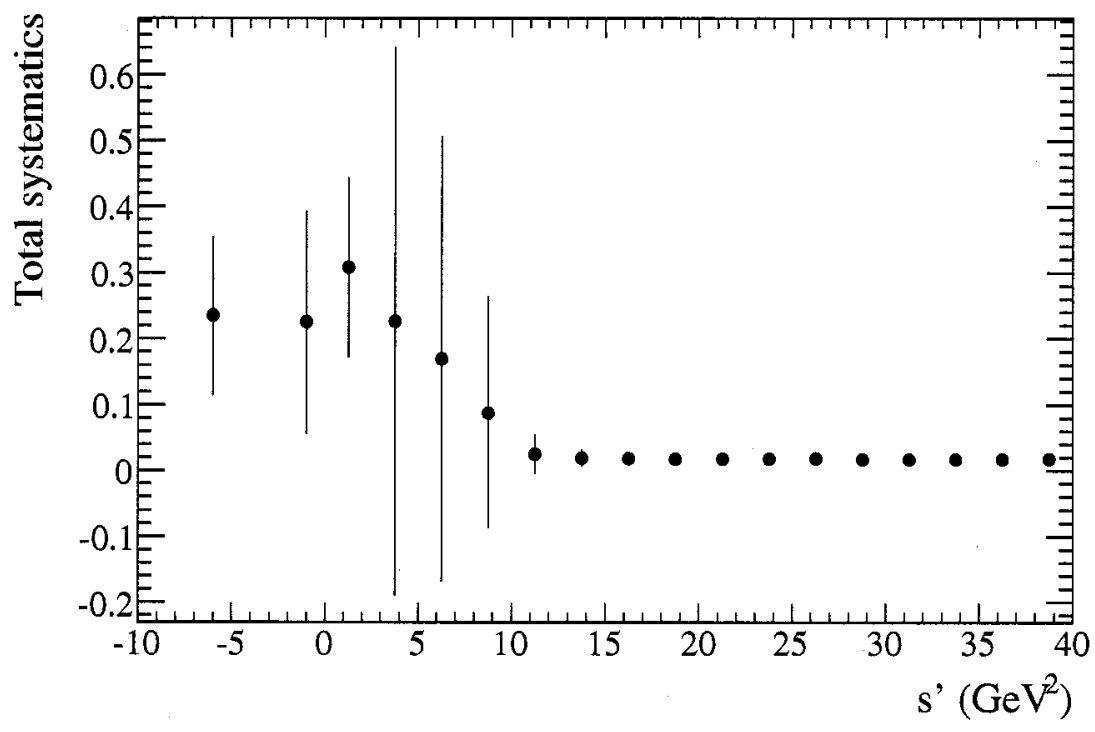

Figure 11.11: Total systematic uncertainties versus $s^{\prime}$ for $\tau^{+} \tau^{-} \gamma$ signal, as fractions of the $\tau^{+} \tau^{-} \gamma$ yield. 


\subsection{Systematic Errors on Background Levels}

The following source of uncertainty are considered for all background samples:

- Production systematics: In the case where a weight is applied to background to correct for background levels (such as $\pi^{0}$ production in continuum, antineutron energy deposition correction, etc.), the systematic error is taken to be half the correction. For $K_{L}^{0}$ and antineutron events, a fixed systematic of $20 \%$ is also added in quadrature to account for the uncertainty in the production crosssection.

- Lateral Shape: As for signal, the error associated with the lateral shape cut is taken to be half the magnitude of the correction determined in Chapter 9.

- Fisher shape: As for signal, the error associated with the Fisher cut is taken to be half the magnitude of the correction determined in Section 9.6.

- Photon Correction: As for signal, events with a real photon not originating from an already-corrected-for process ( $\operatorname{such}$ as $\pi^{0}$ ) have a systematic correction associated with the photon efficiency correction, equal to half the correction value of the correction determined in Section 9.6 as before.

- MC Statistics: The statistical error on the final efficiency in each bin is counted as a systematic error.

In addition, the following errors associated with specific backgrounds are considered:

- $\Upsilon$ decays: This background is estimated at $0.30 \%$ in Section 9.12. A systematic error of $0.15 \%$ is assigned to this value.

- Stale Bhabhas: In Section 9.9, the value $0.30 \pm 0.15 \%$ we obtained for the fraction of this background in the final sample. A $0.15 \%$ systematic error is therefore counted. 
Table 11.2: Values of fixed systematics applied to all $s^{\prime}$ bins.

\begin{tabular}{lc}
\hline \hline Name & Value \\
\hline Integrated Luminosity & $1.1 \%$ \\
$\Upsilon$ decays & $0.15 \%$ \\
Stale Bhabha events & $0.15 \%$ \\
Two-photon events & $2.20 \%$ \\
\hline \hline
\end{tabular}

Table 11.3: Systematic errors on background.

\begin{tabular}{lcccccc}
\hline \hline & $\mathrm{uds}$ & $c \bar{c}$ & $\tau^{+} \tau^{-}$ & $B \bar{B}$ & $\gamma \gamma$ & $e^{+} e^{-} \gamma$ \\
\hline Production & 4.75 & 0.00 & 8.69 & 0.00 & 60.00 & 28.36 \\
$\gamma$ efficiency & 0.00 & 0.00 & 0.00 & 0.00 & 0.75 & 0.81 \\
Lateral moment & 2.61 & 1.54 & 2.33 & 0.00 & 0.00 & 0.00 \\
Fisher & 8.44 & 10.94 & 2.50 & 0.00 & 0.00 & 0.00 \\
MC Statistics & 0.68 & 2.68 & 0.57 & 5.68 & 70.71 & 24.25 \\
Total & 10.06 & 11.36 & 9.36 & 5.68 & 92.74 & 37.33 \\
\hline \hline
\end{tabular}

- Two-Photon physics: The fraction of these events is estimated at $0.8 \%$ in Section 9.13. We again use half this number as systematic uncertainty.

- Residual $e^{+} e^{-} \gamma$ : In Section 9.10 a reweighting procedure is defined to account for residual $e^{+} e^{-} \gamma$ contamination. We use half the difference between the reweighted sample and the original sample as an error on this value.

- Residual $\gamma \gamma$ : As discussed in Section 9.11, the systematic error is set at $0.26 \%$

The last two are counted as production systematics on the $\gamma \gamma$ and $e^{+} e^{-} \gamma$ samples. The fixed systematics values are summarized in Table 11.2. The average values of the errors over the $s^{\prime}$ range are shown in Table 11.3. The $s^{\prime}$ variations of relevant systematic errors are shown for the various background samples in Figs. 11.12-11.15. 

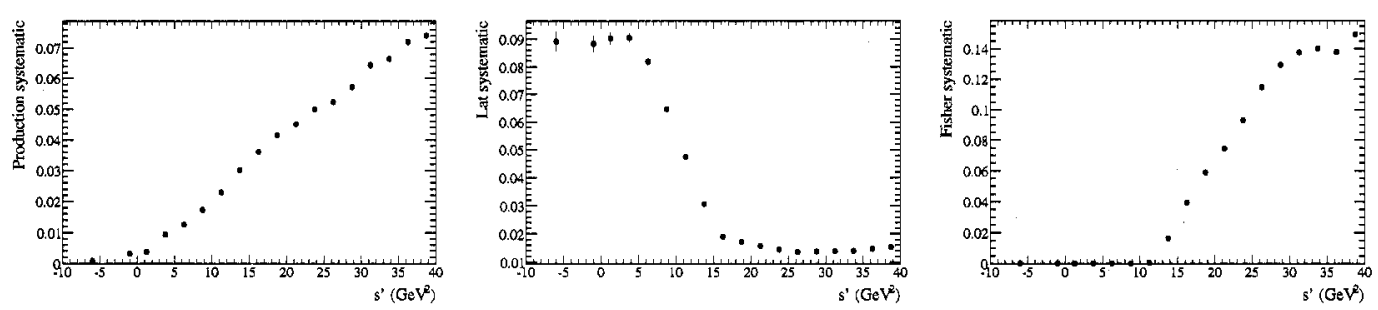

Figure 11.12: Systematic uncertainties versus $s^{\prime}$ for $u d s \mathrm{MC}$, as fractions of the $u d s$ yield. From left to right, the production, lateral moment and event shape systematics are shown
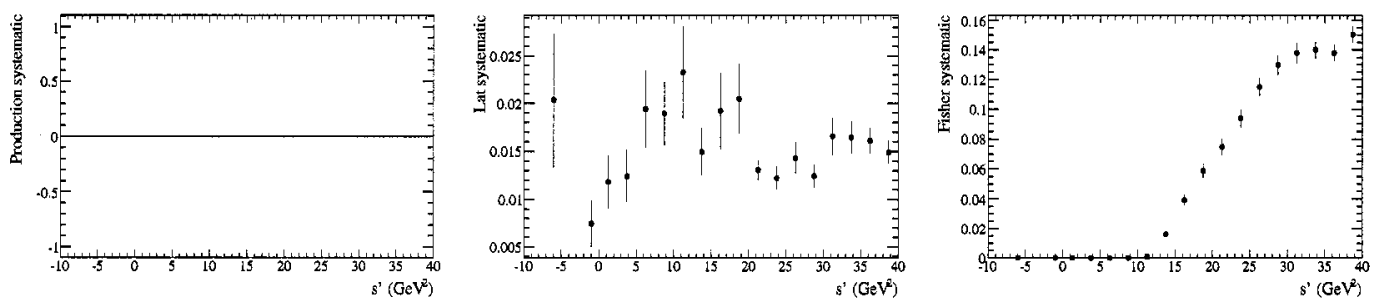

Figure 11.13: Systematic uncertainties versus $s^{\prime}$ for $\bar{c} \bar{c} \mathrm{MC}$, as fractions of the $c \bar{c}$ yield. From left to right, the production, lateral moment and event shape systematics are shown.
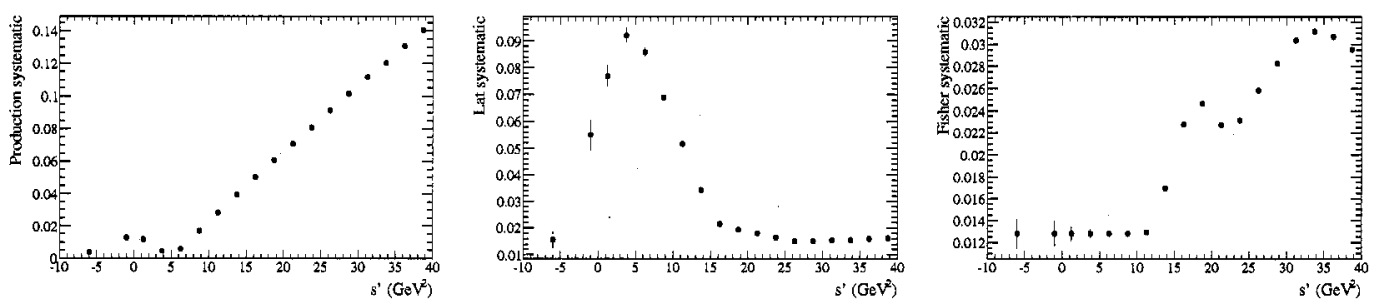

Figure 11.14: Systematic uncertainties versus $s^{\prime}$ for $\tau^{+} \tau^{-} \mathrm{MC}$, as fractions of the $\tau^{+} \tau^{-}$yield. From left to right, the production, lateral moment and event shape systematics are shown. 


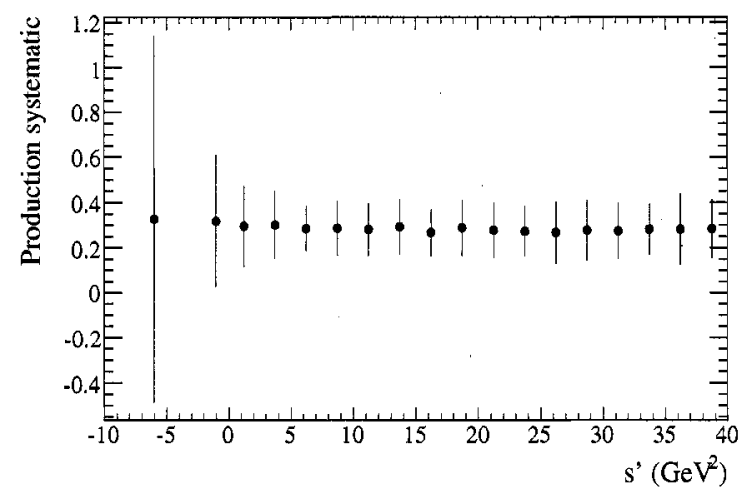

Figure 11.15: Production systematic versus $s^{\prime}$ for radiative Bhabha MC, as fractions of the $e^{+} e^{-} \gamma$ yield.

\subsection{Final Spectrum}

Figs. 11.16 and 11.17 show the $\mathrm{MC}$ background components, scaled to the data integrated luminosity, and Fig. 11.18 shows the same for the $\mu^{+} \mu^{-} \gamma$ and $\tau^{+} \tau^{-} \gamma$ modes.

The raw data spectrum before subtraction is given in Fig. 11.19. The backgroundsubtracted spectrum before efficiency correction is shown in Fig. 11.20. After correction by the efficiency function, we obtain the shape shown in Fig. 11.21. The error bars are include systematic and statistical errors, although the latter are negligible. Table 11.4 shows a breakdown of the systematic error by source. For MC samples, the systematic is taken to be the average of the systematics in each $s^{\prime}$ bins weighted by the shape of final spectrum, and divided by the signal efficiency.

A leading source of systematic uncertainty is the efficiency of the QED vetos, which dominates both the signal efficiency and the $\mu^{+} \mu^{-} \gamma$ yield. The radiative Bhabha yield is another leading source, with the uncertainty originating in the rescaling of Section 9.10. The overall error is $3.3 \%$, with a value that is fairly constant across the $s^{\prime}$ spectrum although it increases slightly at the high- $s^{\prime}$ end. 

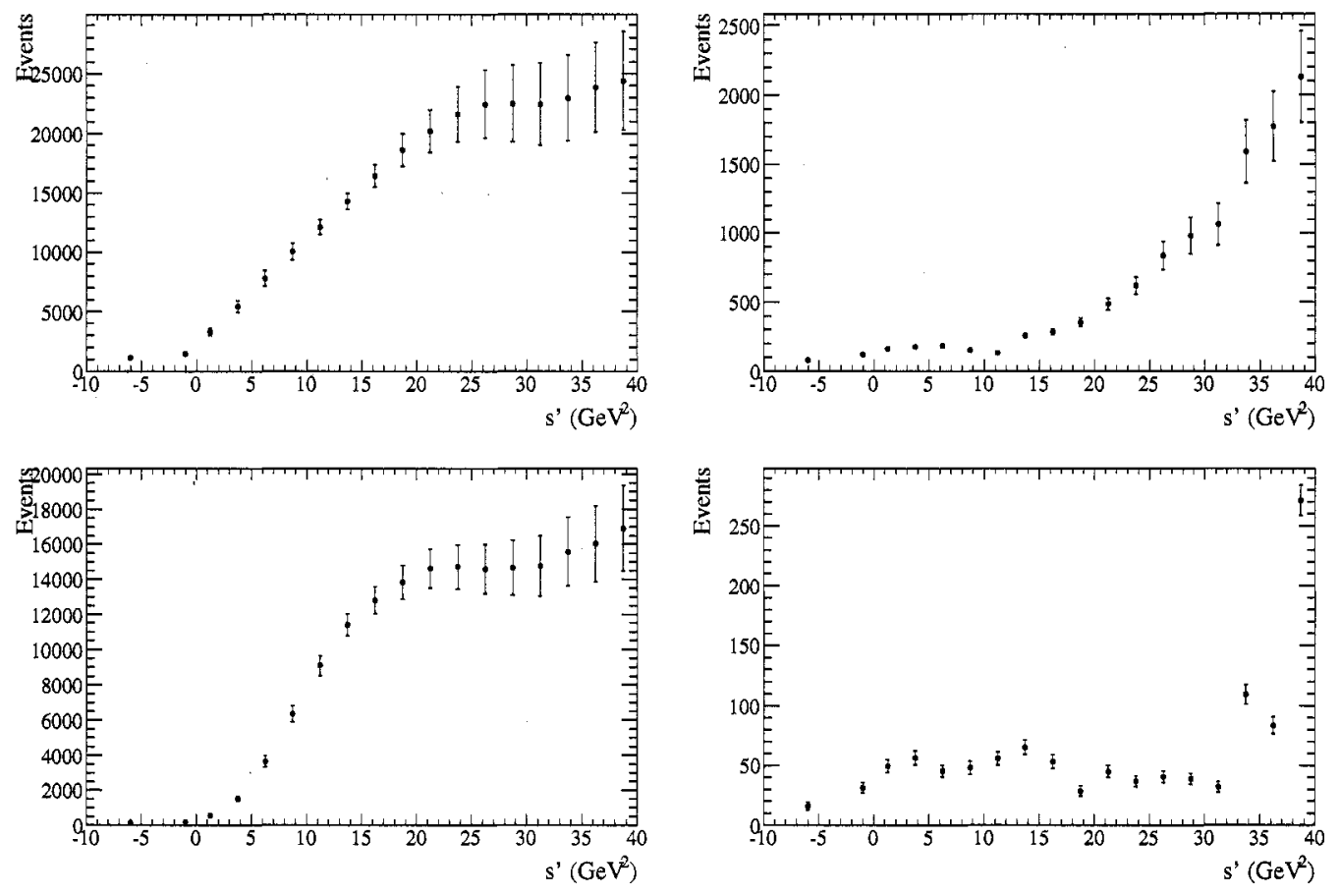

Figure 11.16: MC spectra subtracted from the data, with both systematic and statistical errors, for $u d s$ (top left), $c \bar{c}$ (top right), $\tau^{+} \tau^{-}$(bottom left) and $B \bar{B}$ (bottom right) samples.
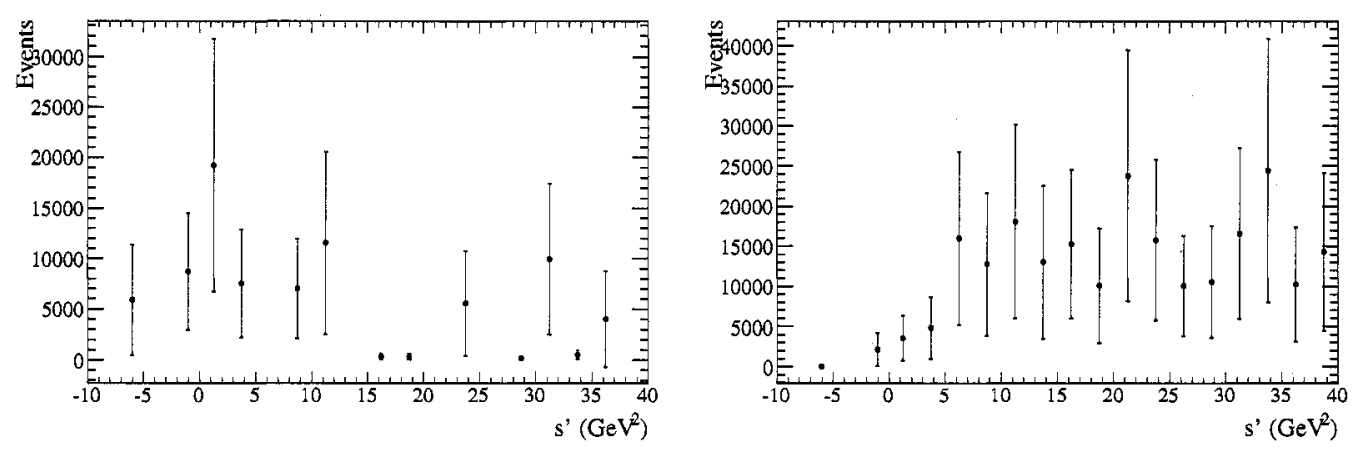

Figure 11.17: MC spectra subtracted from the data, with both systematic and statistical errors, for $\gamma \gamma$ (left) and $e^{+} e^{-} \gamma$ (right) samples. 

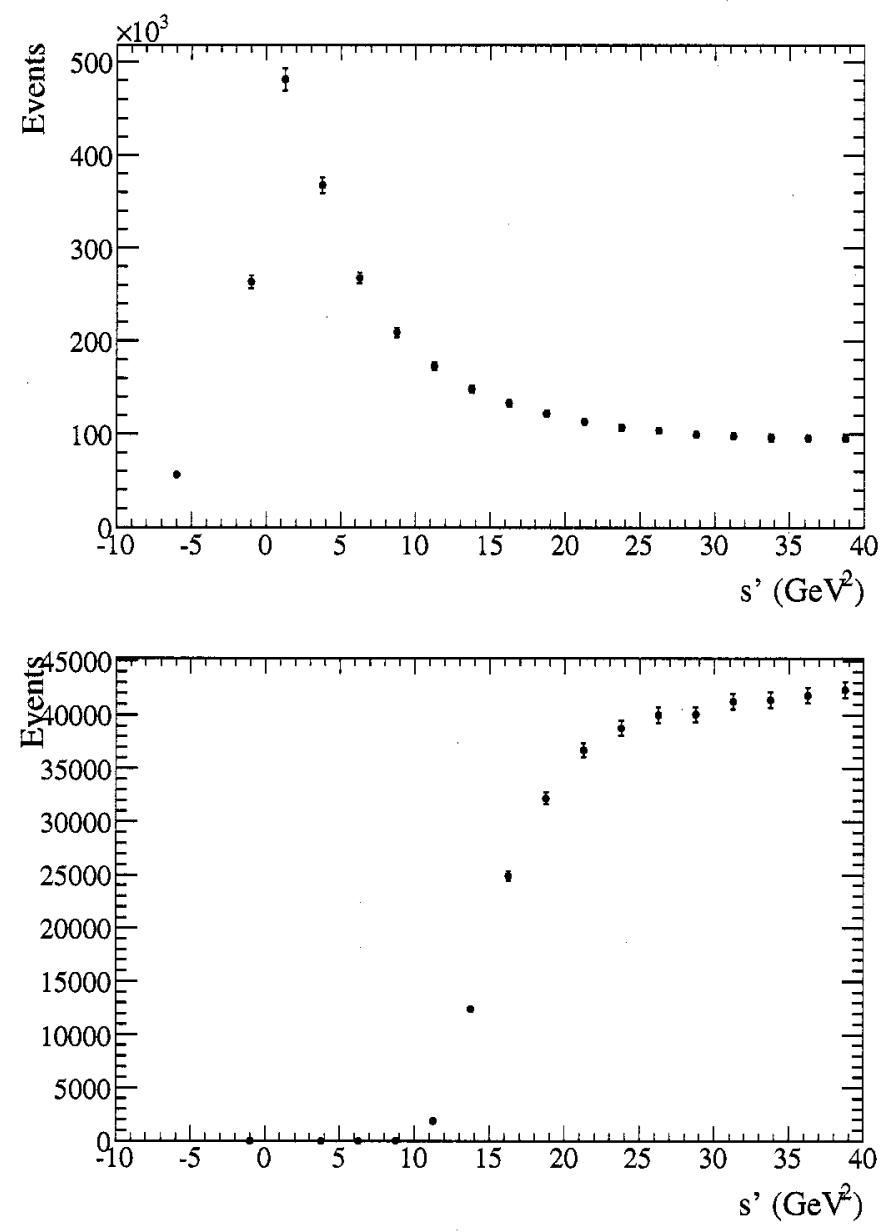

Figure 11.18: MC spectra subtracted from the data, with both systematic and statistical errors, for $\mu^{+} \mu^{-} \gamma$ (top) and $\tau^{+} \tau^{-} \gamma$ (bottom) samples. 


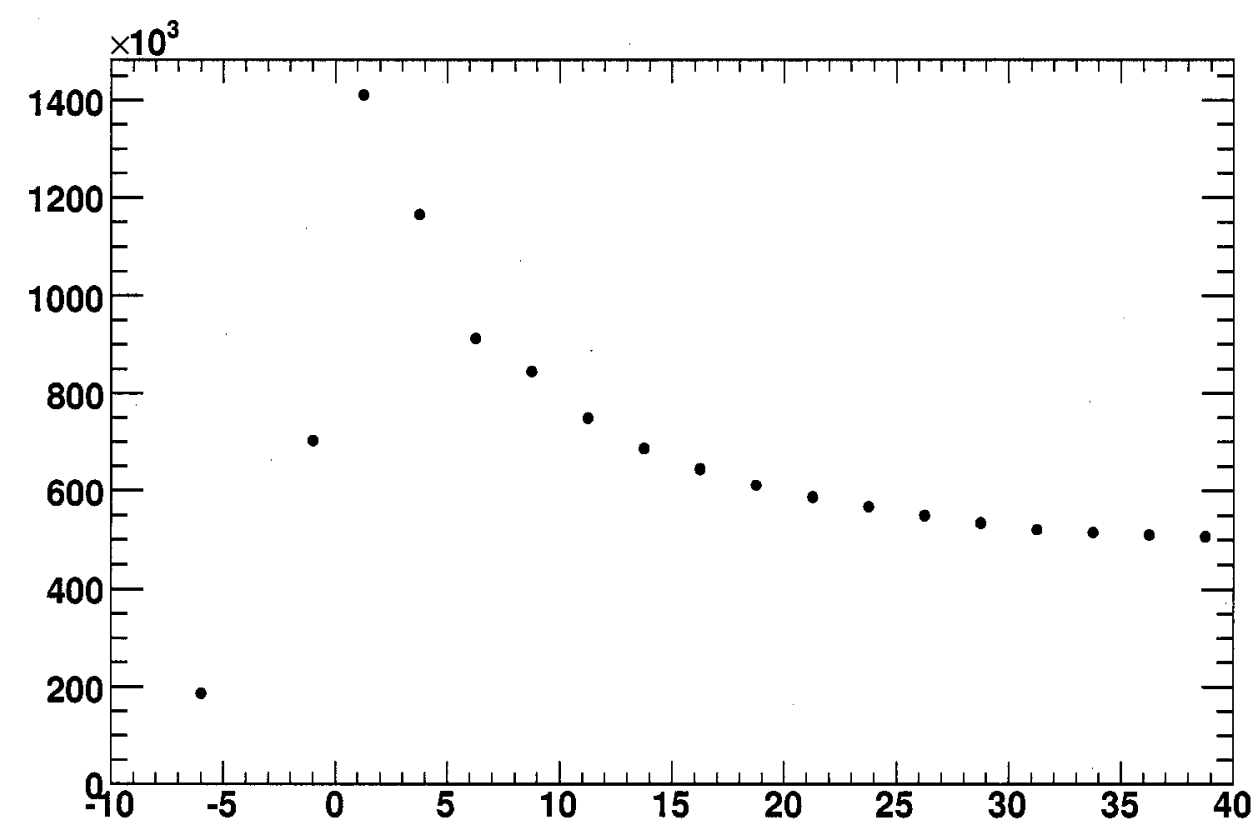

Figure 11.19: Raw data spectrum before background subtraction.

Table 11.4: Breakdown of total systematics by source (\%).

\begin{tabular}{ll}
\hline \hline Data statistics & 0.18 \\
\hline$\mu^{+} \mu^{-} \gamma$ & 1.01 \\
$\tau^{+} \tau^{-} \gamma$ & 0.09 \\
$u d s$ & 0.40 \\
$c \bar{c}$ & 0.02 \\
$\tau^{+} \tau^{-}$ & 0.23 \\
$B \bar{B}$ & 0.00 \\
$\gamma \gamma$ & 0.78 \\
$e^{+} e^{-} \gamma$ & 1.88 \\
Fixed & 2.29 \\
Efficiency & 1.70 \\
Total & 3.99 \\
\hline \hline
\end{tabular}




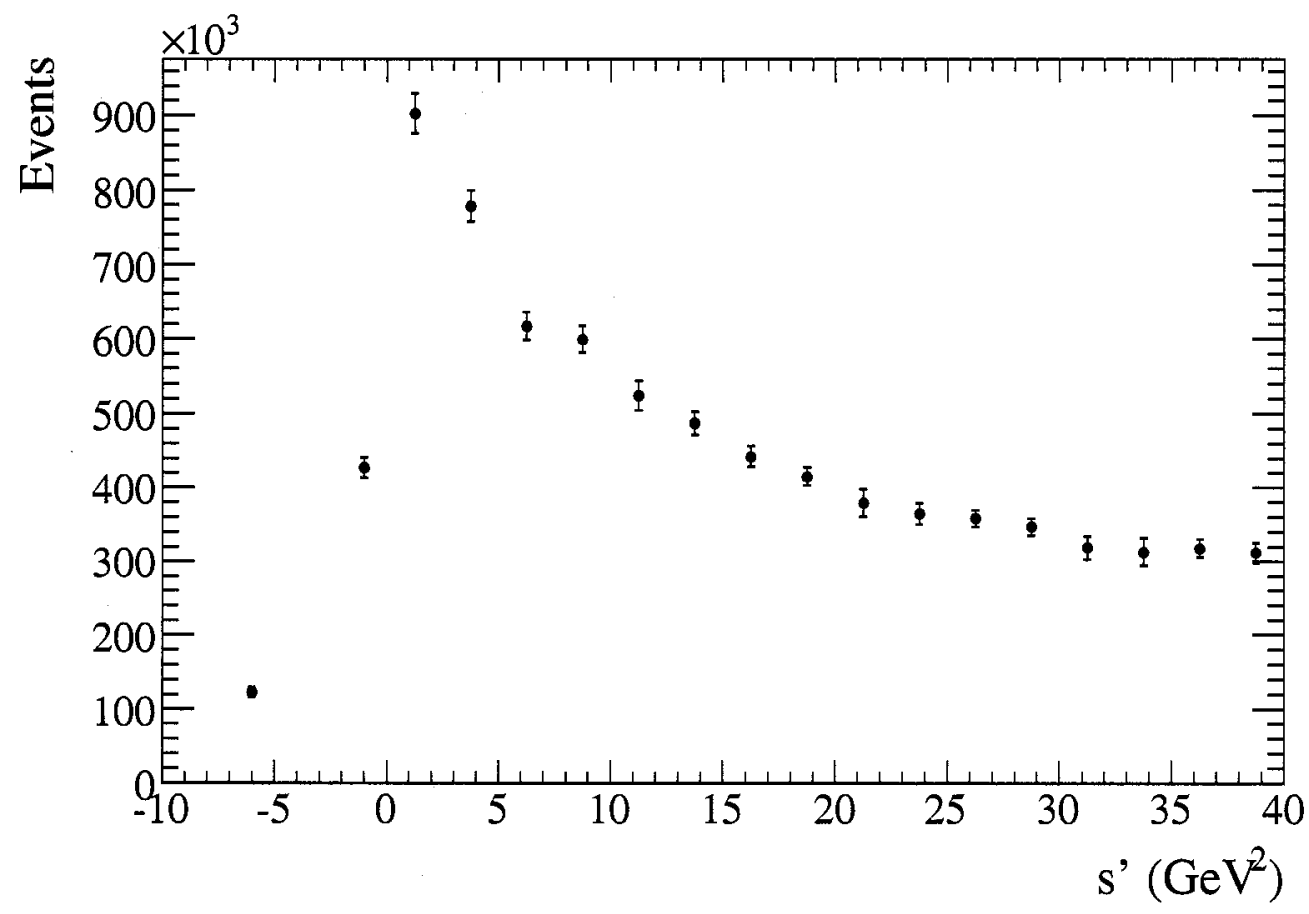

Figure 11.20: Final spectrum after background subtraction with no efficiency correction. 


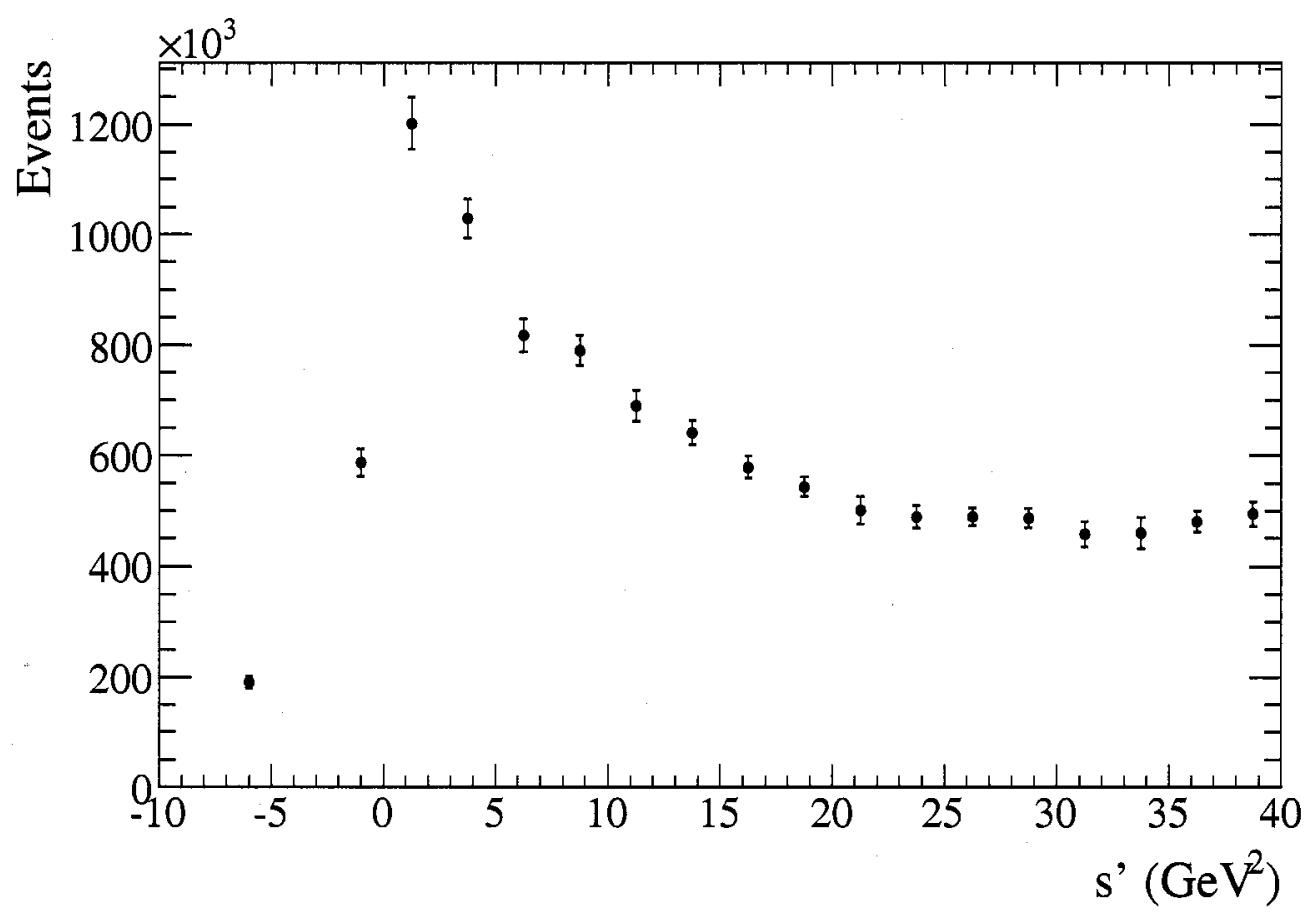

Figure 11.21: Final spectrum after background subtraction and efficiency correction. 


\section{Chapter 12}

\section{Summary and Outlook}

The total cross-section of $e^{+} e^{-} \rightarrow$ hadrons in the region $s^{\prime}<40 \mathrm{GeV}^{2}$ has been measured with a precision of $3.3 \%$ using the ISR technique. The error is dominated by uncertainties on the efficiency of the QED event rejection on signal, and by the amount of radiative Bhabha and two-photon collision background.

Although it is not done in this document, the primary purpose of this measurement is to extract a measurement of $\Delta \alpha_{\text {had }}^{(5)}\left(m_{Z}^{2}\right)$ by computing an integral of the spectrum given in Fig. 11.21, as described in Section 2.3.2.

The weight factor in the integral can be obtained from event generators such as KKMC and PHOKHARA (see Appendix A), with an expected error below 1\%. Since this factor does not vary significantly with $s^{\prime}$, the error on the integral is given to a good approximation by the sum in quadrature of this $1 \%$ error and the $3.3 \%$ error on the $s^{\prime}$ spectrum.

This total error of $3.4 \%$ on the contribution to the $\Delta \alpha_{\text {had }}^{(5)}\left(m_{Z}^{2}\right)$ integral from the region $s^{\prime}<40 \mathrm{GeV}^{2}$ is comparable to the $2.8 \%$ error given in Table 2.3.5 for the same range, which combines the results of many experiments accumulated over a period of 35 years.

Combined with the contributions to $\Delta \alpha_{h a d}^{(5)}\left(m_{Z}^{2}\right)$ from the interval $s^{\prime}>40 \mathrm{GeV}^{2}$, the result described in this work would lead to a precision of $1.6 \%$ on the final value, comparable to the $1.3 \%$ precision of existing measurements. Since the leading sources of systematic uncertainties for this measurement are well-known, further studies are 
likely to lead to lower errors, which may further improve the precision of this technique compared to existing determinations.

However even in its current form the result provides interesting information. Since the systematic errors are of a different nature than those of existing determinations, this result provides a completely independent measurement of the value of $\Delta \alpha_{h a d}^{(5)}\left(m_{Z}^{2}\right)$. By providing a single measurement over the $s^{\prime}<40 \mathrm{GeV}^{2}$, it also avoids the issues associated with the combination of multiple measurements. This result is also complementary to previous measurements in the sense that it is more precise in the region $1<s^{\prime}<40 \mathrm{GeV}^{2}$, whereas it is at present the region $s^{\prime}<1 \mathrm{GeV}^{2}$ that has the smallest errors.

Finally, since the uncertainties associated with this result have little correlation with those of existing measurements a combination could be performed, yielding an error of $2.2 \%$ over $s^{\prime}<40 \mathrm{GeV}^{2}$ and $1.0 \%$ over the entire range.

With the possibility of further improvements to this value, the inclusive ISR technique described in this work therefore offers promising prospects for the measurement of $\Delta \alpha_{h a d}^{(5)}\left(m_{Z}^{2}\right)$. 


\section{Appendix A}

\section{Generation of $e^{+} e^{-} \rightarrow q \bar{q} \gamma$ with the KKMC event generator}

\section{A.1 ISR emission model}

KKMC is a generic $e^{+} e^{-} \rightarrow \bar{f} f(\gamma)$ generator, where $f$ is an arbitrary lepton or quark and an arbitrary number (up to five) of photons can be produced, either as ISR or FSR. It was developed mainly to deal with radiative effects at the $\mathrm{Z}$ pole, but it provides a very complete description of radiative effects which is also valid at $\mathrm{BaBar}$ energies.

The radiative effects are described exactly up to a fixed order, and radiation at higher order is added in the soft/collinear (leading logarithm) approximation. For leptons

KKMC can generate up to 2 photons can be generated exactly (without soft/collinear approximations) in the case of lepton production,

Up to three radiative photons are emitted, and the hadronization of the $q \bar{q}$ system is handled by a dedicated package, RRes. The generator is described in detail in appendix A.

In the case of lepton production, up to 2 photons are generated exactly, and up to 3 more in the leading-logarithm approximation. Each photon can be emitted either in the initial state or the final state, and the ISR/FSR interference term is also taken 
Table A.1: Resonance parameters used in KKMC.

\begin{tabular}{lcccc}
\hline \hline Resonance & mass $(\mathrm{MeV})$ & $\Gamma(\mathrm{MeV})$ & $\Gamma_{\epsilon e}(\mathrm{MeV})$ & Parametrization \\
\hline$\rho$ & 772.6 & 143.7 & $\mathrm{n} / \mathrm{a}$ & Kuehn-Santamaria \\
$\omega$ & 782.78 & 8.68 & $\mathrm{n} / \mathrm{a}$ & Kuehn-Santamaria \\
$\rho^{\prime}$ & 1460 & 310 & $\mathrm{n} / \mathrm{a}$ & Kuehn-Santamaria \\
\hline \hline$\phi$ & 1019.42 & 4.46 & 1.33 & Non-relativistic Breit-Wigner \\
\hline \hline$\psi$ & 3096.87 & 0.087 & 5.26 & Non-relativistic Breit-Wigner \\
$\psi(2 S)$ & 3685.96 & 0.277 & 2.12 & Non-relativistic Breit-Wigner \\
$\psi(3770)$ & 3769.9 & 23.6 & 0.26 & Non-relativistic Breit-Wigner \\
$\psi(4040)$ & 4040 & 52 & 0.75 & Non-relativistic Breit-Wigner \\
$\psi(4160)$ & 4159 & 78 & 0.77 & Non-relativistic Breit-Wigner \\
$\psi(4215)$ & 4415 & 43 & 0.47 & Non-relativistic Breit-Wigner \\
\hline \hline
\end{tabular}

into account at leading-log.

\section{A.2 $R_{\text {had }}$ parameterization}

In the case of quarks, one photon is generated exactly and up to 3 more at leading$\log$, and only ISR is simulated. For quarks, the energy spectrum is simulated using a parameterization of $R_{\text {had }}$ which includes the parton-level continuum terms and the $\omega, \phi, \psi, \psi(2 S), \psi(3770), \psi(4040), \psi(4160)$ and $\psi(4415)$ resonances, as well as $\Upsilon$ resonances which are not used here. The corresponding parameters are shown in Table A.1.

Fig. A.1 shows the $\mathrm{R}$ variations at low $s^{\prime}$ and near the $c \bar{c}$ threshold.

Compared to the recent BES measurements of $R_{\text {had }}$ near the the charmonium threshold [4], the resonances above the $\psi(2 S)$ seem too narrow and too high, and fail to account for the dip in $R_{\text {had }}$ near $\sqrt{s^{\prime}}=4.25 \mathrm{GeV}$. This is the cause of a significant discrepancy between $\mathrm{MC}$ and data in this region.

The pion form factor is described using the Kühn-Santamaria parametrization [79]:

$$
F_{\pi \pi}=\left|\frac{1}{1+\beta}\left[\tilde{\mathcal{B}}_{\rho} \frac{1+\alpha \tilde{\mathcal{B}}_{\omega}}{1+\alpha}+\beta \mathcal{B}_{\rho^{\prime}}\right]\right|^{2}
$$



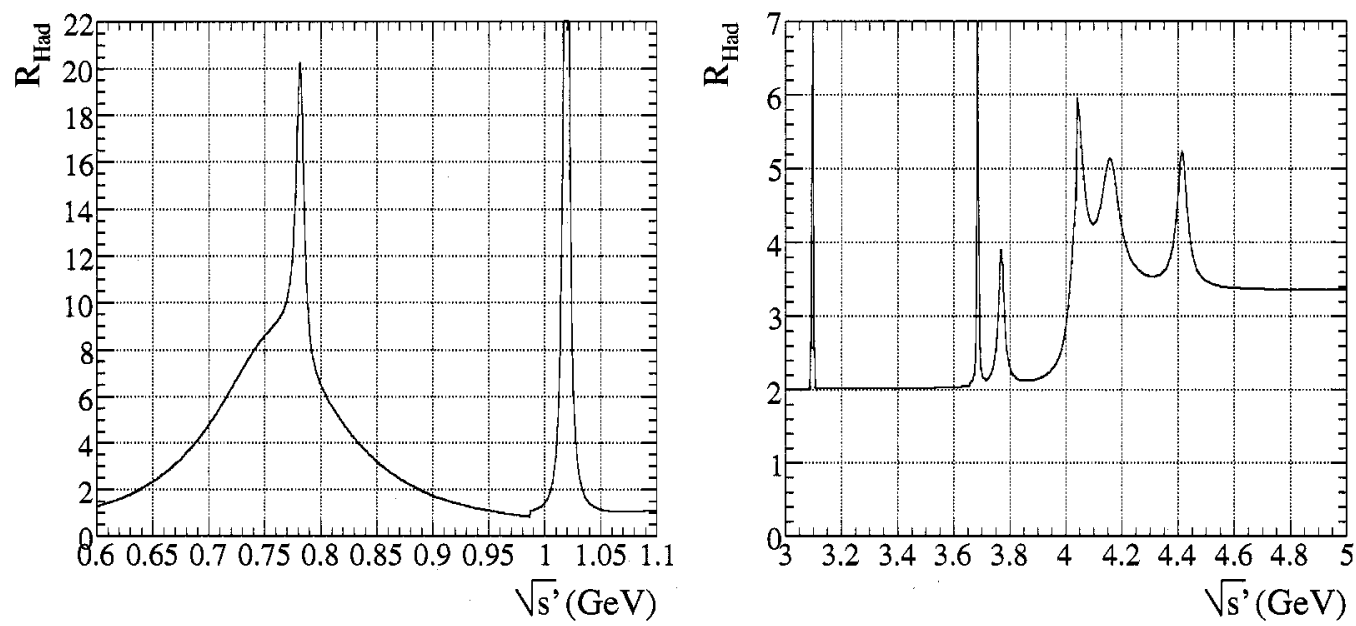

Figure A.1: $R$ function at low $s^{\prime}$ (left) and near the $c \bar{c}$ threshold (right).

where $\mathcal{B}$ refers to a standard non-relativistic Breit-Wigner shape

$$
\mathcal{B}(s, m, \Gamma)=\frac{\Gamma^{2}}{m^{2}-s-i \sqrt{s} \Gamma}
$$

and $\tilde{\mathcal{B}}$ is the relativistic $\mathrm{P}$-wave form given by

$$
\tilde{\mathcal{B}}(m, \Gamma)=\frac{\Gamma^{2}}{m^{2}-s-i \sqrt{s} \Gamma(s)}, \Gamma(s)=\Gamma \frac{m^{2}}{s}\left(\frac{s-4 m_{\pi}^{2}}{m^{2}-4 m_{\pi}^{2}}\right)^{3 / 2}
$$

The values for the masses and widths of $\rho, \omega$ and $\rho^{\prime}$ are given in Table A.1, and for the coefficients the values $\alpha=1.48 \times 10^{-3}$ and $\beta=0.1473$ are used. These values are taken from the fit to the KLOE data reported in Ref. [80]. The form factor is shown in Fig. A.2.

The threshold function $2 /\left(1+e^{-3(s-1)}-1\right.$ is used to model the variations of $R_{\text {had }}$ just above the $\phi$.

The model does not include $\alpha_{s}$ correction to $R_{\text {Had }}$, but these are added by reweighting the continuum events as described in section 2.2.5. The overall shape of the $R$ parametrization is shown in Fig. A.3 


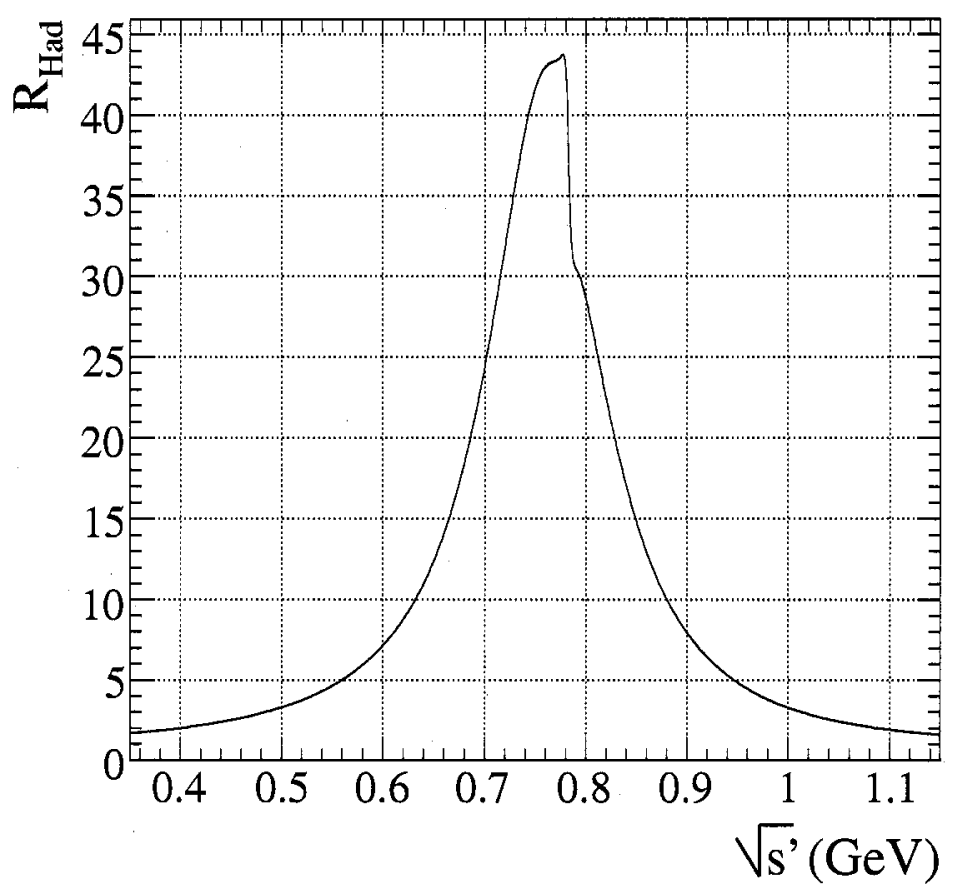

Figure A.2: Pion form-factor parametrization used in KKMC

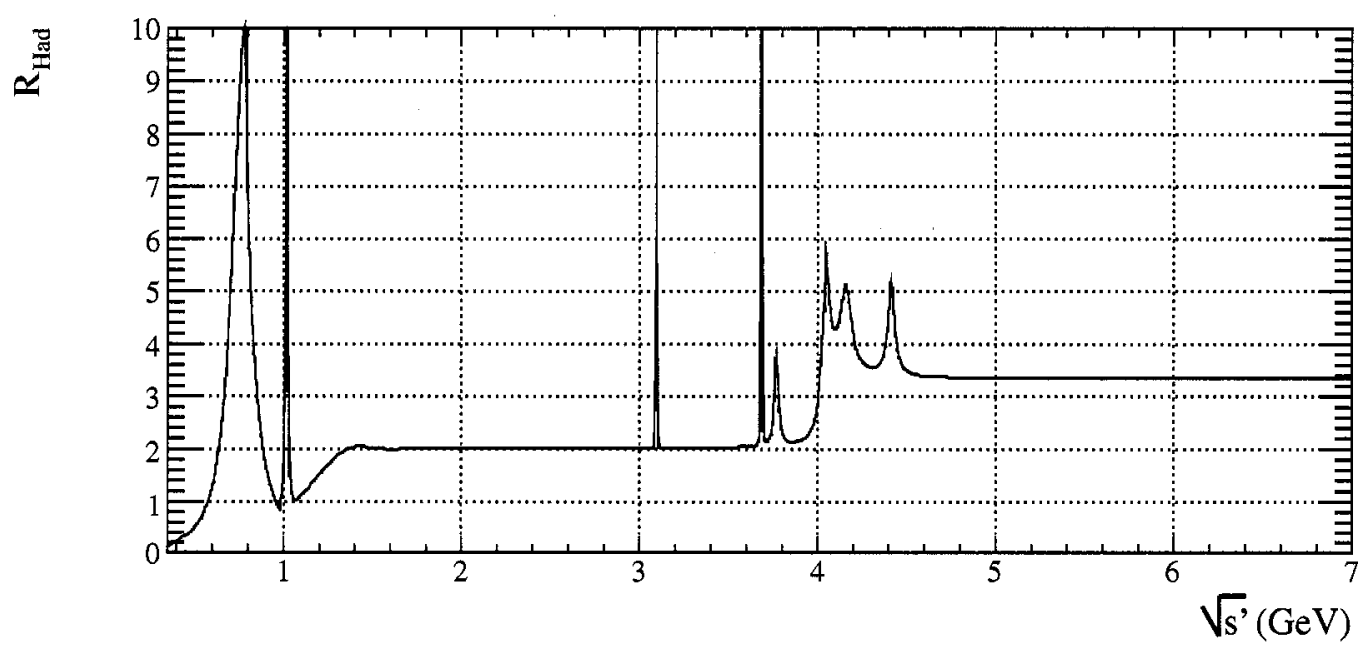

Figure A.3: $\mathrm{R}$ parametrization used in KKMC 


\section{A.3 Hadronization}

In the case of quarks the hadronization is handled by the RRes package, which implements 3 algorithms:

- If the generated $s^{\prime}$ is in the vicinity of the $\rho^{0}, \omega, \phi, \psi$ or $\Upsilon$ resonance, the generator may choose to produce the resonance based on the value of the to peak/continuum ratio at the given energy. The resonance is then passed to JETSET to decay. However this is imprecise since JETSET does not take polarization into account. To improve precision, the $\rho^{0} \rightarrow \pi^{+} \pi^{+}$decay is therefore implemented "by hand" in the generator.

- For $\sqrt{s^{\prime}}>2 \mathrm{GeV}$, the PYTHIA $e^{+} e^{-}$fragmentation routines are used.

- Below $2 \mathrm{GeV}$, the program uses cross-sections parameterized as a function of $\sqrt{s^{\prime}}$ to choose a final state. The modes taken into account are $\pi^{+} \pi^{-} \pi^{0}\left(\pi^{0}\right)$, $\pi^{+} \pi^{-} \pi^{+} \pi^{-}\left(\pi^{0}\right)\left(\pi^{0}\right), 3\left(\pi^{+} \pi^{-}\right), K^{+} K^{-}, K_{S}^{0} K_{L}^{0}, K^{0} K^{p m} \pi^{\mp}$ and $K^{+} K^{-} \pi^{+} \pi^{-}$.

While RRes provides a reasonably accurate description of the $q \bar{q}$ system, there are several problems:

- the cross-section parameterizations disagree strongly with the spectra recently measured by $\mathrm{BaBar}[9]$, and the cross-sections are discontinuous at $2 \mathrm{GeV}$ where PYTHIA kicks in, a shown in Fig. A.4.

- Charmonium resonances below the $c \bar{c}$ threshold are incorrectly simulated: both the $\psi$ and $\psi(2 S)$ peaks are handed to PYTHIA as $\psi$ 's, so that the $\psi(2 S)$ decays are incorrectly simulated (although its mass is correct), while the $\psi(3770)$ is completely absent. However (perhaps to compensate ?), a significant fraction of events below $c \bar{c}$ threshold hadronize to a single $\eta_{c}$, which is forbidden by Cparity. The root cause is the hadronization mechanism used: for a given event, the generator chooses randomly between resonance and continuum production using the relative contribution of each to $R$ at this $s^{\prime}$ value and for the generator quark flavor. For $c \bar{c}$ the only resonance used is the $\psi$, and below $c \bar{c}$ threshold, 


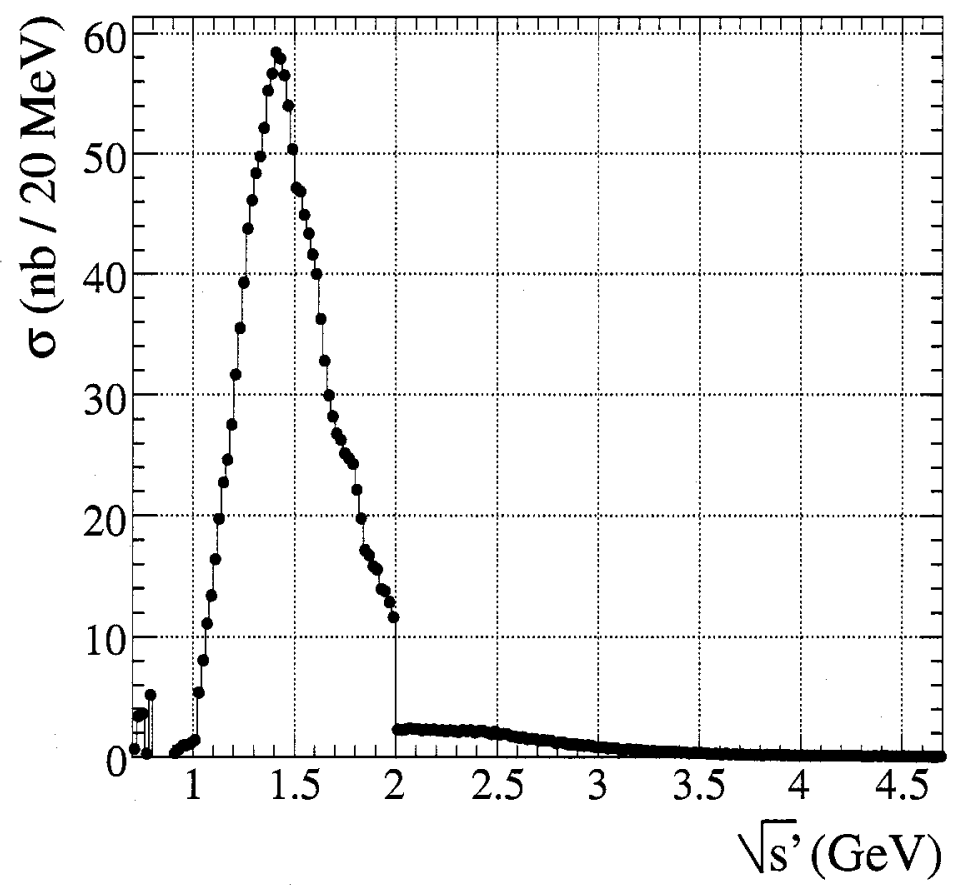

Figure A.4: Cross-section for $e^{+} e^{-} \rightarrow \pi^{+} \pi^{-} \pi^{+} \pi^{-}$used in KKMC. 
where the continuum contribution is zero, any event is automatically counted as $\psi$ since the tails of the $\psi$, however small, are still bigger than the non-existent continuum. This explains why events generated at the $\psi(2 S)$ mass are still counted as $\psi$. For the $\psi(3770)$ and above, which are above $c \bar{c}$ threshold, the events are classified as continuum by KKMC and handed to PYTHIA, which apparently favors the $\eta_{c}$ over the $\psi(3770)$ (spin is not used in PYTHIA). PYTHIA also seems to manipulate the kinematics to produce on-shell $\eta_{c}$ in spite of the fact that these events were originally generated above the $c \bar{c}$ threshold.

- There are significant $s^{\prime}$ binning issues in the narrow resonance peaks, such as $\psi$ and $\psi(2 S)$. Also, the $\rho^{0} \rightarrow \pi^{+} \pi^{-}$mode seems to extend to to relatively high $s^{\prime}$, but also with significant binning effects.

- A curious structure is visible in the $s^{\prime}$ spectrum at $\sqrt{s^{\prime}}=6 \mathrm{GeV}$, for example in Fig. A.5. It is unclear what can be causing it, but the scale of the variations in $s^{\prime}$ is too small for it to matter in this analysis.

It is not entirely clear whether these "features" are due to KKMC itself or to its embedding in the BABARgenerator framework. Due to the inclusiveness of our selection these problems have only a limited impact on the analysis and will not be investigated further.

\section{A.4 Comparison with PHOKHARA}

The PHOKHARA event generator [81] provides an alternative description of the radiative process, with one or two ISR photons simulated exactly and no soft photons. The generator implements $\mu^{+} \mu^{-}$and a number of low-multiplicity exclusive hadronic channels. In order to simulate $e^{+} e^{-} \rightarrow q \bar{q} \gamma$ we use the $\mu^{+} \mu^{-} \gamma$ mode with the following modifications:

- Branching: for each event, PHOKHARA starts by determining whether to generate 1 or 2 photons. The relative fraction to use for the branching is determined by a short test run of 10000 events is performed during the initialization phase. 
This can be easily generalized to

qqbar events by increasing the number of categories from 2 to 8 , with 4 flavors of quarks multiplied by the two possible numbers of photons. The test run is lengthened to 50000 events and the fractions of all 8 categories are determined. For each event, we then start by drawing the number of photons and the quark flavor according to the fractions obtained.

- Cross-section: the differential cross-section for the $e^{+} e^{-} \rightarrow \mu^{+} \mu^{-} \gamma$ is modified to use the mass of the appropriate quark flavor instead of the muon mass. It is multiplied by a factor $R_{\text {had }}^{\text {Corr }}$ ( $s$, flavor equal to $R_{\text {had }}(s)$ divided by the threshold factor $\sqrt{1-\frac{4 m_{q}^{2}}{s}}\left(1+\frac{2 m_{q}^{2}}{s}\right)$ which is already included in the cross-section expression.

- One the $q \bar{q}$ system is generated, it is passed to RRes to perform the same hadronization procedure as for KKMC.

Although the hadronization is identical for both generators, it is interesting to compare the shape of the radiative functions. These are shown in Figs. A.5. To see how much the KKMC radiator differs from the soft-photon approximation, we also show the variations of

$$
\frac{d \sigma_{e^{+} e^{-} \rightarrow q \bar{q} \gamma}}{d s^{\prime}}\left(s, s^{\prime}\right)=\xi\left(s, s^{\prime}\right) \frac{4 \pi \alpha^{2}}{3 s^{\prime}} R_{h a d}\left(s^{\prime}\right)
$$

where $\xi\left(s, s^{\prime}\right)$ is given by Eq. 2.51. Since PHOKHARA has the option to generate either one or two ISR photons, we overlay the distributions for both cases, normalizing to the respective areas of the KKMC and naive distributions(a reliable value of the cross-section is not available in these modes): as expected, the one-photon case is in excellent agreement with the naive shape, while the two-photon case $\mathrm{s}$ in good agreement with KKMC. The difference between KKMC and the naive expectation, of the order of $12 \%$, seems a bit high but is probably due to the contribution of extra soft and collinear photons.

The CM polar angle distribution of the hadronic system is shown in Fig. A.6 

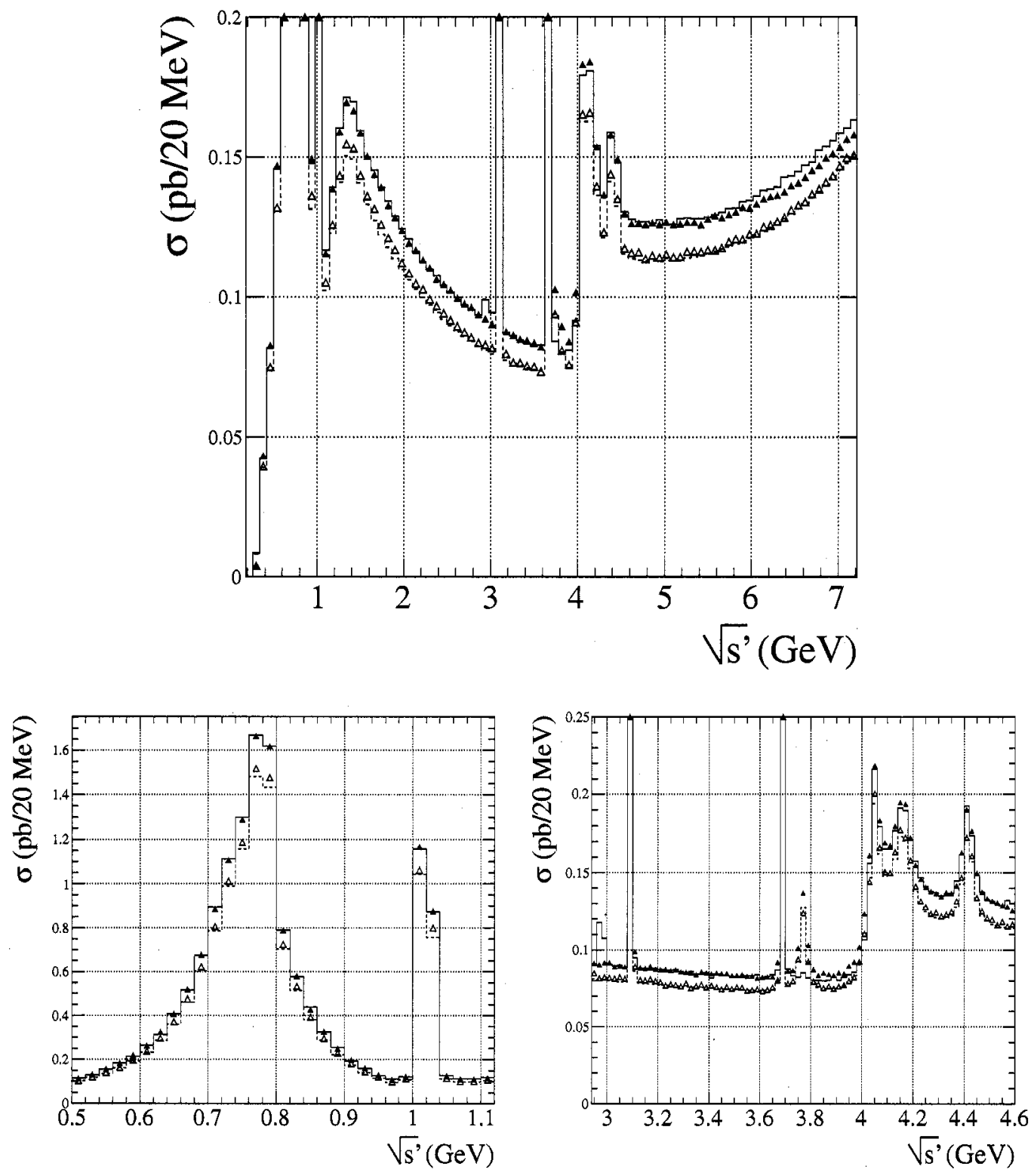

Figure A.5: Generated $s^{\prime}$ spectrum for KKMC (solid line) and the naive expectation of Eq. A.4. The solid triangles show the Phokhara spectrum in the mode with two hard photons enabled, while the empty triangles show the case of only one photon enabled. The plots show the full spectrum (top), the $\rho-\phi$ region (bottom left) and the $c \bar{c}$ threshold region (bottom right). 


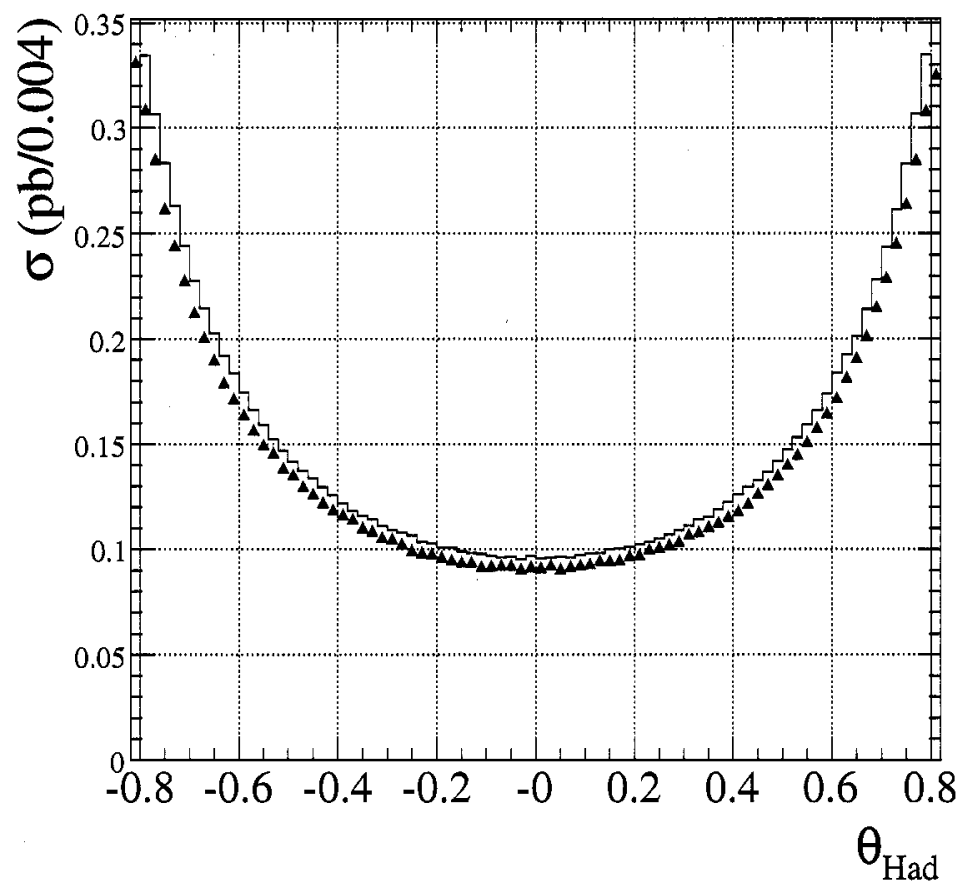

Figure A.6: CM polar angle distribution for the hadronic system in KKMC 


\section{A.5 Comparison of the pion form factor}

The KKMC parameterization of the pion form factor can be checked using the $\pi^{+} \pi^{-}$ generation mode of PHOKHARA. Version 5.0 of the latter uses a more complex form of the Kühn-Santamaria parameterization with a third $\rho$ resonance, but the change with respect to the KKMC version should be minimal. The comparison with the $\pi^{+} \pi^{-}$ events generated by KKMC within the same $\left|\cos \theta_{\text {Had }}^{*}\right|<0.8$ range for the hadronic system is shown in Fig. A.7. The output of both generators are normalized to their reported cross-sections.

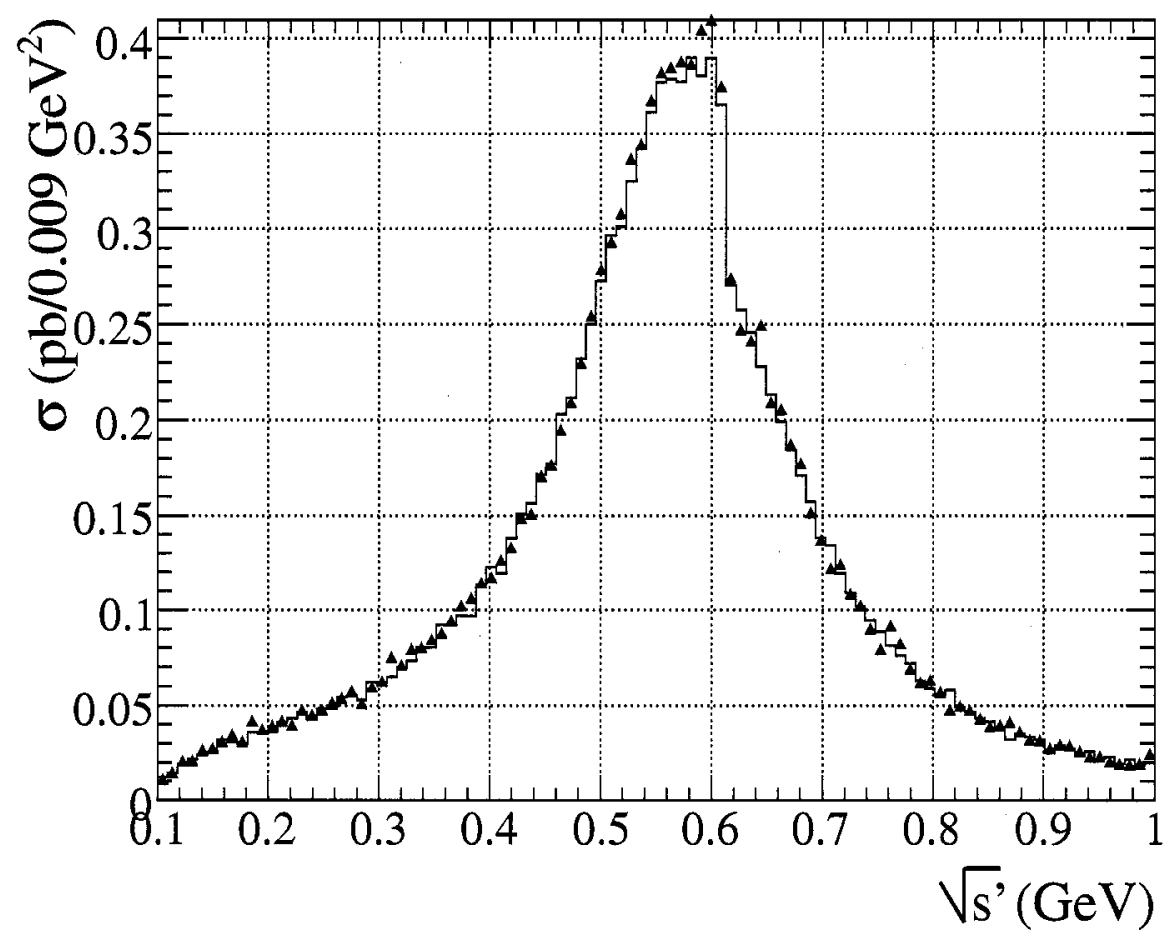

Figure A.7: $s^{\prime}$ distribution for $\pi^{+} \pi^{-}$events as generated by KKMC (solid histogram) and PHOKHARA (triangles).

Excellent agreement is seen in both the shape and overall normalization. 


\section{Appendix B}

\section{Calculation of the Radiator Function $\xi(x)$}

In section 2.3.1, the cross-section for radiating an ISR photon with longitudinal momentum $p_{L}=z \sqrt{s}$ was expressed in terms of the function

$$
\xi\left(s, s^{\prime}\right)=\frac{s^{\prime}}{2 s\left(s-s^{\prime}\right)} \sum_{s^{+}, \lambda} \int \frac{d p_{T}^{2}}{16 \pi^{3}} \frac{e^{2}}{\left(l^{2}-m_{e}^{2}\right)^{2}}\left|J_{E}\right|^{2},
$$

where

$$
\mathcal{J}_{E}\left(p_{1}, k, s, \lambda\right)=\bar{v}_{s^{+}}\left(p_{1}\right) \gamma^{\alpha} \epsilon_{\alpha}^{\lambda}(k) v_{s^{+}}(l),
$$

with $l=p_{1}-k$. The corresponding diagram form is shown in Fig. B.1.

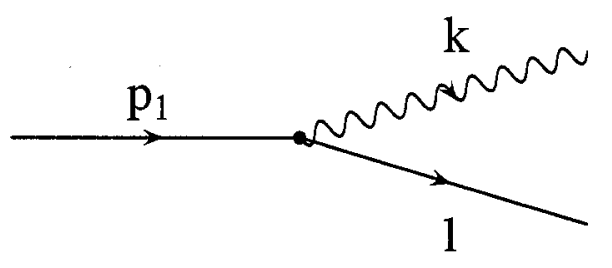

Figure B.1: Diagram for the radiative factor $\xi$

The initial positron and the photon are assumed to be on-shell, so that $p_{1}^{2}=m_{e}^{2}$ 
and $k^{2}=0$. By 4 -momentum conservation the outgoing positron is then off-shell. Taking the $z$ axis to lie in the direction, we call $p_{T}$ the photon transverse momentum and $z$ its longitudinal momentum fraction. We will assume a soft or collinear photon emission, corresponding to $p_{T} \ll P$,

In this approximation, a suitable parametrization for the momenta is

$$
\begin{aligned}
p & =\left(P+\frac{m^{2}}{2 P}, 0,0, P\right) \\
k & =\left(z P+\frac{p_{T}^{2}}{2 z P}, p_{T}, 0, z P\right) \\
l & =\left((1-z) P-\frac{p_{T}^{2}}{2 z P}-\frac{m^{2}}{2 P},-p_{T}, 0,(1-z) P\right),
\end{aligned}
$$

Throughout this calculation only the leading-order mass terms will be accounted for. Then $k^{2}=0$ and $l^{2}=-p T^{2} / z-(1-z) m^{2}$, neglecting terms of order $4\left((m / P)^{4}\right.$, $\left(p_{T} / P\right)^{4}$ and $\left.m^{2} p_{T}^{2} / P^{4}\right)$.

The sums over fermion helicities can usually be calculated using:

$$
\sum_{s} v_{s}(p) \bar{v}_{s}(p)=\not p-m
$$

Unfortunately this expression is exact only if $p^{2}=m^{2}$, so it cannot be used for the outgoing positron. There is however a way out: it turns out that the expression for $\left|J_{E}\right|^{2}$ is insensitive to variations of order $p_{T}^{2} / P^{2}$ and $m^{2} / p^{2}$ in the energy component of the 4-momenta. We are therefore free to use for $l$ the alternate expression

$$
\tilde{l}=\left((1-z) P+\frac{p_{T}^{2}+m^{2}}{2(1-z) P},-p_{T}, 0,(1-z) P\right),
$$

for which $l^{2}=m^{2}$ up to terms of order 4 . We can also modify the expressions for $p$ and $k$ to 


$$
\begin{aligned}
& \tilde{p}=\left(P+\frac{m^{2}}{2 z P}, 0,0, P\right) \\
& \tilde{k}=\left(z P, p_{T}, 0, z P\right)
\end{aligned}
$$

With these expressions,

$$
\begin{aligned}
\frac{1}{2} \sum_{s^{\prime}, s}\left|\mathcal{J}_{E}\right|^{2} & =\frac{e^{2}}{2} \bar{v}_{s^{\prime}}(\tilde{p}) \gamma^{\alpha} v_{s}(l) \bar{v}_{s}(l) \gamma^{\beta} v_{s^{\prime}}(\tilde{p}) \epsilon_{\alpha}^{\lambda}(k) \epsilon_{\beta}^{\lambda}(k) \\
& =\frac{e^{2}}{2} \operatorname{tr}\left[(\tilde{p}-m) \gamma^{\alpha}(\tilde{y}-m) \gamma^{\beta}\right] \epsilon_{\alpha}^{\lambda}(k) \epsilon_{\beta}^{\lambda}(k) \\
& =2 e^{2}\left(p^{\left\{\alpha, \tilde{l}^{\beta}\right\}}-g^{\alpha \beta}\left((p \cdot \tilde{l})-m^{2}\right)\right) \epsilon_{\alpha}^{\lambda}(k) \epsilon_{\beta}^{\lambda}(k)
\end{aligned}
$$

We now need to sum over the photon polarizations. This is usually done using the replacement

$$
\sum_{\lambda} \epsilon_{\alpha}^{\lambda}(k) \epsilon_{\beta}^{\lambda}(k) \rightarrow-g_{\alpha \beta}
$$

This form preserves Lorentz invariance by introducing two fictitious contributions corresponding to longitudinal and timelike polarization directions, in additions to the physical transverse polarizations. These extra contributions have opposite signs, and the Ward identity C.3 ensures that they cancel exactly when B.10 is contracted with a physical amplitude.

In this particular case however, it turns out that this does not work: for the Ward identity to work all articles must be on-shell and 4-momentum must be conserved, and there is no way to fulfill all these conditions at once. In the original parametrization the outgoing positron was off-shell, and the "tilded" momenta violate 4-momentum conservation. One can show that 


$$
\begin{aligned}
& k_{\alpha} \operatorname{tr}\left[(\not p-m) \gamma^{\alpha}(\not y-m) \gamma^{\beta}\right]=2 p^{\beta}\left(l^{2}-m^{2}\right) \\
& k_{\alpha} \operatorname{tr}\left[(\tilde{p}-m) \gamma^{\alpha}(\tilde{y}-m) \gamma^{\beta}\right]=-4 \delta\left[\tilde{l}^{2} \tilde{l}^{\beta}+\tilde{p}^{2} \tilde{p}^{\beta}-\tilde{k}^{2} \tilde{k}^{\beta}\right] \quad(\text { with } \delta=\tilde{p}-\tilde{k}-\tilde{l})
\end{aligned}
$$

so the Ward identity is violated and B.10 cannot be used. In the absence of this simplification, the polarization sum should be restricted "by hand" to lie in the plane transverse to the photon 3-momentum, using the expression

$$
\sum_{\lambda} \epsilon_{\alpha}^{\lambda}(k) \epsilon_{\beta}^{\lambda}(k) \rightarrow\left\{\begin{array}{c}
\delta_{\alpha \beta}-\frac{k_{\alpha} k_{\beta}}{|\mathbf{k}|^{2}} \text { for } 1 \leq \alpha, \beta \leq 3 \\
0 \text { otherwise }
\end{array}\right.
$$

where $\delta_{\alpha \beta}$ is the Kronecker symbol equal to 1 for $1 \leq \alpha=\beta \leq 3$ and 0 otherwise. One can check that $\delta_{\alpha \beta}-\frac{k^{\alpha} k^{\beta}}{|\mathbf{k}|^{2}}$ is the operator that projects out the 2-dimensional subspace normal to $\mathbf{k}$, which is where the physical polarization vectors should lie. This expression has the disadvantage of not being Lorentz-invariant, but calculations are straightforward. We have $\delta_{\alpha \beta} g^{\alpha \beta}=-3, \delta_{\alpha \beta} p^{\left\{\alpha, l^{\beta\}}\right.}=2(\mathbf{p} \cdot 1)$, so using the "tilded" expressions for the momenta we have

$$
\begin{aligned}
\sum_{\lambda} \epsilon_{\alpha}^{\lambda}(k) \epsilon_{\beta}^{\lambda}(k)\left[p^{\left\{\alpha, l^{\beta\}}\right.}-g^{\alpha \beta}\left((p \cdot l)-m^{2}\right)\right] \\
\quad=2\left(p^{0} l^{0}-m^{2}-\frac{(\mathbf{p} \cdot \mathbf{k})(\mathbf{l} \cdot \mathbf{k})}{|\mathbf{k}|^{2}}\right) \\
=(1-z) P^{2}+\frac{1-z}{2 z} m^{2}+\frac{p_{T}^{2}+m^{2}}{2(1-z)}-m^{2}-\left[(1-z) P^{2}-\frac{p_{T}^{2}}{z^{2}}\right] \\
\quad=\frac{1}{z(1-z)}\left[m^{2}\left(z+(1-z)^{2}\right)+p_{T}^{2}\left(\frac{1+(1-z)^{2}}{z}\right)\right]
\end{aligned}
$$

Therefore

$$
\frac{1}{2} \sum_{s^{+}, \lambda}\left|J_{E}\right|^{2}=\frac{2 e^{2}}{z(1-z)}\left[m^{2}\left(z+(1-z)^{2}\right)+p_{T}^{2}\left(\frac{1+(1-z)^{2}}{z}\right)\right]
$$


Plugging this into our main equation, we have

$$
\xi\left(s, s^{\prime}\right)=\frac{e^{2}}{8 \pi^{2} s} \int \frac{d p_{T}^{2}}{\left(l^{2}-m_{e}^{2}\right)^{2}} \frac{1-z}{z}\left[m^{2}\left(z+(1-z)^{2}\right)+p_{T}^{2}\left(\frac{1+(1-z)^{2}}{z}\right)\right] .
$$

Given that for $p_{T} \gg m$

$$
\left(l^{2}-m_{e}^{2}\right)^{2}=\frac{p_{T}^{4}}{z^{2}}+\frac{2}{z}(z-2) m_{e}^{2} p_{T}^{2}
$$

we have

$$
\xi\left(s, s^{\prime}\right)=\frac{\alpha}{2 \pi} \int_{0}^{P} \frac{d p_{T}^{2}}{p_{T}^{2}-K(z) m^{2}} \frac{1+(1-z)^{2}}{\left(z+\frac{p_{T}^{2}}{2 z(1-z) P^{2}}\right)} .
$$

with

$$
K(z)=2(z-2)+\frac{z+(1-z)^{2}}{1+(1-z)^{2}}
$$

The additional terms in the denominators are small and therefore negligible except at the lower integration limits where they cut off the infrared singularities. Introducing $\sqrt{s}=2 P$, we get

$$
\int_{0}^{P} \frac{d p_{T}^{2}}{p_{T}^{2}-K(z) m^{2}} \approx 2 \log \frac{\sqrt{s}}{m}
$$

and

$$
\xi\left(s, s^{\prime}\right)=\frac{\alpha}{\pi s} \log \frac{\sqrt{s}}{m} \frac{1+(1-z)^{2}}{z} .
$$




\section{Appendix C}

\section{Calculation of the Contribution to $e^{+} e^{-} \rightarrow q \bar{q}$ from Double Virtual \\ Photon Exchange}

\section{C.1 Preliminaries: one virtual photon}

\section{C.1.1 Cross-section calculation}

Before tackling the problem of two virtual photons, it is useful to go through a a quick study of the case of one virtual photon.

The matrix elements in this case are well-known. For $e^{+} e^{-} \rightarrow \mu^{+} \mu^{-}$for instance,

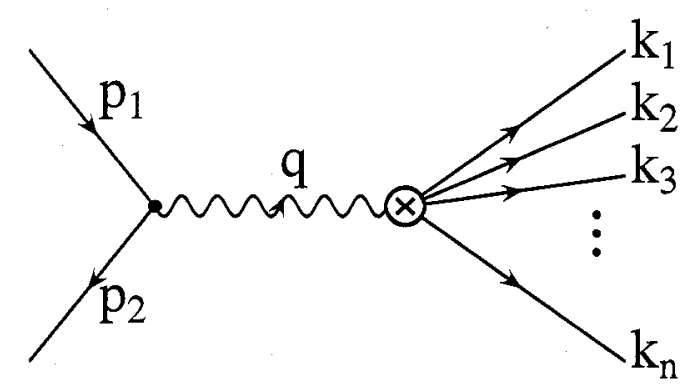

Figure C.1: One-photon exchange diagram for $e^{+} e^{-} \rightarrow F$ 


$$
i \mathcal{M}_{\mu^{+} \mu^{-}}=(-i e)^{2} \bar{v}\left(p_{2}\right) \gamma^{\mu} u\left(p_{1}\right) \frac{-i g_{\mu \nu}}{q^{2}} \bar{v}\left(k_{2}\right) \gamma^{\nu} u\left(k_{1}\right) .
$$

For a more general final state $F$, one can replace the last term by some vector form-factor $h_{F}^{\mu}\left(k_{1} \cdots k_{n}\right)$. Similarly, we can generalize by writing $l_{1}^{\mu}\left(p_{1}, p_{2}\right)$ for the initial state. Then

$$
i \mathcal{M}_{F}=(-i e)^{2} l_{1}^{\mu} \frac{-i g_{\mu \nu}}{q^{2}} h_{F}^{\nu}
$$

Because of the Ward identity, we have

$$
q_{\mu} l_{1}^{\mu}=q_{\mu} h_{F}^{\mu}=0
$$

which will be useful later.

The unpolarized cross-sections are obtained by calculating the spin-averaged matrix element

$$
\left\langle\left|\mathcal{M}_{F}\right|^{2}\right\rangle=\frac{1}{4} \sum_{\text {spins }}\left|\mathcal{M}_{F}\right|^{2}=\frac{e^{4}}{4 s^{2}}\left\langle l_{1}^{\mu} l_{1}^{\nu *}\right\rangle\left\langle h_{F \mu} h_{F \nu}^{*}\right\rangle=\frac{e^{4}}{4 s^{2}} \mathcal{L}_{1}^{\mu \nu} \mathcal{H}_{F \mu \nu}
$$

where $\langle\ldots\rangle$ denotes averaging over spins. The cross-section is given by the phasespace integral

$$
\sigma_{1}\left(e^{+} e^{-} \rightarrow F\right)=\int d \Phi_{n} \frac{(2 \pi)^{4} \delta^{(4)}\left(p_{1}+p_{2}-\sum k_{i}\right)}{4 \sqrt{\left(p_{1} \cdot p_{2}\right)^{2}-\left(m_{1} m_{2}\right)^{2}}}\left\langle\left|\mathcal{M}_{F}\right|^{2}\right\rangle
$$

where

$$
d \Phi_{n}=\prod_{i=1}^{n} \frac{d^{3} \vec{k}_{i}}{(2 \pi)^{3}} \frac{1}{2 \sqrt{\vec{k}_{i}^{2}+m_{i}^{2}}} .
$$

and we have for now ignored any symmetry factors that may arise from identical particles in the final state. Since $\mathcal{L}_{1}^{\mu \nu}$ is independent of the final state we can write, neglecting the mass of the electron, 


$$
\sigma_{1}\left(e^{+} e^{-} \rightarrow F\right)=\frac{e^{4}}{8 s^{3}} \mathcal{L}_{1}^{\mu \nu}\left(p_{1}, p_{2}\right) \int d \Phi_{n}(2 \pi)^{4} \delta^{(4)}\left(p_{1}+p_{2}-\sum k_{i}\right) \mathcal{H}_{F \mu \nu}\left(k_{1} \cdots k_{n}\right)
$$

since $s=\left(p_{1}+p_{2}\right)^{2}=2 p_{1} \cdot p_{2}$. Since all final state momenta are integrated over, the result of the phase-space integral can depend only on $q$ and not the individual $k$ 's: We can therefore write

$$
\mathcal{K}_{F}^{\mu \nu}(q)=\int d \Phi_{n}(2 \pi)^{4} \delta^{(4)}\left(p_{1}+p_{2}-\sum k_{i}\right) \mathcal{H}^{\mu \nu}\left(k_{1} \cdots k_{n}\right)
$$

The Ward identity of Eq. C.3 imposes $q_{\mu} \mathcal{K}_{F}^{\mu \nu}=\mathcal{K}_{F}^{\mu \nu} q_{\nu}=0$. Lorentz covariance then imposes $\mathcal{K}_{F}^{\mu \nu}$ to have the projector form

$$
\mathcal{K}_{F}^{\mu \nu}(q)=\frac{\mathcal{K}_{F}\left(q^{2}\right)}{3}\left(g^{\mu \nu}-\frac{q^{\mu} q^{\nu}}{q^{2}}\right)
$$

where

$$
\mathcal{K}_{F}=g_{\mu \nu} \mathcal{K}_{F}^{\mu \nu}=\int d \Phi_{n}(2 \pi)^{4} \delta^{(4)}\left(p_{1}+p_{2}-\sum k_{i}\right) g_{\mu \nu} \mathcal{H}^{\mu \nu}\left(k_{1} \cdots k_{n}\right)
$$

is only a function of $q^{2}$. This expression is to to contracted with $\mathcal{L}_{1}^{\mu \nu}$, but because $\mathcal{L}_{1}^{\mu \nu}$ also verifies the Ward identity the $q^{\mu} q^{\nu}$ term vanishes when contracted with $\mathcal{L}_{1}^{\mu \nu}$ and we have

$$
\sigma_{1}\left(e^{+} e^{-} \rightarrow F\right)=\frac{e^{4}}{8 s^{3}} \mathcal{L}_{1}^{\mu \nu}\left(p_{1}, p_{2}\right) \mathcal{K}_{F \mu \nu}(q)=\frac{e^{4}}{8 s^{3}} \mathcal{L}_{1}^{\mu \nu}\left(p_{1}, p_{2}\right) \frac{\mathcal{K}_{F}}{3} g_{\mu \nu}=\frac{e^{4}}{8 s^{3}} \frac{\mathcal{L}_{1} \mathcal{K}_{F}}{3}
$$

with $\mathcal{L}_{1}=g_{\mu \nu} \mathcal{L}_{1}^{\mu \nu}$ For massless particles at lowest order

$$
\mathcal{L}_{1}^{\mu \nu}\left(p_{1}, p_{2}\right)=\left\langle\left|\bar{v}\left(p_{2}\right) \gamma^{\mu} u\left(p_{1}\right)\right|^{2}\right\rangle=\operatorname{tr}\left(\not p_{2} \gamma^{\mu} \not p_{1} \gamma^{\nu}\right)
$$

And the trace over vector indices is 


$$
\mathcal{L}_{1}\left(p_{1}, p_{2}\right)=\operatorname{tr}\left(\not \not_{2} \gamma^{\mu} \not_{1} \gamma_{\mu}\right)=-8\left(p_{1} \cdot p_{2}\right)=-4 s
$$

which gives

$$
\sigma_{1}\left(e^{+} e^{-} \rightarrow F\right)=-\frac{e^{4}}{6 s^{2}} \mathcal{K}_{F}=-\frac{8 \pi^{2} \alpha^{2}}{3 s^{2}} \mathcal{K}_{F}
$$

\section{C.1.2 Applications}

- Massless $e^{+} e^{-} \rightarrow \mu^{+} \mu^{-}$scattering: in this case the right side of the diagram is identical to the left side, so that $\mathcal{H}_{\mu^{+} \mu^{-}}=\mathcal{L}_{1}=-4 \mathrm{~s}$. Since this doesn't depend on $k_{1}$ or $k_{2}$, we can integrate the 2-body phase-space by itself, which in the massless case gives $\Phi_{2}=1 / 8 \pi$. We therefore get

$$
\mathcal{K}_{\mu^{+} \mu^{-}}=-4 s \Phi_{2}=-\frac{s}{2 \pi},
$$

which leads to the familiar result

$$
\sigma_{1}\left(e^{+} e^{-} \rightarrow \mu^{+} \mu^{-}\right)=\frac{4 \pi \alpha^{2}}{3 s} .
$$

- Inclusive hadronic production: in this case the cross-section is

$$
\sigma_{1}\left(e^{+} e^{-} \rightarrow \text { hadrons }\right)=\frac{4 \pi \alpha^{2}}{3 s} R(s)
$$

by definition of $R(s)$, so working backward we can calculate

$$
\mathcal{K}_{\text {had }}=-\frac{s}{2 \pi} R(s)
$$

A similar expression can be obtained for an exclusive hadronic final state $H$ by replacing the $R$ function by the "partial" $R_{H}(s)$ giving the shape of the hadronic spectrum in this final state. 

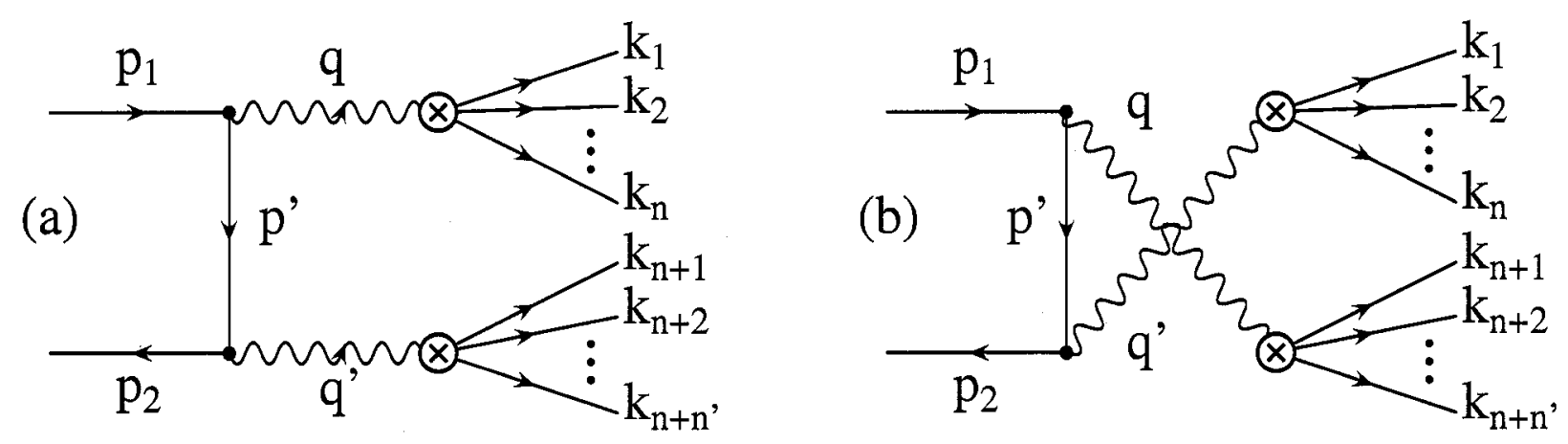

Figure C.2: Two-photon exchange diagrams for $e^{+} e^{-} \rightarrow F F^{\prime}$

The case of a narrow hadronic resonance $V$ is given by the usual Breit-Wigner formula

$$
R_{V}(\omega)=\frac{9}{\alpha^{2}} \mathcal{B}\left(V \rightarrow e^{+} e^{-}\right) \frac{m^{2} \Gamma^{2}}{\left(\omega-m^{2}\right)^{2}+m^{2} \Gamma^{2}},
$$

where $m$ and $\Gamma$ are respectively the mass and width of $V$, and $\mathcal{B}\left(V \rightarrow e^{+} e^{-}\right)$its branching fraction into electrons. In the case of a narrow resonance this form can be approximated with a delta function of equal integral,

$$
R_{V}(\omega)=\frac{9}{\alpha^{2}} \mathcal{B}\left(V \rightarrow e^{+} e^{-}\right) \pi m \Gamma \delta\left(\omega-m^{2}\right)
$$

\section{C.2 Two virtual photons}

Let's now consider the case of two virtual photons, trying to maximize the analogy with the previous section. The key assumption is that the virtual photons decay independently with no interaction between the final-state particles. The corresponding diagrams for arbitrary final states $F$ and $F^{\prime}$ are shown in Fig. C.2. There are two basic diagrams, analogous to the diagrams for $e^{+} e^{-} \rightarrow \gamma \gamma$, which we call "parallel" and "crossed". There may be additional diagrams in the case in which identical particles are produced in the decay of different virtual photons; these complications will be treated in a case-by-case basis when we consider particular final states. 


\section{C.2.1 "Parallel" Diagram}

\section{Cross-section in terms of $\mathcal{K}$ form-factors}

Let's start by computing the "parallel" diagram represented in Figure C.2(a).

$$
i \mathcal{M}_{F}=(-i e)^{4} l_{2}^{\mu \nu} \frac{-i g_{\mu \rho}}{q^{2}} \frac{-i g_{\nu \sigma}}{q^{2}} h_{F}^{\rho} h_{F^{\prime}}^{\sigma}
$$

where $l$ now carries two indices since there are two photons emitted from the initial state.

We now have

$$
\left\langle\left|\mathcal{M}_{F}\right|^{2}\right\rangle=\frac{e^{8}}{4 q^{4} q^{\prime 4}} \mathcal{L}_{2}^{\mu \nu \rho \sigma} \mathcal{H}_{F \mu \rho} \mathcal{H}_{F^{\prime} \nu \sigma}
$$

and

$$
\begin{array}{r}
\sigma_{2}^{\|}\left(e^{+} e^{-} \rightarrow F F^{\prime}\right)=\frac{e^{8}}{8 s} \int d \Phi_{n+n^{\prime}} \frac{1}{q^{4} q^{14}} \mathcal{L}_{2}^{\mu \nu \rho \sigma}\left(p_{1}, p_{2}, p^{\prime}\right)(2 \pi)^{4} \delta^{(4)}\left(p_{1}+p_{2}-\sum k_{i}\right) \\
\mathcal{H}_{F \mu \rho}\left(k_{1} \cdots k_{n}\right) \mathcal{H}_{F^{\prime} \nu \sigma}\left(k_{n+1} \cdots k_{n+n^{\prime}}\right)
\end{array}
$$

with $\mathcal{L}_{2}^{\mu \nu \rho \sigma}=\left\langle l_{2}^{\mu \nu} l_{2}^{\rho \sigma *}\right\rangle$ and the $\mathcal{H}^{\prime}$ 's defined as in the previous section.

The situation is somewhat more complicated that in the one-photon case: to start with, the initial-state tensor now depends on $q$ through $p^{\prime}=p_{1}-q$, so it cannot be taken outside the integral. In addition, the phase-space integration now runs over the entire final state, which covers both $F$ and $F^{\prime}$. To simplify things let's first make them more complicated by inserting

$$
1=\int \frac{d^{4} q}{(2 \pi)^{4}}(2 \pi)^{4} \delta^{(4)}\left(q-\sum_{i=1}^{n} k_{i}\right)
$$

to get 
360APPENDIX C. HADRONIC PRODUCTION FROM TWO VIRTUAL PHOTONS

$$
\begin{array}{r}
\sigma_{2}^{\|}\left(e^{+} e^{-} \rightarrow F F^{\prime}\right)=\frac{e^{8}}{8 s} \int \frac{d^{4} q}{(2 \pi)^{4}} \frac{1}{q^{4} q^{\prime 4}} \mathcal{L}_{2}^{\mu \nu \rho \sigma} \int d \Phi_{n^{\prime}}(2 \pi)^{4} \delta^{(4)} \\
\left(p_{1}+p_{2}-q-\sum_{i=n+1}^{n^{\prime}} k_{i}\right) \mathcal{H}_{F^{\prime} \nu \sigma} \\
\\
\int d \Phi_{n} \mathcal{H}_{F \mu \rho}(2 \pi)^{4} \delta^{(4)}\left(q-\sum_{i=1}^{n} k_{i}\right)
\end{array}
$$

. where we have replaced $\sum_{i=1}^{n} k_{i}$ by $q$ in the first $\delta$ function, and moved $\mathcal{L}_{2}^{\mu \nu \rho \sigma}$ out of the phase-space integral since it depends only on $q$ and not the individual $k_{i}$.

The final state $F$ is now isolated: the rightmost integral has the form of $\mathcal{K}_{F \mu \rho}(q)$ as in Eq. C.8, and using Eq. C.9 and the current conservation property of $\mathcal{L}_{2}$ we can write

$$
\begin{aligned}
& \sigma_{2}^{\|}\left(e^{+} e^{-} \rightarrow F F^{\prime}\right) \\
= & \frac{e^{8}}{8 s} \int \frac{d^{4} q}{(2 \pi)^{4}} \frac{1}{q^{4} q^{\prime 4}} \mathcal{L}_{2}^{\nu \sigma} \int d \Phi_{n^{\prime}}(2 \pi)^{4} \delta^{(4)}\left(p_{1}+p_{2}-q-\sum_{i=n+1}^{n^{\prime}} k_{i}\right) \mathcal{H}_{F^{\prime} \nu \sigma} \frac{1}{3} \mathcal{K}_{F}(q) \\
= & \frac{e^{8}}{24 s} \int \frac{d^{4} q}{(2 \pi)^{4}} \frac{1}{q^{4} q^{\prime 4}} \mathcal{L}_{2}^{\nu \sigma} \mathcal{K}_{F}(q) \int d \Phi_{n^{\prime}}(2 \pi)^{4} \delta^{(4)}\left(p_{1}+p_{2}-q-\sum_{i=n+1}^{n^{\prime}} k_{i}\right) \mathcal{H}_{F^{\prime} \nu \sigma} .
\end{aligned}
$$

with $\mathcal{L}_{2}^{\nu \sigma}=g_{\mu \rho} \mathcal{L}_{2}^{\mu \nu \rho \sigma}$.

The rightmost integral now takes the form of $\mathcal{K}_{F^{\prime} \nu \sigma}\left(p_{1}+p_{2}-q\right)$; using the Ward identity again and setting $\mathcal{L}_{2}=g_{\nu \sigma} \mathcal{L}_{2}^{\nu \sigma}$ we have

$$
\begin{aligned}
\sigma_{2}^{\|}\left(e^{+} e^{-} \rightarrow F F^{\prime}\right) & =\frac{e^{8}}{24 s} \int \frac{d^{4} q}{(2 \pi)^{4}} \frac{1}{q^{4} q^{14}} \mathcal{L}_{2}\left(p_{1}, p_{2}, q\right) \mathcal{K}_{F}(q) \frac{1}{3} \mathcal{K}_{F^{\prime}}\left(p_{1}+p_{2}-q\right) \\
& =\frac{32 \pi^{4} \alpha^{4}}{9 s} \int \frac{d^{4} q}{(2 \pi)^{4}} \frac{1}{q^{4} q^{\prime 4}} \mathcal{L}_{2}\left(p_{1}, p_{2}, q\right) \mathcal{K}_{F}(q) \mathcal{K}_{F^{\prime}}\left(p_{1}+p_{2}-q\right)
\end{aligned}
$$

\section{Change of Integration Variables}

So we now have a very elegant form, essentially a four-dimensional convolution of the final-state form factors for the two virtual photons. At first sight it may seem that 
there are too many parameters: this is essentially a 2-body decay, so 4-momentum conservation should leave free only the usual two angles defining the decay axis. However in this case both virtual photons can have arbitrary masses within the allowed phase space, which gives two additional parameters and explains the 4-dimensional integration. We can write $d^{4} q=q_{0} d^{3} q=q_{0}|\mathbf{q}|^{2} d|\mathbf{q}| d \Omega$, where $d \Omega$ represents the angles and $q_{0},|\mathbf{q}|$ are functions of the masses. From standard 2-body kinematics,

$$
\begin{aligned}
q_{0} & =\frac{s+\omega-\omega^{\prime}}{2 \sqrt{s}} \\
|\mathbf{q}| & =\sqrt{\left(\frac{s+\omega-\omega^{\prime}}{2 \sqrt{s}}\right)^{2}-\omega}=\sqrt{\frac{\left(s-\omega-\omega^{\prime}\right)^{2}-4 \omega \omega^{\prime}}{4 s}}
\end{aligned}
$$

where $\omega=q^{2}$ and $\omega^{\prime}=q^{\prime 2}$. To express the differential form in terms of $\omega$ and $\omega^{\prime}$, we can write unity as

$$
1=\int d \omega \delta\left(\omega-q^{2}\right)=\int d \omega^{\prime} \delta\left(\omega^{\prime}-q^{2}\right)
$$

Then

$$
\begin{aligned}
\int d^{4} q & =\int d \omega \int d^{4} q \delta\left(\omega-q^{2}\right)=\int d \omega d q_{0} d \Omega \int d|\mathbf{q}||\mathbf{q}|^{2} \delta\left(\omega-q_{0}^{2}+|\mathbf{q}|^{2}\right) \\
& =\int d \omega d \Omega d q_{0} \frac{\mathbf{q}}{2}=\int d \omega d \omega^{\prime} d \Omega d q_{0} \frac{\mathbf{q}}{2} \delta\left(\omega^{\prime}-q^{\prime 2}\right) \\
& =\int d \omega d \omega^{\prime} d \Omega d q_{0} \frac{\mathbf{q}}{2} \delta\left(\omega^{\prime}-s-\omega+2 \sqrt{s} q_{0}\right)=\int d \omega d \omega^{\prime} d \Omega \frac{|\mathbf{q}|}{4 \sqrt{s}}
\end{aligned}
$$

Since the $\mathcal{K}_{F\left({ }^{\prime}\right)}(q)$ depend only on $q^{2}$, we can write

$$
\sigma_{2}^{\|}\left(e^{+} e^{-} \rightarrow F F^{\prime}\right)=\frac{2 \alpha^{4}}{9 s} \int d \omega d \omega^{\prime} d \Omega \frac{|\mathbf{q}|}{4 \sqrt{s}} \frac{1}{\omega^{2} \omega^{\prime 2}} \mathcal{L}_{2}\left(\omega, \omega^{\prime}, \Omega\right) \mathcal{K}_{F}(\omega) \mathcal{K}_{F^{\prime}}\left(\omega^{\prime}\right)
$$

Since the only dependence on the solid angle comes from $\mathcal{L}_{2}$, we can rewrite 
362APPENDIX C. HADRONIC PRODUCTION FROM TWO VIRTUAL PHOTONS

$$
\sigma_{2}^{\|}\left(e^{+} e^{-} \rightarrow F F^{\prime}\right)=\frac{\pi \alpha^{4}}{9 s} \int \frac{d \omega}{\omega} \frac{d \omega^{\prime}}{\omega^{\prime}}\left(\frac{\mathcal{K}_{F}(\omega)}{\omega}\right)\left(\frac{\mathcal{K}_{F^{\prime}}\left(\omega^{\prime}\right)}{\omega^{\prime}}\right) \zeta_{\|}\left(\omega, \omega^{\prime}\right)
$$

with

$$
\zeta_{\|}\left(\omega, \omega^{\prime}\right)=\frac{|\mathbf{q}|}{2 \pi \sqrt{s}} \int \mathcal{L}_{2}\left(\omega, \omega^{\prime}, \Omega\right) d \Omega=\frac{|\mathbf{q}|}{\sqrt{s}} \int \mathcal{L}_{2}(\cos \theta) d(\cos \theta)
$$

\section{C.2.2 Both diagrams together}

In the previous section we have obtained a reasonably simple expression for the case of a single diagram. Let's now try to include the other diagram as well. The only difference is that in the crossed diagram the $F$ and $F^{\prime}$ final states attach to the initial fermion line in the opposite order, so we can make the simple replacement

$$
i \mathcal{M}_{F}=-\frac{e^{4}}{q^{2} q^{\prime 2}} l_{2}^{\mu \nu} h_{F \mu} h_{F^{\prime} \nu} \rightarrow-\frac{e^{4}}{q^{2} q^{\prime 2}} l_{2}^{\mu \nu}\left(h_{F \mu} h_{F^{\prime} \nu}+h_{F \nu} h_{F^{\prime} \mu}\right)
$$

so that

$$
\begin{aligned}
\left\langle\left|\mathcal{M}_{F}\right|^{2}\right\rangle & =\frac{e^{8}}{4 q^{4} q^{\prime 4}}\left\langle l_{2}^{\mu \nu} l_{2}^{* \rho \sigma}\right\rangle\left\langle\left(h_{F \mu} h_{F^{\prime} \nu}+h_{F \nu} h_{F^{\prime} \mu}\right)\left(h_{F \rho} h_{F^{\prime} \sigma}+h_{F \sigma} h_{F^{\prime} \rho}\right)^{*}\right\rangle \\
& =\frac{e^{8}}{4 q^{4} q^{\prime 4}} \mathcal{L}_{2}^{\mu \nu \rho \sigma}\left(\mathcal{H}_{F \mu \rho} \mathcal{H}_{F^{\prime} \nu \sigma}+\mathcal{H}_{F \mu \sigma} \mathcal{H}_{F^{\prime} \rho \nu}+\mathcal{H}_{F \rho \nu} \mathcal{H}_{F \mu \sigma}+\mathcal{H}_{F \nu \sigma} \mathcal{H}_{F \mu \rho}\right)
\end{aligned}
$$

We thus get four terms which differ only by their index structure. One can check that for each term the method of section C.2.1 can be reproduced, with the only difference being the way the tensor $\mathcal{L}_{2}^{\mu \nu \rho \sigma}$ is contracted. For the first term, it was shown in section C.2.1 that the appropriate contraction is $\mathcal{L}_{2}^{(1)}=g_{\mu \rho} g_{\nu \sigma} \mathcal{L}_{2}^{\mu \nu \rho \sigma}$. By analogy, the appropriate contractions for the remaining terms are therefore 


$$
\begin{aligned}
\mathcal{L}_{2}^{(2)} & =g_{\mu \sigma} g_{\nu \rho} \mathcal{L}_{2}^{\mu \nu \rho \sigma} \\
\mathcal{L}_{2}^{(3)} & =g_{\rho \nu} g_{\mu \sigma} \mathcal{L}_{2}^{\mu \nu \rho \sigma}=\mathcal{L}_{2}^{(2)} \\
\mathcal{L}_{2}^{(4)} & =g_{\nu \sigma} g_{\mu \rho} \mathcal{L}_{2}^{\mu \nu \rho \sigma}=\mathcal{L}_{2}^{(1)}
\end{aligned}
$$

We can therefore write, as in Eq. C.25,

$$
\sigma_{2}\left(e^{+} e^{-} \rightarrow F F^{\prime}\right)=\frac{\pi \alpha^{4}}{9 s} \int \frac{d \omega}{\omega} \frac{d \omega^{\prime}}{\omega^{\prime}}\left(\frac{\mathcal{K}_{F}(\omega)}{\omega}\right)\left(\frac{\mathcal{K}_{F^{\prime}}\left(\omega^{\prime}\right)}{\omega^{\prime}}\right) \zeta\left(\omega, \omega^{\prime}\right)
$$

with

$$
\zeta\left(\omega, \omega^{\prime}\right)=\frac{|\mathbf{q}|}{2 \pi \sqrt{s}} \int\left(\mathcal{L}_{2}^{(1)}+\mathcal{L}_{2}^{(2)}+\mathcal{L}_{2}^{(3)}+\mathcal{L}_{2}^{(4)}\right) d \Omega=\frac{2|\mathbf{q}|}{\sqrt{s}} \int \mathcal{L}_{2}(\cos \theta) d(\cos \theta)
$$

and

$$
\tilde{\mathcal{L}}_{2}=\mathcal{L}_{2}^{(1)}+\mathcal{L}_{2}^{(2)}
$$

\section{C.2.3 Calculation of $\tilde{\mathcal{L}}_{2}$}

\section{General Form}

The initial-state fermion current consist of two external legs and a virtual electron in the t-channel with momentum $p^{\prime}=p_{1}-q=q^{\prime}-p_{2}$, so ignoring the electron mass,

$$
l_{2}^{\mu \nu}=\bar{v}\left(p_{2}\right) \gamma^{\mu} \frac{\not p^{\prime}}{p^{\prime 2}} \gamma^{\nu} u\left(p_{1}\right)
$$

The spin-averaged modulus-squared of this expression is given by

$$
\mathcal{L}_{2}^{\mu \nu \rho \sigma}=\operatorname{tr}\left(\not p_{2} \gamma^{\mu} \frac{\not p^{\prime}}{p^{2}} \gamma^{\nu} \not p_{1} \gamma^{\sigma} \frac{p^{\prime}}{p^{\prime 2}} \gamma^{\rho}\right)
$$


Fortunately we are only interested in the traces of this quantity, given by

$$
\begin{aligned}
\mathcal{L}_{2}^{(1)} & =\operatorname{tr}\left(p_{2} \gamma^{\mu} \frac{\not p^{\prime}}{p^{\prime 2}} \gamma^{\nu} p_{1} \gamma_{\nu} \frac{\not p^{\prime}}{p^{\prime 2}} \gamma_{\mu}\right)=\frac{4}{p^{4}} \operatorname{tr}\left(\not p_{2} \not p^{\prime} p_{1} \not p^{\prime}\right) \\
& =\frac{16}{p^{\prime 4}}\left[2\left(p_{1} \cdot p^{\prime}\right)\left(p_{2} \cdot p^{\prime}\right)-p^{\prime 2}\left(p_{1} \cdot p_{2}\right)\right]
\end{aligned}
$$

and

$$
\begin{aligned}
\mathcal{L}_{2}^{(2)} & =\operatorname{tr}\left(\not p_{2} \gamma^{\mu} \frac{p^{\prime}}{p^{\prime 2}} \gamma^{\nu} \not p_{1} \gamma_{\mu} \frac{p^{\prime}}{p^{\prime 2}} \gamma_{\nu}\right)=-\frac{2}{p^{4}} \operatorname{tr}\left(\not p_{2} \gamma^{\mu} \not^{\prime} \not p^{\prime} \gamma_{\mu} \not_{1}\right) \\
& =-\frac{8}{p^{\prime 2}} \operatorname{tr}\left(\not \not_{2} \not p_{1}\right)=-\frac{32}{p^{\prime 2}}\left(p_{1} \cdot p_{2}\right)
\end{aligned}
$$

As usual, we define $t=p^{2}=\left(p_{1}-q\right)^{2}=\omega-2 p_{1} \cdot q$. Then $2 p_{2} \cdot q^{\prime}=\omega^{\prime}-t$ and

$$
\begin{aligned}
\mathcal{L}_{2}^{(1)} & =-\frac{8}{t^{2}}\left[(t-\omega)\left(t-\omega^{\prime}\right)+s t\right] \\
\mathcal{L}_{2}^{(2)} & =-\frac{16 s}{t} \\
\mathcal{L}_{2}^{(1)}+\mathcal{L}_{2}^{(2)} & =-\frac{8}{t^{2}}\left[(t-\omega)\left(t-\omega^{\prime}\right)+3 s t\right]
\end{aligned}
$$

In the center of mass frame of the collision, take $\theta$ to be the polar angle of $\mathbf{q}$ with respect to the collision axis. Then

$$
t=\omega-\sqrt{s}\left(q_{0}-|\mathbf{q}| \cos \theta\right)=-\frac{1}{2}\left(s-\omega-\omega^{\prime}\right)-\sqrt{s}|\mathbf{q}| \cos \theta
$$

Maple claims to be able to perform the integral $\int \tilde{\mathcal{L}}_{2} d(\cos \theta)$. However the integral is reported to be divergent if

$$
-1<\frac{\sqrt{s} \sqrt{|\mathbf{q}|^{2}+\omega}-\omega}{\sqrt{s}|\mathbf{q}|}<1 .
$$

If this is the case, it means we can find an $\alpha$ such that 


$$
\cos \alpha=\frac{\sqrt{s} \sqrt{|\mathbf{q}|^{2}+\omega}-\omega}{\sqrt{s}|\mathbf{q}|}
$$

which leads to $\omega-\sqrt{s} / 2\left(q_{0}-|\mathbf{q}| \cos \alpha\right)=0$. So it makes sense: this corresponds to $t(\theta=\alpha)=0$ and it is telling us that if in the course of integrating over $\theta$ the value $t=0$ is reached then the integral diverges, as the $1 / t$ dependence of $\tilde{\mathcal{L}}_{2}$ would indicate.

Assuming therefore that the point $t=0$ is not reached, the integral is convergent and can be written

$$
\int \tilde{\mathcal{L}}_{2} d(\cos \theta)=\frac{8}{|\mathbf{q}| \sqrt{s}}\left(3 s-\omega-\omega^{\prime}\right) \log \frac{\eta_{+}}{\eta_{-}}-32
$$

with

$$
\eta_{ \pm}=\left(q^{0} \pm|\mathbf{q}|\right) \sqrt{s}-\omega=\frac{1}{2}\left(s-\omega-\omega^{\prime}\right) \pm|\mathbf{q}| \sqrt{s} .
$$

and using the relations $s-2 \sqrt{s} q^{0}=\omega^{\prime}-\omega$ and $\eta_{+} \eta_{-}=\omega \omega^{\prime}$. With this expression, we get from Eq. C.29

$$
\zeta\left(\omega, \omega^{\prime}\right)=\frac{16}{s}\left[\left(3 s-\omega-\omega^{\prime}\right) \log \frac{\eta_{+}}{\eta_{-}}-4|\mathbf{q}| \sqrt{s}\right]
$$

\section{Low- $\omega$ approximation}

Since we are mostly interested in the case of smallish $\omega$ and $\omega^{\prime}$, where the hadronic resonances are located, let's try to find an approximation valid in this regime.

We have $q^{0} \pm|\mathbf{q}| \approx q^{0} \pm\left(q^{0}-\frac{w}{2 q^{0}}-\frac{w^{2}}{8\left(q^{0}\right)^{3}}\right)$ so noting that the first-order terms cancel in $\eta_{-}$we have

$$
\begin{aligned}
\eta_{+} & \approx 2 \sqrt{s} q^{0}-2 \omega \approx s \\
\eta_{-} & \approx \frac{\omega s}{2 \sqrt{s} q^{0}}+\frac{\omega^{2} \sqrt{s}}{8\left(q^{0}\right)^{3}}-\omega \approx \frac{\omega s}{s-\omega-\omega^{\prime}}+\frac{\omega^{2} \sqrt{s}}{s^{3 / 2}}-\omega \\
& \approx \frac{\omega^{2}}{s}-\frac{\omega\left(\omega-\omega^{\prime}\right)}{s}=\frac{\omega \omega^{\prime}}{s}
\end{aligned}
$$




$$
\int \tilde{\mathcal{L}}_{2} d(\cos \theta) \stackrel{\omega, \omega^{\prime} \ll s}{\approx} \frac{24 s}{|\mathbf{q}| \sqrt{s}} \log \frac{s^{2}}{\omega \omega^{\prime}}-32 \approx 16\left[3 \log \frac{s^{2}}{\omega \omega^{\prime}}-2\right]
$$

and

$$
\zeta\left(\omega, \omega^{\prime}\right)=16\left[3 \log \frac{s^{2}}{\omega \omega^{\prime}}-2\right]
$$

and the cross-section can finally be expressed as

$$
\sigma_{2}\left(e^{+} e^{-} \rightarrow F F^{\prime}\right)=\frac{16 \pi \alpha^{4}}{9 s} \int \frac{d \omega}{\omega} \frac{d \omega^{\prime}}{\omega^{\prime}}\left(\frac{\mathcal{K}_{F}(\omega)}{\omega}\right)\left(\frac{\mathcal{K}_{F^{\prime}}\left(\omega^{\prime}\right)}{\omega^{\prime}}\right)\left[3 \log \frac{s^{2}}{2 \omega \omega^{\prime}}-2\right]
$$

\section{C.2.4 Cross-Check}

We can cross-check the previous result by calculating $\tilde{\mathcal{L}}_{2}$ directly in the low- $\omega$ approximation. We have

$$
\begin{aligned}
t & \approx \omega-\sqrt{s}\left[q^{0}(1-\cos \theta)+\left(\frac{\omega}{2 q^{0}}+\frac{\omega^{2}}{8\left(q^{0}\right)^{3}}\right) \cos \theta\right] \\
& \approx \omega-\frac{1}{2}\left(s+\omega-\omega^{\prime}\right)(1-\cos \theta)-\cos \theta\left(\omega+\frac{\omega \omega^{\prime}}{s}\right) \\
& \approx-\frac{1-\cos \theta}{2}\left(s-\omega-\omega^{\prime}\right)-\frac{\omega \omega^{\prime}}{s} \cos \theta \approx-\frac{s}{2}\left[1-\left(1-\frac{2 \omega \omega^{\prime}}{s^{2}}\right) \cos \theta\right](C
\end{aligned}
$$

We have kept the second order terms since they are crucial (as they were for $\eta_{-}$) to regularize the divergence in $\tilde{\mathcal{L}}_{2}$ for $\cos \theta=1$. It can be also noted in passing that for non-zero $\omega$ 's, $t<0$ for all $\theta$ and in particular never vanishes, which justifies the convergence of the $\tilde{\mathcal{L}}_{2}$ integral discussed above at least in this particular case.

We can neglect terms of order $\omega$ in the nominator of $\tilde{\mathcal{L}}_{2}$, but not those of order $\omega^{2}$ since they are enhanced by the associated $1 / t^{2}$ divergence. We have 


$$
\begin{aligned}
\tilde{\mathcal{L}}_{2} & \approx-8\left(1+3 \frac{s}{t}+\frac{\omega \omega^{\prime}}{t^{2}}\right) \\
& \approx-8\left[1-\frac{6}{1-\left(1-\frac{2 \omega \omega^{\prime}}{s^{2}}\right) \cos \theta}+\frac{4 \omega \omega^{\prime}}{s^{2}} \frac{1}{\left(1-\left(1-\frac{2 \omega \omega^{\prime}}{s^{2}}\right) \cos \theta\right)^{2}}\right]
\end{aligned}
$$

since

$$
\begin{aligned}
\int_{a}^{b} \frac{d x}{1-(1-\alpha) x} & =\frac{1}{\alpha-1} \log \frac{1-(1-\alpha) b}{1-(1-\alpha) a} \\
\int_{a}^{b} \frac{d x}{(1-(1-\alpha) x)^{2}} & =\frac{b-a}{[1-(1-\alpha) b][1-(1-\alpha) a]}
\end{aligned}
$$

this yields

$$
\int_{\cos \theta_{\min }}^{\cos \theta_{\max }} \tilde{\mathcal{L}}_{2}=8\left[6 \log \frac{\chi_{\min }}{\chi_{\max }}-\left(1+\frac{4 \omega \omega^{\prime}}{s^{2} \chi_{\min } \chi_{\max }}\right)\left(\cos \theta_{\max }-\cos \theta_{\min }\right)\right]
$$

with

$$
\begin{aligned}
& \chi_{\min }=1-\left(1-\frac{2 \omega \omega^{\prime}}{s^{2}}\right) \cos \theta_{\min } \stackrel{\cos \theta_{\min } \rightarrow-1}{\longrightarrow} 2 \\
& \chi_{\max }=1-\left(1-\frac{2 \omega \omega^{\prime}}{s^{2}}\right) \cos \theta_{\max } \stackrel{\cos \theta_{\max } \rightarrow 1}{\longrightarrow} \frac{2 \omega \omega^{\prime}}{s^{2}} .
\end{aligned}
$$

In the limit $\cos \theta_{\min } \rightarrow-1, \cos \theta_{\max } \rightarrow 1$, we recover Eq. C.42, which validates the calculation.

\section{C.2.5 Fiducial Cross-section}

For comparison with experiment we are particularly interested in the fraction of the cross-section in the sensitive region of the detector. This usually corresponds to an interval $\left[\theta_{\min }, \theta_{\max }\right]$ in $\theta$, and from the formulas above we have directly 


$$
\begin{aligned}
\sigma_{2}^{F i d}\left(e^{+} e^{-} \rightarrow F F^{\prime}\right)= & \frac{8 \pi \alpha^{4}}{9 s} \int \frac{d \omega}{\omega} \frac{d \omega^{\prime}}{\omega^{\prime}} \frac{\mathcal{K}_{F}(\omega)}{\omega} \frac{\mathcal{K}_{F^{\prime}}\left(\omega^{\prime}\right)}{\omega^{\prime}} \frac{2|\mathbf{q}|}{\sqrt{s}} \\
& {\left[6 \log \frac{\chi_{\min }}{\chi_{\max }}-\left(1+\frac{4 \omega \omega^{\prime}}{s^{2} \chi_{\min } \chi_{\max }}\right)\left(\cos \theta_{\max }-\cos \theta_{\min }\right)\right] }
\end{aligned}
$$

in particular, in the usual case $\cos \theta_{\min }=-\cos \theta_{\max }$ and for $1-\cos \theta_{\max } \gg \frac{2 \omega \omega^{\prime}}{s^{2}}$ we have

$$
\begin{aligned}
\sigma_{2}^{F i d}\left(e^{+} e^{-} \rightarrow F F^{\prime}\right)= & \frac{16 \pi \alpha^{4}}{9 s} \int \frac{d \omega}{\omega} \frac{d \omega^{\prime}}{\omega^{\prime}} \frac{\mathcal{K}_{F}(\omega)}{\omega} \frac{\mathcal{K}_{F^{\prime}}\left(\omega^{\prime}\right)}{\omega^{\prime}} \\
& {\left[3 \log \frac{1+\cos \theta_{\max }}{1-\cos \theta_{\max }}-\cos \theta_{\max }\right] }
\end{aligned}
$$

It should be noted however that the definition of "fiducial" corresponds here to the momentum $\mathbf{q}$ being in the fiducial volume, not the final decay products. This may be a good approximation in the case of a low- $Q^{2}$ decay of the virtual photon (e.g. $\phi \rightarrow K K)$, but in other cases it may give only a rough estimate of the fiducial cross-section.

\section{C.2.6 Applications}

$e^{+} e^{-} \rightarrow \mu^{+} \mu^{-} \mu^{+} \mu^{-}$

All we need is to plug in the expression for the $\mu^{+} \mu^{-} \mathcal{K}$-factor given in Eq. C.15 into the cross-section expression given by Eq. C.44. Also since the final states are identical, we need only have integrated over the $\theta$ range $[0, \pi / 2]$ to get all possible configurations. By integrating over the full interval $[0, \pi]$ we have effectively counted every configuration twice, so we must insert an additional factor $1 / 2$. We finally get

$$
\sigma_{2}\left(e^{+} e^{-} \rightarrow \mu^{+} \mu^{-} \mu^{+} \mu^{-}\right)=\frac{2 \alpha^{4}}{9 \pi s} \int \frac{d \omega}{\omega} \frac{d \omega^{\prime}}{\omega^{\prime}}\left[3 \log \frac{s^{2}}{2 \omega \omega^{\prime}}-2\right]
$$

Since this expression is valid only in the limit of small $\omega$ 's, we impose an upper 
bound $\omega_{\max }$ on the integral, with $\omega_{\max } \ll s$. Experimentally it means that we should be able to split the final state into two $\left(\mu^{+} \mu^{-}\right)$combinations each with invariant mass squared less than $\omega_{\max }$ (the other two $\left(\mu^{+} \mu^{-}\right)$combinations can have arbitrary masses).

The integral diverges at the infrared limit, so we cut it off at the lower bound by $\left(2 m_{\mu}\right)^{2}$. The integrals can be evaluated analytically and we get

$$
\sigma_{2}\left(e^{+} e^{-} \rightarrow \mu^{+} \mu^{-} \mu^{+} \mu^{-}\right)=\frac{2 \alpha^{4}}{9 \pi s} \log ^{2} \frac{\omega_{\max }}{4 m_{\mu}^{2}}\left(3 \log \frac{s^{2}}{8 \omega_{\max } m_{\mu}^{2}}-2\right)
$$

For an upper bound at the $\psi$ mass, $\omega_{\max }=(3.1 \mathrm{GeV})^{2}$, we get

$$
\sigma_{2}\left(e^{+} e^{-} \rightarrow \mu^{+} \mu^{-} \mu^{+} \mu^{-}\right) \approx 132 \mathrm{fb}
$$

\section{$e^{+} e^{-} \rightarrow$ Hadrons}

In the case of fully hadronic production, we use the $\mathcal{K}$-factors given in Eq. C.18 and get

$$
\sigma_{2}\left(e^{+} e^{-} \rightarrow H H^{\prime}\right)=\frac{2 \alpha^{4}}{9 \pi s} \int \frac{d \omega}{\omega} \frac{d \omega^{\prime}}{\omega^{\prime}}\left[3 \log \frac{s^{2}}{2 \omega \omega^{\prime}}-2\right] R_{H}(\omega) R_{H^{\prime}}\left(\omega^{\prime}\right)
$$

$\mathrm{H}$ and $\mathrm{H}^{\prime}$ can be either inclusive or exclusive hadronic states. However one must be careful about the symmetry factor: if $H \neq H^{\prime}$ then there should be an additional factor of 2 .

For the production of two narrow resonances we therefore get

$$
\sigma_{2}\left(e^{+} e^{-} \rightarrow V V^{\prime}\right)=\frac{18 \pi}{s} \mathcal{B}\left(V \rightarrow e^{+} e^{-}\right) \mathcal{B}\left(V^{\prime} \rightarrow e^{+} e^{-}\right) \frac{\Gamma \Gamma^{\prime}}{m m^{\prime}}\left[3 \log \frac{s^{2}}{2 m^{2} m^{\prime 2}}-2\right]
$$

A channel of particular interest is $e^{+} e^{-} \rightarrow \rho^{0} \phi$. Unfortunately the $\rho$ is not exactly a narrow resonance, but let's forge ahead anyways: we have $m_{\rho}=769 \mathrm{MeV}, \Gamma_{\rho}=$ 
$149 \mathrm{MeV}$ and $\mathcal{B}\left(\rho^{0} \rightarrow e^{+} e^{-}\right)=4.5 \times 10^{-5}, m_{\phi}=1019 \mathrm{MeV}, \Gamma_{\phi}=4.26 \mathrm{MeV}$ and $\mathcal{B}\left(\phi \rightarrow e^{+} e^{-}\right)=3.0 \times 10^{-4}$, which gives (with the additional factor 2 )

$$
\sigma_{2}\left(e^{+} e^{-} \rightarrow \rho^{0} \phi\right) \approx 110 \mathrm{fb}
$$

Due to the finite width of the $\rho$, this result is probably a bit too low: the $\rho$ lineshape actually extends into lower $\omega$ regions where the cross-section is enhanced by $1 / \omega$.

Since the $\phi$ decay have low $Q^{2}$ the fiducial cross-section formula is probably reasonably precise in this mode. We have (with the additional factor 2)

$$
\sigma_{2}^{\text {Fid }}\left(e^{+} e^{-} \rightarrow V V^{\prime}\right)=\frac{36 \pi}{s} \frac{\Gamma_{e e} \Gamma_{e e}^{\prime}}{m m^{\prime}}\left[3 \log \frac{1+\cos \theta_{\max }}{1-\cos \theta_{\max }}-\cos \theta_{\max }\right]
$$

where the $\Gamma_{e e}$ denote the electronic widths, and with a cut at $\cos \theta_{\max }=0.9$ we get

$$
\sigma_{2}^{F i d}\left(e^{+} e^{-} \rightarrow \rho^{0} \phi\right) \approx 35 \mathrm{fb}
$$

and for a cut at $\cos \theta_{\max }=0.8$,

$$
\sigma_{2}^{F i d}\left(e^{+} e^{-} \rightarrow \rho^{0} \phi\right) \approx 25 \mathrm{fb}
$$

Similarly, for $\cos \theta_{\max }=0.8$ we can calculate

$$
\begin{array}{r}
\sigma_{2}^{F i d}\left(e^{+} e^{-} \rightarrow \rho^{0} \rho^{0}\right) \approx 90 \mathrm{fb} \\
\sigma_{2}^{F i d}\left(e^{+} e^{-} \rightarrow \phi \phi\right) \approx 2 \mathrm{fb} \\
\sigma_{2}^{F i d}\left(e^{+} e^{-} \rightarrow \psi \psi\right) \approx 3 \mathrm{fb}
\end{array}
$$




\section{Appendix D}

\section{Factorization of the Radiative Factor in the Total Cross-section}

It was shown in section 2.3.1 that in the soft/collinear photon approximation the amplitude for the ISR process of Fig. 2.16 factorizes into the amplitude for the corresponding non-radiative process times a factor accounting for photon emission. This implied a factorization of the total cross-section, as expressed in Eq. 2.47. This factorization property is important for the extraction of the final results of this analysis, and it is interesting to know how this property is affected by hard photon emission and higher-order corrections.

In fact it can be shown that the factorization is applicable in much better approximations than the leading-order soft-photon case. In Fig. 2.16, let $\mathcal{L}^{\mu}$ be the amplitude for the part to the left of the virtual photon, and $\mathcal{H}^{\mu}$ the part to the right. Let $\mathcal{H}^{\mu \nu}=\mathcal{H}^{\mu} \mathcal{H}^{\dagger \nu}$ and $\mathcal{L}^{\mu \nu}=\left\langle\mathcal{L}^{\mu} \mathcal{L}^{\dagger \nu}\right\rangle$, where \langle\rangle denotes the average over the spins of the initial leptons and the sum over photon polarizations.

Then the cross-section can be written as

$\sigma_{e^{+} e^{-} \rightarrow \gamma X}=\frac{e^{4}}{2 s} \int d \Phi_{n} d \Phi_{\gamma} \frac{1}{q^{4}} \mathcal{L}^{\mu \nu}\left(p_{1}, p_{2}, l\right)(2 \pi)^{4} \delta^{(4)}\left(p_{1}+p_{2}-k-\sum k_{i}\right) \mathcal{H}_{\mu \nu}\left(k_{1} \cdots k_{n}\right)$

where $k$ is the photon momentum, $k_{i}, i=1 \cdots n$ are the momenta of the particles 
in $X$, and $d \Phi_{n}$ and $d \Phi_{\gamma}$ are the phase space elements for $X$ and the photon. Using a similar technique to what is used in Appendix $C$, we write

$$
\sigma_{e^{+} e^{-} \rightarrow \gamma X}=\frac{e^{4}}{2 s} \int d \Phi_{\gamma} \frac{1}{q^{4}} \mathcal{L}^{\mu \nu}\left(p_{1}, p_{2}, l\right) \mathcal{K}_{\mu \nu}\left(p_{1}+p_{2}-q\right)
$$

where $\mathcal{K}$ is defined by Eq. C.8. Using the current conservation properties of Eq. C.9 and $d s^{\prime}=2 \sqrt{s} d E_{\gamma}$ we have

$$
\frac{d \sigma_{e^{+} e^{-} \rightarrow \gamma X}}{d s^{\prime}}\left(s, s^{\prime}\right)=\frac{8 \pi^{2} \alpha^{2}}{3 s^{\prime 2}\left(s-s^{\prime}\right)}\left[\int d \Omega_{\gamma} \mathcal{L}\left(p_{1}, p_{2}, l\right)\right] \mathcal{K}\left(s^{\prime}\right)
$$

For hadronic production, $\mathcal{K}$ is given by Eq. C.18, so that 


\section{Appendix E}

\section{Tracking and Particle Identification Requirements}

\section{E.1 Track requirements}

In most of the analysis the track requirements are the ones described in Section 5.3. However in control samples the standard BABAR GoodTracksLoose requirements are applied, corresponding tracks that:

- Contain at least $12 \mathrm{DCH}$ hits.

- Originate from the interaction region point within $10 \mathrm{~cm}$ along the beam direction and $1.5 \mathrm{~cm}$ in the transverse plane.

- Have minimum transverse momentum: $p_{t}>0.1 \mathrm{GeV} / \mathrm{c}$.

- Have non-zero $B$-vertex fit $\chi^{2}$ probability. 


\section{E.2 Particle Identification (PID)}

\section{E.2.1 Hadron Identification}

The charged particles are identified using corresponding likelihood ratios formed from $d E / d x$ information from the DCH and SVT for $p_{t}<0.7 \mathrm{GeV} / \mathrm{c}$, the measured Cherenkov angle, and the number of photons observed in the DIRC for $p_{t}>$ $0.6 \mathrm{GeV} / \mathrm{c}$.

The likelihood selector calculates a product of likelihoods for each particle hypothesis: $\mathcal{L}_{\text {ipart }}=\mathcal{L}_{\text {ipart }}^{\text {DIRC }} \times \mathcal{L}_{\text {ipart }}^{D C H} \times \mathcal{L}_{\text {ipart }}^{S V T}$. The DCH and SVT Likelihoods are already calculated by comparing the measured $d E / d x$ against the expected $d E / d x$ from the Bethe-Bloch parametrization for each particle hypothesis:

$$
\mathcal{L}^{D C H, S V T}=\frac{d E / d x(\text { measured })-d E / d x(\text { Bethe }- \text { Bloch })}{\sigma},
$$

where $\sigma$ is an error on the measured $d E / d x$ value. The $\mathrm{DCH}$ likelihood is calculated based on a Gaussian Probability Distribution Function (PDF), and the SVT likelihood is calculated based on a Bifurcated Gaussian PDF.

The DIRC likelihood cannot be "calculated" in this way, since there are significant tails in the distributions of the fit Cherenkov angle and the number of photons. To minimize the effect of these tails, a binned likelihood is constructed from the Cherenkov angle (DIRC angle), number of photons $\left(N_{\gamma}\right)$, and track quality (Trkqual). This new likelihood is stored in a lookup table binned in

$$
\begin{aligned}
& \text { ("Momentum in the laboratory frame" }-100 \mathrm{MeV} / \mathrm{c} \text { bins }) \\
& \qquad \times(\text { "DIRC angle" }-3 \text { bins }) \\
& \times\left(\text { ("N }{ }_{\gamma}, \text { Trkqual" }-4 \text { bins }\right) .
\end{aligned}
$$

Only three "DIRC angle" bins, corresponding to the pion, kaon and proton bands are used. The four " $N_{\gamma}$, Trkqual" bins are formed using the Poisson probability for the number of photons observed, the layer of the last DCH hits, and the calorimeter energy deposit. This " $N_{\gamma}$, Trkqual" binning is an attempt to identify problem tracks 
for the DIRC reconstruction. The lookup tables are filled using MC truth-matched tracks ${ }^{1}$. The value of likelihood is the probability that a MC truth-matched track has the measured quantities of that bin. This binned DIRC likelihood is designed to treat the tails in the DIRC response, especially near particle thresholds. It does not separate the DIRC angle bands well at higher momentum, so this binned likelihood is multiplied by a Gaussian DIRC angle likelihood for momentum $>1.5 \mathrm{GeV} / c$.

Finally, the charged particle is assigned to a particular category (electron, muon, pion, kaon or proton) by requiring different cuts on the relevant likelihood ratios.

\section{E.2.2 Electron Identification}

Separation of electrons from muons and charged hadrons rests primarily on information obtained from the electron interaction with the electromagnetic calorimeter (EMC). All BABAR electron identification algorithms require a high-quality charged track (one with at least 12 drift-chamber hits) whose extrapolation to the front face of the EMC matches with an EMC cluster. This algorithm matches clusters within a combined distance in polar angle $(\Delta \Theta)$ and azimuthal separation $(\Delta \Phi)$, between the centroid of the EMC cluster and the impact point of the charged track on the EMC.

One of the primary discriminating variables for electron identification is $E / p$, the ratio of the energy of the EMC cluster to the measured momentum of the matched charged track. Electrons, which should deposit most or all of their energy in the electromagnetic calorimeter, typically have an $E / p$ close to 1, while muons, which, interact with the EMC as minimum ionizing particles, have a small $E / p$, as do noninteracting charged hadrons.

Some fraction of charged hadrons, interacting hadronically with the EMC do deposit a substantially larger fraction of their energy, and may not easily be distinguished from electrons via $E / p$ alone. Both the longitudinal and lateral development of an electromagnetic shower differ from that of a hadronic shower; while the $B A B A R$

\footnotetext{
${ }^{1}$ Truth-matching is an algorithm used to associate a reconstructed track in the Monte Carlo simulation to its true origin.
} 
EMC has no sensitivity to the longitudinal shower development, variables describing the lateral shower shape offer additional discriminating power. In this case, the shower shape variables used include: the number of calorimeter crystals in the reconstructed shower, the lateral moment of the shower (LAT), and a Zernike moment $\left(Z_{42}\right)$, which is sensitive to the azimuthal variation in lateral shower shape. Zernike moments are defined as:

$$
A_{n m}=\sum_{r_{i} \leq R_{0}}^{n} \frac{E_{i}}{E} \cdot f_{n m}\left(\frac{r_{i}}{R_{0}}\right) \cdot e^{-i m \phi_{i}}, \quad R_{0}=15 \mathrm{~cm}
$$

where

$$
f_{n m}\left(\rho_{i} \equiv \frac{r_{i}}{R_{0}}\right)=\sum_{s=0}^{(n-m) / 2} \frac{(-1)^{s}(n-s) ! \rho_{i}^{n-2 s}}{s !((n+m) / 2-s) !((n-m) / 2-s) !} .
$$

At low energies, electron-hadron separation is also enhanced by taking into account both energy loss $(\mathrm{d} E / \mathrm{d} x)$ in the drift chamber and the response of the DIRC using both Cerenkov angle $\theta_{C}$ and the number of photons $N_{C}$.

Two criteria are implemented, a standard criteria (also referred to as "tight") and a loose criteria with higher selection efficiency and cross-feed.

\section{E.2.3 Muon Identification}

Charged tracks identified as muons are required to have a minimum momentum in the laboratory $p_{l a b}>1.0 \mathrm{GeV}$; if the charged track has a matched cluster in the EMC the energy deposited in the EMC is required to be consistent with a minimum ionizing particle, $50 \mathrm{MeV}<E_{c a l}<400 \mathrm{MeV}$. Muon identification, however, is primarily based on quantities characterizing the interaction of the muon with the IFR :

- the number of IFR layers associated with the track has to be $N_{L} \geq 2$.

- the interaction lengths of material traversed by the track has to be $\lambda_{\text {meas }}>2.2$.

- The number of interaction lengths expected for a muon of the measured momentum and angle to traverse is estimated by extrapolating the track up to the last active layer of the IFR. This estimate takes into account the RPC efficiencies 
which are routinely measured and stored. For the difference $\Delta \lambda=\lambda_{\text {exp }}-\lambda_{\text {meas }}$ we require $\Delta \lambda<1.0$, for tracks with momentum greater than $1.2 \mathrm{GeV} / \mathrm{c}$. For track momenta between $0.5 \mathrm{GeV} / c$ and $1.2 \mathrm{GeV} / c$, a variable limit is placed: $\Delta \lambda<\left[\left(p_{l a b}-0.5\right) / 0.7\right]$.

- The continuity of the IFR cluster is defined as $T_{c}=N_{L} /(L-F+1)$, where $L$ and $F$ are the last and first layers with a hit. $T_{c}$ is expected to be 1.0 for muons penetrating an ideal detector whereas it is expected smaller for hadrons. We require $T_{c}>0.3$ for tracks with $0.3<\theta_{l a b}<1.0$ (i.e. in the Forward End Cap to remove beam background).

- The observed number of hit strips in each RPC layer is used to impose the conditions on the average number of hits, $\bar{m}<8$, and the standard deviation, $\sigma_{m}<4$.

- The strip clusters in the IFR layers are combined to form a track and fit to a third degree polynomial, with the quality of the fit selected by the condition $\chi_{\text {fit }}^{2} / D O F<3$. In addition, the cluster centroids are compared to the extrapolated charged track, with the requirement $\chi_{t r k}^{2} / D O F<5$. 


\section{Bibliography}

[1] The ALEPH, DELPHI, L3, OPAL, SLD Collaborations, LEP Electroweak Working Group and SLD Electroweak and Heavy Flavour Groups, hep-ex/0509008, to be published in Physics Reports. M. W. Grünewald, Phys. Rept. 403-404, 189-201 (2004) [hep-ph/0404165]

M. W. Grünewald, Phys. Rept. 322, 125-346 (1999)

http://www.cern.ch/LEPEWWG/

[2] H. Burkhardt and B. Pietrzyk, Phys. Rev. D 72, 057501 (2005)

[3] R. R. Akhmetshin et al., Phys. Lett. B 527, 161-172 (2002)

R. R. Akhmetshin et al., Phys. Lett. B 578, 285-289 (2004)

[4] J. Z. Bai et al., Phys. Rev. Lett. 84, 594 (2000)

J. Z. Bai et al., Phys. Rev. Lett. 88, 101802 (2002)

[5] Peter C. Kim, Charmless Decays of B mesons, SLAC seminar (Sep. 1995).

[6] A. B. Arbuzov, E. A. Kuraev, N. P. Merenkov, L. Trentadue JHEP 9812, 009 (1998)

[7] S. Binner, J. H. Kühn and K. Melnikov, Phys. Lett. B 459, 279 (1999) [hep$\mathrm{ph} / 9902399]$.

[8] B. Aubert et al., Phys. Rev. D 70, 072004 (2004)

[9] B. Aubert et al., Phys. Rev. D 71, 052001 (2005)

[10] B. Aubert et al., Phys. Rev. D 73, 052003 (2006) 
[11] KLOE Collaboration (A. Aloisio et al.), Phys. Lett. B 606, 12-24 (2005) S.E. Muller et al., Nucl. Phys. Proc. Suppl. 126, 335-340 (2004)

[12] Q. R. Ahmad et al. [SNO Collaboration], Phys. Rev. Lett. 87, 071301 (2001) [nucl-ex/0106015].

[13] Y. Fukuda et al. [Super-Kamiokande Collaboration], Phys. Rev. Lett. 81, 1562 (1998) [hep-ex/9807003].

[14] P. C. Rowson, D. Su and S. Willocq, Ann. Rev. Nucl. Part. Sci. 51, 345 (2001) [arXiv:hep-ph/0110168].

[15] Review of Particle Physics, S. Eidelman et al., Phys. Lett. B 592, 1 (2004)

[16] P. Mohr and B. N. Taylor, Rev. Mod. Phys. 77, 1 (2005).

[17] R. S. Van Dyck et al., Phys. Rev. Lett. 59, 26 (1987)

[18] A. Peters et al., Philos. Trans. R. Soc. London, Ser. A 355, (1997)

A. Wicht et al., Phys. Scr. T102, 82 (2002).

[19] P. Cladé et al., Phys. Rev. Lett. 96, 033001 (2006)

[20] ALEPH Collaboration, D. Decamp et al., Z. Phys. C 48 (1990) 365-392

ALEPH Collaboration, D. Decamp et al., Z. Phys. C 53 (1992) 1-20

ALEPH Collaboration, D. Buskulic et al., Z. Phys. C 60 (1993) 71-82

ALEPH Collaboration, D. Buskulic et al., Z. Phys. C 62 (1994) 539-550

ALEPH Collaboration, R. Barate et al., Eur. Phys. J. C 14 (2000) 1-50

DELPHI Collaboration, P. Abreu et al., Nucl. Phys. B 367 (1991) 511-574

DELPHI Collaboration, P. Abreu et al., Nucl. Phys. B 417 (1994) 3-57

DELPHI Collaboration, P. Abreu et al., Nucl. Phys. B 418 (1994) 403-427

DELPHI Collaboration, P. Abreu et al., Eur. Phys. J. C 16 (2000) 371-405

L3 Collaboration, B. Adeva et al., Z. Phys. C 51 (1991) 179-204

L3 Collaboration, O. Adriani et al., Phys. Rept. 236 (1993) 1-146

L3 Collaboration, M. Acciarri et al., Z. Phys. C 62 (1994) 551-576 
L3 Collaboration, M. Acciarri et al., Eur. Phys. J. C 16 (2000) 1-40 OPAL Collaboration, G. Alexander et al., Z. Phys. C 52 (1991) 175-208 OPAL Collaboration, P. D. Acton et al., Z. Phys. C 58 (1993) 219-238 OPAL Collaboration, R. Akers et al., Z. Phys. C 61 (1994) 19-34 OPAL Collaboration, G. Abbiendi et al., Eur. Phys. J. C 19 (2001) 587-651

[21] ALEPH Collaboration, A. Heister et al., Eur. Phys. J. C 20 (2001) 401-430 ALEPH Collaboration, D. Buskulic et al., Z. Phys. C 69 (1996) 183-194 ALEPH Collaboration, D. Buskulic et al., Z. Phys. C 59 (1993) 369-386 ALEPH Collaboration, D. Decamp et al., Phys. Lett. B 265 (1991) 430-444 DELPHI Collaboration, P. Abreu et al., Eur. Phys. J. C 14 (2000) 585-611 DELPHI Collaboration, P. Abreu et al., Z. Phys. C 67 (1995) 183-202 L3 Collaboration, M. Acciarri et al., Phys. Lett. B 429 (1998) 387-398 L3 Collaboration, M. Acciarri et al., Phys. Lett. B 341 (1994) 245-256 L3 Collaboration, O. Adriani et al., Phys. Lett. B 294 (1992) 466-478 OPAL Collaboration, G. Abbiendi et al., Eur. Phys. J. C 21 (2001) 1-21 OPAL Collaboration, G. Alexander et al., Z. Phys. C 72 (1996) 365-375 OPAL Collaboration, R. Akers et al., Z. Phys. C 65 (1995) 1-16 OPAL Collaboration, G. Alexander et al., Phys. Lett. B 266 (1991) 201-217

[22] SLD Collaboration, K. Abe et al., Phys. Rev. Lett. 70 (1993) 2515-2520 SLD Collaboration, K. Abe et al., Phys. Rev. Lett. 73 (1994) 25-29 SLD Collaboration, K. Abe et al., Phys. Rev. Lett. 78 (1997) 2075-2079 SLD Collaboration, K. Abe et al., Phys. Rev. Lett. 84 (2000) 5945-5949

[23] OPAL Collaboration, G. Abbiendi et al., Eur. Phys. J. C 8 (1999) 217-239 DELPHI Collaboration, P. Abreu et al., Eur. Phys. J. C 10 (1999) 415-442 DELPHI Collaboration, J. Abdallah et al., Eur. Phys. J. C 32 (2004) 185-208 ALEPH Collaboration, R. Barate et al., Phys. Lett. B 401 (1997) 150-162 OPAL Collaboration, K. Ackerstaff et al., Eur. Phys. J. C 1 (1998) 439-459 ALEPH Collaboration, R. Barate et al., Eur. Phys. J. C 16 (2000) 597-611 
DELPHI Collaboration, P. Abreu et al., Eur. Phys. J. C 12 (2000) 209-224 SLD Collaboration, K. Abe et al., Phys. Rev. D 71 (2005) 112004 ALEPH Collaboration, R. Barate et al., Phys. Lett. B 401 (1997) 163-175 ALEPH Collaboration, R. Barate et al., Eur. Phys. J. C 4 (1998) 557-570 DELPHI Collaboration, P. Abreu et al., Eur. Phys. J. C 12 (2000) 225-241

[24] ALEPH Collaboration, A. Heister et al., Eur. Phys. J. C 24 (2002) 177-191 ALEPH Collaboration, A. Heister et al., Eur. Phys. J. C 22 (2001) 201-215 DELPHI Collaboration, P. Abreu et al., Z. Phys. C 65 (1995) 569-586 DELPHI Collaboration, J. Abdallah et al., Eur. Phys. J. C 34 (2004) 109-125 L3 Collaboration, O. Adriani et al., Phys. Lett. B 292 (1992) 454-462 L3 Collaboration, M. Acciarri et al., Phys. Lett. B 448 (1999) 152-162 OPAL Collaboration, G. Abbiendi et al., Phys. Lett. B 577 (2003) 18-36 L3 Collaboration, M. Acciarri et al., Phys. Lett. B 439 (1998) 225-236 OPAL Collaboration, G. Abbiendi et al., Phys. Lett. B 546 (2002) 29-47 DELPHI Collaboration, J. Abdallah et al., Eur. Phys. J. C 40 (2005) 1-25 ALEPH Collaboration, R. Barate et al., Phys. Lett. B 434 (1998) 415-425 DELPHI Collaboration, P. Abreu et al., Eur. Phys. J. C 10 (1999) 219-237 OPAL Collaboration, G. Alexander et al., Z. Phys. C 73 (1997) 379-395

[25] SLD Collaboration, K. Abe et al., Phys. Rev. Lett. 83 (1999) 3384-3389 SLD Collaboration, K. Abe et al., Phys. Rev. D 63 (2001) 032005 SLD Collaboration, K. Abe et al., Phys. Rev. Lett. 81 (1998) 942-946 SLD Collaboration, K. Abe et al., Phys. Rev. Lett. 90 (2003) 141804 SLD Collaboration, K. Abe et al., Phys. Rev. Lett. 94 (2005) 091801

[26] UA1 Collaboration, C. Albajar et al., Z. Phys. C 44 (1989) 15 UA2 Collaboration, J. Alitti et al., Phys. Lett. B 276 (1992) 354-364 CDF Collaboration, T. Affolder et al., Phys. Rev. D 64 (2001) 52001-39 CDF Collaboration, F. Abe et al., Phys. Rev. Lett. 75 (1995) 11-16 
CDF Collaboration, F. Abe et al., Phys. Rev. D 52 (1995) 4784-4827

CDF Collaboration, F. Abe et al., Phys. Rev. Lett. 65 (1990) 2243-2246

CDF Collaboration, F. Abe et al., Phys. Rev. D 43 (1991) 2070-2093

D $\varnothing$ Collaboration, B. Abbott et al., Phys. Rev. Lett. 80 (1998) 3008

D $\varnothing$ Collaboration, B. Abbott et al., Phys. Rev. Lett. 84 (2000) 222-227

D $\varnothing$ Collaboration, V. M. Abazov et al., Phys. Rev. D 66 (2002) 012001

D $\varnothing$ Collaboration, B. Abbott et al., Phys. Rev. D 62 (2000) 092006

CDF Collaboration, T. Affolder et al., Phys. Rev. Lett. 85 (2000) 3347-3352

DØ Collaboration, V. M. Abazov et al., Phys. Rev. D 66 (2002) 032008

The CDF Collaboration, the D $\varnothing$ Collaboration, and the Tevatron Electroweak Working Group, Phys. Rev. D 70 (2004) 092008

ALEPH Collaboration, R. Barate et al., Eur. Phys. J. C 17 (2000) 241-261

DELPHI Collaboration, P. Abreu et al., Phys. Lett. B 511 (2001) 159-177

L3 Collaboration, M. Acciarri et al., Phys. Lett. B 454 (1999) 386-398

OPAL Collaboration, G. Abbiendi et al., Phys. Lett. B 507 (2001) 29-46

The LEP Collaborations ALEPH, DELPHI, L3, OPAL, the LEP Electroweak Working Group, and the SLD Electroweak and Heavy Flavour Groups, A combination of preliminary electroweak measurements and constraints on the standard model, Eprint hep-ex/0412015, CERN, 2004

[27] CDF Collaboration, F. Abe et al., Phys. Rev. Lett. 74 (1995) 2626-2631

DØ Collaboration, S. Abachi et al., Phys. Rev. Lett. 74 (1995) 2632-2637

CDF Collaboration, F. Abe et al., Phys. Rev. Lett. 80 (1998) 2779-2784

CDF Collaboration, F. Abe et al., Phys. Rev. Lett. 82 (1999) 271-276

CDF Collaboration, F. Abe et al., Erratum: Phys. Rev. Lett. 82 (1999) 2808-2809

CDF Collaboration, F. Abe et al., Phys. Rev. Lett. 80 (1998) 2767-2772

CDF Collaboration, T. Affolder et al., Phys. Rev. D 63 (2001) 032003

CDF Collaboration, F. Abe et al., Phys. Rev. Lett. 79 (1997) 1992-1997

D $\varnothing$ Collaboration, B. Abbott et al., Phys. Rev. Lett. 80 (1998) 2063-2068

D $\varnothing$ Collaboration, B. Abbott et al., Phys. Rev. D 60 (1999) 052001 
DØ Collaboration, S. Abachi et al., Phys. Rev. Lett. 79 (1997) 1197-1202

D $\varnothing$ Collaboration, B. Abbott et al., Phys. Rev. D 58 (1998) 052001

Dø Collaboration, V. M. Abazov et al., Nature 429 (2004) 638-642

$\mathrm{D} \varnothing$ Collaboration, V. M. Abazov et al., New measurement of the top quark mass in lepton + jets $t$ anti-t events at $D \varnothing$, Eprint hep-ex/0407005, 2004

DØ Collaboration, V. M. Abazov et al., Phys. Lett. B 606 (2005) 25-33

The CDF and D $\varnothing$ Collaborations, and the Tevatron Electroweak Working Group, Combination of $C D F$ and $D \varnothing$ results on the top-quark mass, Eprint hep-ex/0404010, 2004

[28] A. Abulencia et al. [CDF Collaboration], Phys. Rev. Lett. 96, 022004 (2006) [hep-ex/0510049].

V. M. Abazov et al. [D0 Collaboration], Nature 429, 638 (2004) [hepex/0406031].

[29] L3 Collaboration, M. Acciarri et al., Phys. Lett. B 439 (1998) 225-236 ALEPH Collaboration, D. Buskulic et al., Z. Phys. C 71 (1996) 357-378 DELPHI Collaboration, P. Abreu et al., Phys. Lett. B 277 (1992) 371-382 OPAL Collaboration, P. D. Acton et al., Phys. Lett. B 294 (1992) 436-450

[30] Martin Grünewald, Presentation at the 2005 International Europhysics Conference on High Energy Physics, Published in Proceedings of Science.

[31] J. D. Bjorken and E. A. Paschos, Phys. Rev. 185, 1975 (1969).

R. P. Feynman, High Energy Collisions, Proceedings of the Third International Conference, Stony Brook, New York, Pages 237-249 (Gordon and Breach, New York, 1969).

R. P. Feynman, Phys. Rev. Lett. 23, 1415 (1969).

[32] M. Steinhauser, Phys. Lett. B 429, 158 (1998) [hep-ph/9803313]

[33] J. H. Kühn and M. Steinhauser, Phys. Lett. B 437, 425 (1998) [hep-ph/9802241]. 
[34] R. Jost and H. Lehmann, Nuovo Cimento 5, 1598-1608 (1957)

[35] V. A. Novikov, L. B. Okun, M. A. Shifman, A. I. Vainshtein, M. B. Voloshin and V. I. Zakharov, Phys. Repts 41, 1 (1978)

[36] S. G. Gorishny, A Kataev and S. A. Larin, Phys. Lett. B 259, 144 (1991) L. R. Surguladze and M .A Samuel, Phys. Rev. Lett. 66, 560 (1991)

[37] K. G. Chetyrkin and J. H. Kühn, Phys. Lett. B 308, 127 (1993)

[38] K. G. Chetyrkin, B. A. Kniehl, and M. Steinhauser, Phys. Rev. Lett. 79, 2184 (1997)

[39] G. Rodrigo and A. Santamaria, Phys. Lett. B 424, 367-374 (1998) [hepex/9305305]

[40] S. J. Brodsky, C. E. Carlson, R. Suaya, Phys. Rev. D 14, 2264 (1976)

[41] E. Barberio, B. van Eijk, and Z. Was, Comput. Phys. Commun. 66, 115 (1991)

[42] M. Davier A. Höcker, Phys. Lett. B 435, 427 (1998).

[43] M. Davier, S. Eidelman, A. Höcker and Z. Zhang, Eur. Phys. J. C 31, 503 (2003) [arXiv:hep-ph/0308213].

[44] B. Aubert et al. [BABAR Collaboration], Nucl. Instrum. Meth. A 479, 1 (2002) [hep-ex/0105044].

[45] P. Oddone, in Proceedings of the UCLA Workshop: Linear Collider $B \bar{B}$ Factory Conceptual Design, p.243 (World Scientific, 1987)

[46] R. Giles et al. [CLEO Collaboration], Phys. Rev. D 29, 1285 (1984).

[47] The BABAR Collaboration, Phys. Rev. D 72, 032005 (2005)

[48] M. Furman, SLAC-ABC-41-REV

[49] H. Wiedemann, Particle Accelerator Physics I, Springer Verlag (1998) 
[50] An Asymmetric B-Factory based on PEP, SLAC-372, LBL PUB-5303

[51] J. Seeman et al., SLAC-PUB-11727

[52] M. Tigner, IEEE NS-24, 1849 (1977)

[53] KEKB B-factory Design Report, KEK Report 95-7 (1995)

[54] TYVEK, registered trademark of E. I. DuPont de Nemours \& Co., Wilmington, DE, USA.

[55] I. Adam et al. [BABAR-DIRC Collaboration], Nucl. Instrum. Meth. A 538, 281 (2005).

[56] The CLEO Collaboration, CLNS-94-1277

[57] X. D. Chai, Int. J. Mod. Phys. A 20, 3799 (2005).

S. J. Bailey et al., IEEE Trans. Nucl. Sci. 51, 2352 (2004).

[58] F. A. Kirsten et al., BABAR LEvel 1 Drift Chamber and Global Trigger Implementation

[59] S. Gehrig, BABAR Note \# 380

[60] K. Kinoshita, Nucl. Instr. Methods A 276, 242 (1976)

[61] A. Eisner, http://www.slac.stanford.edu/BFROOT/www/Detector/Trigger/soft ware/L3TFilters/filters_bhabha.ps

[62] The BABAR Collaboration, B. Aubert et al., Phys. Rev. Lett.95, 142001 (2005).

[63] H. Kolanoski, Two Photon Physics at $e^{+} e^{-}$Storage Rings (Springer-Verlag, 1984)

[64] Kai Yi, private communication

[65] S. Jadach, B. F. L. Ward and Z. Was, Comput. Phys. Commun. 130, 260 (2000) [hep-ph/9912214].

S. Jadach, B. F. L. Ward, Z. Was, Phys. Rev. D 63, 113009 (2001) [hep$\mathrm{ph} / 0006359]$ 
[66] M. Boonekamp, hep-ph/0111213

[67] S. Jadach, Z. Was, R. Decker, J. H. Kühn, Comput.Phys.Commun.76,361-380 (1993)

[68] S. Jadach, W. Placzek, B.F.L. Ward, Phys. Lett. B 390, 298-308 (1997) [hep$\mathrm{ph} / 9608412]$

[69] The GEANT4 Collaboration, (S. Agostinelli et al., Nucl. Instrum. Meth. A 506, 250-303 (2003)

[70] I. Eschrich, Proceedings of the 10th International Conference on Calorimetry in Particle Physics, p. 658, World Scientific (2002). BABAR Note 550

[71] The BABAR Physics Book, SLAC-R-504

[72] R. A. Fisher, Annals of Eugenics 7, 179-188 (1936)

[73] The CLEO Collaboration, Phys. Rev. D 59052003 (1999)

[74] The CLEO Collaboration, hep-ex/0512061

[75] The CLEO Collaboration, hep-ex/0510015

[76] F. E. Close, An Introduction to Quarks and Partons, Academic Press (1979)

[77] J. M. Jauch and F. Rohrlich, The Theory of Photons and Electrons, AddisonWesley (1955)

[78] H. Burkhardt and B. Pietrzyk, Phys. Lett. B 356 (1995) 398-403

[79] J. H. Kuhn and A. Santamaria, Z. Phys. C 48, 445 (1990).

[80] G. Venanzoni, Invited talk at the Seventh International Workshop on Tau Lepton Physics (TAU02-WE07), Santa Cruz, Ca, USA, Sept 2002 [hep-ex/0210013]

[81] H. Czyż, A. Grzelińska, J. H. Kühn, G. Rodrigo, hep-ph/0512180 H. Czyż, A. Grzelińska, J. H. Kühn, G. Rodrigo, Eur. Phys. J. C 39, 411 (2005) 
[hep-ph/0404078]

H. Czyż, J. H. Kühn, E. Nowak, G. Rodrigo, Eur. Phys. J. C 35, 527 (2004) [hep-ph/0403062]

H. Czyż, A. Grzelińska, J. H. Kühn, G. Rodrigo, Eur. Phys. J. C 33, 333 (2004) [hep-ph/0308312]

H. Czyż, A. Grzelińska, J. H. Kühn, G. Rodrigo, Eur. Phys. J.C 27, 563 (2003) [hep-ph/0212225]

J. H. Kühn, G. Rodrigo, Eur. Phys. J. C 25, 215 (2002) [hep-ph/0204283]

G. Rodrigo, H. Czyż, J. H. Kühn, M. Szopa, Eur. Phys. J. C 24, 71 (2002) [hep-ph/0112184]

G. Rodrigo, A. Gehrmann-De Ridder, M. Guilleaume, J. H. Kühn, Eur. Phys. J. C 22, 81 (2001) [hep-ph/0106132]

[82] CDF Collaboration (F. Abe et al.), Phys. Rev. Lett. 73 (1994) 225-231

CDF Collaboration (F. Abe et al.), Phys. Rev. Lett. 74, 2626-2631 (1995) [hepex/9503002]

[83] The CLEO Collaboration, Phys. Rev. Lett. 85, 520-524 (2000)

[84] F. Low, Phys. Rev. 120, 1860 (1960)

[85] V. E. Balakin et al., Phys. Lett. 34B, 99 (1971) C. Bacci et al., Nuovo Cim. Lett. 3, 709 (1972)

[86] B. Aubert et al. Phys. Rev. Lett. 92, 142002 (2004) [hep-ex/0311038].

[87] B. Aubert et al. [BABAR Collaboration], Phys. Rev. D 69, 011103 (2004) [hepex/0310027].

[88] F. Di Lodovico et al., BABAR Analysis Document \# 765

[89] M. L. Swartz, Phys. Rev. D 53, 5268 (1996) [hep-ph/9509248]. 